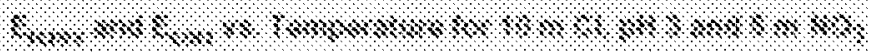

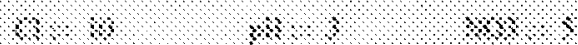

$$
\begin{aligned}
& \text { r } \\
& \text { מ } \\
& \text { ? }
\end{aligned}
$$

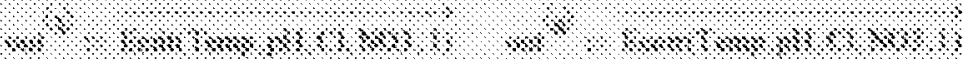

$$
\begin{aligned}
& \text { - } \\
& \text { - }
\end{aligned}
$$

$3,1,1,1,3$,

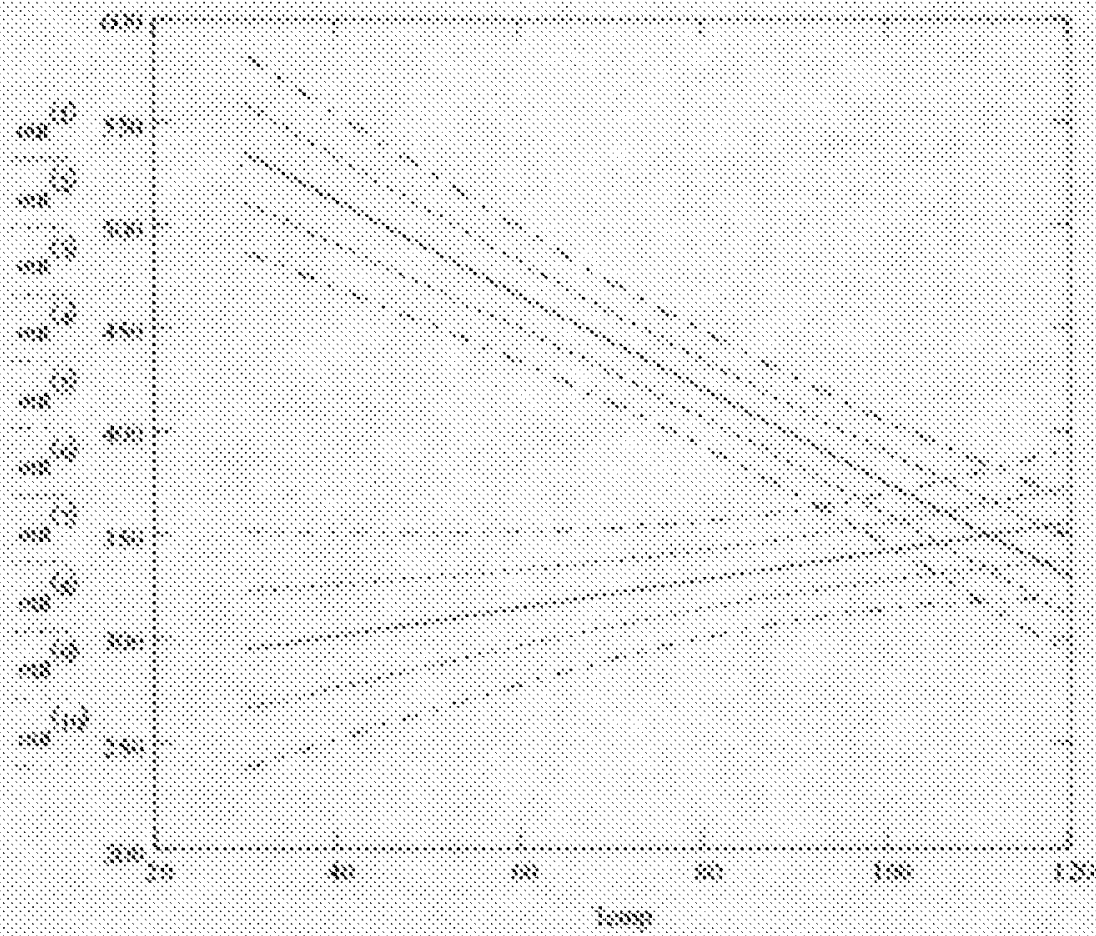




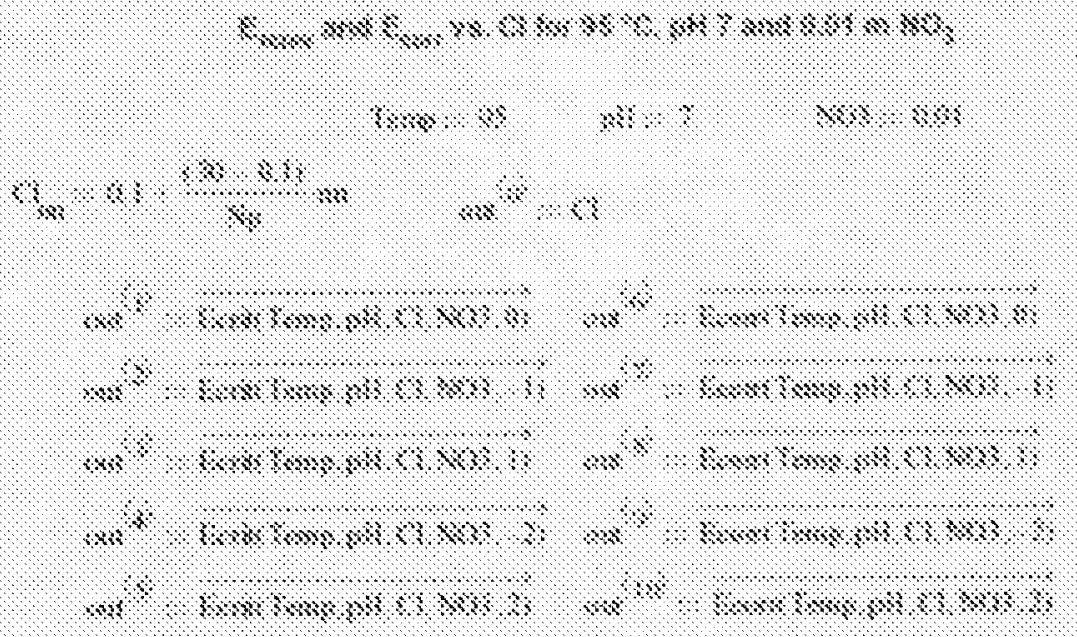

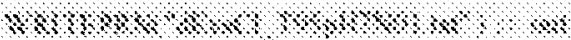

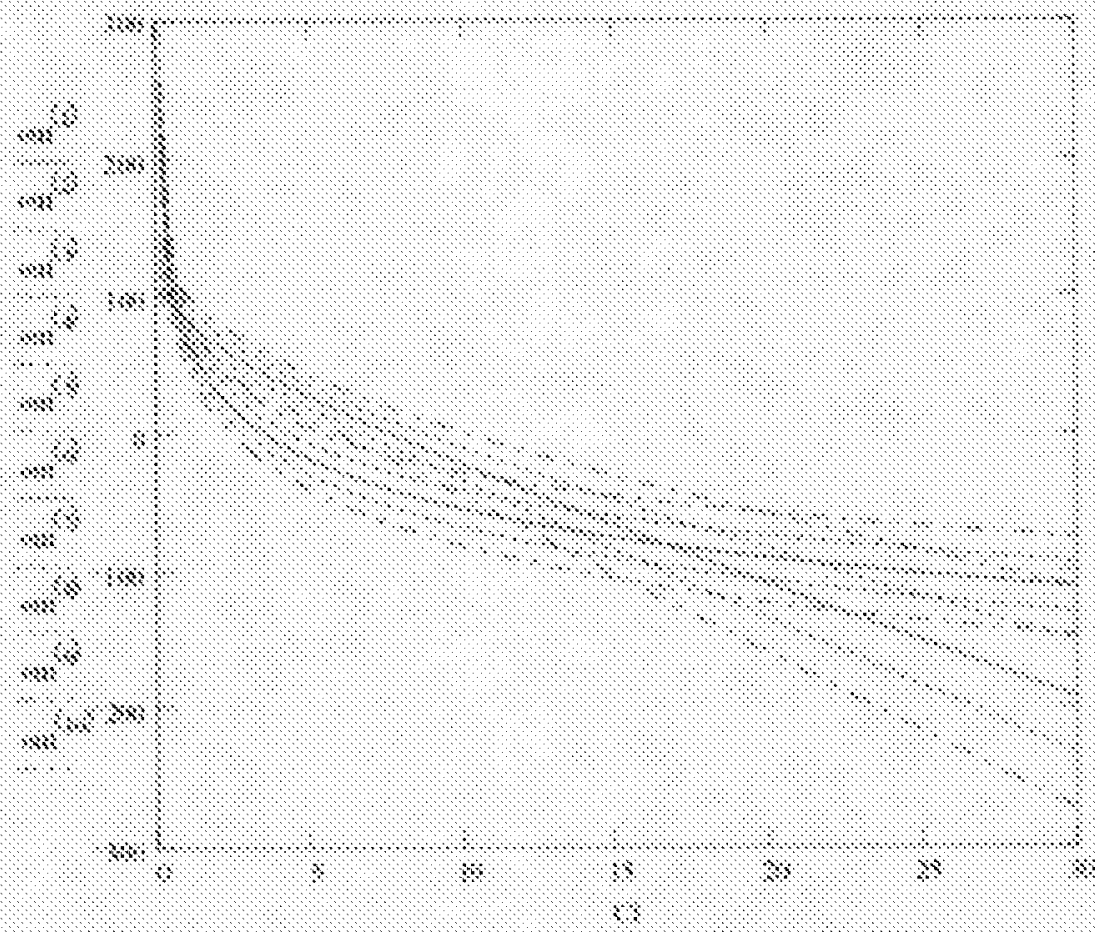




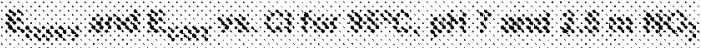

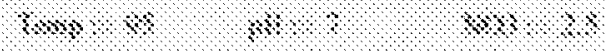

,

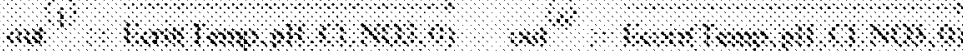

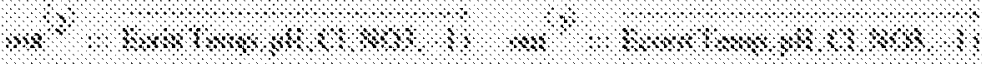

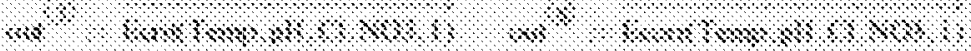

n 1 l

s

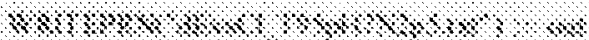

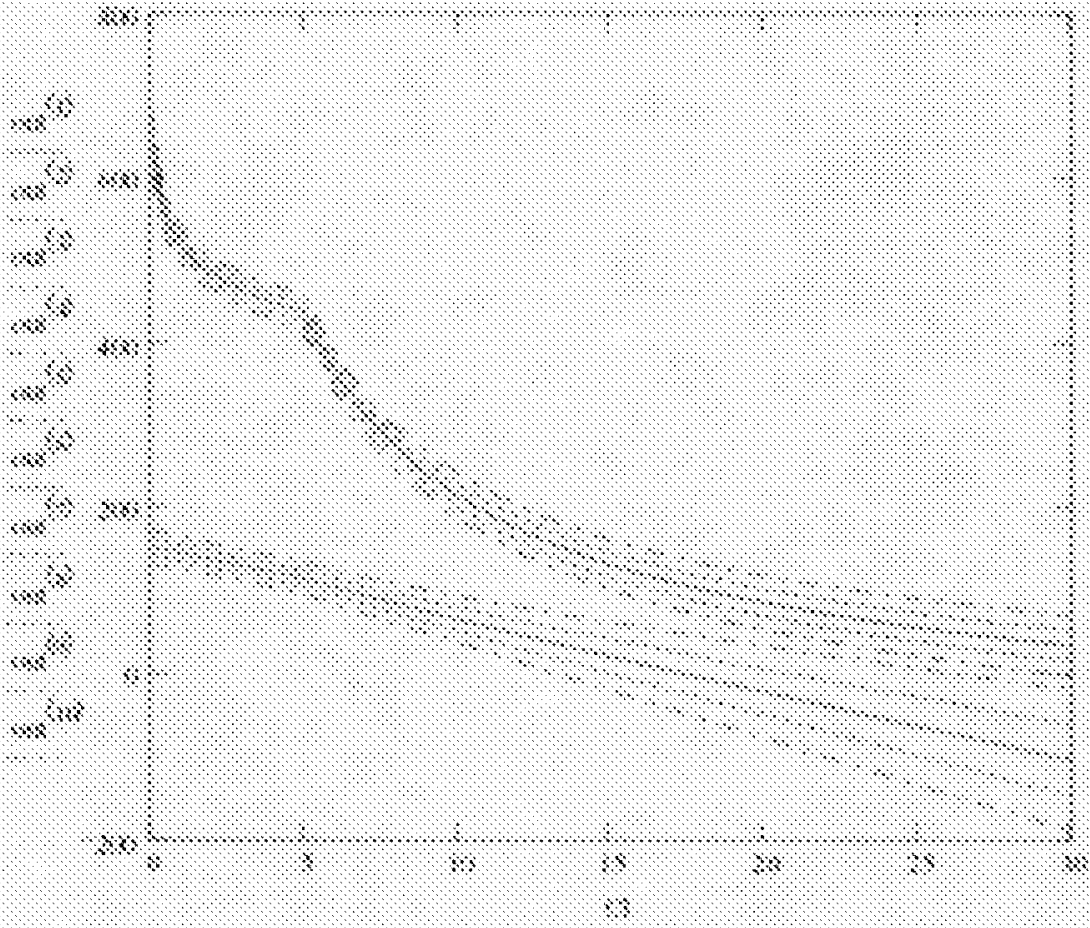




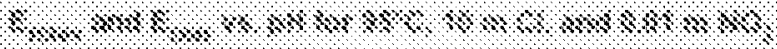

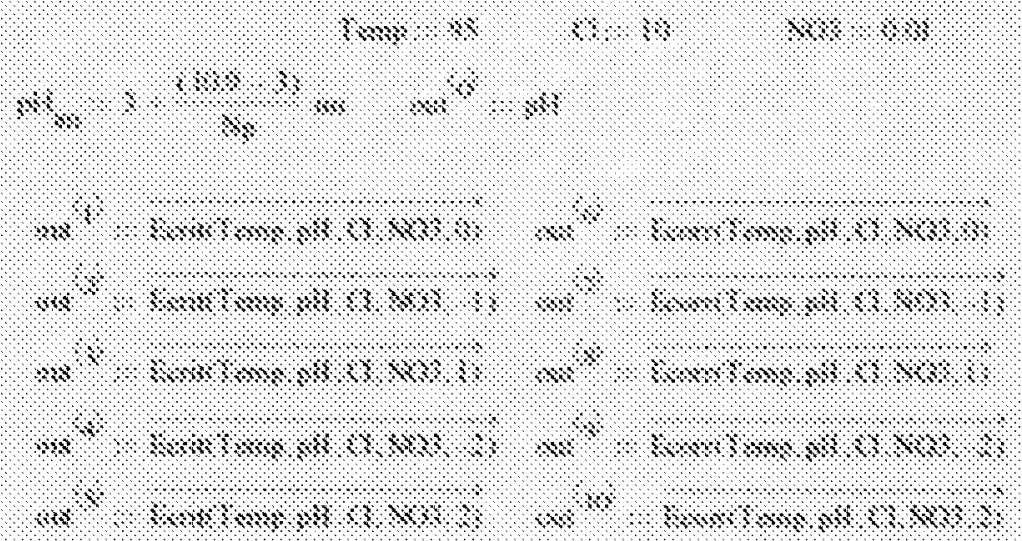

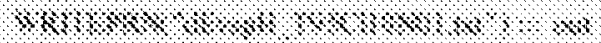

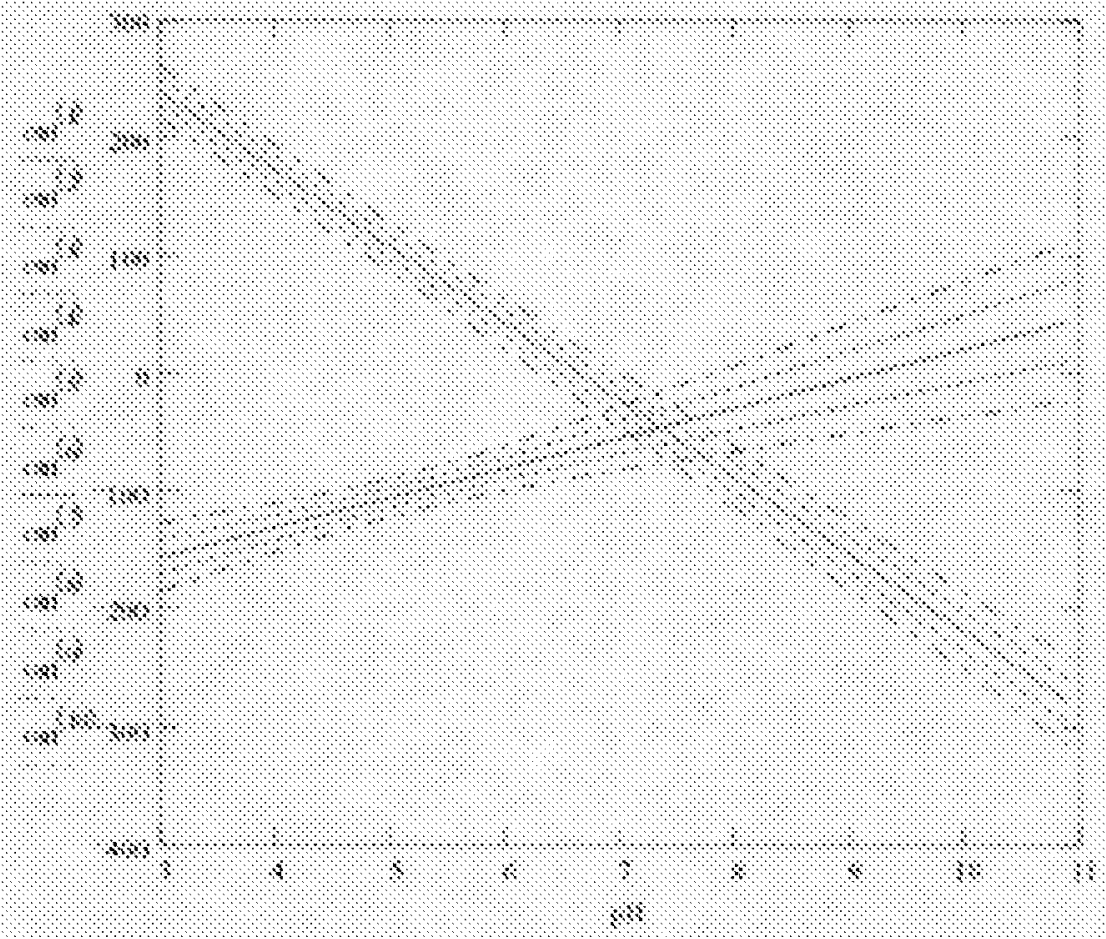




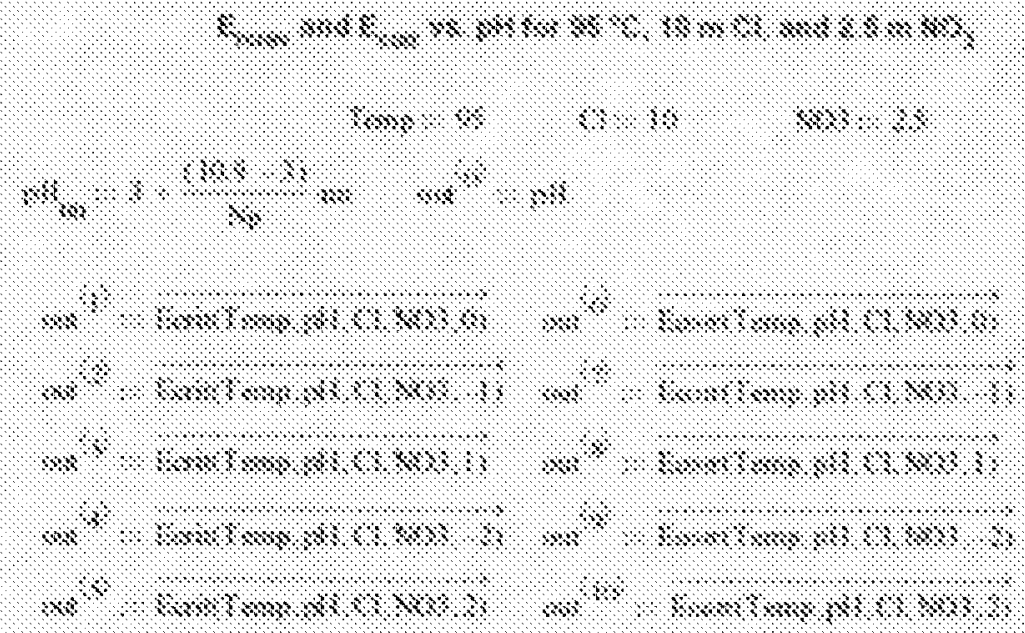

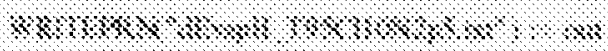

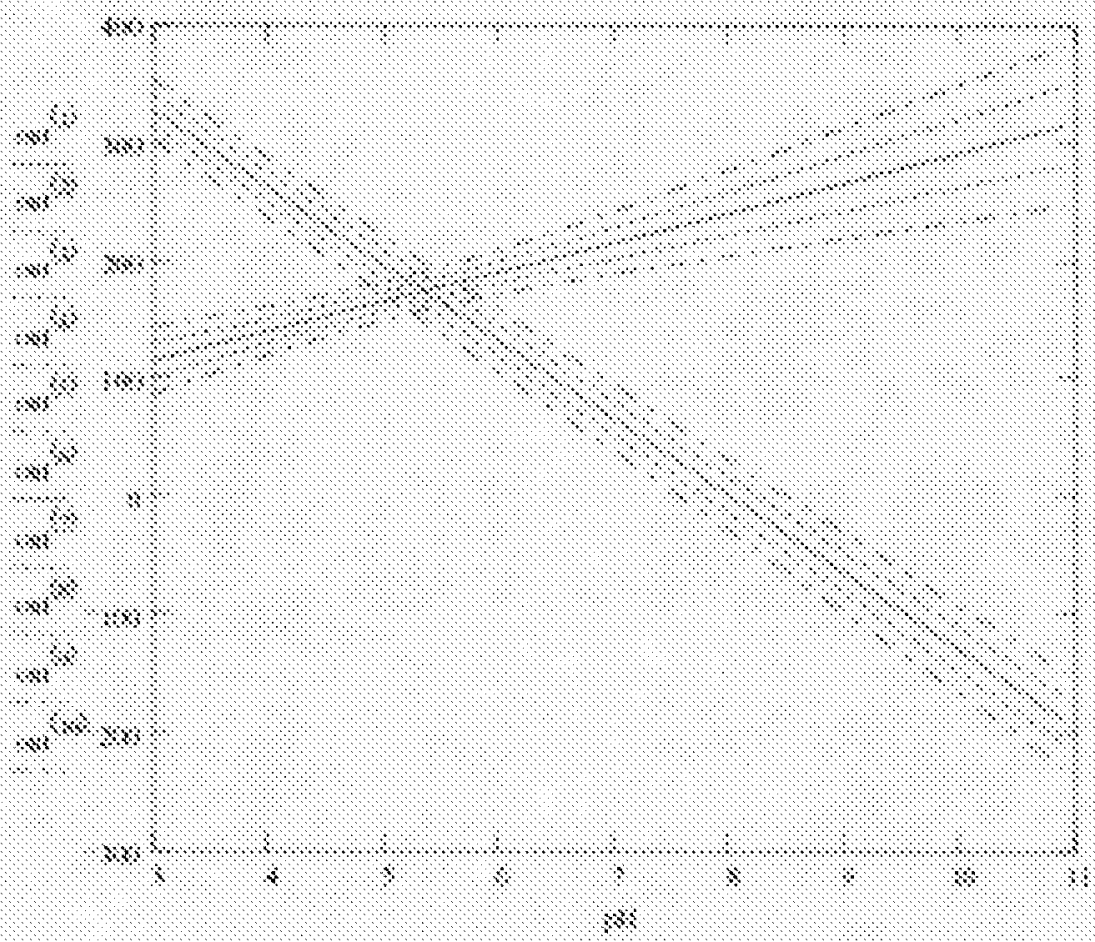




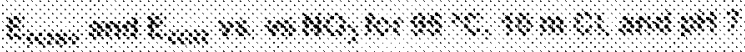

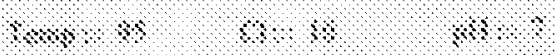

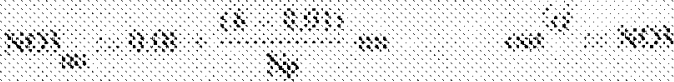

מ

, 3 .

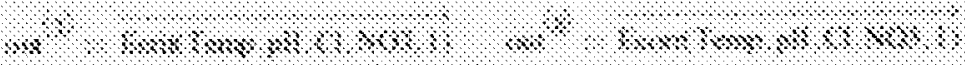

l

l

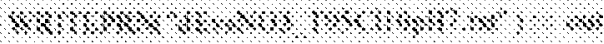

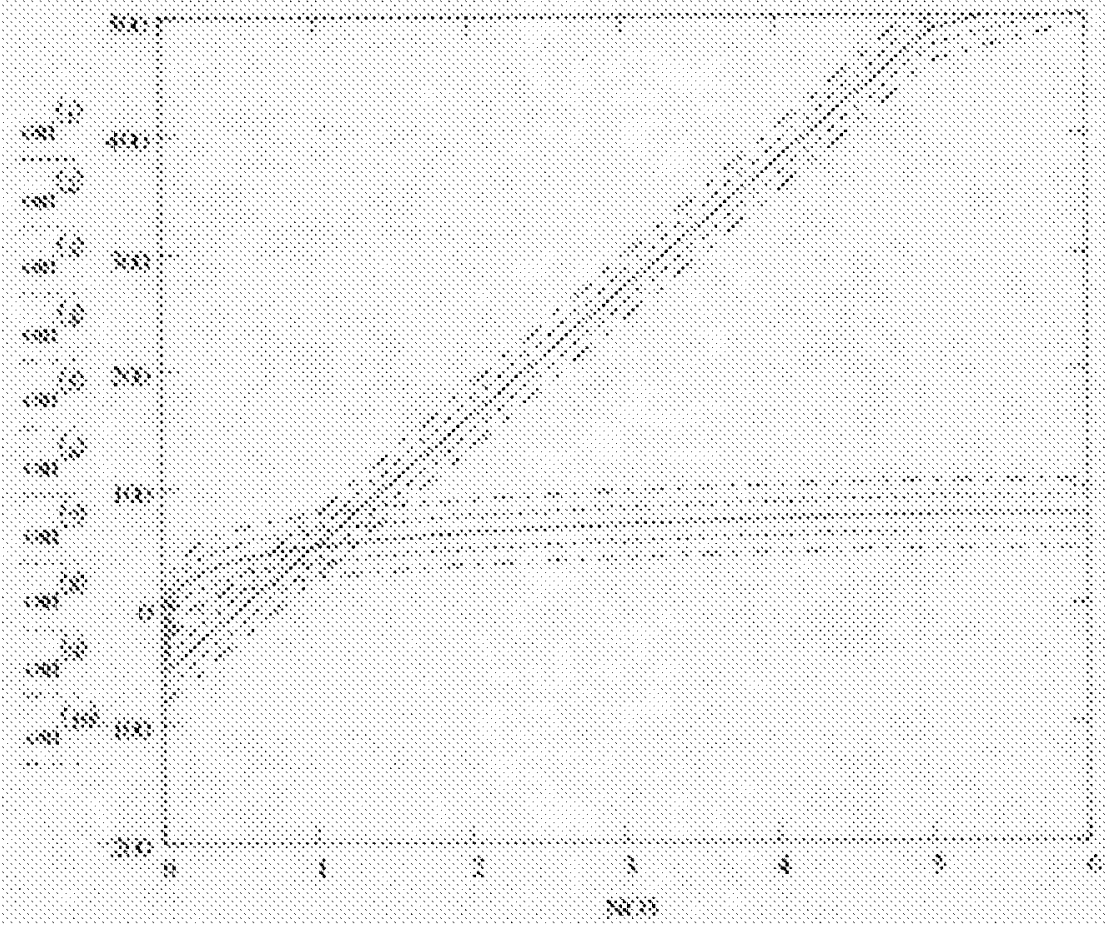




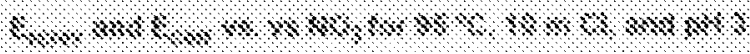

W

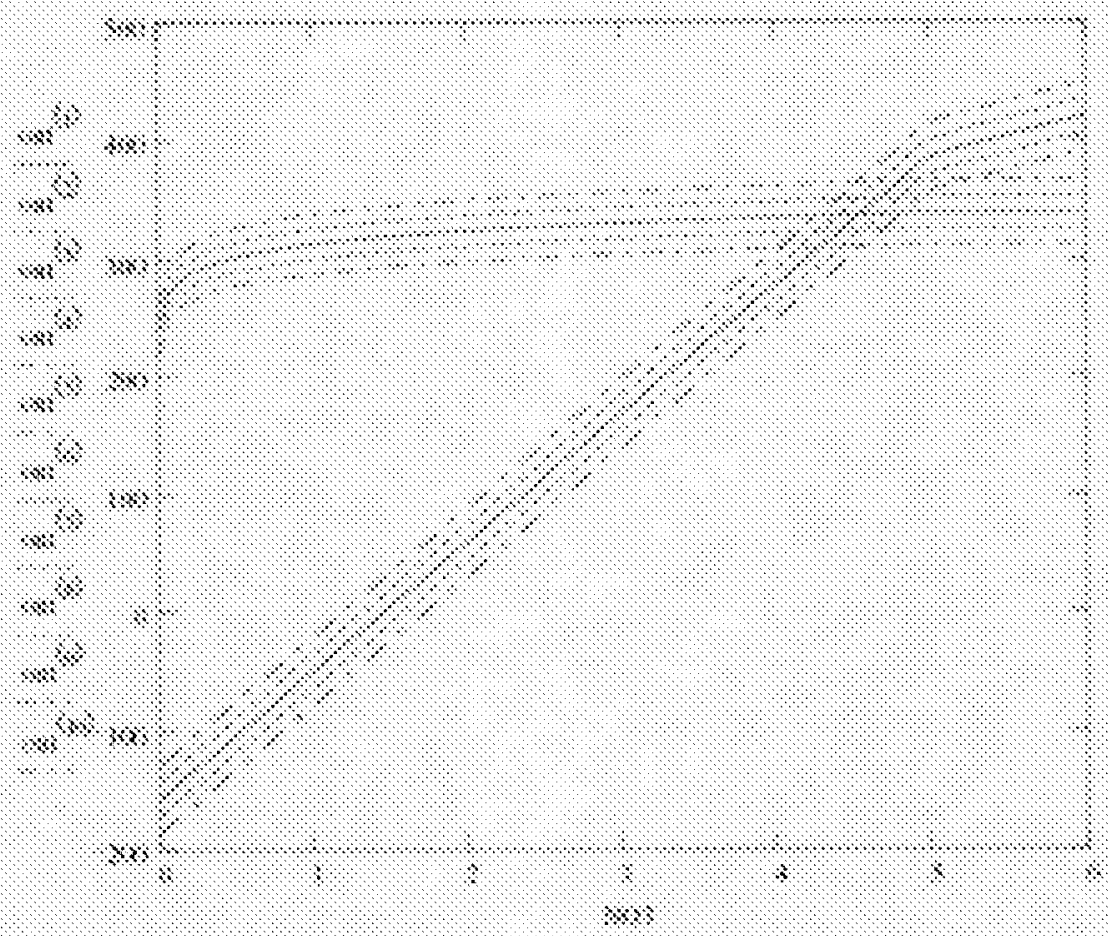


General Corrosion and Localized Corrosion of Waste Package Outer Barrier

\begin{tabular}{|c|c|c|c|c|c|c|c|c|c|c|c|c|c|c|c|c|c|c|}
\hline 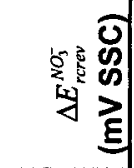 & $\begin{array}{l}\stackrel{\text { N̦ }}{\text { op }} \\
\text { }\end{array}$ & $\begin{array}{l}\text { 吕 } \\
\stackrel{0}{0} \\
\text { ले }\end{array}$ & $\begin{array}{l}\overline{0} \\
\text { त̛ }\end{array}$ & $\begin{array}{l}\stackrel{\sim}{N} \\
\stackrel{2}{\circ}\end{array}$ & $\frac{m}{\frac{m}{1}}$ & $\begin{array}{l}\stackrel{8}{0} \\
\stackrel{0}{0} \\
\text {. }\end{array}$ & $\begin{array}{l}\text { 口 } \\
\ddot{0} \\
\text { पे }\end{array}$ & $\begin{array}{l}\bar{\delta} \\
\text { ळ్ల }\end{array}$ & $\frac{\bar{\sigma}}{\stackrel{\vdots}{N}}$ & 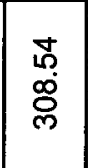 & $\begin{array}{l}\hat{\sigma} \\
0 \\
\hat{0}\end{array}$ & 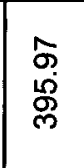 & $\begin{array}{l}\overline{5} \\
\stackrel{5}{\sigma} \\
F\end{array}$ & 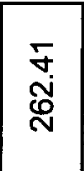 & $\begin{array}{l}J \\
\infty \\
\infty\end{array}$ & 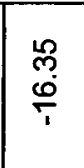 & $\frac{8}{\frac{8}{4}}$ & $\frac{\dddot{T}}{\frac{\pi}{1}}$ \\
\hline 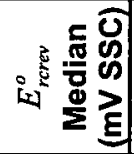 & \begin{tabular}{l}
$\hat{o}$ \\
$\stackrel{0}{1}$ \\
\multirow{1}{*}{}
\end{tabular} & $\begin{array}{l}\forall \\
\stackrel{\infty}{*}\end{array}$ & $\begin{array}{l}g \\
\stackrel{0}{\circ}\end{array}$ & 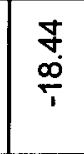 & $\begin{array}{l}\hat{\sigma} \\
\dot{0} \\
T\end{array}$ & $\begin{array}{l}\stackrel{\infty}{8} \\
\stackrel{T}{T}\end{array}$ & $\begin{array}{l}\stackrel{\infty}{0} \\
\stackrel{1}{T}\end{array}$ & $\begin{array}{l}\sigma_{0} \\
\frac{0}{1}\end{array}$ & $\begin{array}{l}\bar{\sigma} \\
\frac{\sigma}{1}\end{array}$ & 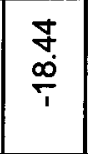 & $\begin{array}{l}\hat{\sigma} \\
\dot{0} \\
\hat{1}\end{array}$ & $\begin{array}{l}5 \\
e \\
\stackrel{0}{1}\end{array}$ & $\begin{array}{l}8 \\
\text { o }\end{array}$ & $\begin{array}{l}8 \\
\text { o } \\
\dot{q}\end{array}$ & $\underset{\substack{++}}{+}$ & 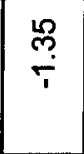 & స్ & $\frac{\infty}{\frac{1}{0}}$ \\
\hline 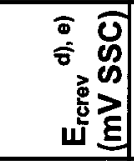 & ఖ & స్ల & 趝 & 古 & প্: & 尽 & స్ల & $\underset{\infty}{\infty}$ & 怘 & 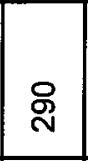 & 8 & $\frac{9}{m}$ & ్ㅠํ & ஜ्ల & 8 & $\stackrel{\infty}{!}$ & นุ & $\frac{\text { N}}{T}$ \\
\hline 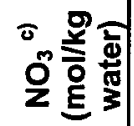 & 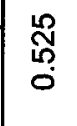 & $\begin{array}{l}\stackrel{\mathscr{N}}{0} \\
\text { గ్ } \\
0\end{array}$ & 䠢 & 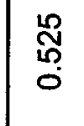 & $\begin{array}{l}\text { Wึ } \\
\text { గ్ర } \\
0\end{array}$ & $\hat{0}$ & $\hat{o}$ & $\stackrel{\text { L }}{\circ}$ & $\stackrel{\text { L }}{\circ}$ & $\stackrel{\mathscr{R}}{\stackrel{2}{\leftarrow}}$ & $\stackrel{\mathbb{R}}{\stackrel{2}{\leftarrow}}$ & $\stackrel{\mathscr{N}}{\leftarrow}$ & $\stackrel{20}{=}$ & $\stackrel{\mathscr{R}}{\leftarrow}$ & $\stackrel{R}{\stackrel{R}{r}}$ & $\stackrel{m}{0}$ & $\stackrel{m}{0}$ & $\stackrel{m}{0}$ \\
\hline 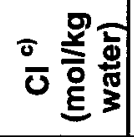 & $\stackrel{n}{n}$ & $\stackrel{n}{n}$ & $\stackrel{\infty}{\infty}$ & 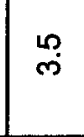 & $\stackrel{\text { L }}{\infty}$ & $\stackrel{\infty}{\infty}$ & $\stackrel{n}{n}$ & $\mathscr{m}$ & $\stackrel{\text { p. }}{m}$ & مֶ & $\ddot{n}$ & $\stackrel{n}{n}$ & קִ & 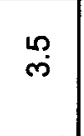 & $\stackrel{n}{n}$ & 0 & 0 & $\omega$ \\
\hline $\begin{array}{c}\overline{\mathbf{a}} \\
\mathrm{x} \\
\mathrm{a}\end{array}$ & $\begin{array}{c}\stackrel{g}{+} \\
\text { م. } \\
\end{array}$ & $\begin{array}{l}\text { g } \\
\text { in }\end{array}$ & 疍 & 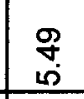 & 疍 & 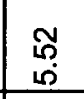 & 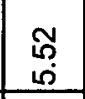 & 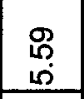 & 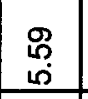 & 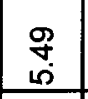 & $\begin{array}{l}\text { go } \\
\text { in } \\
\end{array}$ & $\begin{array}{l}\text { g } \\
\text { in } \\
\end{array}$ & $\begin{array}{l}9 \\
\text { S. } \\
\end{array}$ & 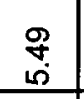 & $\begin{array}{l}9 \\
\text { in } \\
\end{array}$ & ه广 & 苾 & i. \\
\hline - ত⿹ & 음 & 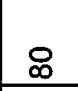 & 8 & \& & $\stackrel{8}{\circ}$ & \& & 8 & \& & \& & : & 움 & $\stackrel{8}{\circ}$ & 8 & 8 & \& & 8 & \& & 음 \\
\hline 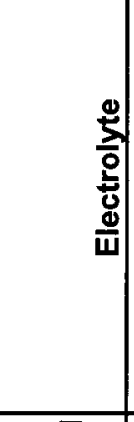 & 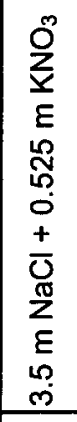 & 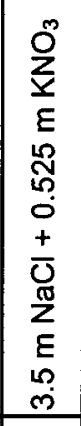 & 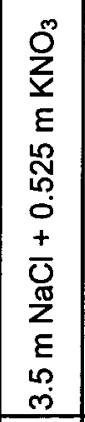 & 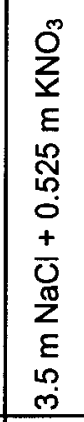 & 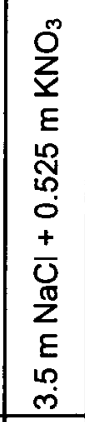 & 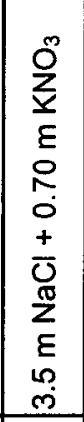 & 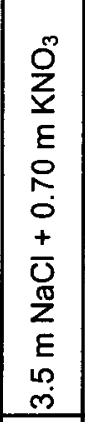 & 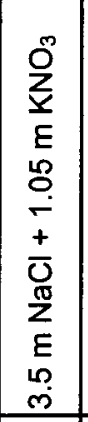 & 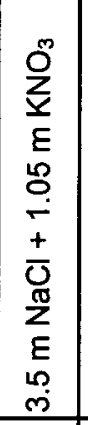 & 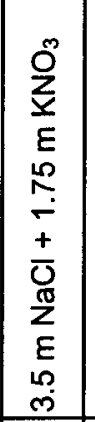 & 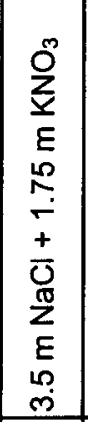 & 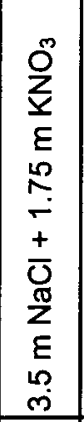 & 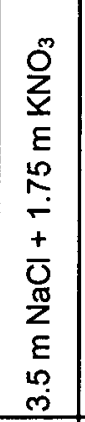 & 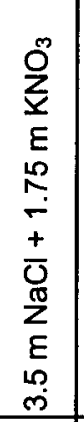 & 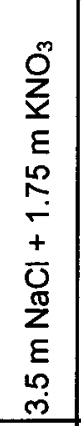 & 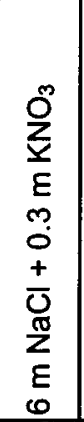 & 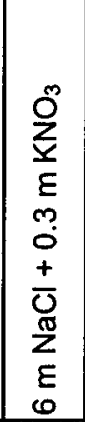 & 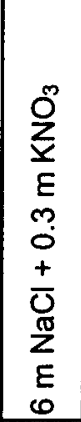 \\
\hline 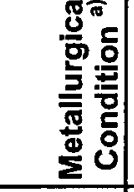 & 岁 & 亭 & 䯧 & 京 & 交 & 交 & 交 & 菖 & 京 & $\underset{\frac{2}{2}}{\frac{3}{2}}$ & 雚 & 交 & 交 & 交 & 京 & 京 & के & 亭 \\
\hline 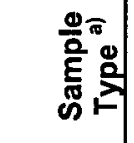 & 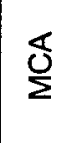 & $\frac{\delta}{\Sigma}$ & $\frac{\overleftarrow{J}}{\Sigma}$ & $\stackrel{\delta}{\Sigma}$ & 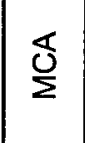 & $\frac{\delta}{\Sigma}$ & 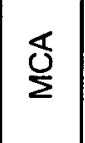 & $\frac{\nwarrow}{\Sigma}$ & $\stackrel{\overleftarrow{J}}{\Sigma}$ & 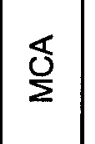 & $\underset{\Xi}{\Sigma}$ & $\stackrel{\overleftarrow{U}}{\Sigma}$ & 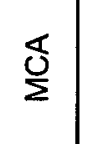 & 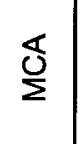 & $\frac{\overleftarrow{J}}{\Sigma}$ & $\underset{\mathcal{J}}{\Sigma}$ & 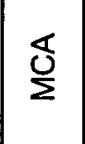 & 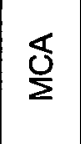 \\
\hline 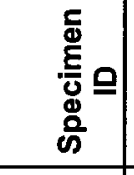 & 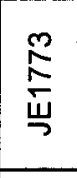 & $\begin{array}{l}\stackrel{\mathscr{N}}{N} \\
\text { 岃 }\end{array}$ & 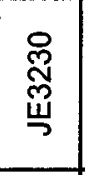 & 总 & 胥 & $\begin{array}{l}\stackrel{8}{\mathscr{N}} \\
\stackrel{\tilde{m}}{丂}\end{array}$ & $\begin{array}{l}\stackrel{\mathscr{U}}{\tilde{W}} \\
\stackrel{\ddot{J}}{J}\end{array}$ & 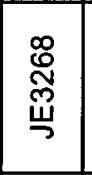 & 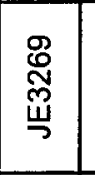 & $\begin{array}{l}\mathscr{N} \\
\stackrel{\tilde{N}}{\mu}\end{array}$ & 总 & 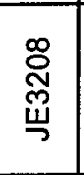 & $\begin{array}{l}\underset{N}{\tilde{N}} \\
\stackrel{\leftrightarrow}{ᄀ}\end{array}$ & 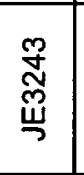 & 怘 & 을 & 空 & $\begin{array}{l}\underset{\sim}{\tilde{N}} \\
\stackrel{\tilde{J}}{J}\end{array}$ \\
\hline $\begin{array}{l}z \\
z \\
\\
8 \\
\frac{\mathbf{g}}{3} \\
\dot{b}\end{array}$ & 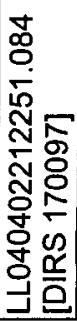 & 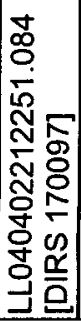 & 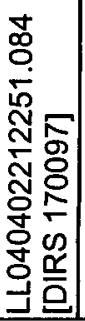 & 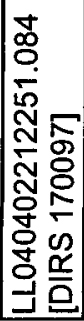 & 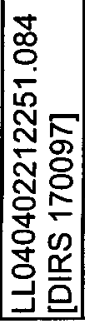 & 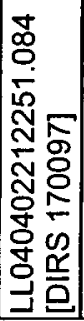 & 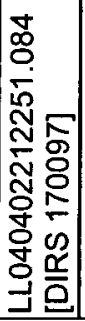 & 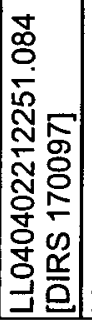 & 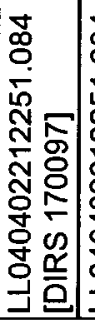 & 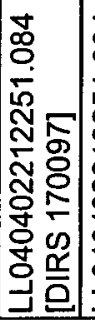 & 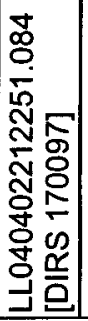 & 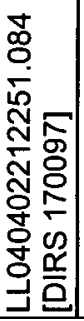 & 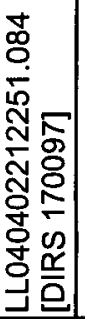 & 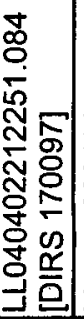 & 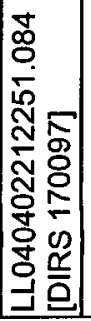 & 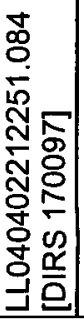 & 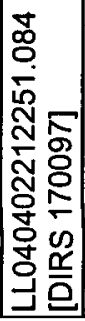 & 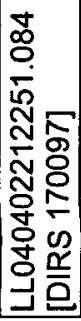 \\
\hline
\end{tabular}


General Corrosion and Localized Corrosion of Waste Package Outer Barrier

\begin{tabular}{|c|c|c|c|c|c|c|c|c|c|c|c|c|c|c|c|c|c|c|}
\hline 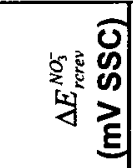 & $\begin{array}{l}\mathscr{P} \\
\infty \\
\dot{y} \\
\dot{y}\end{array}$ & $\begin{array}{l}\mathscr{\leftrightarrow} \\
\stackrel{\varphi}{\leftarrow}\end{array}$ & \begin{tabular}{l}
\multirow{G}{0}{} \\
$\stackrel{\sim}{0}$
\end{tabular} & 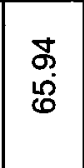 & $\frac{\bar{N}}{\bar{j}}$ & $\frac{m}{\mathscr{D}}$ & $\stackrel{\bar{R}}{\overline{8}}$ & $\begin{array}{l}\hat{m} \\
\text { ֶु. } \\
\text { }\end{array}$ & $\begin{array}{l}\frac{9}{2} \\
\text { O̊ }\end{array}$ & 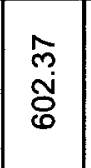 & $\begin{array}{l}\stackrel{\infty}{N} \\
\stackrel{+}{O} \\
\text { Jे }\end{array}$ & $\begin{array}{l}\stackrel{\infty}{N} \\
\stackrel{+}{0} \\
\stackrel{0}{0}\end{array}$ & $\frac{9}{8}$ & $\begin{array}{l}\text { ơ } \\
\stackrel{\leftrightarrow}{\mathbb{N}}\end{array}$ & $\begin{array}{l}\mathscr{\mho} \\
\stackrel{\mathscr{N}}{\square}\end{array}$ & $\underset{\mathscr{O}}{\stackrel{N}{E}}$ & 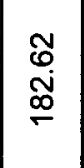 & 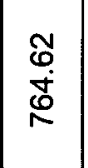 \\
\hline 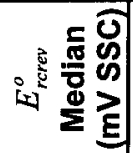 & जิ & $\frac{\infty}{\dot{\varphi}}$ & 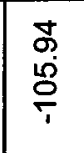 & 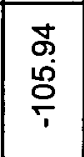 & $\underset{\check{i}}{\bar{\gamma}}$ & $\begin{array}{l}8 \\
\dot{0} \\
\dot{0}\end{array}$ & $\begin{array}{l}80 \\
\text { ¿๋̦ }\end{array}$ & $\stackrel{\hat{m}}{\stackrel{N}{v}}$ & $\begin{array}{l}\frac{9}{0} \\
\frac{0}{1}\end{array}$ & 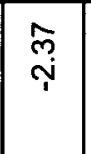 & 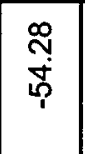 & $\begin{array}{l}\stackrel{\infty}{\sim} \\
\text { țị }\end{array}$ & $\begin{array}{l}\frac{0}{\circ} \\
\frac{0}{1}\end{array}$ & 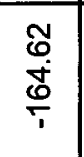 & 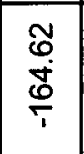 & 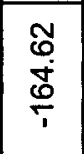 & 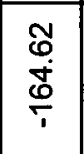 & 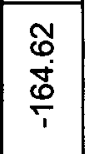 \\
\hline 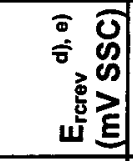 & के & $\begin{array}{l}\infty \\
\infty \\
1\end{array}$ & $\mathscr{\infty}_{1}$ & 욱 & 品 & $\bar{m}$ & ลे & 8 & $\stackrel{\infty}{\stackrel{్}{N}}$ & ৪ & $\stackrel{\mathscr{N}}{\mathscr{N}}$ & 8 & 8 & প్యా & $\bar{y}$ & $\stackrel{m}{\sim}$ & $\stackrel{\infty}{\sim}$ & 8 \\
\hline 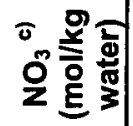 & $\stackrel{m}{0}$ & $\stackrel{m}{0}$ & $\stackrel{\sigma}{0}$ & $\stackrel{\circ}{\circ}$ & ğ & ִூ & ğ & $m$ & $m$ & $m$ & $m$ & $m$ & $m$ & - & - & $m$ & $m$ & 음 \\
\hline 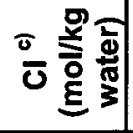 & 0 & 0 & $\omega$ & 0 & 0 & 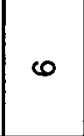 & 0 & 0 & $\boldsymbol{0}$ & 0 & 0 & 0 & 0 & ㅇ & 요 & 오 & 오 & ㅇ \\
\hline $\begin{array}{l}\overline{\mathbf{I}} \\
\mathrm{I} \\
\end{array}$ & هi & $\underset{60}{*}$ & $\begin{array}{c}\hat{n} \\
\text { in }\end{array}$ & $\begin{array}{c}\hat{n} \\
\text { in } \\
i\end{array}$ & $\begin{array}{c}\hat{m} \\
0 \\
0\end{array}$ & $\begin{array}{c}\hat{m} \\
\text { in } \\
i\end{array}$ & $\begin{array}{l}\hat{m} \\
\text { in } \\
\dot{n}\end{array}$ & 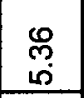 & $\begin{array}{l}0 \\
\text { m. } \\
\text { in }\end{array}$ & $\begin{array}{l}\infty \\
\text { ల్ } \\
i \\
\end{array}$ & 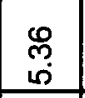 & 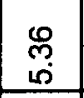 & 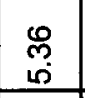 & 芯 & 节 & 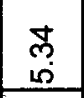 & 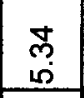 & $\begin{array}{l}\vec{m} \\
\text { Li }\end{array}$ \\
\hline 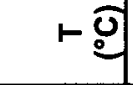 & 8 & $\stackrel{\square}{\circ}$ & 우 & $\stackrel{8}{\circ}$ & 8 & 8 & \& & 8 & 움 & 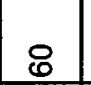 & $\infty$ & $\varnothing$ & $\stackrel{8}{\circ}$ & 음 & 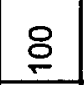 & 음 & 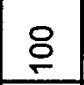 & 응 \\
\hline 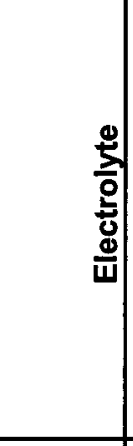 & 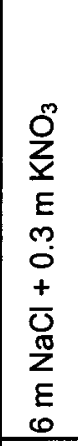 & 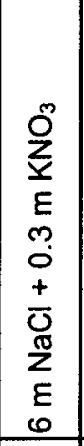 & 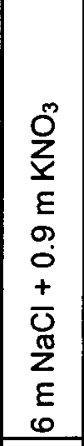 & 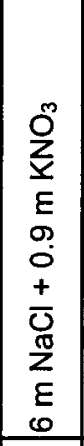 & 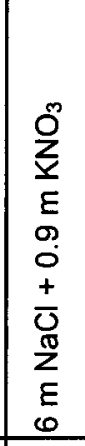 & 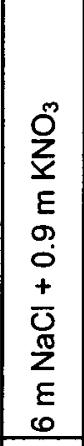 & 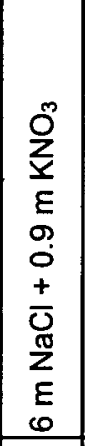 & 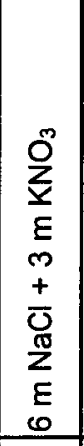 & 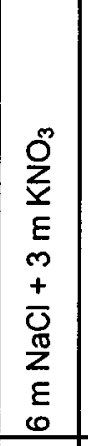 & 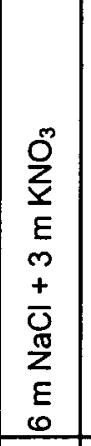 & $\begin{array}{l}O^{\infty} \\
\sum \\
E \\
m \\
+ \\
\bar{L} \\
Z \\
Z \\
E \\
0 \\
\end{array}$ & 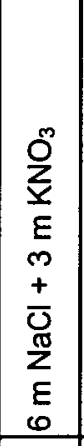 & 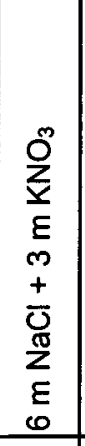 & 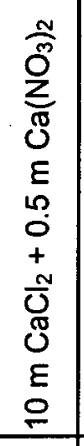 & 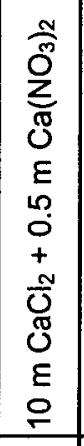 & 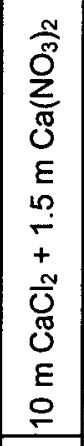 & 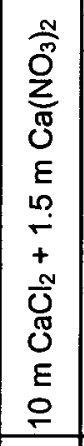 & 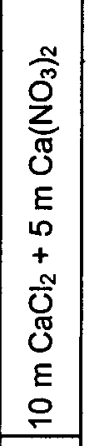 \\
\hline 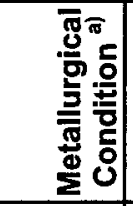 & 3 & 苂 & 㐫 & 京 & 交 & 京 & 交 & 京 & 交 & 高 & 高 & 交 & 京 & 交 & 察 & 亭 & 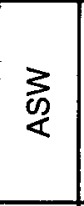 & 交 \\
\hline 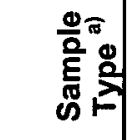 & 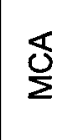 & 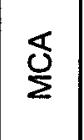 & $\frac{\overleftarrow{J}}{\Sigma}$ & $\underset{\delta}{\Sigma}$ & $\frac{\mathbb{J}}{\Sigma}$ & $\underset{J}{\mathbb{J}}$ & 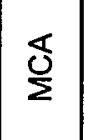 & $\overleftarrow{\delta}$ & $\underset{\delta}{\Sigma}$ & $\frac{\overleftarrow{J}}{\Sigma}$ & $\underset{J}{\Sigma}$ & $\underset{J}{\Sigma}$ & 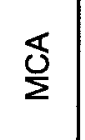 & $\frac{\delta}{\Sigma}$ & $\frac{\delta}{\Sigma}$ & $\frac{\delta}{\Sigma}$ & $\frac{\mathbb{J}}{\Sigma}$ & 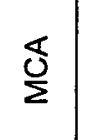 \\
\hline 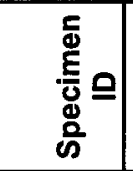 & 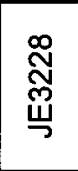 & 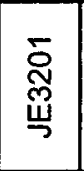 & $\underset{\tilde{W}}{\stackrel{\infty}{\sim}}$ & 惡 & $\begin{array}{l}\stackrel{\text { N }}{\tilde{W}} \\
\stackrel{\leftrightarrow}{7}\end{array}$ & 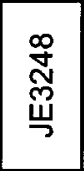 & $\begin{array}{l}\stackrel{\mathscr{N}}{\tilde{N}} \\
\text { 岇 }\end{array}$ & 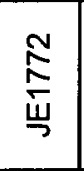 & 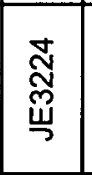 & $\begin{array}{l}\stackrel{\mathscr{N}}{\tilde{N}} \\
\text { Щ }\end{array}$ & $\begin{array}{l}\stackrel{\infty}{\tilde{N}} \\
\stackrel{\tilde{m}}{\sim}\end{array}$ & $\frac{\stackrel{R}{\frac{N}{5}}}{\stackrel{w}{J}}$ & $\begin{array}{l}\text { 岕 } \\
\text { 岃 }\end{array}$ & 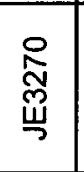 & 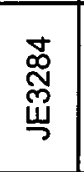 & $\begin{array}{l}\stackrel{p}{N} \\
\stackrel{\tilde{N}}{\longrightarrow}\end{array}$ & 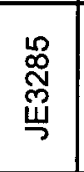 & $\begin{array}{l}\text { 跑 } \\
\text { 岂 }\end{array}$ \\
\hline 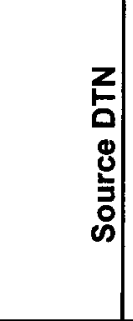 & 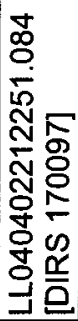 & 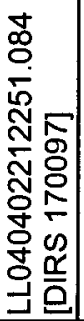 & 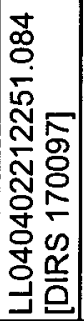 & 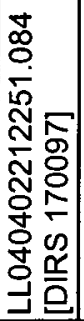 & 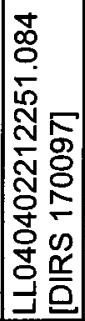 & 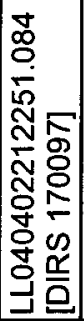 & 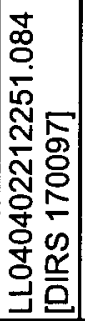 & 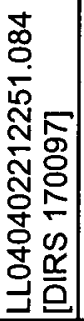 & 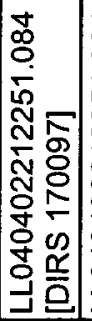 & 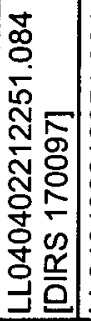 & 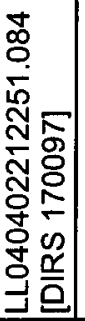 & 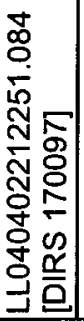 & 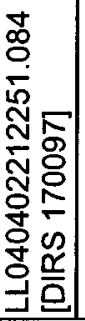 & 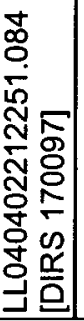 & 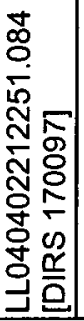 & 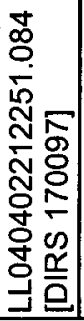 & 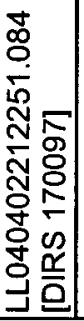 & 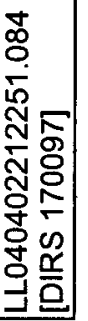 \\
\hline
\end{tabular}


General Corrosion and Localized Corrosion of Waste Package Outer Barrier

\begin{tabular}{|c|c|c|c|c|c|c|c|c|c|c|c|c|c|}
\hline 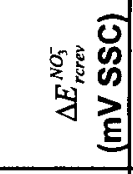 & $\underset{\substack{\infty \\
\infty}}{\stackrel{\infty}{\infty}}$ & $\begin{array}{l}\stackrel{m}{N} \\
\stackrel{0}{\infty} \\
\stackrel{\infty}{\sigma}\end{array}$ & 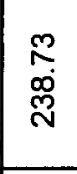 & 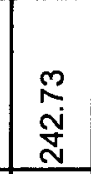 & 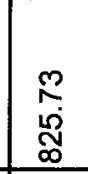 & 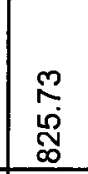 & $\begin{array}{l}\mathscr{O} \\
\ddot{\mathscr{N}} \\
\end{array}$ & 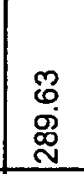 & 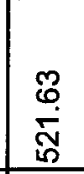 & $\begin{array}{l}\mathscr{8} \\
0 \\
\mathscr{6} \\
\text { ले }\end{array}$ & 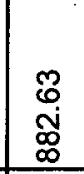 & $\begin{array}{l}\mathscr{P} \\
\stackrel{0}{\sim} \\
\infty \\
\infty\end{array}$ & 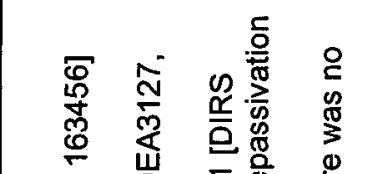 \\
\hline 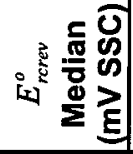 & 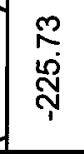 & ִָ & ָ̊. & 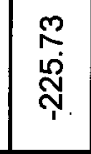 & 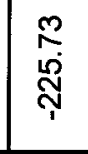 & 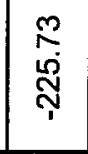 & $\begin{array}{l}\mathscr{\mathscr { O }} \\
\stackrel{\sim}{0} \\
\stackrel{\infty}{\gamma}\end{array}$ & 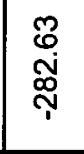 & 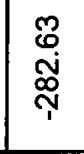 & $\begin{array}{l}\mathscr{\Re} \\
\stackrel{0}{0} \\
\text { ஸे }\end{array}$ & $\begin{array}{l}\mathscr{0} \\
\stackrel{0}{0} \\
\stackrel{\sim}{N}\end{array}$ & $\begin{array}{c}\mathscr{\mathscr { O }} \\
\stackrel{\sim}{\sim} \\
\stackrel{\sim}{1}\end{array}$ & 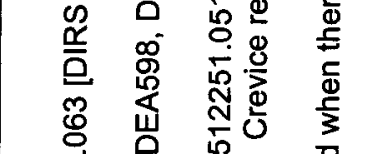 \\
\hline 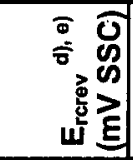 & \& & శ్ & $\stackrel{m}{\longrightarrow}$ & $\stackrel{\approx}{\sigma}$ & 8 & 8 & $\mp$ & $\sim$ & ণ্ণ & 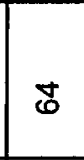 & : & 용 & 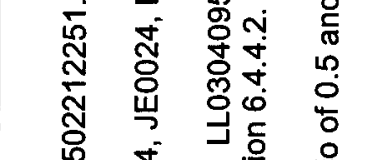 \\
\hline 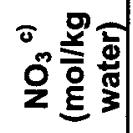 & $\stackrel{\Upsilon}{-}$ & $\stackrel{N}{\leftarrow}$ & $\stackrel{\varphi}{\ddot{m}}$ & $\stackrel{\mathscr{m}}{\ddot{m}}$ & $\cong$ & 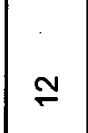 & $\stackrel{\infty}{-}$ & $\stackrel{\infty}{-}$ & نे & ') & $\stackrel{\infty}{\Gamma}$ & $\stackrel{\infty}{\leftarrow}$ & 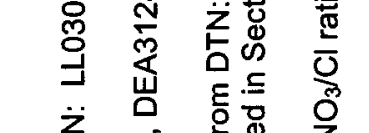 \\
\hline 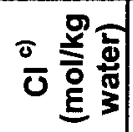 & $\stackrel{\mathbb{N}}{ }$ & $\stackrel{\mathbb{N}}{ }$ & $\stackrel{\mathbb{N}}{ }$ & $\stackrel{\Delta}{\Delta}$ & $\stackrel{\Delta}{\Delta}$ & $\stackrel{\mathbb{N}}{ }$ & 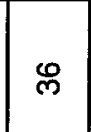 & $\stackrel{m}{\dddot{m}}$ & ల్ల & ल & ஜ্ল & ஜ్ల & 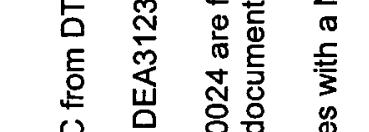 \\
\hline $\begin{array}{l}\bar{a} \\
\mathbf{z} \\
\end{array}$ & $\begin{array}{l} \\
\text { i } \\
\end{array}$ & $\begin{array}{l}\text { ले } \\
\text { in }\end{array}$ & $\begin{array}{l}\text { जे } \\
\text { i. } \\
\text { ம. }\end{array}$ & 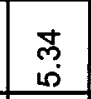 & 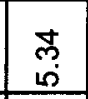 & \begin{tabular}{l}
\multirow{m}{*}{} \\
in \\
\end{tabular} & 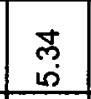 & 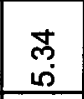 & $\begin{array}{l}\text { mे } \\
\text { in }\end{array}$ & 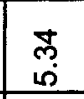 & 芯 & $\begin{array}{l}\text { ले } \\
\text { cin }\end{array}$ & 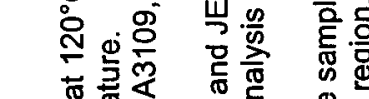 \\
\hline 10 & $\stackrel{\text { ஜి }}{\Gamma}$ & $\stackrel{\text { ஜ }}{\Gamma}$ & ్ㅏㅁ & 욤 & ஜ্ & 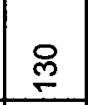 & $\nsubseteq$ & $\stackrel{8}{ }$ & $\stackrel{\square}{\square}$ & 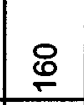 & 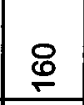 & $\stackrel{8}{\stackrel{0}{0}}$ & 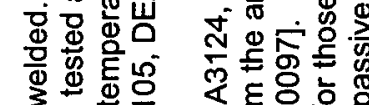 \\
\hline 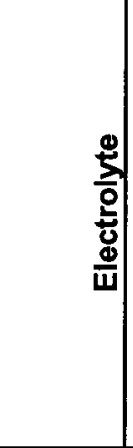 & 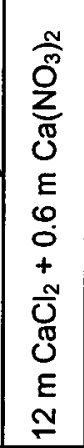 & 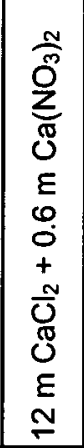 & 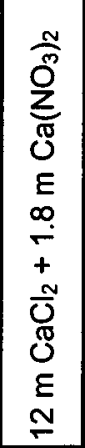 & 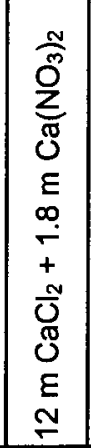 & 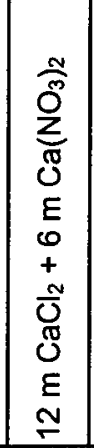 & 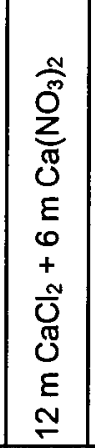 & 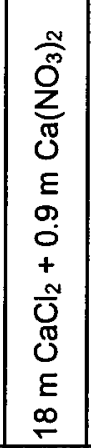 & 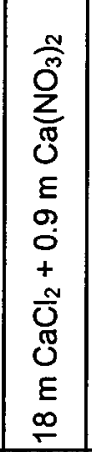 & 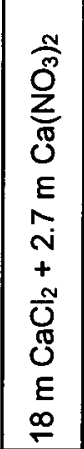 & 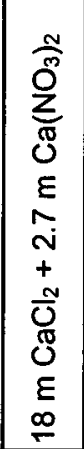 & 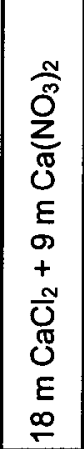 & 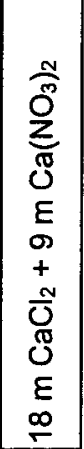 & 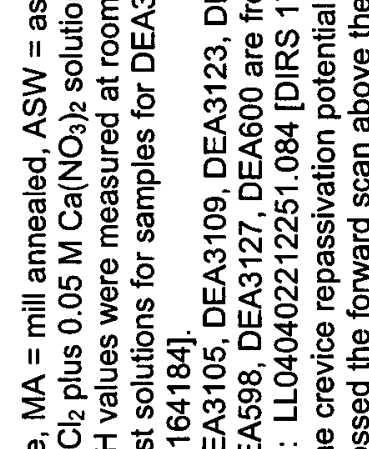 \\
\hline 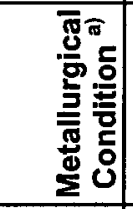 & 交 & 京 & 交 & 高 & 交 & 交 & 高 & 交 & 交 & 高 & 触 & $\sum_{00}^{3}$ & 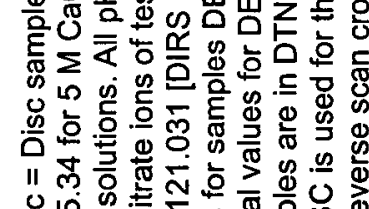 \\
\hline 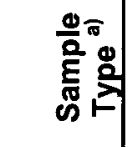 & 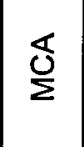 & $\overleftarrow{J}$ & S్ & ญ & ঠ্ & త্ & ঠ્ & S & 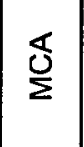 & $\underset{\delta}{\Sigma}$ & ঠ্ড & 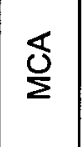 & 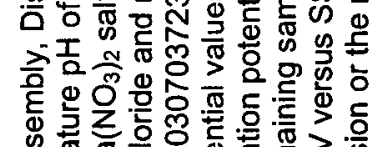 \\
\hline 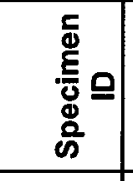 & స్心 & $\underset{\substack{\text { N } \\
\text { 虫 }}}{ }$ & $\underset{\text { N }}{\stackrel{N}{J}}$ & 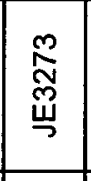 & 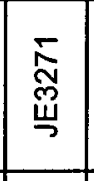 & 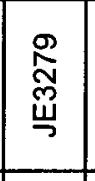 & 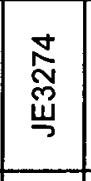 & 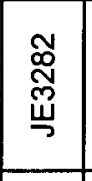 & $\begin{array}{l}\text { \& } \\
\text { ले } \\
\stackrel{\leftrightarrow}{\sim}\end{array}$ & 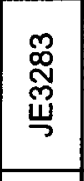 & 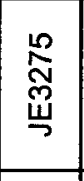 & 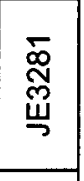 & 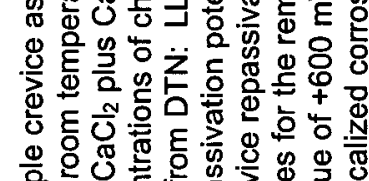 \\
\hline 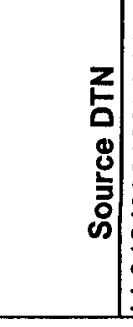 & 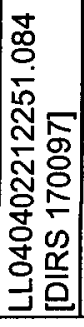 & 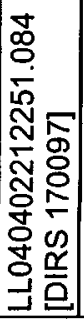 & 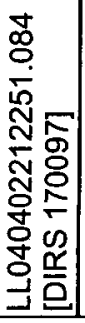 & 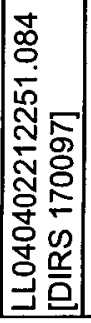 & 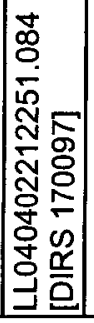 & 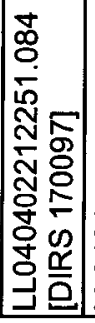 & 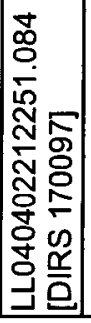 & 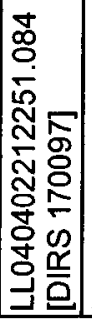 & 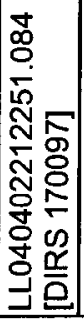 & 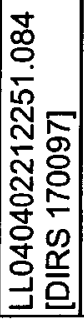 & 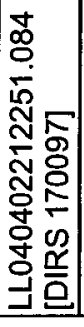 & 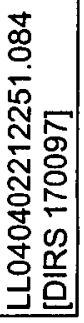 & 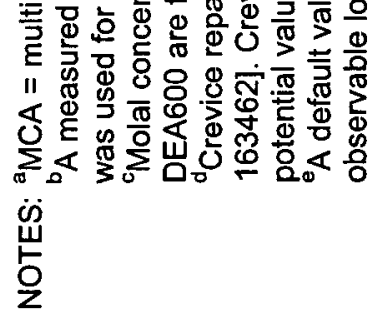 \\
\hline
\end{tabular}


INTENTIONALLY LEFT BLANK 


\begin{abstract}
APPENDIX IX
MATHCAD WORKSHEETS FOR REGRESSION ANALYSIS OF THE CORROSION

POTENTIAL MODEL AND CREVICE REPASSIVATION POTENTIAL MODEL AND FOR LOCALIZED CORROSION SUSCEPTIBILITY ANALYSES
\end{abstract}


INTENTIONALLY LEFT BLANK 
IX. MATHCAD WORKSHEETS FOR REGRESSION ANALYSIS OF THE CORROSION POTENTIAL MODEL AND CREVICE REPASSIVATION POTENTIAL MODEL AND FOR LOCALIZED CORROSION SUSCEPTIBILITY ANALYSES

\section{IX.1 ERCREV_UNCANALYSIS.MCD}

$$
\begin{aligned}
& E_{n \times n}^{a}=a_{2}+a_{1} T+a_{2} p H+a_{1} \log \left(\left[C I^{-}\right]\right)+a_{4} T \times \log \left(\left[C l^{-}\right]\right) \\
& \text {Dat := } \\
& \text { Ercrev_mcd_r1.xls }
\end{aligned}
$$

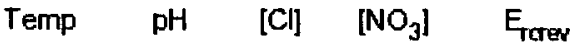

$$
\begin{aligned}
& \text { ('C) (M) (M) (mV SSC) }
\end{aligned}
$$

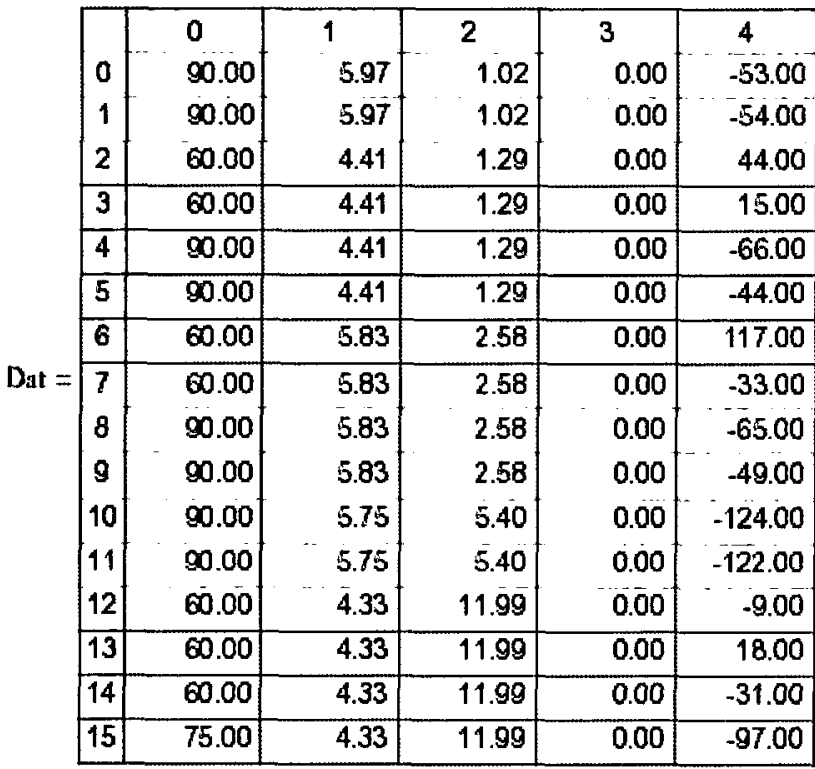

$$
\begin{aligned}
& \mathrm{rY}:=\mathrm{Dat}{ }^{4} \quad-\mathrm{E}_{\mathrm{rcrav}} \\
& \mathrm{rX}^{\langle 0\rangle}=\overrightarrow{\mathrm{Dat}}_{\mathrm{Dat}}^{\vec{\omega}} \quad \begin{array}{l}
\text { Dummy column of } 1 \text { 's - } \\
\text { used to get constant in model }
\end{array} \\
& \mathrm{rX}^{\langle i}:=\mathrm{Dat}^{\langle 0\rangle} \quad \text { Temp } \\
& \mathrm{rX}^{(2)}:=\mathrm{Dat}^{(1)} \quad \mathrm{pH} \\
& \mathrm{rx}(3)=\log \left(\mathrm{Dat} \mathrm{i}^{(2)}\right) \quad \log ([\mathrm{Cl}) \\
& r x^{(4)}:=\overrightarrow{\mathrm{Dat}^{(0)} \cdot \log \left(\mathrm{Dat}{ }^{(2)}\right)} \quad \text { Temp }{ }^{*} \log ([\mathrm{Cl}])
\end{aligned}
$$


Regression Coefficients

$\mathrm{rb}:=\left(\mathrm{r} \mathrm{X}^{\mathrm{T}} \cdot \mathrm{rX}\right)^{-1} \cdot \mathrm{XX}^{\mathrm{T}} \cdot \mathrm{rY} \quad \mathrm{rb}^{\mathrm{T}}=\left(\begin{array}{lllll}214.089 & -3.696 & 25.284 & -252.181 & 1.414\end{array}\right)$

Covariance

$$
\begin{aligned}
& r \Sigma:=\mid \frac{\left(r Y^{T} \cdot r Y-r b^{\top} \cdot r X^{\top} \cdot r Y\right)}{(\operatorname{length}(r Y)-\operatorname{cols}(r X))} \cdot\left(r X^{\top} \cdot r X\right)^{-1} \quad \text { conde }\left(r X^{T} \cdot r X\right)=1.447 \times 10^{6} \\
& \mathrm{r}=\left(\begin{array}{ccccc}
2.198 \mathrm{E}+003 & -1.516 \mathrm{E}+001 & -8.325 \mathrm{E}+001 & -1.805 \mathrm{E}+003 & 1.590 \mathrm{E}+001 \\
-1.516 \mathrm{E}+001 & 2.267 \mathrm{E}-001 & -1.240 \mathrm{E}+000 & 1.877 \mathrm{E}+001 & -1.996 \mathrm{E}-001 \\
-8.325 \mathrm{E}+001 & -1.240 \mathrm{E}+000 & 3.183 \mathrm{E}+001 & -3.237 \mathrm{E}+001 & 7.425 \mathrm{E}-001 \\
-1.805 \mathrm{E}+003 & 1.873 \mathrm{E}+001 & -3.237 \mathrm{E}+001 & 2.907 \mathrm{E}+003 & -2.868 \mathrm{E}+001 \\
1.590 \mathrm{E}+001 & -1.996 \mathrm{E}-001 & 3.425 \mathrm{E}-001 & -2.868 \mathrm{E}+001 & 2.995 \mathrm{E}-001
\end{array}\right) \\
& \begin{array}{lll}
\text { SStot }:=\sum(r Y-\operatorname{mean}(r Y))^{2} & \text { SStot }=1.120 \times 10^{6} & \text { Sum of Squares total } \\
\text { SSres }:=\sum(r Y-r X \cdot r b)^{2} & \text { SSres }=2.690 \times 10^{5} & \text { Sum of Squares residual } \\
\text { SSreg }:=\sum(r X \cdot r b-\operatorname{mean}(r Y))^{2} & \text { SSreg }=8.511 \times 10^{5} & \begin{array}{l}
\text { Sum of Squares regression } \\
\text { or "Explained SS" }
\end{array} \\
\text { Rsq }:=\frac{\text { SSreg }}{\text { SStot }} & \mathrm{Rsq}=0.760 & \text { Coefficient of determination }
\end{array}
\end{aligned}
$$

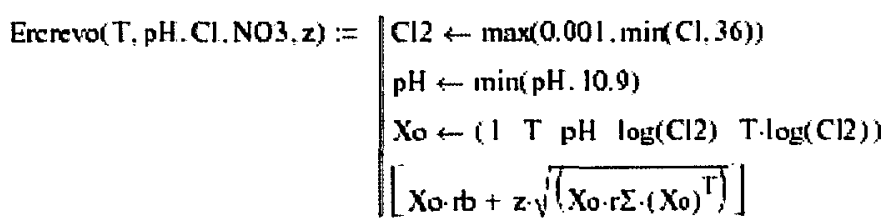$$
\mathrm{Np}:=100 \quad \text { Number of points at which to evaluate is } \mathrm{Np}+1 \text { (indexing starts at ze ro) }
$$$$
\mathrm{nn}:=0 . \mathrm{Np} \quad \text { Range variable takes integer values between zero and } \mathrm{Np}
$$ 


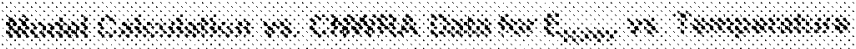
$\$, 0,0,0,0,0,1$,
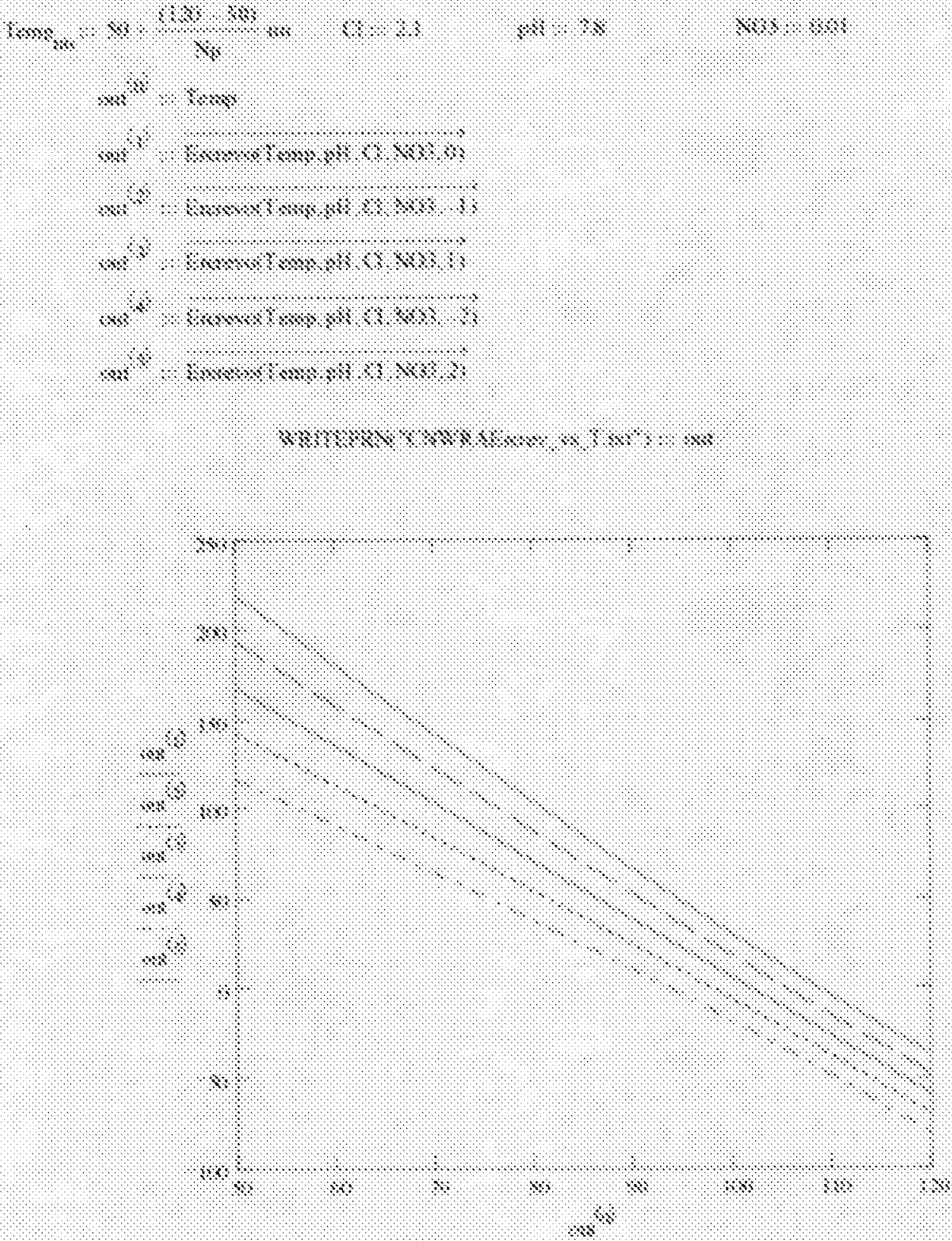
\$

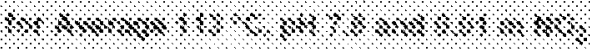

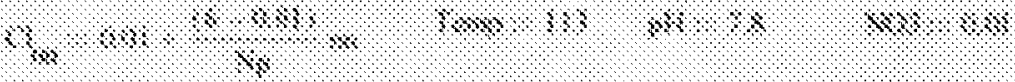

$$
\begin{aligned}
& \text { s. }
\end{aligned}
$$

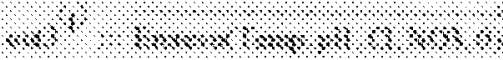

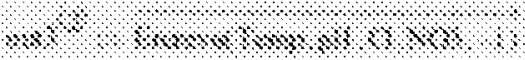

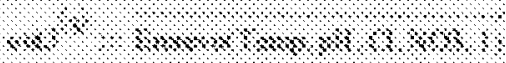

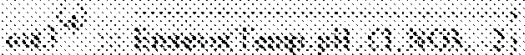

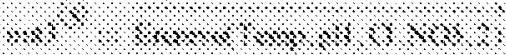

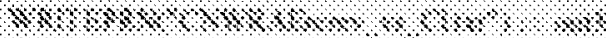

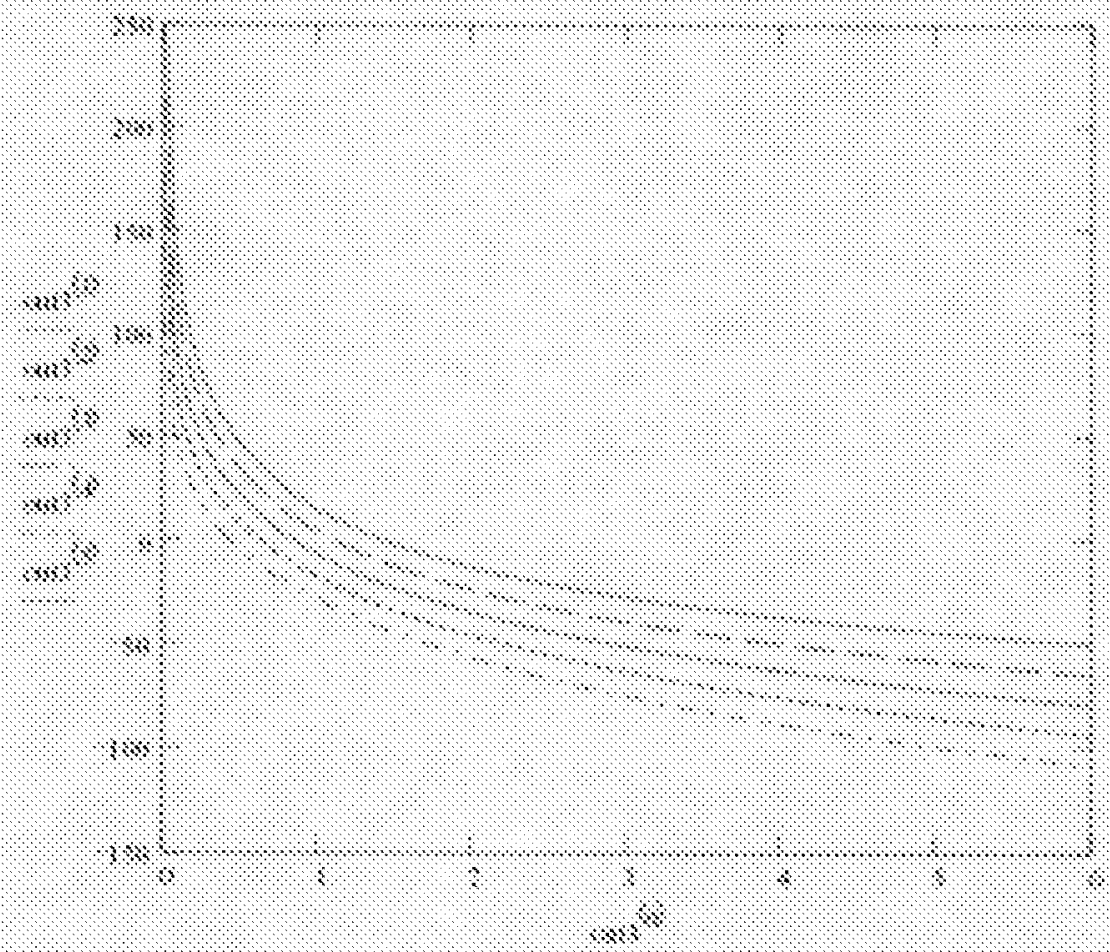




\section{IX.2 DERCREV_UNCANALYSIS.MCD}

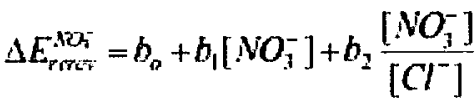

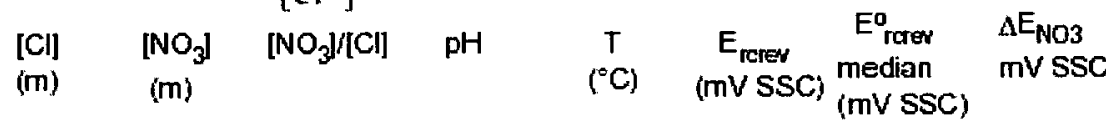

Dat2 :=

\begin{tabular}{|c|c|c|c|c|c|c|c|c|}
\hline & 0 & 1 & 2 & 3 & 4 & 5 & 6 & 7 \\
\hline 0 & 2.57 & 0.03 & 0.01 & 6.47 & 60 & 61 & 87.32 & -26.32 \\
\hline 1 & 2.61 & 0.26 & 0.1 & 6.41 & $\infty 0$ & -72 & -8.57 & -63.43 \\
\hline 2 & $12.50^{\dagger}$ & 1.26 & 0.1 & 4.86 & 105 & 108 & -165.21 & 273.21 \\
\hline 3 & 12.59 & 1.26 & 0.1 & 4.86 & $105^{\circ}$ & 133 & -165.21 & 298.21 \\
\hline 4 & 11.7 & $0 . \overline{1}$ & 0.01 & 5.34 & 120 & -264 & -182.54 & -81.46 \\
\hline 5 & $16.32^{+}$ & 1.64 & 0.1 & 5.34 & $120^{\circ}$ & 37 & -194.47 & 231.47 \\
\hline 6 & $20.82:$ & $2 . \overline{07}$ & 0.1 & 5.34 & $130^{\circ}$ & 32 & -221.5 & 253.5 \\
\hline$?$ & 20.82 & 2.07 & 0.1 & 5.34 & 130 & 24 & -221.5 & 245.5 \\
\hline 8 & 1 & 0.05 & 0.05 & 5.38 & 60 & 93.9 & 128.36 & 34.46 \\
\hline 9 & 1 & 0.05 & 0.05 & 5.38 & 100 & -119.1 & $\begin{array}{l}-19.48 \\
\end{array}$ & -99.62 \\
\hline 10 & 1 & 0.05 & 0.05 & 5.38 & 80 & -51.8 & 54.44 & -106.24 \\
\hline 11 & 1 & 0.05 & 0.05 & 5.38 & 100 & -122.4 & -19.48 & -102.92 \\
\hline $12+$ & 1 & 0.05 & 0.05 & 5.38 & 6 & 348.2 & 128.36 & 219.84 \\
\hline $13^{+}$ & $1+$ & 0.05 & 0.05 & 5.38 & 80 & 26.2 & 54.44 & -28.24 \\
\hline 14 & $1 \dagger$ & 0.15 & 0.15 & 5.13 & 60 & $30 \overline{2} .58$ & 122.04 & $1 \overline{80.54}$ \\
\hline 15 & $1 t$ & 0.15 & 0.15 & 5.13 & $80^{\circ}$ & 289.9 & 49.12 & 241.78 \\
\hline
\end{tabular}

$r Y 2:=D_{a 12}(j) \quad \Delta E_{N O 3}$

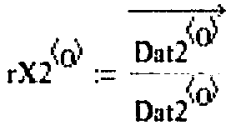

$$
\begin{aligned}
& \text { Dummy column of } 1 \text { 's . } \\
& \text { used to get constant in model } \\
& \mathbf{r X 2}{ }^{\langle 1\rangle}:=\mathrm{Dat2}(\hat{}) \quad\left[\mathrm{NO}_{3}\right] \\
& \left.\mathrm{rX2}^{\langle 2\rangle}=\frac{\overrightarrow{\mathrm{Dat}_{2}\langle\hat{i}}}{\mathrm{Dat}_{2}\langle\alpha\rangle} \quad\left[\mathrm{NO}_{3}\right] / \mathrm{Cl}\right]
\end{aligned}
$$


Regression Coefficients

$\mathrm{rb} 2:=\left(\mathrm{rX}_{2}{ }^{\mathrm{T}} \cdot{ }_{\mathrm{rXX}}\right)^{-1}{ }_{\mathrm{r}} \times 2^{\mathrm{T}} \cdot \mathrm{rY} 2 \quad \mathrm{rb} 2{ }^{\mathrm{T}}=\left(\begin{array}{lll}22.589 & 33.748 & 749.745\end{array}\right)$

Covariance

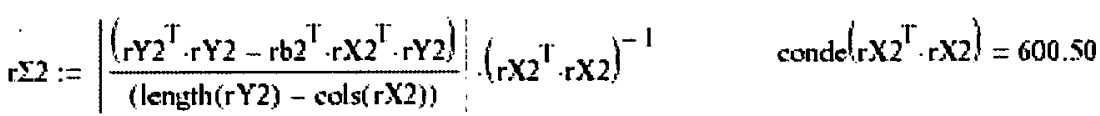

$r \Sigma 2=\left(\begin{array}{ccc}5.814 E+002 & -1.737 E+000 & -1.542 E+003 \\ -1.737 E+000 & 2.683 E+001 & -2.291 E+002 \\ -1.542 E+003 & -2.291 E+002 & 9.119 E+003\end{array}\right)$

$\begin{array}{lll}\text { SStot2 }:=\sum(\mathrm{rY} 2-\operatorname{mean}(\mathrm{rY} 2))^{2} & \mathrm{SStot} 2=5417140.14 & \text { Sum of Squares total } \\ \mathrm{SSres} 2:=\sum(\mathrm{rY} 2-\mathrm{rX2} \cdot \mathrm{rb} 2)^{2} & \mathrm{SSres} 2=1574242.09 & \text { Sum of Squares residual } \\ \mathrm{SSreg} 2:=\sum(\mathrm{rX} 2 \cdot \mathrm{rb} 2-\operatorname{menn}(\mathrm{rY} 2))^{2} & \mathrm{SSres} 2=3842898.05 & \begin{array}{l}\text { Sum of Squares regression } \\ \text { or "Explained SS" }\end{array} \\ \mathrm{Rsq} 2:=\frac{\text { SSreg2 }}{\text { SStot2 }} & \mathrm{Rsq} 2=0.709 & \text { Coefficient of determination }\end{array}$

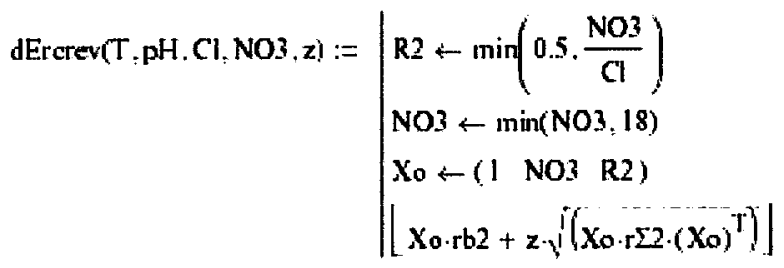

$N_{p}:=100 \quad$ Number of points at which to evaluate is $N p+1$ (indexing starts at zero)

$\mathrm{nn}:=0 . . \mathrm{Np} \quad$ Range variable takes integer values between zero and $\mathrm{Np}$ 


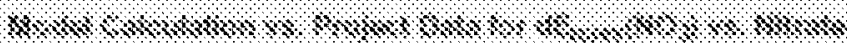
$3,0,0,1,0,1, \%$
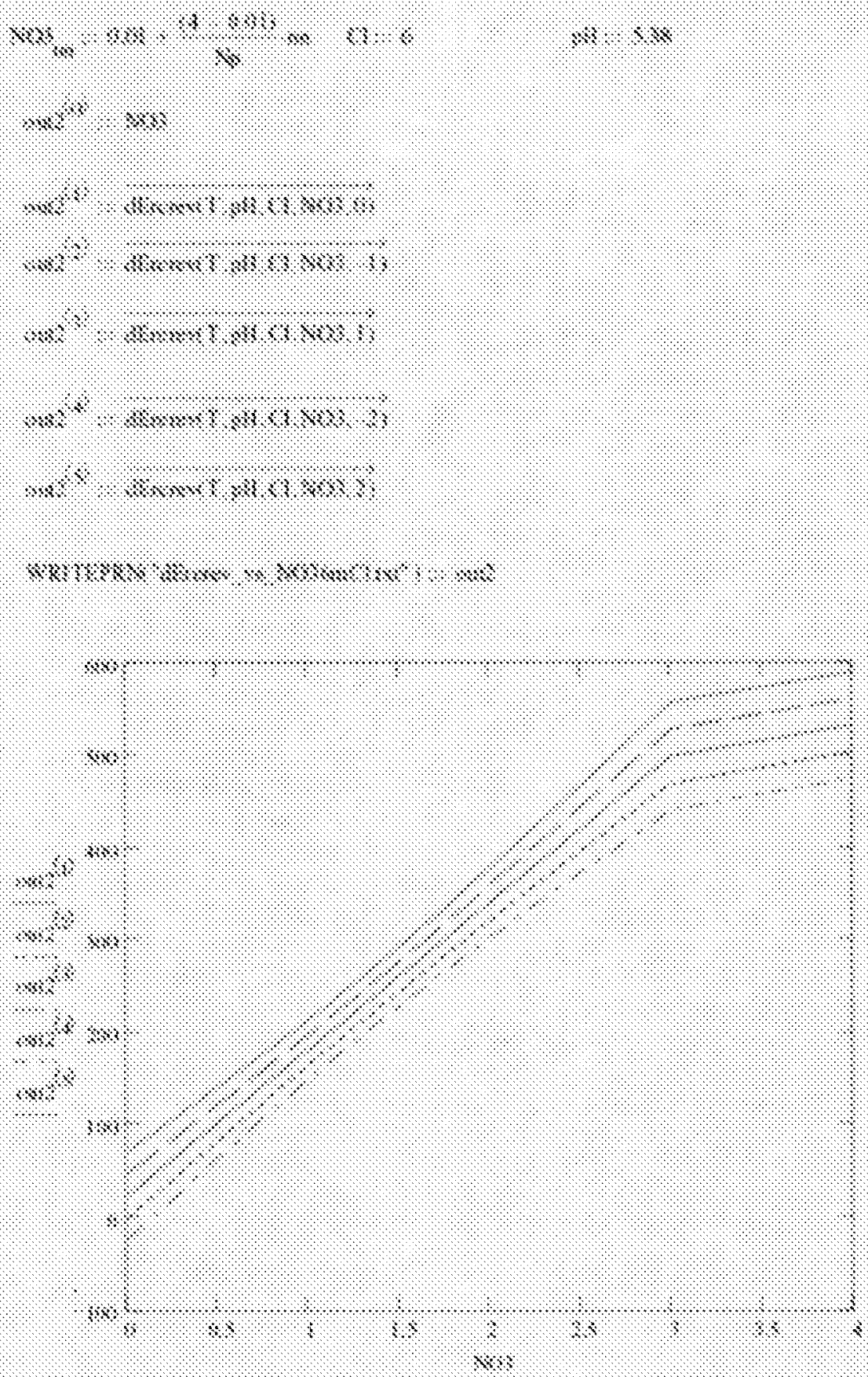


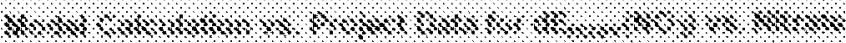

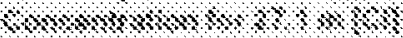

$$
3+3+2
$$

$$
\begin{aligned}
& \text { - } \\
& \text { \$ } \\
& \text {. } \\
& \text { \$ } 1 \text { + }
\end{aligned}
$$

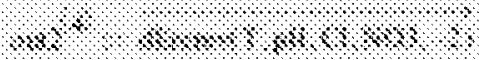

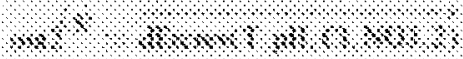

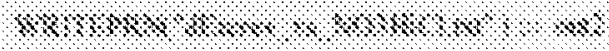

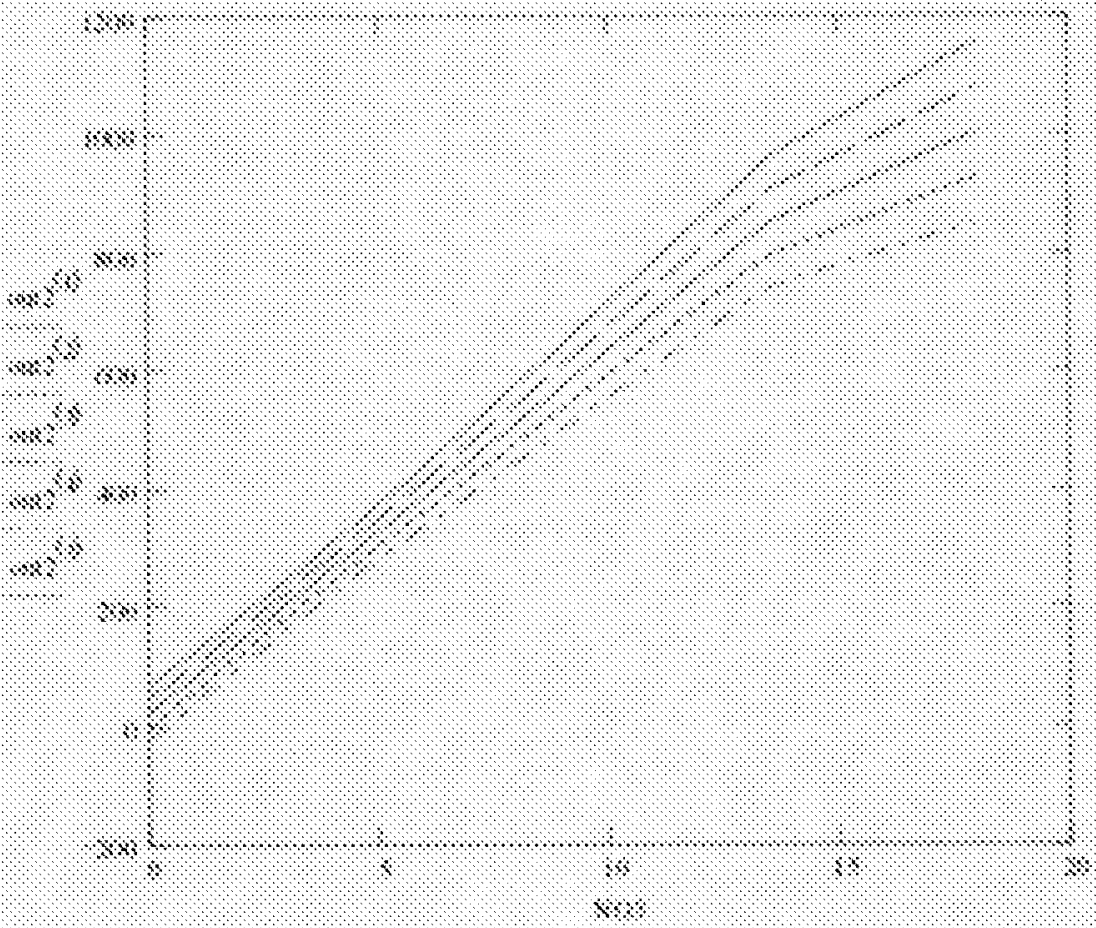




\section{IX.3 ECORR UNCANALYSIS.MCD}

$$
\begin{aligned}
& E_{\mathrm{row}}=c_{\mathrm{a}}+c_{1} T+c_{2} \mathrm{pH}+c_{3}\left[\mathrm{Cl}^{-}\right]+c_{4} \log \left(\frac{\left[\mathrm{NO}_{3}^{-}\right]}{\left[\mathrm{Cl}^{-}\right]}\right) \\
& \begin{array}{lllll}
\text { Temp } & \mathrm{pH} & {[\mathrm{Cl}]} & {\left[\mathrm{NO}_{3}\right]} & \mathrm{E}_{\mathrm{odr}}
\end{array} \\
& \begin{array}{llll}
\left({ }^{\circ} \mathrm{C}\right) & (\mathrm{m}) & (\mathrm{m}) & \text { (mV SCC) }
\end{array}
\end{aligned}
$$

\begin{tabular}{|c|c|c|c|c|c|}
\hline & 0 & 1 & 2 & 3 & 4 \\
\hline 0 & 90.000 & 10.910 & 0.214 & 0.114 & -32.000 \\
\hline 1 & 90.000 & $10.910^{?}$ & 0.214 & 0.114 & -55.000 \\
\hline 2 & 60.000 & $3.140^{-1}$ & 0.765 & 0.372 & 380.000 \\
\hline 3 & 60.000 & $3.140^{\circ}$ & $0.765^{\circ}$ & 0.372 & 408.000 \\
\hline 4 & 60.000 & 9.660 & $3.300 \cdot 10^{-3}$ & $9.730 \cdot 10-4$ & 54.200 \\
\hline 5 & 60.000 & 9.660 & $3.300 \cdot 10^{-3}$ & $9.730 \cdot 10^{-4}$ & 31.100 \\
\hline 6 & 90.000 & 8.500 & $3.250 \cdot 10^{-3}$ & $3.130 \cdot 10^{-4}$ & 81.300 \\
\hline 7 & 90.000 & 8.500 & $3.250^{0} 10^{-3}$ & $3.130 \cdot 10^{-4}$ & 108.000 \\
\hline 8 & 120.000 & 4.920 & $11.988^{\prime}$ & $1.000 \cdot 10^{-B}$ & -126.000 \\
\hline 9 & 120.000 & 4.920 & 11.988 & $1.000 \cdot 10^{-8}$ & -139.000 \\
\hline 10 & 120.000 & $4.920^{\circ}$ & $11.988^{-}$ & $1.000 \cdot 10^{-8}$ & -150.000 \\
\hline 11 & 120.000 & 4.920 & $11.988^{\circ}$ & $1.000^{10}-8$ & -126.000 \\
\hline 12 & 120.000 & $4.920^{\circ}$ & $11.888^{\circ}$ & $1.000 .10 \cdot 8$ & -133.000 \\
\hline 13 & $\infty 0.000$ & $4.170^{\circ}$ & $0.809^{i}$ & 0.402 & 387.000 \\
\hline 14 & 90.000 & $2.800^{\circ}$ & 0.782 & 0.400 & 401.000 \\
\hline 15 & 90.000 & 2.800 & 0.782 & 0.400 & 400.000 \\
\hline 16 & 90.000 & 2.800 & 0.782 & 0.400 & 401.000 \\
\hline 17 & 90.000 & 2.800 & 0.782 & 0.400 & 401.000 \\
\hline 18 & 90.000 & 2.800 & 0.782 & 0.400 & 401.000 \\
\hline
\end{tabular}

$$
\begin{aligned}
& \left.r Y 3:=D_{a t 3^{4}}{ }^{4}\right\rangle
\end{aligned}
$$

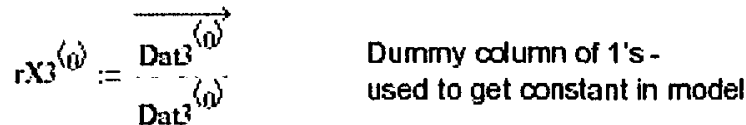

$$
\begin{aligned}
& \mathrm{rXo}^{(1)}:=\mathrm{Dat}^{(0)} \quad \text { Temp }{ }^{\mathrm{C}} \mathrm{C} \\
& \mathrm{rX3}^{(2)}=\mathrm{Dat}^{\left(\mathrm{S}^{3}\right.} \quad \mathrm{pH} \\
& \mathrm{rX3}^{(3)}:=\mathrm{Dat} 3^{(2)} \quad[\mathrm{Cl}] \\
& \mathrm{rX}^{\langle 4\rangle}:=\log \left(\begin{array}{l}
\overrightarrow{\mathrm{Dat}_{3}(3)} \\
\left.\mathrm{Dat}^{\langle 3}\right)
\end{array} \quad \log ([\mathrm{NO}] /[\mathrm{Cl}])\right.
\end{aligned}
$$


Regression Coefficients

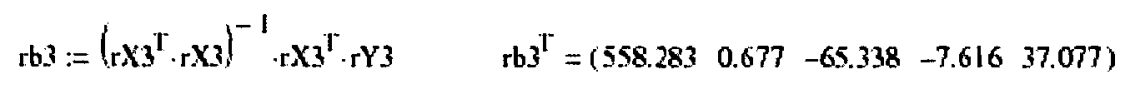

Covariance

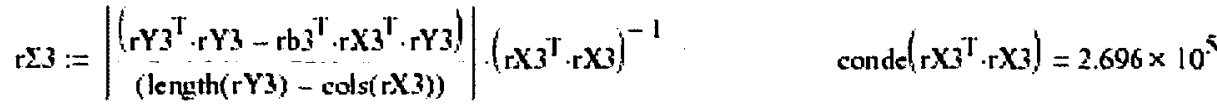

$$
\begin{aligned}
& r \Sigma 3=\left(\begin{array}{ccccc}
1.307 \mathrm{E}+003 & -1.345 \mathrm{E}+001 & -2.595 \mathrm{E}+001 & 3.751 \mathrm{E}+000 & -2.149 \mathrm{E}+001 \\
-1.245 \mathrm{E}+001 & 1.708 \mathrm{E}-001 & -8.595 \mathrm{E}-002 & -1.678 \mathrm{E}-001 & 2.599 \mathrm{E}-001 \\
-2.595 \mathrm{E}+001 & -8.595 \mathrm{E}-002 & 6.033 \mathrm{E}+000 & 7.349 \mathrm{E}-001 & 6.611 \mathrm{E}-001 \\
3.751 \mathrm{E}+000 & -1.678 \mathrm{E}-001 & 7.349 \mathrm{E}-001 & 2.501 \mathrm{E}+000 & 1.510 \mathrm{E}+000 \\
-2.149 \mathrm{E}+001 & 2.599 \mathrm{E}-001 & 6.611 \mathrm{E}-001 & 1.510 \mathrm{E}+000 & 5.969 \mathrm{E}+000
\end{array}\right)
\end{aligned}
$$

$$
\begin{aligned}
& \text { SStot } 3:=\sum(\mathrm{rY} 3-\operatorname{mean}(\mathrm{rY} 3))^{2} \quad \text { SStot } 3=3.476 \times 10^{6} \quad \text { Sum of Squares total } \\
& \text { SSres3 := } \sum(\mathrm{rY} 3-\mathrm{rX3} \cdot \mathrm{rb} 3)^{2} \quad \text { SSres3 }=1.844 \times 10^{5} \quad \text { Sum of Squares residual } \\
& \text { SSreg } 3:=\sum\left(\mathrm{rX} 3 \cdot \mathrm{rb} 3-\text { mean }(\mathrm{rY} 3)^{2} \quad \text { SSreg } 3=3.291 \times 10^{6} \quad\right. \text { Sum of Squares regression } \\
& R_{s q 3}:=\frac{\text { SSreg } 3}{\text { SStot3 }} \quad R_{s q 3}=0.947 \quad \text { Coefficient of determination }
\end{aligned}
$$

Ecorr(T.pH.Cl. NO3.z):= ${ }^{C} \mathrm{Cl} 2 \leftarrow \max (0.001 . \min (\mathrm{Cl} .36)) \quad \sqrt{\mathrm{Rsq}^{3}}=0.973$

$$
\begin{aligned}
& \mathrm{pH} \leftarrow \min (\mathrm{pH}, 10.9) \\
& \mathrm{R} 2 \leftarrow \min \left(0.5, \frac{0.001}{\mathrm{Cl}}\right) \text { if } \mathrm{NO3}<0.001 \\
& \min \left(0.5 \cdot \frac{\mathrm{NO} 3}{\mathrm{Cl}}\right) \text { otherwise } \\
& \mathrm{Xc} \leftarrow(1 \mathrm{~T} \mathrm{pHC12} \log (\mathrm{R} 2))
\end{aligned}
$$

$\mathrm{Np}:=100 \quad$ Number of points at which to evaluate is $\mathrm{Np}+1$ (indexing starts at zero)

$\mathrm{nn}:=0 . \mathrm{Np} \quad$ Range variable takes integer values between zero and $\mathrm{Np}$ 

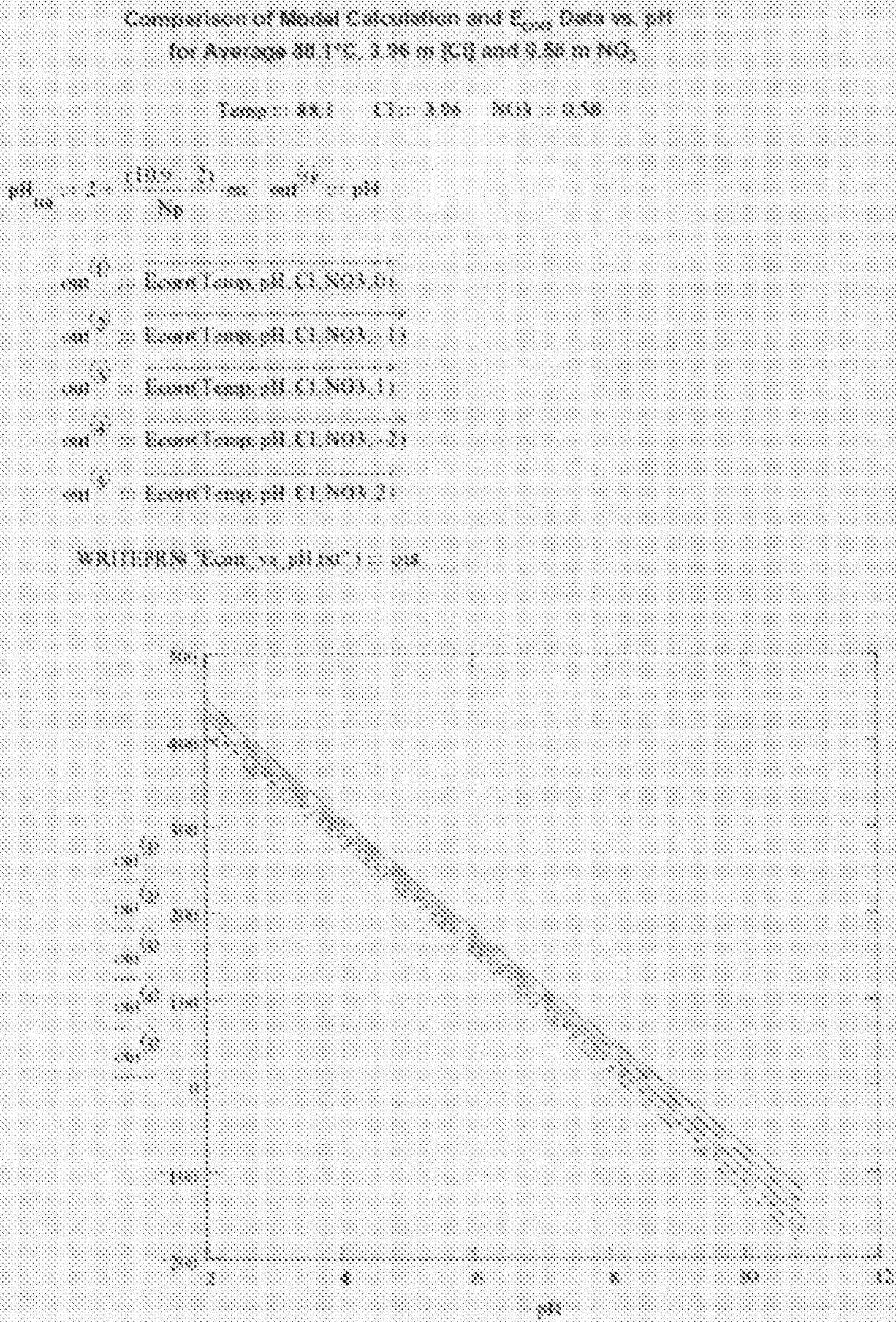


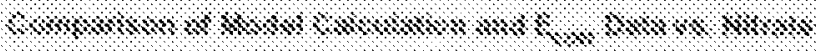

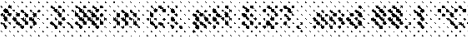
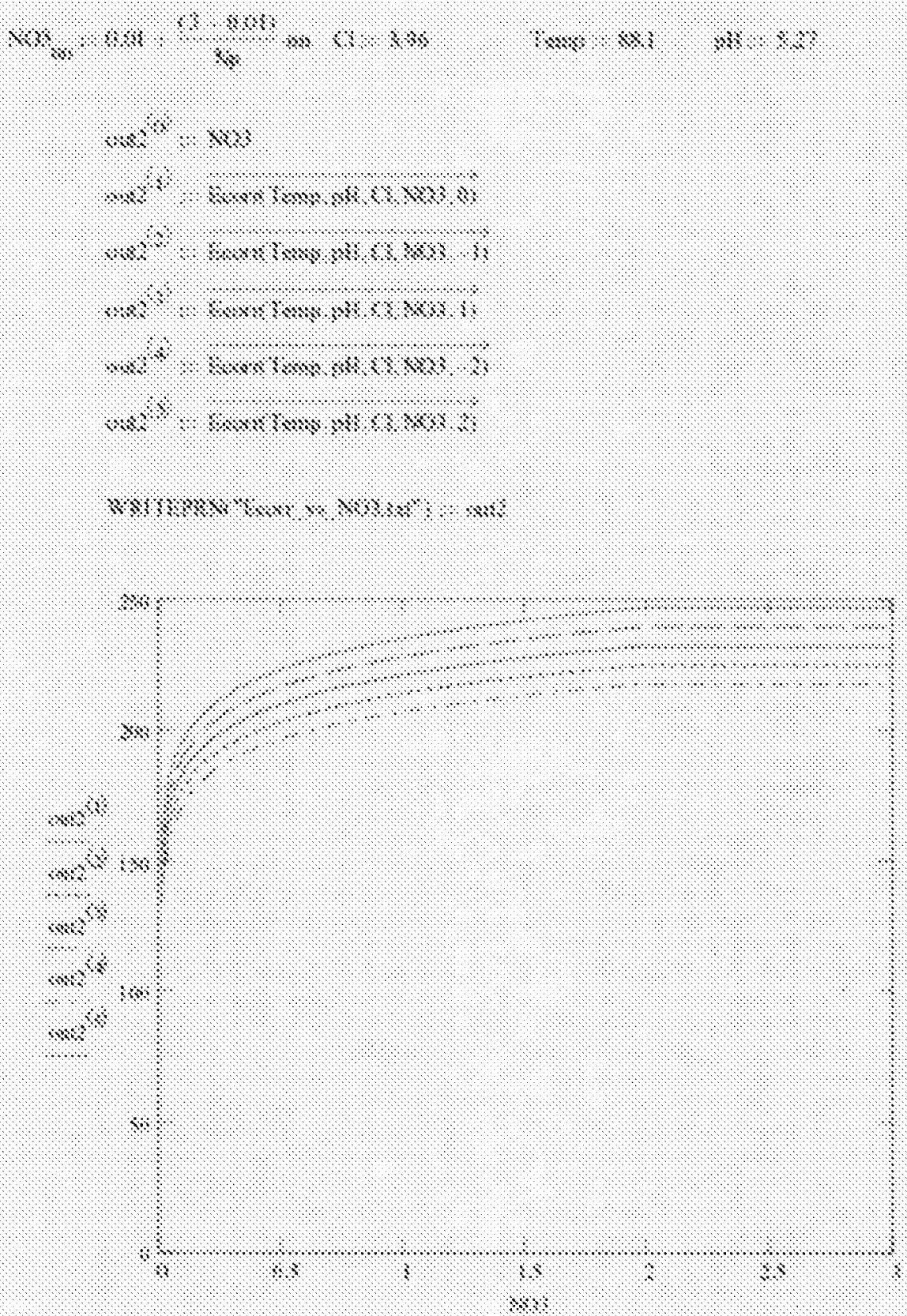


\section{IX.4 ERCREV_V_ECORR.MCD}

$E_{m v}^{o}=a_{0}+a_{1} T+a_{2} p H+a_{3} \log \left(\left[C l^{-}\right]\right)+a_{4} T \times \log \left(\left[C l^{-}\right]\right)$

Regression coefficients and covariance matirx are:

$$
\begin{aligned}
& \mathrm{\omega}:=\left(\begin{array}{c}
214.089 \\
-3.696 \\
25.284 \\
-252.181 \\
1.414
\end{array}\right) \quad \mathrm{I}:=\left(\begin{array}{ccccc}
2.198 \mathrm{E}+003 & -1.516 \mathrm{E}+001 & -8.325 \mathrm{E}+001 & -1.805 \mathrm{E}+003 & 1.590 \mathrm{E}+001 \\
-1.516 \mathrm{E}+001 & 2.267 \mathrm{E}-001 & -1.240 \mathrm{E}+000 & 1.877 \mathrm{E}+001 & -1.996 \mathrm{E}-001 \\
-8.325 \mathrm{E}+001 & -1.240 \mathrm{E}+000 & 3.183 \mathrm{E}+001 & -3.237 \mathrm{E}+001 & 7.425 \mathrm{E}-001 \\
-1.805 \mathrm{E}+003 & 1.877 \mathrm{E}+001 & -3.237 \mathrm{E}+001 & 2.907 \mathrm{E}+003 & -2.868 \mathrm{E}+001 \\
1.590 \mathrm{E}+001 & -1.996 \mathrm{E}-001 & 7.425 \mathrm{E}-001 & -2.868 \mathrm{E}+001 & 2.995 \mathrm{E}-001
\end{array}\right) \\
& \Delta E_{\mathrm{ran}}^{\mathrm{No}_{3}}=b_{a}+b_{1}\left[\mathrm{NO}_{3}^{-}\right]+b_{2} \frac{\left[\mathrm{NO}_{3}^{-}\right]}{\left[\mathrm{Cl}^{-}\right]}
\end{aligned}
$$

Regression coefficients are:

$$
\begin{aligned}
& \mathrm{rb} 2:=\left(\begin{array}{l}
22.589 \\
33.748 \\
749.745
\end{array}\right) \\
& E_{\text {iesr }}=c_{a}+c_{1} T+c_{2} p H+c_{3}\left[\mathrm{Cl}^{-}\right]+c_{4} \log \left(\frac{\left.\mathrm{NO}_{3}^{-}\right]}{\left[\mathrm{Cl}^{-}\right]}\right)
\end{aligned}
$$

Regression coefficients and covariance matirx are:

$$
\mathrm{rb3}:=\left(\begin{array}{c}
558.283 \\
0.677 \\
-65.338 \\
-7.616 \\
37.077
\end{array}\right) \quad \Sigma \Sigma 3:=\left(\begin{array}{ccccc}
1.307 \mathrm{E}+003 & -1.345 \mathrm{E}+001 & -2.595 \mathrm{E}+001 & 3.751 \mathrm{E}+000 & -2.149 \mathrm{E}+001 \\
-1.345 \mathrm{E}+001 & 1.708 \mathrm{E}-001 & -8.595 \mathrm{E}-002 & -1.678 \mathrm{E}-001 & 2.599 \mathrm{E}-001 \\
-2.595 \mathrm{E}+001 & -8.595 \mathrm{E}-002 & 6.033 \mathrm{E}+000 & 7.349 \mathrm{E}-001 & 6.61 \mathrm{EE}-001 \\
3.751 \mathrm{E}+000 & -1.678 \mathrm{E}-001 & 7.349 \mathrm{E}-001 & 2.501 \mathrm{E}+000 & 1.510 \mathrm{E}+\infty 00 \\
-2.149 \mathrm{E}+001 & 2.599 \mathrm{E}-001 & 6.611 \mathrm{E}-001 & 1.510 \mathrm{E}+000 & 5.969 \mathrm{E}+\infty 00
\end{array}\right)
$$




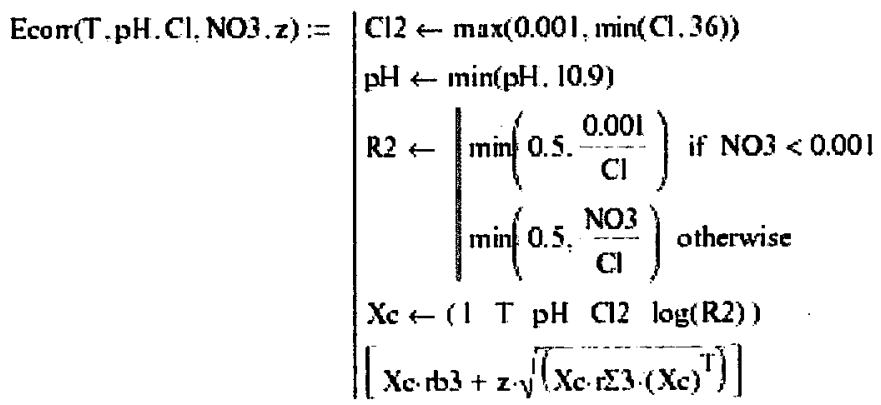

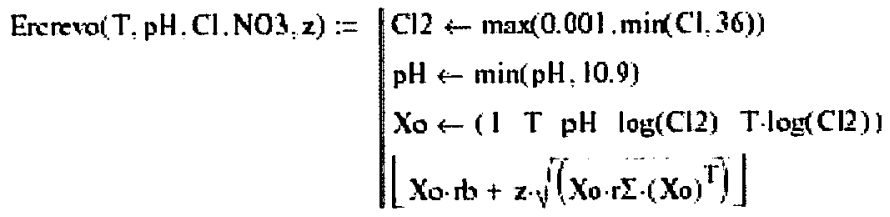

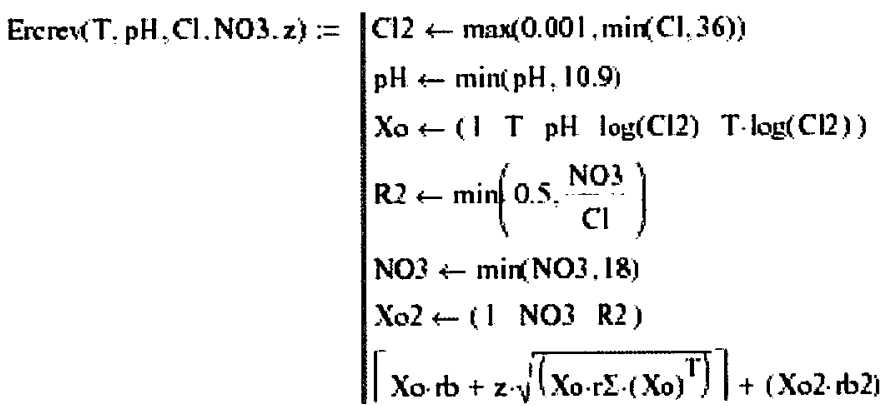

Ecrit(T.pH.Cl.NO3,z):= $\begin{aligned} & \text { Ercrevo(T.pH.Cl.NO3.z) if NO3 }<0.001 \\ & \text { Ercren(T.pH.Cl.NO3.z) otherwise }\end{aligned}$

$N p:=100$

Number of points at which to evaluate is Np+1 (indexing starts at zero)

$\mathrm{nn}:=0 . . \mathrm{Np}$

Range variable takes integer values between zero and Np 


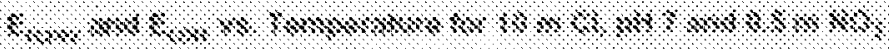
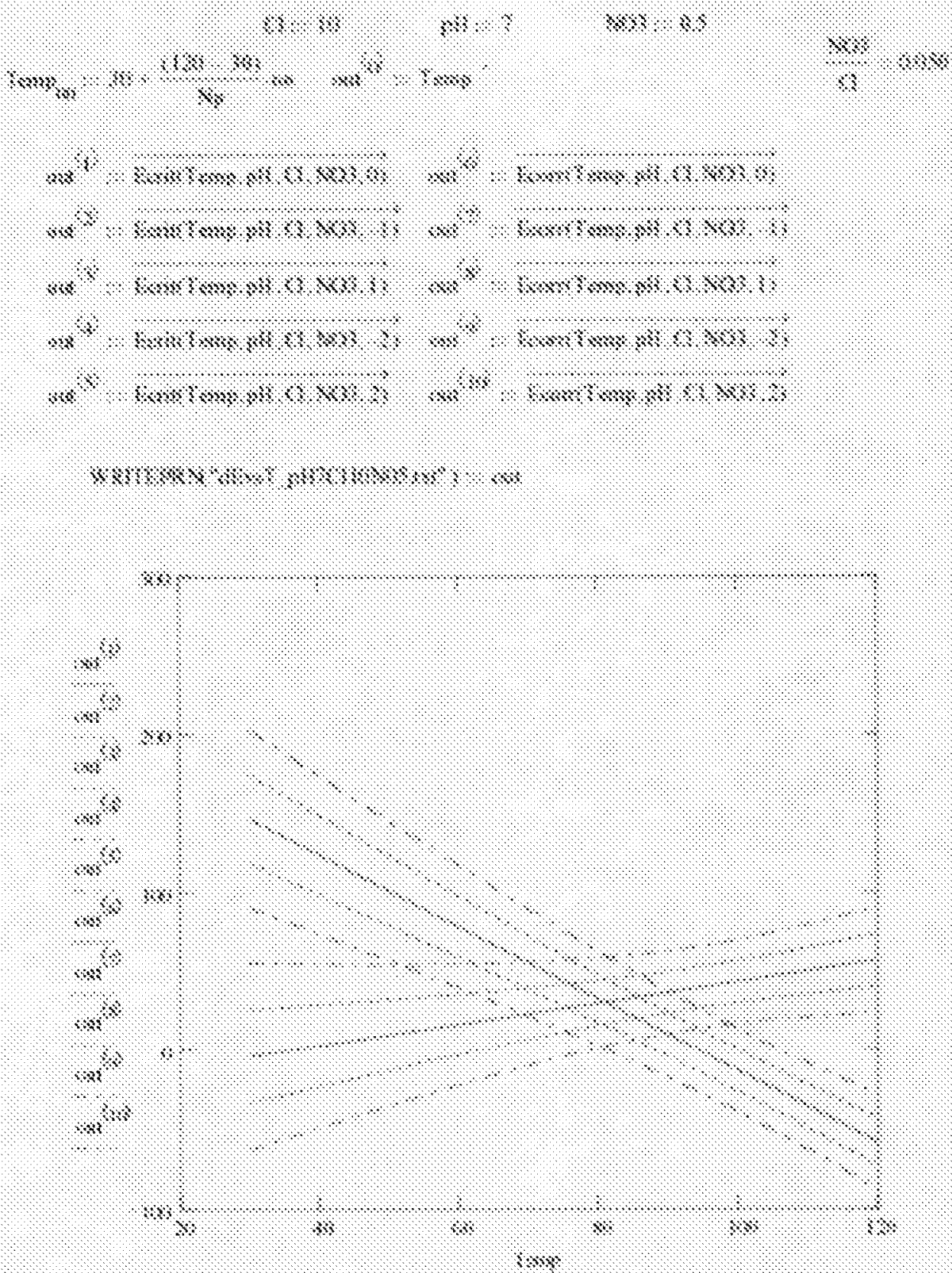


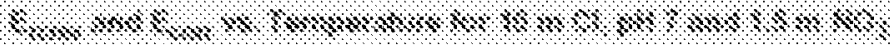

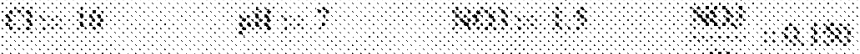

$$
\begin{aligned}
& \text { r } \\
& \text { l } \\
& \text { - } \\
& \text { l } \\
& \text { - } \\
& \text { \$ }
\end{aligned}
$$

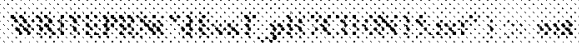

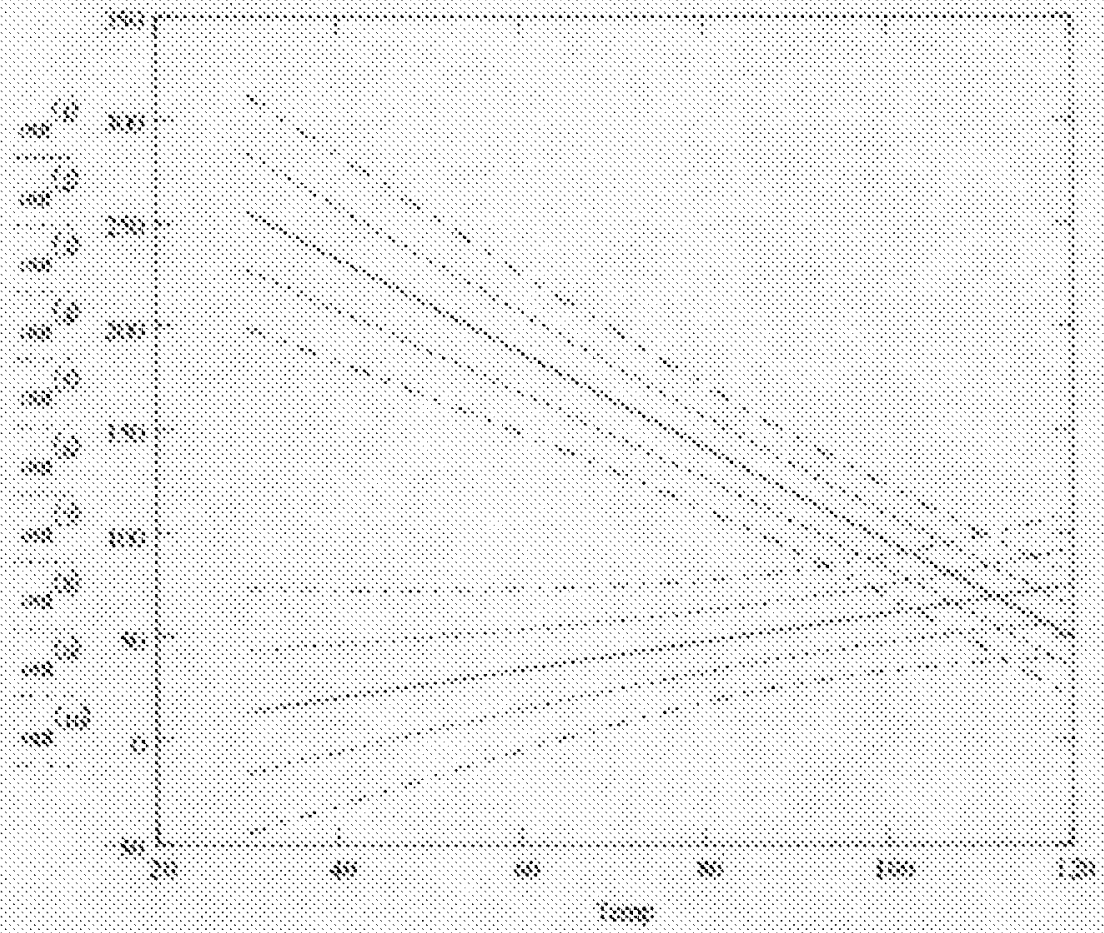




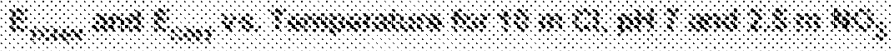

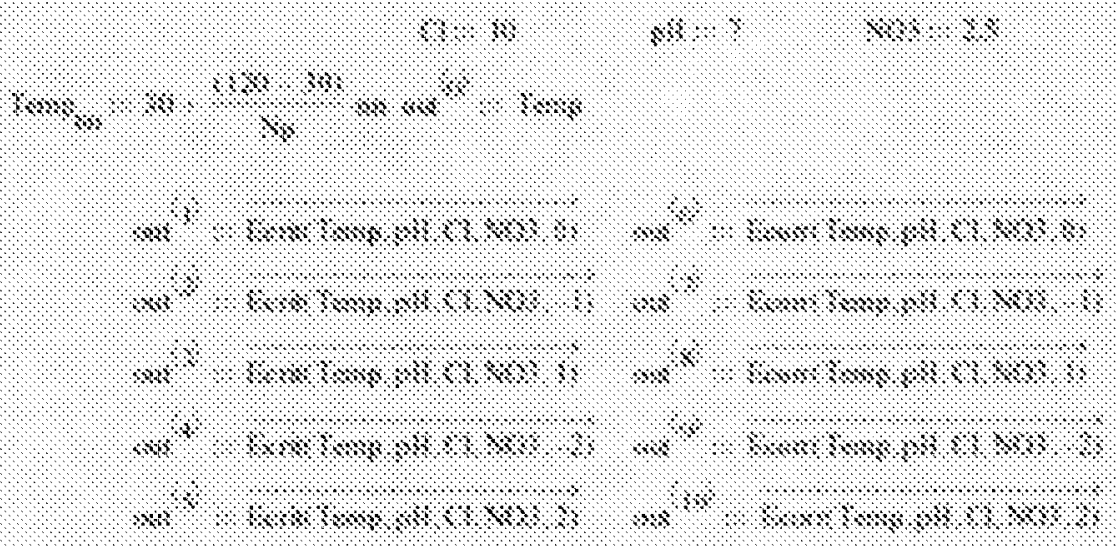

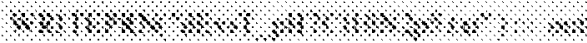

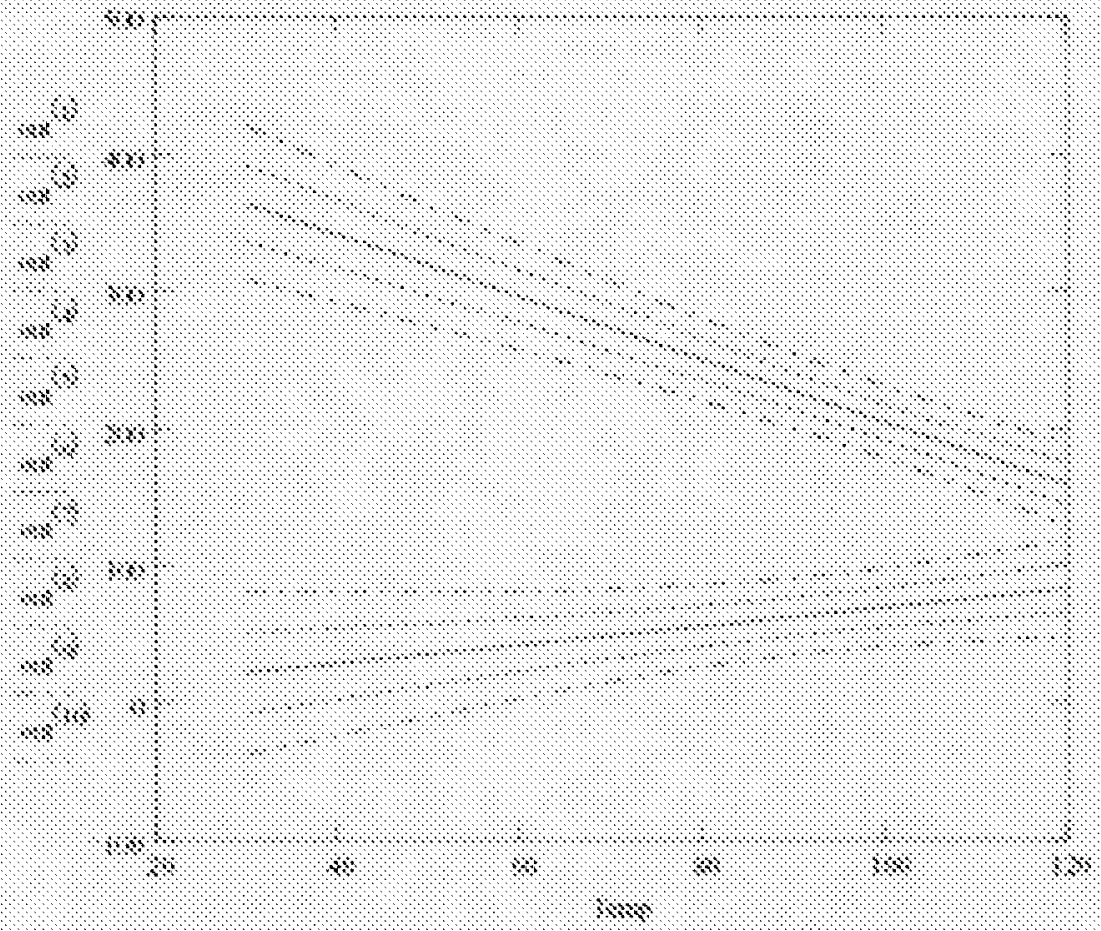




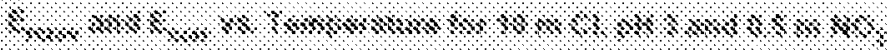

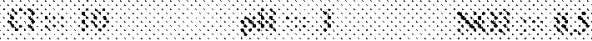

$$
\begin{aligned}
& \text { - } \\
& \text { - }
\end{aligned}
$$

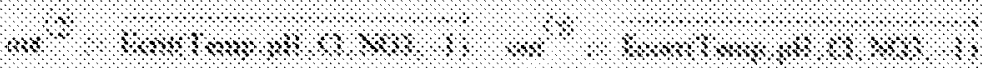

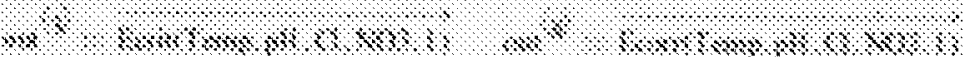

$$
\begin{aligned}
& \text { s } \\
& \text { s }
\end{aligned}
$$

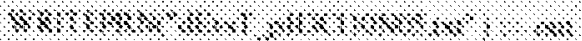

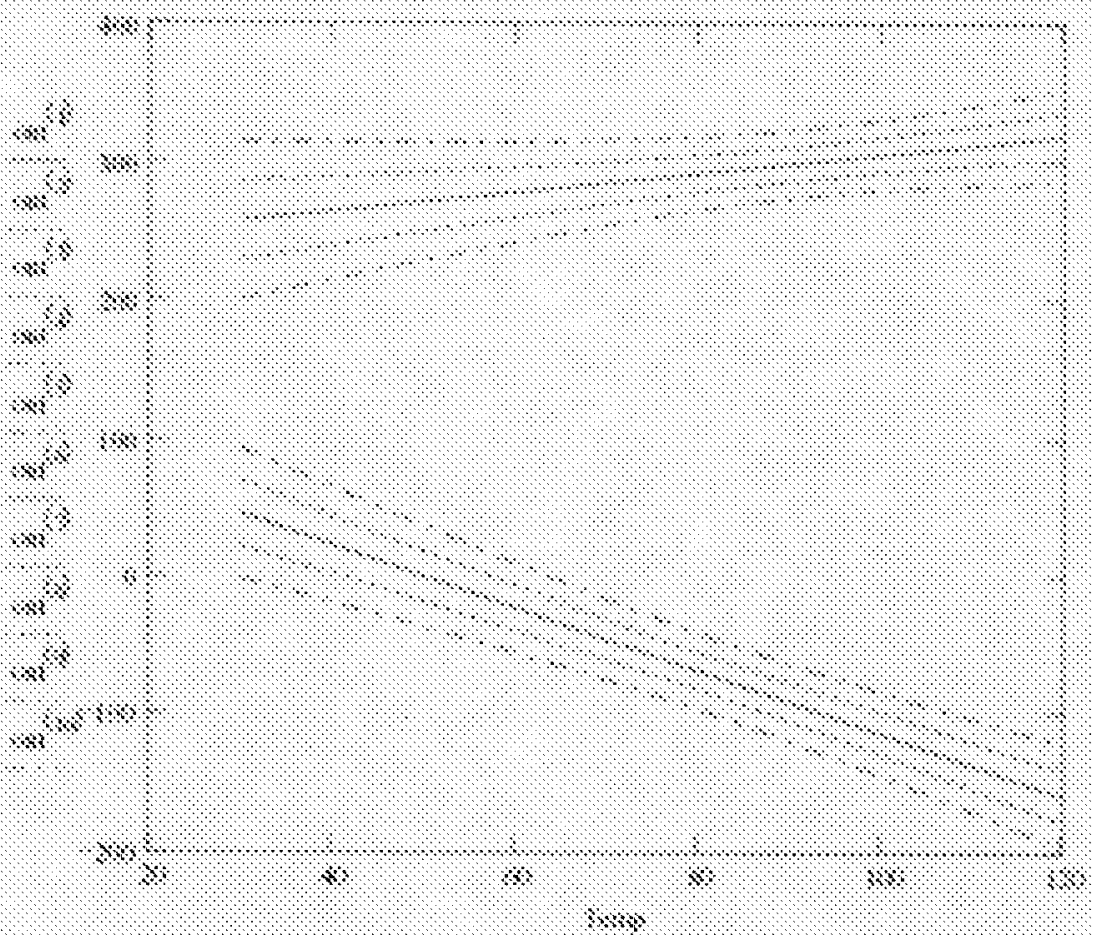




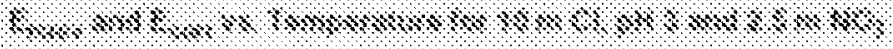

$$
\begin{aligned}
& \text { ४ } \\
& \text {. } \\
& \text { - } \\
& \text { - } \\
& \text { _ } \\
& \text { - } 1 \text { l. } \\
& \text { - }
\end{aligned}
$$

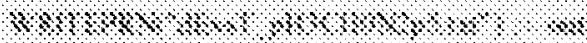

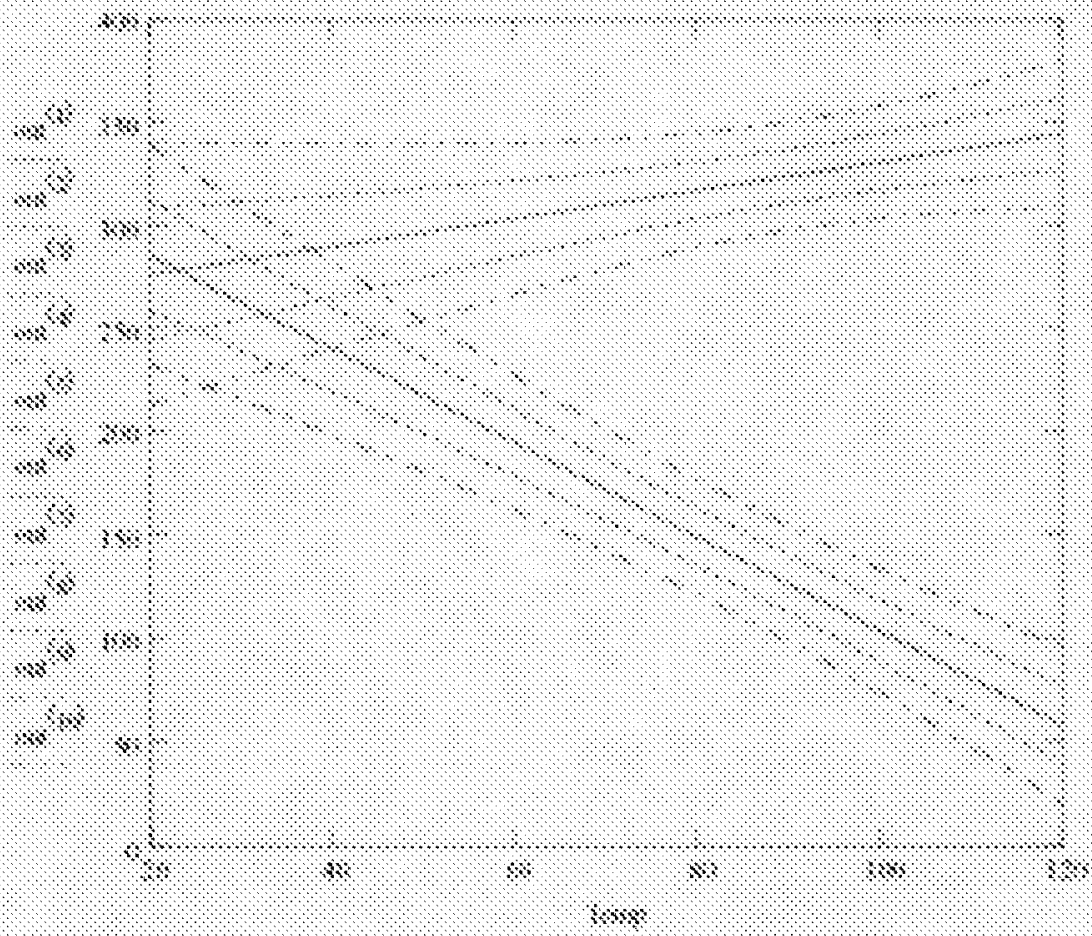


General Corrosion and Localized Corrosion of Waste Package Outer Barrier

\begin{tabular}{|c|c|c|c|c|c|c|c|c|c|c|c|c|c|c|c|c|c|c|c|c|c|c|c|c|c|}
\hline 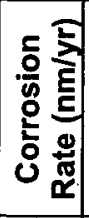 & & 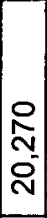 & $\begin{array}{c}\mathbb{N} \\
\infty \\
\infty \\
\infty \\
\infty\end{array}$ & $\left|\begin{array}{c|}8 \\
0 \\
\infty \\
\infty\end{array}\right|$ & $\stackrel{\infty}{\sigma}$ & $\stackrel{\text { \& }}{\circ}$ & ळ్ & $\bar{\Sigma}$ & 点 & $\stackrel{8}{\square}$ & 芯 & $\stackrel{\bar{\alpha}}{\stackrel{-}{\circ}}$ & 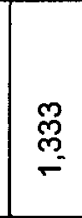 & $\stackrel{m}{0}$ & চ্ & $\frac{N}{\sigma}$ & $\frac{8}{\mathscr{\rho}}$ & $\frac{\varphi}{\sigma}$ & $\frac{8}{8}$ & $\underset{\infty}{\infty}$ & & $=$ & & & $\begin{array}{l} \\
\end{array}$ \\
\hline 짐 & & $\frac{\nabla}{\square}$ & $\mid \begin{array}{l}\nabla \\
\dot{0}\end{array}$ & $\mid \begin{array}{l} \pm \\
\dot{0}\end{array}$ & $\begin{array}{l}\bar{\infty} \\
\infty \\
\dot{0}\end{array}$ & $\begin{array}{l}\bar{\infty} \\
0 \\
0\end{array}$ & $\begin{array}{l}\bar{\infty} \\
\infty \\
0 \\
\end{array}$ & 뭇 & סั & ઼ָ & fo & $\begin{array}{l}\text { f } \\
0\end{array}$ & $\underset{8}{\tilde{f}}$ & $\begin{array}{l}\mathbb{J} \\
\dot{ \pm}\end{array}$ & $\underset{J}{\mathbb{O}}$ & $\begin{array}{l}J \\
0 \\
\dot{y}\end{array}$ & 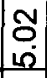 & $\begin{array}{c}\widetilde{O} \\
0 \\
10\end{array}$ & $\begin{array}{c}2 \\
0 \\
-10\end{array}$ & $\frac{m}{6}$ & $\begin{array}{c}m \\
0 \\
0\end{array}$ & 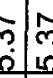 & $\begin{array}{c}n \\
\vdots \\
0\end{array}$ & & $\left.\right|_{10} ^{N}$ \\
\hline $5 \stackrel{9}{5}$ & 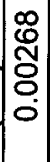 & 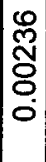 & 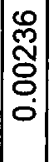 & 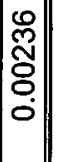 & 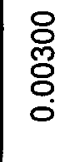 & 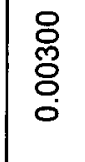 & 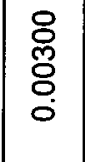 & $\begin{array}{l}8 \\
8 \\
8 \\
\\
0\end{array}$ & 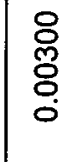 & 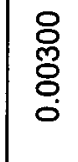 & 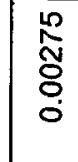 & 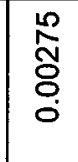 & 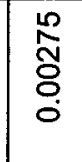 & 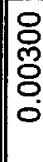 & $\begin{array}{l}8 \\
\varnothing \\
\\
\\
\end{array}$ & $\begin{array}{l} \\
\\
\\
0 \\
0 \\
0\end{array}$ & $\begin{array}{l} \\
\\
\\
0 \\
0 \\
0 \\
\end{array}$ & $\begin{array}{l}8 \\
8 \\
\varnothing \\
0 \\
0 \\
0\end{array}$ & $\begin{array}{l}8 \\
8 \\
0 \\
0 \\
0\end{array}$ & 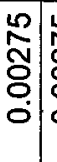 & 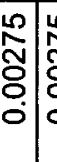 & 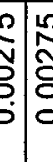 & 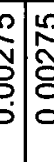 & & 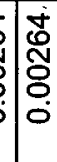 \\
\hline है & & 욤 & 10 & 욤 & 8 & 8 & 8 & 8 & 8 & 8 & 8 & 8 & 8) & 8 & 8 & 8 & 8 & 8 & 8 & ৪ & ৪) & 5 & s. & $\stackrel{2}{\circ}$ & $\%$ \\
\hline : & & $\begin{array}{l}\frac{N}{0} \\
\mathcal{D} \\
\vdots \\
\delta\end{array}$ & $\left|\begin{array}{c}\mathbb{N} \\
\mathbb{D} \\
0 \\
\vdots \\
\vdots\end{array}\right|$ & $\mid$ & 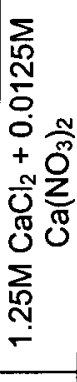 & 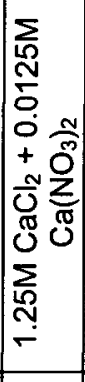 & 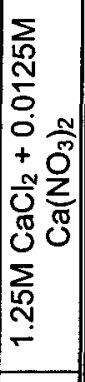 & 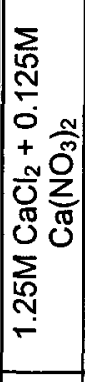 & 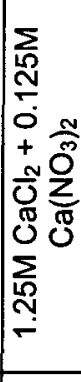 & 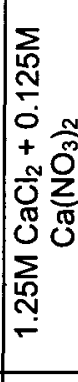 & 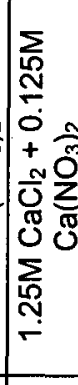 & 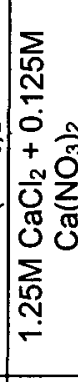 & 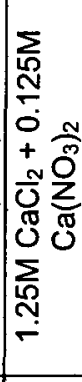 & 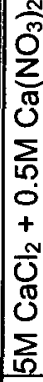 & 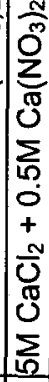 & 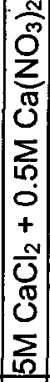 & \begin{tabular}{|c|}
0 \\
0 \\
0 \\
0 \\
0 \\
$\sum$ \\
10 \\
0 \\
0 \\
+ \\
0 \\
0 \\
0 \\
0 \\
$\sum_{10}$ \\
\end{tabular} & 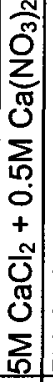 & 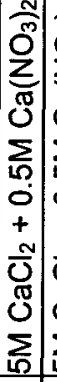 & 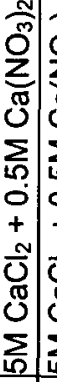 & 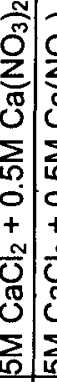 & 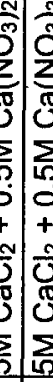 & 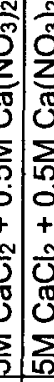 & 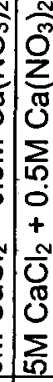 & 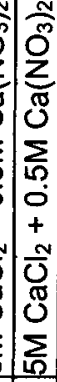 \\
\hline 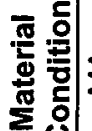 & $\sum$ & 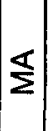 & 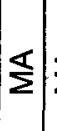 & $\frac{\mathbb{s}}{\Sigma}$ & $£$ & $\sum$ & $\stackrel{\mathbb{L}}{\Sigma}$ & 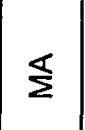 & $\stackrel{\mathbb{S}}{\Sigma}$ & 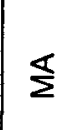 & $\Sigma$ & $\S$ & 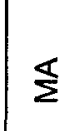 & $\frac{\S}{\Sigma}$ & $\Sigma$ & $\Sigma$ & $\frac{\varsigma}{\Sigma}$ & 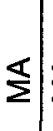 & $\Sigma$ & $\stackrel{\Sigma}{\Sigma}$ & $\Sigma \mid \S$ & 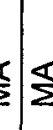 & $\frac{5}{\Sigma}$ & 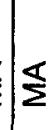 & $\sum$ \\
\hline 尊 & & 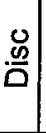 & $\mid$ & . & $\frac{J}{S}$ & $\frac{\overleftarrow{S}}{\Sigma}$ & 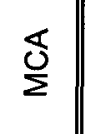 & $\stackrel{\delta}{\delta}$ & $\stackrel{\delta}{S}$ & $\stackrel{\delta}{\delta}$ & $\underset{J}{\Sigma}$ & $\underset{\delta}{\Sigma}$ & $\stackrel{\delta}{\Sigma}$ & $\frac{\delta}{\Sigma}$ & 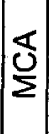 & $\mid$ & 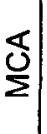 & $\frac{\delta}{\delta}$ & $\mathbb{S}_{\Sigma}^{\mathbb{S}}$ & 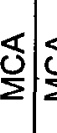 & $\frac{\delta}{\delta} \mid \overleftarrow{c}$ & $\frac{\delta}{\Sigma}$ & $\frac{1}{S}$ & $\frac{\delta}{\Sigma}$ & $\frac{\delta}{S}$ \\
\hline 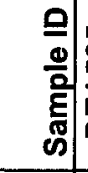 & 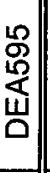 & & $\begin{array}{l}\mathscr{9} \\
\stackrel{0}{0} \\
\stackrel{4}{0} \\
\end{array}$ & 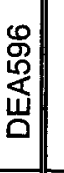 & 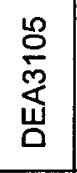 & 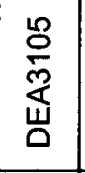 & 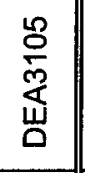 & 号 & $\begin{array}{l}\stackrel{\infty}{0} \\
\text { 岗 } \\
\text { 岁 }\end{array}$ & 号 & 竞 & 总 & $\frac{9}{\frac{8}{m}}$ & 容 & $\mid \begin{array}{l}\frac{5}{0} \\
\frac{\operatorname{mon}}{\omega} \\
0\end{array}$ & 富 & $\begin{array}{l}\frac{m}{0} \\
\frac{\operatorname{m}}{2} \\
\frac{u}{0}\end{array}$ & 空 & 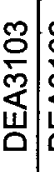 & 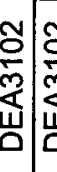 & 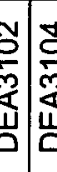 & $\frac{\square}{d}$ & 客 & 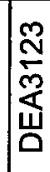 & 离 \\
\hline 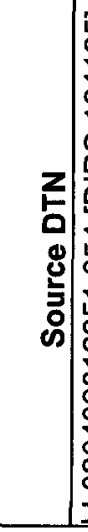 & 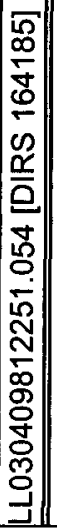 & 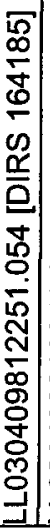 & 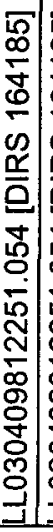 & 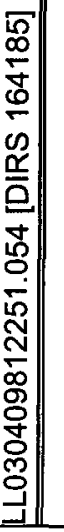 & 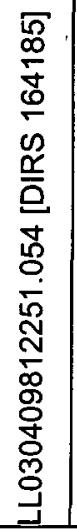 & 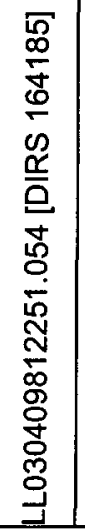 & 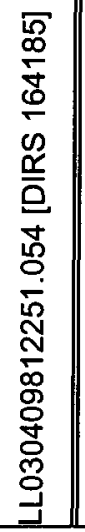 & 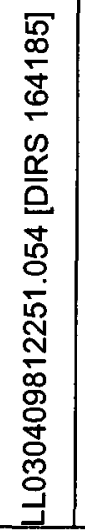 & 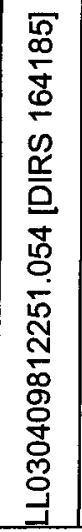 & 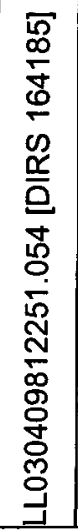 & 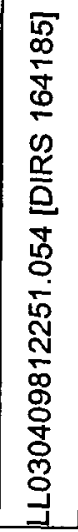 & 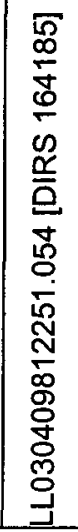 & 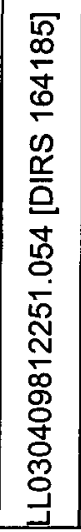 & 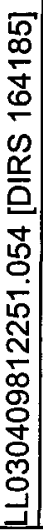 & 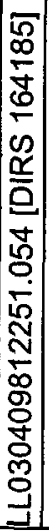 & 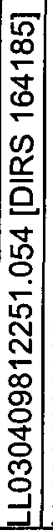 & 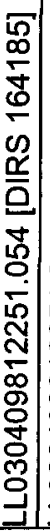 & 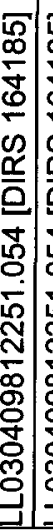 & 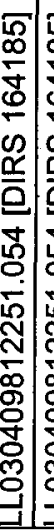 & 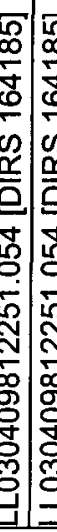 & 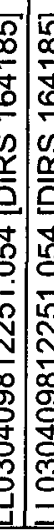 & 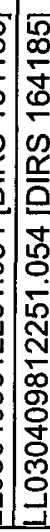 & 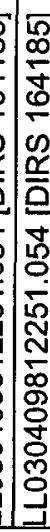 & 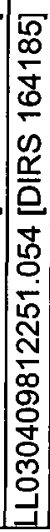 & 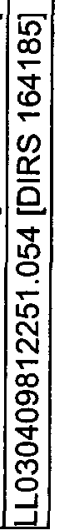 \\
\hline
\end{tabular}




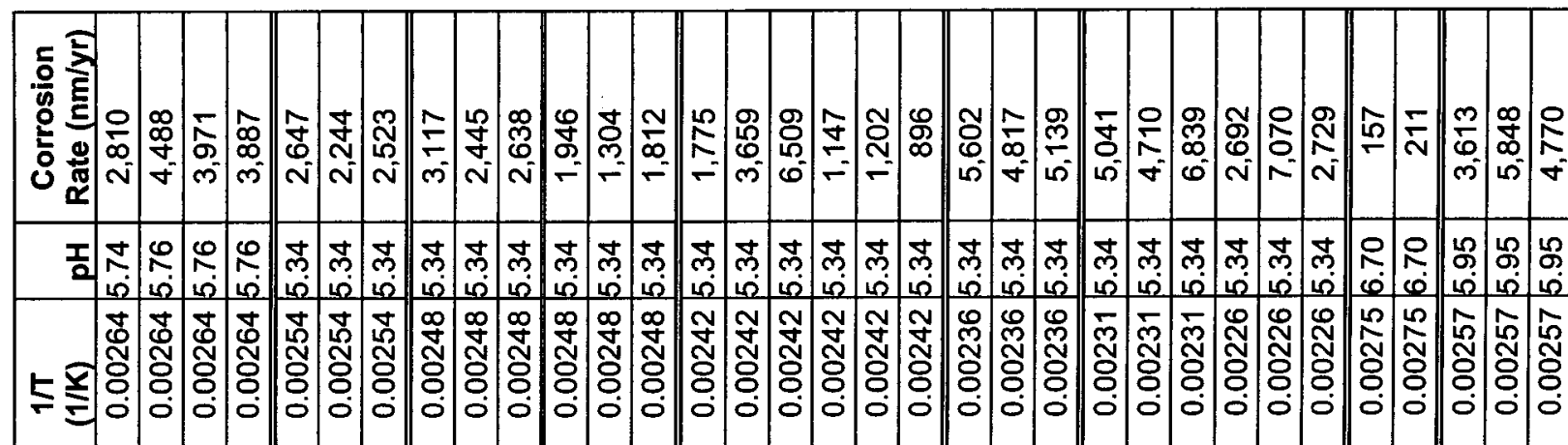

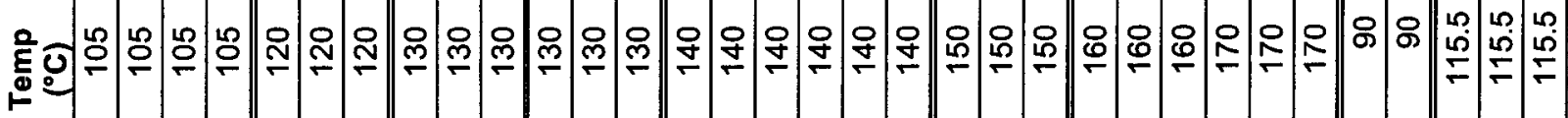

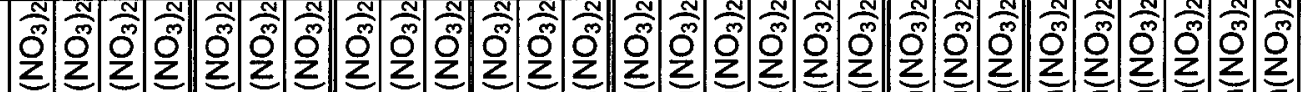

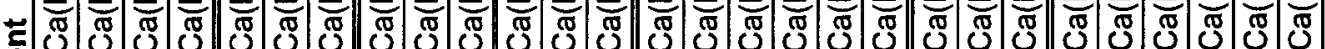

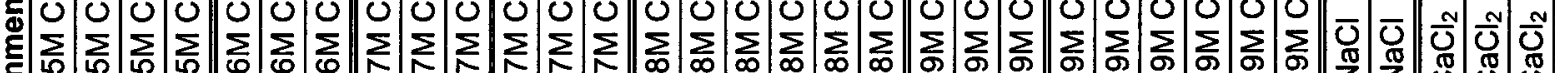

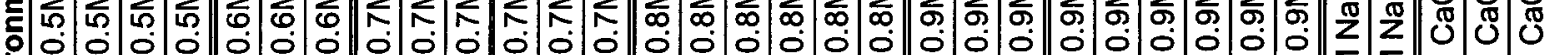

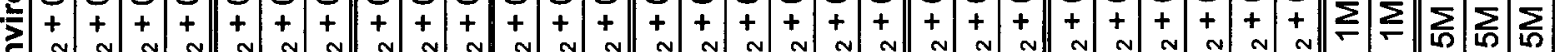

w

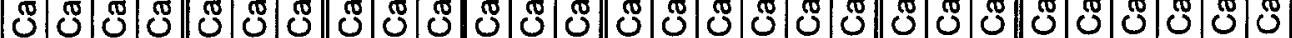

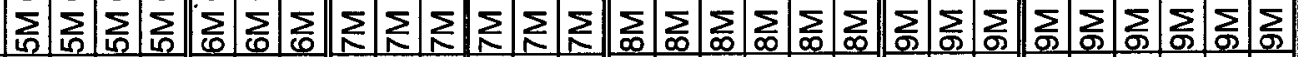

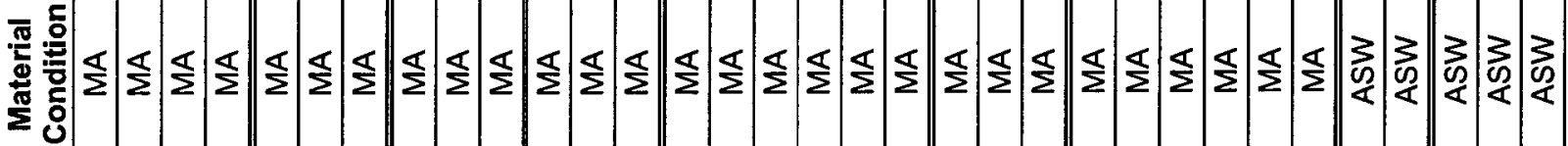

응 :

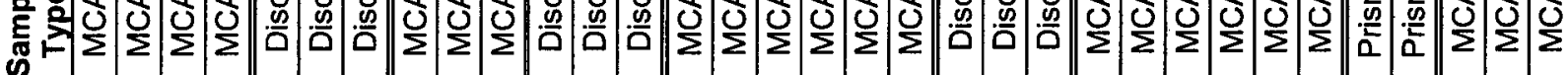

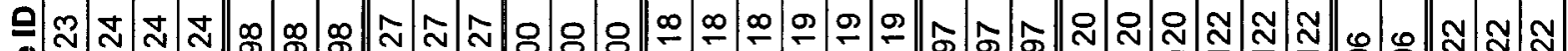

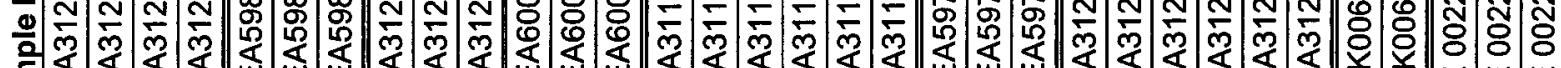
हึ

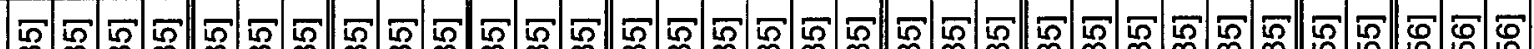

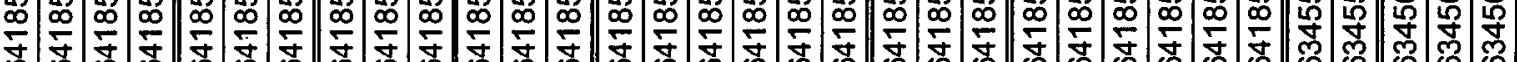
Oे

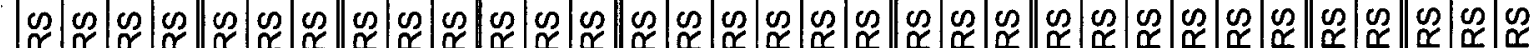

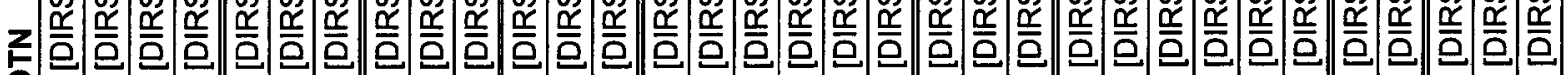

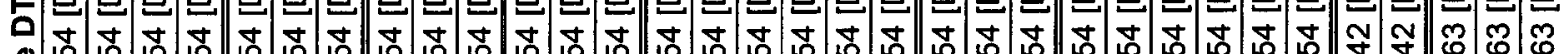
\&

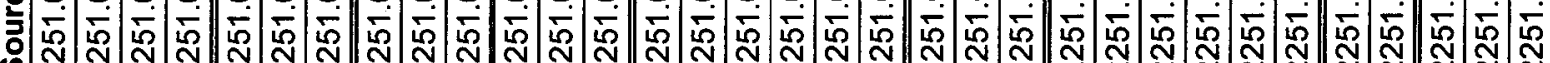

m 定

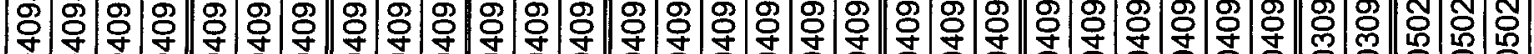

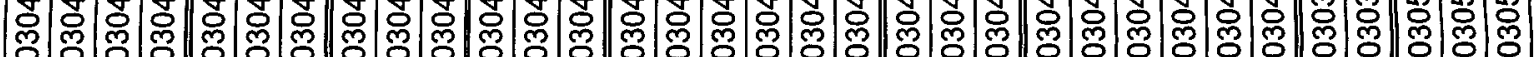

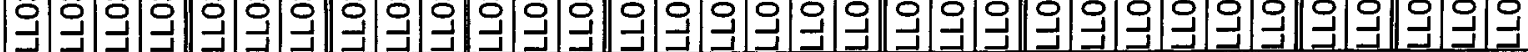




\begin{tabular}{|c|c|c|c|c|c|c|c|c|c|c|c|c|c|c|c|c|c|c|c|c|c|c|c|c|c|c|c|}
\hline 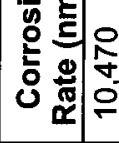 & & & 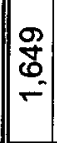 & 怘 & 今̂ & $\begin{array}{l}\mathscr{8} \\
\stackrel{0}{-}\end{array}$ & & E & 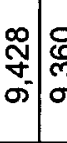 & $\begin{array}{lll} \\
0 \\
0\end{array}$ & $\left.\right|_{\infty} ^{\infty}$ & $\mid \begin{array}{l}\mathscr{\infty} \\
\mathbb{8} \\
\sim \\
\sim\end{array}$ & & : & $\mid$ & & & \begin{tabular}{l}
$f$ \\
$\stackrel{f}{t}$ \\
\hdashline
\end{tabular} & 告 & ్ి & ్ֻలి & $\begin{array}{l}3 \\
=\end{array}$ & $\mid \begin{array}{l}\infty \\
\substack{\infty \\
\sim}\end{array}$ & 占 & & $=\mid \begin{array}{c}\bar{N} \\
\mathfrak{N}\end{array}$ & $\left|\begin{array}{c}0 \\
\frac{9}{5} \\
0\end{array}\right|$ \\
\hline & & 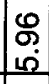 & $\mid \begin{array}{c}m \\
0 \\
0\end{array}$ & \begin{tabular}{|c|c}
2 \\
0 \\
0 \\
\end{tabular} & $\begin{array}{c}0 \\
2 \\
0\end{array}$ & $\begin{array}{c}\infty \\
\infty \\
0 \\
0\end{array}$ & \begin{tabular}{lll} 
& \multicolumn{1}{c}{} \\
$\infty$ & & \\
0 & 0 &
\end{tabular} & 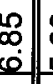 & 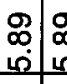 & 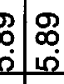 & 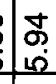 & 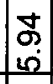 & 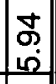 & $\begin{array}{l}1 \\
0 \\
0 \\
0\end{array}$ & 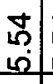 & 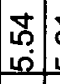 & 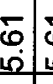 & 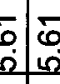 & ס & \begin{tabular}{|c|}
9 \\
0 \\
0 \\
\end{tabular} & \begin{tabular}{c|c}
9 & \multicolumn{1}{c}{} \\
& \\
\end{tabular} & & \begin{tabular}{|l}
0 \\
0 \\
0 \\
0 \\
\end{tabular} & & 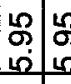 & 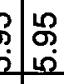 & $\underset{\substack{N\\
}}{\mathcal{N}}$ \\
\hline$=\stackrel{\bar{Y}}{=}$ & & 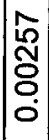 & 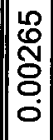 & $\mid$ & $\mid$ & 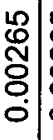 & & 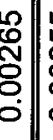 & 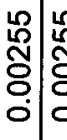 & 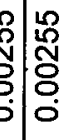 & 莡 & 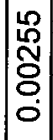 & 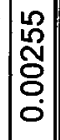 & $\mid$ & 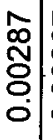 & 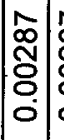 & 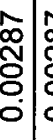 & 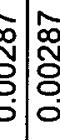 & 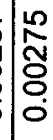 & 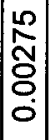 & 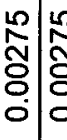 & 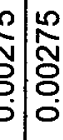 & 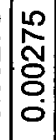 & 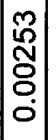 & 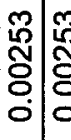 & 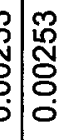 & 芯 \\
\hline
\end{tabular}

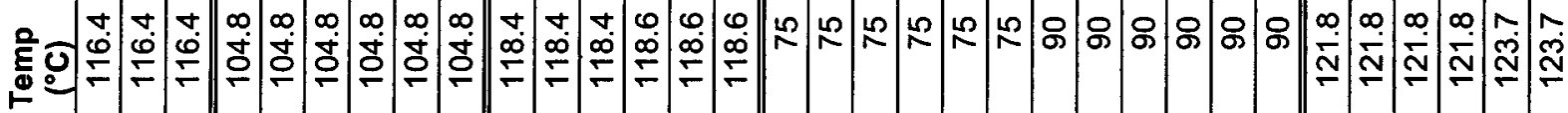

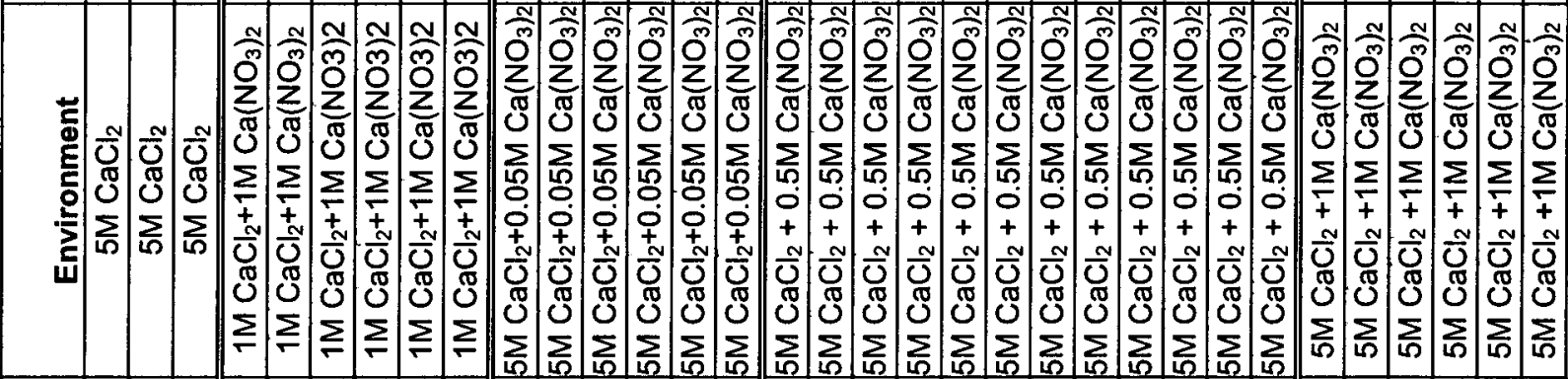

증

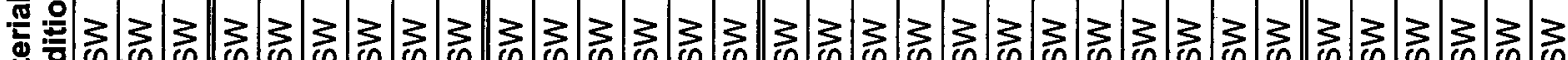
要

:

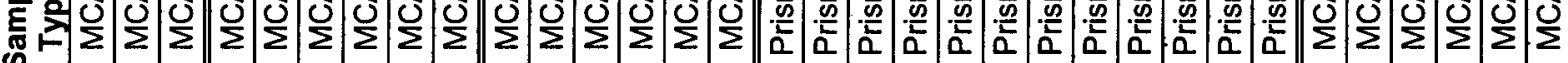

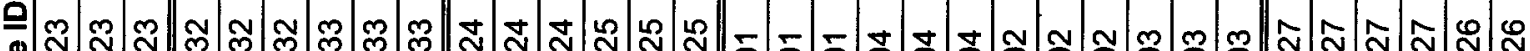
ఊ

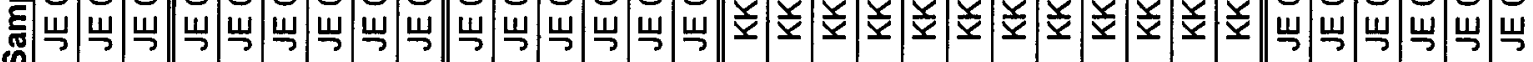

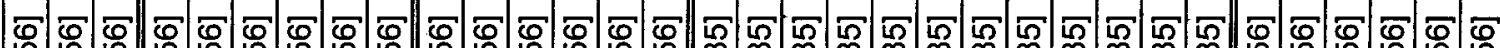
乌ᄂ రీ

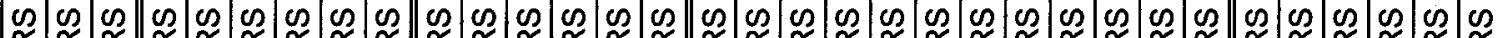

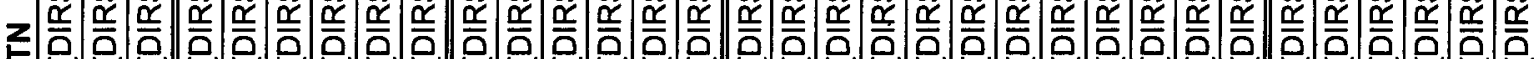
\&:

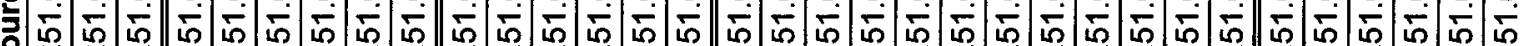
药

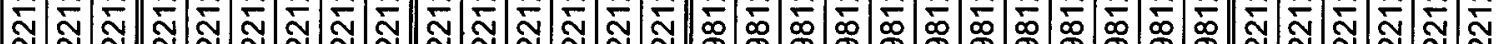
สิก

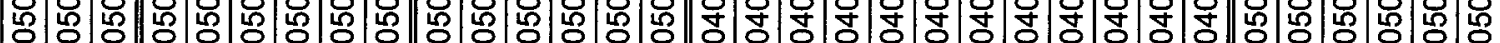

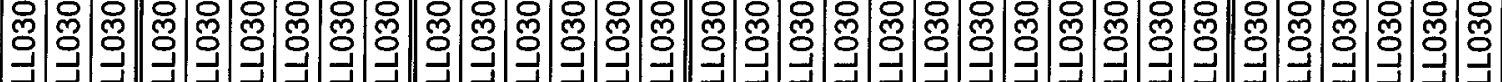




\begin{tabular}{|c|c|c|c|c|c|c|c|c|c|c|c|c|c|c|c|c|c|c|c|}
\hline 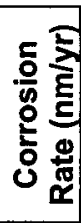 & & $\begin{array}{c}\stackrel{m}{m} \\
m^{\prime}\end{array}$ & $\begin{array}{l}\text { 莴 } \\
\text { N }\end{array}$ & $\begin{array}{l}\mathbb{R} \\
\text { S్ } \\
\text { ') }\end{array}$ & 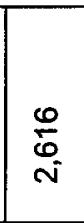 & \begin{tabular}{l}
$\bar{\delta}$ \\
\multirow{\infty}{N}{} \\
$\sim$
\end{tabular} & $\begin{array}{l}\mathscr{L} \\
\end{array}$ & $\hat{\sigma}$ & $\underset{\infty}{\stackrel{\rho}{\infty}}$ & 莡 & 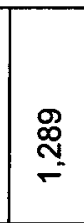 & 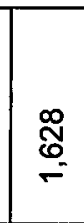 & $\stackrel{0}{\circ}$ & $\begin{array}{l}\mathscr{D} \\
\stackrel{\mathscr{D}}{N}\end{array}$ & 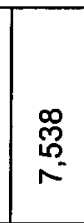 & 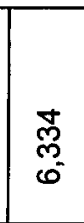 & $\begin{array}{l}\mathscr{D} \\
\stackrel{\text { }}{*}\end{array}$ & 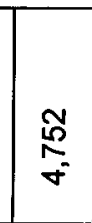 & $\begin{array}{l}\mathscr{8} \\
\stackrel{8}{0} \\
+\end{array}$ \\
\hline 곰 & $\begin{array}{c}N \\
0 \\
0\end{array}$ & 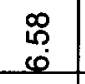 & 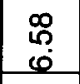 & 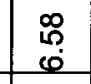 & 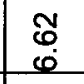 & $\begin{array}{l}\mathbb{N} \\
0 \\
\oplus\end{array}$ & 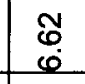 & $\begin{array}{r}8 \\
0 \\
0 \\
0\end{array}$ & $\begin{array}{r}8 \\
8 \\
0 \\
0\end{array}$ & $\begin{array}{l}\mathscr{8} \\
\stackrel{0}{\circ} \\
\end{array}$ & 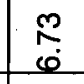 & 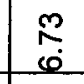 & $\begin{array}{c}\frac{\pi}{9} \\
6\end{array}$ & $\stackrel{\circ}{0}$ & $\begin{array}{l}\hat{0} \\
0 \\
0\end{array}$ & $\begin{array}{l}0 \\
0 \\
0\end{array}$ & $\frac{5}{6}$ & $\frac{6}{6}$ & $\frac{\omega n}{6}$ \\
\hline $5 \underline{\Xi}$ & 令 & 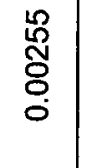 & 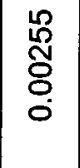 & $\begin{array}{l}\stackrel{L}{\circ} \\
\text { ه్ర } \\
0 \\
0\end{array}$ & 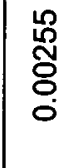 & 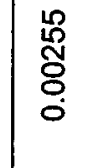 & 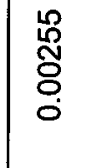 & 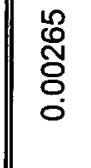 & 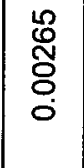 & 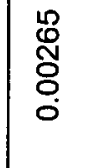 & $\begin{array}{l}\stackrel{0}{0} \\
\text { Oั } \\
0\end{array}$ & $\begin{array}{l}\stackrel{8}{0} \\
\overparen{O} \\
0 \\
0\end{array}$ & 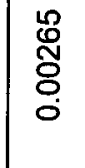 & $\begin{array}{l}\stackrel{\leftrightarrow}{\circ} \\
\text { ర్ర } \\
0\end{array}$ & 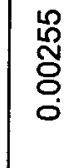 & 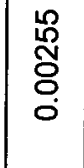 & $\begin{array}{l}\text { 苂 } \\
\text { ᄋ్ }\end{array}$ & 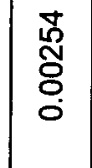 & 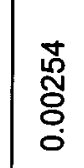 \\
\hline$\stackrel{\circ}{\varepsilon} 0$ & $\stackrel{\sim}{\vec{N}}$ & $\begin{array}{l}\stackrel{\infty}{\infty} \\
\stackrel{\infty}{=}\end{array}$ & $\begin{array}{l}\stackrel{\infty}{\infty} \\
\stackrel{\infty}{\leftarrow}\end{array}$ & $\begin{array}{l}\infty \\
\stackrel{\infty}{=}\end{array}$ & $\begin{array}{l}\stackrel{O}{O D} \\
\stackrel{0}{F}\end{array}$ & $\stackrel{\circ}{\stackrel{\circ}{\rightleftharpoons}}$ & $\begin{array}{l}\stackrel{0}{\circ} \\
\stackrel{2}{\rightleftharpoons}\end{array}$ & $\begin{array}{l}\infty \\
\stackrel{\infty}{\circ}\end{array}$ & $\begin{array}{l}\infty \\
\stackrel{0}{\circ}\end{array}$ & $\begin{array}{l}\infty \\
\dot{0} \\
\stackrel{0}{0}\end{array}$ & $\begin{array}{l}\infty \\
\stackrel{0}{0}\end{array}$ & $\begin{array}{l}\infty \\
\stackrel{0}{0}\end{array}$ & $\begin{array}{l}\infty \\
\stackrel{0}{\circ}\end{array}$ & 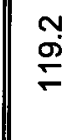 & $\stackrel{\text { Nִ }}{\stackrel{2}{\rightleftharpoons}}$ & Nָ & 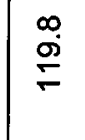 & $\stackrel{\infty}{\stackrel{\infty}{\sigma}}$ & 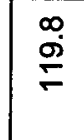 \\
\hline 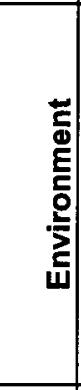 & 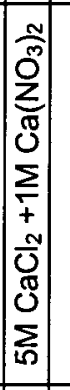 & $\begin{array}{l}\text { N } \\
\mathbb{N} \\
\sum_{i \infty}\end{array}$ & $\begin{array}{l}\frac{N}{0} \\
\mathbb{N} \\
\sum_{i \infty}\end{array}$ & $\begin{array}{l}\bar{N} \\
\mathbb{N} \\
\sum_{i \infty}\end{array}$ & $\begin{array}{l}\bar{N} \\
\mathbb{N} \\
\sum_{i \infty}\end{array}$ & $\begin{array}{l}\bar{N} \\
\mathbb{J} \\
\sum_{i \infty}\end{array}$ & $\begin{array}{l}\bar{N} \\
\mathbb{N} \\
\sum_{L S}\end{array}$ & 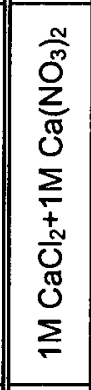 & 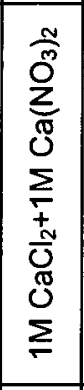 & 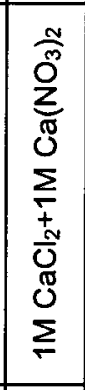 & 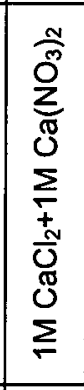 & 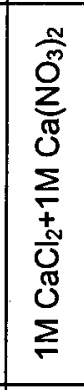 & 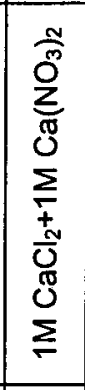 & 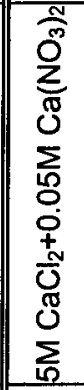 & 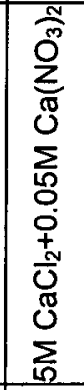 & 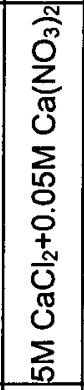 & 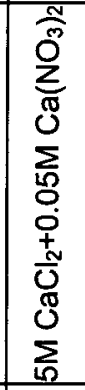 & 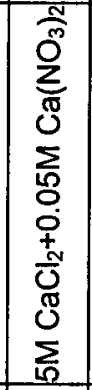 & 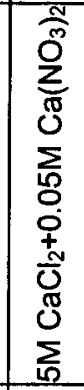 \\
\hline 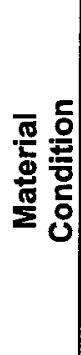 & 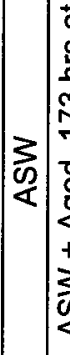 & 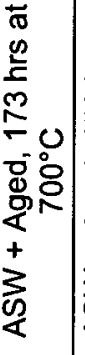 & 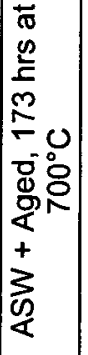 & 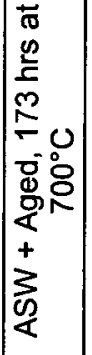 & 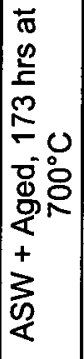 & 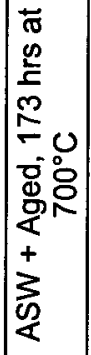 & 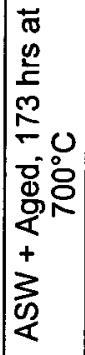 & 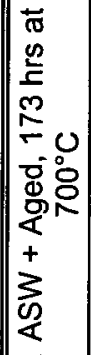 & 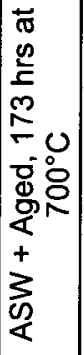 & 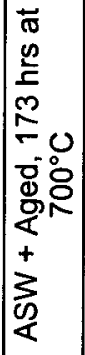 & 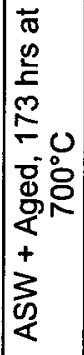 & 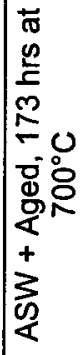 & 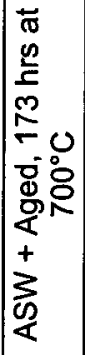 & 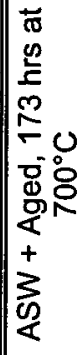 & 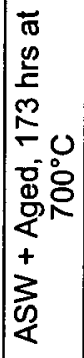 & 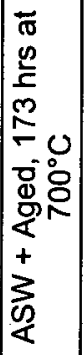 & 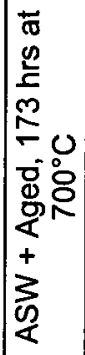 & 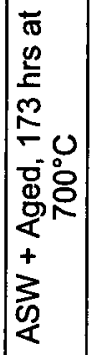 & 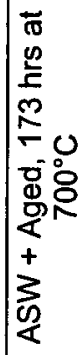 \\
\hline $\begin{array}{l}\frac{0}{0} \\
\text { 윯 }\end{array}$ & 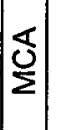 & $\underset{\Sigma}{\overleftarrow{J}}$ & $\underset{\Sigma}{\mathbb{S}}$ & $\stackrel{\mathbb{S}}{\Sigma}$ & $\stackrel{\delta}{\Sigma}$ & 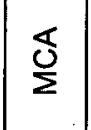 & $\frac{\delta}{\Sigma}$ & $\stackrel{\delta}{\Sigma}$ & 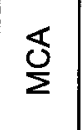 & $\frac{\delta}{\Sigma}$ & $\stackrel{\overleftarrow{J}}{\Sigma}$ & $\frac{\nwarrow}{\Sigma}$ & $\stackrel{\delta}{\delta}$ & 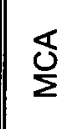 & $\frac{\nwarrow}{\Sigma}$ & 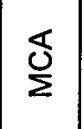 & $\frac{\delta}{\Sigma}$ & 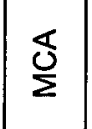 & 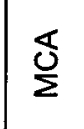 \\
\hline 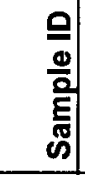 & 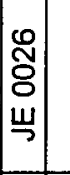 & $\begin{array}{l}\overline{5} \\
\text { 익 }\end{array}$ & $\begin{array}{l}\frac{5}{8} \\
\\
\end{array}$ & $\begin{array}{l}\overline{8} \\
\text { 崩 }\end{array}$ & $\begin{array}{l}\stackrel{N}{8} \\
\stackrel{\leftrightarrow}{2}\end{array}$ & $\begin{array}{l}\text { N } \\
\text { 을 }\end{array}$ & 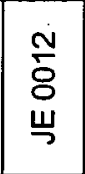 & $\begin{array}{l}\text { 옹 } \\
\text { 号 }\end{array}$ & $\begin{array}{l}\text { 옹 } \\
\text { 익 }\end{array}$ & $\begin{array}{l}8 \\
8 \\
8 \\
\text { 山 }\end{array}$ & $\begin{array}{l}\text { 옹 } \\
\text { 억 }\end{array}$ & $\begin{array}{l}\text { 옹 } \\
\text { 익 }\end{array}$ & $\begin{array}{l}\text { 응 } \\
\text { 억 }\end{array}$ & $\begin{array}{l}\frac{0}{8} \\
\stackrel{8}{\prime}\end{array}$ & $\begin{array}{l}\frac{0}{8} \\
\ddot{ }\end{array}$ & $\begin{array}{l}\stackrel{0}{8} \\
\stackrel{8}{3}\end{array}$ & $\begin{array}{l}\frac{10}{8} \\
8 \\
山\end{array}$ & $\begin{array}{l}\frac{10}{8} \\
\stackrel{\mu}{7}\end{array}$ & $\begin{array}{l}\frac{6}{8} \\
\text { ய }\end{array}$ \\
\hline $\begin{array}{l}\mathbf{z} \\
\mathbf{z} \\
\mathbf{5} \\
\mathbf{8} \\
\frac{0}{5} \\
\overline{8} \\
\dot{8} \\
\end{array}$ & 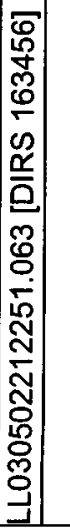 & 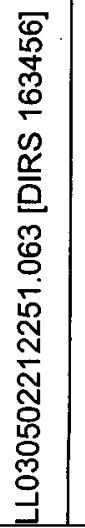 & 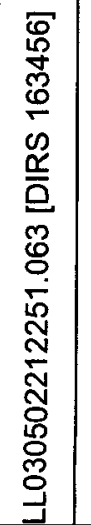 & 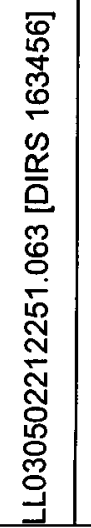 & 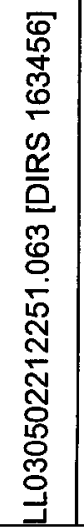 & 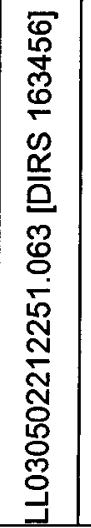 & 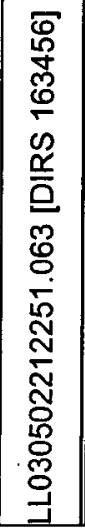 & 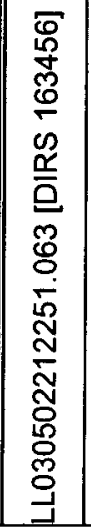 & 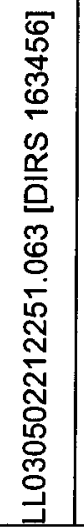 & 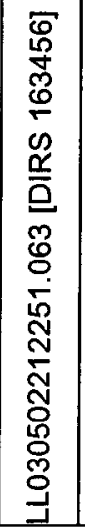 & 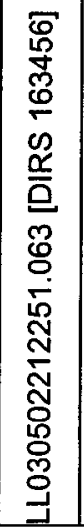 & 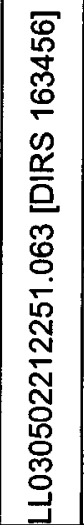 & 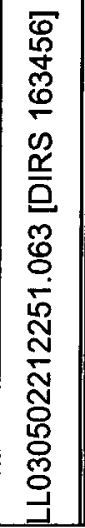 & 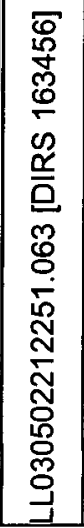 & 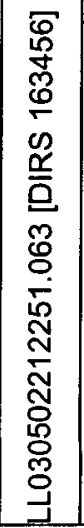 & 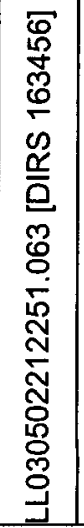 & 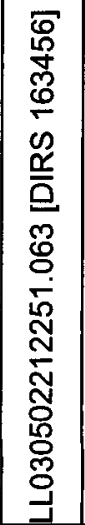 & 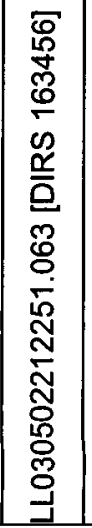 & 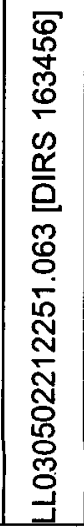 \\
\hline
\end{tabular}


General Corrosion and Localized Corrosion of Waste Package Outer Barrier

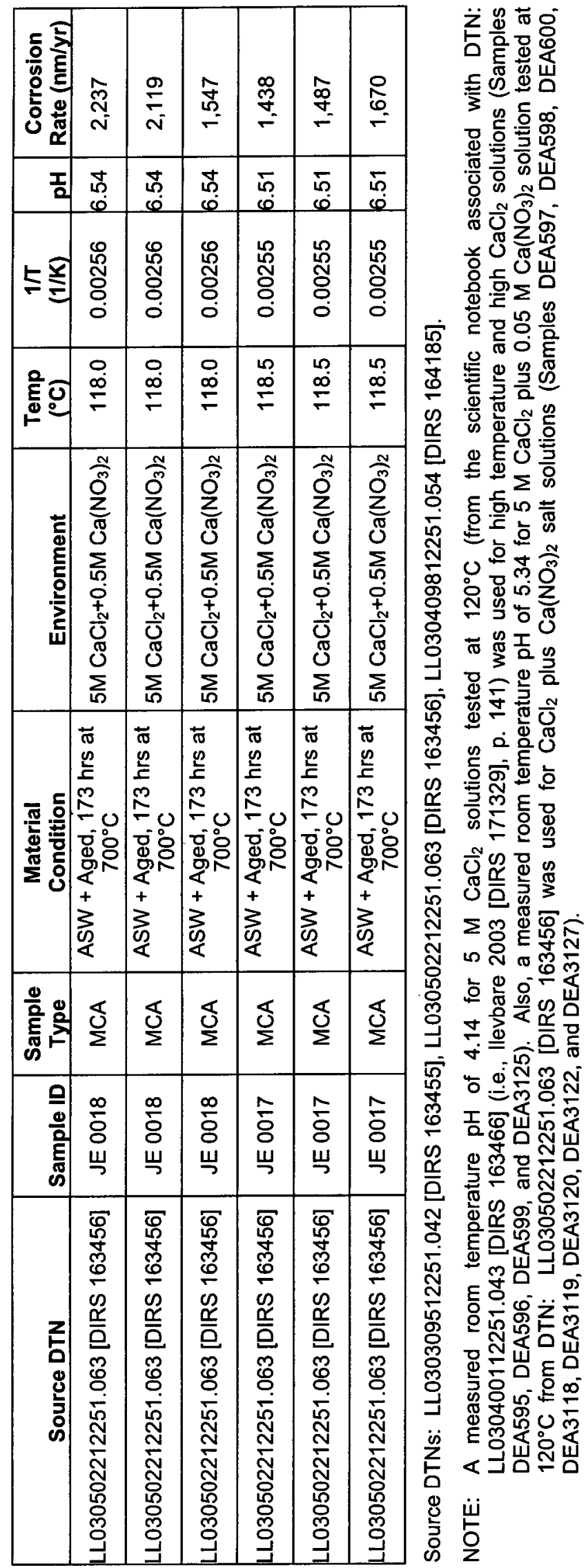




\section{APPENDIX V \\ LONG-TERM STEADY-STATE CORROSION POTENTIAL MEASUREMENTS OF ALLOY 22 SAMPLES USED IN THE CORROSION POTENTIAL MODEL AND ANALYSIS}


INTENTIONALLY LEFT BLANK 
General Corrosion and Localized Corrosion of Waste Package Outer Barrier

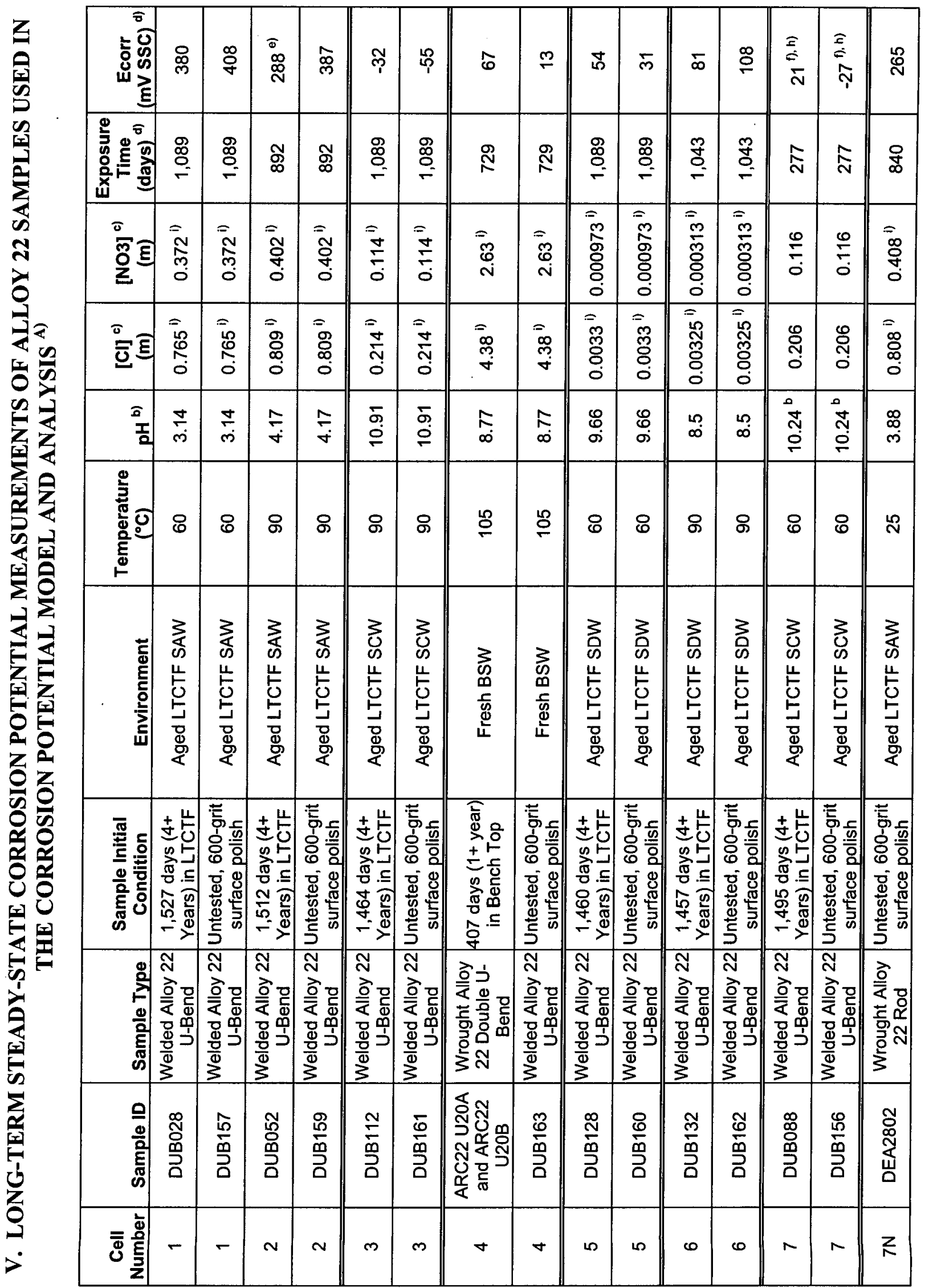


General Corrosion and Localized Corrosion of Waste Package Outer Barrier

\begin{tabular}{|c|c|c|c|c|c|c|c|c|c|c|c|c|c|c|c|c|c|c|}
\hline 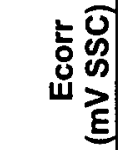 & 占 & 㐫 & $\stackrel{\stackrel{2}{\leftarrow}}{\leftarrow}$ & $\stackrel{\text { mి }}{\stackrel{1}{1}}$ & $\stackrel{\circ}{\frac{1}{4}}$ & $\stackrel{\mathscr{N}}{T}$ & 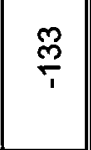 & $\bar{q}$ & 암 & চ্ণ & $\check{q}$ & ঠ & & 号 & \& & 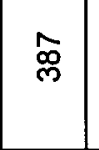 & $\underset{\infty}{N}$ & $\begin{array}{l}0 \\
\stackrel{0}{m}\end{array}$ \\
\hline 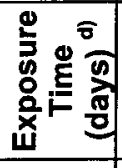 & 吕 & 옹 & ষ্ঠ & 苂 & 苂 & 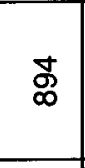 & ষ্ঠ & $\underset{\infty}{\infty}$ & $\underset{\infty}{\infty}$ & $\underset{\infty}{\infty}$ & $\underset{\infty}{\infty}$ & $\underset{\infty}{\stackrel{\infty}{\infty}}$ & $\underset{\infty}{\stackrel{\infty}{\infty}}$ & $\underset{\infty}{\infty}$ & $\underset{\infty}{\infty}$ & & జ్లు & 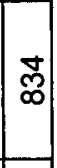 \\
\hline 官司 & $\begin{array}{l}= \\
\text { o } \\
\text { ơ } \\
0\end{array}$ & $\begin{array}{l}\text { o } \\
\text { \& } \\
\stackrel{+}{0}\end{array}$ & 0 & 0 & 0 & 0 & 0 & ت্ं & $\stackrel{\nabla}{\circ}$ & $\stackrel{\square}{+\Delta}$ & $=$ & ت্t & $\stackrel{+}{\circ}$ & $\stackrel{0}{*}$ & $\stackrel{\nabla}{\circ}$ & $\begin{array}{l}\overline{\mathscr{N}} \\
\stackrel{N}{+} \\
\dot{0}\end{array}$ & 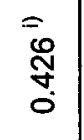 & 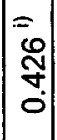 \\
\hline$\overline{\overline{0}} \overline{\mathrm{O}}$ & $\begin{array}{l}= \\
\overline{0} \\
0 \\
0 \\
0 \\
0\end{array}$ & $\begin{array}{l}= \\
\infty \\
\infty \\
\infty \\
0\end{array}$ & 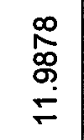 & $\begin{array}{l}\stackrel{\infty}{\infty} \\
\stackrel{\infty}{\infty} \\
\stackrel{5}{\leftarrow}\end{array}$ & 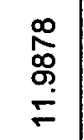 & $\begin{array}{l}\stackrel{\infty}{\infty} \\
\stackrel{\infty}{o} \\
\stackrel{+}{=}\end{array}$ & 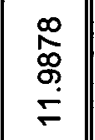 & $\begin{array}{l}= \\
\stackrel{\sim}{\alpha} \\
\stackrel{0}{0} \\
\tilde{0}\end{array}$ & $\begin{array}{c}\overline{\tilde{\alpha}} \\
\stackrel{\alpha}{\sim} \\
\stackrel{0}{0}\end{array}$ & $\begin{array}{c}\bar{\alpha} \\
\stackrel{\alpha}{0} \\
0\end{array}$ & $\begin{array}{c}\bar{\sim} \\
\stackrel{\infty}{\infty} \\
\stackrel{0}{0}\end{array}$ & $\begin{array}{c}\stackrel{\sim}{\tilde{\alpha}} \\
\stackrel{0}{0} \\
0\end{array}$ & $\begin{array}{c}\overline{\tilde{D}} \\
\stackrel{\infty}{\infty} \\
\stackrel{0}{0}\end{array}$ & 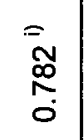 & 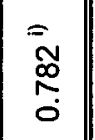 & 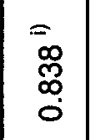 & 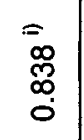 & $\begin{array}{l}= \\
\infty \\
0 \\
0 \\
0 \\
0\end{array}$ \\
\hline $\begin{array}{c}a \\
\frac{1}{2}\end{array}$ & $\begin{array}{l}\infty \\
\infty \\
\dot{m}\end{array}$ & $\begin{array}{l}\infty \\
\infty \\
\end{array}$ & 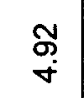 & $\underset{\sim}{\mathscr{\gamma}}$ & $\underset{\sim}{\mathscr{\gamma}}$ & $\begin{array}{l}\text { S̆ } \\
\text { + }\end{array}$ & $\underset{\mathscr{\sigma}}{\mathscr{\sim}}$ & $\stackrel{\infty}{\sim}$ & $\stackrel{\infty}{\sim}$ & $\stackrel{\infty}{\sim}$ & $\stackrel{\infty}{\sim}$ & 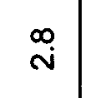 & $\stackrel{\infty}{\text { N }}$ & $\stackrel{\infty}{\sim}$ & $\stackrel{\infty}{\mathbf{N}}$ & $\begin{array}{l}\infty \\
\infty \\
\end{array}$ & \begin{tabular}{l}
$\mathscr{M}$ \\
\hdashline \\
\end{tabular} & $\begin{array}{l}\mathscr{m} \\
\infty \\
\end{array}$ \\
\hline 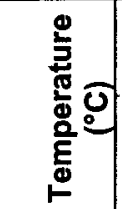 & $\stackrel{\sim}{N}$ & : & $\stackrel{ }{\stackrel{2}{ }}$ & $\stackrel{\text { ㅇ }}{\sim}$ & 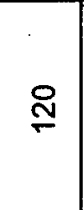 & $\stackrel{\text { ণ }}{\text { Tे }}$ & ని & ৪) & 8 & ৪ு & ৪ & ৪) & 요 & ৪ా & ৪ & 요 & 요 & ৪) \\
\hline 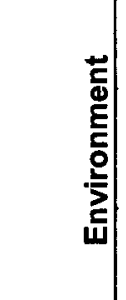 & 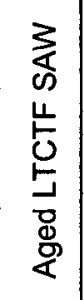 & 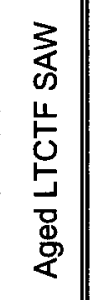 & $\begin{array}{l}\bar{N} \\
\bar{J} \\
\sum_{0}\end{array}$ & $\begin{array}{l}\bar{N} \\
\sum_{\pi} \\
\sum_{0}\end{array}$ & $\begin{array}{l}\bar{N} \\
\mathbb{N} \\
\sum_{10}\end{array}$ & $\begin{array}{l}\bar{N} \\
\sum_{0}^{N} \\
0\end{array}$ & $\begin{array}{l}\bar{N} \\
\bar{N} \\
\sum_{10}\end{array}$ & $\begin{array}{l}3 \\
\frac{1}{5} \\
\frac{5}{8} \\
\frac{d}{4}\end{array}$ & 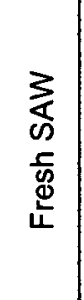 & 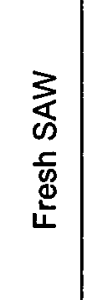 & $\begin{array}{l}\vdots \\
\frac{5}{5} \\
\frac{1}{5} \\
\frac{d}{4}\end{array}$ & 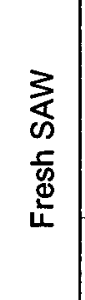 & $\begin{array}{l}\frac{3}{4} \\
0 \\
\frac{1}{\tilde{3}} \\
\frac{d}{4}\end{array}$ & $\begin{array}{l}3 \\
\frac{5}{5} \\
\frac{1}{0} \\
\frac{0}{4}\end{array}$ & 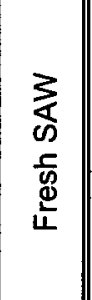 & 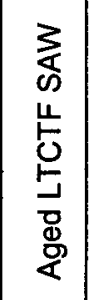 & 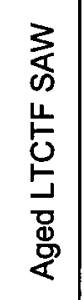 & 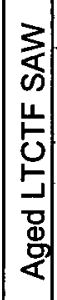 \\
\hline 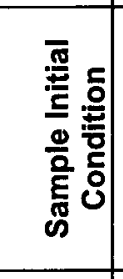 & 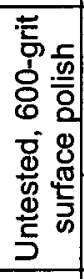 & 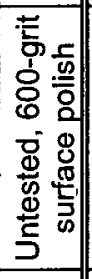 & 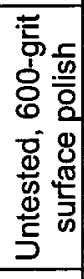 & 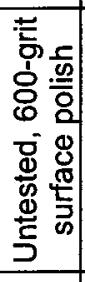 & 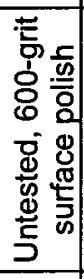 & 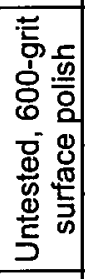 & 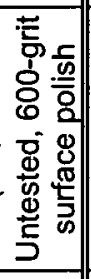 & 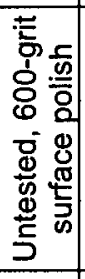 & 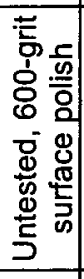 & 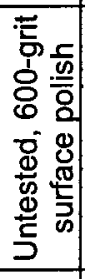 & 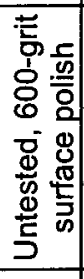 & 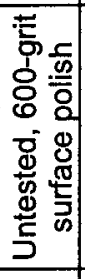 & 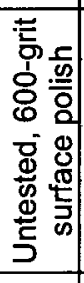 & 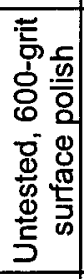 & 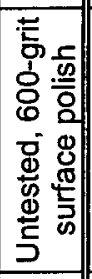 & 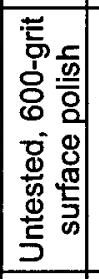 & 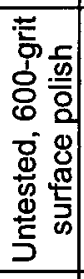 & 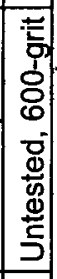 \\
\hline 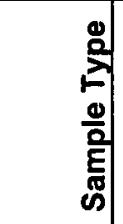 & 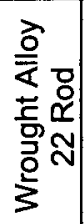 & 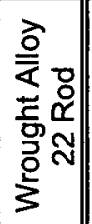 & 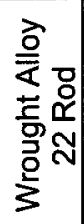 & 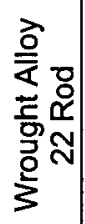 & 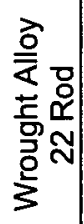 & 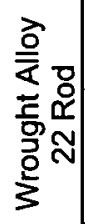 & 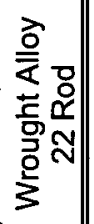 & 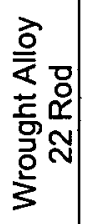 & 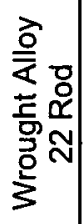 & 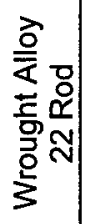 & 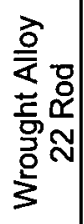 & 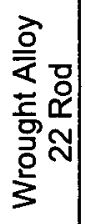 & 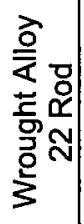 & 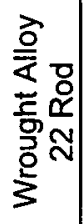 & 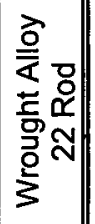 & 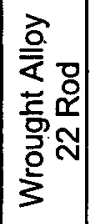 & 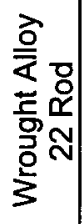 & 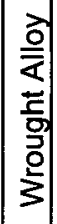 \\
\hline $\begin{array}{l}\text { 음 } \\
\text { 응 } \\
\text { 홈 } \\
\text { ஸे }\end{array}$ & 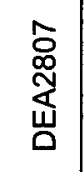 & 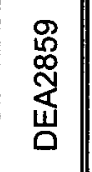 & $\frac{\stackrel{L}{O}}{\frac{1}{4}}$ & 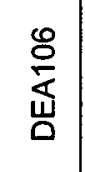 & 容 & $\frac{\infty}{\frac{0}{4}}$ & $\frac{8}{\frac{8}{\overleftarrow{L}}}$ & 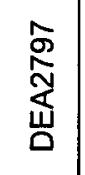 & 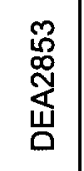 & 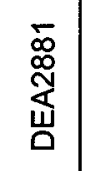 & 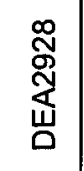 & 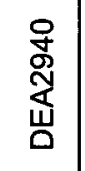 & 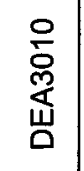 & 离 & 商 & 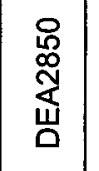 & 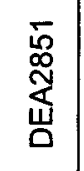 & 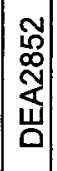 \\
\hline$\overline{\bar{\delta}} \frac{\overline{\mathrm{g}}}{\mathrm{g}}$ & $z$ & $z$ & $\infty$ & $\infty$ & $\infty$ & $\infty$ & $\infty$ & $\sigma$ & $\sigma$ & $\sigma$ & $\sigma$ & $\sigma$ & $\sigma$ & $\sigma$ & $\sigma$ & 으 & 우 & 우 \\
\hline
\end{tabular}


General Corrosion and Localized Corrosion of Waste Package Outer Barrier

\begin{tabular}{|c|c|c|c|c|c|c|c|c|c|c|c|c|c|c|c|c|c|}
\hline 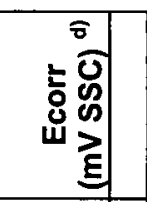 & 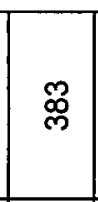 & 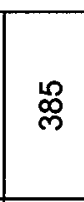 & $\frac{10}{m}$ & $\frac{\infty}{\infty}$ & జ్ల & 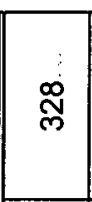 & 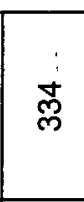 & ભ్లా & 芯 & ఖ్ల & 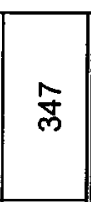 & $\stackrel{\infty}{+}$ & $\stackrel{\infty}{\stackrel{\infty}{N}}$ & in & $\stackrel{+}{\circ}$ & $\Phi$ & 유 \\
\hline 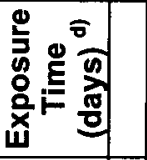 & 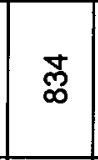 & జ్ల & 苂 & ঙ্లি & 心్ల & กู & ָ̃ & ָิ & $\mathbb{N}$ & 总 & 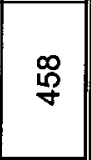 & 今̊ & 今̊ & જ̊ & 용 & 苞 & 莴 \\
\hline 畐司 & 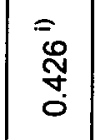 & 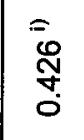 & 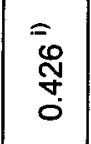 & $\begin{array}{l}\overline{\mathscr{N}} \\
\stackrel{y}{0} \\
0\end{array}$ & $\begin{array}{l}= \\
\stackrel{0}{0} \\
\text { Yे } \\
0\end{array}$ & 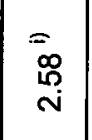 & 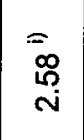 & 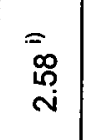 & 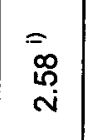 & 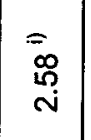 & $\begin{array}{l}= \\
\infty \\
\stackrel{\infty}{0} \\
\text { in }\end{array}$ & $\frac{\mathscr{8}}{\stackrel{\mathscr{\infty}}{\sigma}}$ & $\frac{\mathscr{\infty}}{\frac{\infty}{0}}$ & $\frac{\infty}{\frac{\infty}{\leftarrow}}$ & $\frac{\mathscr{0}}{\frac{0}{\sigma}}$ & $\frac{\infty}{\stackrel{\infty}{c}}$ & $\frac{\mathscr{\infty}}{\stackrel{\infty}{\tau}}$ \\
\hline 흔혀 & 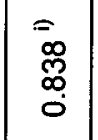 & 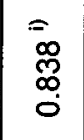 & 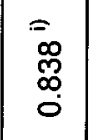 & $\begin{array}{l}= \\
\infty \\
\infty \\
\infty \\
0 \\
0\end{array}$ & 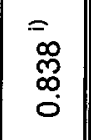 & 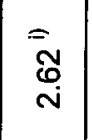 & তั & 응 & 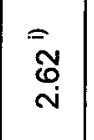 & 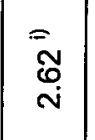 & 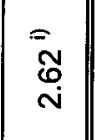 & $\begin{array}{l}\stackrel{\Xi}{\circ} \\
\stackrel{8}{\rightleftarrows}\end{array}$ & 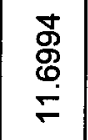 & $\begin{array}{l}\stackrel{\Xi}{8} \\
\stackrel{g}{\rightleftarrows} \\
\end{array}$ & $\begin{array}{l}\stackrel{\Xi}{\circ} \\
\stackrel{8}{0} \\
\stackrel{-}{=}\end{array}$ & 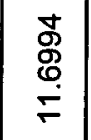 & $\begin{array}{l}\stackrel{\square}{\circ} \\
\stackrel{0}{\circ} \\
\end{array}$ \\
\hline $\begin{array}{l}\bar{a} \\
\text { 工 } \\
\end{array}$ & $\begin{array}{l}\mathscr{M} \\
\infty \\
\text { ウె }\end{array}$ & $\begin{array}{l}\mathscr{M} \\
\infty \\
\text { m }\end{array}$ & 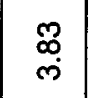 & $\begin{array}{l}\mathscr{M} \\
\infty \\
\text { mi }\end{array}$ & $\begin{array}{l}\mathscr{M} \\
\infty \\
\text { mi }\end{array}$ & $\stackrel{\mathscr{\rho}}{\ddot{m}}$ & $\stackrel{\bullet}{\ddot{m}}$ & $\ddot{~}$ & $\stackrel{\mathscr{0}}{\oplus}$ & $\stackrel{\mathscr{O}}{\oplus}$ & $\stackrel{\mathscr{m}}{\ddot{m}}$ & ڤึ & 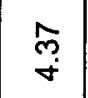 & $\stackrel{\widehat{m}}{\text { mे }}$ & $\stackrel{\hat{m}}{\sigma}$ & $\stackrel{\widehat{m}}{\text { q }}$ & $\stackrel{\widehat{m}}{\leftrightarrow}$ \\
\hline 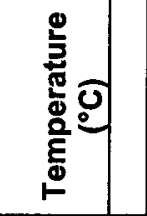 & Яి & ৪) & 요 & পு & 요 & ৪ & ৪ & ৪) & ৪ా & 요 & পᄋ & ஜి & க) & ৪ి & 요 & 용 & 용 \\
\hline 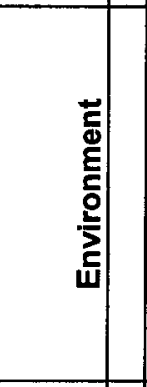 & 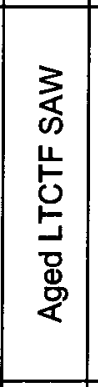 & 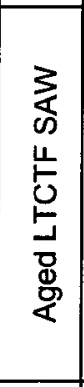 & 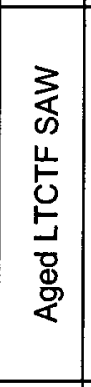 & 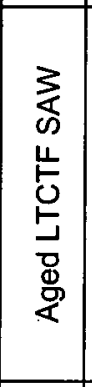 & 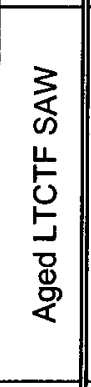 & 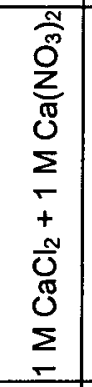 & 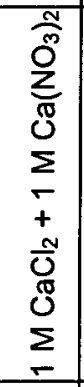 & 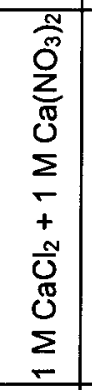 & 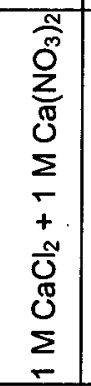 & 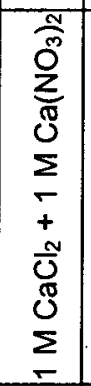 & 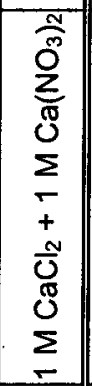 & 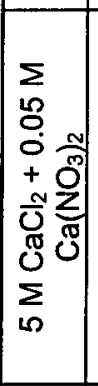 & 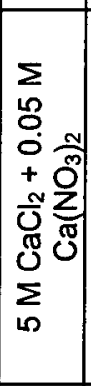 & $\begin{array}{l}\sum_{0} \\
0 \\
0 \\
+\frac{N}{0} \\
+0 \\
0 \\
0 \\
0 \\
\sum_{0} \\
0\end{array}$ & $\begin{array}{l}\sum_{0} \\
0 \\
0 \\
+0 \\
+0 \\
\overline{0} \\
0 \\
0 \\
\sum_{\infty} \\
0\end{array}$ & $\begin{array}{l}\sum \\
0 \\
0 \\
0 \\
+0 \\
0 \\
0 \\
0 \\
0 \\
\sum_{0}^{0} \\
0 \\
0\end{array}$ & 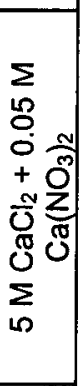 \\
\hline 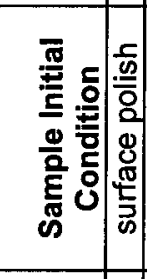 & 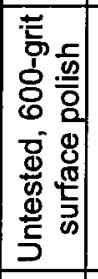 & 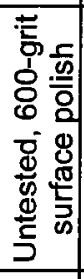 & 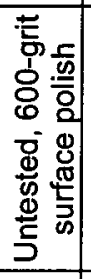 & 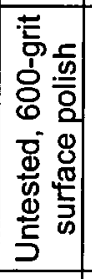 & 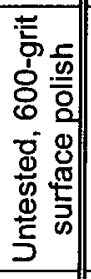 & 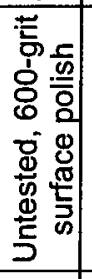 & 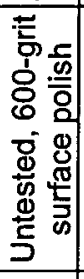 & 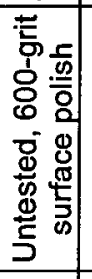 & 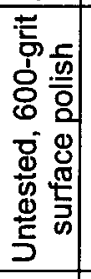 & 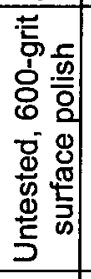 & 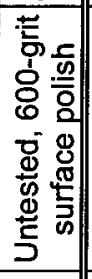 & 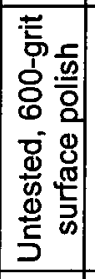 & 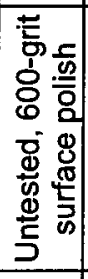 & 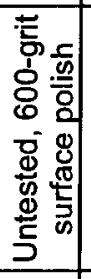 & 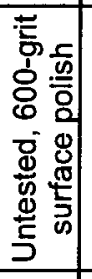 & 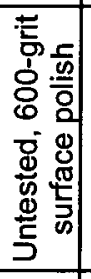 & 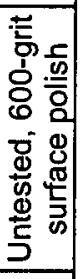 \\
\hline 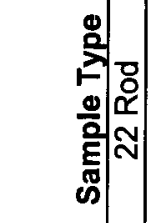 & 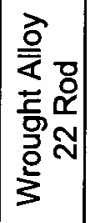 & 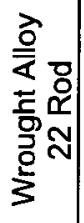 & 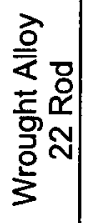 & 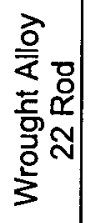 & 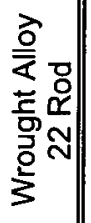 & 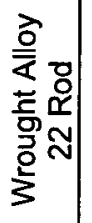 & 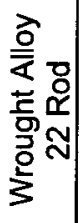 & 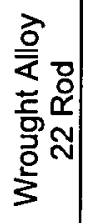 & 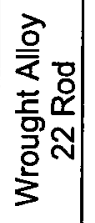 & 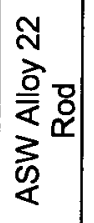 & 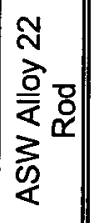 & 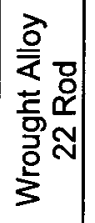 & 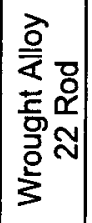 & 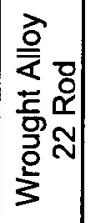 & 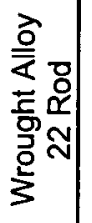 & 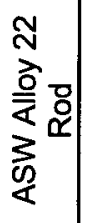 & 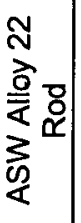 \\
\hline $\begin{array}{l}\text { 응 } \\
\text { 응 } \\
\text { 융 }\end{array}$ & $\begin{array}{l}\text { 芯 } \\
\stackrel{0}{0} \\
\text { 岁 }\end{array}$ & 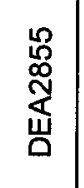 & 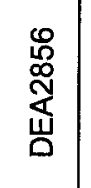 & 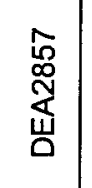 & 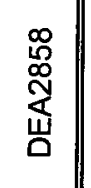 & $\begin{array}{l}\hat{o} \\
\text { o } \\
\text { 㟔 }\end{array}$ & 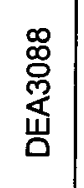 & 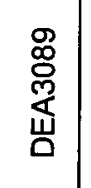 & $\begin{array}{l}\stackrel{8}{\circ} \\
\stackrel{\mathscr{m}}{\overleftarrow{\leftrightarrow}} \\
\text { 岁 }\end{array}$ & $\begin{array}{l}\text { ్ֶరి } \\
\text { జ్ }\end{array}$ & 芯 & 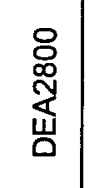 & 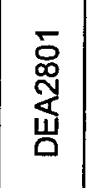 & $\begin{array}{l}\text { ஜ } \\
\text { 品 } \\
\text { 岌 }\end{array}$ & 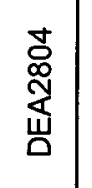 & 弚 & 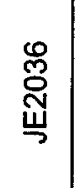 \\
\hline 㐫 & 우 & 으 & 으 & 우 & 으 & $\stackrel{m}{\sim}$ & $\stackrel{m}{=}$ & $\stackrel{m}{?}$ & $\stackrel{m}{2}$ & $\stackrel{m}{=}$ & $\stackrel{m}{\longrightarrow}$ & \pm & 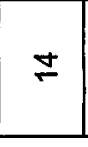 & 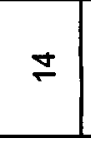 & $\stackrel{\nabla}{ }$ & 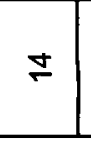 & $\underset{\leftarrow}{ }$ \\
\hline
\end{tabular}


General Corrosion and Localized Corrosion of Waste Package Outer Barrier

\begin{tabular}{|c|c|c|c|c|c|c|c|c|c|c|c|c|c|c|c|c|c|c|}
\hline 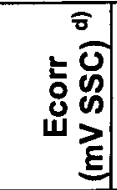 & $\stackrel{\infty}{\text { W }}$ & $\stackrel{8}{\circ}$ & g) & வ & సి & $\stackrel{\text { N }}{\text { N }}$ & ָু & 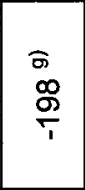 & $\begin{array}{l}\frac{6}{10} \\
\stackrel{1}{2} \\
\frac{1}{1}\end{array}$ & $\underset{T}{\stackrel{0}{T}}$ & $\frac{\sigma}{\frac{\pi}{5}}$ & $\begin{array}{l}\text { ब. } \\
\infty \\
\& \\
\end{array}$ & $\frac{0}{8}$ & \& & $\stackrel{\infty}{\stackrel{\leftrightarrow}{q}}$ & $\underset{\nabla}{\nabla}$ & $\underset{\forall}{\forall}$ & $\frac{\nabla}{\square}$ \\
\hline 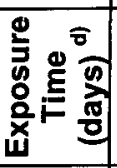 & :্̊口: & 吕 & : & : & 芯 & 落 & 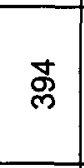 & 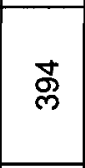 & 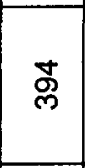 & ఫ్ల & 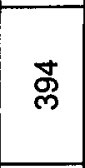 & హ్ల & $\underset{m}{\stackrel{R}{m}}$ & $\underset{m}{\stackrel{2}{m}}$ & 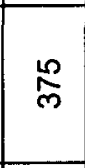 & $\underset{\sim}{\stackrel{R}{m}}$ & $\underset{m}{\stackrel{2}{\rho}}$ & $\stackrel{10}{\frac{1}{m}}$ \\
\hline 容馬 & 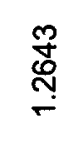 & $\begin{array}{l}\stackrel{?}{ \pm ্} \\
\stackrel{N}{ }\end{array}$ & $\begin{array}{l}\text { ֻ } \\
\stackrel{\text { ș }}{\sigma}\end{array}$ & 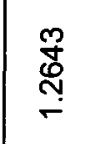 & & 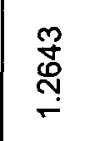 & $\begin{array}{l}\overline{0} \\
\overline{0}\end{array}$ & $\begin{array}{l}\overline{8} \\
\dot{8} \\
\dot{0}\end{array}$ & $\begin{array}{l}\overline{0} \\
\dot{0} \\
\dot{0}\end{array}$ & $\begin{array}{l}\overline{0} \\
\dot{8} \\
\dot{0}\end{array}$ & $\begin{array}{l}\bar{\phi} \\
\overline{0} \\
\end{array}$ & $\frac{\overline{8}}{8}$ & $\stackrel{+}{\circ}$ & $\stackrel{\nabla}{\stackrel{\nabla}{0}}$ & $\bar{\sigma}$ & $\stackrel{=}{\circ}$ & ت্t. & $=$ \\
\hline$\overline{\overline{0}} \widehat{\bar{g}}$ & 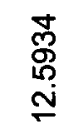 & 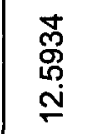 & 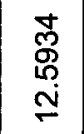 & 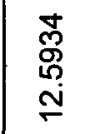 & 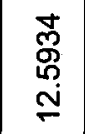 & 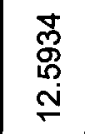 & 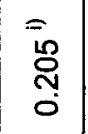 & 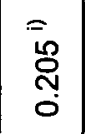 & 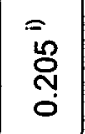 & 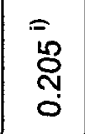 & ํㅠㅁ & 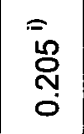 & 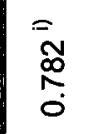 & 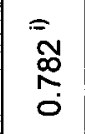 & $\begin{array}{l}\bar{\sim} \\
\stackrel{\infty}{0} \\
\stackrel{0}{0} \\
0\end{array}$ & 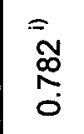 & $\begin{array}{l}\overline{\mathcal{N}} \\
\stackrel{\alpha}{0} \\
\stackrel{0}{0}\end{array}$ & $\begin{array}{l}= \\
\tilde{\mathcal{N}} \\
\stackrel{\alpha}{0} \\
0\end{array}$ \\
\hline $\begin{array}{l}\overline{\mathbf{a}} \\
\overline{\mathbf{2}}\end{array}$ & $\begin{array}{l}\stackrel{9}{o} \\
\stackrel{+}{*}\end{array}$ & $\stackrel{\infty}{\stackrel{\infty}{+}}$ & $\begin{array}{l}\stackrel{9}{O} \\
\stackrel{+}{+}\end{array}$ & 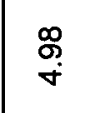 & 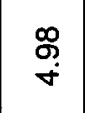 & $\begin{array}{l}\stackrel{\infty}{o} \\
+\end{array}$ & $\begin{array}{l}\text { J } \\
\stackrel{\circ}{\circ}\end{array}$ & $\begin{array}{l}\text { 茄 } \\
\stackrel{\circ}{\circ}\end{array}$ & $\begin{array}{l}\text { 호 } \\
\stackrel{\circ}{\circ}\end{array}$ & $\begin{array}{l}\stackrel{ }{\circ} \\
\stackrel{0}{\circ}\end{array}$ & $\begin{array}{l}\text { 음 } \\
\stackrel{\circ}{\circ}\end{array}$ & $\begin{array}{l}\text { هั } \\
\stackrel{\circ}{\circ}\end{array}$ & $\underset{\mathrm{o}}{\mathrm{N}}$ & 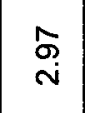 & $\begin{array}{l}\hat{\sigma} \\
\stackrel{i}{ }\end{array}$ & $\underset{\mathrm{s}}{\mathrm{s}}$ & ডু & 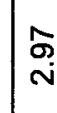 \\
\hline 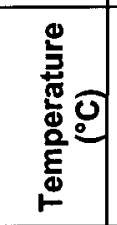 & ৪ & ৪ & ৪ & ৪) & ৪্ & ৪) & 요 & ৪) & ৪) & 8 & ৪ & 용 & ৪৪ & 요 & ৪্ & ৪ & 요 & ৪ \\
\hline 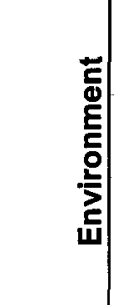 & 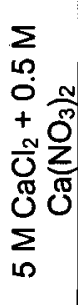 & 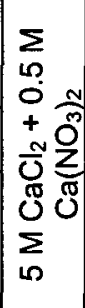 & 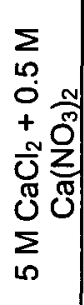 & 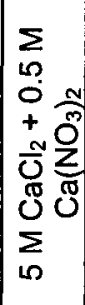 & 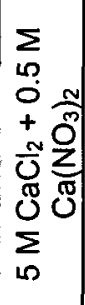 & 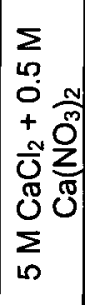 & $\begin{array}{l}3 \\
3 \\
0 \\
\bar{y} \\
\frac{5}{4} \\
\frac{1}{4}\end{array}$ & 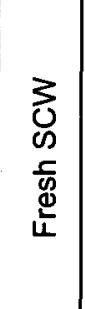 & 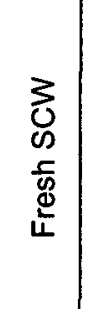 & 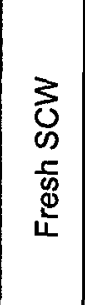 & $\begin{array}{l}3 \\
\text { o } \\
0 \\
\frac{1}{5} \\
\frac{0}{4}\end{array}$ & 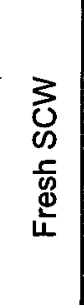 & 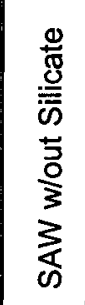 & 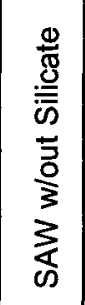 & 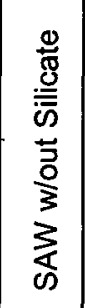 & 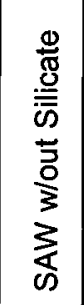 & 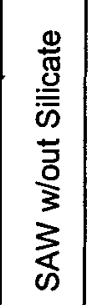 & 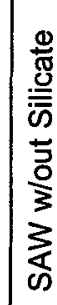 \\
\hline 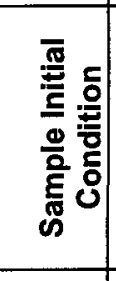 & 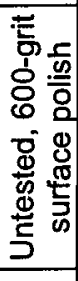 & 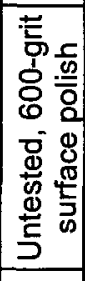 & 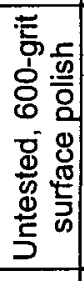 & 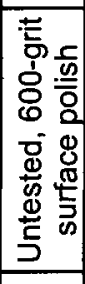 & 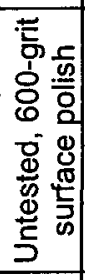 & 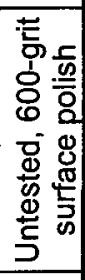 & 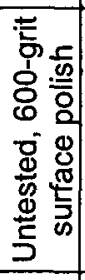 & 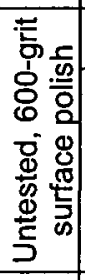 & 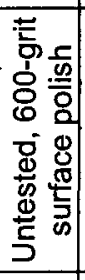 & 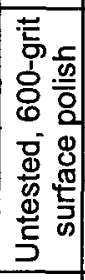 & 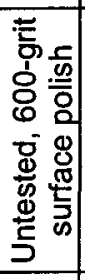 & 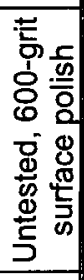 & 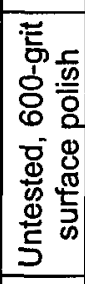 & 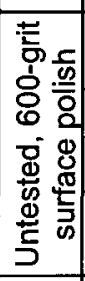 & 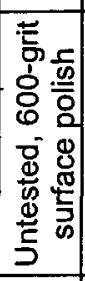 & 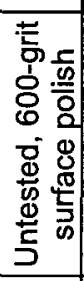 & 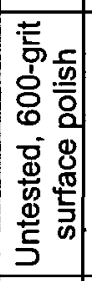 & 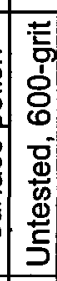 \\
\hline 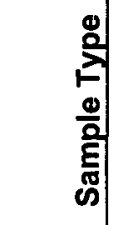 & 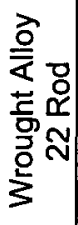 & 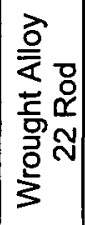 & 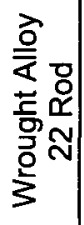 & 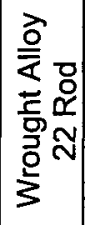 & 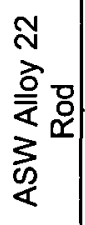 & 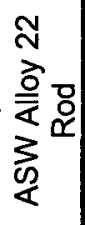 & 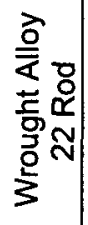 & 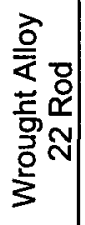 & 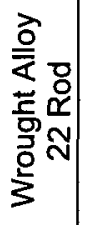 & 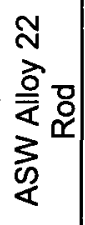 & 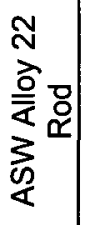 & 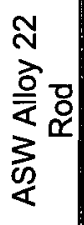 & 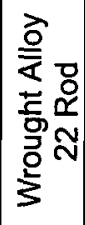 & 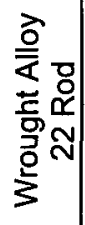 & 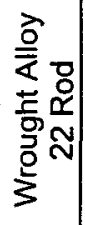 & 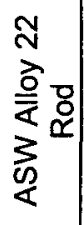 & 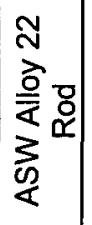 & 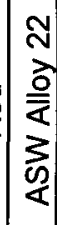 \\
\hline $\begin{array}{l}\frac{0}{0} \\
\frac{0}{0} \\
\overline{5} \\
\mathscr{n}\end{array}$ & 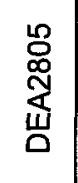 & $\begin{array}{l}\mathscr{O} \\
\stackrel{0}{0} \\
\stackrel{\leftrightarrow}{0}\end{array}$ & $\begin{array}{l}\stackrel{\infty}{\circ} \\
\stackrel{\leftrightarrow}{\dddot{y}} \\
\text { 岁 }\end{array}$ & \begin{tabular}{l} 
吕 \\
\multirow{2}{*}{} \\
岃
\end{tabular} & 司 & 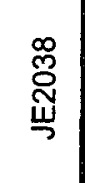 & $\begin{array}{l}\text { 옹 } \\
\stackrel{\sim}{\mathbb{1}} \\
\text { 岁 }\end{array}$ & 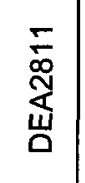 & 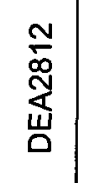 & 突 & 월 & 竎 & 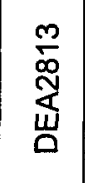 & 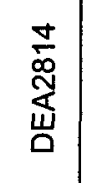 & 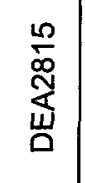 & $\begin{array}{l}\text { Y్ } \\
\text { జ్ㄱ }\end{array}$ & 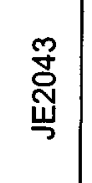 & $\begin{array}{l}\text { 寸 } \\
\text { ய్ }\end{array}$ \\
\hline$\overline{\bar{g}} \frac{\overline{\mathbf{g}}}{\mathrm{g}}$ & $\stackrel{2}{2}$ & $\stackrel{20}{2}$ & $\stackrel{2}{2}$ & $\stackrel{20}{2}$ & $\stackrel{2}{2}$ & $\stackrel{2}{2}$ & $\stackrel{\varphi}{0}$ & 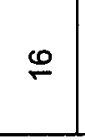 & 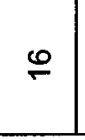 & 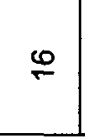 & $\stackrel{\varphi}{0}$ & $\stackrel{\oplus}{ }$ & $\approx$ & 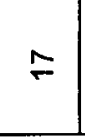 & 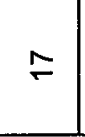 & $\mp$ & $\approx$ & 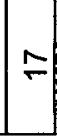 \\
\hline
\end{tabular}




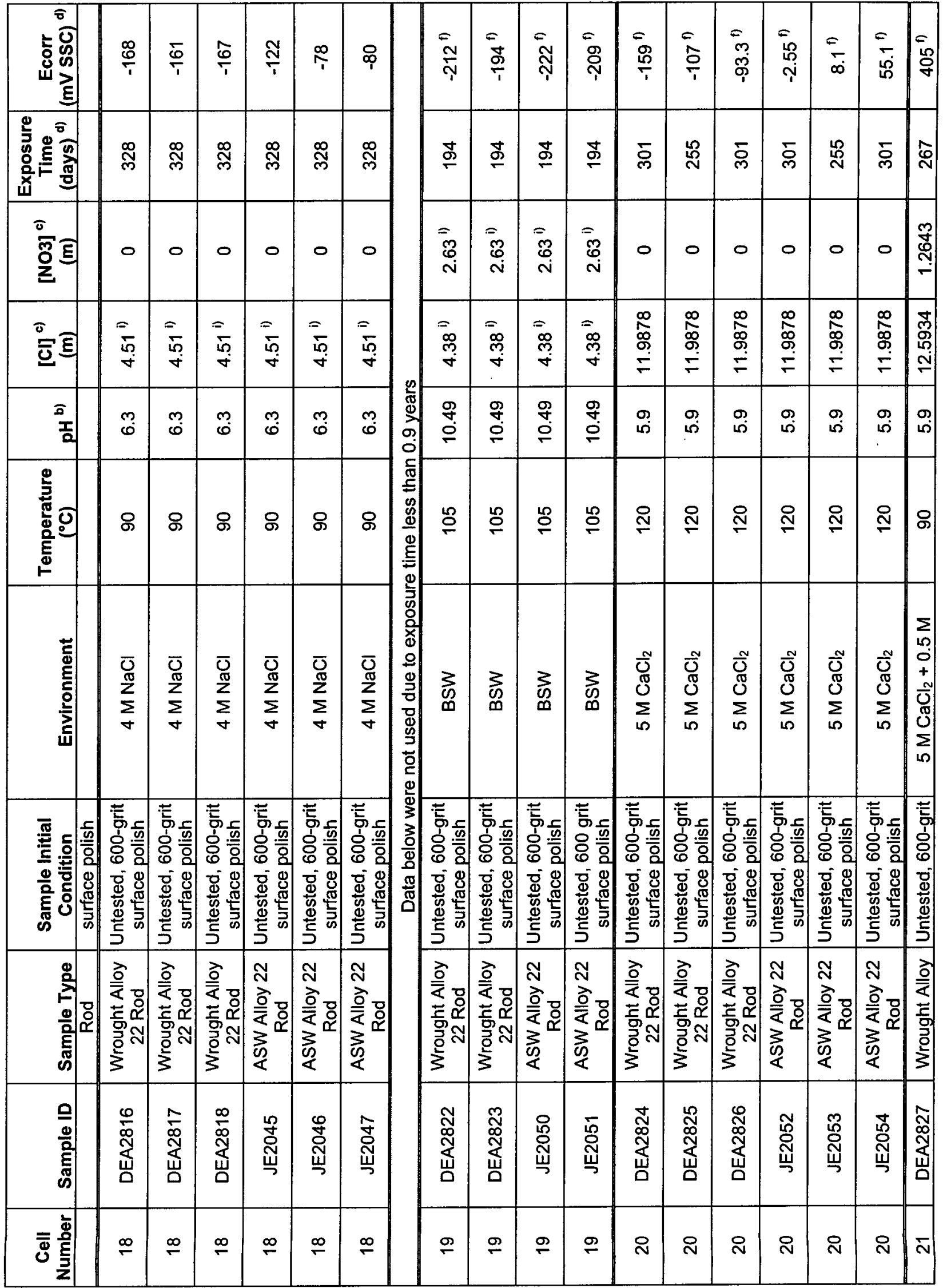


General Corrosion and Localized Corrosion of Waste Package Outer Barrier

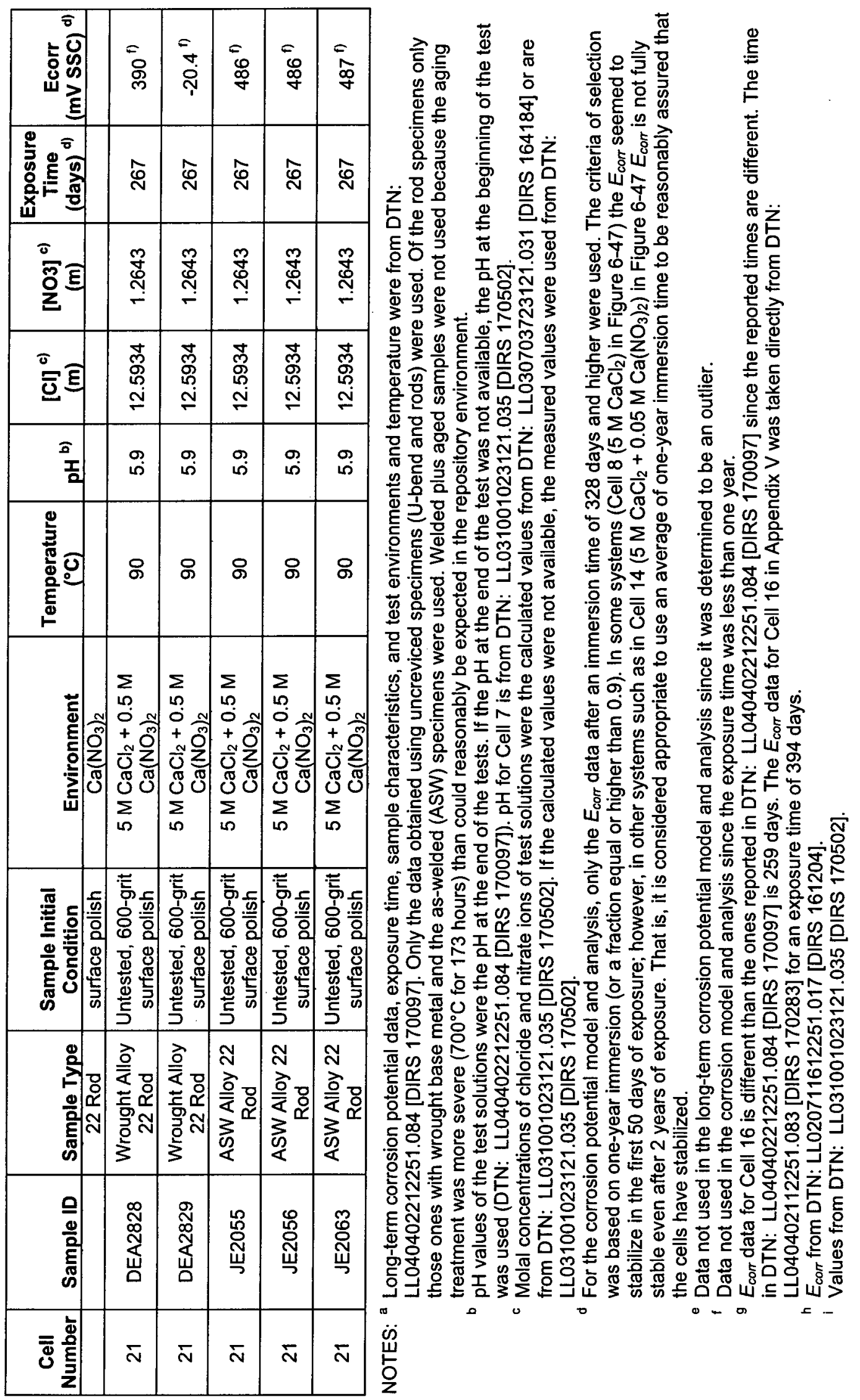




\section{APPENDIX VI}

PROJECT ALLOY 22 CREVICE REPASSIVATION POTENTIAL DATA USED IN THE CRITICAL POTENTIAL MODEL IN THE ABSENCE OF NITRATE IONS 


\section{INTENTIONALLY LEFT BLANK}


General Corrosion and Localized Corrosion of Waste Package Outer Barrier

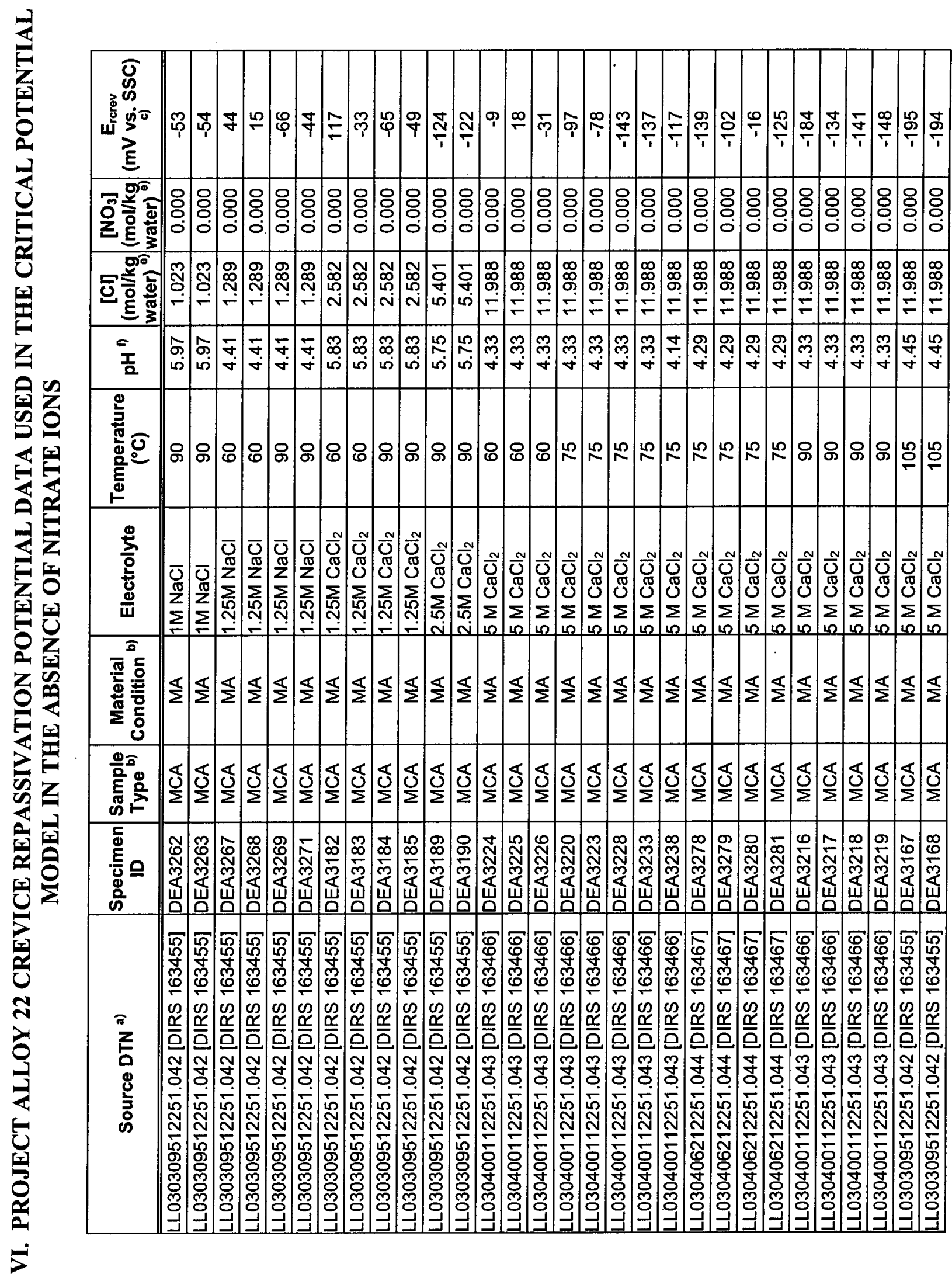




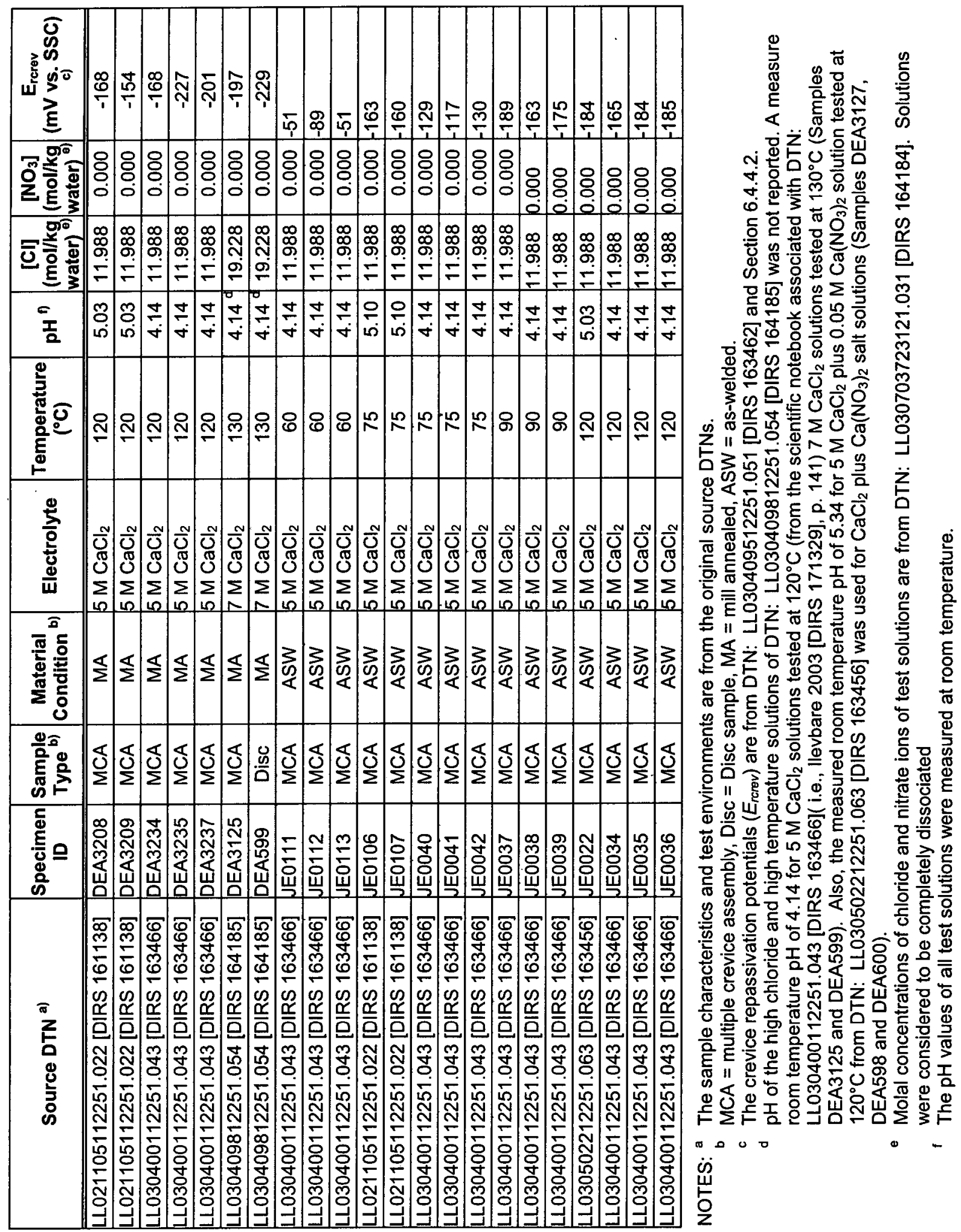




\section{APPENDIX VII}

CNWRA ALLOY 22 CREVICE REPASSIVATION POTENTIAL DATA USED IN THE CRITICAL POTENTIAL MODEL IN THE ABSENCE OF NITRATE IONS 
INTENTIONALLY LEFT BLANK 


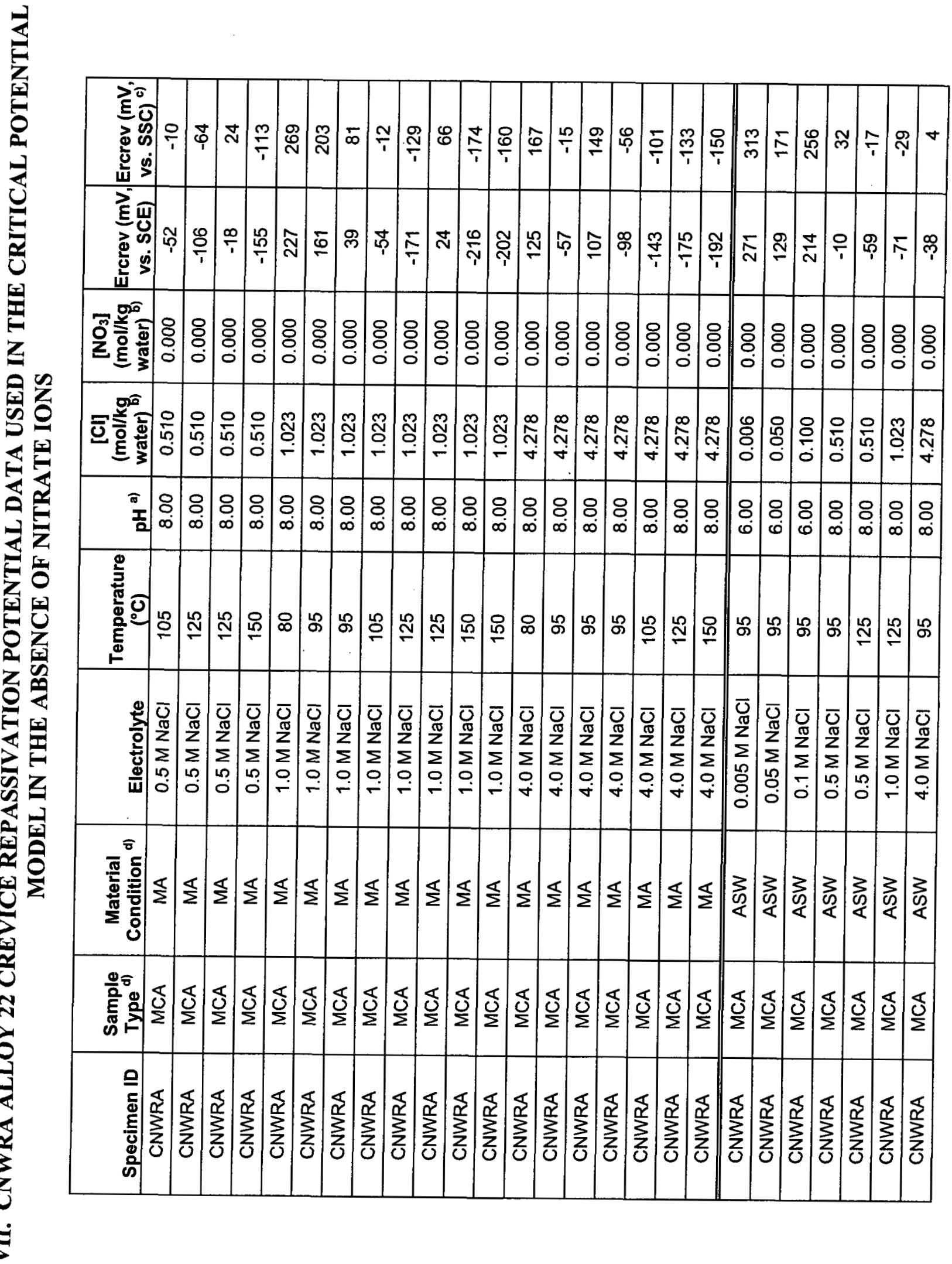




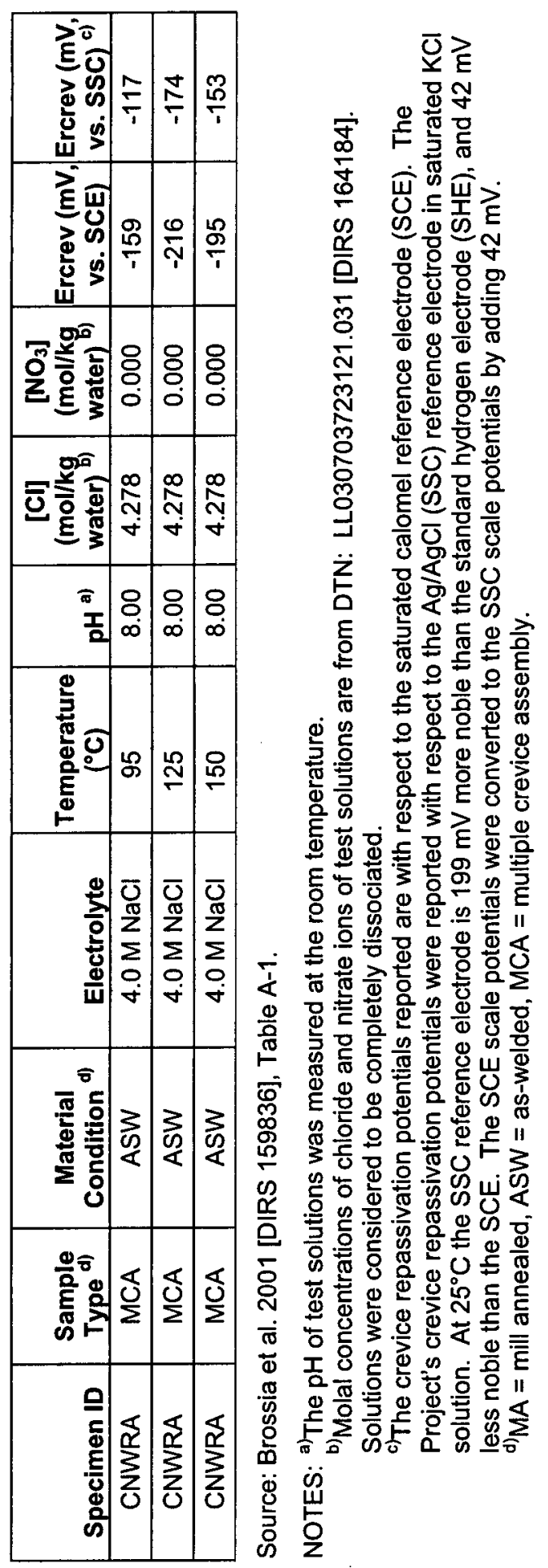


APPENDIX VIII

PROJECT ALLOY 22 CREVICE REPASSIVATION POTENTIAL DATA USED IN THE CRITICAL POTENTIAL MODEL IN THE PRESENCE OF NITRATE IONS 
INTENTIONALLY LEFT BLANK 
General Corrosion and Localized Corrosion of Waste Package Outer Barrier

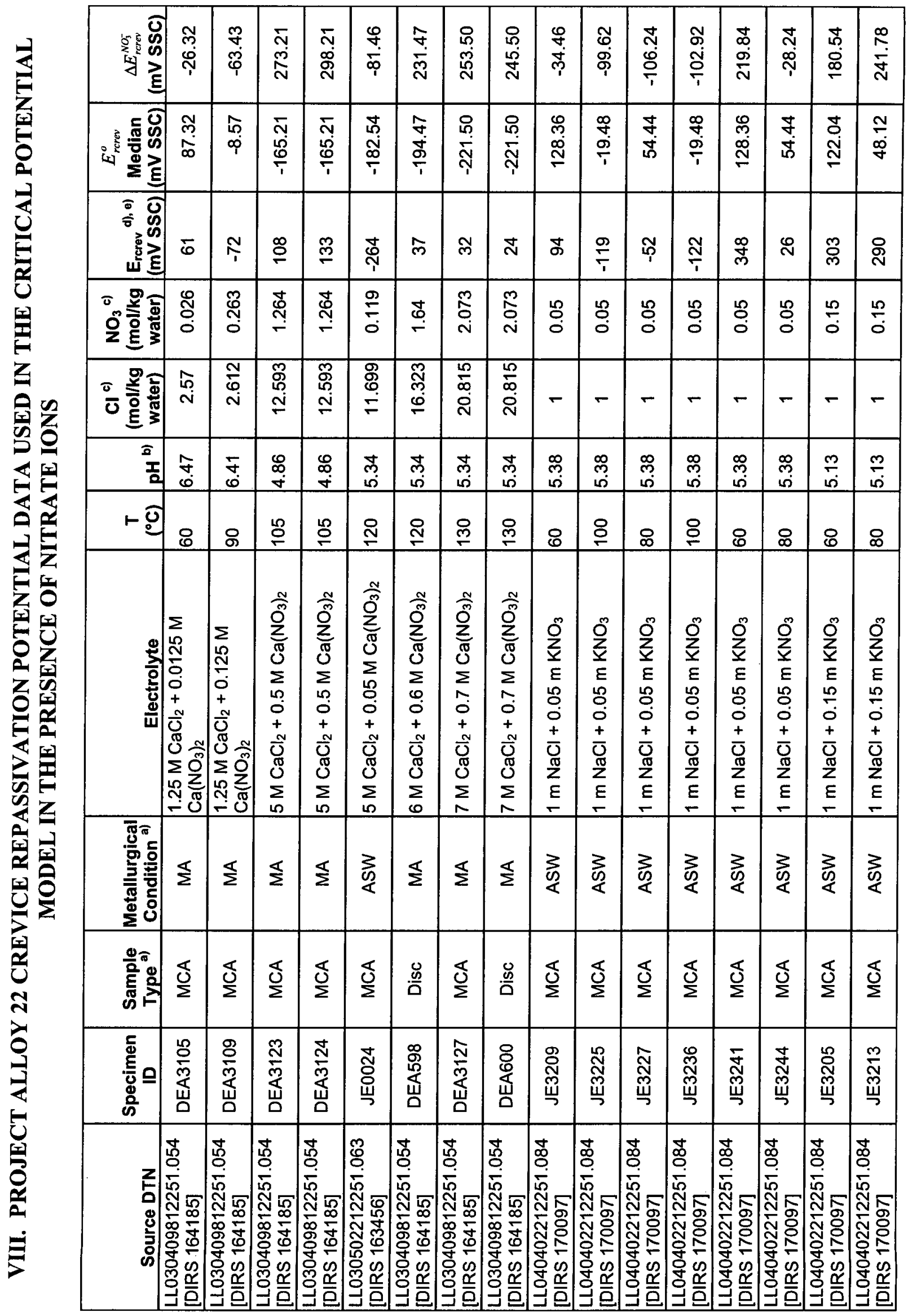


General Corrosion and Localized Corrosion of Waste Package Outer Barrier

\begin{tabular}{|c|c|c|c|c|c|c|c|c|c|c|c|c|c|c|c|c|c|c|}
\hline 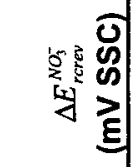 & 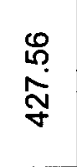 & 品 & $\frac{8}{\dot{y}}$ & $\begin{array}{l}8 \\
\text { के } \\
\text { p }\end{array}$ & $\frac{\mathscr{\infty}}{\stackrel{N}{N}}$ & $\stackrel{\mathscr{0}}{\stackrel{\oplus}{*}}$ & $\begin{array}{l}\text { 感 } \\
\text { مै }\end{array}$ & $\begin{array}{l}\infty \\
\infty \\
\stackrel{8}{8} \\
\text { D }\end{array}$ & $\begin{array}{l}\text { 우 } \\
10 \\
\infty \\
\infty\end{array}$ & $\frac{L_{n}}{N}$ & $\begin{array}{l}8 \\
\stackrel{0}{4} \\
\text { T }\end{array}$ & $\begin{array}{l}\frac{\nabla}{\dot{\phi}} \\
\stackrel{\phi}{\varphi}\end{array}$ & $\frac{\hbar}{\dot{\varphi}}$ & $\begin{array}{l}\hat{N} \\
\stackrel{\hat{\varphi}}{1}\end{array}$ & ثั & $\begin{array}{l}\underset{\infty}{\infty} \\
\stackrel{N}{T} \\
\stackrel{T}{T}\end{array}$ & 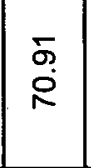 & $\begin{array}{l}\bar{n} \\
\stackrel{\infty}{*} \\
\text { }\end{array}$ \\
\hline 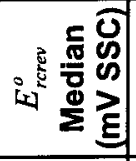 & $\begin{array}{l}\stackrel{\Xi}{\mathbb{N}} \\
\stackrel{N}{ }\end{array}$ & $\frac{N}{\stackrel{\infty}{q}}$ & 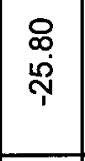 & 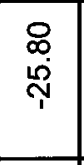 & 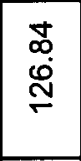 & $\begin{array}{l}\mathbb{\$} \\
\dot{0} \\
\stackrel{N}{ }\end{array}$ & $\begin{array}{l}\text { న్ } \\
\text { గై }\end{array}$ & ్ָ & $\frac{8}{\grave{1}}$ & $\frac{8}{\frac{8}{1}}$ & 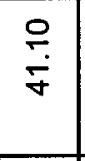 & $\begin{array}{l}\mathscr{9} \\
\stackrel{\rho}{\rho} \\
\stackrel{1}{1}\end{array}$ & $\frac{\mathscr{m}}{\stackrel{T}{\leftarrow}}$ & $\underset{\stackrel{P}{*}}{\stackrel{P}{\leftarrow}}$ & \begin{tabular}{l}
$\mathscr{9}$ \\
$\stackrel{0}{*}$ \\
\multirow{T}{*}{}
\end{tabular} & $\begin{array}{l}8 \\
\dot{q} \\
\dot{q}\end{array}$ & $\begin{array}{l}\bar{\sigma} \\
\dot{\sigma} \\
\frac{\sigma}{1}\end{array}$ & 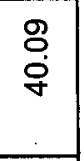 \\
\hline 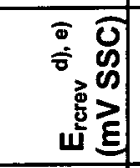 & 品 & ల్లి & $\stackrel{10}{\sim}$ & $\stackrel{8}{\$}$ & 疋 & 8 & ్ㅇㅇ & 芦 & 导 & 芯 & $\uparrow$ & స్ల & $\frac{T}{T}$ & 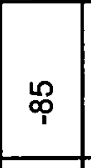 & $\frac{+}{1}$ & $\stackrel{m}{T}$ & 足 & $\stackrel{\text { : }}{\text { ऽ }}$ \\
\hline 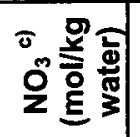 & $\frac{10}{0}$ & $\frac{n}{0}$ & $\frac{n}{0}$ & $\frac{10}{\circ}$ & $\stackrel{10}{0}$ & مْ & مْ & مٌ & $\stackrel{10}{0}$ & $\stackrel{0}{0}$ & $\frac{\stackrel{R}{2}}{0}$ & $\stackrel{\frac{n}{5}}{5}$ & $\stackrel{\stackrel{n}{\leftarrow}}{\circ}$ & $\stackrel{\stackrel{R}{f}}{\circ}$ & $\stackrel{\stackrel{n}{2}}{\circ}$ & $\frac{\stackrel{R}{\leftarrow}}{\sigma}$ & $\stackrel{\mathscr{n}}{0}$ & $\begin{array}{l}\mathbb{W} \\
\text { గ్ర } \\
0\end{array}$ \\
\hline 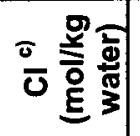 & - & $r$ & $r$ & - & - & - & $\sigma$ & - & - & - & $\stackrel{n}{n}$ & $\stackrel{n}{\infty}$ & $\ddot{\infty}$ & $\stackrel{n}{\infty}$ & $\stackrel{n}{n}$ & $\stackrel{n}{n}$ & $\stackrel{n}{n}$ & $\stackrel{n}{n}$ \\
\hline $\begin{array}{l}\mathbf{a} \\
\mathbf{1} \\
\end{array}$ & $\frac{m}{60}$ & $\frac{m}{5}$ & $\frac{m}{2}$ & $\frac{m}{5}$ & $\begin{array}{l}\widetilde{N} \\
\stackrel{\omega}{\infty} \\
\end{array}$ & 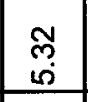 & 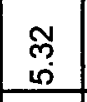 & $\begin{array}{l}\text { ల్ } \\
\text { Lే }\end{array}$ & $\begin{array}{l}\text { ले } \\
\text { ஸे }\end{array}$ & $\begin{array}{c}\widetilde{N} \\
\text { î. } \\
\end{array}$ & 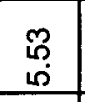 & 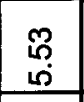 & 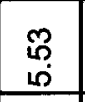 & $\begin{array}{l}\text { B. } \\
\text { in } \\
0\end{array}$ & 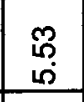 & $\begin{array}{l}\text { g } \\
\text { in } \\
\end{array}$ & 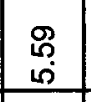 & $\begin{array}{l}\stackrel{9}{+} \\
\leftarrow \circ \\
\end{array}$ \\
\hline$\vdash$ 임 & 8 & $\infty$ & $\stackrel{ }{\circ}$ & $\stackrel{8}{\circ}$ & 8 & 8 & \& & \& & 음 & $\stackrel{8}{\circ}$ & 8 & 옴 & 8 & வ & 웅 & 8 & வ & 8 \\
\hline 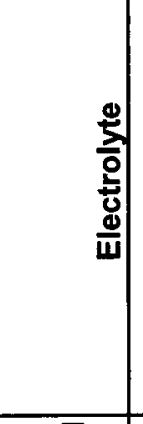 & 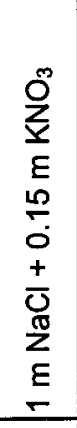 & 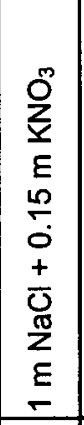 & 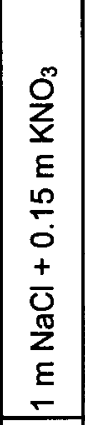 & 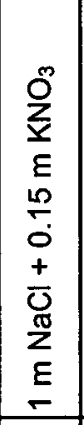 & 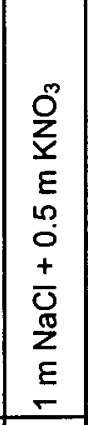 & 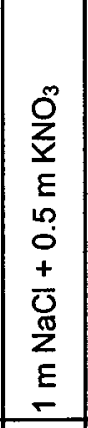 & 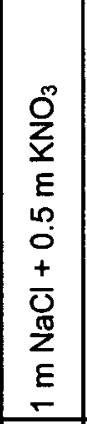 & 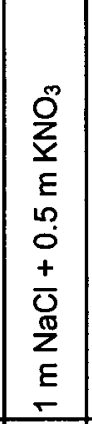 & 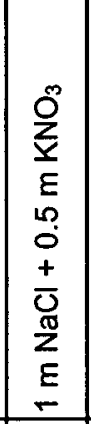 & 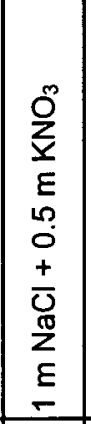 & 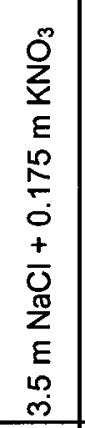 & 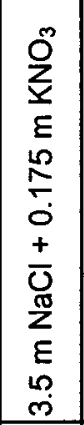 & 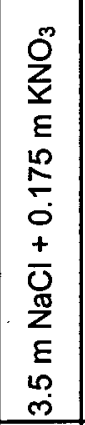 & 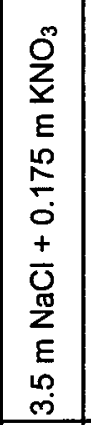 & 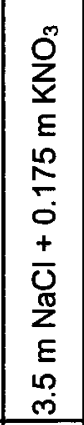 & 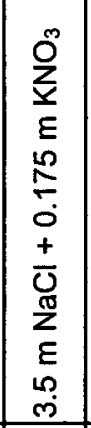 & 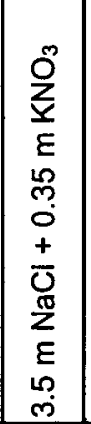 & 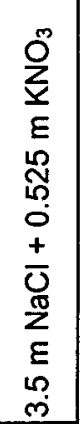 \\
\hline 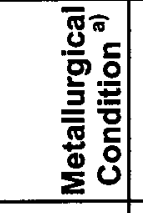 & 交 & 亭 & 离 & 亭 & 变 & 雚 & 紊 & 交 & 交 & 交 & 交 & 交 & 䓠 & 高 & 离 & 交 & 毫 & 帝 \\
\hline 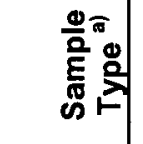 & 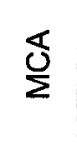 & $\frac{\delta}{\Sigma}$ & $\underset{\Xi}{\Sigma}$ & $\frac{\delta}{\Sigma}$ & 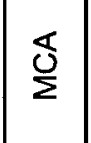 & $\frac{\delta}{\Sigma}$ & 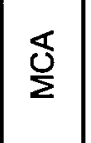 & 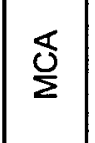 & $\frac{\delta}{\Sigma}$ & $\frac{\pi}{\Sigma}$ & $\frac{\delta}{\Sigma}$ & 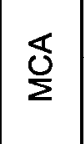 & $\underset{\delta}{\Sigma}$ & $\frac{\delta}{\Sigma}$ & $\overleftarrow{\delta}$ & $\frac{\overleftarrow{S}}{\Sigma}$ & $\frac{\delta}{\Sigma}$ & $\frac{\nwarrow}{\Sigma}$ \\
\hline 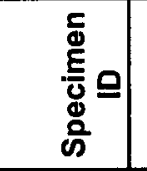 & $\begin{array}{l}\underset{\sim}{\tilde{N}} \\
\stackrel{\Xi}{J}\end{array}$ & $\underset{\stackrel{D}{N}}{\stackrel{\mathscr{m}}{\sim}}$ & $\begin{array}{l}\text { ্ָ } \\
\text { 岃 }\end{array}$ & $\begin{array}{l}\text { ్ָ } \\
\stackrel{\tilde{m}}{\longrightarrow}\end{array}$ & 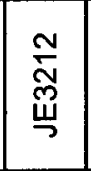 & 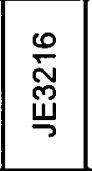 & $\stackrel{\infty}{\stackrel{\infty}{\tilde{N}}}$ & $\underset{\stackrel{\mathscr{W}}{\tilde{W}}}{\stackrel{\mathscr{W}}{丂}}$ & $\stackrel{\mathscr{W}}{\tilde{W}}$ & 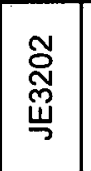 & $\frac{\bar{T}}{\frac{T}{J}}$ & 胥 & 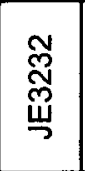 & 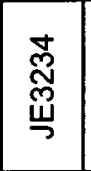 & $\begin{array}{l}\stackrel{\mathscr{N}}{\tilde{W}} \\
\stackrel{\tilde{W}}{J}\end{array}$ & 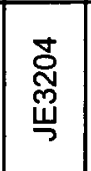 & 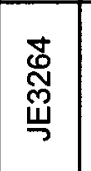 & 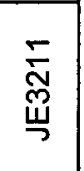 \\
\hline 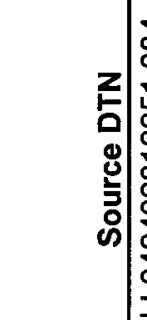 & 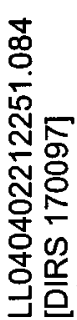 & 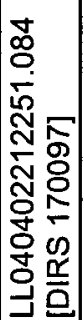 & 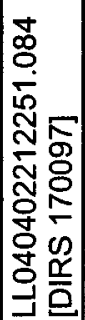 & 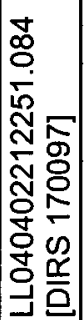 & 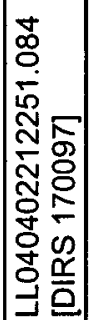 & 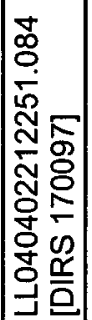 & 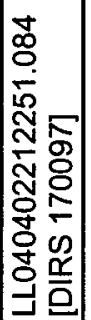 & 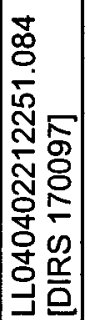 & 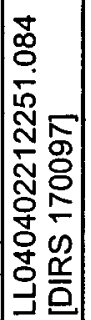 & 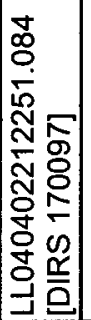 & 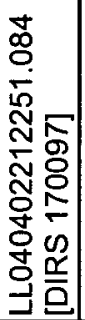 & 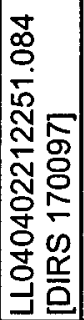 & 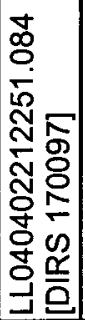 & 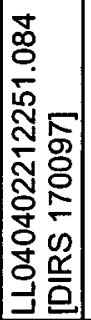 & 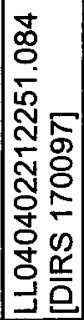 & 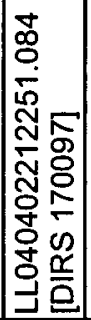 & 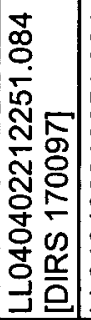 & 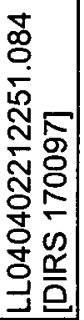 \\
\hline
\end{tabular}


156800 Plinski, M.J. 2001. Waste Package Operations Fabrication Process Report. TDREBS-ND-000003 REV 02. Las Vegas, Nevada: Bechtel SAIC Company. ACC: MOL.20011003.0025.

100817 Pourbaix, M. 1974. Atlas of Electrochemical Equilibria in Aqueous Solutions. Houston, Texas: National Association of Corrosion Engineers. TIC: 208955.

169631 Pulvirenti, A.L.; Needham, K.M.; Adel-Hadadi, M.A.; Barkatt, A.; Marks, C.R.; and Gorman, J.A. 2004. "Multi-Phase Corrosion of Engineered Barrier Materials." Corrosion/2004, 59th Annual Conference \& Exposition, March 28-April 1, 2004, New Orleans. Paper No. 04694. Houston, Texas: NACE International. TIC: 255943.

162237 Rebak, R.B.; Edgecumbe Summers, T.S.; Lian, T.; Carranza, R.M.; Dillman, J.R.; Corbin, T.; and Crook, P. 2002. "Effect of Thermal Aging on the Corrosion Behavior of Wrought and Welded Alloy 22." Corrosion/2002, 57th Annual Conference \& Exposition, April 7-11, 2002, Denver, Colorado. Paper No. 02542. Houston, Texas: NACE International. TIC: 254582.

159370 Revie, R.W., ed. 2000. Uhlig's Corrosion Handbook. 2nd Edition. New York, New York: John Wiley \& Sons. TIC: 248360.

162259 Sawyer, D.T. and Roberts, J.L., Jr. 1974. "II. Reference Electrodes." Experimental Electrochemistry for Chemists. Pages 34-60. New York, New York: John Wiley \& Sons. TIC: 254739.

110246 Scully, J.R.; Hudson, J.L.; Lunt, T.; Ilevbare, G.; and Kehler, B. 1999. Localized Corrosion Initiation and Transition to Stabilization in Alloys 625 and C-22. Charlottesville, Virginia: University of Virginia. TIC: 246630.

154513 Scully, J.R.; Ilevbare, G.; and Marks, C. 2001. Passivity and Passive Corrosion of Alloys 625 and 22. SEAS Report No. UVA/527653/MSE01/103. Charlottesville, Virginia: University of Virginia, School of Engineering \& Applied Science. TIC: 248056.

162238 Sharland, S.M.; Naish, C.C.; Taylor, K.J.; and Marsh, G.P. 1994. "An Experimental and Modelling Study of the Localized Corrosion of Carbon Steel Overpacks for the Geological Disposal of Radioactive Waste." Life Prediction of Corrodible Structures. Parkins, R.N., ed. Volume 1. Pages 402-418. Houston, Texas: NACE International. TIC: 254835.

168164 Smailos, E. 1993. "Corrosion of High-Level Waste Packaging Materials in Disposal Relevant Brines." Nuclear Technology, 104, 343-350. La Grange Park, Illinois: American Nuclear Society. TIC: 249836.

159774 Smailos, E. and Köster, R. 1987. "Corrosion Studies on Selected Packaging Materials for Disposal of High Level Wastes." Materials Reliability in the Back End of the Nuclear Fuel Cycle, Proceedings of a Technical Committee Meeting, Vienna, 2-5 
September 1986. IAEA TECHDOC-421, 7-24. Vienna, Austria: International Atomic Energy Agency. TIC: 252877.

105941 Stedinger, J.R.; Vogel, R.M.; and Foufoula-Georgiou, E. 1993. "Frequency Analysis of Extreme Events." Chapter 18 of Handbook of Hydrology. Maidment, D.R., ed. New York, New York: McGraw-Hill. TIC: 236568.

162243 Stoecker, J.G., II. 1987. "Evaluation of Microbiological Corrosion.” In Corrosion, Volume 13, Pages 314-315 of Metals Handbook. 9th Edition. Metals Park, Ohio: ASM International. TIC: 209807.

162260 Taylor, B.N. and Kuyatt, C.E. 1994. Guidelines for Evaluating and Expressing the Uncertainty of NIST Measurement Results. NIST Technical Note 1297. 1994 Edition. Gaithersburg, Maryland: National Institute of Standards and Technology, Physics Laboratory. TIC: 254588.

110250 Treseder, R.S.; Baboian, R.; and Munger, C.G. 1991. NACE Corrosion Engineer's Reference Book. 156-181. Houston, Texas: NACE International. TIC: 245834.

154741 Urquidi, M. and Macdonald, D.D. 1985. "Solute-Vacancy Interaction Model and the Effect of Minor Alloying Elements on the Initiation of Pitting Corrosion." Journal of the Electrochemical Society, 132, (3), 555-558. New York, New York:

Electrochemical Society. TIC: 249843.

162245 Vetter, K.J. and Strehblow, H.H. 1974. "Pitting Corrosion in an Early Stage and its Theoretical Implications." Localized Corrosion, U.R. Evans Conference on Localized Corrosion, December 6-10, 1971, Williamsburg, Virginia. International Corrosion Conference Series, NACE-3. Pages 240-251. Houston, Texas: Natioanl Asscociation of Corrosion Engineers. TIC: 254803.

138928 Walsh, D.W. 1999. "The Effects of Microstructure on MIC Susceptibility in High Strength Aluminum Alloys." Corrosion 99. Paper No. 187, 1-13. Houston, Texas: NACE International. TIC: 246549.

114895 Welsch, G.; Smialek, J.L.; Doychak, J.; Waldman, J.; and Jacobson, N.S. 1996. “High Temperature Oxidation and Properties." Chapter 2 of Oxidation and Corrosion of Intermetallic Alloys. Welsch, G. and Desai, P.D.; eds. West Lafayette, Indiana: Purdue University. TIC: 245280.

154743 Zhang, L. and Macdonald, D.D. 1998. "Segregation of Alloying Elements in Passive Systems-I. XPS Studies on the Ni-W System." Electrochimica Acta, 43, (18), 26612671. New York, New York: Pergamon Press. TIC: 249845.

154742 Zhang, L. and Macdonald, D.D. 1998. "Segregation of Alloying Elements in Passive Systems-II. Numerical Simulation." Electrochimica Acta, 43, (18), 2673-2685. New York, New York: Pergamon Press. TIC: 249846. 


\subsection{CODES, STANDARDS, REGULATIONS, AND PROCEDURES}

15660510 CFR 63. Energy: Disposal of High-Level Radioactive Wastes in a Geologic Repository at Yucca Mountain, Nevada. Readily available.

AP-2.14Q, Rev. 3, ICN 0. Document Review. Washington, D.C.: U.S. Department of Energy, Office of Civilian Radioactive Waste Management. ACC:

DOC.20030827.0018.

AP-2.22Q, Rev. 1, ICN 1. Classification Analyses and Maintenance of the Q-List. Washington, D.C.: U.S. Department of Energy, Office of Civilian Radioactive Waste Management. ACC: DOC.20040714.0002.

AP-2.27Q, Rev. 1, ICN 4. Planning for Science Activities. Washington, D.C.: U.S. Department of Energy, Office of Civilian Radioactive Waste Management. ACC: DOC.20040610.0006.

AP-3.15Q, Rev. 4, ICN 5. Managing Technical Product Inputs. Washington, D.C.: U.S. Department of Energy, Office of Civilian Radioactive Waste Management. ACC: DOC.20040812.0004.

AP-SIII.10Q, Rev. 2, ICN 7. Models. Washington, D.C.: U.S. Department of Energy, Office of Civilian Radioactive Waste Management. ACC: DOC.20040920.0002.

AP-SV.1Q, Rev. 1, ICN 1. Control of the Electronic Management of Information. Washington, D.C.: U.S. Department of Energy, Office of Civilian Radioactive Waste Management. ACC: DOC.20040308.0001.

100497 ASTM B 575-94. 1994. Standard Specification for Low-Carbon Nickel-MolybdenumChromium, Low-Carbon Nickel-Chromium-Molybdenum, and Low-Carbon NickelChromium-Molybdenum-Tungsten Alloy Plate, Sheet, and Strip. Philadelphia, Pennsylvania: American Society for Testing and Materials. TIC: 237683.

105725 ASTM C 1174-97. 1998. Standard Practice for Prediction of the Long-Term Behavior of Materials, Including Waste Forms, Used in Engineered Barrier Systems (EBS) for Geological Disposal of High-Level Radioactive Waste. West Conshohocken, Pennsylvania: American Society for Testing and Materials. TIC: 246015.

169968 ASTM E 178-02. 2002. Standard Practice for Dealing with Outlying Observations. West Conshohocken, Pennsylvania: American Society for Testing and Materials. TIC: 256164.

103515 ASTM G 1-90 (Reapproved 1999). 1999. Standard Practice for Preparing, Cleaning, and Evaluating Corrosion Test Specimens. West Conshohocken, Pennsylvania: American Society for Testing and Materials. TIC: 238771. 
163908 ASTM G 102-89 (Reapproved 1999). 1989. Standard Practice for Calculation of Corrosion Rates and Related Information from Electrochemical Measurements. West Conshohocken, Pennsylvania: American Society for Testing and Materials. TIC: 249897.

138911 ASTM G 3-89 (Reapproved 1999). 1989. Standard Practice for Conventions Applicable to Electrochemical Measurements in Corrosion Testing. West Conshohocken, Pennsylvania: American Society for Testing and Materials. TIC: 247076 .

137688 ASTM G 30-94. 1994. Standard Practice for Making and Using U-Bend StressCorrosion Test Specimens. Philadelphia, Pennsylvania: American Society for Testing and Materials. TIC: 246890.

117479 ASTM G 5-94. 1994. Standard Reference Test Method for Making Potentiostatic and Potentiodynamic Anodic Polarization Measurements. Philadelphia, Pennsylvania: American Society for Testing and Materials. TIC: 231902.

163907 ASTM G 59-97. 1998. Standard Test Method for Conducting Potentiodynamic Polarization Resistance Measurements. West Conshohocken, Pennsylvania: American Society for Testing and Materials. TIC: 249897.

127897 ASTM G 61-86 (Reapproved 1998). 1987. Standard Test Method for Conducting Cyclic Potentiodynamic Polarization Measurements for Localized Corrosion Susceptibility of Iron-, Nickel-, or Cobalt-Based Alloys. West Conshohocken, Pennsylvania: American Society for Testing and Materials. TIC: 246716.

LP-SI.11Q-BSC, Rev. 0, ICN 0. Software Management. Washington, D.C.: U.S. Department of Energy, Office of Civilian Radioactive Waste Management. ACC: DOC.20040225.0007.

169585 TIP-CM-51 Rev. 0, Change Notice CM-51-0-4. Long Term Corrosion Test Facility Specimen De-Scaling Procedure for Alloy 22, Alloy C4, Titanium Alloys, TiGr 7, TiGr 12, and TiGr 16 Specimens. Livermore, California: Lawrence Livermore National Laboratory. ACC: MOL.20030927.0180.

\subsection{SOURCE DATA, LISTED BY DATA TRACKING NUMBER}

161204 LL020711612251.017. Open Circuit Potential Tests. Submittal date: 10/11/2002.

161135 LL020903812251.019. Comparison of Weight Changes Resulting from the Reaction of Deposited Calcium Chloride on Alloy-22, Platinum, and Glass Substrates at $150 \mathrm{C}$ and $22.5 \%$ Relative Humidity. Submittal date: 10/01/2002.

161138 LL021105112251.022. Electrochemical Behavior of Alloy 22 in Simulated Acidic Concentrated Water (SAW), Simulated Concentrated Water (SCW) and $5 \mathrm{M} \mathrm{CaCl} 2$ Solutions. Submittal date: 12/11/2002. 
163775 LL030308812251.017. The Effect of Temperature on the Reaction Rate of Calcium Chloride on the Surface of Alloy-22 at $22.5 \%$ Relative Humidity and Atmospheric Pressure. Submittal date: 05/07/2003.

163455 LL030309512251.042. Low Temperature Operating Mode Electrochemical Test Data: Corrosion Potential, Linear Polarization, and Cyclic Polarization Tests of Alloy 22 in Oxalic Acid, $\mathrm{NaF}, \mathrm{NaCl}, \mathrm{NaF}+\mathrm{NaCl}$, and $\mathrm{CaCl}_{2}$ Solutions Recorded at 30, 45, 60, 75, 90 and 105 Deg C. Submittal date: 05/22/2003.

163466 LL0304001 12251.043. Electrochemical Behavior of Multiple Crevice Assembly (MCA) Alloy 22 Specimens in 5M CaCl2. Submittal date: 04/07/2003.

163467 LL030406212251.044. Alloy 22 in 5M CaCl2 at 75C - Supplemental. Submittal date: 04/08/2003.

163469 LL030406412251.045. Transmission Electron Microscopy (TEM) Images of Oxide Film on Air-Oxidized Alloy22 (UNS N06022) Samples Aged at 550C. Submittal date: $04 / 11 / 2003$.

163462 LL030409512251.051. Electrochemical Analysis of Alloy 22 - CaCl2 Data at Various Temperatures, Surface Conditions, and Electrolyte Solutions. Submittal date: 05/23/2003.

164185 LL030409812251.054. Electrochemical Data of Alloy 22 in CaCl2 Solutions-Effect of Nitrate. Submittal date: 06/26/2003.

163712 LL030412512251.057. LTCTF Corrosion Rate Calculations for Five-Year Exposed Alloy C22 Specimens Cleaned Under TIP-CM-51. Submittal date: 05/28/2003.

163456 LL030502212251.063. Corrosion Potential, Polarization Resistance, and Cyclic Polarization in Several Environments at Several Temperatures for Alloy 22.

Submittal date: 05/20/2003.

164184 LL030703723121.031. Conversion of Corrosion Testing Solutions from Molar to Molal Concentration Units. Submittal date: 07/10/2003.

170502 LL031001023121.035. Conversion of Corrosion Testing Solutions from Molar to Molal Concentration Units (II). Submittal date: 04/23/2004.

170221 LL040303612251.078. Microbiology Influenced Corrosion (MIC) Effects on Microcosm-Incubated Alloy 22. Submittal date: 03/29/2004.

170283 LL040402112251.083. Corrosion Potential and Critical Potential for Alloy 22. Submittal date: 05/19/2004.

170097 LL040402212251.084. Critical and Corrosion Potential for Alloy 22 (N06022). Submittal date: 05/26/2004. 
170222 LL040402912251.085. Microbiologically Induced Corrosion (MIC) Linear Polarization Corrosion Rate of Alloy 22 Weldments for Sterile and Non-Sterile Coupons. Submittal date: 04/20/2004.

171362 LL040803112251.117. Target Compositions of Aqueous Solutions Used for Corrosion Testing. Submittal date: 08/14/2004.

138343 LL991203505924.094. Approach and Supporting Data for MIC Modeling. Submittal date: $12 / 13 / 1999$.

170760 MO0407SEPFEPLA.000. LA FEP List. Submittal date: 07/20/2004.

\subsection{OUTPUT DATA, LISTED BY DATA TRACKING NUMBER}

MO0409MWDULCMW.000. Updated Localized Corrosion Model of the Waste Package Outer Barrier. Submittal date: 09/17/2004.

MO0409MWDUGCMW.000. Updated General Corrosion Model of the Waste Package Outer Barrier. Submittal date: 09/17/2004. 
APPENDIX I

SUMMARY OF THE ELECTROCHEMICAL CORROSION TEST PROCEDURES TO GENERATE INPUT DATA FOR ANALYSES AND MODELS IN THIS REPORT 
INTENTIONALLY LEFT BLANK 


\section{SUMMARY OF THE ELECTROCHEMICAL CORROSION TEST PROCEDURES TO GENERATE INPUT DATA FOR ANALYSES AND MODELS IN THIS REPORT}

This appendix summarizes the electrochemical corrosion tests that were employed to generate input data for the analyses and models of the WPOB documented in this report. The typical sequence for electrochemical testing was as follows:

- Grind and polish the testing specimen to eliminate residual effects of fabrication. The surface finishing of the test specimens corresponded to abrasive 600-grit paper. This grinding was done within one hour prior of immersing the test specimen in the electrolyte solution. The testing cell is as described in ASTM G 5-94 (1994 [DIRS 117479]).

- After immersion of the specimen in the electrolyte solution of interest, the free corrosion potential of the specimen was monitored for 24 hours. The value of potential at the end of this 24-hour period was called the corrosion potential $\left(E_{\text {corr }}\right)$. In a few cases, the corrosion potential was monitored for shorter times ( 1 or 2 hours).

- After this 24-hour period, at least three polarization resistance (PR) tests were carried out according to ASTM G 59-97. The value of the slope of the polarization resistance curve $\left(R_{p}\right)$ was used to calculate the general corrosion rate (ASTM G 59-97 1998 [DIRS 163907] and G 102-89 1989 [DIRS 163908]).

- After the polarization resistance tests, one cyclic potentiodynamic polarization (CPP) test was carried out according to ASTM G 61-86 (1987 [DIRS 127897]). The cyclic polarization curve yielded the value of the critical potential for localized corrosion of the WPOB.

Details of the tests are provided in the corresponding Scientific Notebooks cited in the Data Tracking Numbers (DTNs) of the input data documented in Section 4.1.1.

\section{I.1 DETERMINATION OF CORROSION RATES FROM POLARIZATION RESISTANCE TESTS}

The polarization resistance test was carried out using the default set-up of the potentiostat and its software. The test consists of ramping the potential at a rate of $0.167 \mathrm{mV} / \mathrm{sec}(0.6 \mathrm{~V} / \mathrm{hr})$ (consistent with ASTM G 59-97 1998 [DIRS 163907]) while recording the current applied to the specimen. The potential scan was started $20 \mathrm{mV}$ below $E_{\text {corr }}$ and finished $20 \mathrm{mV}$ above $E_{\text {corr }}$. The value of $E_{\text {corr }}$ chosen by the instrument for this scan is the $E_{\text {corr }}$ value 10 seconds before the scan was started (default set up). The resulting data consists of applied values of potential $(E)$ and applied values of current $(I)$. If these data are plotted $E$ versus $I$ on a linear scale, a more or less straight line is obtained. The slope of this line is called the resistance to polarization or $R_{p}$. ASTM G 59-97 (1998 [DIRS 163907]) and ASTM G 102-89 (1989 [DIRS 163908]) explain the reasoning behind the use of this slope in calculating instantaneous corrosion rates. To calculate the corrosion rates, besides the slope $R_{p}$, several other data are needed. These data include, (1) the exposed area of the specimen, (2) the values of the Tafel slopes, (3) the Faraday constant (charge passed by one mole of electrons), (4) the value of the equivalent weight of the tested 
alloy and (5) the density of the tested alloy. The last two data are needed to use the Faraday conversion of current to penetration rate.

The calculation performed in the current report was done considering the following.

- The exposed area of the specimens: For multiple crevice assembly (MCA) samples, the area changed according to the length of the immersed specimen in the electrolyte solution. The exposed area of samples with other geometries (e.g., prism, rod and disc) also varies from sample to sample.

- The cathodic and anodic Tafel slopes are both equal to $0.12 \mathrm{~V} / \mathrm{dec}$ ade. That is, it is considered that it takes $0.12 \mathrm{~V}$ to change the current on the specimen by one order of magnitude. This is a default setting of the Gamry software.

- The charge passed by one mole of electrons is 96,486 C (Lide 1991 [DIRS 131202], Table 1, p. I-1).

- The equivalent weight (EW) for Alloy 22 (N06022) is calculated considering that the alloy dissolves stoichiometrically, and it depends on the valence for the dissolved elements. The value used was taken from Table 1 of ASTM G 102 (1989 [DIRS 163908]), third oxidation state. That is $\mathrm{EW}=23.28$.

- The density of Alloy 22 (N06022) is $8.69 \mathrm{~g} / \mathrm{cm}^{3}$ (Haynes International 1997 [DIRS 100896], p. 13).

The value of $R_{p}$ in ohm- $\mathrm{cm}^{2}$ can be calculated by plotting the curve and estimating its slope. This can be done manually. The Gamry software has a program to calculate the corrosion rate directly from the acquired data. The calculation of $R_{p}$ (which is used to calculate the corrosion rate) can be accomplished by using the entire range of potentials $(40 \mathrm{mV})$ or a specific predetermined range around the potential for zero applied current $\left(E_{c o r r}\right)$. Since in most environments the plots of potential versus current in the linear scale were not straight (linear), a smaller range of potential around $E_{\text {corr }}$ was chosen (ASTM G 3-89 1989 [DIRS 138911]). The range chosen in our calculations was $10 \mathrm{mV}$ below $E_{\text {corr }}$ and $10 \mathrm{mV}$ above $E_{\text {corr }}$. To perform the calculation, the $E_{\text {corr }}$ at 10 seconds before the test was selected and from this value $10 \mathrm{mV}$ were subtracted $\left(E_{s}=E_{\text {corr }}\right.$ $-10 \mathrm{mV})$. This potential was the starting potential for the calculation range $\left(E_{s}\right)$. Similarly the finish potential point $\left(E_{f}\right)$ was chosen as $E_{f}=E_{c o r r}+10 \mathrm{mV}$.

$$
i_{\text {corr }}=10^{6} \frac{B}{R_{p}}
$$

where $i_{\text {corr }}$ is the corrosion current density in $\mu \mathrm{A} / \mathrm{cm}^{2}$, and $R_{p}$ is the slope in ohm- $\mathrm{cm}^{2} . B$ is the Stern-Geary coefficient and is related to the anodic and cathodic Tafel slopes as follows (ASTM G-59-97 1998 [DIRS 163907]).

$$
B=\frac{b_{a} \cdot b_{c}}{2.303\left(b_{a}+b_{c}\right)}
$$




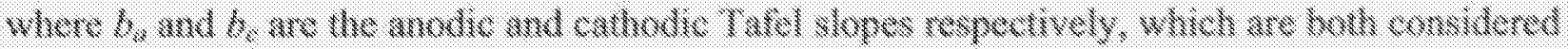

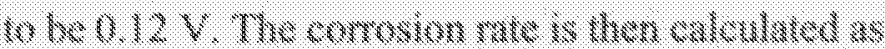

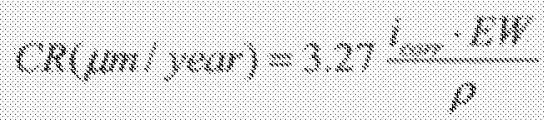

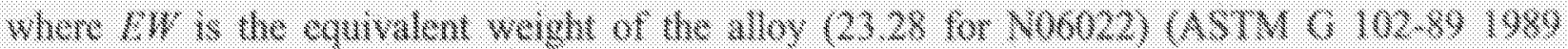

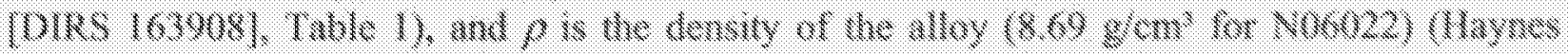
International 1907 [DIRS 100806), p. (3).

\subsection{DFTLRMINATION OF TUE CRITICAL POTEWTIA. OR RWPASWY ATION

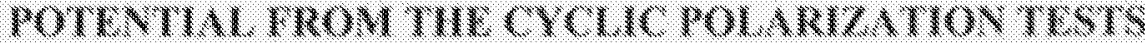

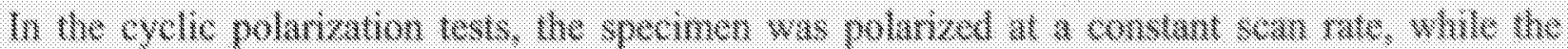

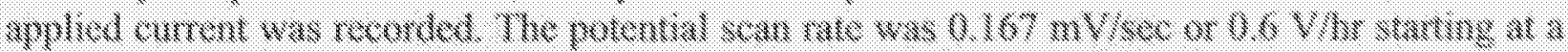
poternal of $150 \mathrm{~m}$ V helow L

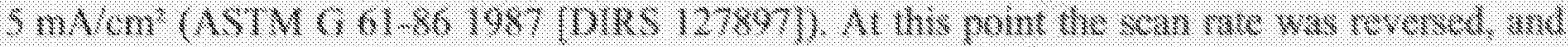

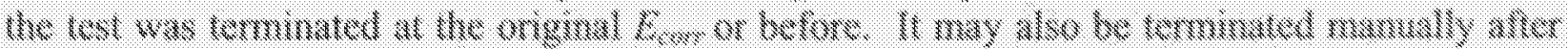
He reversing surwe intersects the forward curve.

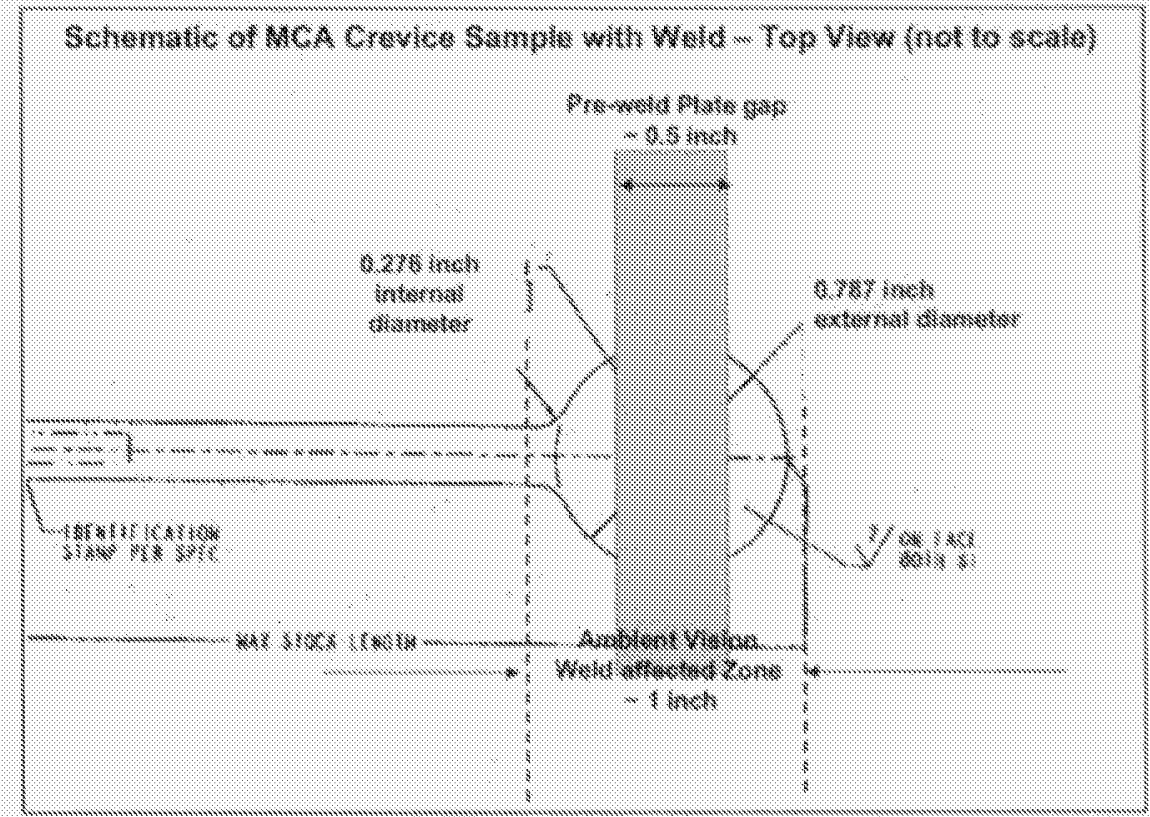

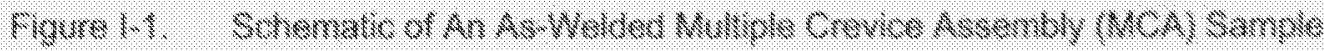




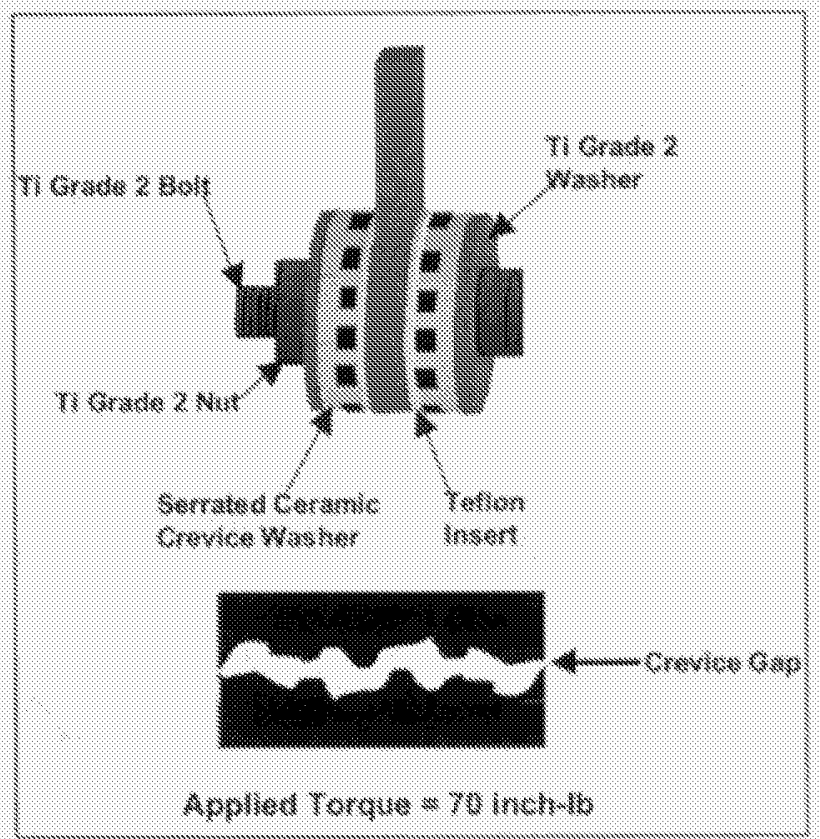

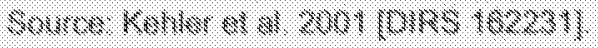

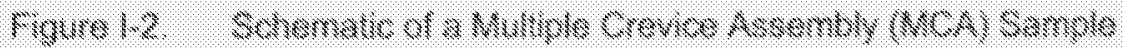

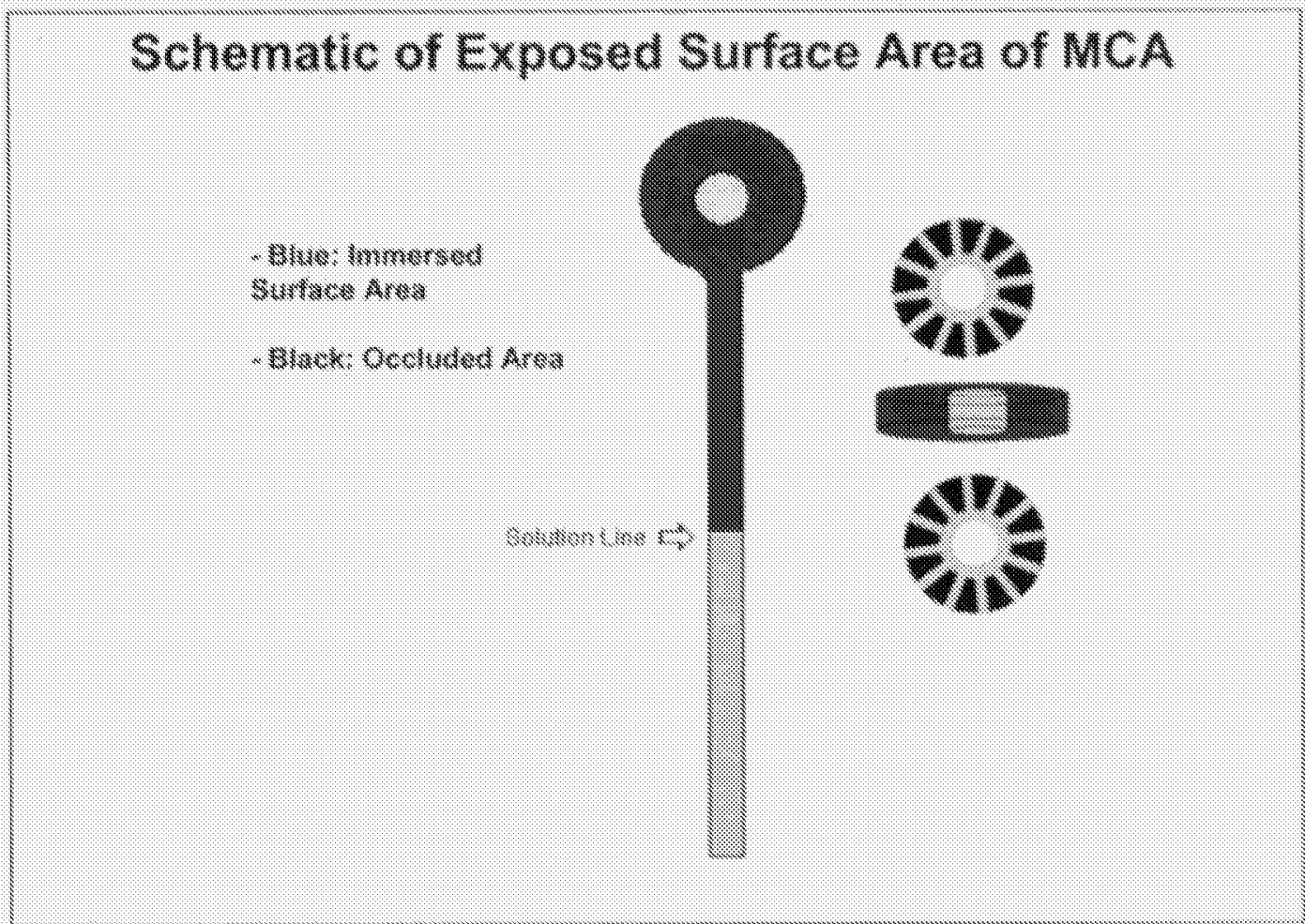

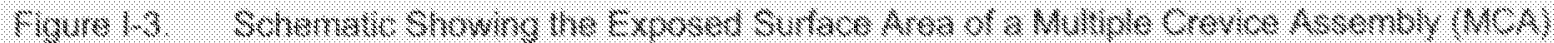

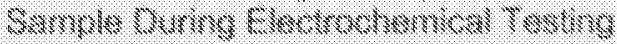




\section{APPENDIX II \\ GENERAL CORROSION RATES OF ALLOY 22 CREVICE SAMPLES BASED ON WEIGHT-LOSS MEASUREMENTS AFTER 5-YEAR EXPOSURE IN THE LONG- TERM CORROSION TEST FACILITY}


INTENTIONALLY LEFT BLANK 
General Corrosion and Localized Corrosion of Waste Package Outer Barrier

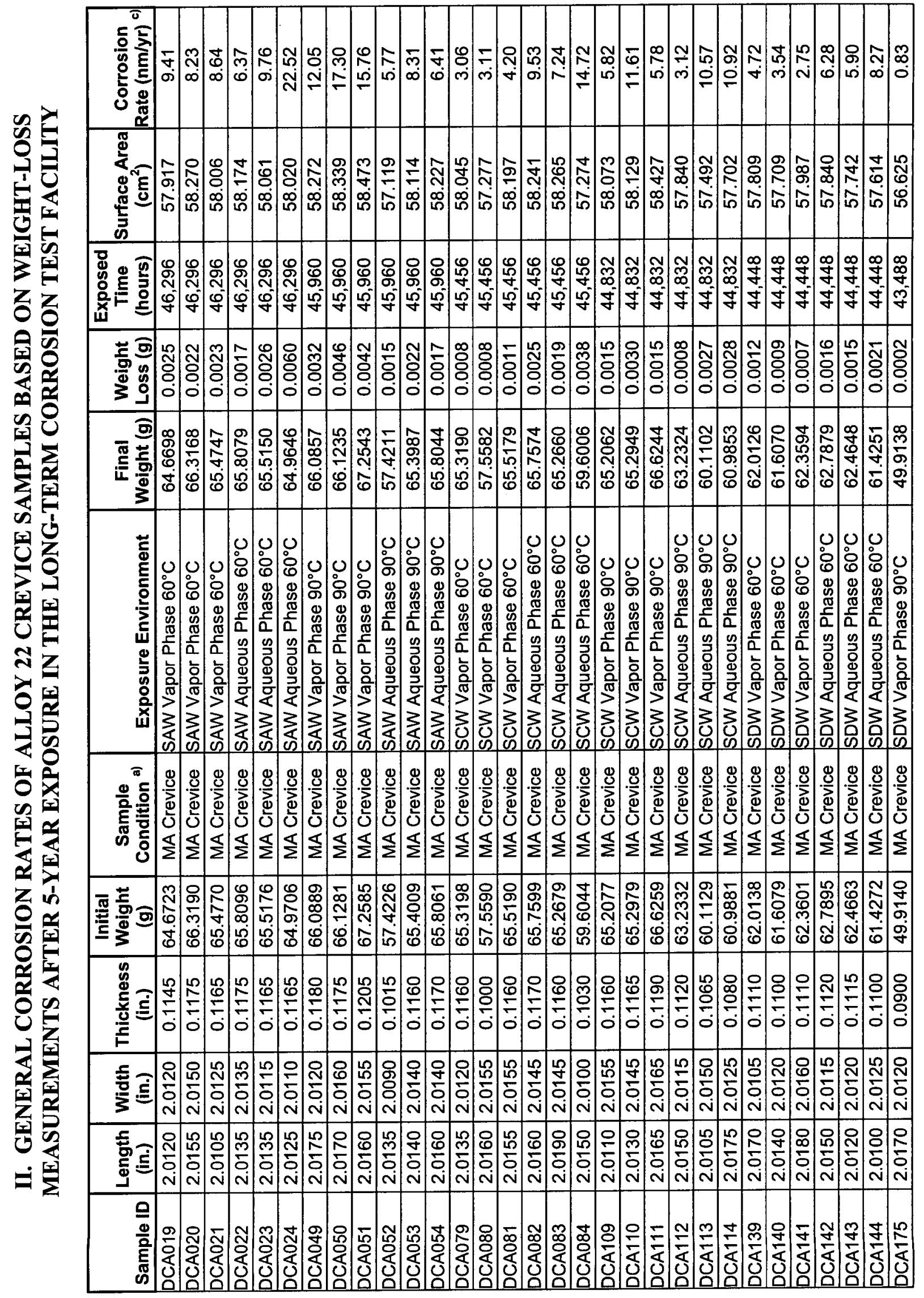


General Corrosion and Localized Corrosion of Waste Package Outer Barrier

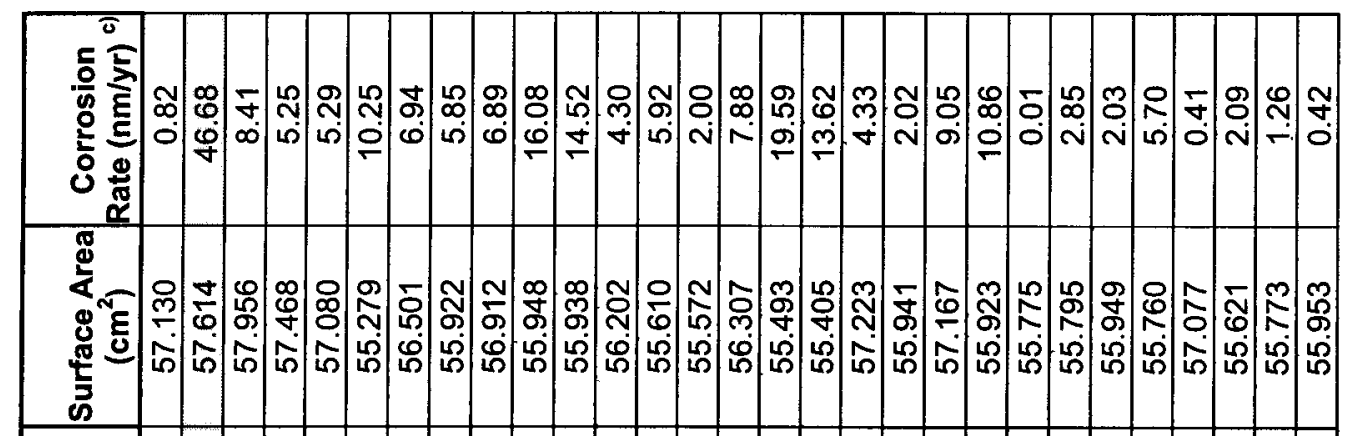

疍 莒

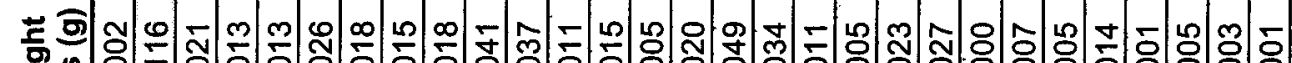

产

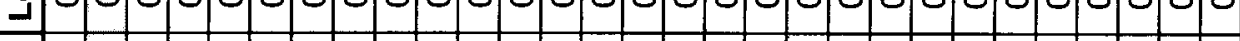

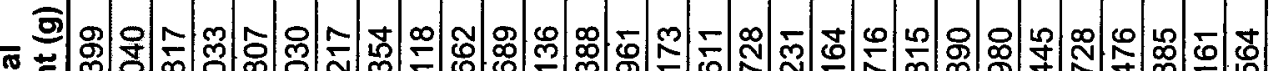

至

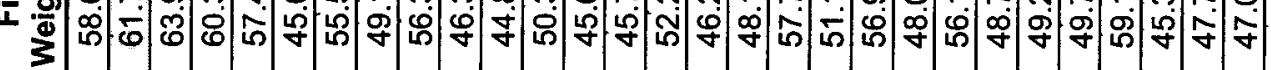

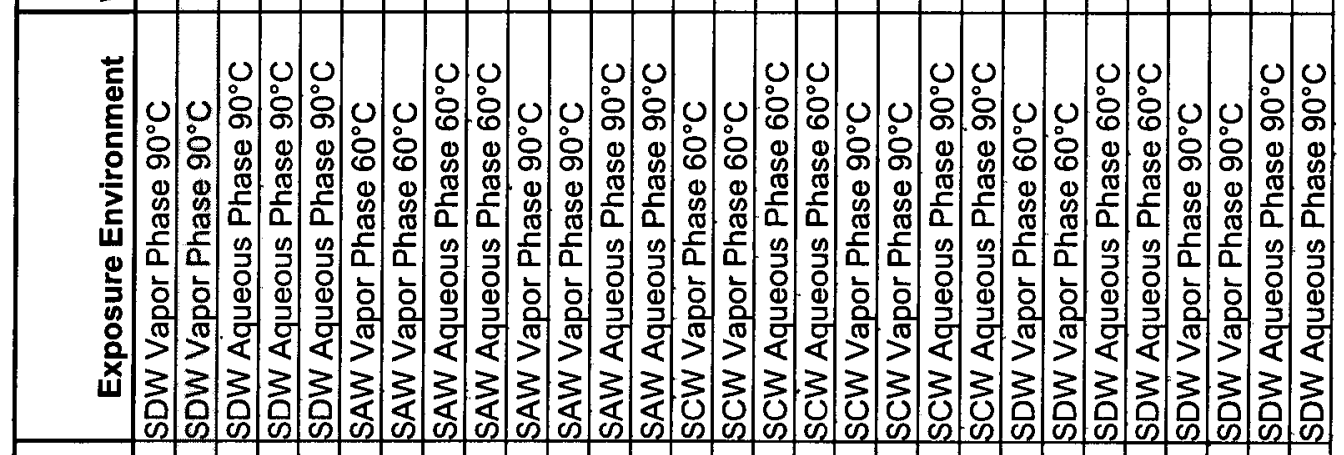

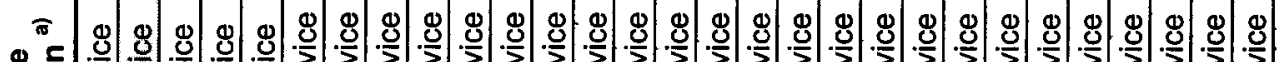

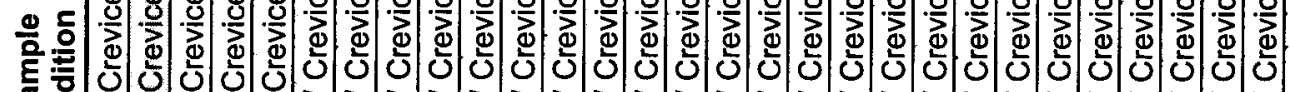

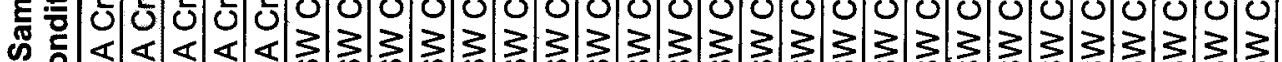

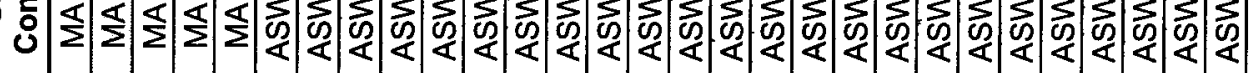

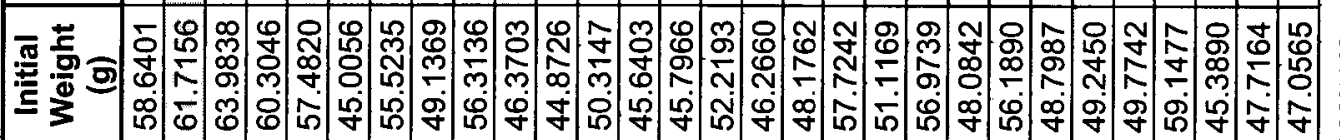

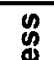

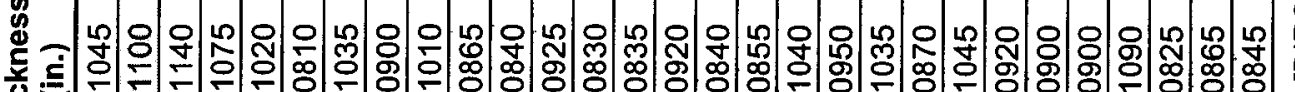

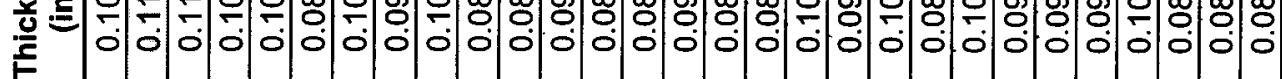

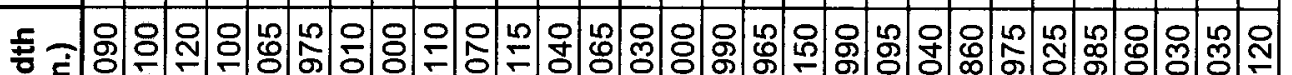

క

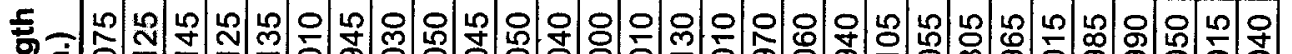

兽

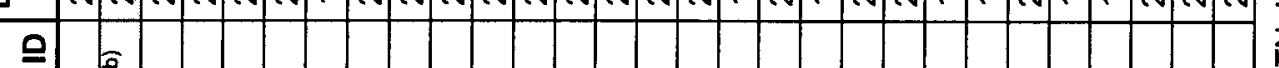

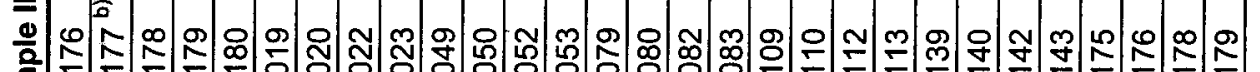

悹

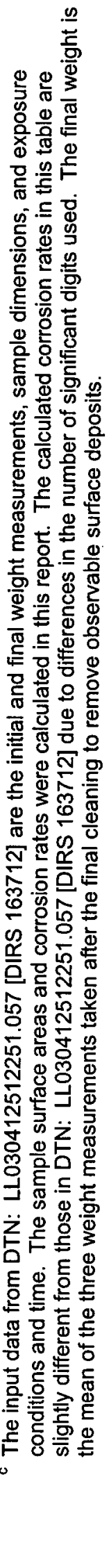




\begin{abstract}
APPENDIX III
GENERAL CORROSION RATE OF ALLOY 22 WEIGHT-LOSS SAMPLES BASED ON THE WEIGHT-LOSS MEASUREMENT AFTER 5-YEAR EXPOSURE IN THE LONGTERM CORROSION TEST FACILITY.
\end{abstract}


INTENTIONALLY LEFT BLANK 


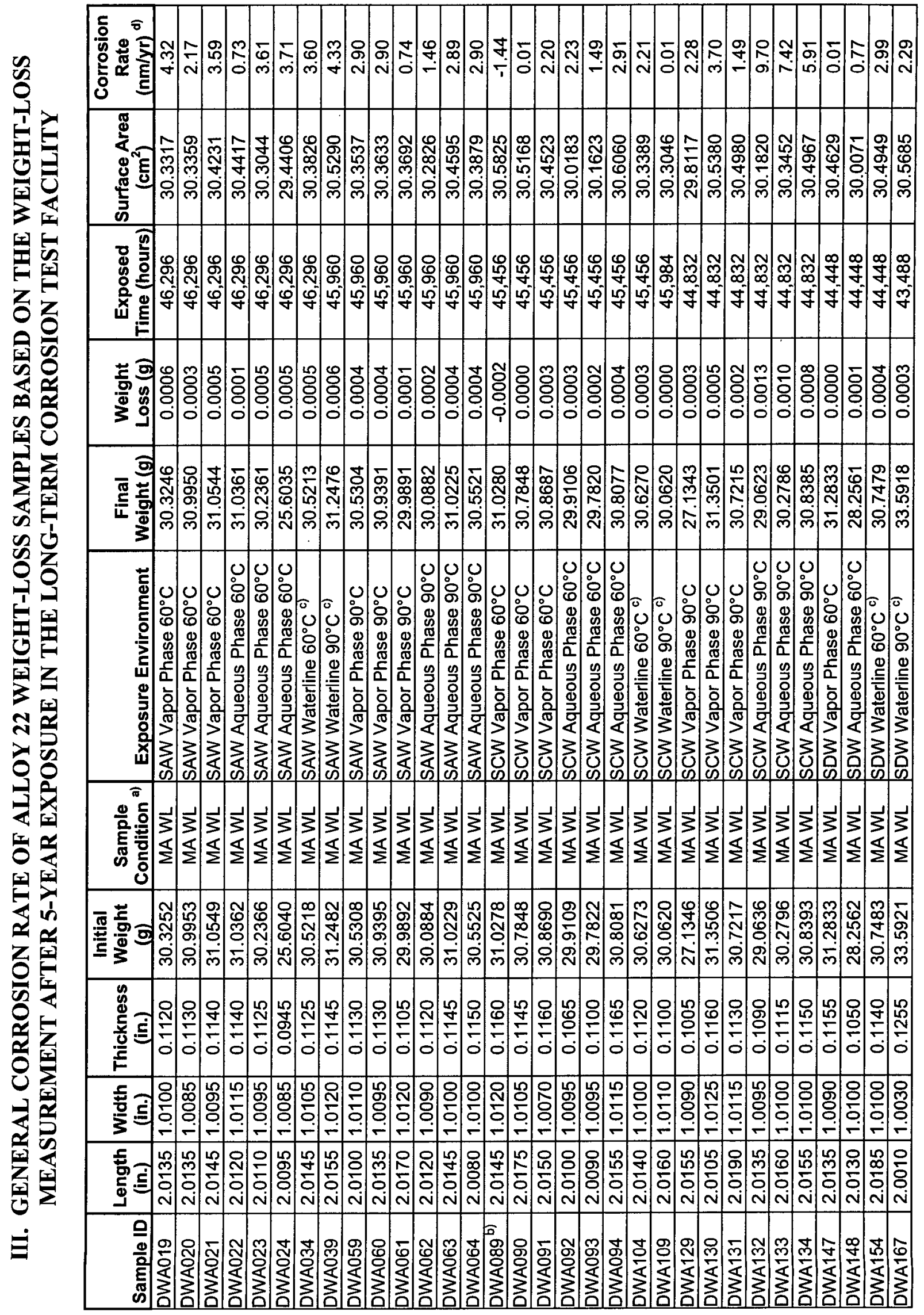


General Corrosion and Localized Corrosion of Waste Package Outer Barrier

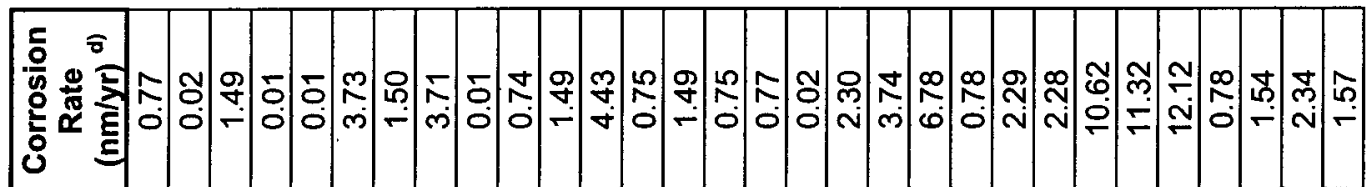

\$

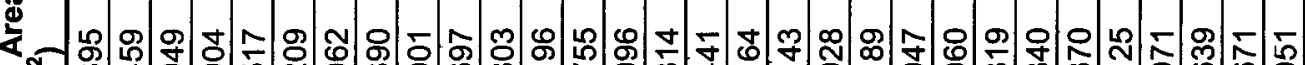

\&

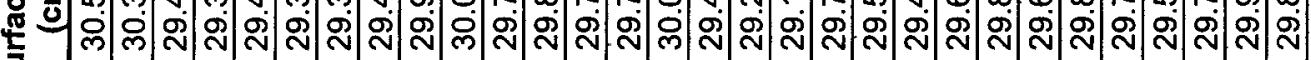
ब)

ఫ : 希 1.

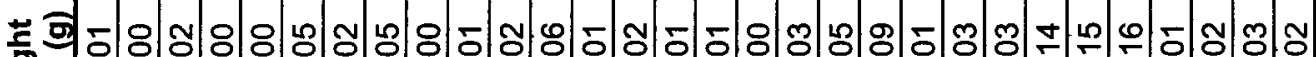

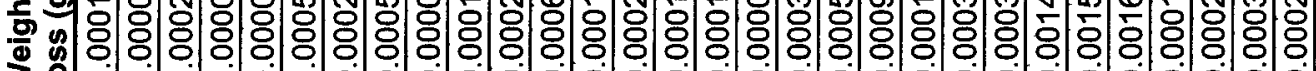
క气

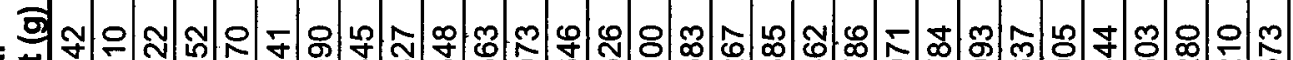

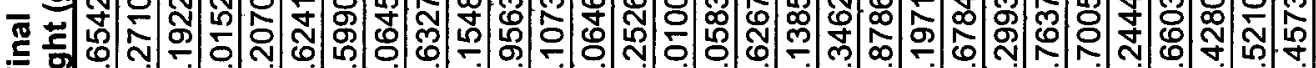

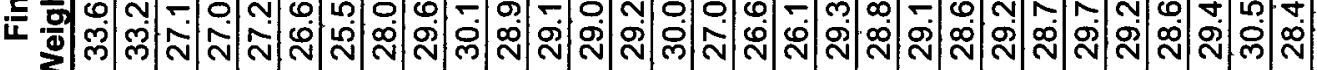

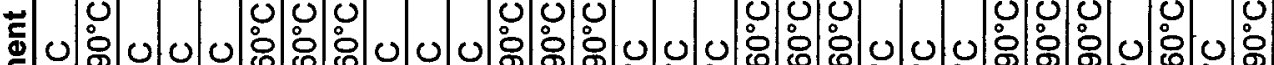

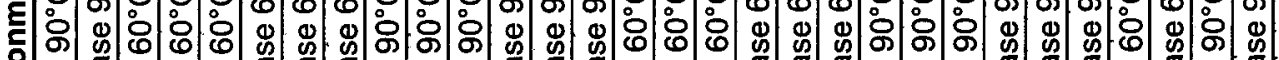

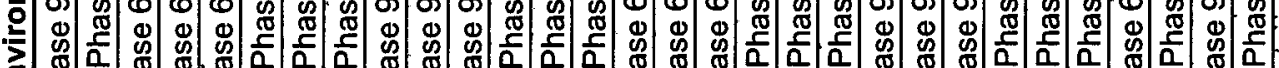

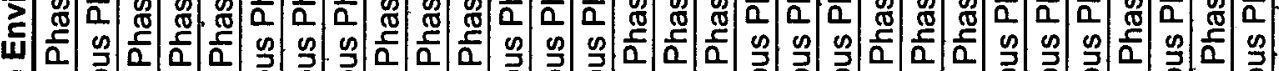

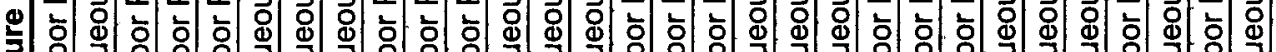

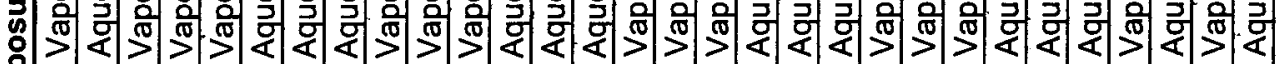

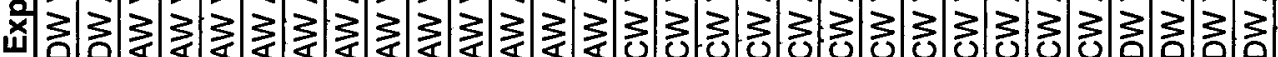

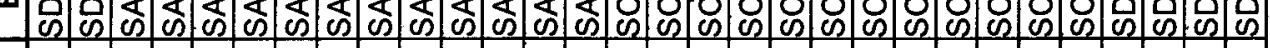

(

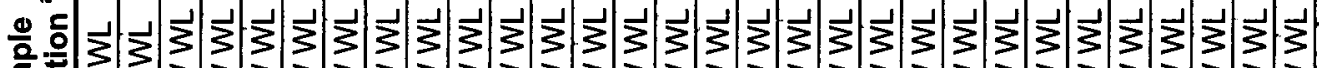

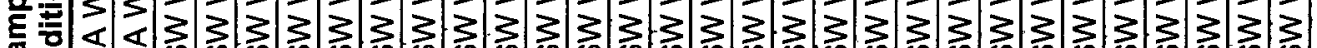

कू

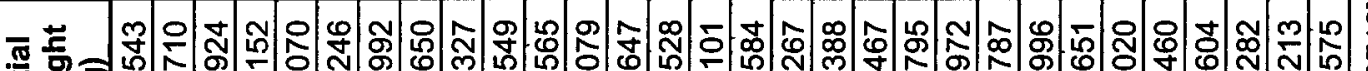

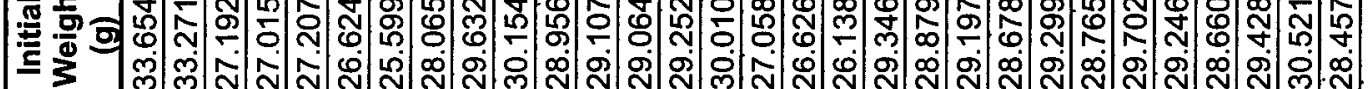
m m N N N N N N N N N N N N N N N

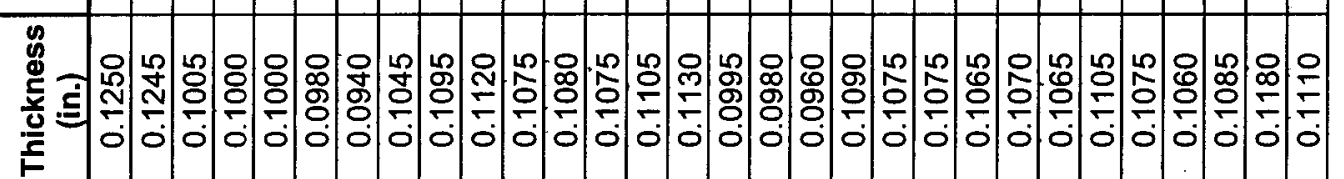

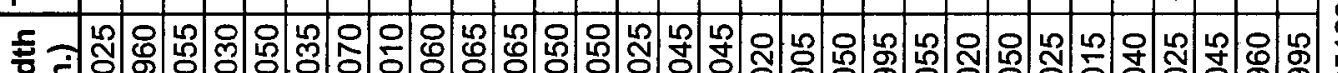

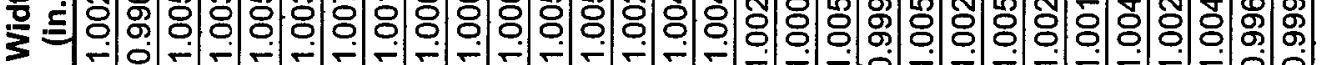

占-

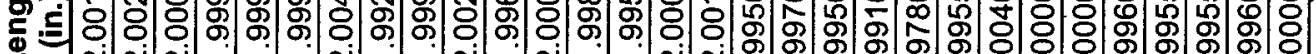

으

- +2 -

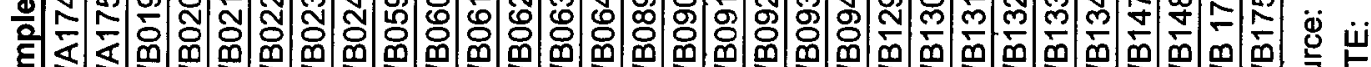

ह่ 


\section{APPENDIX IV}

CORROSION RATE OF ALLOY 22 AS A FUNCTION OF TEMPERATURE CALCULATED FROM POLARIZATION RESISTANCE MEASUREMENTS 
INTENTIONALLY LEFT BLANK 


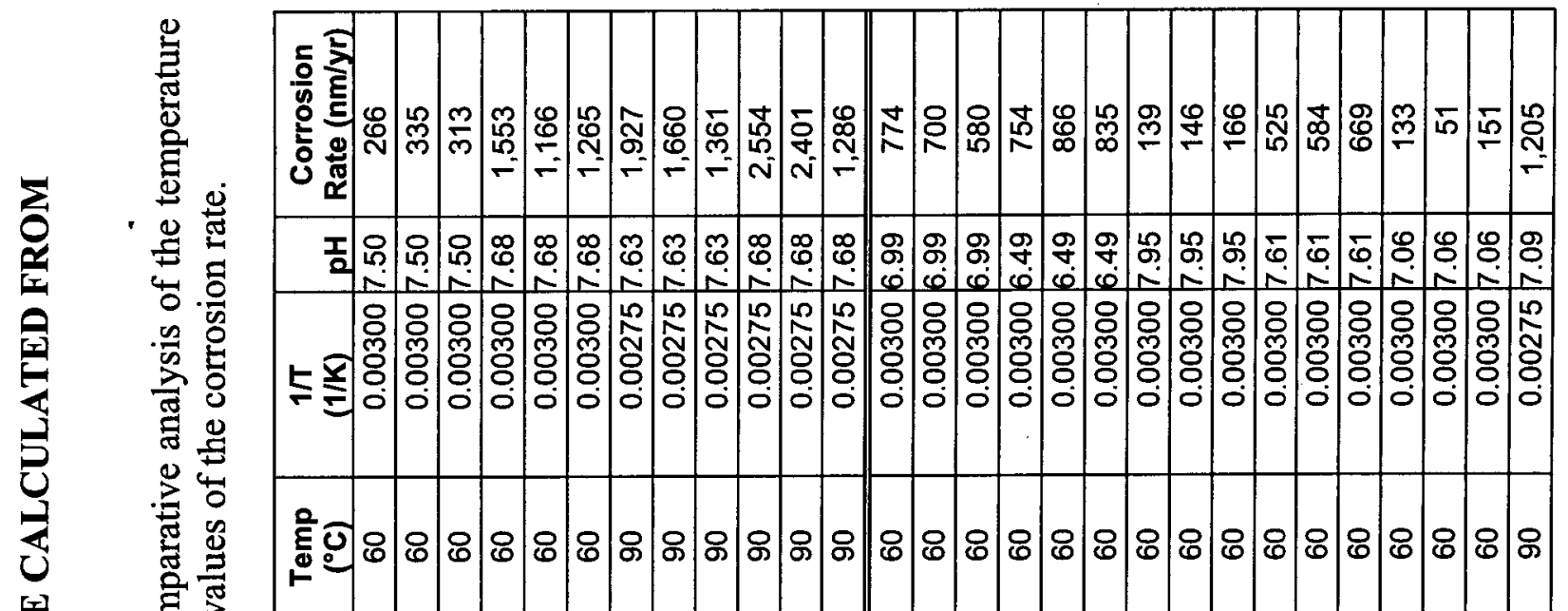

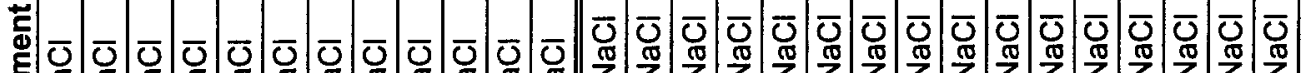

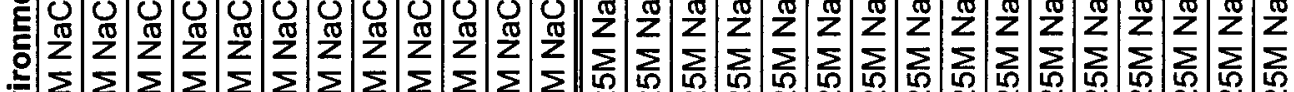

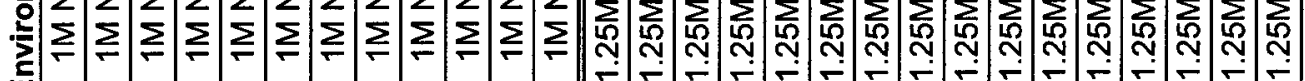

$\sum \frac{1}{2} \frac{0}{\pi}$

된

들 롤

元四 응

电艺 总它

$\bigcup \sum_{0}$

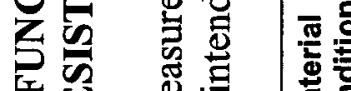

四 回

4 르

«䒘 导志

N

父出 娄

뭉은

$\gtrless \ll$ 莡

四 昰

되 을

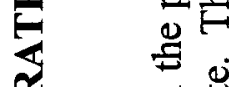

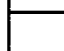

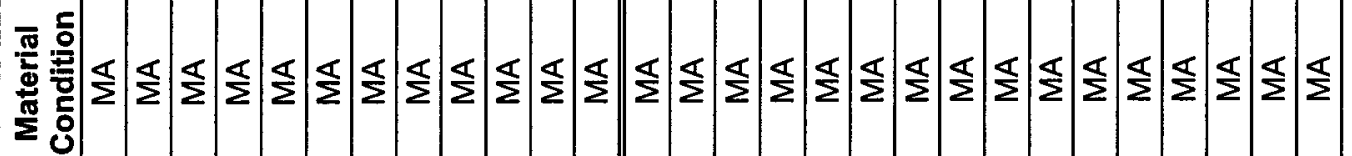

冡:

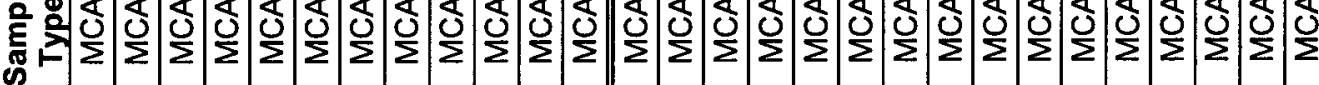

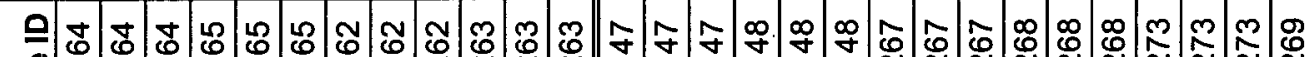

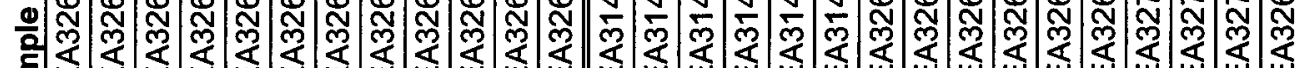

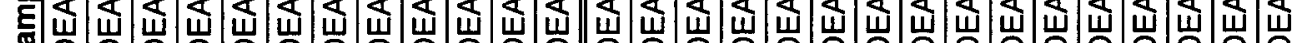

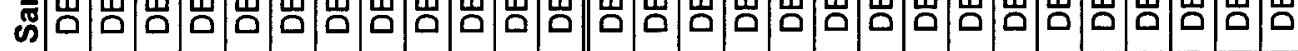

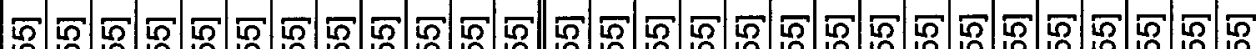

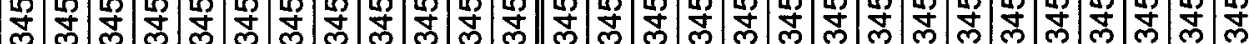

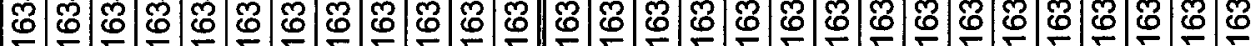

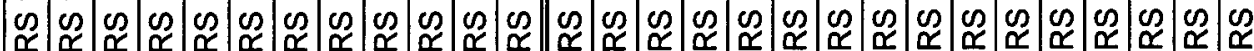

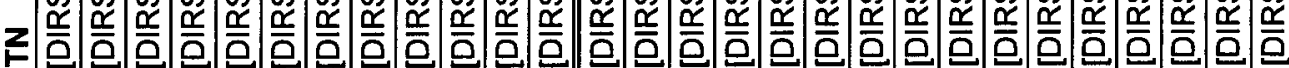

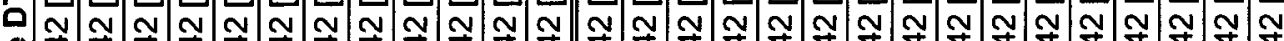
ปั

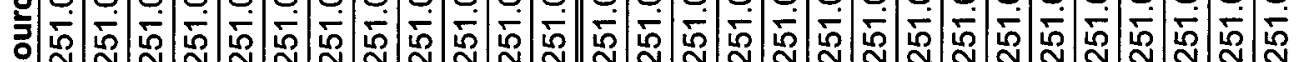
ต ลิก 产 ठे

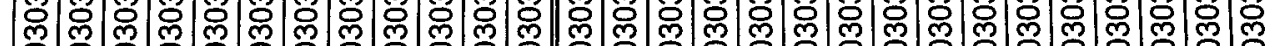




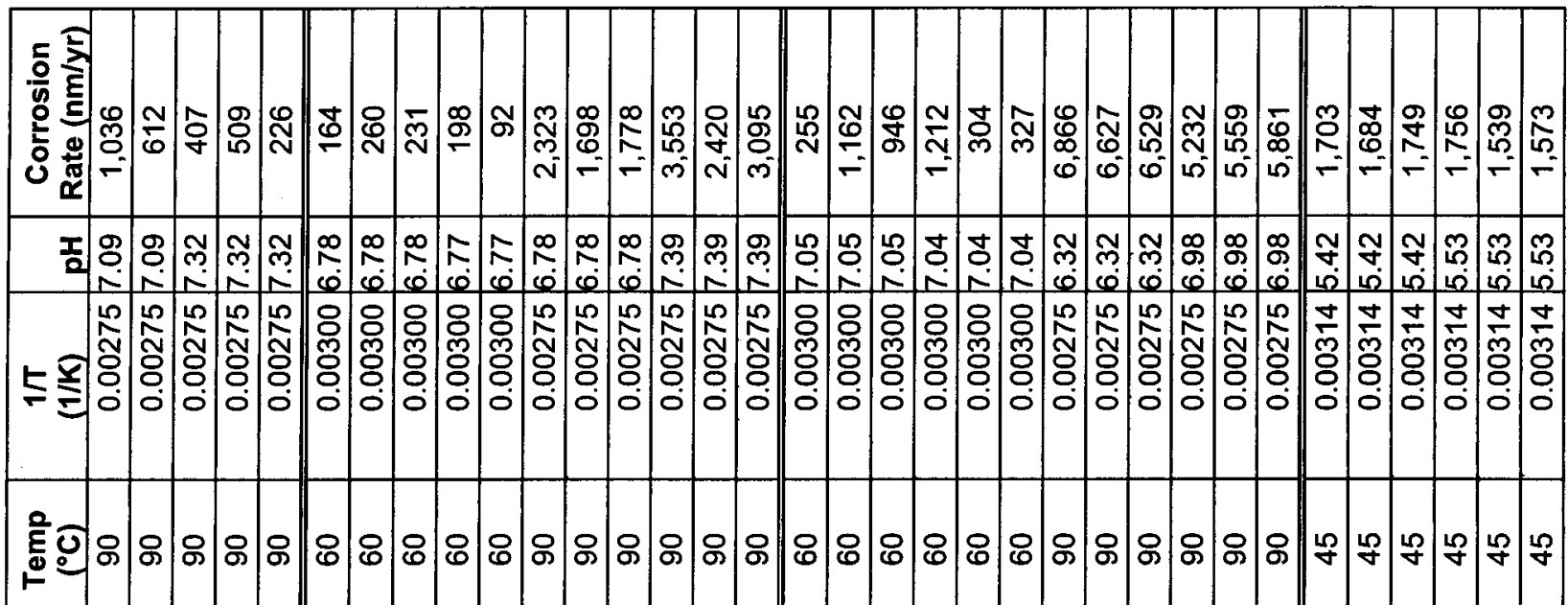

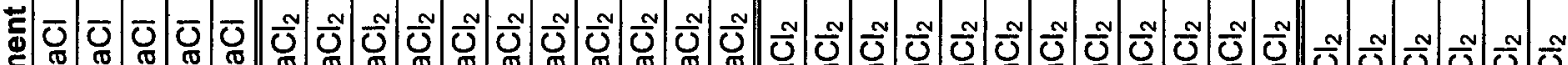
E

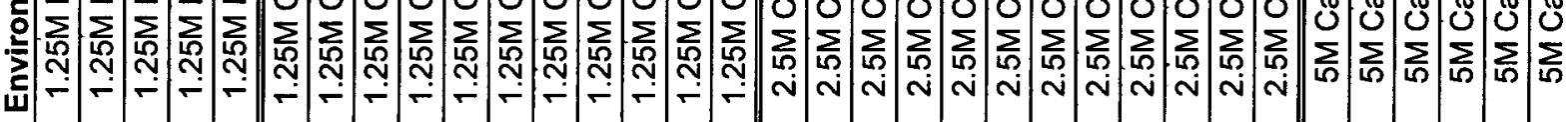
w

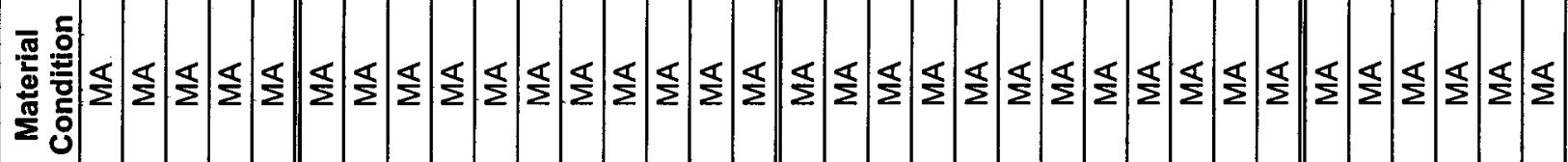

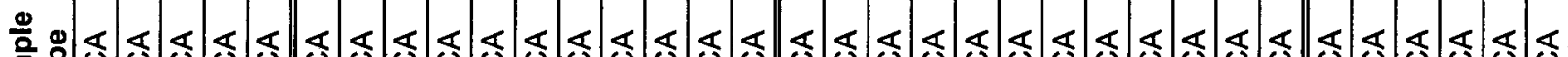

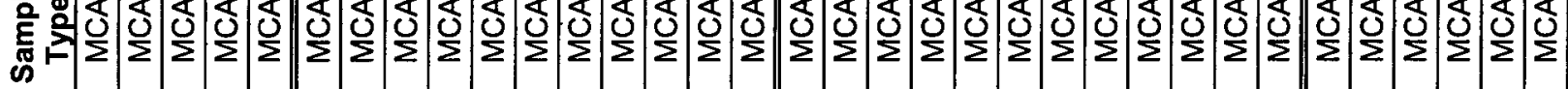

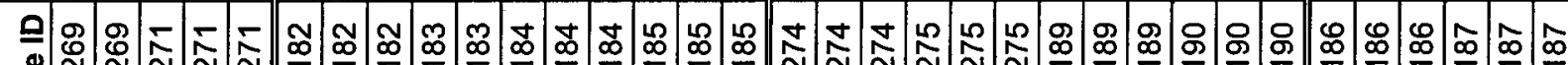
。ำ

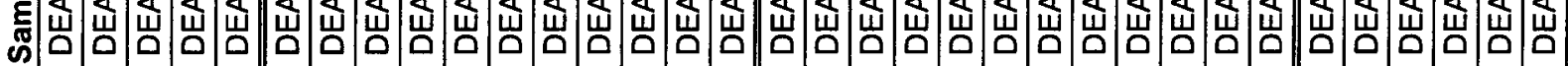

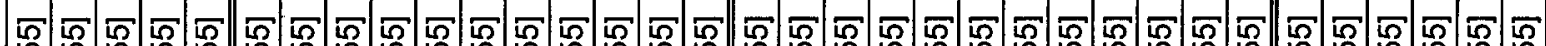

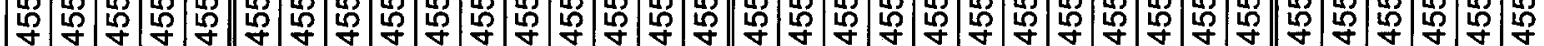
ర్లి

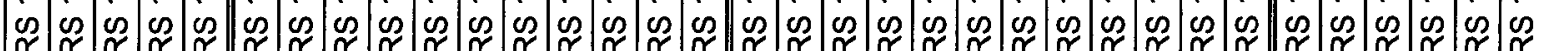

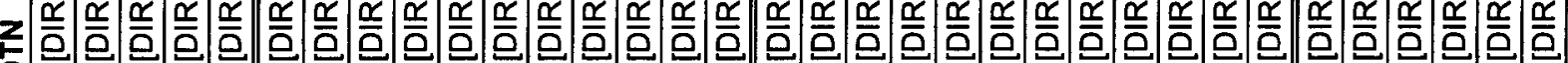
$D$
$\mathcal{O}$

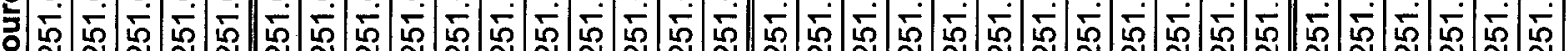

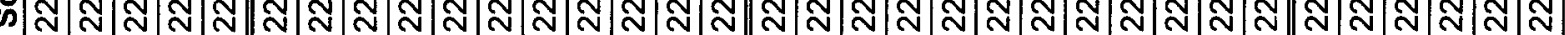

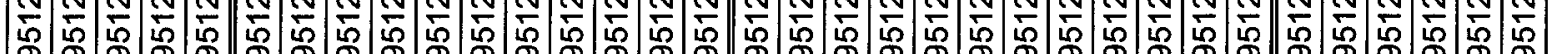

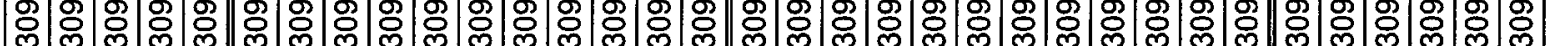

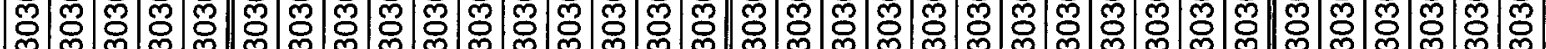

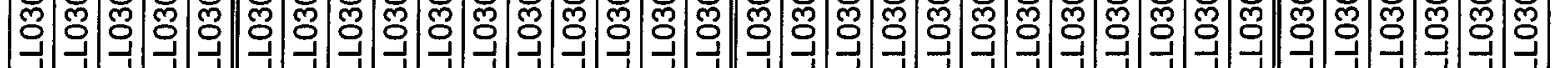




\begin{tabular}{|c|c|c|c|c|c|c|c|c|c|c|c|c|c|c|c|c|c|c|c|c|c|c|c|c|c|c|c|c|c|c|c|}
\hline 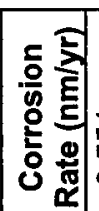 & & 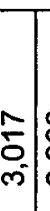 & 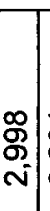 & 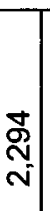 & & $\begin{array}{l}\mathcal{F} \\
\text { W } \\
\text { N }\end{array}$ & $\mid \begin{array}{c}\mathbb{5} \\
\tilde{m} \\
\infty^{\circ}\end{array}$ & & $\frac{5}{\frac{5}{60}}$ & $\mid \begin{array}{l}\infty \\
0 \\
0 \\
\infty\end{array}$ & $\begin{array}{l}\mathscr{0} \\
\mathbb{m} \\
m \\
m\end{array}$ & $\mid \begin{array}{l}m \\
\tilde{m} \\
m\end{array}$ & 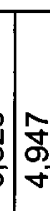 & & $\underset{\sim}{\stackrel{*}{m}}$ & $\mid \begin{array}{l}\widehat{\infty} \\
0 \\
\sigma \\
\sigma\end{array}$ & $\mid \begin{array}{l}8 \\
8 \\
0 \\
5 \\
5\end{array}$ & $\left|\begin{array}{c}\infty \\
\mathscr{m} \\
10 \\
\omega^{\prime}\end{array}\right|$ & $\left|\begin{array}{l}0 \\
\frac{0}{N} \\
\sigma \\
\sigma\end{array}\right|$ & 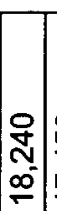 & $\begin{array}{l}0 \\
8 \\
5 \\
5 \\
5\end{array}$ & 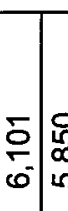 & 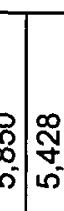 & 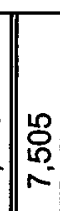 & $\left|\begin{array}{c}\frac{\pi}{6} \\
10\end{array}\right|$ & 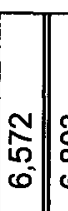 & \begin{tabular}{|c|c} 
& \\
0 & \\
0 & \\
0 & \\
0 & $c$
\end{tabular} & \begin{tabular}{l|l} 
\\
$y$ \\
0 \\
0
\end{tabular} & 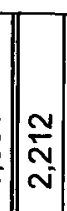 & & $\stackrel{8}{0}$ \\
\hline 짐 & & $\begin{array}{l}0 \\
0 \\
0\end{array}$ & $\begin{array}{l}0 \\
0 \\
0\end{array}$ & \begin{tabular}{l|}
10 \\
$\Omega$ \\
10 \\
10
\end{tabular} & & $\mid$\begin{tabular}{l}
$L$ \\
\multicolumn{2}{l|}{} \\
10
\end{tabular} & $\frac{m}{n}$ & 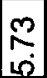 & $\stackrel{2}{2}$ & 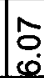 & $\mid \begin{array}{c}0 \\
0\end{array}$ & ${ }_{0}^{0}$ & $\mid \begin{array}{l}\infty \\
0 \\
0 \\
0\end{array}$ & 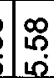 & & $\underset{D}{N}$ & $\frac{10}{\pi}$ & $\left|\begin{array}{c}N \\
0\end{array}\right|$ & $\left|\begin{array}{c}\nabla \\
0\end{array}\right|$ & & $\frac{ \pm}{\dot{0}}$ & $\begin{array}{c}\mathrm{N} \\
\dot{0}\end{array}$ & \begin{tabular}{c|c}
$\frac{N}{0}$ & $\frac{N}{c}$ \\
0
\end{tabular} & & & 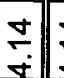 & & & & & $\frac{\dot{q}}{\dot{q}}$ \\
\hline $5 \underline{9}$ & 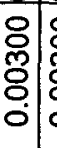 & $\begin{array}{l} \\
\\
\\
\\
\end{array}$ & 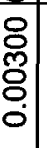 & 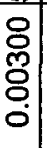 & . & 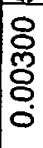 & 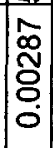 & 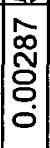 & 旋 & 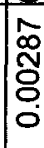 & 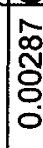 & 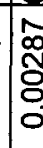 & 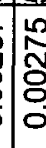 & مू & & స్ & 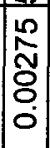 & 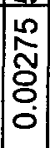 & 芯 & 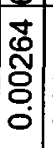 & 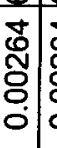 & 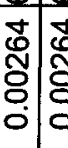 & 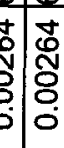 & 离 & 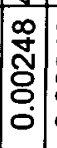 & 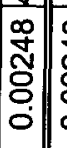 & 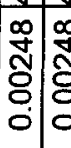 & 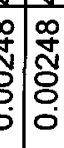 & 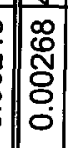 & $\mid \begin{array}{l}\infty \\
\stackrel{0}{0} \\
0 \\
0 \\
0\end{array}$ & 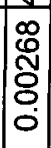 \\
\hline
\end{tabular}

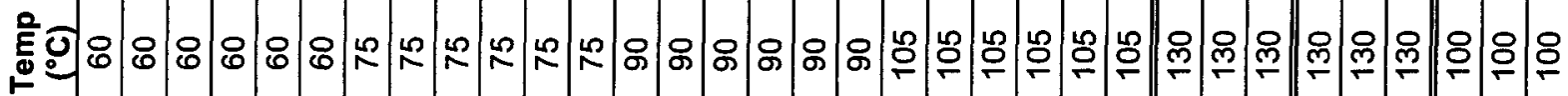

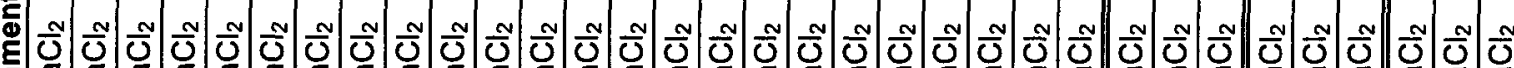
ప i

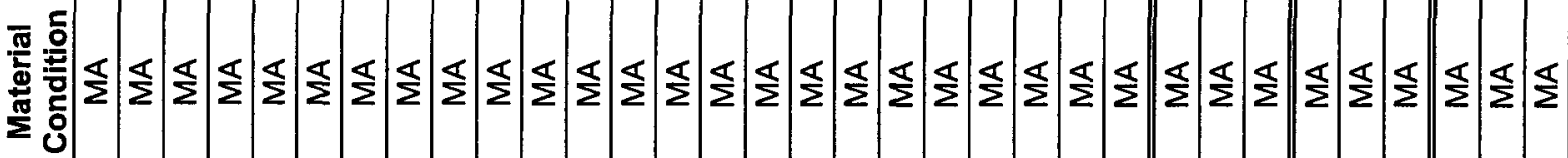

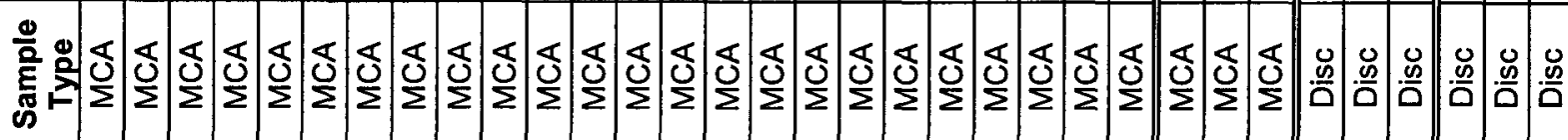

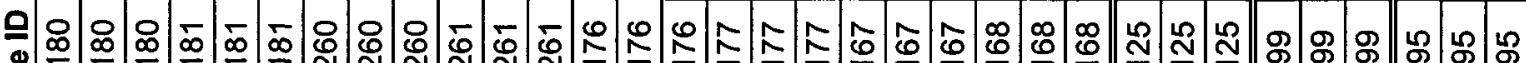

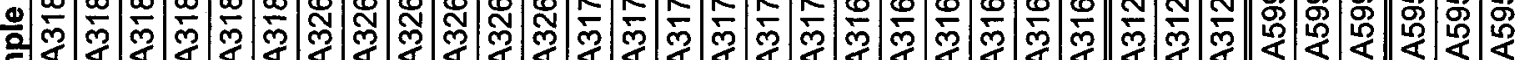

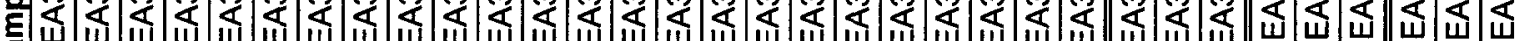

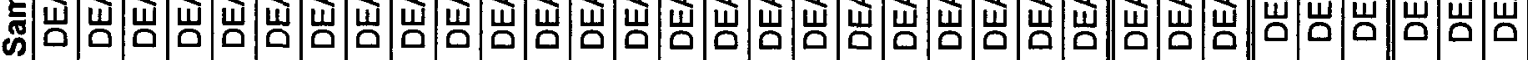

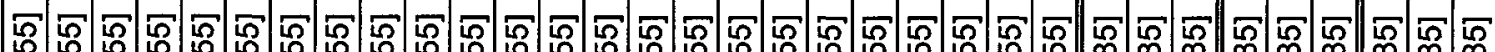

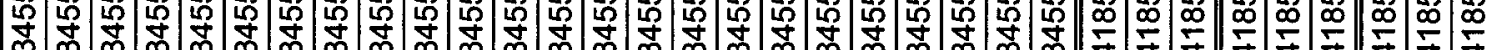

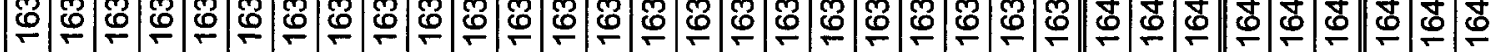

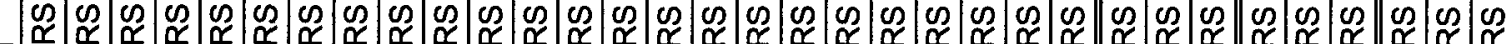

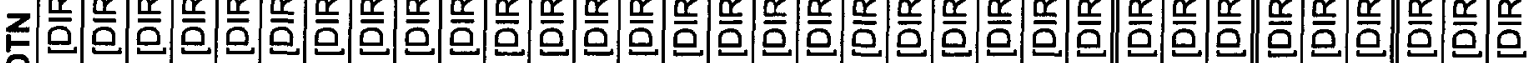
J

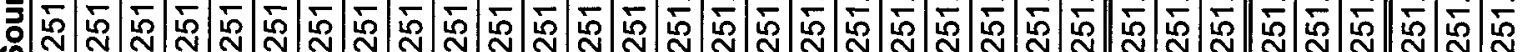
N N N N N N N N N N N N N N N N N N N N N N N N N N N $N$ N

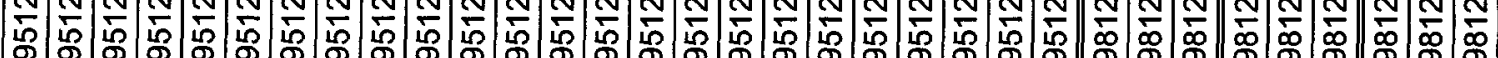

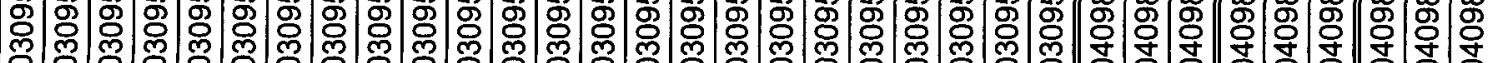

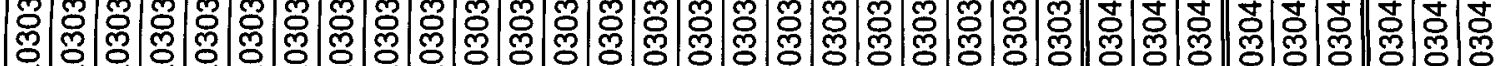

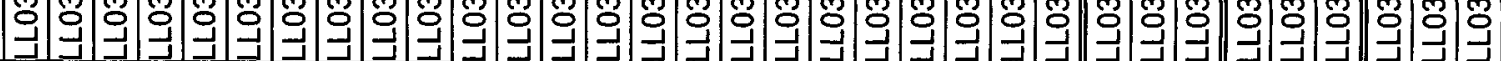


reported at 18 months was $300 \mu \mathrm{m}$, which, assuming all pit growth occurred in 6 months, corresponds to a pit growth rate of $600 \mu \mathrm{m} / \mathrm{yr}$. Also, pitting was not observed in Brine 3 (the $\mathrm{NaCl}$-based brine), which is likely to be more representative of repository conditions than $\mathrm{MgCl}_{2}$-based brines, after 18 months of testing although pitting may have initiated if the testing period were longer.

In addition, the localized corrosion penetration model assumes that, when it occurs, localized corrosion of the WPOB propagates at a (time-independent) constant rate (Assumption 5.4). This assumption is highly conservative because it is known that the localized corrosion rate decreases with time, and this is particularly more likely under discontinuous and tortuous thin water film conditions expected on the waste package surface in the postclosure repository. Section 6.4.4.8.2 provides further discussion on the above issues

Based on the above discussion, the range of propagation rates used in the localized corrosion model of WPOB is reasonable and consistent with the literature data on relevant corrosionresistant alloys for the conditions expected in the repository. Therefore, Activities One and Two have been met.

\subsection{SUMMARY OF MODEL VALIDATION}

In light of the above discussion, it is concluded that the general and localized corrosion models for the WPOB and their output corroborate well with those reported in the scientific literature. It is also clear from the above discussion that the validation activities performed for building confidence in the model have sufficiently strong scientific bases, and that all of the validation activities used to determine that the required level of confidence in the model have been achieved have been met. 


\section{CONCLUSIONS}

\subsection{BASE-CASE MODEL SUMMARY}

This report documents the analyses and models for general and localized corrosion of the waste package outer barrier (WPOB). The purpose of the general and localized corrosion models are to analyze degradation of the Alloy 22 waste package outer barrier by general and localized corrosion processes under the expected repository environmental conditions over the repository performance period. The general and localized corrosion models include several submodels, which account for dry oxidation, aqueous general corrosion, microbially influenced corrosion (MIC), crevice corrosion initiation, and crevice corrosion growth. A model overview is graphically presented in Figure 8-1.

Corrosion performance of the WPOB depends on the integrity of the thin adherent passive film formed on the alloy surface in contact with the exposure environments in the repository. The extremely low general corrosion rates and excellent resistance to localized corrosion of the WPOB in the repository intimately depend on the long-term stability of the passive film on the surface of the barrier. The passivity of Alloy 22 was evaluated by examining the oxide layers formed in a mixed-salt environment at $95^{\circ} \mathrm{C}$ (Section 6.4.1.2). The surface analysis data indicated that the oxide layers responsible for passivity of Alloy 22 consist of chromium oxide $\left(\mathrm{Cr}_{2} \mathrm{O}_{3}\right)$ containing nickel. The collected data indicated that (1) the passive films are very protective and stable, (2) contributions from metal corrosion become extremely small, and (3) redox reactions from the species in solution are stable.

Dry oxidation is not a performance limiting process of the WPOB under the exposure conditions expected in the repository and is not considered for the waste package performance analysis (Section 6.4.2). Aging and phase instability of Alloy 22 is not expected to significantly impact the WPOB corrosion performance under the thermal conditions expected in the repository; therefore, this process is not considered in the waste package performance analysis (Section 6.4.6).

General corrosion of the WPOB occurs when the RH at the waste package surface is equal to or greater than the $\mathrm{RH}$ threshold $\left(R H_{\text {threshold }}\right.$ ) for corrosion initiation (Assumption 5.1). The general corrosion rate of the WPOB is a function of temperature, expressed with an activation energy using a modified Arrhenius relation (Section 6.4.3.4). Because of very low general corrosion rates of the WPOB for the conditions expected in the repository, waste package performance is not limited by general corrosion during the regulatory time period. As a bounding and conservative analysis, for a constant waste package surface temperature of $150^{\circ} \mathrm{C}$, the median penetration depth by general corrosion over 10,000 years, using the median general corrosion rate of $51.8 \mathrm{~nm} / \mathrm{yr}$, is about $518 \mu \mathrm{m}$, which is less than 3 percent of the total thickness of the WPOB $(20 \mathrm{~mm})$. An even more conservative approach is to use the general corrosion rate of $256 \mathrm{~nm} / \mathrm{yr}\left(99.99^{\text {th }}\right.$ percentile rate at $\left.150^{\circ} \mathrm{C}\right)$, for which the total penetration depth by general corrosion is about $2,560 \mu \mathrm{m}$ after 10,000 years, which is less than 13 percent of the total thickness of the WPOB. This bounding analysis demonstrates that the waste package performance in the repository is not limited by general corrosion. 
The WPOB is subject to MIC when the relative humidity at the waste package surface is equal to or greater than 90 percent (Section 6.4.5). The MIC effect is represented by an enhancement to the abiotic general corrosion rate of the WPOB.

Localized corrosion of the WPOB is modeled with a crevice corrosion initiation model and a propagation model (Section 6.4.4). The initiation model considers that crevice corrosion of the WPOB occurs when the steady-state corrosion potential $\left(E_{\text {corr }}\right)$ is equal to or greater than the crevice repassivation potential $\left(E_{\text {rcrev }}\right)$, that is, $\Delta E\left(=E_{\text {rcrev }}-E_{\text {corr }}\right) \leq 0$. The WPOB is not subject to crevice corrosion if the solution contacting the waste package has a neutral to alkaline $\mathrm{pH}$ and contains significant concentrations of inhibitive ions such as nitrate. The WPOB is potentially susceptible to crevice corrosion if an acidic chloride-containing solution with relatively low concentrations of inhibitive ions contacts the waste package while it is at elevated temperatures. However, once the waste package cools and the solutions contacting the waste package become less concentrated and less aggressive, localized corrosion is not expected to initiate.

Additional details of the model summary are given in the following sections.

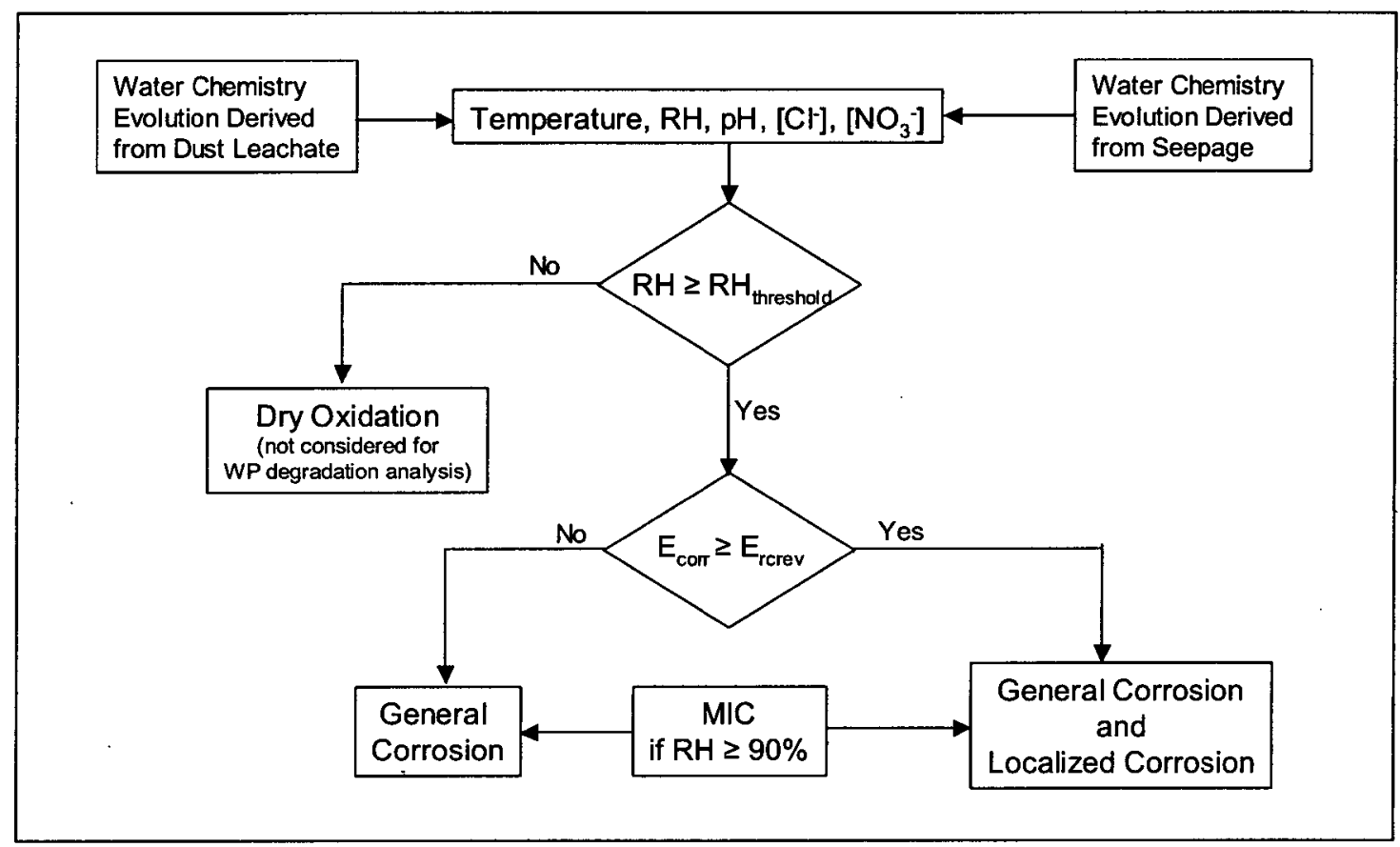

Figure 8-1. Schematic Representation of General Corrosion and Localized Corrosion Model of the Waste Package Outer Barrier 


\subsection{WASTE PACKAGE OUTER BARRIER GENERAL CORROSION MODEL OUTPUTS}

General corrosion of the WPOB occurs when the RH at the waste package surface is equal to or greater than the $\mathrm{RH}$ threshold for corrosion initiation $\left(R H_{\text {threshold }}\right)$ (Assumption 5.1). The base-case general corrosion model for the WPOB (Section 6.4.3.4) is based on a temperature dependence of the corrosion process, represented by an activation energy using a modified Arrhenius relation. The model is expressed as follows:

$$
\ln \left(R_{T}\right)=\ln \left(R_{o}\right)+C_{1}\left(\frac{1}{T}-\frac{1}{333.15}\right)
$$

where $R_{T}$ is the temperature-dependent general corrosion rate in $\mathrm{nm} / \mathrm{yr}, T$ is absolute temperature in Kelvin, and $R_{o}$ and $C_{l}$ are constants. The temperature dependence term $\left(C_{l}\right)$ was obtained from short-term polarization resistance data for Alloy 22 specimens tested for a range of sample configurations, metallurgical conditions, and exposure conditions. The temperature dependence term is normally distributed with a mean of $-3116.47 \mathrm{~K}$ and a standard deviation of $296.47 \mathrm{~K}$. The activation energy was estimated to be $25.9 \pm 2.5 \mathrm{~kJ} / \mathrm{mol} . R_{o}$ is a Weibull distribution $(s=8.88 \mathrm{~nm} / \mathrm{yr}, b=1.62$, and $l=0)$ that was fit to the general corrosion rate distribution derived from weight-loss data of the 5-year exposed crevice specimens. The 5-year data were considered to represent the distribution of long-term general corrosion rates of the WPOB at $60^{\circ} \mathrm{C}$.

The entire variance in $R_{o}$ represents variability in the general corrosion process. The general corrosion rate variability is applied among the modeled waste packages and local areas on the surface of an individual waste package. The entire variance in the temperature dependence term $\left(C_{l}\right)$ is due to uncertainty, and the uncertainty is limited to \pm 3 standard deviations. This treatment of temperature dependence accounts for over 99.7 percent of the variance in this term.

The general corrosion model developed in this report (Section 6.4.3.4) was developed using data in both mixed ionic environments and data from simple salt solutions including highly concentrated chloride brines and chloride brines containing nitrate ions. In Section 7.2, the general corrosion model is validated against data obtained at temperatures as high as $150^{\circ} \mathrm{C}$. Therefore, the general corrosion model should be applicable over all repository exposure environments.

The general corrosion model is applied to the conditions in which a stable aqueous water film can exist on the waste package surface. The WPOB is considered to be subject to MIC when the relative humidity at the WPOB surface is equal to or greater than 90 percent (Section 6.4.5). The effect of MIC on general corrosion of the WPOB is represented by an enhancement factor applied to the general corrosion rate determined from Equation 8-1. The MIC enhancement factor is uniformly distributed between 1 and 2 , and the entire variance of the distribution is due to uncertainty.

The technical product outputs of the general corrosion model analysis are documented in the output DTN: MO0409MWDUGCMW.000 and summarized in Table 8-1. 
Table 8-1. Summary of General Corrosion Model Output for Waste Package Outer Barrier

\begin{tabular}{|c|c|c|c|c|c|}
\hline \multirow[b]{2}{*}{ Output Name } & \multirow{2}{*}{$\begin{array}{c}\text { Output } \\
\text { Description }\end{array}$} & \multirow[b]{2}{*}{ DTN } & \multicolumn{3}{|c|}{ Output Uncertainty } \\
\hline & & & $\begin{array}{l}\text { Source of } \\
\text { Uncertainty }\end{array}$ & $\begin{array}{l}\text { Uncertainty } \\
\text { Distribution }\end{array}$ & $\begin{array}{c}\text { Characteristic } \\
\text { Values }\end{array}$ \\
\hline $\begin{array}{l}\text { Parameter } R_{0} \text { of } \\
\text { temperature } \\
\text { dependent } \\
\text { general } \\
\text { corrosion model, } \\
\text { Equation 8-1. }\end{array}$ & $\begin{array}{l}\text { Weibull distribution } \\
\text { (scale factor } s= \\
8.88 \mathrm{~nm} / \mathrm{yr} \text {, shape } \\
\text { factor } b=1.62 \text {, } \\
\text { and location factor / } \\
=0 \text { ). } \\
\text { (Section } 6.4 .3 .4 \text { ) }\end{array}$ & $\begin{array}{l}\text { MO0409MWDUGCMW. } \\
000\end{array}$ & Measurement & $\begin{array}{l}\text { No } \\
\text { uncertainty } \\
\text { (entire } \\
\text { variance due } \\
\text { to variability) }\end{array}$ & N/A \\
\hline $\begin{array}{l}\text { Parameter } C_{1} \text { of } \\
\text { temperature } \\
\text { dependent } \\
\text { general } \\
\text { corrosion model, } \\
\text { Equation 8-1. }\end{array}$ & $\begin{array}{l}\text { Normal distribution } \\
\text { (mean -3116.47 K, } \\
\text { s.d. } 296.47 \mathrm{~K} \text { ). } \\
\text { Limited to } \pm 3 \\
\text { standard } \\
\text { deviations. } \\
\text { (Section 6.4.3.4) }\end{array}$ & $\begin{array}{c}\text { MO0409MWDUGCMW. } \\
000\end{array}$ & Measurement & $\begin{array}{l}\text { Entire } \\
\text { distribution }\end{array}$ & N/A \\
\hline $\begin{array}{l}\text { MIC } \\
\text { enhancement } \\
\text { factor }\end{array}$ & $\begin{array}{l}\text { Uniform distribution } \\
(1,2) \text {. } \\
\text { (Section } 6.4 .5)\end{array}$ & $\begin{array}{c}\text { MO0409MWDUGCMW. } \\
000\end{array}$ & Measurement & $\begin{array}{l}\text { Entire } \\
\text { distribution }\end{array}$ & N/A \\
\hline $\begin{array}{l}\text { MIC initiation } \\
\text { threshold }\end{array}$ & $\begin{array}{l}\text { Relative humidity } \\
\text { threshold for MIC } \\
\text { initiation. } \\
\text { (Section 6.4.5) }\end{array}$ & $\begin{array}{l}\text { MO0409MWDUGCMW. } \\
000\end{array}$ & N/A & N/A & $90 \%$ \\
\hline
\end{tabular}

\subsection{WASTE PACKAGE OUTER BARRIER LOCALIZED CORROSION MODEL OUTPUTS}

Localized corrosion of the WPOB is modeled with two model components: an initiation model and a propagation model (Section 6.4.4).

\subsubsection{Waste Package Outer Barrier Localized Corrosion Initiation Outputs}

Localized corrosion of the WPOB initiates when the steady-state corrosion potential $\left(E_{\text {corr }}\right)$ is equal to or greater than a critical potential $\left(E_{\text {critical }}\right)$, that is, $\Delta \mathrm{E}\left(=E_{\text {critical }}-E_{\text {corr }}\right) \leq 0$. As a conservative measure, the localized corrosion initiation model uses the crevice repassivation potential $\left(E_{\dot{r} c r e v}\right)$ as the critical potential. The crevice repassivation potential $\left(E_{r c r e v}\right)$ is expressed as follows.

$$
E_{\text {rerev }}=E_{\text {rerev }}^{o}+\Delta E_{\text {rcrev }}^{N O_{3}^{-}}
$$

where $E_{\text {rcrev }}^{o}$ is the crevice repassivation potential in the absence of inhibitive nitrate ions, and $\Delta E_{r c r e v}^{N O_{3}^{-}}$is the crevice repassivation potential changes resulting from the inhibiting effect of nitrate ion in solution. The potentials are in $\mathrm{mV}$ versus SSC. 
The crevice repassivation potential of the WPOB in the absence of inhibitive nitrate ion is expressed as follows:

$$
E_{\text {rerev }}^{o}=a_{o}+a_{1} T+a_{2} p H+a_{3} \log \left(\left[C l^{-}\right]\right)+a_{4} T \times \log \left(\left[C l^{-}\right]\right)
$$

where $a_{o}, a_{1}, a_{2}, a_{3}$, and $a_{4}$ are constants, $T$ is the temperature $\left({ }^{\circ} \mathrm{C}\right)$, and $\left[\mathrm{Cl}^{-}\right]$is the chloride ion concentration in molal (moles $/ \mathrm{kg}$ water). The value of the coefficients and their uncertainty $\left( \pm 1\right.$ standard deviation) of the model parameters are $a_{0}=214.089 \pm 46.880, a_{1}=-3.696 \pm 0.476$, $a_{2}=25.284 \pm 5.641, a_{3}=-252.181 \pm 53.912$, and $a_{4}=1.414 \pm 0.547$. The variance of the model is calculated via a covariance matrix (Section 6.4.4.3), and the entire variance is due to uncertainty. The uncertainty of the coefficients is limited to \pm 2 standard deviations.

The effect of nitrate ion on the crevice repassivation potential is represented as follows:

$$
\Delta E_{\text {rcrev }}^{N O_{3}^{-}}=b_{o}+b_{1}\left[\mathrm{NO}_{3}^{-}\right]+b_{2} \frac{\left[\mathrm{NO}_{3}^{-}\right]}{\left[\mathrm{Cl}^{-}\right]}
$$

where $b_{o}, b_{1}$ and $b_{2}$ are constants, $\left[\mathrm{NO}_{3}^{-}\right]$is the nitrate ion concentration in molal (moles $/ \mathrm{kg}$ water), and other parameters are defined as before. The parameter coefficients and their uncertainty ( \pm 1 standard deviation) are: $b_{o}=22.589 \pm 24.113, b_{1}=33.748 \pm 5.180$, and $b_{2}=$ $749.745 \pm 95.491$.

The long-term steady-state corrosion potential model for the WPOB is expressed as follows:

$$
E_{\text {corr }}=c_{o}+c_{1} T+c_{2} p H+c_{3}\left[\mathrm{Cl}^{-}\right]+c_{4} \log \left(\frac{\left[\mathrm{NO}_{3}^{-}\right]}{\left[\mathrm{Cl}^{-}\right]}\right)
$$

where $E_{\text {corr }}$ is the long-term steady-state corrosion potential in $\mathrm{mV}$ versus $\mathrm{SSC}, c_{o}, c_{1}, c_{2}, c_{3}$, and $c_{4}$ are coefficients of the model parameters, and other parameters are defined as before. The model was evaluated by fitting it to the long-term corrosion potential data. The estimated regression coefficients and their uncertainty ( \pm 1 standard deviation) are: $c_{o}=558.283 \pm 36.156$, $c_{1}=0.677 \pm 0.413, c_{2}=-65.338 \pm 2.456, c_{3}=-7.616 \pm 1.581$, and $c_{4}=37.077 \pm 2.443$. The variance of the model is calculated via the covariance matrix (Section 6.4.4.5), and the entire variance is due to uncertainty. As with the crevice repassivation potential model, the uncertainty of the coefficients of the corrosion potential model is limited to \pm 2 standard deviations.

The empirical correlations used in the waste package outer barrier localized corrosion initiation model for the long-term corrosion potential $\left(E_{\text {corr }}\right)$ and crevice repassivation potential $\left(E_{\text {rcrev }}\right)$ are expressed as functions of temperature, $\mathrm{pH}$, chloride ion concentration, and nitrate ion concentration (Sections 6.4.4.3 and 6.4.4.5). Based on the range of environmental conditions in which the input data were obtained (Appendices V, VI, and VII) and the model validation activities (Sections 7.2.2 and 7.2.3), the application of the waste package outer barrier localized corrosion initiation model can be summarized as follows. 
Like general corrosion, localized corrosion requires the presence of a liquid water film on the waste package surface. To implement the waste package outer barrier localized corrosion initiation model, the following criteria are applied in a stepwise fashion:

1. If aqueous brine chemistry causes the initiation of localized corrosion, then localized corrosion continues to propagate regardless of changes in the bulk chemical exposure environment. This is a conservative modeling assumption made because no detailed model of the chemistry evolution of the crevice solution is available at this time.

2. If the exposure temperature exceeds $160^{\circ} \mathrm{C}$ and a water film is present on the waste package surface, then localized corrosion initiates.

Localized corrosion initiated as a result of this criterion is reevaluated in accordance with Criterion 3 (below) when the exposure temperature drops below $160^{\circ} \mathrm{C}$.

3. If the exposure temperature exceeds $120^{\circ} \mathrm{C}$ but is less than or equal to $160^{\circ} \mathrm{C}$ then,

a) If the nitrate-to-chloride ion ratio is 0.5 (or greater), no localized corrosion will occur, or

b) If the nitrate-to-chloride ion ratio is less than 0.5 , then localized corrosion initiates and continues to propagate regardless of changes in the bulk chemical exposure environment (Criterion 1).

4. If the exposure temperature is greater than or equal to $20^{\circ} \mathrm{C}$ and less than or equal to $120^{\circ} \mathrm{C}$ then the empirical correlations for the long-term corrosion potential $\left(E_{c o r r}\right)$ and crevice repassivation potential $\left(E_{\text {rcrev }}\right)$ (Sections 6.4.4.3 and 6.4.4.5) are evaluated in accordance with the following implementation rules. If localized corrosion is determined to initiate, then localized corrosion continues to occur regardless of changes in the bulk chemical exposure environment (Criterion 1)

a) If the nitrate-to-chloride ion ratio in the environment exceeds 0.5 , then evaluate $E_{\text {rcrev }}$ and $E_{\text {corr }}$ at a nitrate-to-chloride ion ratio of 0.5 .

b) If the molality of chloride ion in the environment exceeds 36 molal, then evaluate $E_{\text {rcrev }}$ and $E_{c o r r}$ at a molality of chloride ion of 36 molal.

If the molality of chloride ion is less than 0.001 molal, then evaluate $E_{r c r e v}$ and $E_{c o r r}$ at a molality of chloride ion of 0.001 molal.

c) If the molality of nitrate ion in the environment exceeds 18 molal, then evaluate $E_{\text {rcrev }}$ and $E_{c o r r}$ at a molality of nitrate ion of $18 \mathrm{molal}$.

If the molality of nitrate ion is less than 0.001 molal, then $E_{\text {rcrev }}=E_{\text {rcrev }}^{o}$ (i.e., the crevice repassivation potential in the absence of nitrate ions) and evaluate $E_{\text {corr }}$ at a molality of nitrate ion of 0.001 molal.

d) If the $\mathrm{pH}$ in the environment exceeds 10.9 , then evaluate $E_{\text {rcrev }}$ and $E_{\text {corr }}$ at a $\mathrm{pH}$ of 10.9 . 
If the $\mathrm{pH}$ in the environment is less than 2.8 , then initiate localized corrosion.

Nitrate ions inhibit localized corrosion initiation Section 6.4.4.3). In addition, carbonate and sulfate ions may have an inhibitive effect on localized corrosion. Therefore, because only nitrate ions are accounted for in the model, the results for solutions with significant amounts of other potentially inhibitive ions in addition to nitrate ions are conservative. The model results for the beneficial effects of the inhibitive ions combined with alkaline $\mathrm{pH}$ conditions of the typical carbonate-containing waters in the repository are consistent with the experimental observations on the immunity of Alloy 22 to localized corrosion in those waters (Section 7.2.3).

The entire variance of the crevice corrosion initiation model (i.e., crevice repassivation potential model and corrosion potential model) is due to uncertainty. Variability in the crevice repassivation potential and corrosion potential among the waste packages to be modeled is represented with the temporally and spatially varying waste package temperature and water chemistry contacting the waste packages. In the absence of specific information regarding local environments on the waste package, the area affected by localized corrosion due to seepage water can be based on the fraction of the waste package surface that is exposed to seepage. Similarly, in the absence of specific information on the areal distribution of localized corrosion attack, the area affected by localized corrosion that may occur under deliquescent salt conditions can be conservatively bounded by the area exposed to dust deposition (i.e., the entire waste package surface).

The crevice corrosion initiation model is used exclusively for evaluating the long-term localized corrosion susceptibility of the WPOB and is not intended for short-term transient behavior.

The technical product outputs of the crevice corrosion initiation model analysis are documented in the output DTN: MO0409MWDULCMW.000 and summarized in Table 8-2.

\subsubsection{Localized Corrosion Propagation Model Outputs}

The localized corrosion penetration model assumes that, when it occurs, localized corrosion propagates at a (time-independent) constant rate (Assumption 5.4). This is a highly conservative assumption because it is known that the localized corrosion rate decreases with time (Section 6.4.4.8.2). Also, in general, localized corrosion tends to arrest or die shortly after initiation.

Due to the outstanding corrosion resistance of Alloy 22, very little data exist for localized corrosion under the conditions expected in the repository. The literature data for localized corrosion of relevant alloys that were considered for the current localized penetration rate model are for extremely corrosive conditions not expected in the repository. Those extreme penetration rates found in the literature were used to bound localized corrosion rates of Alloy 22 under repository conditions.

The technical product outputs of the crevice corrosion propagation model analysis are documented in the output DTN: MO0409MWDULCMW.000 and summarized in Table 8-2. 
Table 8-2. Summary of Localized Corrosion Model Output for Waste Package Outer Barrier

\begin{tabular}{|c|c|c|c|c|c|}
\hline \multirow[b]{2}{*}{ Output Name } & \multirow[b]{2}{*}{$\begin{array}{l}\text { Output } \\
\text { Description }\end{array}$} & \multirow[b]{2}{*}{ DTN } & \multicolumn{3}{|c|}{ Output Uncertainty } \\
\hline & & & $\begin{array}{l}\text { Source of } \\
\text { Uncertainty }\end{array}$ & $\begin{array}{c}\text { Uncertainty } \\
\text { Distribution } \\
\text { (if } \\
\text { applicable) }\end{array}$ & $\begin{array}{c}\text { Characteristic } \\
\text { Values (if } \\
\text { applicable) }\end{array}$ \\
\hline $\begin{array}{l}5 \text { coefficients of } \\
\text { crevice } \\
\text { repassivation } \\
\text { potential model } \\
\text { without nitrate } \\
\text { ions, Equation } \\
8-3\end{array}$ & $\begin{array}{l}\text { Mean } a_{0}=214.089 \\
\text { Mean } a_{1}=-3.696 \\
\text { Mean } a_{2}=25.284 \\
\text { Mean } a_{3}=-252.181 \\
\text { Mean } a_{4}=1.414 \\
\text { Covariance matrix } \\
\text { given in Equation } \\
\text { 6-33. Coefficient } \\
\text { distributions are } \\
\text { limited to } \pm 2 \\
\text { standard } \\
\text { deviations. }\end{array}$ & $\begin{array}{l}\text { MO0409MWDULCMW } \\
.000\end{array}$ & Measurement & $\begin{array}{l}\text { Entire } \\
\text { distribution }\end{array}$ & N/A \\
\hline $\begin{array}{l}3 \text { coefficients of } \\
\text { crevice } \\
\text { repassivation } \\
\text { potential change } \\
\text { model due to } \\
\text { nitrate ions, } \\
\text { Equation } 8-4\end{array}$ & $\begin{array}{l}b_{o}=22.589 \\
b_{1}=33.748 \\
b_{2}=749.745\end{array}$ & $\begin{array}{c}\text { MO0409MWDULCMW } \\
.000\end{array}$ & N/A & N/A & N/A \\
\hline $\begin{array}{l}5 \text { coefficients of } \\
\text { long-term } \\
\text { corrosion } \\
\text { potential model, } \\
\text { Equation 8-5 }\end{array}$ & $\begin{array}{l}\text { Mean } c_{o}=558.283 \\
\text { Mean } c_{1}=0.677 \\
\text { Mean } c_{2}=-65.338 \\
\text { Mean } c_{3}=-7.616 \\
\text { Mean } c_{4}=37.077 \\
\text { Covariance matrix } \\
\text { given in Equation } \\
\text { 6-37. Coefficient } \\
\text { distributions are } \\
\text { limited to } 2 \\
\text { standard } \\
\text { deviations. }\end{array}$ & $\begin{array}{c}\text { MO0409MWDULCMW } \\
.000\end{array}$ & Measurement & $\begin{array}{l}\text { Entire } \\
\text { distribution }\end{array}$ & $N / A$ \\
\hline $\begin{array}{l}\text { Crevice } \\
\text { corrosion } \\
\text { propagation rate } \\
\text { (Table 6-8) }\end{array}$ & $\begin{array}{l}\text { Log-uniform } \\
\text { distribution, } \\
0^{\text {th }} \text { percentile }= \\
12.7 \mu \mathrm{m} / \mathrm{yr} \\
50^{\text {th }} \text { percentile }= \\
127 \mu \mathrm{m} / \mathrm{yr} \\
100^{\text {th }} \text { percentile }= \\
1270 \mu \mathrm{m} / \mathrm{yr}\end{array}$ & $\begin{array}{c}\text { MO0409MWDULCMW } \\
.000\end{array}$ & $\begin{array}{l}\text { Conceptual } \\
\text { model, data }\end{array}$ & $\begin{array}{l}\text { Entire } \\
\text { distribution }\end{array}$ & $\mathrm{N} / \mathrm{A}$ \\
\hline
\end{tabular}

\subsection{YUCCA MOUNTAIN REVIEW PLAN ACCEPTANCE CRITERIA}

The Yucca Mountain Review Plan (YMRP) contains Acceptance Criteria (AC) intended to establish the basis for the review of the material contained in the License Application. As this report serves, in part, as the basis for the License Application, it is important to show how the information contained herein addresses each of the applicable YMRP Acceptance Criteria.

The YMRP Acceptance Criteria applicable to this report are identified in Technical Work Plan for: Regulatory Integration Modeling and Analysis of the Waste Form and Waste Package 
(BSC 2004 [DIRS 171583], Table 3-1). For each applicable criterion, the criterion is quoted in italics, followed by pointers to where within the report the information addressing the criterion can be found. In some cases, the criterion is only partially addressed in this report. A demonstration of full compliance requires a review of multiple reports.

\subsubsection{System Description and Demonstration of Multiple Barriers (NRC 2003 [DIRS 163274], Section 2.2.1.1.3)}

The waste package meets the definition of a barrier in 10 CFR 63.2 [DIRS 156605].

\section{Acceptance Criterion 1 - Identification of Barriers is Adequate.}

Barriers relied on to achieve compliance with 10 CFR 63.113(b), as demonstrated in the total system performance assessment, are adequately identified, and are clearly linked to their capability. The barriers identified include at least one from the engineered system and one from the natural system.

The system that this model report addresses is the engineered barrier system (EBS), and the barrier that this model report addresses is the waste package (WP). The waste package barrier functions are addressed throughout this report and more specifically in Sections 1.3 and 6.3. This report does not address natural systems.

\section{Acceptance Criterion 2 - Description of Barrier Capability is Acceptable.}

The capability of the identified barriers to prevent or substantially reduce the rate of movement of water or radionuclides from the Yucca Mountain repository to the accessible environment, or prevent the release or substantially reduce the release rate of radionuclides from the waste is adequately identified and described:

(1) The information on the time period over which each barrier performs its intended function, including any changes during the compliance period, is provided;

(2) The uncertainty associated with barrier capabilities is adequately described;

(3) The described capabilities are consistent with the results from the total system performance assessment; and

(4) The described capabilities are consistent with the definition of a barrier at 10 CFR 63.2.

The waste package barrier contributes to waste isolation by keeping water away from the waste for its lifetime and, when breached, by reducing the contact of water with the waste and radionuclide release rate from the waste. The waste package barrier functions are addressed throughout this report and more specifically in Sections 1.3 and 6.3. The models to describe these capabilities are summarized in Sections 8.1, 8.2, and 8.3. Uncertainties associated with the waste package barrier's capabilities are also summarized in Sections 8.1, 8.2, and 8.3. The waste package degradation models and their associated uncertainties are consistent with the models 
used in TSPA-LA. The waste package meets the definition of a barrier in 10 CFR 63.2 [DIRS 156605].

Acceptance Criterion 3 - Technical Basis for Barrier Capability Is Adequately Presented.

The technical bases are consistent with the technical basis for the performance assessment. The technical basis for assertions of barrier capability is commensurate with the importance of each barrier's capability and the associated uncertainties.

The technical basis for the barrier capability is documented in Sections 6.4.2, 6.4.3, 6.4.4, 6.4.5, and 6.4.6. Section 6.4.3 documents the technical basis for the general corrosion model, Section 6.4.4 for the localized corrosion model, and Section 6.4.5 for the microbially influenced corrosion (MIC) model. Section 6.4.2 documents the technical basis that the degradation of the WPOB by dry oxidation is negligible under the repository thermal conditions, and, therefore, is not included in the waste package degradation analysis. Section 6.4 .6 documents the technical basis that aging and phase instability of Alloy 22 will not significantly affect the corrosion performance of the WPOB in the repository and, therefore, is not included in the waste package degradation analysis. The technical bases are consistent with the corresponding technical bases in performance assessment because the waste package degradation models are used as input to TSPA-LA.

\subsubsection{Degradation of Engineered Barriers (NRC 2003 [DIRS 163274], Section 2.2.1.3.1.3)}

\section{Acceptance Criterion 1 - System Description and Model Integration are Adequate}

(1) TSPA adequately incorporates important design features, physical phenomena and couplings and uses consistent assumptions throughout the degradation of engineered barriers abstraction process.

(2) Abstraction uses assumptions, technical bases, data and models that are appropriate and consistent with [those used] in other related abstractions.

(3) The descriptions of the engineered barriers, design features, degradation processes, physical phenomena, and couplings that may affect the degradation of the engineered barriers are adequate.

(4) Initial and boundary conditions are propagated consistently throughout the abstraction process.

(5) Sufficient technical basis for the inclusion [and exclusion] of FEPS are provided;

(6) Adequate technical bases are provided, for selecting the design criteria, that mitigate any potential impact of in-package criticality on repository performance, including considering all features, events, and processes that may increase the reactivity of the system inside the waste package. 
(7) Guidance in NUREG 1297 and NUREG 1298 [re: Expert Elicitation] are followed.

The physical phenomena, factors (including design features, environmental factors, and their coupling) are described in Section 6.3 as part of the conceptual model discussion for general and localized corrosion of the WPOB. The models developed in this report are adequately incorporated into TSPA-LA. Integration of the submodels for WPOB corrosion degradation analysis (general corrosion model, MIC model, crevice repassivation potential model, corrosion potential model, and localized corrosion penetration model) is also described in Section 6.3. Throughout this report, the analyses use assumptions, technical bases, input data and models that appropriately reflect the design of the waste package and the humid air and groundwater media that may come in contact with the waste package. Assumptions used in this report are addressed in Section 5. The data, technical bases and models are addressed in Sections 4.1 and 6. This information is used in a manner that is consistent with other abstractions of processes associated with the degradation of the waste package barrier. Initial and boundary conditions are propagated consistently throughout the abstraction process as described in Sections 1.2, 6, and 8 where the waste package degradation models and ranges of application are discussed.

The features, events, and processes (FEPs) treated in this report are identified in Section 6.2. Sufficient technical basis for the inclusion of FEPs (Table 6-1) are provided in Section 6.3 as part of the conceptual model discussion for general and localized corrosion of the WPOB and Sections 6.4.3, 6.4.4, and 6.4.5. Section 6.4.3 documents the technical basis for inclusion of general corrosion, Section 6.4.4 for inclusion of localized corrosion, and Section 6.4.5 for inclusion of microbially influenced corrosion (MIC).

Those sections of the acceptance criterion that relate to the selection of design criteria are not applicable to this report because design criteria are not selected in this report. Those sections of the acceptance criterion that relate to the use of expert elicitation are not applicable to this report because expert elicitation was not used in this report.

\section{Acceptance Criterion 2 - Data Are Sufficient for Model Justification}

(1) Parameters used to evaluate the degradation of EBS are adequately justified and describes how the data were used, interpreted, and appropriately synthesized into the parameters.

(2) Sufficient data have been collected to establish initial and boundary conditions;

(3) Data on the degradation of the engineered barriers are based on laboratory measurements, site-specific field measurements, industrial and/or natural analogs and tests designed to replicate anticipated conditions. As appropriate, sensitivity or uncertainty analyses are provided and are shown to be adequate to determine the possible need for additional data.

(4) Degradation models for the applicable processes are adequate. 
Section 4.1.1 documents the input data and their use in the model analysis. Appendices II to VIII document the numerical values of the data used in the model development and analysis. The input data and parameters used to evaluate the performance of the waste package were obtained from controlled sources and were adequately justified for their intended use (Section 4.1.1). Section 4.1.1 and Appendices II to VIII show that sufficient data have been collected to establish initial and boundary conditions for the models developed in this report. The data used was based on laboratory measurements under testing conditions designed to replicate anticipated repository exposure conditions. Degradation models for the processes relevant to degradation of the waste package were given appropriate consideration (Section 6) and were found to be adequate for their intended use (Section 7).

\section{Acceptance Criterion 3 - Data Uncertainty is Characterized and Propagated through the Model Abstraction}

(1) Models use parameter values, assumed ranges, probability distributions and/or bounding assumptions that are technically defensible, reasonably account for uncertainties and variabilities, and do not result in under-representation of the risk estimate.

(2) Appropriate parameters, based on techniques that may include laboratory experiments, field measurements, industrial analogs and process-level modeling studies conducted under conditions relevant to the range of environmental conditions within the waste package emplacement drifts are used.

(3) Assumed range of values and probability distributions for parameters used in conceptual and process-level models are not likely to underestimate the actual degradation and failure of engineered barriers.

(4) Appropriate methods were used for nondestructive examination of fabricated engineered barriers to assess the type, size, and location of fabrication defects that may lead to premature failure as a result of rapidly initiated engineered barrier degradation. Specify and justify the allowable distribution of fabrication defects in the engineered barriers, and assesses the effects of defects that cannot be detected on the performance of the engineered barriers.

(5) Where sufficient data do not exist, the definition of parameter values and conceptual models is based on appropriate use of other sources, such as expert elicitation.

Each of the models developed in this report use parameter values, assumed ranges, probability distributions or technically defensible bounding assumptions reasonably account for uncertainties and variabilities, and do not result in under-representation of the risk estimate. The various models developed in this report use data and parameters developed based on laboratory experiments (Section 4.1.1 and Appendices II to VIII) or bounding assumptions, or both, that are technically defensible and reasonably account for uncertainties and variabilities. The effects of data uncertainties on the parameter ranges and uncertainty distributions in the models developed in this report are discussed in Sections 6.4.3, 6.4.4, and 6.4.5. 
Section 6.4.3 discusses the effects of data uncertainties on the general corrosion model developed in this report. Uncertainties in the data used for the general corrosion model analysis (5-year weight-loss measurements and short-term polarization resistance measurements) were characterized and quantified, and propagated through the general corrosion model abstraction (Section 6.4.3). The treatment of general corrosion in this report is not likely to under-represent the risk estimate from general corrosion. For example, the general corrosion model uses (at a given temperature) a constant rate of uniform penetration (Assumption 5.2) based on laboratory weight-loss measurements from samples with creviced geometries exposed for five years in repository relevant solutions. This assumption is conservative because the general corrosion rate of alloys decreases with time (Figure 7-1). Therefore, it is not likely that the general corrosion model developed in this report will under-represent the risk estimate from general corrosion.

Section 6.4.4 discusses the effects of data uncertainties on the localized corrosion model developed in this report. Uncertainties in the data used for the localized corrosion model analysis (crevice repassivation potentials, and long-term steady-state corrosion potentials) were characterized and quantified, and propagated through the localized corrosion model abstraction (Section 6.4.4). A conservative bounding approach, based on the literature data for Alloy 22 in highly corrosive environments, was used to capture the uncertainty in the localized corrosion rate of Alloy 22 (Section 6.4.4). The treatment of localized corrosion in this report is not likely to under-represent the risk estimate from localized corrosion. For example, the localized corrosion model developed in this report uses a localized corrosion initiation criterion based on crevice corrosion (Assumption 5.3). This treatment is conservative because initiation thresholds (in terms of exposure parameters such as temperature and solution chemistry) for crevice corrosion are lower than for pitting (another type of localized corrosion attacking boldly exposed surfaces). Furthermore, the localized corrosion model uses a localized corrosion rate, which is a time independent constant rate (Assumption 5.4). This assumption is conservative because localized corrosion penetration rates decrease with time. Therefore, it is not likely that the localized corrosion model developed in this report will underrepresent the risk estimate from localized corrosion.

Section 6.4.5 discusses the effects of data uncertainties on the microbially influenced corrosion (MIC) model developed in this report. The treatment of MIC in this report is not likely to underrepresent the risk estimate from MIC. The MIC RH initiation threshold is a reasonable representation of the conditions necessary for microbes to be active in the repository environment. Relevant data were analyzed to determine the effects of microbial action on corrosion rates. Given that the effect of MIC is a multiplier on the general corrosion rate (discussed above as a conservative representation), the treatment of MIC in this report is unlikely to under-represent the risk estimate from MIC.

Those sections of the acceptance criterion that relate to nondestructive examination of fabricated engineered barriers are not applicable to this report because no analyses of nondestructive examination of fabricated engineered barriers were analyzed in this report. Those sections of the acceptance criterion that relate to the use of other sources, such as expert elicitation, are not applicable to this report because no other sources were used in the creation of this report. 


\section{Acceptance Criterion 4 - Model Uncertainty is Characterized and Propagated Through the Model Abstraction}

(1) Alternative modeling approaches of features, events, and processes are considered and are consistent with available data and current scientific understanding and the results and limitations are appropriately considered in the abstraction.

(2) Consideration of conceptual model uncertainty is consistent with available site characterization data, laboratory experiments, ... and the treatment of uncertainty does not result in under-estimation of the risk estimate.

(3) Alternative modeling approaches, consistent with available data and current scientific understanding, are used and the modeling results are evaluated using tests and analyses that are sensitive to the processes modeled.

The uncertainties in the general corrosion and localized corrosion models are addressed through the qualitative assessment of alternative conceptual models (Sections 6.4.3.5 and 6.4.4.8), which were consistent with available data and current scientific understanding. Although these alternative models are not used in TSPA-LA, they are used, where applicable, for model validation in Section 7. Consideration of uncertainties of the models developed in this report is an integral part of the model development and validation. Conceptual model uncertainty is consistent with the information that has been developed through laboratory experiments (Section 4.1.1 and Appendices II to VIII). As discussed in Acceptance Criterion 3, the treatment of uncertainty is unlikely to result in under-representation of the risk estimates.

\section{Acceptance Criterion 5 - Model Abstraction Output Is Supported By Objective Comparisons}

(1) Models implemented in this total system performance assessment abstraction provide results consistent with output from detailed process-level models and or empirical observations (laboratory and field testing, and/or natural analogs).

(2) Numerical corrosion models used to calculate the lifetimes of the engineered barriers are adequate representations, considering the associated uncertainties in the expected long-term behaviors, the range of conditions (including residual stresses), and the variability in engineered barrier fabrication processes (including welding);

(3) Evidence is sufficient to show that models will not underestimate the actual degradation and failure of engineered barriers.

(4) Mathematical degradation models are based on the same environmental parameters, material factors, assumptions and approximations shown to be appropriate for closely analogous applications. 
(5) Accepted and well documented procedures are used to construct and test the numerical models that simulate the EBS chemical environment and degradation of engineered barriers

(6) Sensitivity analyses or bounding analyses are provided to support the abstraction of degradation of engineered barriers that cover ranges consistent with the site data, field or laboratory experiments and tests, and industrial analogs.

The results of waste package degradation models developed in this report are implemented in the TSPA-LA as specified in this report, therefore the TSPA-LA model results are consistent with output from this report. The models developed in this report were compared with laboratory data used to develop the models in Section 6. The models developed in this report were compared with laboratory and/or literature data not used in model development in Section 7. As discussed in Acceptance Criterion 3, there is sufficient evidence that the models developed in this report will not underestimate the actual degradation and failure of engineered barriers. The mathematical models developed in this report were based on data collected using materials (Alloy 22) and exposure environments relevant to those expected in the repository. The models developed in this report were constructed following the accepted and well-documented AP-SIII.10Q, Models. 
INTENTIONALLY LEFT BLANK 


\section{INPUTS AND REFERENCES}

\subsection{DOCUMENTS CITED}

163034 Agarwal, D.C. 2000. "Nickel and Nickel Alloys." Chapter 45 of Uhlig's Corrosion Handbook. 2nd Edition. Revie, R.W., ed. New York, New York: John Wiley \& Sons. TIC: 248360.

163903 Akashi, M.; Nakayama, G.; and Fukuda, T. 1998. "Initiation Criteria for Crevice Corrosion of Titanium Alloys Used for HLW Disposal Overpack." Corrosion in Advanced Materials and Systems, Proceedings of Corrosion/98 Research Topical Symposium. Paper No. 158. Houston, Texas: NACE International. TIC: 254584.

170360 Andresen, P.L.; Kim, Y.J.; Emigh, P.W.; Catlin, G.M.; and Martiniano, P.J. 2003. GE GRC Final Report. BSC PO 2450-100-PO-10508. Las Vegas, Nevada: Bechtel SAIC Company. ACC: MOL.20040520.0076.

165442 Angeliu, T.M. 2001. "Microstructural Characterization of L-Grade Stainless Steels Relative to the IGSCC Behavior in BWR Environments." Corrosion/2001 56th Annual Conference \& Exposition, March 11-16, 2001, Houston, Texas, USA. Paper No. 01121 . Houston, Texas: NACE International. TIC: 254941.

133378 ASM International. 1987. Corrosion. Volume 13 of $A S M$ Handbook. Formerly 9th Edition, Metals Handbook. Materials Park, Ohio: ASM International. TIC: 240704.

154510 Baker, E.A. 1988. "Long-Term Corrosion Behavior of Materials in the Marine Atmosphere." Degradation of Metals in the Atmosphere, Proceedings of the Symposium on Corrosion of Metals, Philadelphia, Pennsylvania, 12-13 May 1986. Dean, S.W. and Lee, T.S., eds. ASTM STP 965. Pages 125-144. Philadelphia, Pennsylvania: American Society for Testing and Materials. TIC: 224019.

158781 Beavers, J.A.; Devine, T.M., Jr.; Frankel, G.S.; Jones, R.H.; Kelly, R.G.; Latanision, R.M.; and Payer, J.H. 2002. Final Report, Waste Package Materials Performance Peer Review Panel, February 28, 2002. Las Vegas, Nevada: Waste Package Materials Performance Peer Review Panel. ACC: MOL.20020614.0035.

164137 Böhni, H. 2000. "Localized Corrosion of Passive Metals.” Chapter 10 of Uhlig's Corrosion Handbook. Revie, R.W., ed. 2nd Edition. New York, New York: John Wiley \& Sons. TIC: 248360.

128141 Borenstein, S.W. and White, D.C. 1989. "Influence of Welding Variables on Microbiologically Influenced Corrosion of Austenitic Stainless Steel Weldments." Corrosion 89, April 17-21, 1989, New Orleans, Louisiana. Paper Number 183. Pages 183/1-183/13. Houston, Texas: National Association of Corrosion Engineers. TIC: 246583. 
159836 Brossia, C.S.; Browning, L.; Dunn, D.S.; Moghissi, O.C.; Pensado, O.; and Yang, L. 2001. Effect of Environment on the Corrosion of Waste Package and Drip Shield Materials. CNWRA 2001-003. San Antonio, Texas: Center for Nuclear Waste Regulatory Analyses. TIC: 252324.

155950 BSC (Bechtel SAIC Company) 2001. FY 01 Supplemental Science and Performance Analyses, Volume 1: Scientific Bases and Analyses. TDR-MGR-MD-000007 REV 00 ICN 01. Las Vegas, Nevada: Bechtel SAIC Company. ACC: MOL.20010801.0404; MOL.20010712.0062; MOL.20010815.0001.

158966 BSC 2002. The Enhanced Plan for Features, Events, and Processes (FEPs) at Yucca Mountain. TDR-WIS-PA-000005 REV 00. Las Vegas, Nevada: Bechtel SAIC Company. ACC: MOL.20020417.0385.

169131 BSC 2004. Abstraction of Drift Seepage. MDL-NBS-HS-000019, Rev. 01. Las Vegas, Nevada: Bechtel SAIC Company.

169983 BSC 2004. Aging and Phase Stability of Waste Package Outer Barrier. ANL-EBSMD-000002, Rev. 02. Las Vegas, Nevada: Bechtel SAIC Company.

168489 BSC 2004. D\&E / PA/C IED Emplacement Drift Configuration and Environment. 800-IED-MGR0-00201-000-00B. Las Vegas, Nevada: Bechtel SAIC Company. ACC: ENG.20040326.0001.

169480 BSC 2004. D\&E / PA/C IED Typical Waste Package Components Assembly. 800IED-WIS0-00201-000-00E. Las Vegas, Nevada: Bechtel SAIC Company. ACC: ENG.20040517.0007.

169860 BSC 2004. Engineered Barrier System: Physical and Chemical Environment Model. ANL-EBS-MD-000033, Rev. 03. Las Vegas, Nevada: Bechtel SAIC Company.

161237 BSC 2004. Environment on the Surfaces of the Drip Shield and Waste Package Outer Barrier. ANL-EBS-MD-000001, Rev. 01. Las Vegas, Nevada: Bechtel SAIC Company.

169991 BSC 2004. Evaluation of Potential Impacts of Microbial Activities on Drift Chemistry. ANL-EBS-MD-000038, Rev. 01. Las Vegas, Nevada: Bechtel SAIC Company.

169997 BSC 2004. FEPs Screening of Processes and Issues in Drip Shield and Waste Package Degradation. ANL-EBS-PA-000002, Rev. 03. Las Vegas, Nevada: Bechtel SAIC Company.

169565 BSC 2004. Multiscale Thermohydrologic Model. ANL-EBS-MD-000049, Rev. 02. Las Vegas, Nevada: Bechtel SAIC Company.

168361 BSC 2004. Q-List. 000-30R-MGR0-00500-000-000 REV 00. Las Vegas, Nevada: Bechtel SAIC Company. ACC: ENG.20040721.0007. 
170992 BSC 2004. Safety Classification of SSCs and Barriers. 000-00C-MGR0-01000-00000A. Las Vegas, Nevada: Bechtel SAIC Company. ACC: ENG.20040721.0005.

169985 BSC 2004. Stress Corrosion Cracking of the Drip Shield, the Waste Package Outer Barrier, and the Stainless Steel Structural Material. ANL-EBS-MD-000005, Rev. 02. Las Vegas, Nevada: Bechtel SAIC Company.

171583 BSC 2004. Technical Work Plan For: Regulatory Integration Modeling and Analysis of the Waste Form and Waste Package. TWP-WIS-MD-000009 REV 00 ICN 01. Las Vegas, Nevada: Bechtel SAIC Company. ACC: DOC.20040910.0001.

166275 Canori, G.F. and Leitner, M.M. 2003. Project Requirements Document. TER-MGRMD-000001 REV 02. Las Vegas, Nevada: Bechtel SAIC Company.

ACC: DOC.20031222.0006.

165441 Chen, H.-L.; Evans, K.J.; Hackel, L.A.; Rankin, J.E.; Yamamoto, R.M.; Demma, A.G.; Dewald, A.T.; Lee, M.J.; and Hill, M.R. 2002. Mitigation of Tensile Weld Stresses in Alloy 22 Using Laser Peening. UCRL-ID-151055. Livermore, California: Lawrence Livermore National Laboratory. ACC: MOL.20030911.0252.

163885 Cleveland, W.S. 1993. Visualizing Data. Summit, New Jersey: Hobart Press. TIC: 254702.

100349 CRWMS M\&O 1998. Waste Package Degradation Expert Elicitation Project. Rev. 1. Las Vegas, Nevada: CRWMS M\&O. ACC: MOL.19980727.0002.

102933 CRWMS M\&O 1999. Waste Package Materials Properties. BBA000000-017170210-00017 REV 00. Las Vegas, Nevada: CRWMS M\&O.

ACC: MOL.19990407.0172.

153802 CRWMS M\&O 2000. Waste Package Degradation Process Model Report. TDRWIS-MD-000002 REV 00 ICN 02. Las Vegas, Nevada: CRWMS M\&O. ACC: MOL.20001228.0229.

171539 DOE (U.S. Department of Energy) 2004. Quality Assurance Requirements and Description. DOE/RW-0333P, Rev. 16. Washington, D.C.: U.S. Department of Energy, Office of Civilian Radioactive Waste Management.

ACC: DOC.20040907.0002.

140067 Drever, J.I. 1997. The Geochemistry of Natural Waters, Surface and Groundwater Environments. 3rd Edition. Upper Saddle River, New Jersey: Prentice Hall.

TIC: 246732.

162213 Dunn, D.S. and Brossia, C.S. 2002. "Assessment of Passive and Localized Corrosion Processes for Alloy 22 as a High-Level Nuclear Waste Container Material." Corrosion/2002, 57th Annual Conference \& Exposition, April 7-11, 2002, Denver, Colorado. Paper No. 02548. Houston, Texas: NACE International. TIC: 254579. 
164495 Dunn, D.S.; Cragnolino, G.A.; and Sridhar, N. 2000. "An Electrochemical Approach to Predicting Long-Term Localized Corrosion of Corrosion-Resistant High-Level Waste Container Materials." Corrosion, 56, (1), 90-104. Houston, Texas: National Association of Corrosion Engineers. TIC: 254836.

171452 Dunn, D.S.; Cragnolino, G.A.; Pan, Y.M.; and Tang, L.T. 2004. "Effect of Fabrication Processes on Alloy 22 Corrosion Resistance." Corrosion/2004, 59th Annual Conference \& Exposition, March 28-April 1, 2004, New Orleans. Paper No. 04698. Houston, Texas: NACE International. TIC: 255943.

166948 Dunn, D.S.; Daruwalla, D.; and Pan, Y.-M. 2003. Effect of Fabrication Processes on Material Stability-Characterization and Corrosion. CNWRA 2004-01. San Antonio, Texas: Center for Nuclear Waste Regulatory Analyses. TIC: 255881.

154481 Dunn, D.S.; Pan, Y-M.; and Cragnolino, G.A. 1999. Effects of Environmental Factors on the Aqueous Corrosion of High-Level Radioactive Waste ContainersExperimental Results and Models. CNWRA 99-004. San Antonio, Texas: Center for Nuclear Waste Regulatory Analyses. TIC: 246615.

164138 Dunn, D.S.; Yang, L.; Pan, Y.-M.; and Cragnolino, G.A. 2003. "Localized Corrosion Susceptibility of Alloy 22." Corrosion/2003, 58th Annual Conference \& Exposition, March 16-20, 2003, San Diego, California. Paper No. 03697. Houston, Texas: NACE International. TIC: 254387.

168172 Else, T.A.; Pantle, C.R.; and Amy, P.S. 2003. "Boundaries for Biofilm Formation: Humidity and Temperature." Applied and Environmental Microbiology, 69, (8), 5006-5010. Washington, D.C.: American Society for Microbiology. TIC: 255916.

128233 Enos, D.G. and Taylor, S.R. 1996. "Influence of Sulfate-Reducing Bacteria on Alloy 625 and Austenitic Stainless Steel Weldments." Corrosion Science, 52, (11), 831843. Houston, Texas: National Association of Corrosion Engineers. TIC: 246554.

158069 EPRI (Electric Power Research Institute) 2002. Evaluation of the Proposed HighLevel Radioactive Waste Repository at Yucca Mountain Using Total System Performance Assessment, Phase 6. EPRI TR-1003031. Palo Alto, California: Electric Power Research Institute. TIC: 252239.

117697 Estill, J.C. 1998. "Long-Term Corrosion Studies.” 2.2 of Engineered Materials Characterization Report. McCright, R.D., ed. UCRL-ID-119564 Volume 3 Rev.1.1. Livermore, California: Lawrence Livermore National Laboratory. ACC: MOL.19981222.0137.

163849 Estill, J.C.; Hust, G.A.; and Rebak, R.B. 2003. "Long Term Corrosion Potential Behavior of Alloy 22." Corrosion/2003, 58th Annual Conference \& Exposition, March 16-20, 2003, San Diego, California. Paper No. 03688. Houston, Texas: NACE International. TIC: 254387. 
164857 Evans, K.J. and Rebak, R.B. 2002. "Passivity of Alloy 22 in Concentrated Electrolytes Effect of Temperature and Solution Composition." Corrosion Science, $A$ Retrospective and Current Status in Honor of Robert P. Frankenthal, Proceedings of the International Symposium. Frankel, G.S.; Isaacs, H.S.; Scully, J.R.; and Sinclair, J.D., eds. Proceedings Volume 2002-13. 344-354. Pennington, New Jersey: Electrochemical Society. TIC: 254801.

112115 Evans, M.; Hastings, N.; and Peacock, B. 1993. Statistical Distributions. 2nd Edition. New York, New York: John Wiley \& Sons. TIC: 246114.

100890 Fontana, M.G. 1986. Corrosion Engineering. 3rd Edition. New York, New York: McGraw-Hill. TIC: 240700.

164140 Frankel, G.S. 2002. "Localized Corrosion Phenomenology and Controlling Parameters." Section 6 of Waste Package Materials Performance Peer Review, A Compilation of Special Topic Reports. Wong, F.M.G. and Payer, J.H., eds. Las Vegas, Nevada: Waste Package Materials Performance Peer Review Panel. ACC: MOL.20020731.0138.

162216 Frankel, G.S. 1998. "Pitting Corrosion of Metals, A Review of the Critical Factors." Journal of the Electrochemical Society, 145, (6), 2186-2198. Pennington, New Jersey: Electrochemical Society. TIC: 254587.

164141 Frankel, G.S. and Kelly, R.G. 2002. "Passivity-Induced Ennoblement." Section 11 of Waste Package Materials Performance Peer Review, A Compilation of Special Topic Reports. Wong, F.M.G. and Payer, J.H., eds. Las Vegas, Nevada: Waste Package Materials Performance Peer Review Panel. ACC: MOL.20020731.0138.

100859 Gdowski, G.E. 1991. Survey of Degradation Modes of Four Nickel-ChromiumMolybdenum Alloys. UCRL-ID-108330. Livermore, California: Lawrence Livermore National Laboratory. ACC: NNA.19910521.0010.

100893 Gruss, K.A.; Cragnolino, G.A.; Dunn, D.S.; and Sridhar, N. 1998. "Repassivation Potential for Localized Corrosion of Alloys 625 and C22 in Simulated Repository Environments." Proceedings of Corrosion 98, March 22-27, 1998, San Diego, California. Pages 149/1 to 149/15. Houston, Texas: NACE International. TIC: 237149.

105750 Hack, H.P. 1983. "Crevice Corrosion Behavior of Molybdenum-Containing Stainless Steels in Seawater." Materials Performance, 22, (6), 24-30. Houston, Texas: NACE International. TIC: 245826.

100814 Harrar, J.E.; Carley, J.F.; Isherwood, W.F.; and Raber, E. 1990. Report of the Committee to Review the Use of J-13 Well Water in Nevada Nuclear Waste Storage Investigations. UCID-21867. Livermore, California: Lawrence Livermore National Laboratory. ACC: NNA.19910131.0274. 
100896 Haynes International. 1997. Hastelloy C-22 Alloy. Kokomo, Indiana: Haynes International. TIC: 238121.

100897 Haynes International. 1997. Hastelloy Alloy C-276. Haynes International Product Brochure. Kokomo, Indiana: Haynes International. TIC: 238832.

162220 Horn, J. and Jones, D. 2002. "Microbiologically Influenced Corrosion: Perspectives and Approaches." Manual of Environmental Microbiology. 2nd Edition. Hurst, C.J., ed. Pages 1072-1083. Washington, D.C.: ASM Press. TIC: 254738.

160670 Hua, F.; Sarver, J.; Jevec, J.; and Gordon, G. 2002. "General Corrosion Studies of Candidate Container Materials in Environments Relevant to Nuclear Waste Repository." Corrosion/2002, 57th Annual Conference \& Exposition, April 7-11, 2002, Denver, Colorado. Paper No. 02530. Houston, Texas: NACE International. TIC: 252067.

162256 Hua, F.H. 2002. Testing of Candidate Container Materials to Resolve Post-VA Waste Package Materials Issues, DE-AC08-01RW12101, Final Report. RDD:02:43761-301000:02. Alliance, Ohio: McDermott Technology. ACC: MOL.20030709.0142.

162221 Hunkeler, F. and Boehni, H. 1983. "Pit Growth Measurements on Stainless Steels." Passivity of Metals and Semiconductors, Proceedings of the Fifth International Symposium on Passivity, Bombannes, France, May 30-June 3, 1983. Froment, M., ed. Pages 655-660. New York, New York: Elsevier. TIC: 236283.

171329 Ilevbare, G. 2003. Electrochemical Polarization Studies of Localized Corrosion. Partial Submittal for Scientific Notebook SN-LLNL-SCI-463-V1. Pages 125-160 ACC: MOL.20040423.0227; MOL.20040423.0229.

162222 Ishikawa, H.; Honda, A.; and Sasaki, N. 1994. "Long Life Prediction of Carbon Steel Overpacks for Geological Isolation of High-Level Radioactive Waste." Life Prediction of Corrodible Structures. Parkins, R.N., ed. Volume 1. Pages 454-483. Houston, Texas: NACE International. TIC: 254834.

164087 Jain, V.; Dunn, D.; Sridhar, N.; and Yang, L. 2003. "Effect of Measurement Methods and Solution Chemistry on the Evaluation of the Localized Corrosion of Candidate High-Level Waste Container Materials." Corrosion/2003, 58th Annual Conference \& Exposition, March 16-20, 2003, San Diego, California. Paper No. 03690. Houston, Texas: NACE International. TIC: 254387.

162225 Jayaweera, P.; Priyantha, N.; Macdonald, D.D.; Engelhard, G.; and Davydov, A. 2003. Deterministic Prediction of Localized Corrosion Damage to Alloy C-22 HLNW Canisters. SRI Project 10333. Menlo Park, California: SRI International.

TIC: 253900.

169906 Jones, D.A. 1992. Principles and Prevention of Corrosion. 1st Edition. New York, New York: Macmillan. TIC: 242631. 
155193 Kain, R.M. 1987. "Evaluation of Crevice Corrosion.” In Corrosion, Volume 13, Pages 303-310 of ASM Handbook. Formerly 9th Edition, Metals Handbook. Materials Park, Ohio: ASM International. TIC: 240704.

162230 Kehler, B.A.; Ilevbare, G.O.; and Scully, J.R. 1999. "Comparison of the Crevice Corrosion Resistance of Alloys 625 and C22." Passivity and Localized Corrosion, An International Symposium in Honor of Professor Norio Sato. Seo, M.; MacDougall, B.; Takahashi, H.; and Kelly, R.G., eds. 99-27, 644-654. Pennington, New Jersey: Electrochemical Society. TIC: 254581.

162231 Kehler, B.A.; Ilevbare, G.O; and Scully, J.R. 2001. "Crevice Corrosion Stabilization and Repassivation Behavior of Alloy 625 and Alloy 22." Corrosion, 57, (12), 10421065. Houston, Texas: NACE International. TIC: 254305.

100051 Langmuir, D. 1997. Aqueous Environmental Geochemistry. Upper Saddle River, New Jersey: Prentice Hall. TIC: 237107.

164856 Lian, T.; Estill, J.C.; Hust, G.A.; Fix, D.V.; and Rebak, R.B. 2002. "Passive Corrosion Behavior of Alloy 22 in Multi-Ionic Aqueous Environments."

Transportation, Storage, and Disposal of Radioactive Materials-2002-Presented at the 2002 ASME Pressure Vessels and Piping Conference, Vancouver, British Columbia, Canada, August 5-9, 2002. Hafner, R.S.; Farmer, J.C.; Hensel, S.J.; Lake, W.H., Jr. and Smith, A.C., eds. PVP-Vol. 449. Pages 67-74. New York, New York: American Society of Mechanical Engineers. TIC: 254800.

110238 Lian, T.; Martin, S.; Jones, D.; Rivera, A.; and Horn, J. 1999. "Corrosion of Candidate Container Materials by Yucca Mountain Bacteria." Corrosion 99, 54th Annual Conference and Expo, San Antonio, Texas, April 25-30, 1999. Paper No. 476. Houston, Texas: NACE International. TIC: 245833.

131202 Lide, D.R., ed. 1991. CRC Handbook of Chemistry and Physics. 72nd Edition. Boca Raton, Florida: CRC Press. TIC: 3595.

167921 Lloyd, A.C.; Shoesmith, D.W.; McIntyre, N.S.; and Noël, J.J. 2003. "Effects of Temperature and Potential on the Passive Corrosion Properties of Alloys C22 and C276." Journal of the Electrochemical Society, 150, (4), B120-B130. New York, New York: Electrochemical Society. TIC: 255963.

154718 Lorang, G.; Jallerat, N.; Vu Quang, K.; and Langeron, J.-P. 1990. “AES Depth Profiling of Passive Overlayers Formed on Nickel Alloys." Surface and Interface Analysis, 16, 325-330. Chichester, England: John Wiley \& Sons. TIC: 249830.

154720 Macdonald, D.D. 1992. "The Point Defect Model for the Passive State." Journal of the Electrochemical Society, 139, (12), 3434-3449. Manchester, New Hampshire: Electrochemical Society. TIC: 249804. 
154721 Macdonald, D.D. 1999. "Passivity-The Key to Our Metals-Based Civilization." Pure and Applied Chemistry, 71, (6), 951-978. Oxford, England: Blackwell Science.

TIC: 249795.

154738 Marcus, P. and Maurice, V. 2000. "Passivity of Metals and Alloys." Chapter 3 of Corrosion and Environmental Degradation. Schütze, M., ed. Volume I. Materials Science and Technology Volume 19. New York, New York: Wiley-VCH.

TIC: 249831.

162234 Marsh, G.P.; Taylor, K.J.; and Harker, A.H. 1991. The Kinetics of Pitting Corrosion of Carbon Steel Applied to Evaluating Containers for Nuclear Waste Disposal. SKB TR-91-62. Stockholm, Sweden: Svensk Kärnbränsleförsörjning AB. TIC: 206582.

114637 McCright, R.D. 1998. Corrosion Data and Modeling, Update for Viability Assessment. Volume 3 of Engineeréd Materials Characterization Report. UCRL-ID119564, Rev. 1.1. Livermore, California: Lawrence Livermore National Laboratory. ACC: MOL.19980806.0177.

152193 McGuire, R.; Vlasity, J.; Kessler, J.; Long, A.; Childs, S.; Ross, B.; Schwartz, F.; Shoesmith, D.; Kolar, M.; Apted, M.; Zhou, W.; Sudicky, E.; Smith, G.; Kozak, M.; Salter, P.; Klos, R.; Venter, A.; Stenhouse, M.; Watkins, B.; and Little, R. 1998. Alternative Approaches to Assessing the Performance and Suitability of Yucca Mountain for Spent Fuel Disposal. EPRI TR-108732. Palo Alto, California: Electric Power Research Institute. TIC: 248813.

162235 Mughabghab, S.F. and Sullivan, T.M. 1989. "Evaluation of the Pitting Corrosion of Carbon Steels and Other Ferrous Metals in Soil Systems." Waste Management, 9, (4), 239-251. Elmsford, New York: Pergamon Press. TIC: 254591.

162251 Newman, R.C. 1985. "The Dissolution and Passivation Kinetics of Stainless Alloys Containing Molybdenum-I. Coulometric Studies of $\mathrm{Fe}-\mathrm{Cr}$ and $\mathrm{Fe}-\mathrm{Cr}-\mathrm{Mo}$ Alloys." Corrosion Science, 25, (5), 331-339. New York, New York: Elsevier. TIC: 254590.

162250 Newman, R.C. and Franz, E.M. 1984. "Growth and Repassivation of Single Corrosion Pits in Stainless Steel." Corrosion, 40, (7), 325-330. Houston, Texas: National Association of Corrosion Engineers. TIC: 254580.

163274 NRC (U.S. Nuclear Regulatory Commission) 2003. Yucca Mountain Review Plan, Final Report. NUREG-1804, Rev. 2. Washington, D.C.: U.S. Nuclear Regulatory Commission, Office of Nuclear Material Safety and Safeguards. TIC: 254568.

162236 Papoulis, A. 1965. Probability, Random Variables, and Stochastic Processes. New York, New York: McGraw-Hill. TIC: 254701.

166944 Pensado, O.; Dunn, D.S.; Cragnolino, G.A.; and Jain, V. 2002. Passive Dissolution of Container Materials-Modeling and Experiments. CNWRA 2003-01. San Antonio, Texas: Center for Nuclear Waste Regulatory Analyses. TIC: 254056. 
In Evaluation of the Impact of Microbial Activities on Drift Chemistry (BSC 2004 [DIRS 169991], Section 7.1), based on different nutritional requirements, microorganisms are categorized into one of two groups:

1) Heterotrophs, which use organic carbon compounds as their carbon and energy source, and

2) Autotrophs, which derive their energy from the oxidation of inorganic compounds and cell carbon from $\mathrm{CO}_{2}$.

In the repository, heterotrophic microbial activities will be limited by the lack of a significant organic carbon supply; autotrophic microbial activities will be limited by the lack of available inorganic electron donors in the oxic repository environment (BSC 2004 [DIRS 169991], Section 7.1).

The following environmental factors will severely limit microbial activities in the repository (BSC 2004 [DIRS 169991], Section 7.1):

- The in-drift temperatures during the thermal pulse created by radioactive decay will exceed the temperature tolerance of all known microbes for a significant portion of the repository time, thus exerting a sterilization effect on microorganisms.

- An oxic environment will prevail in the repository over the growth-permissive period and, therefore, prevent the generation and accumulation of reduced inorganic species that are the prerequisite for autotrophic metabolism.

- Microbial incubation experiments have demonstrated that water availability is the primary limiting factor for microbial growth in the repository. The relative humidity and the liquid-water saturation degree in the repository are predicted to be low, thus further limiting microbial activities.

- Evaporation of seepage waters in the low relative humidity environment will result in brine solutions with ionic strengths higher than 10 molar, which is an environment in which only a few halophiles may be able to survive.

- Phosphate and organic carbon are important limiting factors for microbial growth in the repository. The extremely low organic carbon supply in the repository will limit heterotrophic microbial activities.

- Because of elevated temperatures, radiation fields, low humidity, and low nutrient supplies, the formation of biofilms in the drift is unlikely.

Due to the aforementioned environmental constraints, microbial activity in the repository will be limited, and its impacts on drift chemistry will be insignificant (BSC 2004 [DIRS 169991], Section 7.1). Evaluation of the Impact of Microbial Activities on Drift Chemistry (BSC 2004 [DIRS 169991], Section 7.1) also states that: 
- Yucca Mountain groundwater contains only a trace concentration of organic carbon, which is negligible compared to the total carbonate concentration in the solution. Furthermore, an autotrophic microbial reaction, if it occurs, will not generate $\mathrm{CO}_{2}$, because it is a $\mathrm{CO}_{2}$-fixation process, and the accumulated biomass will probably be recycled back to $\mathrm{CO}_{2}$, resulting in zero $\mathrm{CO}_{2}$ accumulation or depletion. Even if all the organic carbon were converted to carbon dioxide, the perturbation to the water chemistry is negligible. Therefore, the impact of microbial activity on water chemistry $(\mathrm{pH}$ and total $\mathrm{CO}_{2}$ ) will be negligible.

- In an oligotrophic environment such as the repository, microbial activities are limited, and the concentrations of the microbially generated low molecular weight organic acids are expected to be very low. The complexation effect of these organic acids on radionuclide dissolution is negligible.

- Since an oxic environment will prevail in the repository, actinide precipitation due to reduction from higher to lower valence states will be unlikely. For the same reason, microbial activity will not reductively dissolve $\mathrm{Fe}(\mathrm{III})$ oxides generated from oxic metal corrosion and, therefore, will not change the availability of sorption sites for radionuclides in the repository. Other biotransformation processes (such as dissolution due to oxidation reactions, production of sequestering agents, and biosorption) may occur, but their impacts on radionuclide mobility will be insignificant, due to a low microbial activity in the repository.

- Due to the oxic environment, denitrification is inhibited. Microbial consumption of nitrate will be unlikely.

- A long-distance biocolloidal transport is unlikely in a subsurface environment, especially in an unsaturated zone. Field measurements indicate that a majority of microbial cells are attached to solid matrix. Through biosorption and bioaccumulation, microbes actually tend to immobilize radionuclides in subsurface systems. It is expected that biocolloids generated in the drift will be filtered out and readsorbed within the invert or the unsaturated zone.

Based on this discussion, the effects of MIC on waste package degradation will be minimal (BSC 2004 [DIRS 169991], Section 7.1). Specifically, the following factors will limit, if not completely prevent, microbially influenced corrosion (BSC 2004 [DIRS 169991], Section 7.1):

- Oxic environments will prevail in the repository over the regulatory time period. Therefore, sulfate reduction - an anaerobic reaction pathway generally considered to be responsible for MIC - will be unlikely to occur. For the same reason, microbial oxidation of sulfide will also be unlikely due to lack of reduced sulfur species in the repository.

- The repository conditions of low relative humidity, elevated temperature, high radiation dose, and limited nutrient supplies all together will prevent the formation of biofilms, which are generally required for MIC. 
Although microbes can be active at relative humidity as low as $75 \%$, most microbes can thrive only when relative humidity is above $90 \%$ (BSC 2004 [DIRS 169991], Section 7.1). In the TSPA calculations, a threshold for microbially influenced corrosion of $90 \%$ relative humidity is used (BSC 2004 [DIRS 169991], Section 7.1). This choice for the threshold for microbially influenced corrosion likely leads to an overestimate of MIC effect, because the other factors such as elevated temperature, high radiation dose, and limited nutrient supplies will also limit microbial activity, for which microbes will require even higher relative humidity to maintain their active metabolism (BSC 2004 [DIRS 169991], Section 7.1). On this basis, the MIC factor is applied to the WPOB general corrosion rate only when the relative humidity at the WPOB surface is greater than or equal to 90 percent (i.e., the activity of water is greater than or equal to 0.90 ).

Results of a recent study on the biofilms formation on Alloy 22 coupons by the microbes obtained from the repository tuff rocks (Else et al. 2003 [DIRS 168172]) indicate that the above $\mathrm{RH}$ threshold for MIC without considering the effect of temperature is highly conservative. The study showed that $100 \% \mathrm{RH}$ and $30^{\circ} \mathrm{C}$ were the optimal conditions for bacterial attachment and biofilm formation on Alloy 22 coupon surfaces. At decreased $\mathrm{RH}$ values, ranging from $32 \%$ to $84 \%$ at $30^{\circ} \mathrm{C}$, culturable counts were below or near the level of detection for all metal types studied. It was shown that as temperature increases (to $60^{\circ} \mathrm{C}$ or $70^{\circ} \mathrm{C}$ ) or RH decreases to below $100 \%$, biofilm formation on Alloy 22 coupon surfaces was impeded. The study concluded that the boundary conditions for the microbe colonization on the metal surfaces required $100 \% \mathrm{RH}$ and $30^{\circ} \mathrm{C}$ for even minimal biofilms production (Else et al. 2003 [DIRS 168172]).

Environmental factors that affect levels of bacterial growth include temperature and radiation. These factors, however, are closely coupled to $\mathrm{RH}$; as temperature and radiation decrease in the repository, $\mathrm{RH}$ is predicted to increase. At the same time, while there are some types of microorganisms that can survive elevated temperatures $\left(\geq 120^{\circ} \mathrm{C}\right)$ and high-radiation doses, if there is no available water, then microbial activity is completely prevented. Thus, because water availability is the primary limiting factor and this factor is coupled to other less critical limiting factors, water availability (as expressed by $\mathrm{RH}$ ) was used as the primary gauge of microbial activity.

It has been observed that nickel-based alloys such as Alloy 22 are highly resistant to microbially influenced corrosion (Lian et al. 1999 [DIRS 110238]). Furthermore, microbial growth in the repository will be limited by the availability of nutrients such as organic carbon, nitrogen, or phosphorous (BSC 2004 [DIRS 169991], Section 7.1). There are no standard tests designed specifically to investigate the susceptibility of an engineering alloy to MIC (Stoecker 1987 [DIRS 162243]). One commonly used method to evaluate the effect of MIC is to test the alloy of interest in-situ (in the field) using the same variables as for the intended application. However, testing in the laboratory with live organisms can provide more controlled conditions of various environmental variables, and sterile controls can be incorporated to better assess MIC-specific effects (Horn and Jones 2002 [DIRS 162220]). The latter approach was used to evaluate the effects of microbiological processes on general corrosion of the WPOB.

The Project has conducted MIC testing in which Alloy 22 coupons were incubated with sterile or nonsterilized Yucca Mountain rock in simulated concentrated (10×) groundwater for almost 5 years (DTN: LL040303612251.078 [DIRS 170221]). Scanning Electron Microscope (SEM) images of unexposed, sterile control, and nonsterile samples are shown in Figure 6-67. Alloy 22 
samples exposed to microbial environments showed submicron-sized degradation after almost 5 years of exposure; similar degradation was not seen in sterile controls after 43 months of exposure. Although the surface morphology differs between sterile and nonsterile samples, the submicron-sized degradation morphology does not have the macroscopic appearance of pitting corrosion, which tends to result in larger pit diameters (e.g., Smailos 1993 [DIRS 168164], Figures 2 and 3). Note that grinding marks are evident on both sterile and nonsterile sample surfaces even after about 5 years of exposure. Such a corrosion surface morphology would better be described as enhanced general corrosion especially at higher submicron-sized degradation densities.

For general corrosion of the WPOB, the effect of MIC can be described as follows;

$$
C R_{M I C}=C R_{s t} \cdot f_{M I C}
$$

where $C R_{M I C}$ is the general corrosion rate in the presence of microorganisms, $C R_{s t}$ is the general corrosion rate of the alloy in the absence of microorganisms, and $f_{M I C}$ is the MIC factor. If $f_{M I C}$ is greater than one, there is an enhancement of the corrosion rate of the alloy as a consequence of the presence or activity of microorganisms.

Lian et al. (1999 [DIRS 110238]) has shown that MIC can enhance corrosion rates of Alloy 22 by a factor of two. Measurements for Alloy 22 are shown in Table 6-10. This study used 12 strains of Yucca Mountain bacteria, including acid, slime, and sulfide producers, as well as iron-oxidizing bacteria. The growth medium was supplemented with $0.5 \%$ glucose and $0.75 \%$ protease peptone in $100 \mathrm{x}$ concentrated simulated J-13 well water Lian et al. (1999 [DIRS 110238]). The MIC factor $f_{M I C}$ is calculated as the ratio of corrosion rates (microbes to sterile) from the table. The value of $f_{M I C}$ for Alloy 22 in sterile media is one $\left(f_{M I C}=1\right)$, whereas the value of $f_{M I C}$ for Alloy 22 in inoculated media is larger $\left(f_{M I C}=2\right)$. The MIC factor, $f_{M I C}$, is uniformly distributed between 1 and 2 , and the variance of this distribution is entirely due to uncertainty.

Table 6-10. Alterations in Corrosion Rates and Potentials of Mill-Annealed Alloy 22 Associated with Microbial Degradation at Room Temperature

\begin{tabular}{|c|c|c|c|}
\hline \multirow[b]{2}{*}{ Tested Sample Initial Condition } & \multirow{2}{*}{$\begin{array}{l}\text { Average Corrosion Rate } \\
(\mu \mathrm{m} / \mathrm{yr})\end{array}$} & \multicolumn{2}{|c|}{ Corrosion Potential $E_{\text {corr }}\left(V_{S C E}\right)$} \\
\hline & & Initial & Endpoint \\
\hline Alloy $22+$ Yucca Mountain Microbes & 0.022 & -0.440 & -0.252 \\
\hline Sterile Alloy 22 & 0.011 & -0.260 & -0.200 \\
\hline
\end{tabular}

Source: DTN: LL991203505924.094 [DIRS 138343]. 
$+3,30,0$

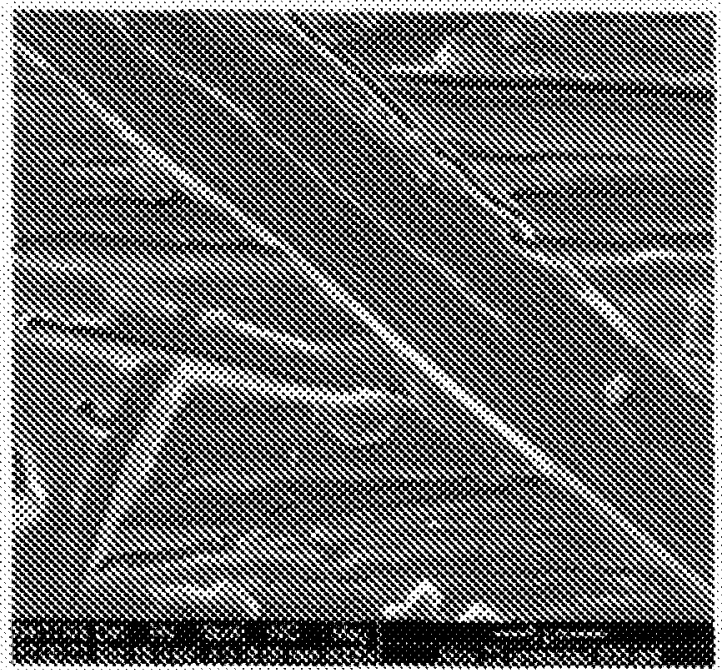

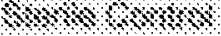

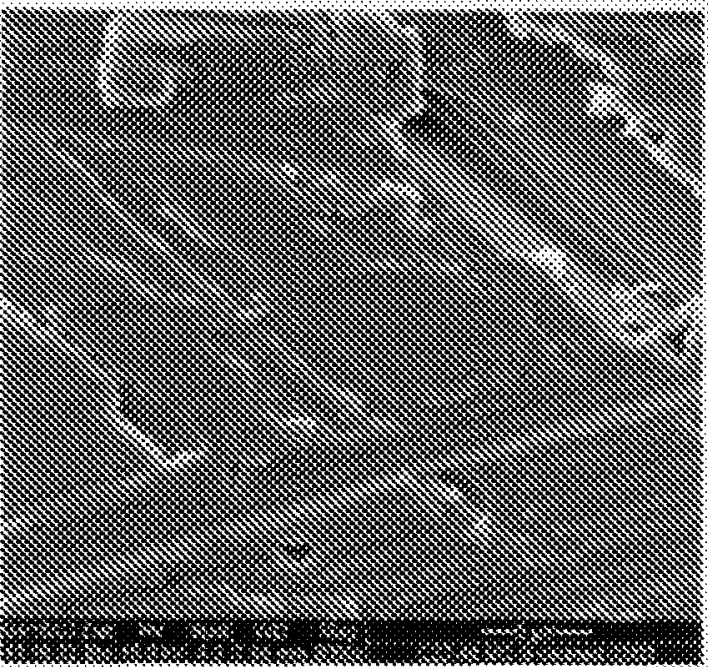

\$, $8,0, \mathrm{k}$

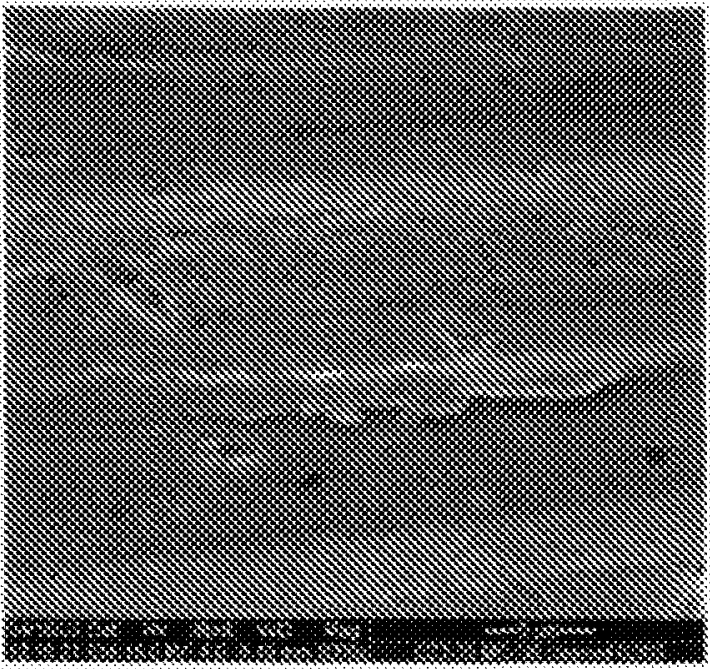

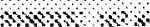

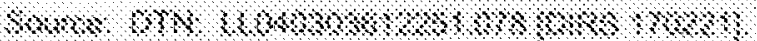

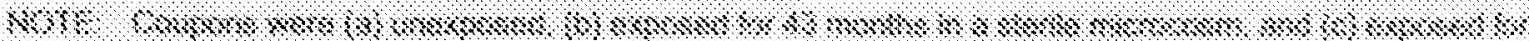

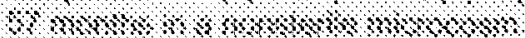

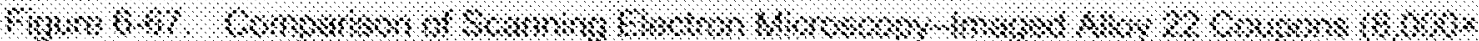

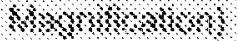

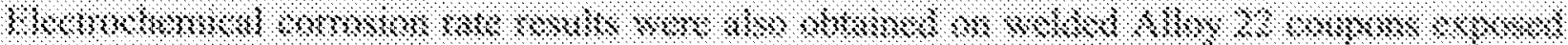

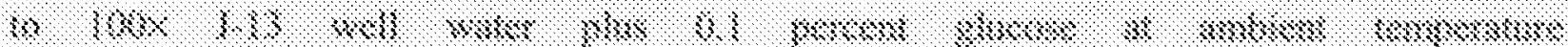

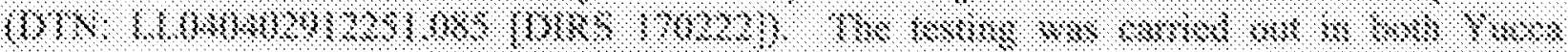

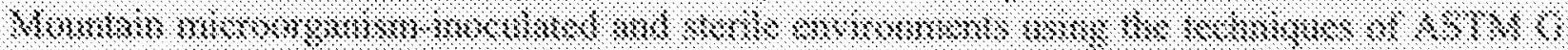

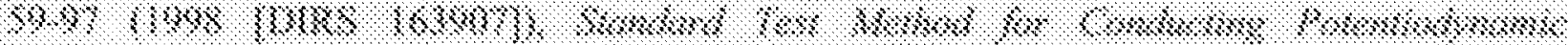

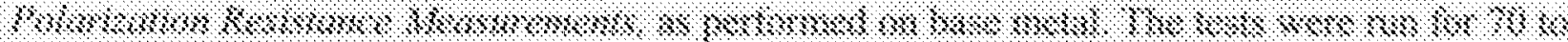

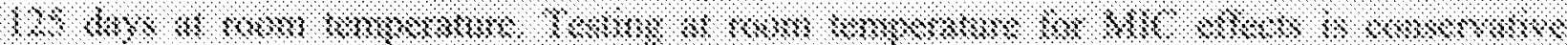

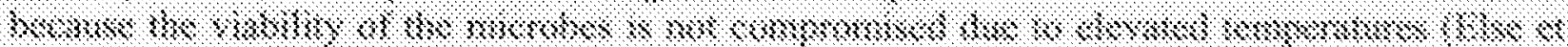

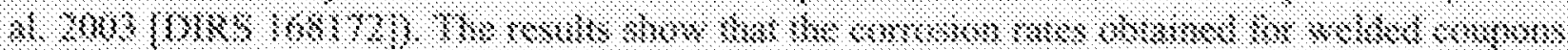


in nonsterile environments were only slightly higher than those in sterile environments (Figure 6-68) and consistent with the corrosion enhancement factor obtained from the nonwelded coupon data shown in Table 6-10. The data also show that the corrosion rates for welded coupons are essentially the same as those for nonwelded coupons.

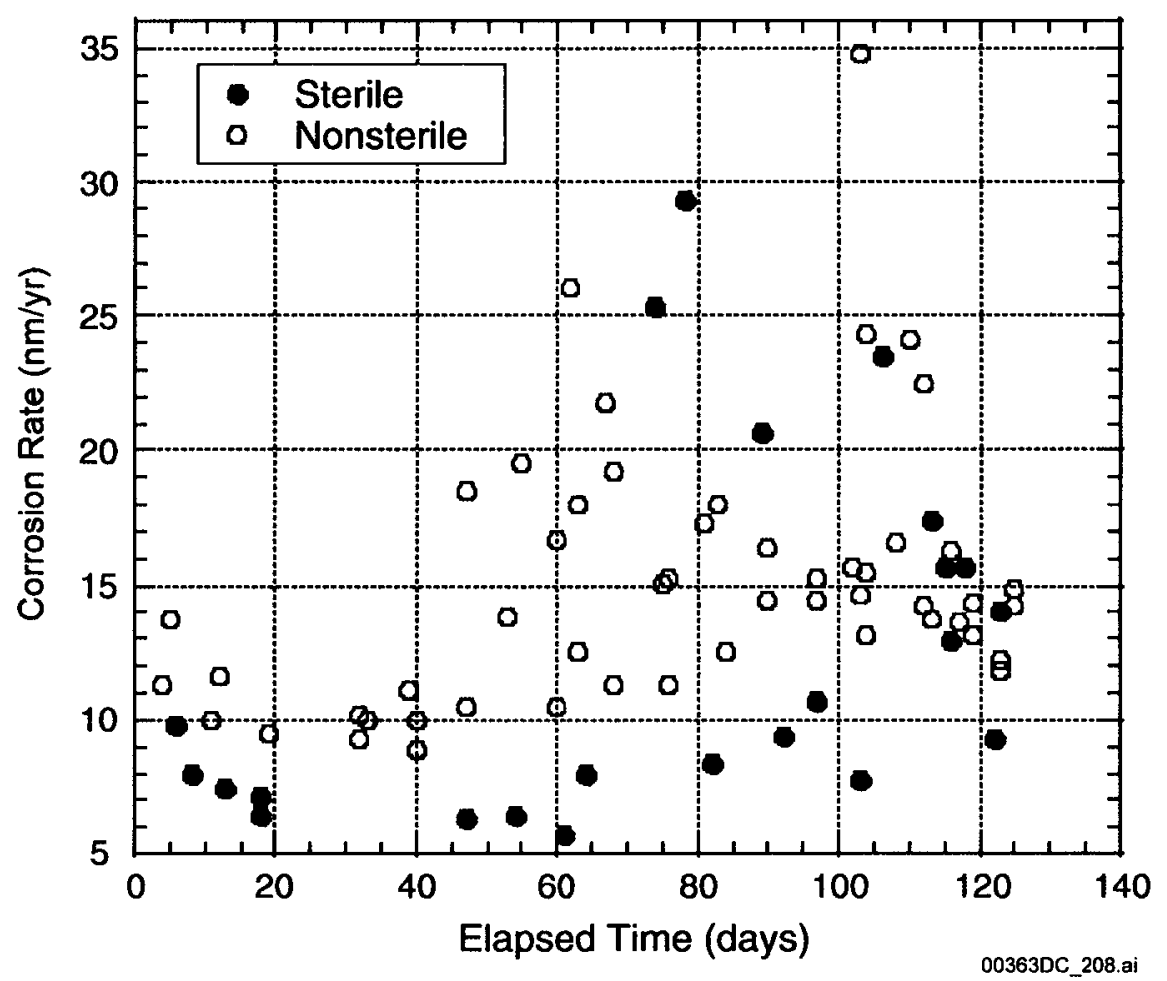

Source: DTN: LL040402912251.085 [DIRS 170222].

Figure 6-68. Corrosion Rates Determined by Polarization Resistance Testing of Welded Alloy 22 Coupons

Not all areas are equivalent on any given waste package with respect to bacterial colonization. It is well documented that bacteria preferentially colonize on weldments, and heat-affected zones (Borenstein and White 1989 [DIRS 128141]; Walsh 1999 [DIRS 138928]; Enos and Taylor 1996 [DIRS 128233]). Conservatively, the MIC factor is applied to the entire waste package surface area. The treatment is conservative because the effects of MIC are not being applied only to the welded regions where bacteria preferentially colonize.

Determination of a critical mass of total bacteria required to cause MIC is not an issue that needs to be addressed in the MIC model. Bacterial densities in Yucca Mountain rock have been determined to be on the order of $10^{4}$ to $10^{5} \mathrm{cells} / \mathrm{gm}$ of rock (BSC 2004 [DIRS 169991], Section 6.3). In absolute terms, this is almost certainly above the threshold required to cause MIC. A more germane concern is the types of bacteria present, their abundance, and how their relative numbers are affected when water is available for growth. Corrosion rates will be affected (at least on some waste package materials) for example, if organic acid producers outcompete sulfate reducers or inorganic acid producers for available nutrients when water is sufficient to support growth. No data are currently available regarding the composition of the bacterial community over the changing environmental conditions anticipated during repository 
evolution. Instead, this issue has been addressed in the current model by determining overall corrosion rates under a standardized set of conditions, in the presence and absence of a defined set of characterized Yucca Mountain bacteria.

\subsubsection{Effect of Aging and Phase Instability on Corrosion}

As specified in the waste package design and fabrication specification (Plinski 2001 [DIRS 156800], Section 8.1), the WPOB base metal and all fabrication welds (not including the welds for the closure lids) are fully annealed before the waste packages are loaded with waste. Analysis documented in Aging and Phase Stability of Waste Package Outer Barrier (BSC 2004 [DIRS 169983], Section 8) has shown that phase instabilities are not expected in Alloy 22 base metal and welded material due to the thermal hydrologic exposure profiles in the repository. Nonthermal stress mitigation processes, currently planned for the outer lid closure weld, may introduce cold work into the material. Angeliu (2001 [DIRS 165442]) observed that unmitigated Stainless Steel Type 316NG weldments can contain up to 20 percent cold work due to weld shrinkage and differential thermal expansion. Given that the phase instability analysis included welded samples, the effects of cold work have been included in the analysis as well.

Project data show that the corrosion properties of aged welds are comparable to those of unaged welds. In order to analyze the effect of thermal aging on corrosion of Alloy 22, three metallurgical conditions of Alloy 22 were studied at the LTCTF using the multiple crevice assembly (MCA) samples: mill annealed, as-welded, and as-welded plus thermally aged (at $700^{\circ} \mathrm{C}$ for 173 hours). The samples were tested in $5 \mathrm{M} \mathrm{CaCl}_{2}$ and $5 \mathrm{M} \mathrm{CaCl}_{2}+0.5 \mathrm{M}$ $\mathrm{Ca}\left(\mathrm{NO}_{3}\right)_{2}$ solutions with the test temperatures up to $120^{\circ} \mathrm{C}$. As described in Appendix I, after being immersed in the test solution in an open-circuit condition for 24 hours, the polarization resistance of the samples was measured.

Comparison of the calculated corrosion rates of the mill-annealed (MA), as-welded (ASW), and as-welded plus thermally aged samples are shown in Figure 6-69 for $5 \mathrm{M} \mathrm{CaCl}_{2}$ solutions and Figure 6-70 for $5 \mathrm{M} \mathrm{CaCl}_{2}+0.5 \mathrm{M} \mathrm{Ca}\left(\mathrm{NO}_{3}\right)_{2}$ solutions. The corrosion rate data are listed in Appendix IV. As discussed earlier (Sections 4.1.1.4 and 6.4.3.4), corrosion rates from polarization resistance measurements are only for comparative analysis of the effect of thermal aging on corrosion rates; the tests are not intended to be used to obtain absolute values of the corrosion rate. The absolute values of the general corrosion rates of Alloy 22 are determined from weight-loss measurements as discussed in Section 6.4.3.4. The mill-annealed MCA samples in $5 \mathrm{M} \mathrm{CaCl}_{2}$ solutions at differing temperatures were considered as the baseline condition for the analysis. The baseline condition rates were compared with those of the ASW and ASW plus thermally aged MCA samples tested in the same electrolyte solution condition. A data trend-line was drawn for the baseline condition data for an easier comparison with the ASW and ASW plus thermally aged sample data. The comparison shown in Figure 6-69 clearly shows that there is no significant enhancement of the corrosion rate due to welding or thermal aging of the welded samples for the tested conditions. 


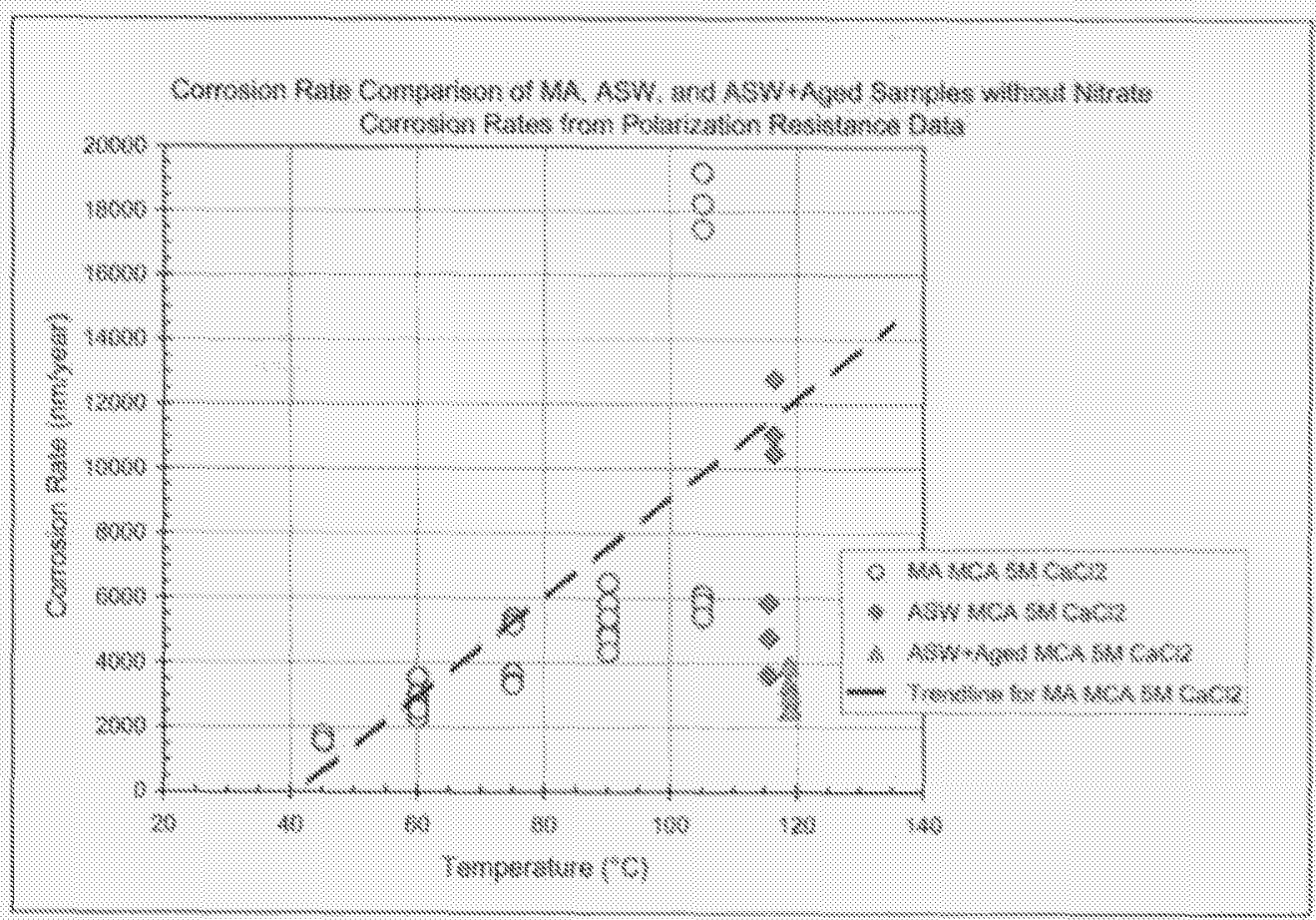

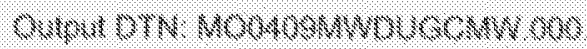

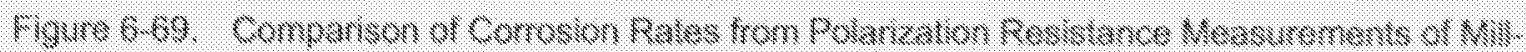

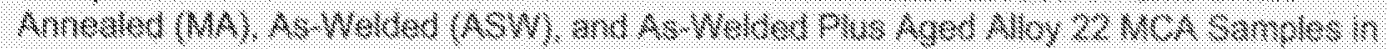
5 M CaCl, Brines at Varying Temperdures

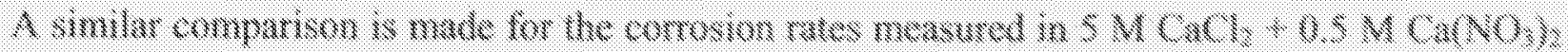

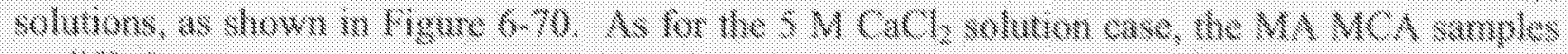

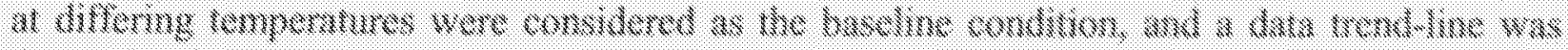

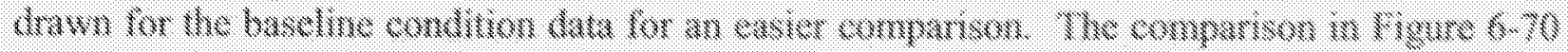

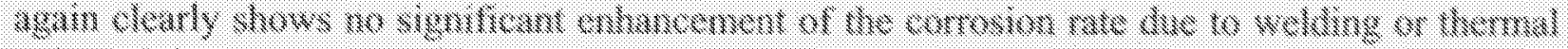

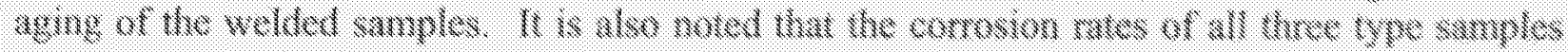

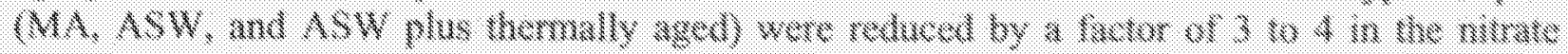

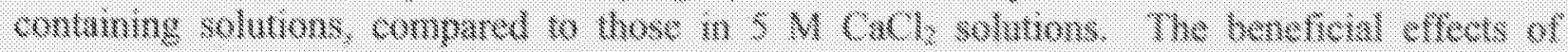

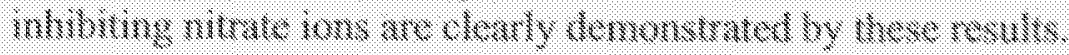




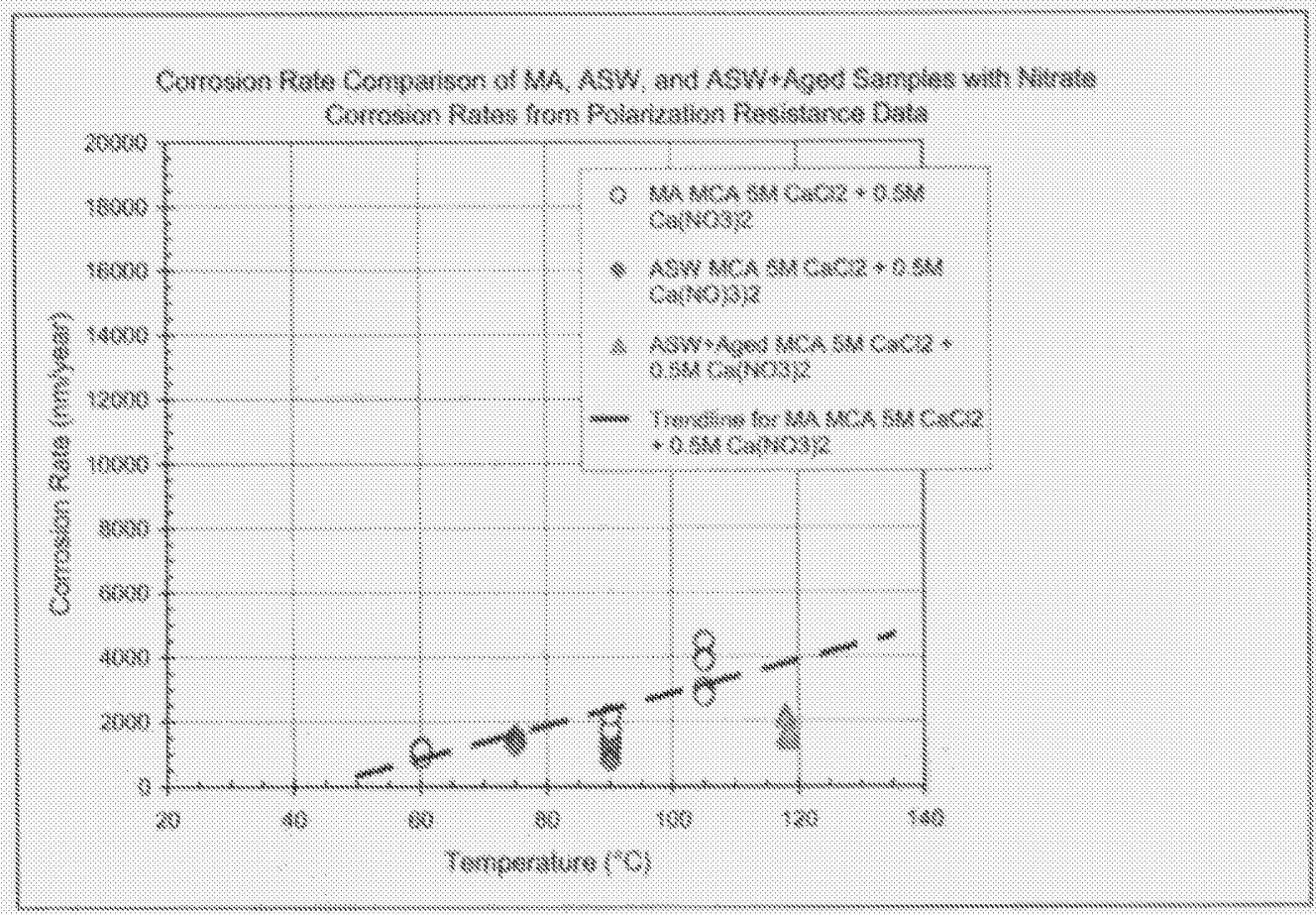

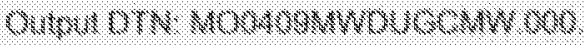

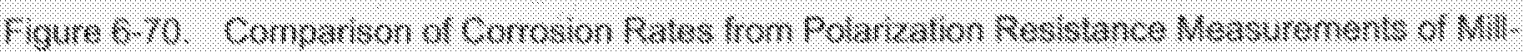

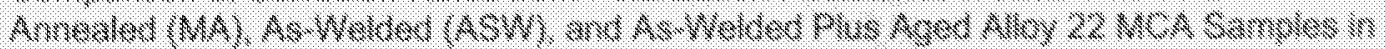

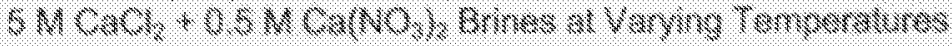

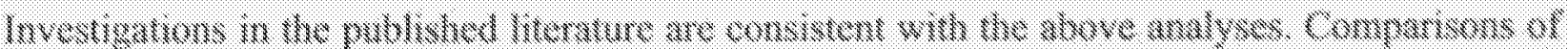

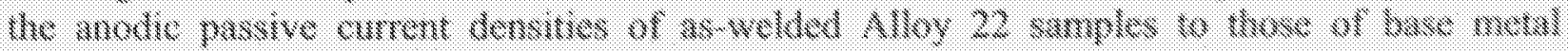

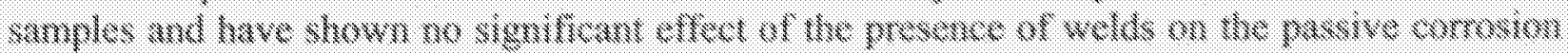

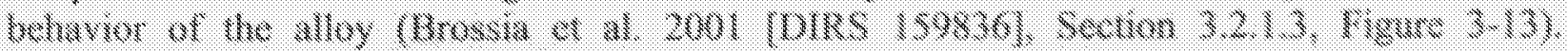

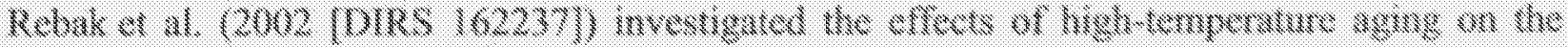

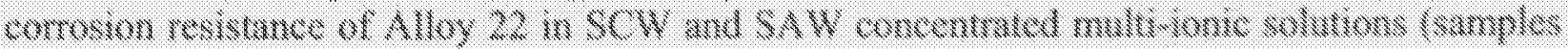

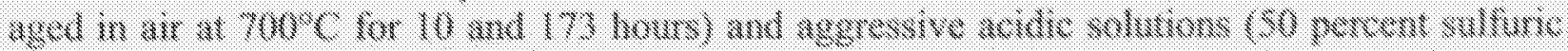

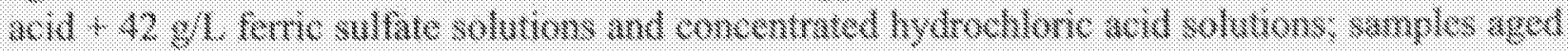

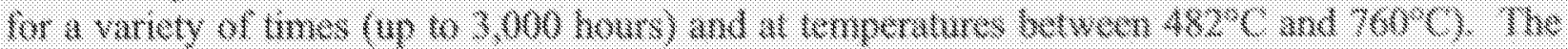

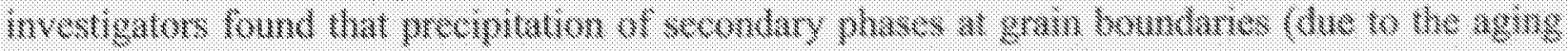

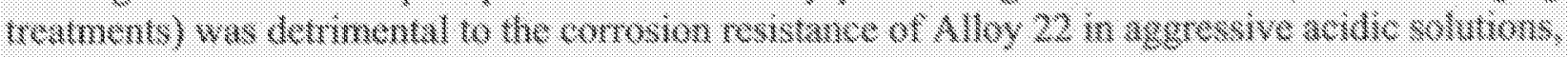

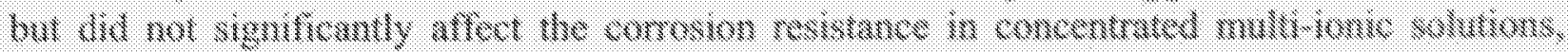

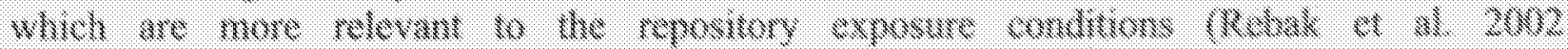

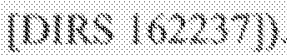

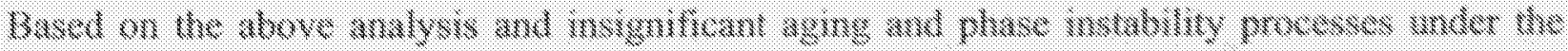

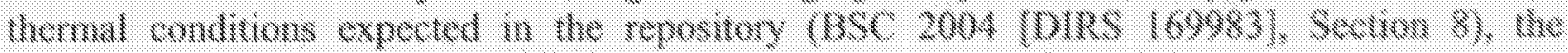

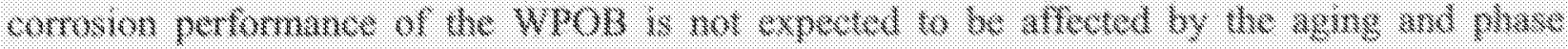

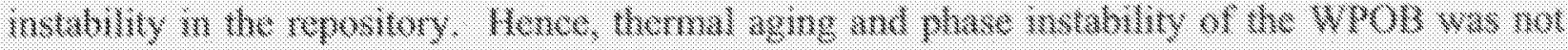

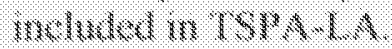


INTENTIONALLY LEFT BLANK 


\section{VALIDATION}

Models described in this report are expected to adequately predict general and localized corrosion processes of the WPOB under the exposure conditions expected in the repository for a period of at least 10,000 years. This long time of application makes it difficult to validate these models in the usual way (i.e., by comparison of model predicted values with those observed experimentally for the whole range of time) (ASTM C 1174-97 1998 [DIRS 105725], Sections 19.3 and 20.4). Consequently, by validating the input parameter values used and comparing these parameters and model predictions to available peer-reviewed and qualified Project data, these models were validated. As required by AP-2.27Q and stated in the technical work plan (TWP) for this activity (BSC 2004 [DIRS 171583], Table 2-1), the level of confidence (i.e., Level of Validation) required for the models developed in this report is Level III meaning the Level of Model Importance is high. A high level of confidence in the WPOB general and localized corrosion models will be obtained by building confidence in the methods used to develop the models and corroborating or validating the model output values and alternate conceptual models with those available in the peer reviewed scientific literature.

\subsection{CONFIDENCE BUILDING DURING MODEL DEVELOPMENT TO ESTABLISH SCIENTIFIC BASIS AND ACCURACY FOR INTENDED USE}

For Level III validation, the development of the model should be documented in accordance with the requirements of Section 5.3.2(b) of AP-SIII.10Q. The development of the general and localized corrosion models for the waste package outer barrier was conducted according to these criteria, as discussed below:

1. Selection of input parameters and/or input data, and a discussion of how the selection process builds confidence in the model. [AP-SIII.10Q 5.3.2(b) (1) and AP-2.27Q Attachment 3 Level I (a)]

The inputs to the general and localized corrosion models for the waste package outer barrier have all been obtained from controlled sources (Table 4-1, Section 4.1.1), including discussion about selection of input and design parameters. Model assumptions have been described in Section 5. Detailed discussion about model concepts can be found throughout Section 8 and particularly in Section 6.3. Thus, this requirement can be considered satisfied.

2. Description of calibration activities, and/or initial boundary condition runs, and/or run convergences, simulation conditions set up to span the range of intended use and avoid inconsistent outputs, and a discussion of how the activity or activities build confidence in the model. Inclusion of a discussion of impacts of any non-convergence runs [(APSIII.10Q 5.3.2(b)(2) and AP-2.27Q Attachment 3 Level I (e)].

Discussion of initial and boundary conditions are described in Sections 1.2, 6, and 8 where the waste package degradation models and ranges of application are discussed. Sections 6.4.3 and 6.4.4 provide discussion of various model results (i.e., those of convergence runs). Discussion about nonconvergence runs is not applicable to this report because none were encountered. Thus, this requirement can also be considered satisfied. 
3. Discussion of the impacts of uncertainties to the model results including how the model results represent the range of possible outcomes consistent with important uncertainties. [(AP-SIII.10Q 5.3.2(b)(3) and AP-2.27Q Attachment 3 Level 1 (d) and $(f)]$.

Uncertainties associated with the waste package barrier's capabilities are summarized in Sections 8.1, 8.2, and 8.3. More detailed discussions of the impact of uncertainties on the model results developed in this report are found in Sections 6.4.3, 6.4.4, and 6.4.5.

Section 6.4.3 discusses the effects of data uncertainties on the general corrosion model developed in this report. Uncertainties in the data used for the general corrosion model analysis (5-year weight loss measurements and short-term polarization resistance measurements) were characterized and quantified, and propagated through the general corrosion model abstraction (Section 6.4.3). Section 6.4.4 discusses the effects of data uncertainties on the localized corrosion model developed in this report. Uncertainties in the data used for the localized corrosion model analysis (crevice repassivation potentials, and long-term steady-state corrosion potentials) were characterized and quantified, and propagated through the localized corrosion model abstraction (Section 6.4.4). A conservative bounding approach, based on the literature data for Alloy 22 in highly corrosive environments, was used to capture the uncertainty in the localized corrosion rate of Alloy 22 (Section 6.4.4). Section 6.4.5 discusses the effects of data uncertainties on the microbially influenced corrosion (MIC) model developed in this report.

4. Formulation of defensible assumptions and simplifications. [AP-2.27Q Attachment 3 Level I (b)].

Discussion of assumptions and simplifications are provided in Section 5 with appropriate technical bases for their use.

5. Consistency with physical principles, such as conservation of mass, energy, and momentum. [AP-2.27Q Attachment 3 Level I (c)]

Consistency with physical principles is demonstrated by the conceptual and mathematical formulation in Section 6.3 and 6.4, respectively.

\subsection{CONFIDENCE BUILDING AFTER MODEL DEVELOPMENT TO SUPPORT THE SCIENTIFIC BASIS OF THE MODEL}

Four validation activities were developed to ensure the required level of confidence in these models for their stated purposes has been achieved. Four validation activities are documented in the TWP for this activity (BSC 2004 [DIRS 171583], Table 2-1). The validation activities were developed to ensure the required level of confidence in these models has been achieved for their stated purposes are:

Activity One: Show that the modeled propagation rates of general corrosion and localized corrosion of the waste package are reasonable and consistent with rates determined by alternative techniques or alternative models for the conditions expected in the repository. 
Activity Two: Show that the modeled propagation rates of general corrosion and localized corrosion of the waste package reasonable and consistent with rates from literature data and natural or industrial analogues of relevant corrosion resistant alloys for the conditions expected in the repository.

Activity Three: Show that the response of the correlations for $E_{\text {corr }}$ and $E_{\text {critical }}$ of the waste package are reasonable and consistent with literature data on relevant corrosion resistant alloys and available analogues for the conditions expected in the repository.

Activity Four: Show that the response of the correlations for $E_{c o r r}$ and $E_{\text {critical }}$ of the waste package consistent with other alternative models for localized corrosion initiation for the conditions expected in the repository.

The criterion for satisfying these validation activities is that the corroborating data must match qualitatively (BSC 2004 [DIRS 171583], Table 2-1). These validation activities are consistent with the model validation techniques given in AP-SIII.10Q, Models, Sections 5.3.2.c)2) and 5.3.2.c)3). A detailed description of the validation of the WPOB general and localized corrosion models in light of these validation activities follows.

\subsubsection{General Corrosion Model of the Waste Package Outer Barrier}

Validation of the general corrosion model of the WPOB requires meeting the salient parts of Activities One and Two.

Activity One: $\quad$ Show that the modeled propagation rates of general corrosion and localized corrosion of the waste package are reasonable and consistent with rates determined by alternative techniques or alternative models for the conditions expected in the repository.

Activity Two: Show that the modeled propagation rates of general corrosion and localized corrosion of the waste package reasonable and consistent with rates from literature data and/or natural analogues and/or industrial analogues of relevant corrosion resistant alloys for the conditions expected in the repository.

As described in Section 6.4.3.4, the base-case general corrosion model for the WPOB is based on a temperature dependence of the corrosion process, represented by an activation energy using the natural logarithmic form of a modified Arrhenius relation. The model is expressed as follows:

$$
\ln \left(R_{T}\right)=\ln \left(R_{o}\right)+C_{1}\left(\frac{1}{T}-\frac{1}{333.15}\right)
$$

$R_{T}$ is temperature-dependent general corrosion rate in $\mathrm{nm} / \mathrm{yr}, T$ is temperature in Kelvin, and $R_{o}$ and $C_{l}$ are constants. The temperature dependence term $\left(C_{l}\right)$ was obtained from short-term polarization resistance data for Alloy 22 specimens tested for a range of sample configurations, metallurgical conditions, and exposure conditions. See Section 6.4.3.4 for details of the model derivation and parameter evaluation. A normally distributed temperature-dependence term with 
a mean of $-3,116.47 \mathrm{~K}$ and a standard deviation of $296.47 \mathrm{~K}$ was chosen. The activation energy was estimated to be $25.9 \pm 2.5 \mathrm{~kJ} / \mathrm{mol}$. The general corrosion rate distribution $\left(R_{o}\right)$ derived from the weight-loss data of the 5-year crevice specimens was fit to a Weibull distribution ( $s=8.88 \mathrm{~nm} / \mathrm{yr}, b=1.62$, and $l=0 \mathrm{~nm} / \mathrm{yr}$ ). This distribution is considered to represent the distribution of long-term general corrosion rates of the WPOB at $60^{\circ} \mathrm{C}$. The median ( $50^{\text {th }}$ percentile) rate of $R_{o}$ is $7.08 \mathrm{~nm} / \mathrm{yr}$, and the $99.999^{\text {th }}$ percentile rate is $40.1 \mathrm{~nm} / \mathrm{yr}$.

The validation of the general corrosion model consists of two parts: evaluation of the activation energy value (or the temperature dependence term, $C_{1}$ ) of the model and evaluation of the general corrosion rates predicted by the model. As discussed below, the evaluation was performed by comparing the aforementioned model properties with the literature data for corrosion-resistant alloys similar to Alloy 22 measured in repository relevant environmental conditions.

Temperature dependence of the passive corrosion rate of Alloy 22 was also reported by other investigators. Based on the passive current densities of Alloy 22 measured in $5 \mathrm{M} \mathrm{LiCl}$ solutions with small amounts of [ $\left.\mathrm{SO}_{4}^{-}\right]$and $\left[\mathrm{NO}_{3}^{-}\right]$added (Scully et al. 2001 [DIRS 154513], Table 4, Section 1.4), an activation energy of $36 \mathrm{~kJ} / \mathrm{mol}$ was estimated (BSC 2001 [DIRS 155950], Section 7.3.5.3). The same analysis also estimated an activation energy of $32 \mathrm{~kJ} / \mathrm{mol}$ from the measured passive current densities of Alloy 22 polarized at $350 \mathrm{mV}$ vs SSC in a solution of $1 \mathrm{M}$ $\mathrm{NaCl}$ and $0.1 \mathrm{M} \mathrm{H}_{2} \mathrm{SO}_{4}$ (Lloyd et al. 2003 [DIRS 167921]). EPRI (2002 [DIRS 158069], Section 5.3.2) estimated an activation energy of $19 \mathrm{~kJ} / \mathrm{mol}$ for Alloy C-4 from the corrosion rates from weight-loss measurements of the alloy measured over a period of 3 to 5 years at temperatures in the range of $90^{\circ} \mathrm{C}$ to $200^{\circ} \mathrm{C}$ in saturated $\mathrm{Mg}^{2+}$-dominated brines (Smailos et al. 1987 [DIRS 159774]). A recent study sponsored by the Project estimated activation energies of $26.8 \mathrm{~kJ} / \mathrm{mol}$ and $24.9 \mathrm{~kJ} / \mathrm{mol}$ for annealed Alloy 22 and welded Alloy 22 samples, respectively. The activation energies were determined from corrosion rates based on the weight-loss measurements in $\mathrm{BSW}$ solutions at temperatures from $60^{\circ} \mathrm{C}$ to $105^{\circ} \mathrm{C}$ for eight weeks (Hua 2002 [DIRS 162256], p. 90, Table 8-2).

An analysis of the effect of temperature on the passive corrosion of Alloy 22, conducted by researchers at the CNWRA, reported a mean apparent activation energy of $44.7 \mathrm{~kJ} / \mathrm{mol}$ with a standard deviation of $5.5 \mathrm{~kJ} / \mathrm{mol}$, based on potentiostatic anodic current measurements of smooth cylindrical specimens polarized at $100 \mathrm{mV}_{\mathrm{SCE}}$ in de-aerated $0.028 \mathrm{M} \mathrm{NaCl}$ solutions (Pensado et al. 2002 [DIRS 166944], Section 4.3). In the tests, the temperature was decreased from $95^{\circ} \mathrm{C}$ to $80^{\circ} \mathrm{C}$ to $60^{\circ} \mathrm{C}$ to $40^{\circ} \mathrm{C}$ to $25^{\circ} \mathrm{C}$ at defined times over a total test duration of about 900 hours. The higher activation energy of the above study than that of the base-case general corrosion model in this report may have been caused by the combined effects of the exposure time and the order of the temperature changes (i.e., decrease from high temperature to low temperature). This test approach would yield higher passive corrosion rates at the higher temperatures and lower passive corrosion rates at lower temperatures, thus giving a higher activation energy than the approach used by Lloyd et al. (2003 [DIRS 167921]) in which the test temperature changed from low to high in the potentiostatic passive current measurements. Another factor to consider is that CNWRA researchers (Dunn and Brossia 2002 [DIRS 162213]) found a temperature dependence of the passive corrosion rate of Alloy 22 in low salinity solutions $\left(0.028 \mathrm{M}\left[\mathrm{Cl}^{-}\right]\right)$, but no temperature dependence at higher salinities $\left(4.0 \mathrm{M}\left[\mathrm{Cl}^{-}\right]\right)$, which 
are more relevant to the repository. The temperature dependence resulting from studies at higher chloride concentrations (e.g., Scully et al. 2001 [DIRS 154513]; Lloyd et al. 2003 [DIRS 167921]; EPRI 2002 [DIRS 158069]; Hua 2002 [DIRS 162256]) are more relevant. Considering these factors, the apparent activation energy value and its standard deviation reported by Pensado et al. (2002 [DIRS 166944], Section 4.3) is not considered relevant.

In another study, CNWRA researchers reported a calculated activation energy of $46.3 \mathrm{~kJ} / \mathrm{mol}$ for the temperature dependence of Alloy 22 general corrosion in $0.028 \mathrm{M} \mathrm{NaCl}$ over the temperature range between $25^{\circ} \mathrm{C}$ and $95^{\circ} \mathrm{C}$ (Dunn et al. 2004 [DIRS 171452]), and an activation energy of $49.6 \mathrm{~kJ} / \mathrm{mol}$ in a 35 -percent $\mathrm{MgCl}_{2}$ solution for the temperature range between $40^{\circ} \mathrm{C}$ and $120^{\circ} \mathrm{C}$. The activation energy obtained in the dilute chloride solution $(0.028 \mathrm{M} \mathrm{NaCl})$ is not considered relevant as discussed previously. The Alloy 22 corrosion rates in the 35-percent $\mathrm{MgCl}_{2}$ solution were more than an order of magnitude greater than those obtained in the $0.028 \mathrm{M} \mathrm{NaCl}$ solution indicating that the aggressive $\mathrm{MgCl}_{2}$ solution may have disrupted the stability of the passive film (Dunn et al. 2004 [DIRS 171452]) leading to active (rather than passive) corrosion. On this basis, the activation energy obtained in the 35-percent $\mathrm{MgCl}_{2}$ solution is not relevant to the passive corrosion conditions for which the general corrosion model was developed.

As indicated by the literature data cited above, the temperature dependence of general corrosion of Alloy 22 and other similar corrosion-resistant Ni-Cr-Mo alloys is about the same as that used in the base-case general corrosion model in this report considering the differing conditions (especially solution chemistry and sample condition) employed in the tests. Also, as shown by the 5-year weight-loss data (Section 6.4.3.2, Appendices II and III) and the corrosion rates from the polarization resistance measurements (Section 6.4.3.4, Appendix IV), there is no significant dependence of the Alloy 22 general corrosion rate on metallurgical conditions and sample geometry. In addition, the activation energy of the Alloy 22 general corrosion rate obtained in this report is similar to the results of the other investigators cited above. It is noted that the activation energies of general corrosion rate of highly corrosion-resistant $\mathrm{Ni}-\mathrm{Cr}-\mathrm{Mo}$ alloys are similar regardless of the exposure time in the test environments. That is, the temperature dependence of the Alloy 22 general corrosion rate does not change significantly as the general corrosion rate decreases with the exposure time.

Because of the extremely low corrosion rates of Alloy 22, there are little data for Alloy 22 in the scientific literature that could be used to evaluate the general corrosion model. However, similar passive corrosion behavior has also been observed for $\mathrm{Ni}-\mathrm{Cr}-\mathrm{Mo}$ corrosion-resistant alloys. For example, Alloy C (UNS N06455) is found to retain a very thin passive film, indicated by the mirror-like surface finish observed after 44 years of exposure at Kure Beach to a marine environment (i.e., salt air with alternate wetting and drying, as well as the presence of surface deposits) (Baker 1988 [DIRS 154510], p. 134 and Table 6). More recent examination of specimens from this alloy after more than 50 years of exposure indicates that the samples continue to maintain a mirror-like finish and passive film behavior (McCright 1998 [DIRS 114637], Figure ES-1). Under these same conditions, the less corrosion-resistant Alloy 600 exhibited a corrosion rate of $8 \mathrm{~nm} / \mathrm{yr}$ after 36 years of exposure. This long-term corrosion rate is consistent with the model prediction.

The $50^{\text {th }}, 95^{\text {th }}$ and $99.99^{\text {th }}$ (reasonable upper bound) percentile rates at $25^{\circ} \mathrm{C}$ for the mean activation energy $(25.91 \mathrm{~kJ} / \mathrm{mol})$ predicted by the general corrosion model are $2.4,5.8$, 
and $11.7 \mathrm{~nm} / \mathrm{yr}$, respectively (Figure $6-26 \quad 25^{\circ} \mathrm{C}$ model result CDF; DTN: MO0409MWDUGCMW.000). For the lower bound activation energy $(18.53 \mathrm{~kJ} / \mathrm{mol})$ those rates predicted by the model are $3.2,8.0$, and $15.9 \mathrm{~nm} / \mathrm{yr}$ respectively (Figure $6-2825^{\circ} \mathrm{C}$ model result CDF; DTN: MO0409MWDUGCMW.000). As discussed above, the general corrosion behavior of corrosion-resistant $\mathrm{Ni}-\mathrm{Cr}$-Mo alloys are similar. These long-term results corroborate the expected excellent long-term passive behavior of Alloy 22 under chloride-containing aqueous environments relevant to repository exposure conditions.

The model validation for intermediate and elevated temperature conditions was performed by comparing the model results to the recently reported weight-loss measurements of MA and ASW Alloy $22 \mathrm{MCA}$ specimens after exposure to the $\mathrm{BSW}$ solutions at temperatures from $60^{\circ} \mathrm{C}$ to $105^{\circ} \mathrm{C}$ over a period of 8 weeks (Hua et al. 2002 [DIRS 160670]). From the 8-week weight-loss measurements, an average general corrosion rate of $75 \mathrm{~nm} / \mathrm{yr}$ at $60^{\circ} \mathrm{C}$ and $300 \mathrm{~nm} / \mathrm{yr}$ at $105^{\circ} \mathrm{C}$ were reported for the Alloy $22 \mathrm{MCA}$ specimens. The model calculated $50^{\text {th }}, 95^{\text {th }}$ and $99.99^{\text {th }}$ (reasonable upper bound) percentile rates at $60^{\circ} \mathrm{C}$ for the mean activation energy $(25.91 \mathrm{~kJ} / \mathrm{mol}$ ) are $7.1,17.5$, and $35.0 \mathrm{~nm} / \mathrm{yr}$ respectively. For the $105^{\circ} \mathrm{C}$ condition, the model calculated $50^{\text {th }}$, $95^{\text {th }}$, and $99.99^{\text {th }}$ percentile rates are $21.6,53.2$, and $106 \mathrm{~nm} / \mathrm{yr}$ respectively for the mean activation energy $(25.91 \mathrm{~kJ} / \mathrm{mol})$, and $29.6,73.1$, and $146 \mathrm{~nm} / \mathrm{yr}$ respectively for the upper bound activation energy $(33.29 \mathrm{~kJ} / \mathrm{mol})$. Although the upper bound rates of the model are consistent with the data, it appears the model predicted general corrosion rates are generally lower than the 8-week exposure data. However, considering the shorter-term nature of the data (compared to the 5-year data used in the model), the model results are in a good agreement with the data obtained in this repository relevant exposure environment.

The model predictions for high temperature chloride-containing brines are also consistent with general corrosion rate data for surface-welded Alloy C-4 samples tested at $150^{\circ} \mathrm{C}$ in $\mathrm{NaCl}$-rich brines (Smailos 1993 [DIRS 168164], Table IV). The samples corroded uniformly under the test conditions. As discussed above, the general corrosion behavior of corrosion-resistant Ni-Cr-Mo alloys are similar. A mean general corrosion rate of $70 \mathrm{~nm} / \mathrm{yr}$ and $60 \mathrm{~nm} / \mathrm{yr}$ was reported for 12-month and 18-month test periods, respectively. The reported general corrosion rates are the average values of five samples. For the same $\mathrm{NaCl}$-rich brines containing $6 \times 10^{-4} \mathrm{M} \mathrm{Na} \mathrm{Na}_{2} \mathrm{~S}$, the reported mean general corrosion rates of the surface-welded alloy were $510 \mathrm{~nm} / \mathrm{yr}$ and $120 \mathrm{~nm} / \mathrm{yr}$ for 12-month and 18-month test periods, respectively.

The model calculated $50^{\text {th }}, 95^{\text {th }}$ and $99.99^{\text {th }}$ percentile rates at $150^{\circ} \mathrm{C}$ for the mean activation energy $\left(25.91 \mathrm{~kJ} / \mathrm{mol}\right.$ ) are 52,128 , and $256 \mathrm{~nm} / \mathrm{yr}$, respectively (Figure 6-26 $150^{\circ} \mathrm{C}$ model result CDF; DTN: MO0409MWDUGCMW.000). For the upper bound activation energy $(33.29 \mathrm{~kJ} / \mathrm{mol})$, the model calculated $50^{\text {th }}, 95^{\text {th }}$ and $99.99^{\text {th }}$ percentile rates at $150^{\circ} \mathrm{C}$ are 91,225 , and $451 \mathrm{~nm} / \mathrm{yr}$, respectively (Figure $6-27150^{\circ} \mathrm{C}$ model result CDF; DTN: MO0409MWDUGCMW.000). The $150^{\circ} \mathrm{C}$ model results for the lower-bound activation energy $\left(18.53 \mathrm{~kJ} / \mathrm{mol}\right.$ ) are 29,73 , and $145 \mathrm{~nm} / \mathrm{yr}$, respectively (Figure $6-28150^{\circ} \mathrm{C}$ model result CDF; DTN: MO0409MWDUGCMW.000). As demonstrated above, the predicted general corrosion rates for high-temperature chloride-containing brines are consistent with literature data for Alloy C-4 whose general corrosion behavior is expected to be similar to Alloy 22 for the conditions expected in the repository. 
The general corrosion rate is temperature dependent, and for a given temperature, it is assumed constant (i.e., time independent) (Assumption 5.2). Therefore, for a given temperature, the depth of penetration or thinning of the WPOB by general corrosion is equal to the general corrosion rate at that temperature, multiplied by the time duration that the waste package surface is at that temperature. However, general corrosion rates of metals and alloys tend to decrease with time. The dependence of the general corrosion rate of Alloy 22 on the exposure time is shown in Figure 7-1 for the mean general corrosion rates of Alloy 22 at $90^{\circ} \mathrm{C}$, measured with different test techniques (potentiostatic polarization, polarization resistance, and weight-loss measurements) for exposure times up to 5 years. Given the differences between the techniques used to determine corrosion rates presented in the figure, some variation about the central trend line is to be expected. A trend-line was drawn for better visualization of the data trend. The data shown in the figure are summarized in Table 7-1.

The exposure time ranges from one day to 5-plus years exposure at the LTCTF. Each data point for the 6-month, 1-year, and 2-year weight-loss measurements in the figure is the mean of at least 144 samples (CRWMS M\&O 2000 [DIRS 153802], p. 3-39). The 5-year general corrosion rate is the mean of the weight-loss measurements of 59 crevice samples from the LTCTF (Table 6-6 and Section 6.4.3.4). The mean general corrosion rate of the crevice samples after 5-year exposure at the LTCTF was $0.0072 \mu \mathrm{m} / \mathrm{yr}$, and the standard deviation was $0.005 \mu \mathrm{m} / \mathrm{yr}$. It is noted that the corrosion rates measured by short-term electrochemical techniques provide corroboration of the rates from weight-loss method. The trend of decreasing general corrosion rate with time is consistent with the expected corrosion behavior of passive alloys such as Alloy 22 under repository-type aqueous conditions. The current conservative approach for the constant (time-independent) general corrosion rate at a given temperature in the waste package degradation analysis provides an additional confidence for the general corrosion model.

A comparison of the rates obtained from the temperature-dependent general corrosion model with the rates from alternative techniques from the scientific literature shows that Activities One and Two have been met. In addition, the conservative approach used in calculating the penetration depth by general corrosion over time would provide an additional confidence to the model and the modeling approach.

Table 7-1. Summary of Mean General Corrosion Rates of Alloy 22 at $90^{\circ} \mathrm{C}$ Versus Exposure Time

\begin{tabular}{|c|c|c|c|}
\hline \multicolumn{2}{|c|}{ Exposure Time } & \multirow{2}{*}{$\begin{array}{l}\text { Mean Rate } \\
(\mu \mathrm{m} / \mathrm{yr})\end{array}$} & \multirow{2}{*}{ Sources } \\
\hline (years) & (days) & & \\
\hline 0.0027 & 1 & 0.460 & $\begin{array}{l}\text { Lian et al. } 2002 \text { [DIRS 164856], Table 3, potentiostatic polarization technique } \\
\text { at } 100 \mathrm{mV} \text { versus SSC applied potential in } S A W, 90^{\circ} \mathrm{C}, \mathrm{N}_{2} \text { purge. }\end{array}$ \\
\hline 0.0027 & 1 & 1.250 & $\begin{array}{l}\text { Lian et al. } 2002 \text { [DIRS 164856], Table } 3 \text {, potentiostatic polarization technique } \\
\text { at } 100 \mathrm{mV} \text { versus SSC applied potential in } S C W, 90^{\circ} \mathrm{C}, \mathrm{N}_{2} \text { purge. }\end{array}$ \\
\hline 0.019 & 7 & 0.100 & $\begin{array}{l}\text { Evans and Rebak } 2002 \text { [DIRS 164857], Figure 2, polarization resistance } \\
\text { technique, after } 1 \text { week in open-circuit potential in SAW, } 90^{\circ} \mathrm{C} \text {, air purge. }\end{array}$ \\
\hline 0.154 & 56 & 0.182 & $\begin{array}{l}\text { Hua } 2002 \text { [DIRS 162256], p. 97, calculated from regression fit for 56-day } \\
\text { weight loss in BSW, CR (MPY) }=31.3^{\star} \exp (-25300 / R T) \text {. }\end{array}$ \\
\hline 0.50 & 183 & 0.050 & CRWMS M\&O 2000 [DIRS 153802] (p. 3-39), LTCTF weight-loss data \\
\hline 1.00 & 365 & 0.030 & CRWMS M\&O 2000 [DIRS 153802] (p. 3-39), LTCTF weight-loss data \\
\hline 2.30 & 840 & 0.010 & CRWMS M\&O 2000 [DIRS 153802] (p. 3-39), LTCTF weight-loss data \\
\hline 5.06 & 1,846 & 0.007 & Section 6.4 .3 .3 , Table $6-6$ \\
\hline
\end{tabular}




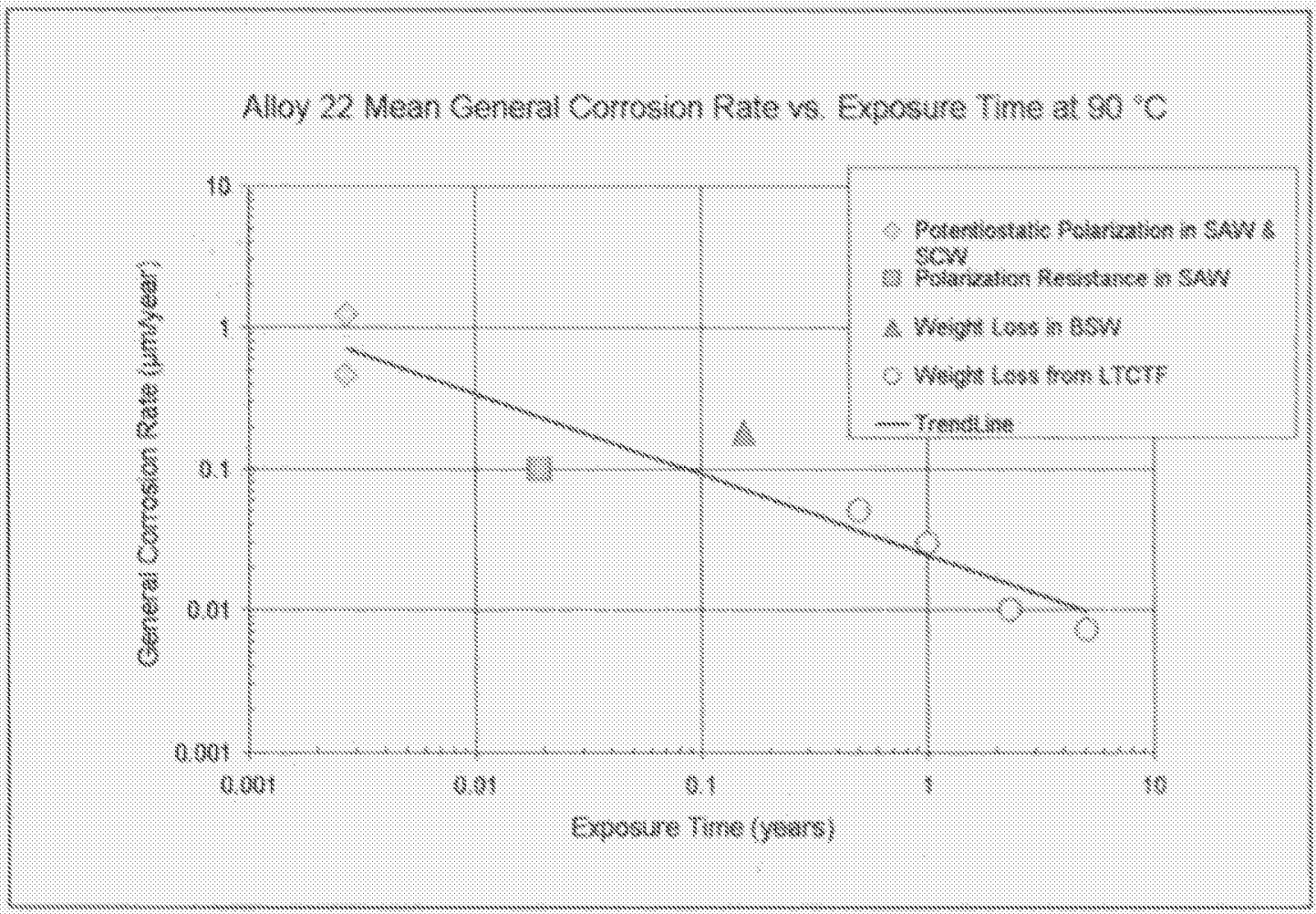

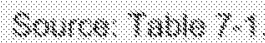

Figure 7.1. Decrease of the Menen General Corrosion Rate of Alby 22 wht Mines

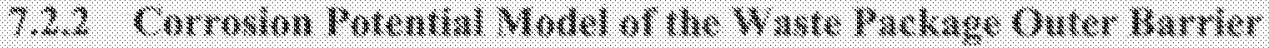

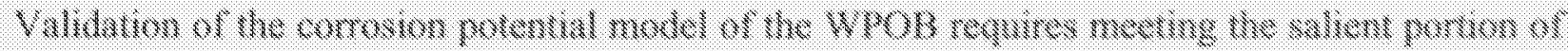
i.

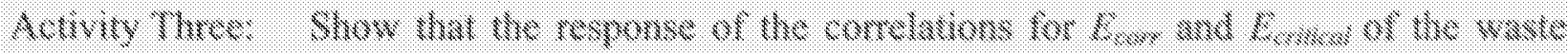

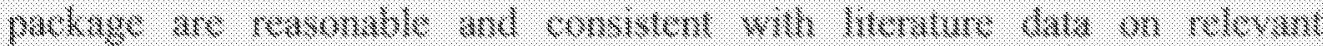

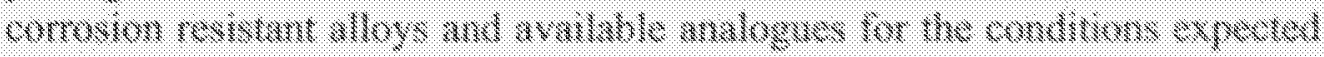

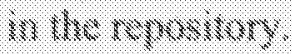

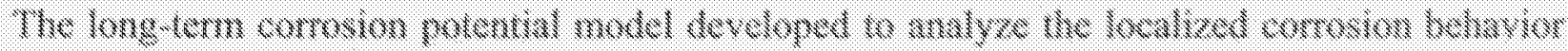
of the WPOH under the condinons expected an the repowitury in expresed as follown:

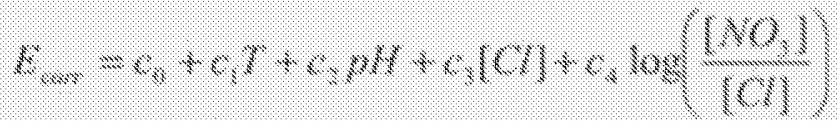

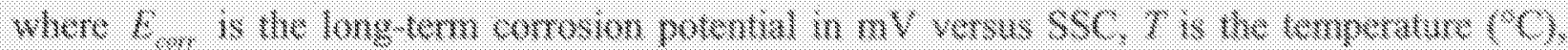

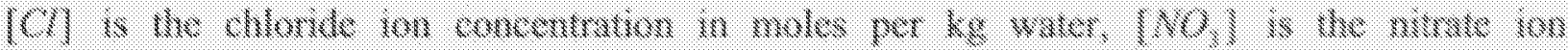

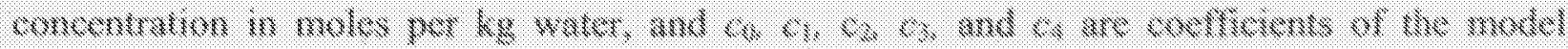

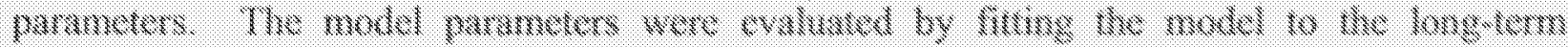

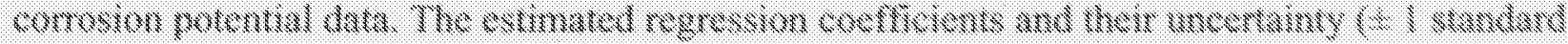

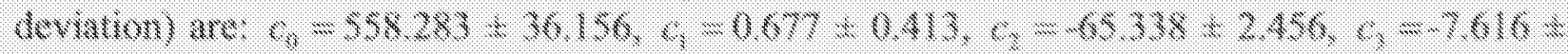


1.581 , and $c_{4}=37.077 \pm 2.443$. This model is to estimate the long-term steady-state opencircuit corrosion potential of Alloy 22 for a range of exposure conditions related to the repository. The model is not intended to predict short-term corrosion potential because the corrosion potential of Alloy 22 evolves with time under the conditions relevant to the repository.

Few data exist for the steady-state corrosion potential of Alloy 22 for conditions related to the repository that can be used to evaluate the corrosion potential model. A study sponsored by the U.S. Department of Energy Nuclear Energy Research Initiative published measurements of the open-circuit corrosion potential of Alloy 22 under air-saturated conditions in a saturated $\mathrm{NaCl}$ solution $(\mathrm{pH}=3)$ at $80^{\circ} \mathrm{C}$ for a period of 200 days (Jayaweera et al. 2003 [DIRS 162225], pp. 9-18 to 9-22, Figure 9.13). The chloride ion concentration of the solution was about 6.2 molal. The measured corrosion potential at the end of the testing (200 days) ranges from about 160 to $250 \mathrm{mV}$ versus standard hydrogen electrode. For these exposure conditions, the mean value of the corrosion potential from the long-term corrosion potential model is $229 \mathrm{mV} \mathrm{SSC}$, and \pm 2 standard deviation values are 250 and $207 \mathrm{mV} \mathrm{SSC}$, respectively. At $25^{\circ} \mathrm{C}\left(77^{\circ} \mathrm{F}\right)$, the $\mathrm{Ag} / \mathrm{AgCl}$ scale with the reference electrode in saturated $\mathrm{KCl}$ solution is more noble than the standard hydrogen electrode scale by $199 \mathrm{mV}$ (Sawyer and Roberts 1974 [DIRS 162259], pp. 39 to 45, Table 2-4). Therefore, the model-predicted corrosion potentials in the standard hydrogen electrode scale are $428 \mathrm{mV}$ (mean), $449 \mathrm{mV}$ (plus 2 standard deviations), and $406 \mathrm{mV}$ (minus 2 standard deviations). Although the lower-bound of the model-predicted corrosion potential (406 mV versus standard hydrogen electrode) is higher than Jayaweera et al.'s (2003 [DIRS 162225], pp. 9 to 18 ) results (160 to $250 \mathrm{mV}$ versus standard hydrogen electrode) by about $150 \mathrm{mV}$, the model prediction is considered a reasonably good match considering that the investigators indicated that the corrosion potential had likely not reached steady state. In addition, overestimates of the corrosion potential by the model are conservative because, for a given condition, the overestimates could result in localized corrosion initiation under conditions in which it would not be observed.

Dunn et al. (2003 [DIRS 166948], Figures 4-17 and 4-18) reported the corrosion potential data of Alloy 22 in air-saturated $0.028 \mathrm{M} \mathrm{NaCl}$ solutions as a function of immersion time for approximately 60 days. These data were also used to validate the corrosion potential model. The corrosion potentials appeared to stabilize after 60 days of testing, but there were significant spreads of data for the near-neutral and alkaline conditions. Comparing to the longer-term corrosion potential data of Alloy 22 samples tested in air-saturated and more concentrated $\mathrm{NaCl}$ solutions (Jayaweera et al. 2003 [DIRS 162225], pp. 9-18 to 9-22, Figure 9.13), it is likely that the corrosion potentials may have not yet reached the steady state after 60 days of testing. The corrosion potential data after 60 days of testing, read from the figures, are summarized in the first four rows of Table 7-2, along with the environmental condition inputs to the model calculations and model predicted corrosion potentials. Because the model predicted corrosion potential is in the SSC scale, the reported corrosion potential data in the SCE scale were converted to the SSC scale by adding $42 \mathrm{mV}$. The fifth row in Table 7-2 contains one corrosion potential observation measured from an Alloy 22 crevice corrosion test specimen exposed to an air-saturated $4 \mathrm{M} \mathrm{Cl}^{-}$ solution (Dunn et al. 2003 [DIRS 164138], pp. 2 and 5). The specimen was periodically removed and examined for signs of localized corrosion (Dunn et al. 2003 [DIRS 164138], p. 5). During over 750 days of testing, no localized corrosion was observed (Dunn et al. 2003 [DIRS 164138], p. 5). Over the last 200 days of testing the corrosion potential reached a 
maximum value near $-100 \mathrm{mV}_{\mathrm{SCE}}$. As summarized in Table $7-2$, the model predictions are consistent with the cited literature data.

Table 7-2. Summary of Model Validation Analysis for the Corrosion Potential Model

\begin{tabular}{|c|c|c|c|c|c|}
\hline \multirow{2}{*}{$\begin{array}{c}\text { Test Condition, and Data } \\
\text { Source a) }\end{array}$} & \multirow{2}{*}{$\begin{array}{c}\text { Measured } \\
E_{\text {corr }} \\
\left(\mathrm{mV}_{\text {scE }}\right)\end{array}$} & \multirow{2}{*}{$\begin{array}{l}\text { Measured } E_{\text {corr }} \\
\quad\left(\mathrm{mV}_{\mathrm{ssc}}\right)^{\mathrm{b})}\end{array}$} & \multirow{2}{*}{$\begin{array}{l}\text { Environmental Condition } \\
\text { Inputs to Model } \\
\text { Calculation }\end{array}$} & \multicolumn{2}{|c|}{$E_{\text {corr }}$ Model ${ }^{c)}$} \\
\hline & & & & $\begin{array}{c}\text { Mean } \\
\left(\mathrm{mV}_{\text {ssc }}\right)\end{array}$ & \pm 2 s.d. $\left(\mathrm{mV}_{\mathrm{ssc}}\right)$ \\
\hline $\begin{array}{l}\text { Alloy } 22 \text { thermally } \\
\text { oxidized; a air-saturated } \\
0.028 \mathrm{M} \mathrm{NaCl}, 0.16 \mathrm{mM} \\
\mathrm{NO}_{3}{ }^{\circ}, 95^{\circ} \mathrm{C}, \mathrm{pH} 10.6 \text { to } \\
11.7 \text { (Dunn et al. } 2003 \\
\text { [DIRS } 166948 \text { ], Figure } \\
4-17 \text { ) }\end{array}$ & $\begin{array}{l}-300 \text { to } \\
-100\end{array}$ & -258 to -58 & \multirow{2}{*}{$\begin{array}{l}0.028 \mathrm{~m}[\mathrm{Cl}], 0.00016 \mathrm{~m} \\
{\left[\mathrm{NO}_{3}\right], 95^{\circ} \mathrm{C}, \mathrm{pH} 11.2} \\
\text { (mid-point) }\end{array}$} & \multirow{2}{*}{-143} & \multirow{2}{*}{$\begin{array}{l}-175 \text { (-2 s.d.) } \\
-112 \text { (+2 s.d.) }\end{array}$} \\
\hline $\begin{array}{l}\text { Alloy } 22 \text { polished; air- } \\
\text { saturated } 0.028 \mathrm{M} \\
\mathrm{NaCl}, 0.16 \mathrm{mM} \mathrm{NO}_{3}^{\circ} \\
95^{\circ} \mathrm{C}, \mathrm{pH} 10.6 \text { to } 11.7 \\
\text { (Dunn et al. } 2003 \text { [DIRS } \\
\text { 166948], Figure 4-17) }\end{array}$ & -200 to 0 & -158 to 42 & & & \\
\hline $\begin{array}{l}\text { Alloy } 22 \text { thermally } \\
\text { oxidized; air-saturated } \\
0.028 \mathrm{M} \mathrm{NaCl}, 0.16 \mathrm{mM} \\
\mathrm{NO}_{3}^{-}, 95^{\circ} \mathrm{C}, \mathrm{pH} 8.2 \text { to } \\
9.3 \text { (Dunn et al. } 2003 \\
\text { [DIRS } 166948 \text { ], Figure } \\
\text { 4-18) }\end{array}$ & $\begin{array}{l}-120 \text { to } \\
70\end{array}$ & -78 to 112 & $\begin{array}{l}0.028 m[\mathrm{Cl}], 0.00016 \mathrm{~m} \\
{\left[\mathrm{NO}_{3}\right], 95^{\circ} \mathrm{C}, \mathrm{pH} 8.8} \\
\text { (mid-point) }\end{array}$ & -6 & $\begin{array}{l}-30(-2 \text { s.d. }) \\
18(+2 \text { s.d. })\end{array}$ \\
\hline $\begin{array}{l}\text { Alloy } 22 \text { thermally } \\
\text { oxidized; air-saturated } \\
0.028 \mathrm{M} \mathrm{NaCl}, 0.16 \mathrm{mM} \\
\mathrm{NO}_{3}{ }^{-}, 95^{\circ} \mathrm{C}, \mathrm{pH} 2.7 \text { to } \\
2.8 \text { (Dunn et al. } 2003 \\
\text { [DIRS 166948], Figure } \\
\text { 4-18) }\end{array}$ & $\begin{array}{l}250 \text { to } \\
270\end{array}$ & 292 to 312 & $\begin{array}{l}0.028 \mathrm{~m}[\mathrm{Cl}], 0.00016 \mathrm{~m} \\
{\left[\mathrm{NO}_{3}\right], 95^{\circ} \mathrm{C}, \mathrm{pH} 2.75} \\
\text { (mid-point) }\end{array}$ & 389 & $\begin{array}{l}365 \text { (-2 s.d.) } \\
413(+2 \text { s.d.) }\end{array}$ \\
\hline $\begin{array}{l}\text { Alloy } 22 \text { crevice } \\
\text { specimen, air saturated } \\
4 \mathrm{M} \mathrm{Cl}^{-}, 0.16 \mathrm{mM} \mathrm{NO}_{3}^{-} \text {, } \\
95^{\circ} \mathrm{C} \text { (Dunn et al. [DIRS } \\
164138 \text { ], p. } 2 \text { and } 5 \text { ) }\end{array}$ & -100 & -58 & $\begin{array}{l}4.51 \mathrm{~m}[\mathrm{Cl}]^{\mathrm{d})}, 0.00016 \\
m\left[\mathrm{NO}_{3}\right], 95^{\circ} \mathrm{C}, \mathrm{pH} 6.3^{\mathrm{d})}\end{array}$ & 41 & $\begin{array}{l}25 \text { (-2 s.d.) } \\
57 \text { (+2 s.d.) }\end{array}$ \\
\hline
\end{tabular}

NOTES: ${ }^{\text {a) }}$ Some Alloy 22 samples were thermally oxidized at $200^{\circ} \mathrm{C}$ for 30 days.

b) At $25^{\circ} \mathrm{C}$ the saturated calomel reference electrode (SCE) is $42 \mathrm{mV}$ more noble than the silver-silver chloride reference electrode (SSC). The SCE scale potentials were converted to the SSC scale potentials by adding $42 \mathrm{mV}$.

c) The values in these columns are obtained by substituting the environmental condition inputs into the equation used to determine the corrosion potential (Ecorr (T,pH,Cl,NO3,z) in Appendix IX on p. IX-14). z = 0 gives the mean, $z= \pm 2$ gives the \pm 2 s.d. values.

d) $\mathrm{pH}$ of 6.3 and $\mathrm{Cl}^{\prime}$ molality from Cell 18 in Appendix $\mathrm{V}(4 \mathrm{M} \mathrm{NaCl}$ solution).

A comparison of the long-term corrosion potential obtained from the long-term corrosion potential model with the long-term corrosion potential data of Alloy 22 measured independently has shown that the salient portion of Activity Three relating to the long-term corrosion potential model has been met. 


\subsubsection{Critical Potential Model of the Waste Package Outer Barrier}

Validation of the critical potential model of the WPOB requires meeting the salient portion of Activity Three.

Activity Three: Show that the response of the correlations for $E_{\text {corr }}$ and $E_{\text {critical }}$ of the waste package are reasonable and consistent with literature data on relevant corrosion resistant alloys and available analogues for the conditions expected in the repository.

$E_{\text {critical }}$ can be defined as a threshold potential above which the current density or corrosion rate of Alloy 22 increases significantly and irreversibly above the general corrosion rate of the passive metal. As a conservative measure, the base-case localized corrosion model uses the crevice repassivation potential $\left(E_{\text {rcrev }}\right)$ as the critical potential for the localized corrosion initiation analysis. The crevice repassivation potential $\left(E_{\text {rcrev }}\right)$ is expressed as follows.

$$
E_{\text {rcrev }}=E_{\text {rcrev }}^{o}+\Delta E_{\text {rcrev }}^{N O_{3}^{-}}
$$

where $E_{r c r e v}^{o}$ is the crevice repassivation potential in the absence of inhibitive nitrate ions, and $\Delta E_{r c r e v}^{N O_{3}^{-}}$is the crevice repassivation potential change resulting from the inhibiting effect of nitrate ion in solution. The crevice repassivation potential of Alloy 22 in the absence of inhibitive nitrate ion is expressed as follows.

$$
E_{\text {rcrev }}^{o}=a_{o}+a_{1} T+a_{2} p H+a_{3} \log ([C l])+a_{4} T \times \log ([C l])
$$

where $a_{o}, a_{1}, a_{2}, a_{3}$, and $a_{4}$ are constants, $T$ is the temperature $\left({ }^{\circ} \mathrm{C}\right)$, and $[C l]$ is the chloride ion concentration in moles per $\mathrm{kg}$ water. The value of the coefficients and their uncertainty ( \pm 1 standard deviation) of the model parameters from the least square fitting were determined to be: $a_{0}=214.089 \pm 46.880, a_{1}=-3.696 \pm 0.476, a_{2}=25.284 \pm 5.641, a_{3}=-252.181 \pm 53.912$, and $a_{4}=1.414 \pm 0.547$.

The effect of nitrate ion on the crevice repassivation potential is represented by the following functional form:

$$
\Delta E_{\text {rerev }}^{N \mathrm{NO}_{3}^{-}}=b_{o}+b_{1}\left[\mathrm{NO}_{3}\right]+b_{2} \frac{\left[\mathrm{NO}_{3}\right]}{[\mathrm{Cl}]}
$$

where $[\mathrm{Cl}]$ is the chloride ion concentration in moles per $\mathrm{kg}$ water, $\left[\mathrm{NO}_{3}\right]$ is the nitrate ion concentration in moles per $\mathrm{kg}$ water, and $b_{o}, b_{1}$, and $b_{2}$ are constant. The parameter coefficients and their uncertainty ( \pm 1 standard deviation) resulting from the fitting procedure were determined to be: $b_{o}=22.589 \pm 24.113, b_{1}=33.748 \pm 5.180$, and $b_{2}=749.745 \pm 95.491$.

There are limited data in the scientific literature for the crevice repassivation potential of Alloy 22 for the conditions relevant to the repository. Even for those literature data that may be 
applicable, it is difficult to use those data to evaluate the critical potential model because they were obtained using different criteria and/or measurement approaches (Section 6.4.4.1).

There are three crevice repassivation potential data points for Alloy 22 previously reported by the investigators at the CNWRA for the NRC (Dunn et al. 1999 [DIRS 154481], Figures 3-6 and 3-9). These data were not included in Effect of Environment on the Corrosion of Waste Package and Drip Shield Materials (Brossia et al. 2001 [DIRS 159836]) and not included in the crevice repassivation model development. These data are summarized in Table 7-3. The crevice repassivation potentials were read from the figures referenced. As shown in the table, the model predicted crevice repassivation potentials of Alloy 22 agree with the measured data within $\pm 100 \mathrm{mV}$. This represents an acceptable level of agreement between the model and the measured data particularly in light of the subjective nature of the selection of critical potentials from CPP curves and the dynamic nature of the measurement technique (Section 6.4.4.1). Also, according to the localized corrosion initiation model developed in this report, localized corrosion would initiate under these essentially pure chloride exposure conditions (e.g., Figure 6-61 for $95^{\circ} \mathrm{C}, 10 \mathrm{~m}$ chloride, and $0.01 \mathrm{~m}$ nitrate).

A comparison of the crevice repassivation potential obtained from the long-term corrosion potential model with the crevice repassivation potential data of Alloy 22 measured independently has shown that the salient portion of Activity Three relating to the crevice repassivation model has been met.

Table 7-3. Summary of Model Validation Analysis for Crevice Repassivation Potential Model

\begin{tabular}{|c|c|c|c|c|c|}
\hline \multirow{2}{*}{$\begin{array}{l}\text { Exposure } \\
\text { Environment and } \\
\text { Data Source }\end{array}$} & \multirow{2}{*}{$\begin{array}{c}\text { Measured } \\
E_{\text {rcrov }} \\
\left(\mathrm{mV}_{\text {sCE }}\right)\end{array}$} & \multirow{2}{*}{$\begin{array}{c}\text { Measured } \\
E_{\text {rerov }} \\
\left(\mathrm{mV}_{\text {ssc }}\right)^{\text {a) }}\end{array}$} & \multirow{2}{*}{$\begin{array}{l}\text { Environmental Condition } \\
\text { Inputs to Model Calculation }\end{array}$} & \multicolumn{2}{|c|}{$E_{\text {rerov }}$ Model ${ }^{b)}$} \\
\hline & & & & Mean $\left(m V_{s s c}\right)$ & \pm 2 s.d. $\left(\mathrm{mV}_{\mathrm{ssc}}\right)$ \\
\hline $\begin{array}{l}0.5 \mathrm{M} \mathrm{NaCl}, 10 \mathrm{ppm} \\
\mathrm{NO}_{3}, \mathrm{pH} 8,175^{\circ} \mathrm{C} \\
\text { (Dunn et al. } 1999 \\
\text { [DIRS 154481], } \\
\text { Figure 3-9) }\end{array}$ & -275 & -233 & $\begin{array}{c}0.51 \mathrm{~m}[\mathrm{Cl}], 0.00016 \mathrm{~m} \\
{\left[\mathrm{NO}_{3}\right], \mathrm{pH} 8,175^{\circ} \mathrm{C}}\end{array}$ & -229 & $\begin{array}{l}-143 \text { (+2 s.d.) } \\
-315(-2 \text { s.d.) }\end{array}$ \\
\hline $\begin{array}{l}9 \mathrm{M} \mathrm{LiCl}, 10 \mathrm{ppm} \\
\mathrm{NO}_{3}, \mathrm{pH} 5.6,95^{\circ} \mathrm{C} \\
\text { (Dunn et al. } 1999 \\
\text { [DIRS 154481], } \\
\text { Figure 3-7) }\end{array}$ & -230 & -188 & $\begin{array}{l}11 \mathrm{~m}[\mathrm{Cl}] \text { (estimated) }{ }^{\mathrm{c}} \text {, } \\
0.00016 \mathrm{~m}\left[\mathrm{NO}_{3}\right], \mathrm{pH} 5.6 \\
95^{\circ} \mathrm{C}\end{array}$ & -118 & $\begin{array}{l}-100 \text { (+2 s.d.) } \\
-136(-2 \text { s.d.) }\end{array}$ \\
\hline $\begin{array}{l}11 \mathrm{M} \mathrm{LiCl}, 10 \mathrm{ppm} \\
\mathrm{NO}_{3}, \mathrm{pH} 5.6,95^{\circ} \mathrm{C} \\
\text { (Dunn et al. } 1999 \\
\text { [DIRS 154481], } \\
\text { Figure 3-7) } \\
\end{array}$ & -260 & -218 & $\begin{array}{c}13 \mathrm{~m}[\mathrm{Cl}]\left(\text { estimated) }{ }^{\mathrm{c})}\right. \\
0.00016 \mathrm{~m}\left[\mathrm{NO}_{3}\right], \mathrm{pH} 5.6 \\
95^{\circ} \mathrm{C}\end{array}$ & -127 & $\begin{array}{l}-108(+2 \text { s.d.) } \\
-146(-2 \text { s.d.) }\end{array}$ \\
\hline
\end{tabular}

NOTES: ${ }^{\text {a }}$ At $25^{\circ} \mathrm{C}$ the saturated calomel reference electrode (SCE) is $42 \mathrm{mV}$ more noble than the silver-silver chloride reference electrode (SSC). The SCE scale potentials were converted to the SSC scale potentials by adding $42 \mathrm{mV}$.

b The values in these columns are obtained by substituting the environmental condition inputs into the equation used to determine the critical potential $(\mathrm{Ecrit}(\mathrm{T}, \mathrm{pH}, \mathrm{Cl}, \mathrm{NO}, \mathrm{z})$ in Appendix IX on $\mathrm{p}$. IX-14). $\mathrm{z}=0$ gives the mean, $z= \pm 2$ gives the \pm 2 s.d. values.

$c$ The values of chloride ion molality are estimated. Given that these values are not direct input to this report, estimation of the chloride ion molality is acceptable for this usage. 


\subsubsection{Localized Corrosion Initiation Model of the Waste Package Outer Barrier}

The discussion in this section considers the correlations for $E_{\text {rcrev }}$ and $E_{\text {corr }}$ (or more accurately their difference, $\Delta E=E_{\text {rcrev }}-E_{\text {corr }}$ ) and compares the predicted results with experimental data/observations and the alternative conceptual model discussed in Section 6.4.4.8.1. These discussions relate to Activities Three and Four.

Activity Three: Show that the response of the correlations for $E_{c o r r}$ and $E_{\text {critical }}$ of the waste package are reasonable and consistent with literature data on relevant corrosion resistant alloys and available analogues for the conditions expected in the repository.

Activity Four: Show that the response of the correlations for $E_{\text {corr }}$ and $E_{\text {critical }}$ of the waste package consistent with other alternative models for localized corrosion initiation for the conditions expected in the repository.

Additional model validation was performed by comparing the model predictions for localized corrosion susceptibility with relevant observations. As discussed in Section 6.4.3 for the WPOB general corrosion model analysis, Alloy 22 crevice samples were tested for over 5 years in three different solutions (SDW, SCW, and SAW) in the long-term corrosion testing facility (LTCTF). None of the crevice samples has suffered localized corrosion attack after being tested for over 5 years. Also Dunn et al. (2003 [DIRS 164138], pp. 2 and 5) exposed an Alloy 22 crevice corrosion test specimen to an air saturated $4 \mathrm{M} \mathrm{Cl}^{-}$solution for over 750 days. The specimen was periodically removed and examined for signs of localized corrosion (Dunn et al. 2003 [DIRS 164138], p. 5). During over 750 days of testing, no localized corrosion was observed (Dunn et al. 2003 [DIRS 164138], p. 5). These observations are used for model validation of the localized corrosion initiation model (i.e., crevice repassivation potential model in conjunction with the corrosion potential model).

The analysis results are summarized in Table 7-4. As indicated by the results in the table, the model predicts no localized corrosion occurrence for the exposure conditions of the 5-year LTCTF crevice samples. The model predicts that localized corrosion will occur in the air saturated $4 \mathrm{M} \mathrm{Cl}^{-}$solution. These results show that the crevice corrosion initiation model (crevice repassivation potential model, in conjunction with the corrosion potential model) adequately predicts the crevice corrosion susceptibility of Alloy 22 and is conservative in some cases.

Table 7-4. Comparison of Model Prediction for Localized Corrosion Susceptibility with Experimental Observations of Alloy 22 Crevice Samples Tested for Over 5 Years in LTCTF

\begin{tabular}{|c|c|c|c|c|c|c|c|}
\hline \multicolumn{5}{|c|}{ Long-Term Immersion Test Results } & \multicolumn{3}{|c|}{ Model Results } \\
\hline $\begin{array}{c}\text { Exposure } \\
\text { Environment }\end{array}$ & $\begin{array}{c}\text { Crevice } \\
\text { Corrosion } \\
\text { Observation }\end{array}$ & $\mathrm{pH}^{\mathrm{a}}$ & $\begin{array}{c}{[\mathrm{Cl}]} \\
\text { (moles/kg }_{\text {water) }}\end{array}$ & $\begin{array}{c}{ }_{\left.\mathrm{NO}_{3}\right]}{ }^{(\text {moles } / \mathrm{kg}} \\
\text { water) }^{\mathrm{a}}\end{array}$ & $\begin{array}{c}\text { Mean } E_{\text {corr }} \\
(m V \text { vs. } \\
\text { SSC) }\end{array}$ & $\begin{array}{c}\text { Mean } E_{\text {rcrev }} \\
\text { (mV vs. } \\
\text { SSC) })^{b}\end{array}$ & $\begin{array}{c}\text { Mean } \Delta E \\
\left(E_{\text {rcrev }}-E_{\text {corr }}\right) \\
\text { (mV vs. SSC) }\end{array}$ \\
\hline $\mathrm{SDW}, 90^{\circ} \mathrm{C}$ & No & 8.5 & 0.00325 & 0.000313 & 45 & 407 & 362 \\
\hline $\mathrm{SCW}, 90^{\circ} \mathrm{C}$ & No & 10.91 & 0.214 & 0.114 & -106 & 642 & 748 \\
\hline SAW, $90^{\circ} \mathrm{C}$ & No & 3.83 & 0.838 & 0.426 & 351 & 400 & 49 \\
\hline $\begin{array}{l}\text { Alloy } 22 \text { crevice } \\
\text { specimen, air }\end{array}$ & No & 6.3 & 4.51 & 0.00016 & 41 & -55 & -96 \\
\hline
\end{tabular}




\begin{tabular}{|c|c|c|c|c|c|c|c|}
\hline \multicolumn{5}{|c|}{ Long-Term Immersion Test Results } & \multicolumn{3}{|c|}{ Model Results } \\
\hline $\begin{array}{c}\text { Exposure } \\
\text { Environment }\end{array}$ & $\begin{array}{c}\text { Crevice } \\
\text { Corrosion } \\
\text { Observation }\end{array}$ & $\mathrm{pH}^{\mathrm{a}}$ & $\begin{array}{c}{[\mathrm{Cl}]} \\
\text { (moles } / \mathrm{kg} \\
\text { water) }^{\mathrm{a}}\end{array}$ & $\begin{array}{c}{\left[\mathrm{NO}_{3}\right]} \\
(\mathrm{moles} / \mathrm{kg} \\
\text { water) }\end{array}$ & $\begin{array}{c}\text { Mean } E_{\text {corr }} \\
(\mathrm{mV} \text { vs. } \\
\text { SSC) }\end{array}$ & $\begin{array}{c}\text { Mean } E_{\text {rcrev }} \\
(m V \text { vs. } \\
\text { SSC) }\end{array}$ & $\begin{array}{c}\text { Mean } \Delta E \\
\left(E_{\text {rcrev }}-E_{\text {corr }}\right) \\
\text { (mV vs. SSC) }\end{array}$ \\
\hline $\begin{array}{c}\text { saturated } 4 \mathrm{M} \\
\mathrm{Cl}-, 0.16 \mathrm{mM} \\
\mathrm{NO}_{3}^{\circ}, 95^{\circ} \mathrm{C} \\
\text { (Dunn et al. } \\
\text { [DIRS } 164138 \text {, } \\
\text { Do. } 2 \text { and 5) }\end{array}$ & & & & & & & \\
\hline
\end{tabular}

NOTES: SDW = simulated dilute water, SCW = simulated concentrated water, SAW = simulated acidified water.

${ }^{a}$ The $\mathrm{pH}$ values and molal concentrations are from the values in Appendix $V$ for aged SDW (Cell 6), SCW

(Cell 3), SAW (Cell 10), and $4 \mathrm{M} \mathrm{NaCl}$ (Cell 18) solutions. For the very dilute $0.16 \mathrm{mM} \mathrm{NO}_{3}^{-}$concentration, the molar value is used.

${ }^{b} E_{\text {corr }}$ was calculated using Equation 7-2, and $E_{\text {rcrev }}$ using Equation 7-3.

Smailos (1993 [DIRS 168164]) tested Alloy C-4 (a nickel-based alloy similar to Alloy 22 (ASTM B 575-94 [DIRS 100497])) in two aggressive $\mathrm{MgCl}_{2}$-based brines and one NaCl-based brine at $150^{\circ} \mathrm{C}$ for up to 18 months. The compositions of these brines are listed in Table 7-5. It was found that localized corrosion (pitting) was observed in Brines 1 and $2\left(\mathrm{MgCl}_{2}\right.$-based), but not in Brine 3 ( $\mathrm{NaCl}$-based).

Table 7-5. Compositions and pH Values of Salt Brines Used in Smailos 1993 [DIRS 168164]

\begin{tabular}{|c|c|c|c|c|c|c|c|c|c|}
\hline & \multicolumn{8}{|c|}{ Weight Percent } & \multirow[b]{2}{*}{$\begin{array}{c}\mathrm{pH} \\
\left(\operatorname{at}^{\circ} 25^{\circ} \mathrm{C}\right)\end{array}$} \\
\hline Brine & $\mathrm{NaCl}$ & $\mathrm{KCl}$ & $\mathrm{MgCl}_{2}$ & $\mathrm{MgSO}_{4}$ & $\mathrm{CaCl}_{2}$ & $\mathrm{CaSO}_{4}$ & $\mathrm{~K}_{2} \mathrm{SO}_{4}$ & $\mathrm{H}_{2} \mathrm{O}$ & \\
\hline 1 & 1.4 & 4.7 & 26.8 & 1.4 & 0 & 0 & 0 & 65.7 & 4.6 \\
\hline 2 & 0.31 & 0.11 & 33.03 & 0 & 2.25 & 0.005 & 0 & 64.3 & 4.1 \\
\hline 3 & 25.9 & 0 & 0 & 0.16 & 0 & 0.21 & 0.23 & 73.5 & 6.5 \\
\hline
\end{tabular}

Source: Smailos 1993 [DIRS 168164], Table II.

The model predicts localized corrosion will occur for all three brines (the nitrate-to-chloride ion ratio is less than 0.50 and the exposure temperature is between $120^{\circ} \mathrm{C}$ and $160^{\circ} \mathrm{C}$ (Section 6.4.4.6.7)). This result may be conservative for Brine 3 (NaCl-based); however, localized corrosion may have initiated in Brine 1 if the test duration had exceeded 18 months.

Section 6.4.4.8.1 documents that temperature can be used as an alternative parameter to determine susceptibility to localized corrosion. Values of critical pitting temperature and critical crevice corrosion temperature have been measured for Alloy 22, in relatively pure concentrated chloride solutions (i.e., high salinity, $\left[\mathrm{Cl}^{-}\right]=24,300 \mu \mathrm{g} / \mathrm{g}$, and a high $\left[\mathrm{Cl}^{-}\right]$to $\left[\mathrm{SO}_{4}{ }^{2-}\right]$ ratio) (Haynes International 1997 [DIRS 100897], p. 9; McGuire et al. 1998 [DIRS 152193], Section 5.1.2). Under these highly corrosive conditions, the critical crevice corrosion temperature for Alloy 22 was measured to be $102^{\circ} \mathrm{C}$. The use of a critical temperature-based model is not advisable in TSPA-LA because it does not account for the effects of electrochemical characteristics of the solution contacting the metal, particularly those of important corrosion inhibiting anions such as nitrate ions present in the groundwater at the repository. Also, the localized corrosion initiation model developed in this report utilizes long-term corrosion potential data while the critical crevice corrosion temperature testing used a 100-hour exposure period. Furthermore, several of the graphs in Section 6.4.4.6 (e.g., Figure 
6-53, Figure 6-54, Figure 6-57, and Figure 6-58) indicate that the localized corrosion initiation model predicts the possibility for localized corrosion initiation at temperatures below $102^{\circ} \mathrm{C}$. This observation gives further confidence in the localized corrosion initiation model.

The model validation documented in this section has shown that Activity Four has been met and provides additional confidence that Activity Three has been met.

\subsubsection{Localized Corrosion Penetration Model of the Waste Package Outer Barrier}

Validation of the localized corrosion penetration rate model of the WPOB requires meeting the salient parts of Activities One and Two.

Activity One: Show that the modeled propagation rates of general corrosion and localized corrosion of the waste package are reasonable and consistent with rates determined by alternative techniques or alternative models for the conditions expected in the repository.

Activity Two: Show that the modeled propagation rates of general corrosion and localized corrosion of the waste package reasonable and consistent with rates from literature data and/or natural analogues and/or industrial analogues of relevant corrosion resistant alloys for the conditions expected in the repository.

Due to the outstanding corrosion resistance of Alloy 22, very little data exist for such localized corrosion under the conditions expected in the repository. The literature data for localized corrosion of relevant alloys that were considered for the current localized penetration rate model (Section 6.4.4.7) are for extremely corrosive conditions not expected in the repository. Those extreme penetration rates found in the literature were used to bound localized corrosion rates of Alloy 22 under repository conditions.

In the development of the localized corrosion penetration model, it was noted that the Alloy 22 average corrosion rate in a highly aggressive 10 percent ferric chloride crevice corrosion test solution was about $12.7 \mu \mathrm{m} / \mathrm{yr}$. The average corrosion rate of Alloy C-276 and Alloy C-4 (Ni-Cr-Mo alloys similar to Alloy 22) in the same test solution were $1.4 \mathrm{mils} / \mathrm{yr}(35.6 \mu \mathrm{m} / \mathrm{yr})$ and $20 \mathrm{mils} / \mathrm{yr}(508 \mu \mathrm{m} / \mathrm{yr}$ ), respectively (Haynes International 1997 [DIRS 100897], p. 8). These values fall within the range of rates used for the Alloy 22 localized corrosion penetration model as well as illustrating that Alloy 22 is more corrosion resistant than these alloys. In a solution composed of 7 volume percent $\mathrm{H}_{2} \mathrm{SO}_{4}, 3$ volume percent $\mathrm{HCl}, 1$ weight percent $\mathrm{FeCl}_{3}$, and 1 weight percent $\mathrm{CuCl}_{2}$, a penetration rate of $610 \mu \mathrm{m} / \mathrm{yr}$ measured by weight loss was observed for Alloy C-276 at $102^{\circ} \mathrm{C}$ (Gdowski 1991 [DIRS 100859], Table 23). This value also falls within the range of rates used for the Alloy 22 localized corrosion penetration model. Smailos (1993 [DIRS 168164]) tested Alloy C-4 in two aggressive $\mathrm{MgCl}_{2}$-based brines and one $\mathrm{NaCl}$-based brine at $150^{\circ} \mathrm{C}$ for up to 18 months. The compositions of these brines are listed in Table 7-5. It was found that localized corrosion (pitting) was observed in Brines 1 and 2 (the $\mathrm{MgCl}_{2}$-based brines) but not in Brine 3 (the $\mathrm{NaCl}$-based brine). Maximum pit depths between 300 and $900 \mu \mathrm{m}$ were observed after 18 months of testing (Smailos 1993 [DIRS 168164], Table III). These values correspond to pit penetration rates of between 200 and $600 \mu \mathrm{m} / \mathrm{yr}$. These values fall within the range of rates used for the Alloy 22 localized corrosion penetration model. Pitting was not observed in Brine 1 at 12 months and the maximum pit depth 


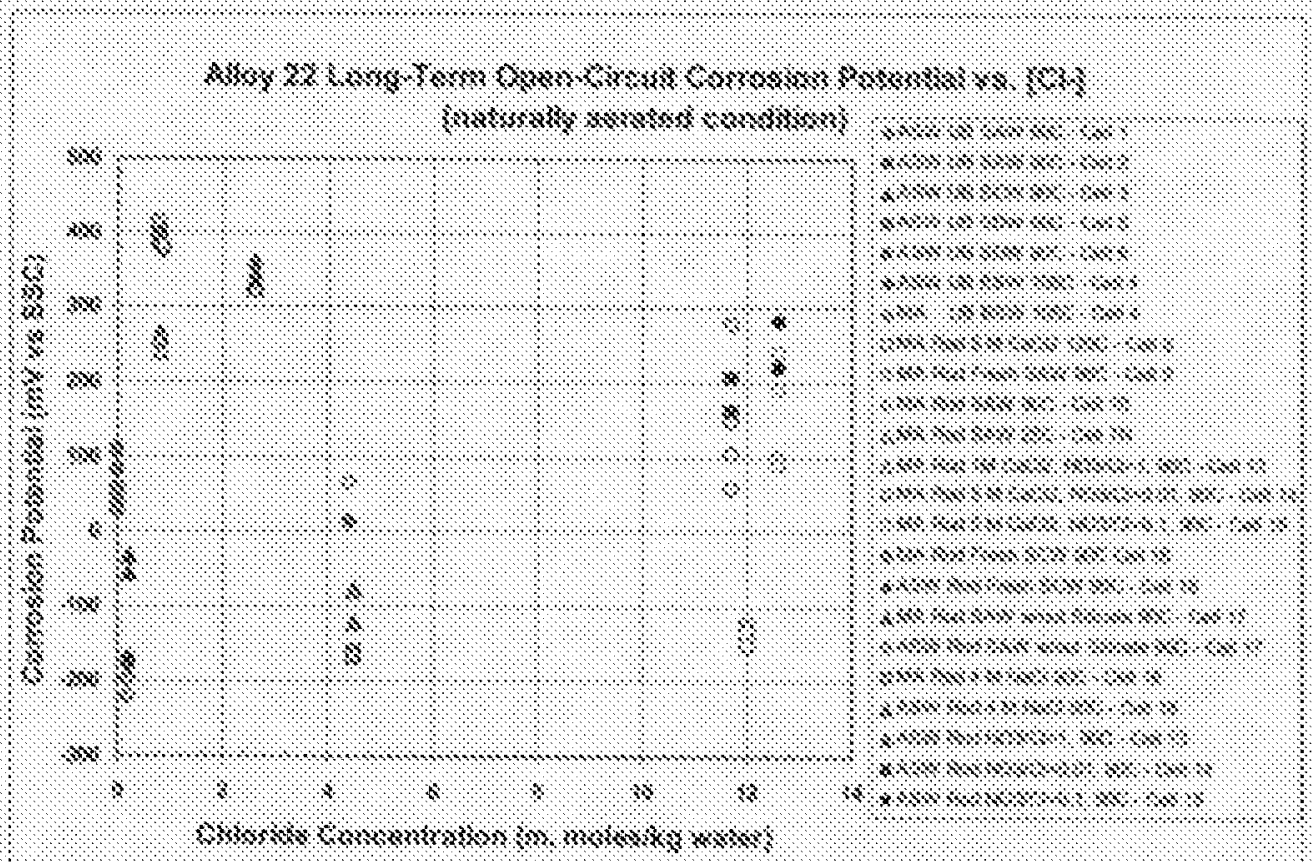

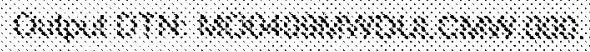

K

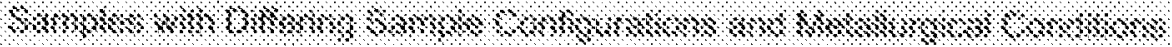

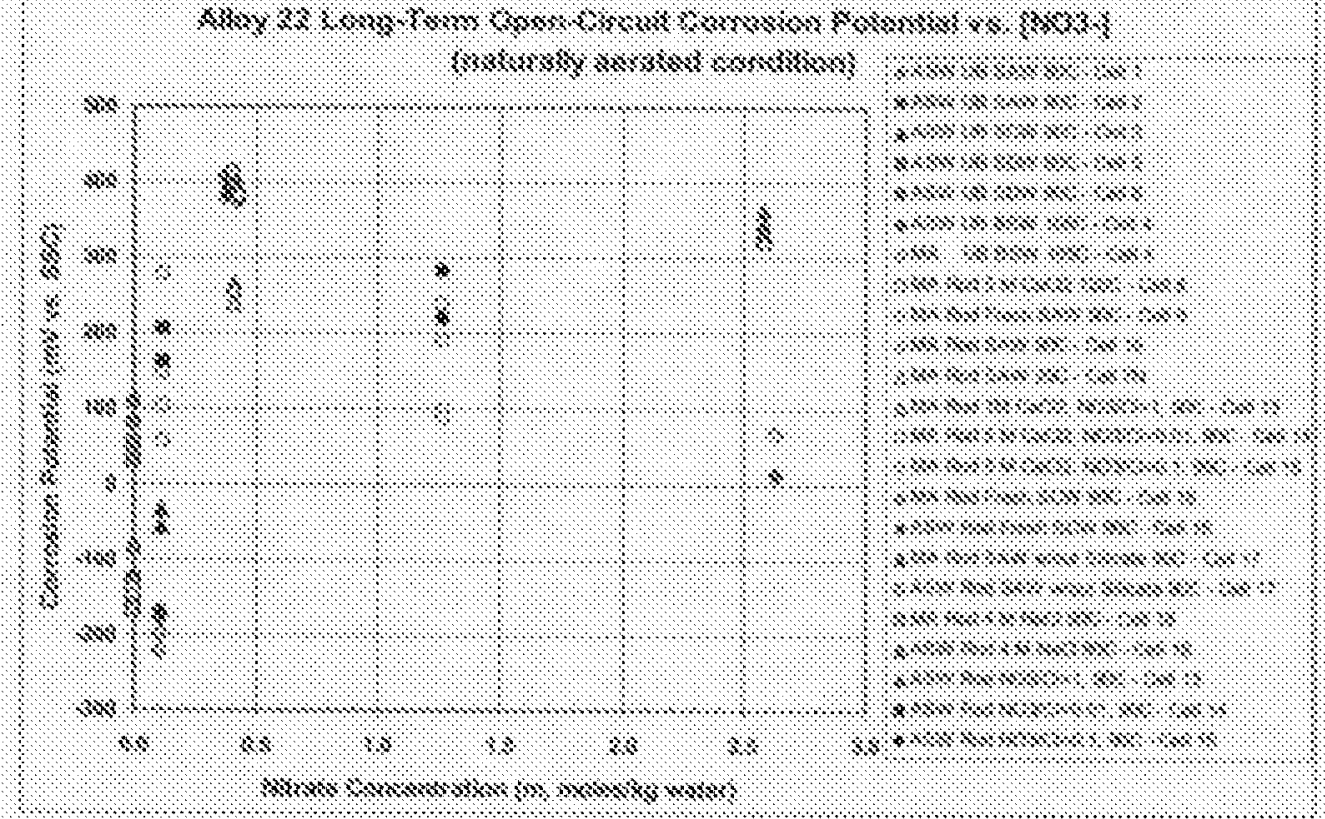

$\$, \$, \ldots, \ldots, \ldots$,

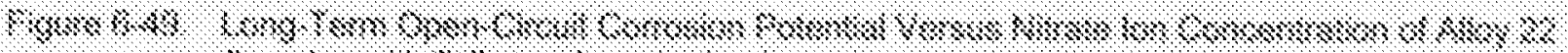

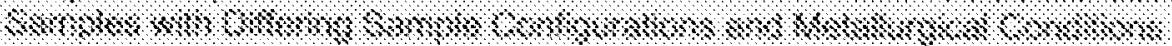




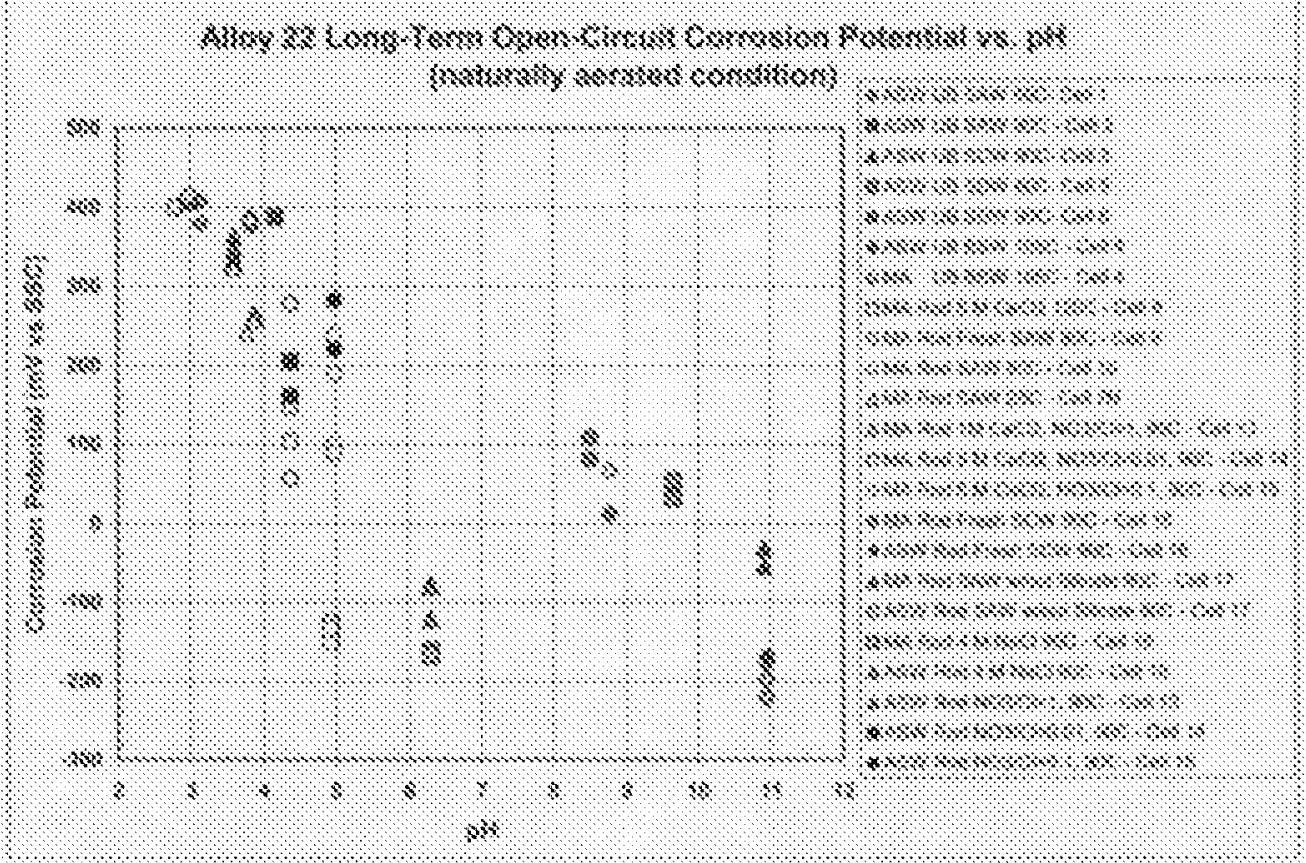

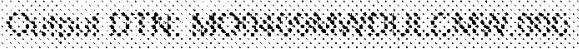

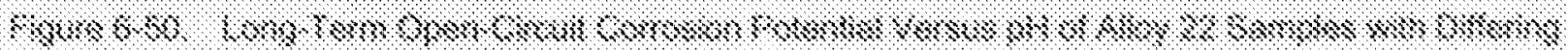

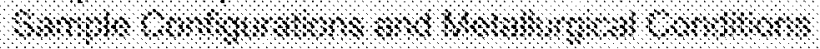

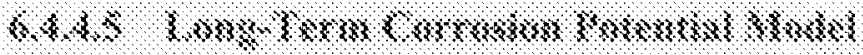

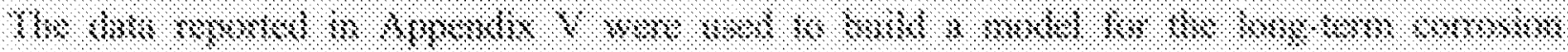

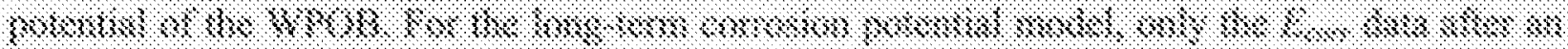

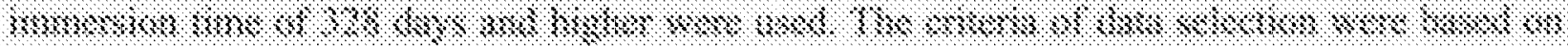

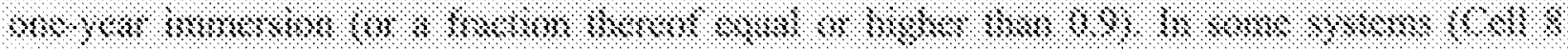

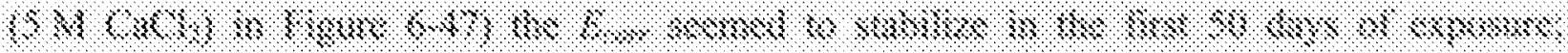

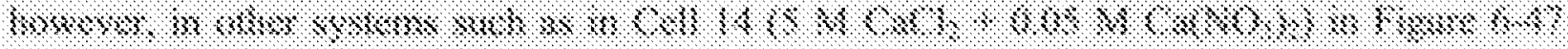

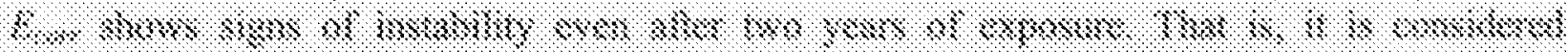

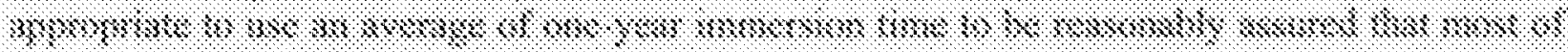

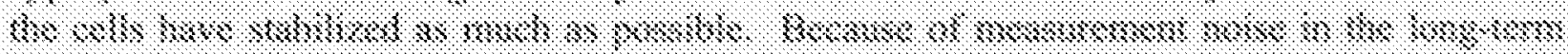

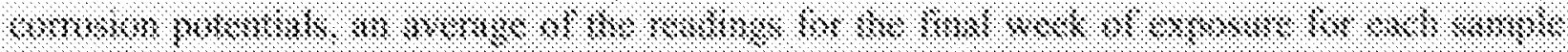

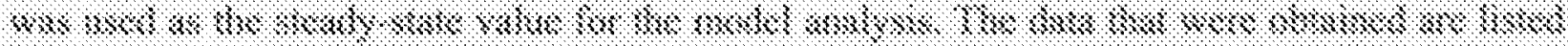

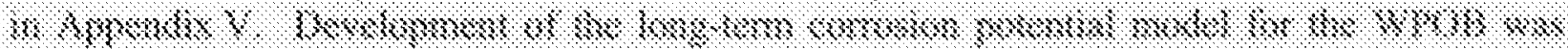

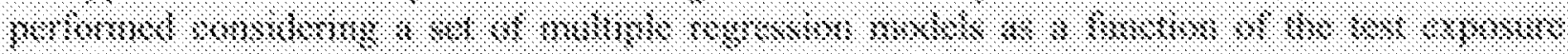

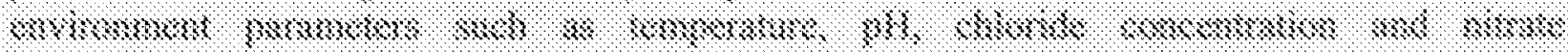
$306 \% \times 8 \times 8 \times 6 \%$

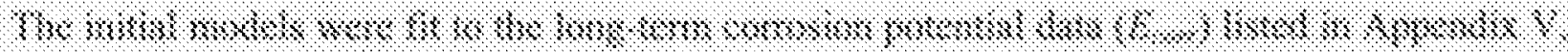

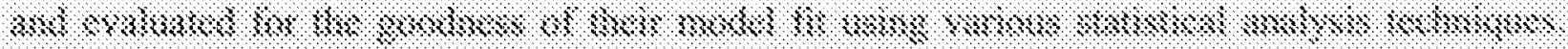

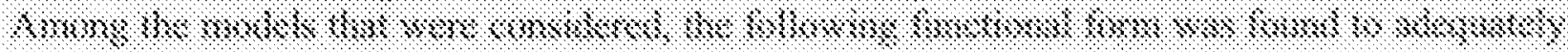

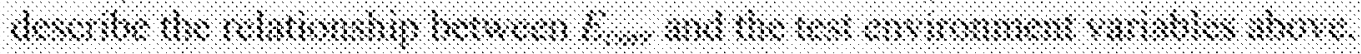




$$
E_{\text {corr }}=c_{o}+c_{1} T+c_{2} p H+c_{3}\left[\mathrm{Cl}^{-}\right]+c_{4} \log \left(\frac{\left[\mathrm{NO}_{3}^{-}\right]}{\left[\mathrm{Cl}^{-}\right]}\right)
$$

where $T$ is the temperature $\left({ }^{\circ} \mathrm{C}\right),\left[\mathrm{Cl}^{-}\right]$is the chloride ion concentration in molality $(\mathrm{m}$, moles $/ \mathrm{kg}$ water), $\left[\mathrm{NO}_{3}^{-}\right]$is the molal nitrate ion concentration, and $c_{o}, c_{1}, c_{2}, c_{3}$, and $c_{4}$ are coefficients of the model parameters. Using the method of least squares, the above model was fitted to the data in Appendix V. The model fitting was performed using Mathcad version 11.1, and the Mathcad worksheet for the model fitting is found in the output DTN: MO0409MWDULCMW.000 and in Appendix IX.3. For the data with no nitrate ions, a very small value of $10^{-8} \mathrm{~m}$ was used for the nitrate ion concentration in the regression analysis.

The estimated regression coefficients and their uncertainty ( \pm 1 standard deviation) are: $c_{0}=558.283 \pm 36.156, c_{1}=0.677 \pm 0.413, c_{2}=-65.338 \pm 2.456, c_{3}=-7.616 \pm 1.581$, and $c_{4}=37.077 \pm 2.443$. The covariance matrix resulting from the fitting procedure was determined to be:

$$
C V=\left[\begin{array}{ccccc}
1.307 \times 10^{3} & -1.345 \times 10^{1} & -2.595 \times 10^{1} & 3.751 \times 10^{0} & -2.149 \times 10^{1} \\
-1.345 \times 10^{1} & 1.708 \times 10^{-1} & -8.595 \times 10^{-2} & -1.678 \times 10^{-1} & 2.599 \times 10^{-1} \\
-2.595 \times 10^{1} & -8.595 \times 10^{-2} & 6.033 \times 10^{0} & 7.349 \times 10^{-1} & 6.611 \times 10^{-1} \\
3.751 \times 10^{0} & -1.678 \times 10^{-1} & 7.349 \times 10^{-1} & 2.501 \times 10^{0} & 1.510 \times 10^{0} \\
-2.149 \times 10^{1} & 2.599 \times 10^{-1} & 6.611 \times 10^{-1} & 1.510 \times 10^{0} & 5.969 \times 10^{0}
\end{array}\right] \text { Eq. 6-37) }
$$

The correlation coefficient $R$ for the fit is 0.973 , and the coefficient of determination $R^{2}$ is 0.947 . An $R^{2}$ value of 0.947 indicates that the regression model fits the experimental data very well.

The entire variance of the model is due to the measurement uncertainty. The same logic as the crevice repassivation potential data (i.e., ASTM G 61-86 (1987 [DIRS 127897]) discussed in Section 6.4.4.3.1) was used to quantify the uncertainty of the long-term corrosion potential data. As with the crevice repassivation potential model, the uncertainty in the steady-state corrosion potential model is limited to the range between \pm 2 standard deviations of the mean-this includes 95 percent of all variation provided random variations are the only source of error. This uncertainty range is sufficient as the data are highly reproducible (Estill et al. 2003 [DIRS 163849]).

For the waste package degradation analysis in the repository, variability in the corrosion potential among waste packages is represented by the temporally and spatially varying waste package temperature and chemistry of water contacting the waste package. The purpose of this long-term corrosion potential model is to estimate the long-term steady-state open-circuit corrosion potential of Alloy 22 for a range of exposure conditions related to the repository. The model is not intended for short-term transient conditions.

The model results and the steady-state corrosion potential data are shown in Figure 6-51 and Figure 6-52 as a function of $\mathrm{pH}$ and nitrate concentration, respectively. The steady state $E_{\text {corr }}$ values are the same as those shown in Figure 6-48 through Figure 6-50. Note the model results 
are for the average values of the test environment parameters (temperature, chloride concentration and $\mathrm{pH}$ ) of the source data. The $E_{\text {corr }}$ data for the test conditions off the average values, therefore, are not bound within \pm 2 standard deviations of the model mean. The figures show that the steady-state corrosion potential is a strong function of both $\mathrm{pH}$ and nitrate concentration (at low nitrate concentrations).

Figure 6-51 shows a strong dependence of the steady-state corrosion potential on $\mathrm{pH}$. The model predicts that the corrosion potential changes at a rate of about $65 \mathrm{mV}$ per $\mathrm{pH}$ unit. This model behavior represents the effect of ennoblement in $E_{c o r r}$ of the alloy in acidic conditions as seen in the SAW solutions (Figure 6-46). Other investigators also reported such an ennoblement of Alloy 22 in acidic conditions (Dunn et al. 2003 [DIRS 164138], Figures 8 and 9; Jayaweera et al. 2003 [DIRS 162225], Section 9.3.2, Figure 9.13). The corrosion potential of Alloy 22 is expected to drop sharply for very low $\mathrm{pH}$ conditions because the passive film on the alloy would become unstable in such extreme acidic conditions and the alloy would become more active. The current model does not represent this.

As shown in Figure 6-52, for the mean chloride ion concentration of $3.96 \mathrm{~m}$, the corrosion potential increases as the nitrate ion concentration increases for low nitrate concentrations, but has little dependence on nitrate concentration for higher nitrate concentrations. To be consistent with the evaluation method for $E_{\text {rcrev }}$ in Section 6.4.4.3.2, if the exposure nitrate-to-chloride ion concentration ratio exceeds 0.5 , the value 0.5 is used in evaluating the model. Nitrate is an oxidizing anion and, therefore, promotes the passivation of Alloy 22, thus increasing the $E_{\text {corr }}$. The model prediction represents the experimental data well. It can be seen from the coefficient of the temperature term that the corrosion potential is a weak function of temperature and increases slightly with temperature. This may be due to passive film becoming more defect-free at higher temperatures (Lloyd et al. 2003 [DIRS 167921]) as the defect repair processes in the passive film could become accelerated as temperature increases. Other variables such as $\mathrm{pH}$ and nitrate concentration seem more important in controlling the $E_{\text {corr }}$ of Alloy 22 than the temperature. 


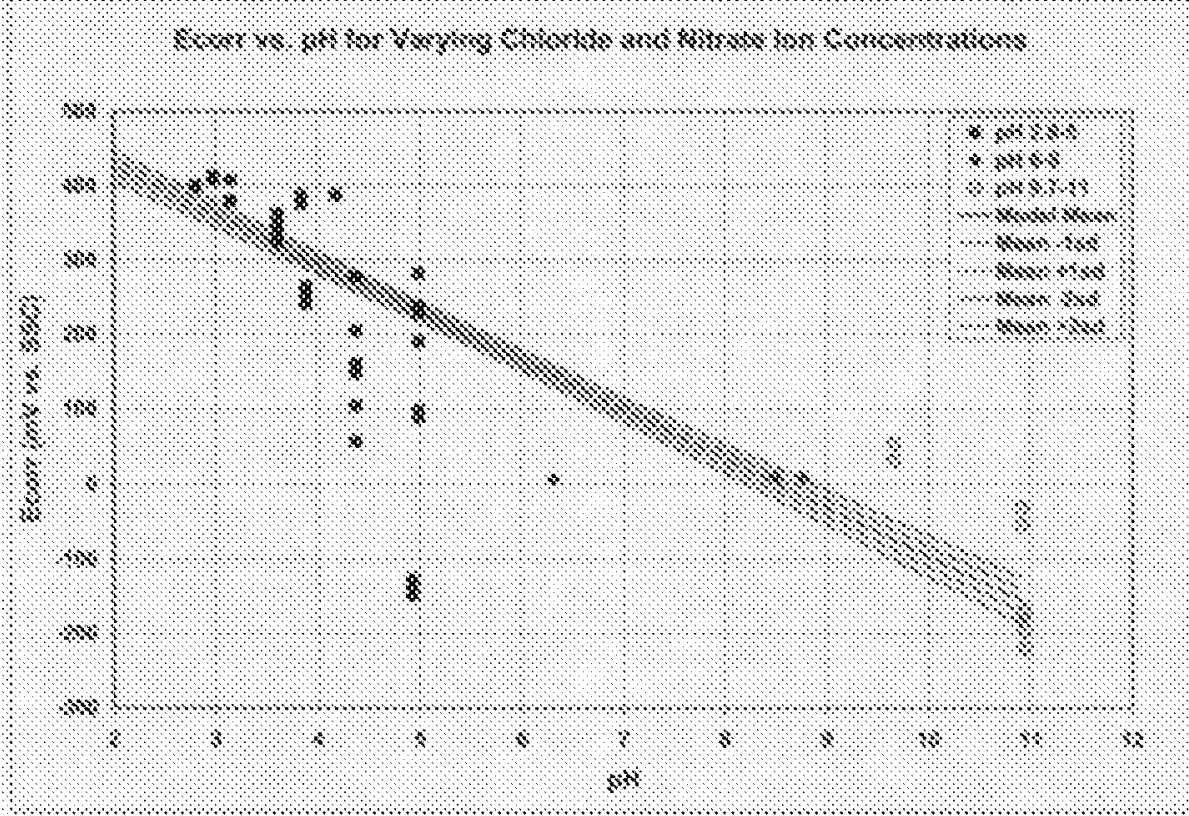

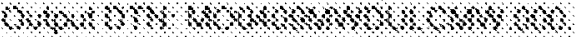

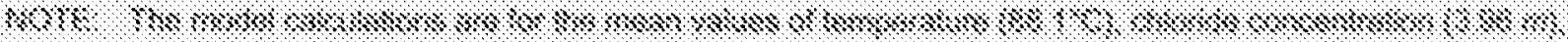

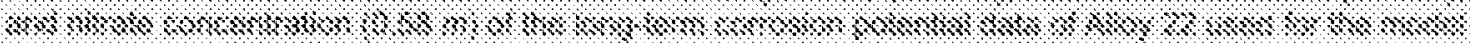
$\$, \$, 0$,

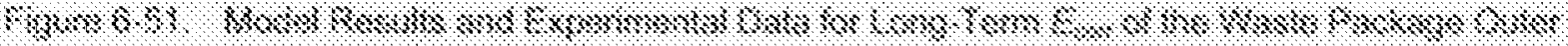
$\$, 18,4,8,8$

$\$, \$, \$, \ldots, \$, \$ n$

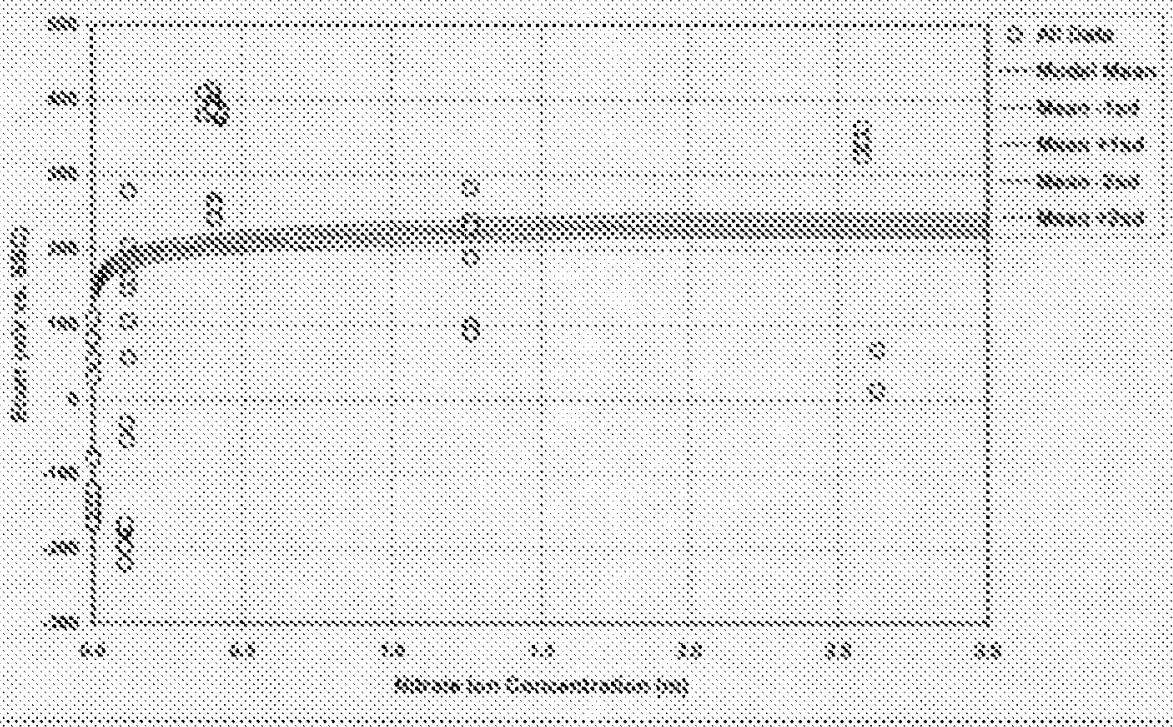

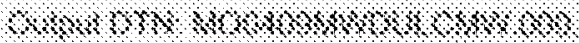

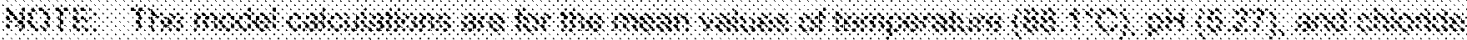

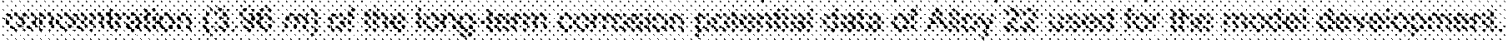

H

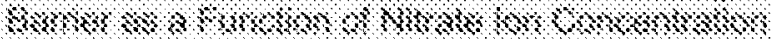




\subsubsection{Analysis of Localized Corrosion Initiation Model}

In the localized corrosion initiation model, localized corrosion of the WPOB occurs when the open-circuit corrosion potential $\left(E_{c o r r}\right)$ is equal to or greater than a critical potential (the crevice repassivation potential $\left(E_{\text {rcrev }}\right)$ in the current model), that is, $\Delta \mathrm{E}\left(=E_{\text {rcrev }}-E_{c o r r}\right) \leq 0$. This conceptual model of localized corrosion initiation is widely accepted by the corrosion community and has been published extensively (Böhni 2000 [DIRS 164137], Section B; Dunn et al. 2000 [DIRS 164495]; Dunn et al. 2003 [DIRS 164138]; Frankel 1998 [DIRS 162216]; Frankel 2002 [DIRS 164140]; Frankel and Kelly 2002 [DIRS 164141]; Beavers et al. 2002 [DIRS 158781], Section 8.3). For the current localized corrosion initiation model a conservative measure, the crevice repassivation potential $\left(E_{\text {rcrev }}\right)$, was used for the critical potential. For the same or similar metallurgical conditions (mill annealed or as-welded), the localized corrosion initiation model components (i.e., $E_{\text {corr }}$ and $E_{\text {rcrev }}$ ) could be affected by the exposure condition (temperature, $\mathrm{pH}$, chloride ion concentration, nitrate ion concentration).

This section documents the analysis of the localized corrosion initiation model for long-term localized corrosion susceptibility of the WPOB for a range of environmental conditions of interest. The analyses were performed by comparing the crevice repassivation potential model results with the long-term corrosion potential model results, as described above. The analyses were conducted with Mathcad version 11.1 (the Mathcad worksheet files are included in the output DTN: MO0409MWDULCMW.000). The localized corrosion initiation model is intended to be used to analyze the long-term localized corrosion susceptibility of the WPOB, and not for analysis of short-term transient behavior.

\subsection{Localized Corrosion Susceptibility Versus Temperature for 10 Molal Chloride and pH 7 Condition with Varying Nitrate Concentrations.}

Figure 6-53 shows the model results for the localized corrosion susceptibility of the WPOB for a neutral-pH concentrated chloride-containing brine $(10 \mathrm{~m}$ (moles/ $\mathrm{kg}$ water) chloride) with a very low nitrate ion concentration $\left(\mathrm{NO}_{3} / \mathrm{Cl}\right.$ ratio $\left.=0.05\right)$. The mean critical temperature for localized corrosion (crevice corrosion) for this condition is about $81{ }^{\circ} \mathrm{C}$. The upper bound of the critical temperature, obtained from the intersection of the +2 standard deviation confidence bound of the crevice repassivation potential and the -2 standard deviation confidence bound of the corrosion potential, is about $99^{\circ} \mathrm{C}$. The lower bound of the critical temperature, obtained from the intersection of the -2 standard deviation confidence bound of the crevice repassivation potential and the +2 standard deviation confidence bound of the corrosion potential, is about $51^{\circ} \mathrm{C}$.

As shown in Figure 6-54, with an increase of nitrate concentration to $1.5 \mathrm{~m}\left(\mathrm{NO}_{3} / \mathrm{Cl}\right.$ ratio $=0.15$ ), the mean critical temperature increases to about $112^{\circ} \mathrm{C}$, and the lower bound critical temperature is about $94^{\circ} \mathrm{C}$.

With a further increase of nitrate concentration to $2.5 m\left(\mathrm{NO}_{3} / \mathrm{Cl}\right.$ ratio $\left.=0.25\right)$, the model predicts that crevice corrosion is not possible below $120^{\circ} \mathrm{C}$ (Figure 6-55), which is above or at least close to the boiling temperature of a 10 molal chloride-containing solution. Therefore, it is concluded that for neutral $\mathrm{pH}$ chloride-containing brines, the WPOB will not undergo localized corrosion when the nitrate ion concentration is higher than $2.5 \mathrm{~m}$. 


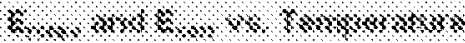

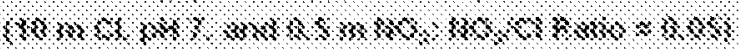

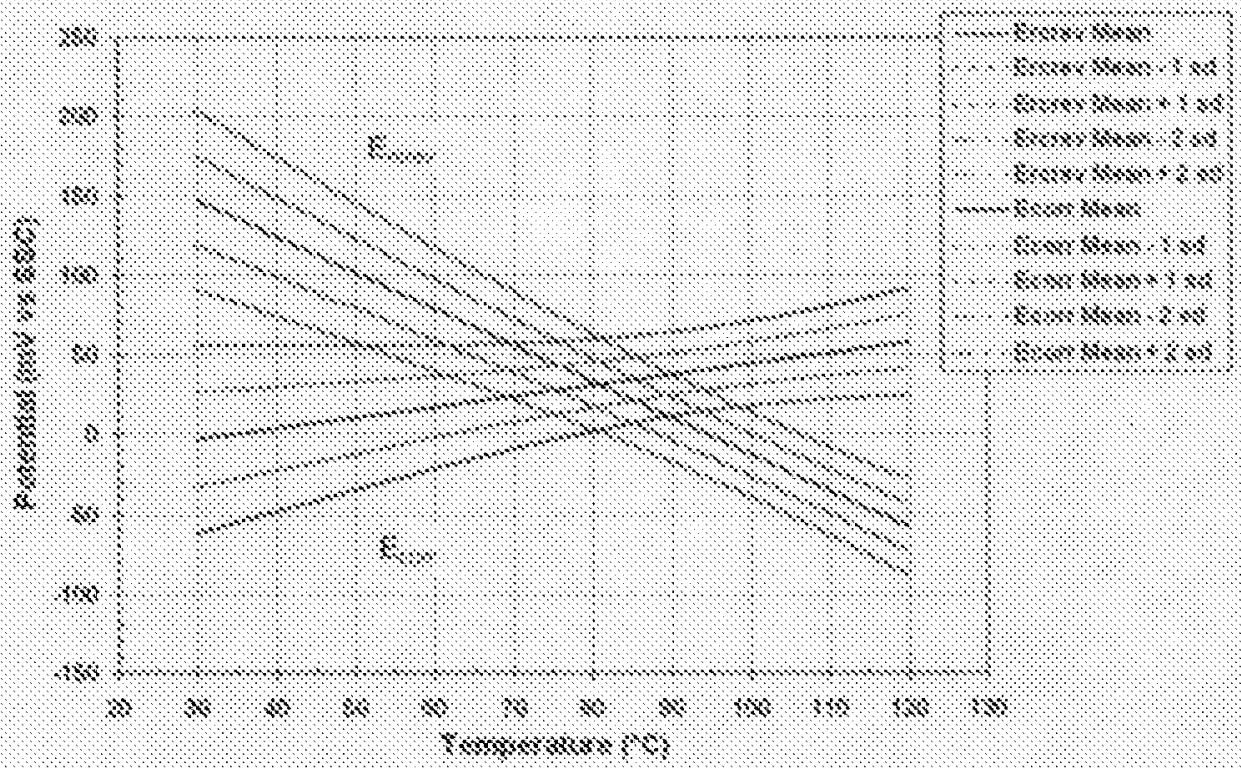

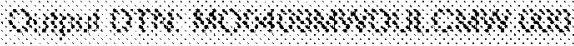

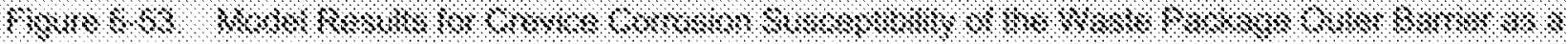

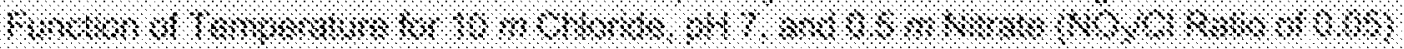

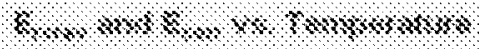

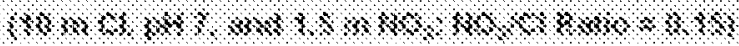

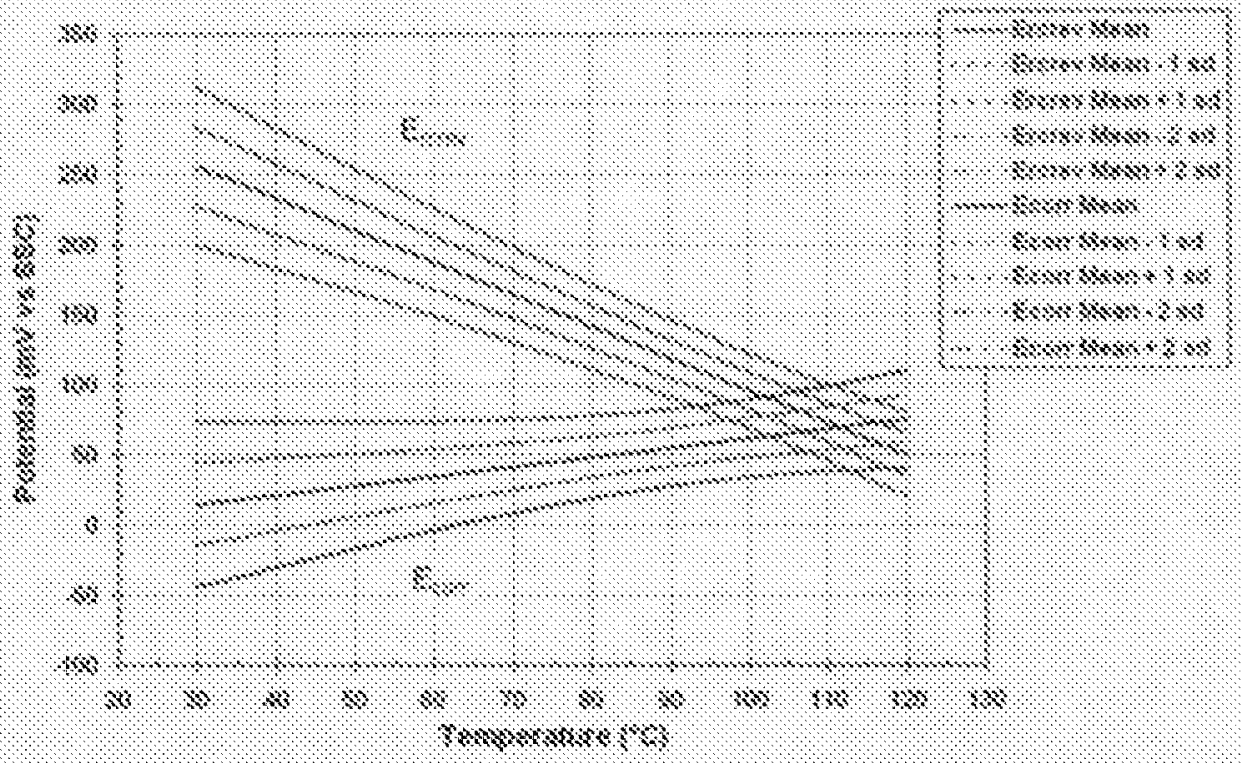

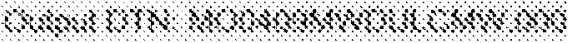

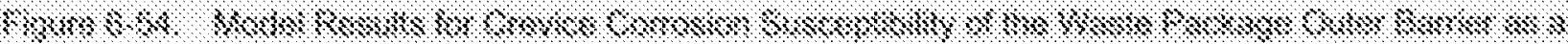

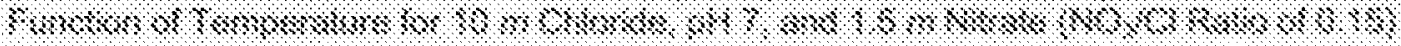




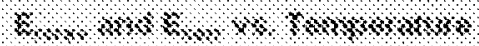

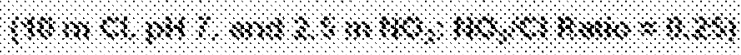

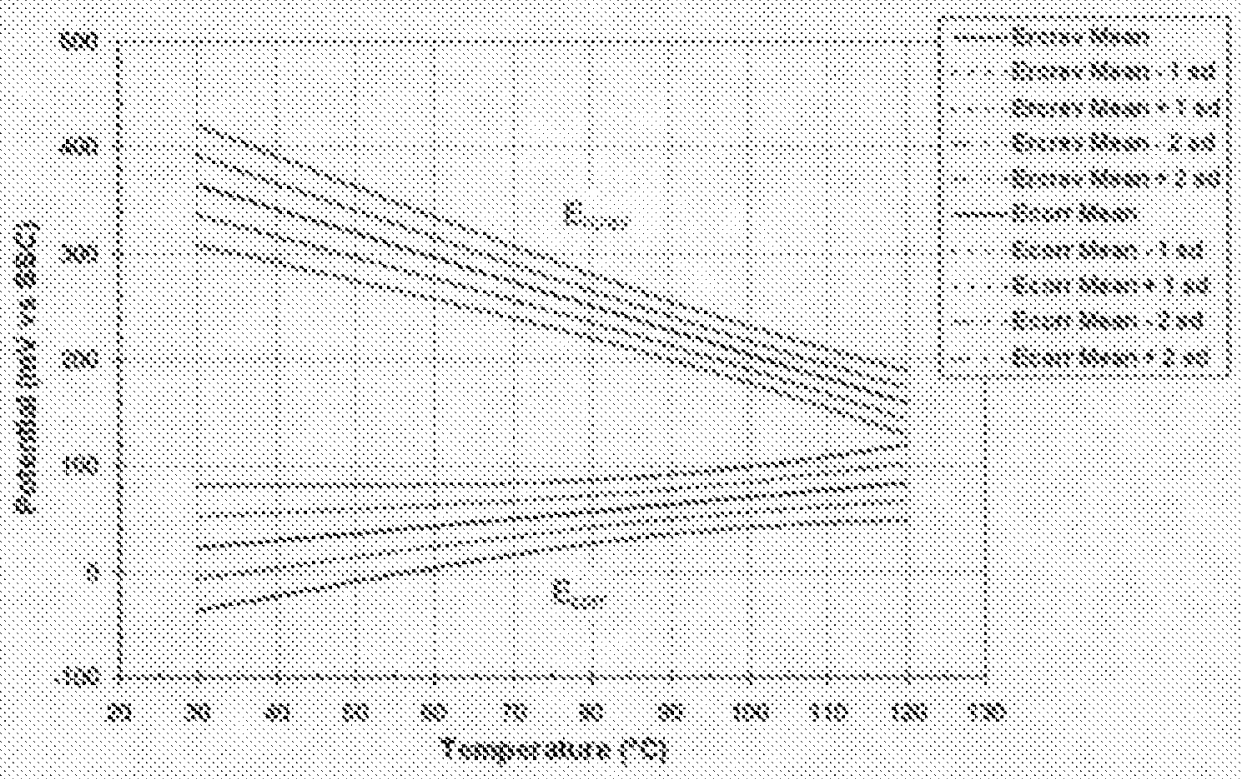

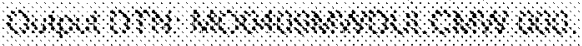

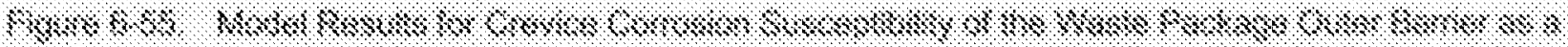

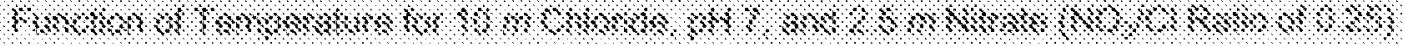

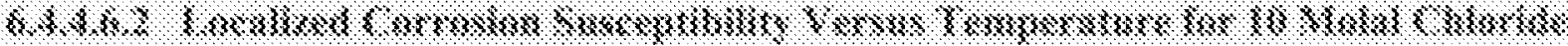

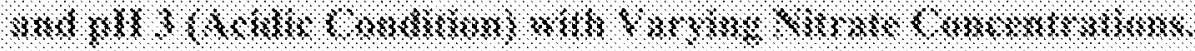

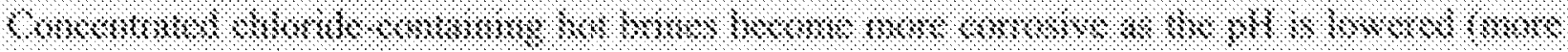

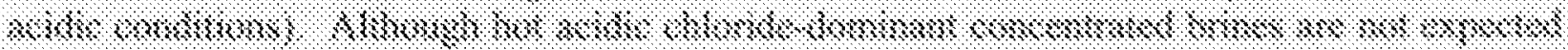

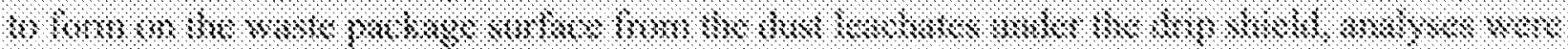

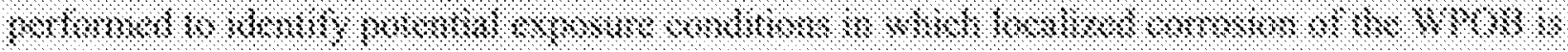
$18 \times 18$

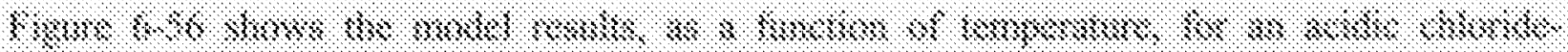

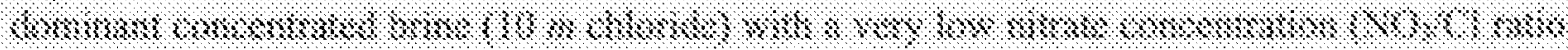

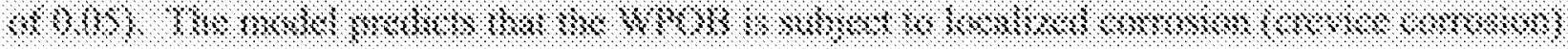

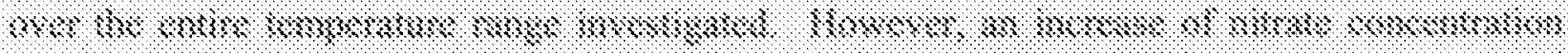

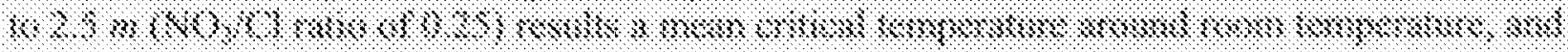

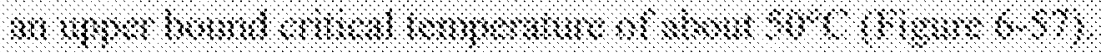

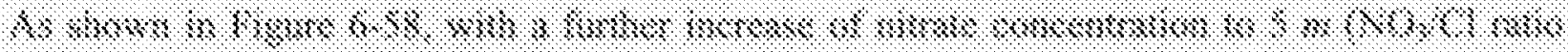

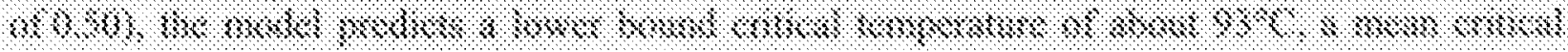
B

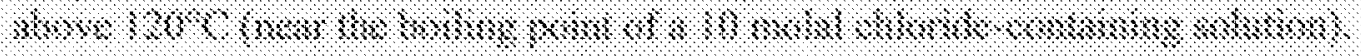


$2,0,0,0,0,0,0,0$

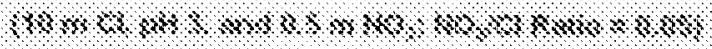

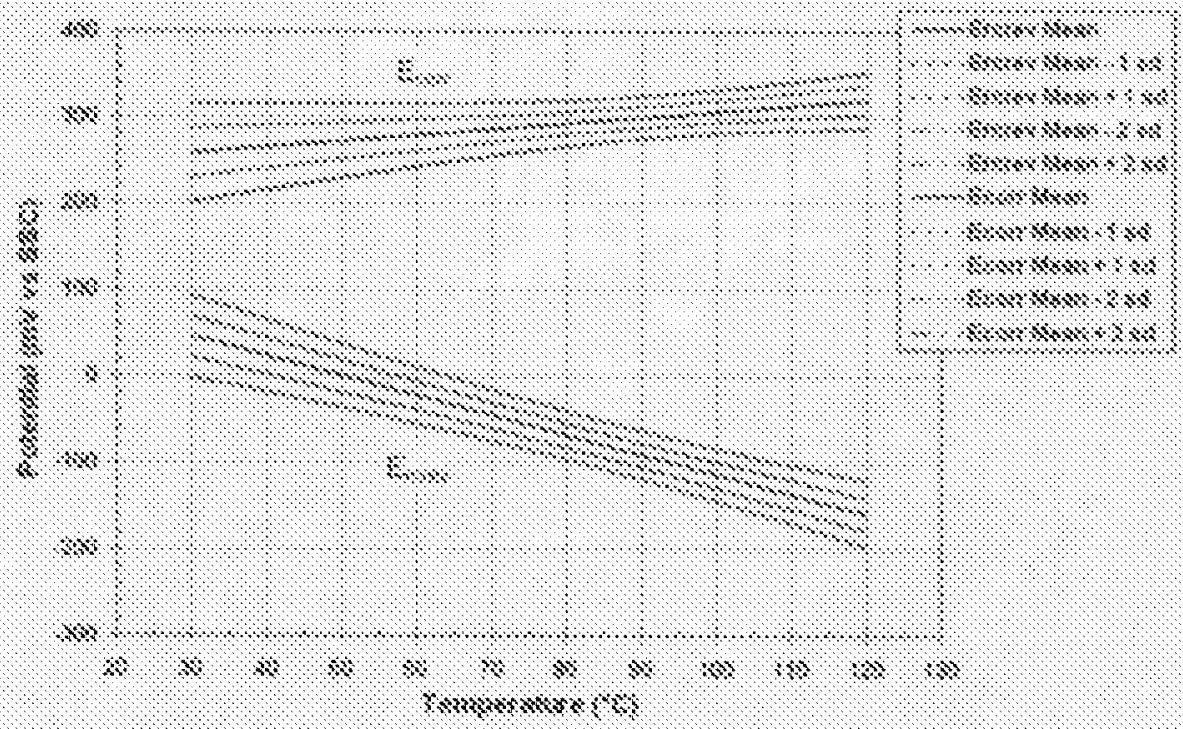

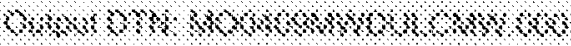

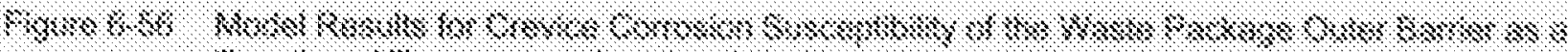

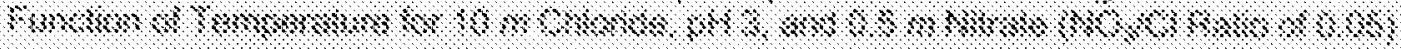

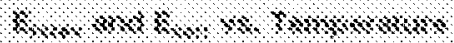

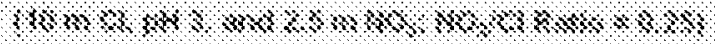

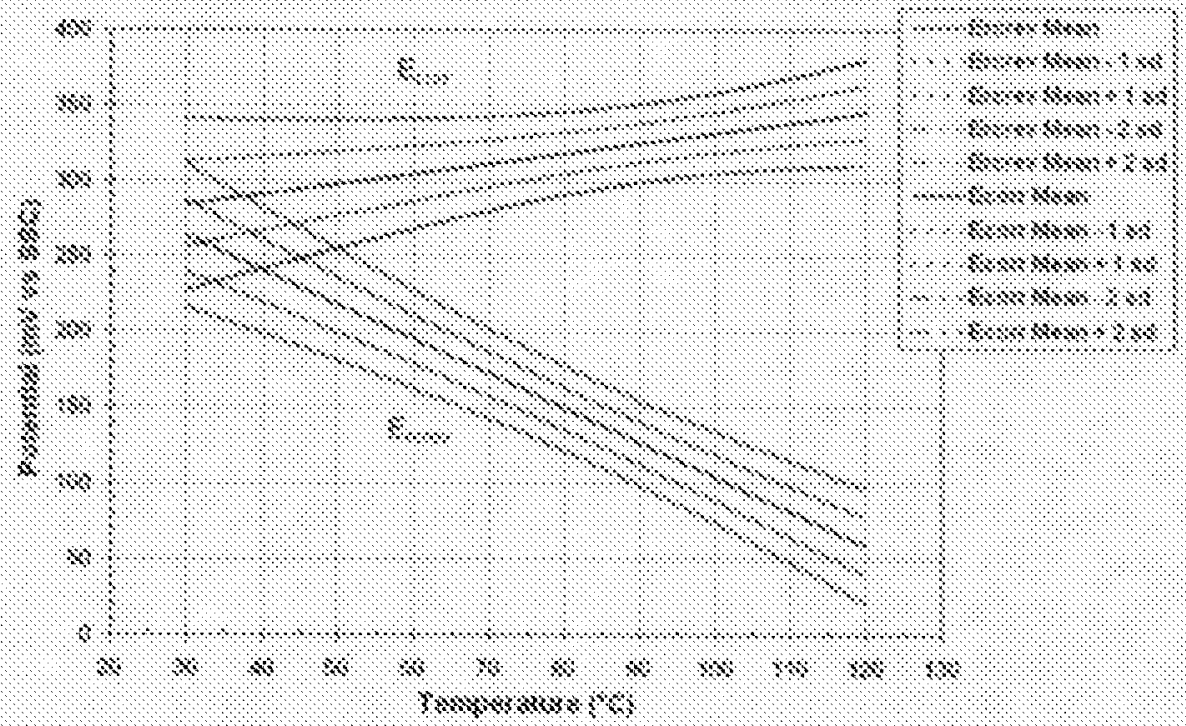

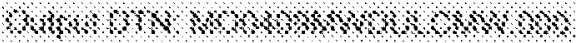

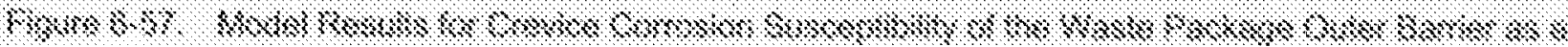

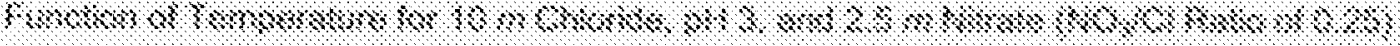


$0,1,1,8,8,8$

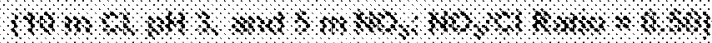

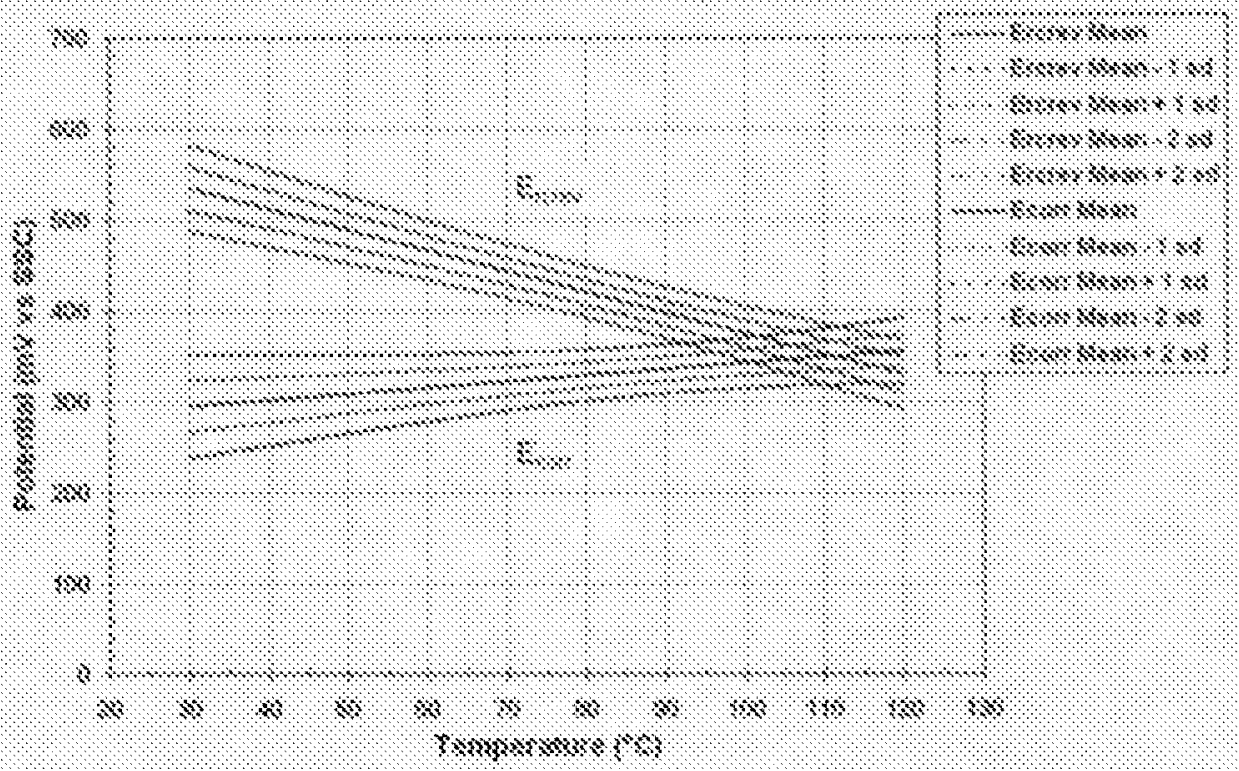

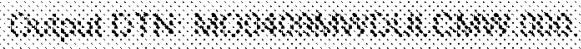

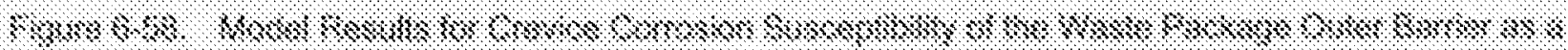

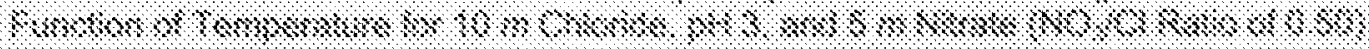

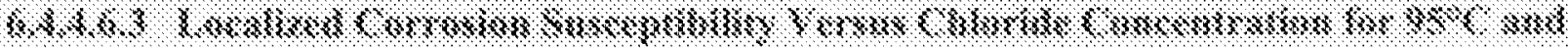

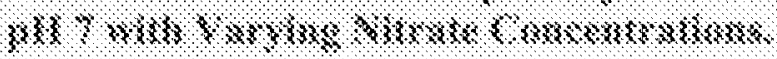

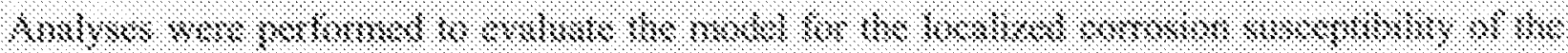

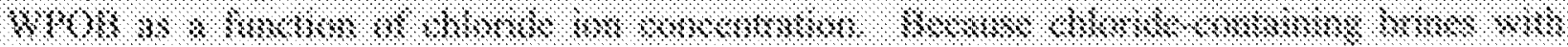

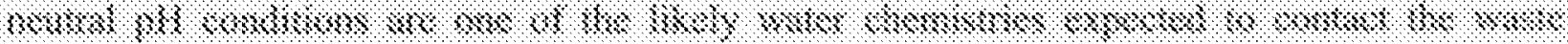

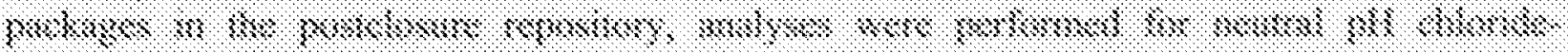

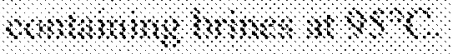

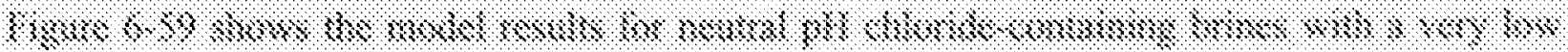

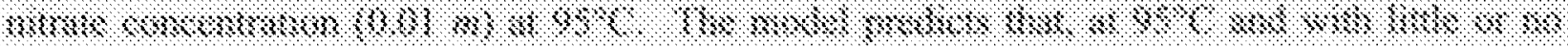

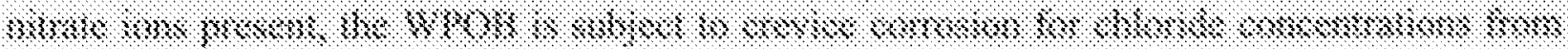

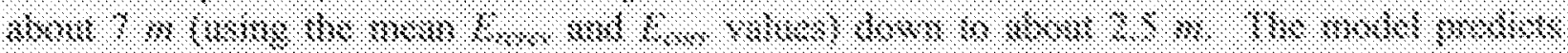

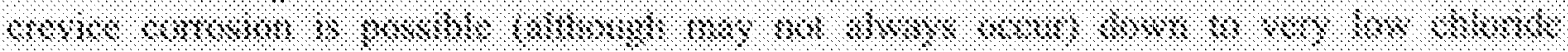

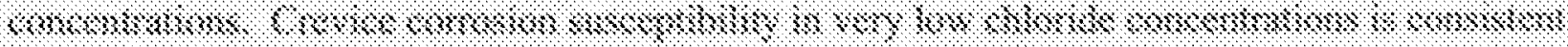

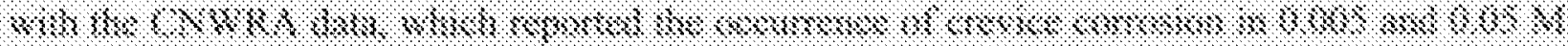

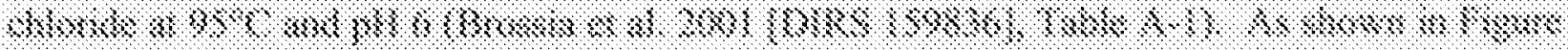

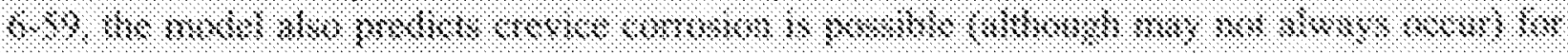

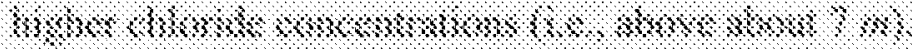

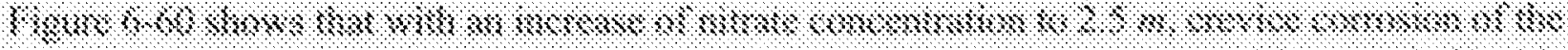

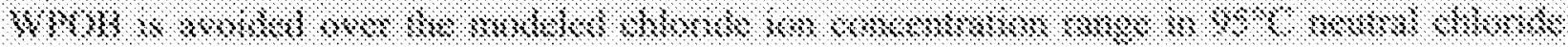

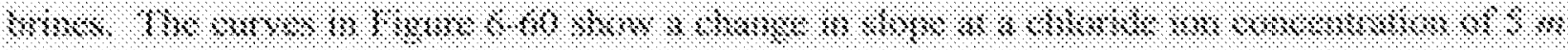

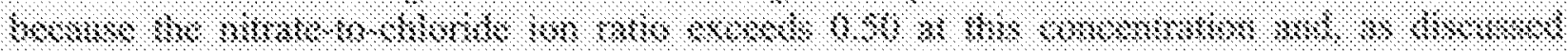




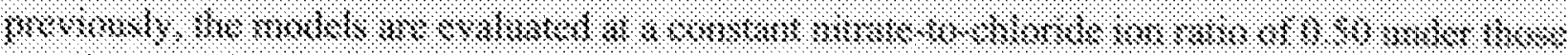

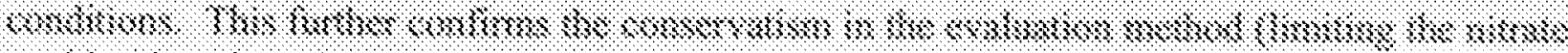

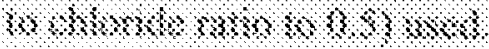

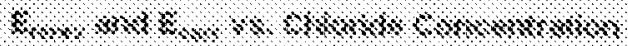

$3,1,0,18,1$,

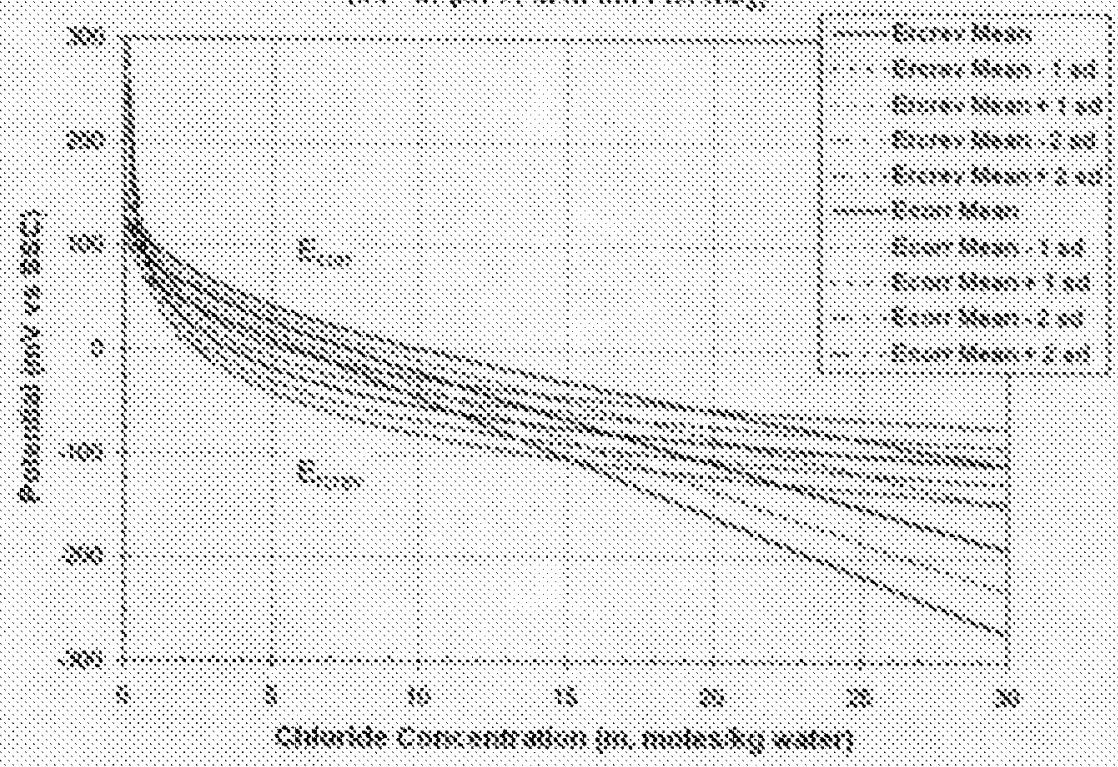

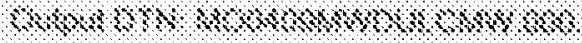

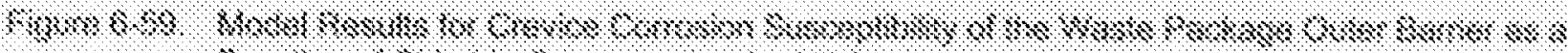

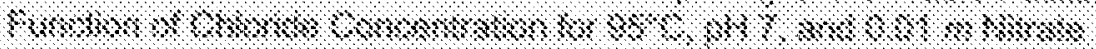




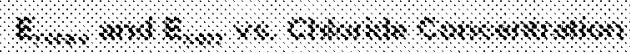

$3,1,13,8,8$

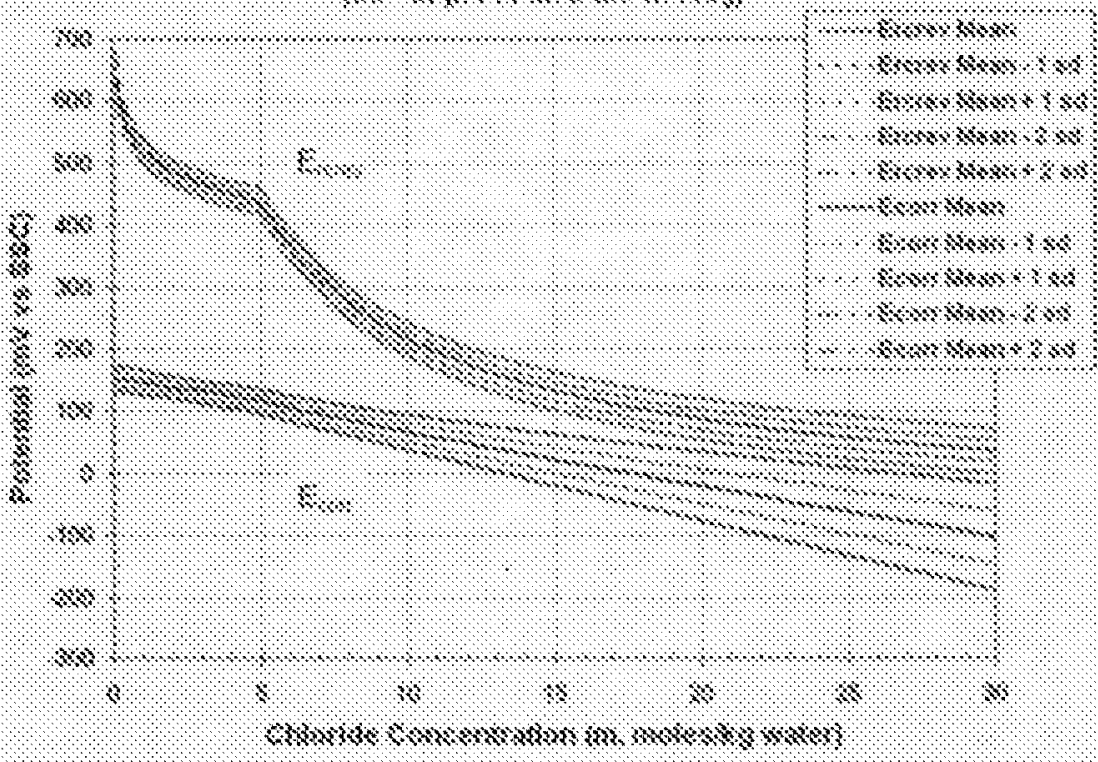

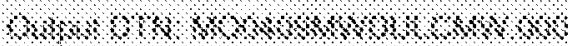

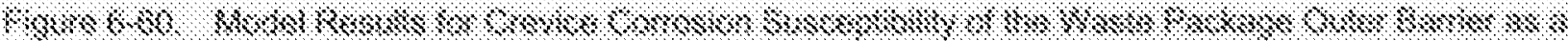

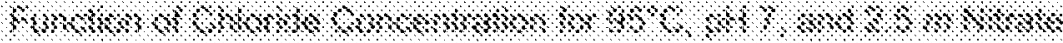

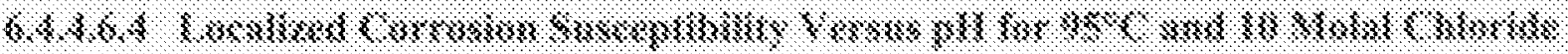

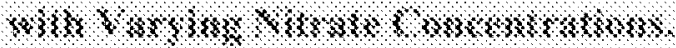

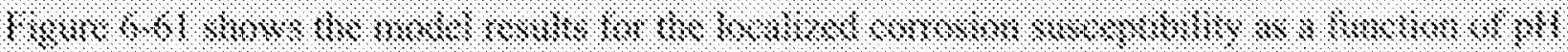

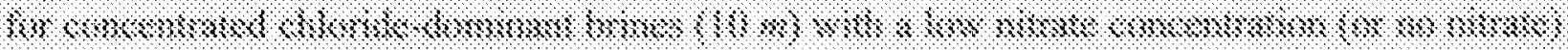

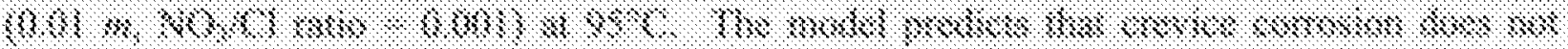

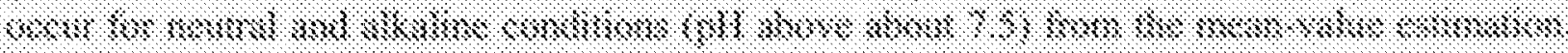

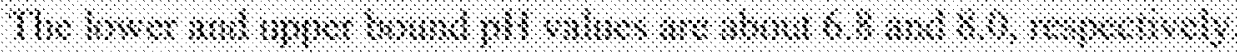

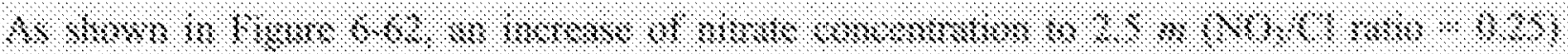

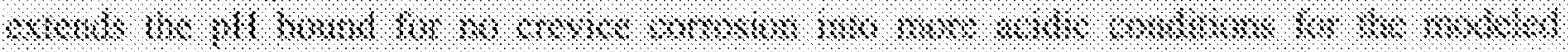

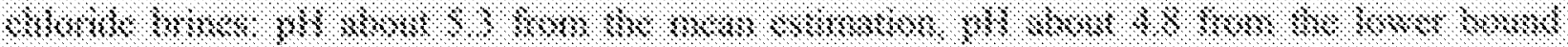

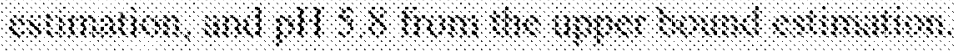




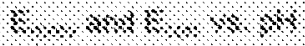

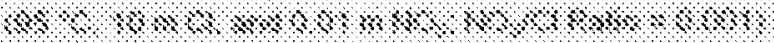

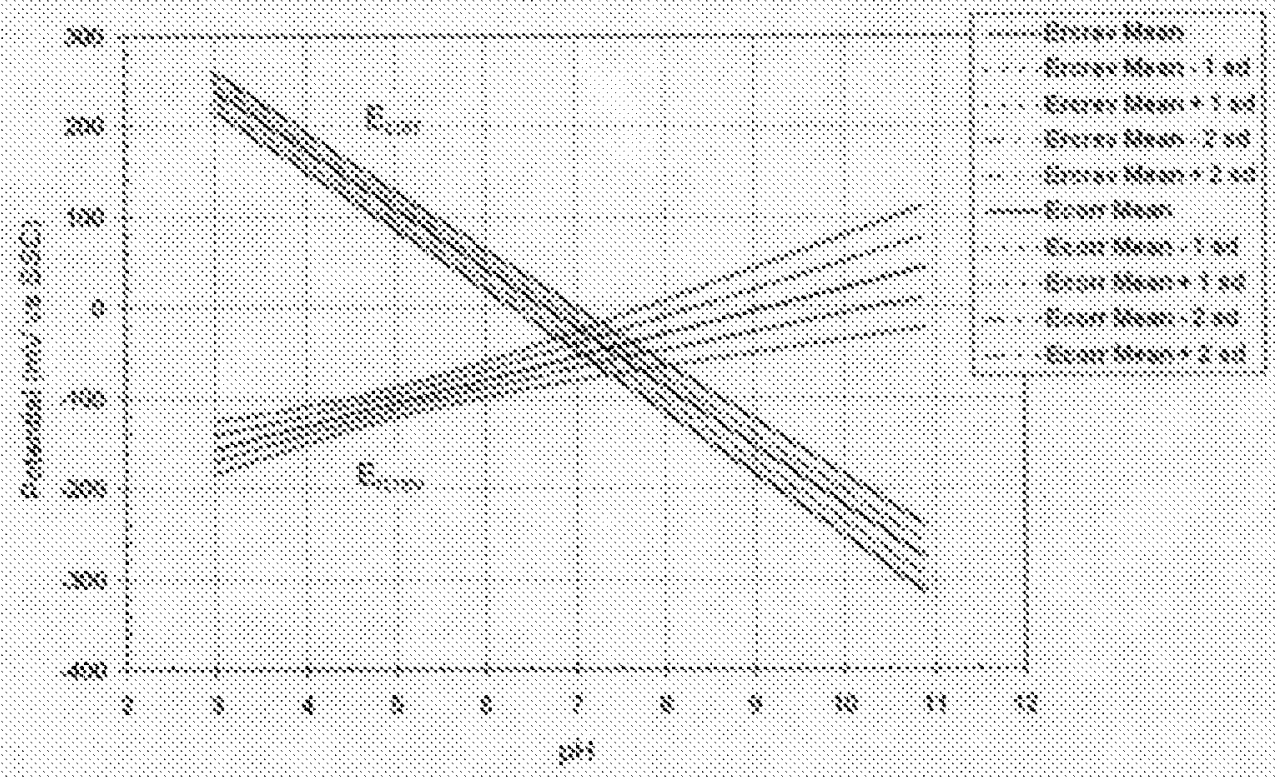

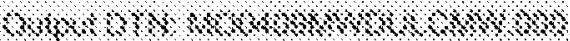

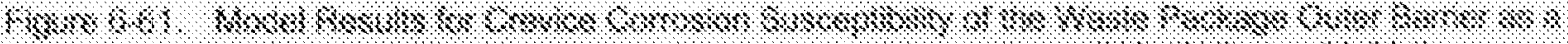

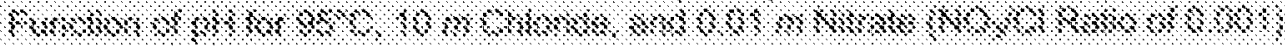

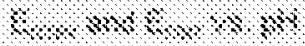

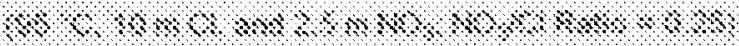

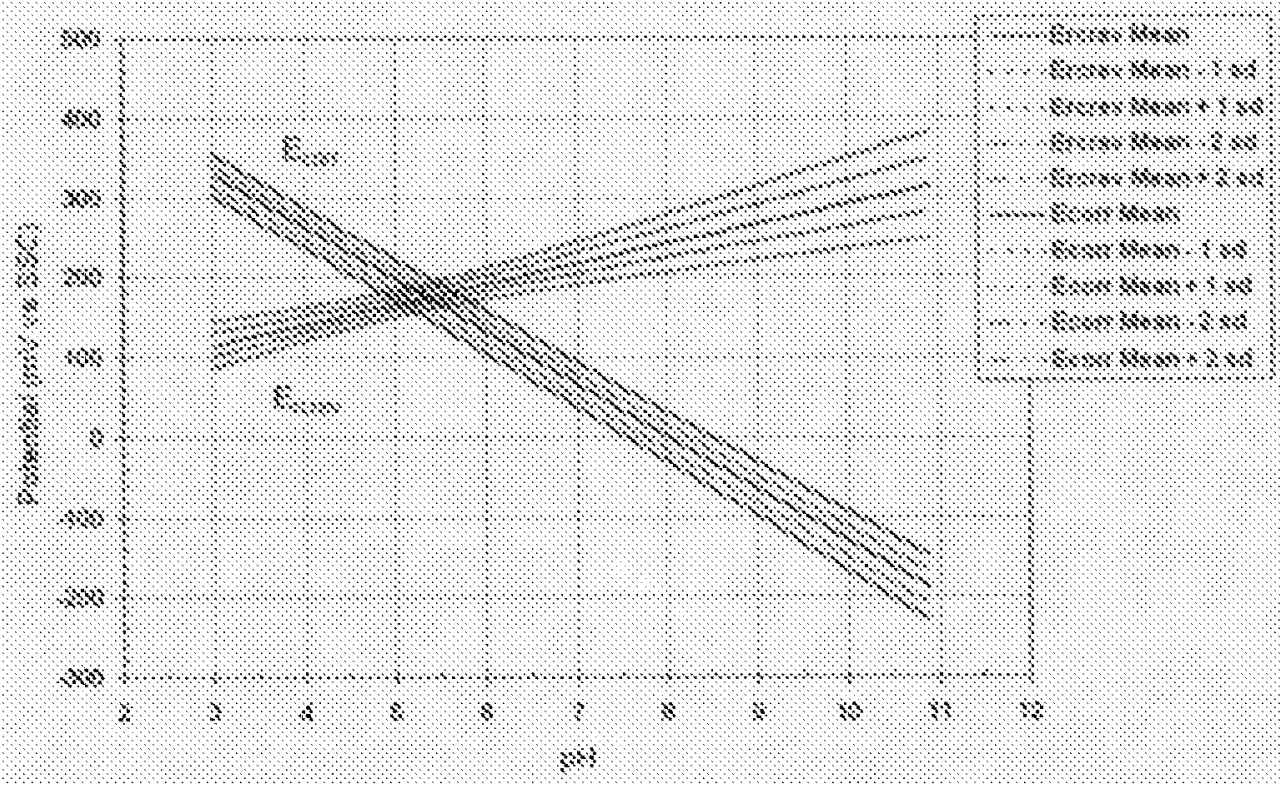

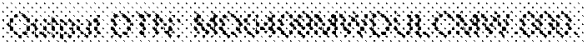

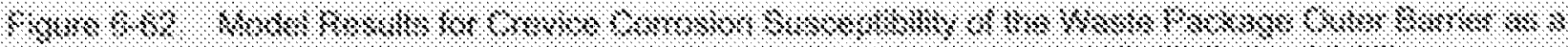

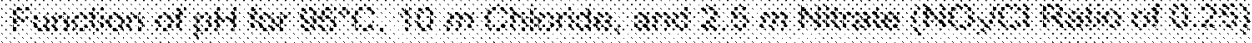




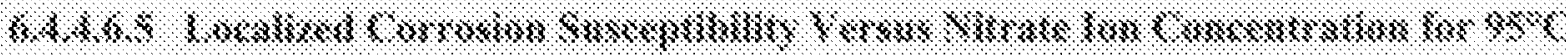

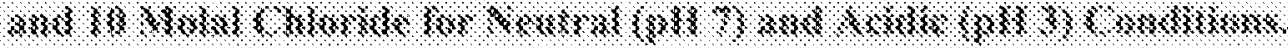

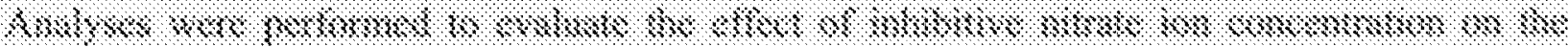

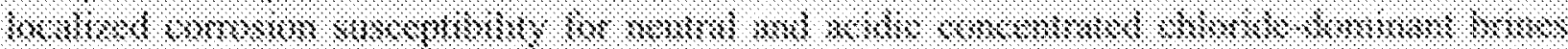

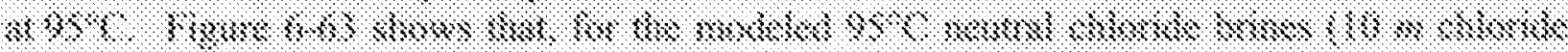

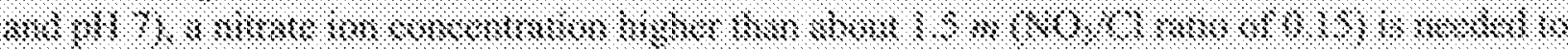

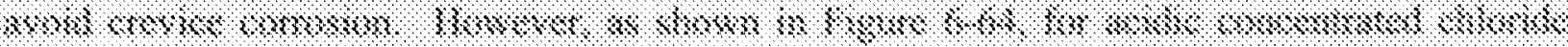

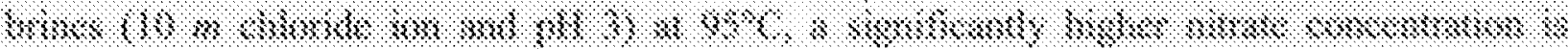

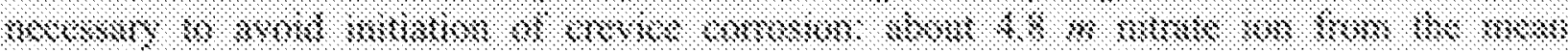

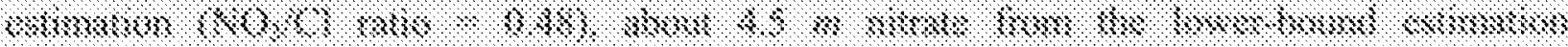

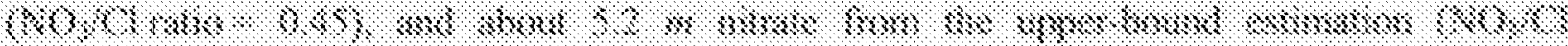
४४४४।

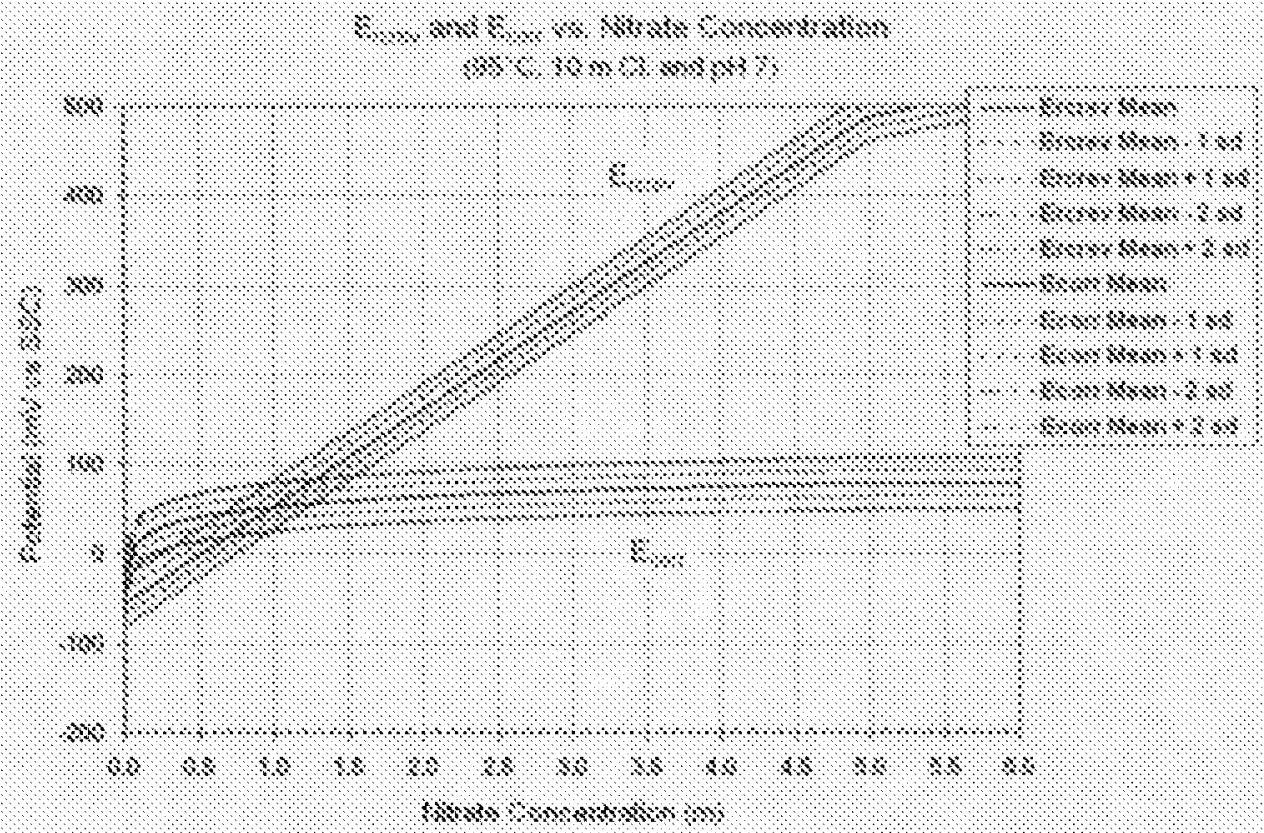

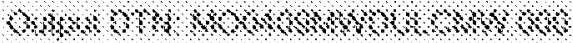

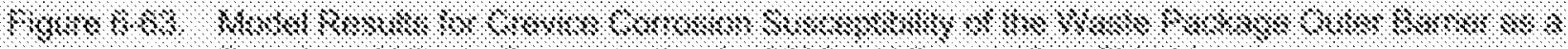

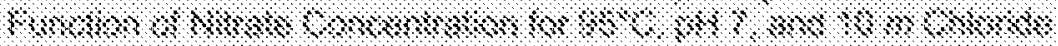




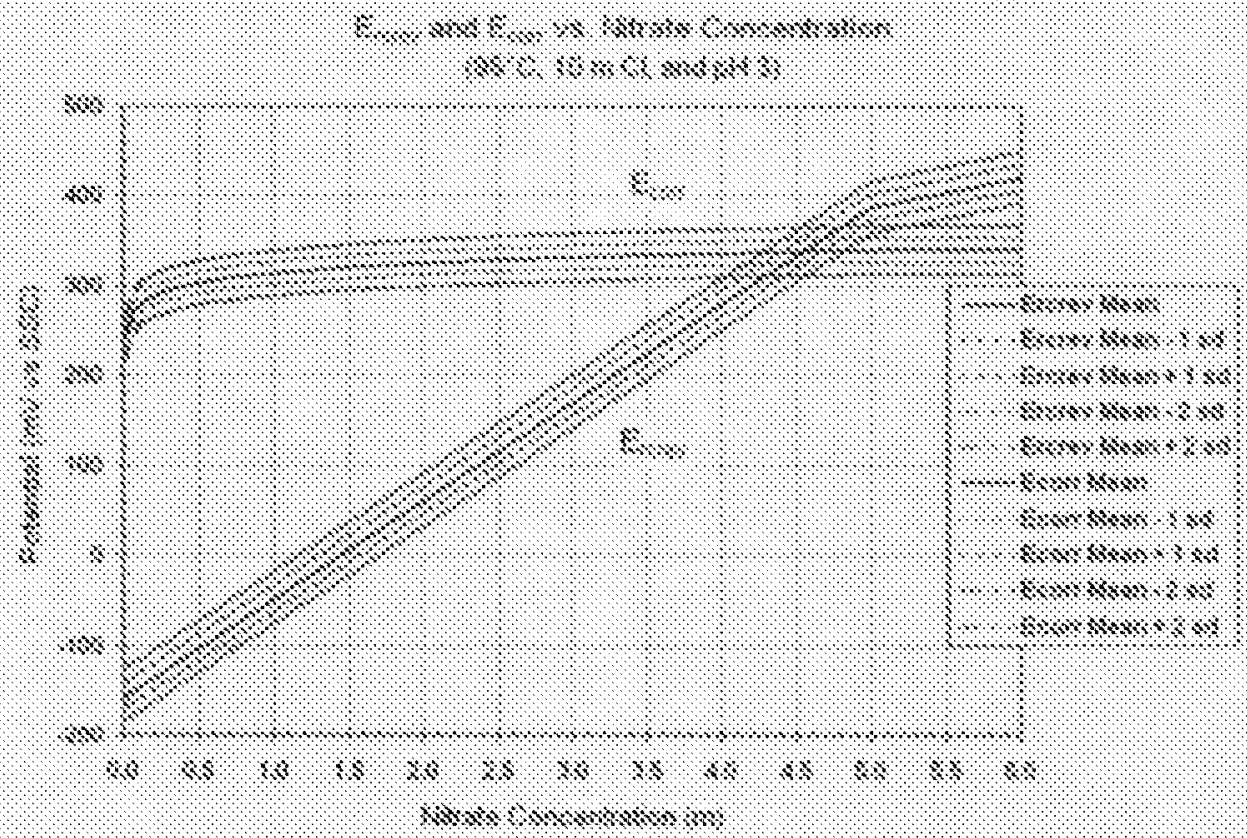

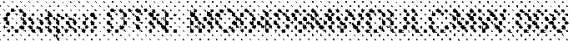

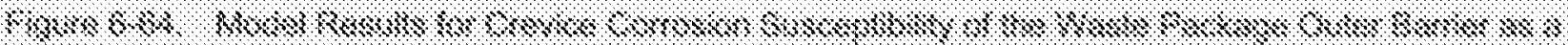

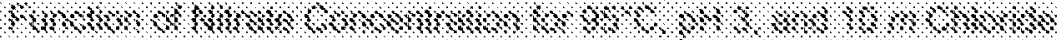

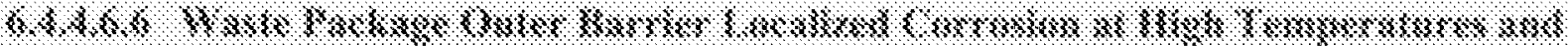

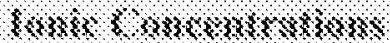

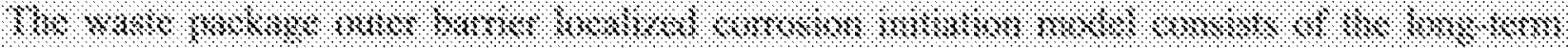

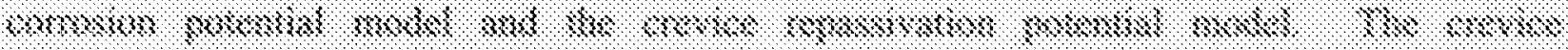

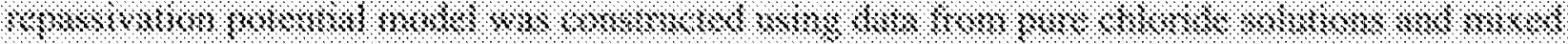

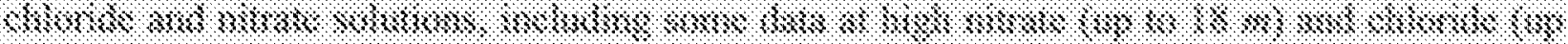

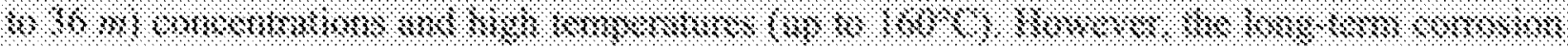

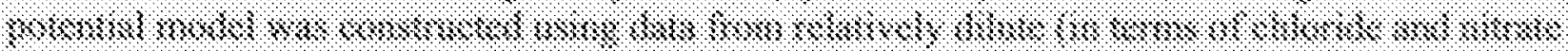

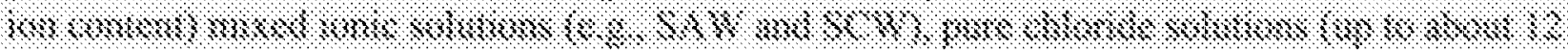

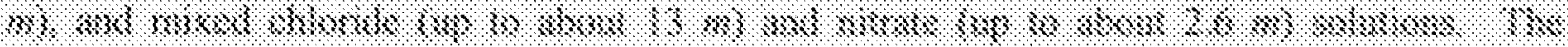

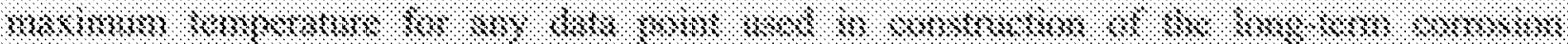

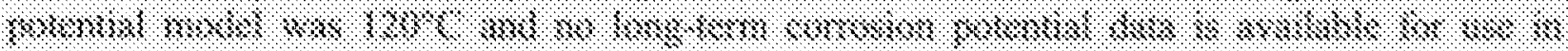

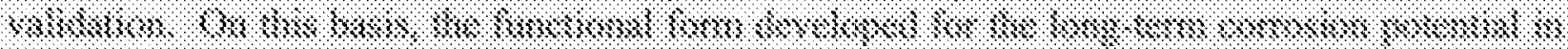

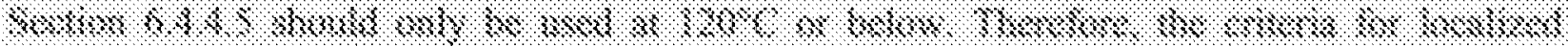

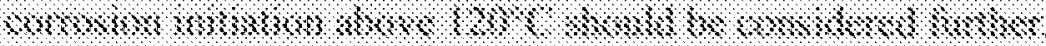

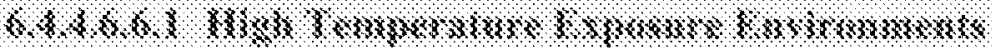

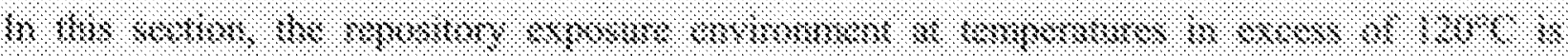

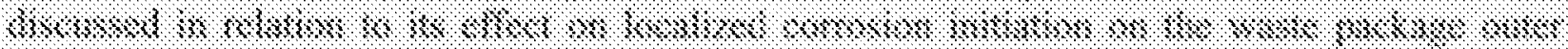

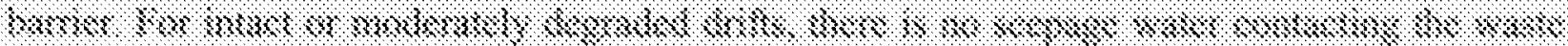

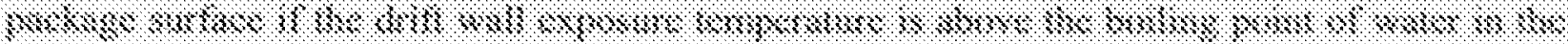

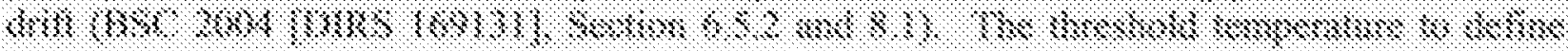


boiling is $100^{\circ} \mathrm{C}$ at the drift wall (BSC 2004 [DIRS 169131], Section 6.5.2). The waste package surface temperature is $120^{\circ} \mathrm{C}$ or below when the drift wall exposure temperature is $100^{\circ} \mathrm{C}$ or below (e.g., BSC 2004 [DIRS 169565], Figures 6.3-66 and 6.3-68). For collapsed drifts during the period of above boiling rock temperatures, ambient rock water boils off in the rubble material and in-drift flux perturbations give rise to moderate reflux of condensate in the upper half of collapsed drifts (BSC 2004 [DIRS 169131], Section 6.5.3, and 8.1). However, water drainage down to the waste packages is not possible, a result of the vaporization barrier forming near the waste package (BSC 2004 [DIRS 169131], Section 6.5.3). The vaporization and reflux processes cease after a few hundred to more than thousand years and the TH conditions slowly approach steady-state (ambient) behavior (BSC 2004 [DIRS 169131], Section 6.5.3). During this transition phase, the fluxes in the lower half of the collapsed drift remain zero at all times (BSC 2004 [DIRS 169131], Section 6.5.3). Therefore, the relevant chemistries to consider for waste package surface temperatures above $120^{\circ} \mathrm{C}$ are those which result from the interaction of water condensation with dusts films which may be present on the waste package surface (dust leachates).

For water to be present on the waste package surface at temperatures above $120^{\circ} \mathrm{C}$, the water must be a concentrated solution such that the vapor pressure of the solution and the relative humidity (RH) of the water is reduced below that of pure water. Figure 6-65 (BSC 2004 [DIRS 161237], Figure 3) shows the maximum RH (equivalent to the activity) for water to be present at a given exposure temperature. The figure shows, for example, that at $120^{\circ} \mathrm{C}$, the $\mathrm{RH}$ must be 45 percent or less for water to be present on the waste package surface with lower RH required at higher temperatures. For water to exist at such a low relative humidities, the water must be in a concentrated solution with other ions. Dust leachates at temperatures above $120^{\circ} \mathrm{C}$ (below 45 percent $\mathrm{RH}$ ) have high nitrate ion concentrations (on the order of $10 \mathrm{~m}$ nitrate) (BSC 2004 [DIRS 169860], Figure 6.13-39) and low chloride to nitrate ion ratios (BSC 2004 [DIRS 169860], Figure 6.13-38) (i.e., high nitrate-to-chloride ion ratios). Given the inhibiting effect of nitrate ions and the nitrate-to-chloride ion ratio on localized corrosion initiation (Section 6.4.4.3.2), localized corrosion initiation would not be highly probable in these exposure environments.

Another consideration relevant to the exposure environment at higher temperatures is acid gas volatility. Pulvirenti et al. (2004 [DIRS 169631]) have reported generating acid gas volatiles including $\mathrm{HCl}$ and $\mathrm{HNO}_{3}$ by concentrating (through boiling) various repository relevant solutions. These researchers trapped and condensed the volatiles and measured $\mathrm{pH}$ values less than 1. If these volatiles disperse to the drift wall and either diffuse or advect into it or be neutralized by reaction with the surrounding rock, as is likely in the open repository system, then acid gas volatility will limit the possibility of development of low $\mathrm{pH}$ (acidic conditions) on the waste package surface. Thermogravimetric experiments were conducted in which an Alloy 22 sample surface was coated with an aerosol film of calcium chloride (DTN: LL030308812251.017 [DIRS 163775], LL020903812251.019 [DIRS 161135]). 


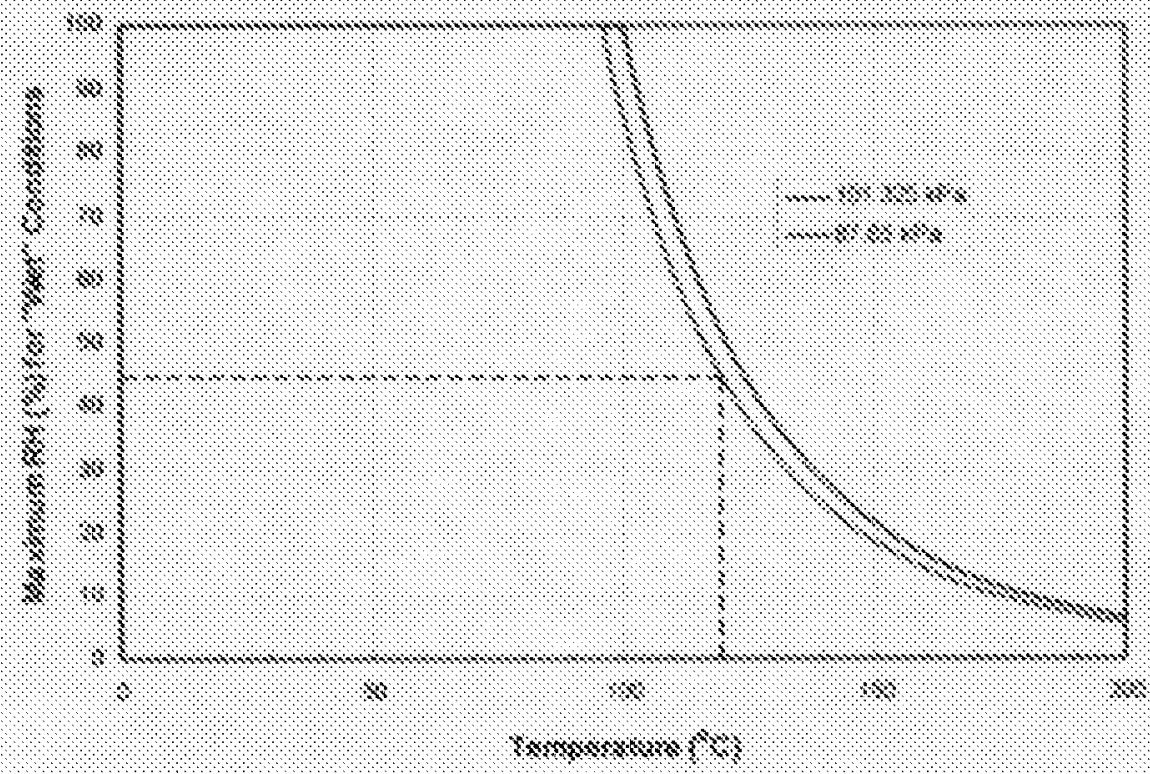

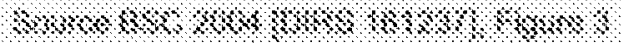

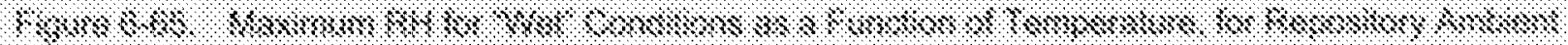

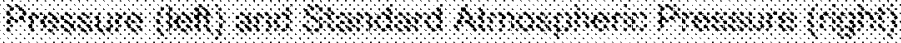

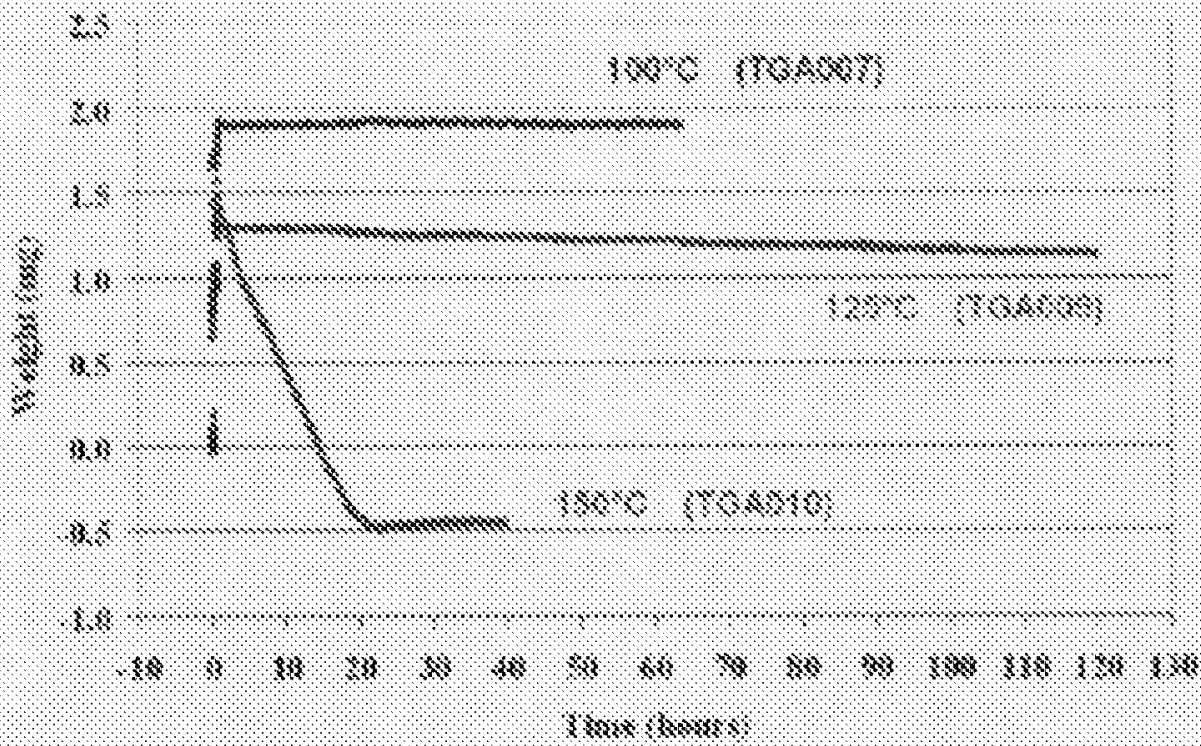

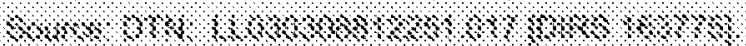

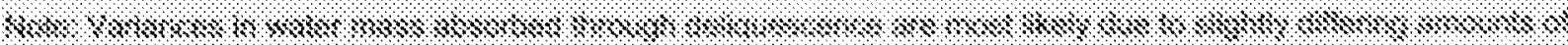

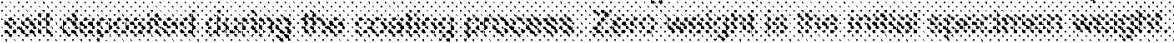

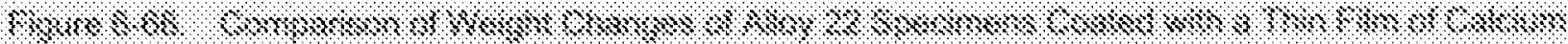

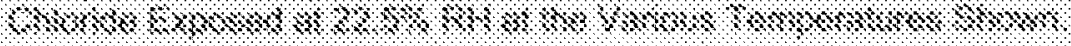

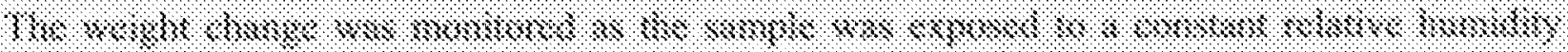
४४ ४

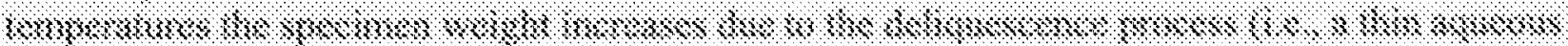

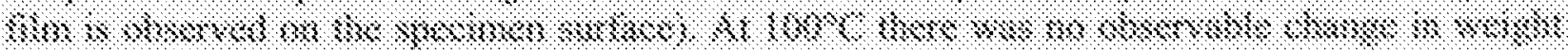


following the initial increase, and the specimen surface remained wet and visually unchanged throughout the test. At $125^{\circ} \mathrm{C}$ a steady weight loss was observed for more than 600 hours. During this time, the specimen surface was observed to slowly become dry and precipitate formation was observed. At $150^{\circ} \mathrm{C}$ a sharp decline in weight was observed following initial deliquescence, and reaction completion was reached in $\sim 24$ hours. Subsequent analyses indicated that the preciptates formed did not contain metal ions, suggesting the weight changes observed were not due to electrochemical reactions and no evidence of localized attack was observed. The precipitation reaction was coupled with the loss of chloride as $\mathrm{HCl}$ gas (DTN: LL020903812251.019 [DIRS 161135], Readme.pdf). Thus, experimental evidence suggestes that acid gas volatility limits the possible formation of low $\mathrm{pH}$ (acidic conditions) from evolution of calcium chloride-based brines at $125^{\circ} \mathrm{C}$ and $150^{\circ} \mathrm{C}$, but may not at $100^{\circ} \mathrm{C}$.

\subsection{Corrosion Data Relevant to High Temperature Exposure Environments}

The previous section discussed the repository exposure environment at temperatures in excess of $120^{\circ} \mathrm{C}$. This section discusses relevant data collected under these types of exposure conditions. Table 6-8 shows the high nitrate and chloride concentration data used in construction of the crevice repassivation model in the first 20 rows. The table also contains nine rows of data not used in construction of the $E_{\text {rcrev }}$ model, which has nitrate-to-chloride ion ratios above 0.50 . The table shows the electrolyte composition (including chloride and nitrate ion molal concentrations), nitrate-to-chloride ion ratio, $\mathrm{pH}$, temperature, the measured crevice repassivation potential, and the crevice repassivation potential model results at their minimum (-2 standard deviation (sd)) and maximum $(+2 \mathrm{sd})$ values. The measured crevice repassivation potentials agree well with the model results although the crevice repassivation potential model results are generally lower than the measured values (i.e., the model is generally conservative). One exception to this observation is of note. The two measured crevice repassivation potentials in the $10 \mathrm{~m} \mathrm{CaCl}_{2}+$ $1.5 \mathrm{~m} \mathrm{Ca}\left(\mathrm{NO}_{3}\right)_{2}$ solution at $100^{\circ} \mathrm{C}$ are reported to be 13 and $18 \mathrm{mV} \mathrm{SSC}$ and the model predicts that the crevice repassivation to be between 50 and $94 \mathrm{mV} \mathrm{SSC}$. The small difference between the model results and measured values is negligible for the purposes of modeling.

It can also be seen from Table 6-8 that when the nitrate-to-chloride ion ratio exceeds 0.50 , the crevice repassivation potential is generally not reported (two crevice repassivation potential measurements of 740 and $781 \mathrm{mV}$ SSC shown in the table correspond to cross over of the reverse scan at potentials above the passive region). As discussed in Section 6.4.4.1, it is reasonable to use a value of $+600 \mathrm{mV} \mathrm{SSC}$ when the crevice repassivation potential is not reported in tests such as these. The highest long-term corrosion potentials measured in the data used to construct the long-term corrosion potential model were about $+415 \mathrm{mV} \mathrm{SSC} \mathrm{(measured}$ in acidic SAW solutions with nitrate-to-chloride ion ratios of about 0.5 ). As can be seen from Figure 6-51, the effect of increasing $\mathrm{pH}$ is to decrease the measured long-term corrosion potential. Therefore, it is reasonable to expect that the long-term corrosion potential will never exceed about $+415 \mathrm{mV} \mathrm{SSC}$ in the repository environment. From the data in Table 6-8, when the nitrate-to-chloride ion ratio is 0.50 or above at high temperatures $\left(>120^{\circ} \mathrm{C}\right)$, and thus at high chloride and/or nitrate ion concentrations, the crevice repassivation potential is high enough to preclude localized corrosion initiation. 
General Corrosion and Localized Corrosion of Waste Package Outer Barrier

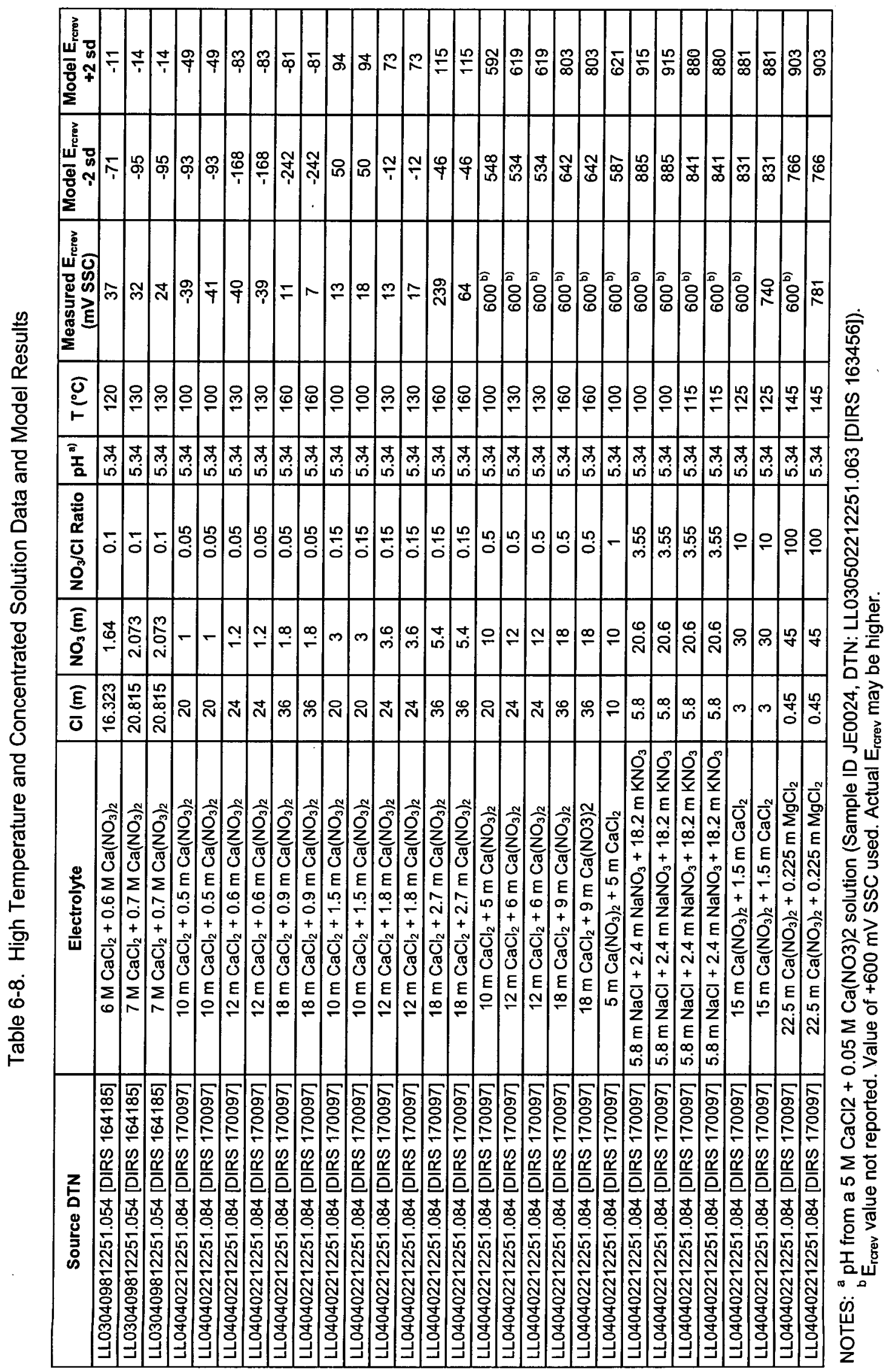




\subsection{Implementation of the Waste Package Outer Barrier Localized Corrosion Initiation Model}

As discussed in the previous sections, the crevice repassivation potential model was constructed using data from pure chloride solutions and mixed chloride and nitrate solutions, including some data at high nitrate (up to $18 \mathrm{~m}$ ) and chloride (up to $36 \mathrm{~m}$ ) concentrations and high temperatures (up to $160^{\circ} \mathrm{C}$ ). However, the long-term corrosion potential model was constructed using data from relatively dilute (in terms of chloride and nitrate ion content) mixed ionic solutions (e.g., SAW and SCW), pure chloride solutions (up to about $12 \mathrm{~m}$ ), and mixed chloride (up to about $13 \mathrm{~m}$ ) and nitrate (up to about $2.6 \mathrm{~m}$ ) solutions. The maximum temperature for any data point used in construction of the long-term corrosion potential model was $120^{\circ} \mathrm{C}$. The lack of measured long-term corrosion potential data in the high temperature $\left(>120^{\circ} \mathrm{C}\right)$ regime indicates it would be prudent not to make use of the developed functional forms to predict localized corrosion initiation under these conditions. Like general corrosion, localized corrosion requires the presence of a liquid water film on the waste package surface. To implement the waste package outer barrier localized corrosion initiation model, the following criteria are applied in a stepwise fashion:

1. If aqueous brine chemistry causes the initiation of localized corrosion, then localized corrosion continues to propagate regardless of changes in the bulk chemical exposure environment. This is a conservative modeling assumption made because no detailed model of the chemistry evolution of the crevice solution is available at this time.

2. If the exposure temperature exceeds $160^{\circ} \mathrm{C}$ and a water film is present on the waste package surface, then localized corrosion initiates.

Localized corrosion initiated as a result of this criterion is reevaluated in accordance with Criterion 3 (below) when the exposure temperature drops below $160^{\circ} \mathrm{C}$.

3. If the exposure temperature exceeds $120^{\circ} \mathrm{C}$ but is less than or equal to $160^{\circ} \mathrm{C}$ then,

a) If the nitrate-to-chloride ion ratio is 0.5 (or greater), no localized corrosion will occur, or

b) If the nitrate-to-chloride ion ratio is less than 0.5 , then localized corrosion initiates and continues to propagate regardless of changes in the bulk chemical exposure environment (Criterion 1).

4. If the exposure temperature is greater than or equal to $20^{\circ} \mathrm{C}$ and less than or equal to $120^{\circ} \mathrm{C}$ then the empirical correlations for the long-term corrosion potential $\left(E_{\text {corr }}\right)$ and crevice repassivation potential $\left(E_{\text {rcrev }}\right)$ (Sections 6.4.4.3 and 6.4.4.5) are evaluated in accordance with the following implementation rules. If localized corrosion is determined to initiate, then localized corrosion continues to occur regardless of changes in the bulk chemical exposure environment (Criterion 1)

a) If the nitrate-to-chloride ion ratio in the environment exceeds 0.5 , then evaluate $E_{\text {rcrev }}$ and $E_{\text {corr }}$ at a nitrate-to-chloride ion ratio of 0.5 . 
b) If the molality of chloride ion in the environment exceeds 36 molal, then evaluate $E_{\text {rcrev }}$ and $E_{\text {corr }}$ at a molality of chloride ion of 36 molal.

If the molality of chloride ion is less than 0.001 molal, then evaluate $E_{\text {rcrev }}$ and $E_{c o r r}$ at a molality of chloride ion of 0.001 molal.

c) If the molality of nitrate ion in the environment exceeds 18 molal, then evaluate $E_{\text {rcrev }}$ and $E_{\text {corr }}$ at a molality of nitrate ion of 18 molal.

If the molality of nitrate ion is less than 0.001 molal, then $E_{\text {rcrev }}=E_{\text {rcrev }}^{o}$ (i.e., the crevice repassivation potential in the absence of nitrate ions) and evaluate $E_{\text {corr }}$ at a molality of nitrate ion of 0.001 molal.

d) If the $\mathrm{pH}$ in the environment exceeds 10.9, then evaluate $E_{\text {rcrev }}$ and $E_{\text {corr }}$ at a pH of 10.9 .

If the $\mathrm{pH}$ in the environment is less than 2.8 , then initiate localized corrosion.

The chemical limits specified in Criterion 4 are conservative. Limiting the chloride-to-nitrate ratio used in evaluation of the model to 0.5 is conservative because $E_{\text {rcrev }}$ increases markedly with nitrate ion concentration, at a given chloride ion concentration, $\mathrm{pH}$ and temperature, while $E_{\text {corr }}$ is relatively insensitive to nitrate ion concentration (at higher nitrate ion concentrations) (e.g., Figure 6-64). Limiting the chloride-to-nitrate ratio to 0.5 limits the beneficial effect of nitrate concentration in the localized corrosion initiation model as does limiting the nitrate ion concentration to 18 molal (consistent with the highest nitrate ion concentration at which $E_{\text {rcrev }}$ data was obtained). Both $E_{\text {rcrev }}$ and $E_{\text {corr }}$ decrease with chloride ion concentration, at a given nitrate ion concentration, temperature, and $\mathrm{pH}$, although $E_{\text {corr }}$ decreases at a faster rate than $E_{\text {rcrev }}$ at higher chloride ion concentrations (e.g., Figure 6-59). Therefore, limiting the chloride ion concentration to 36 molal (consistent with the highest chloride ion concentration at which $E_{\text {rcrev }}$ data was obtained) is conservative for determination of localized corrosion initiation (i.e., $\Delta E=E_{\text {rcrev }}-E_{\text {corr }} \leq 0$ ). At lower chloride ion concentrations, $E_{\text {rcrev }}$ generally decreases at a faster rate than $E_{\text {corr }}$; therefore, the lower bound chloride ion concentration is limited to 0.001 molal. Also, the functional forms for $E_{\text {corr }}$ (Equation 6-36) and $\Delta E_{\text {rcrev }}^{N O_{3}^{-}}$(Equation 6-34) contain nitrate-to-chloride ratios that increase at unrealistically high rates at low $(<0.001$ molal $)$ chloride ion concentrations. It is necessary to impose a lower limit $(0.001 \mathrm{molal})$ to the nitrate ion concentration as the functional form for $E_{\text {corr }}$ involves a logarithm of nitrate ion concentration which is undefined (approaches - - ) at zero nitrate ion concentration. The use of a lower limit nitrate ion concentration is conservative for evaluating $E_{\text {rcrev }}$ (i.e., $\Delta E_{\text {rcrev }} \mathrm{NO}_{3}^{-}$is always positive in Equation 6-31). $E_{\text {rcrev }}$ increases with increasing $\mathrm{pH}$ at constant temperature and nitrate and chloride ion concentrations, and $E_{\text {corr }}$ decreases with increasing $\mathrm{pH}$ at constant temperature and nitrate and chloride ion concentrations (e.g., Figure 6-62). It is conservative to use an upper limit on $\mathrm{pH}$ of 10.9 (consistent with the highest $\mathrm{pH}$ at which $E_{\text {corr }}$ data was obtained), because $E_{\text {rcrev }}$ is restricted from increasing further and $E_{c o r r}$ from decreasing further at higher $\mathrm{pH}$ values). Similarly, it is conservative to initiate localized corrosion at the lower limiting $\mathrm{pH}$ of 2.8 (consistent with the lowest $\mathrm{pH}$ at which $E_{\text {corr }}$ data was obtained). 


\subsubsection{Localized Corrosion Penetration Rate Model}

If the corrosion potential of the WPOB exceeds the critical potential, the WPOB is considered to be subject to localized (crevice) corrosion, and penetration of the barrier by localized corrosion is modeled. Due to the outstanding corrosion resistance of Alloy 22, very little data exists for localized corrosion penetration rates under repository-relevant exposure conditions. A reasonable lower bound for the localized corrosion propagation rate of Alloy 22 would be its average corrosion rate in a highly aggressive $10 \%$ ferric chloride crevice corrosion test solution. According to vendor data (Haynes International 1997 [DIRS 100897], p. 8), the average corrosion rate of Alloy 22 in a $10 \%$ ferric chloride solution at $75^{\circ} \mathrm{C}$ is $0.5 \mathrm{mils} / \mathrm{yr}$ or $12.7 \mu \mathrm{m} / \mathrm{yr}$. A reasonable upper bound for Alloy 22 localized corrosion penetration rates would be corrosion rates in concentrated $\mathrm{HCl}$ solutions at elevated temperatures. Alloy 22 corrosion rates between 5 and $50 \mathrm{mils} / \mathrm{yr}$ (i.e., between 127 and 1,270 $\mu \mathrm{m} / \mathrm{yr}$ ) (Haynes International 1997 [DIRS 100896], p. 12) were obtained under these conditions. Although higher corrosion rates are shown in the cited reference, the chosen rates are high enough to adequately represent the localized corrosion penetration rate for modeling purposes. A 20 -mm-thick Alloy 22 barrier would be penetrated in 16 to 160 years using the chosen penetration rates (i.e., these rates are high enough for their intended purpose). Generally, the "true" localized corrosion penetration rates in local areas may be higher than those measured by weight loss. However, the literature data mentioned above were from short-term tests, and are, therefore, conservative for use in long-term localized corrosion degradation analysis because the rate of localized corrosion penetration tends to decrease with time (Section 6.4.4.8.2).

Based on these data, the localized corrosion penetration rates for the WPOB are modeled in a range from 12.7 to $1,270 \mu \mathrm{m} / \mathrm{yr}$ with the median value of $127 \mu \mathrm{m} / \mathrm{yr}$, as shown in Table 6-9. A log-uniform distribution between the bounds was chosen for the localized corrosion penetration rate. The basis for this selection is that the penetration rate values from the literature span three orders of magnitude, and the percentiles provided are consistent with a log-uniform distribution. This distribution is based on data that bounds those extreme penetration rates found in the literature and are a highly conservative representation of localized corrosion rates of Alloy 22 for the exposure conditions expected in the postclosure repository. The entire variance in the penetration rate is due to uncertainty.

Table 6-9. Distribution of Localized Corrosion Rates for Alloy 22

\begin{tabular}{|c|c|}
\hline Percentile & Localized Corrosion Rate $(\mu \mathrm{m} / \mathrm{yr})$ \\
\hline $0^{\text {th }}$ & 12.7 \\
\hline $50^{\text {th }}$ & 127.0 \\
\hline $100^{\text {th }}$ & $1,270.0$ \\
\hline
\end{tabular}

Output DTN: MO0409MWDULCMW.000.

\subsubsection{Alternative Conceptual Models for Localized Corrosion}

Alternative conceptual models (ACMs) are based on modeling assumptions and simplifications different from those employed in the base-case model. An important reason for considering ACMs is to help build confidence that changes in modeling assumptions or simplifications will not change conclusions regarding subsystem and total system performance. Conceptual model 
uncertainty results from sparse observational data and a lack of available information to corroborate or refute plausible alternative interpretations of the subsystem and the processes occurring within the subsystem. This section discusses the ACMs for the localized corrosion models of the waste package outer barrier (WPOB).

\subsection{Localized Corrosion Susceptibility of the Waste Package Outer Barrier}

Localized corrosion of the WPOB is modeled with two model components: an initiation model and a propagation model. Localized corrosion of the WPOB occurs when the open-circuit corrosion potential $\left(E_{c o r r}\right)$ is equal to or greater than a critical potential (the crevice repassivation potential $\left(E_{\text {rcrev }}\right)$ in this report), that is, $\Delta \mathrm{E}\left(=E_{\text {rcrev }}-E_{\text {corr }}\right) \leq 0$. This conceptual model of localized corrosion initiation is widely accepted by the corrosion community and has been published extensively (Böhni 2000 [DIRS 164137], Section B; Dunn et al. 2000 [DIRS 164495]; Dunn et al. 2003 [DIRS 164138]; Frankel 1998 [DIRS 162216]; Frankel 2002 [DIRS 164140]; Frankel and Kelly 2002 [DIRS 164141]; Beavers et al. 2002 [DIRS 158781], Section 8.3). Exposure conditions in the repository will evolve with time, making it necessary to know $E_{c o r r}$ and $E_{\text {rcrev }}$ as a function of this evolution. This requires that a database of $E_{\text {rcrev }}$ values covering this range of conditions, plus a model for the evolution of $E_{\text {corr }}$ with exposure time are required.

An alternative parameter that can be used to determine susceptibility to localized corrosion is temperature. The evolution of waste package temperature with time can be calculated with reasonable certainty. This evolution, coupled with knowledge of the critical temperature for the initiation of localized corrosion (pitting/crevice corrosion) can then be used to determine when these processes may occur.

The use of critical temperatures in this manner was well documented (Frankel 1998 [DIRS 162216]), and values of critical pitting temperature (CPT) and critical crevice corrosion temperature (CCT) have been measured for a series of alloys, including Alloy 22, in relatively pure concentrated chloride solutions (i.e., high salinity, $\left[\mathrm{Cl}^{-}\right]=24,300 \mu \mathrm{g} / \mathrm{g}$, and a high $\left[\mathrm{Cl}^{-}\right]$to [SO $\left.{ }_{4}{ }^{2-}\right]$ ratio) (Haynes International 1997 [DIRS 100897], p. 9; McGuire et al. 1998 [DIRS 152193], Section 5.1.2). However, the test conditions are not directly relevant to the potential environments on the waste package surface. Under these highly corrosive conditions, the CCT for Alloy 22 was measured to be $102^{\circ} \mathrm{C}$, and for Alloy 276 to be $80^{\circ} \mathrm{C}$. The CPT for Alloy 22 was greater than $150^{\circ} \mathrm{C}$, and that for Alloy 276 was $150^{\circ} \mathrm{C}$.

The critical temperature-based model is not considered in the TSPA-LA because it does not account for the effects of electrochemical characteristics of the solution contacting the metal, particularly those of important corrosion inhibiting anions such as nitrate and sulfate ions present in the groundwater at the repository.

\subsection{Time-Dependent Growth Law for Localized Corrosion}

The base-case model assumes that, when localized corrosion of the WPOB occurs, it propagates at a (time-independent) constant rate (Assumption 5.4). This assumption is highly conservative because it is known that the localized corrosion rate decreases with time (CRWMS M\&O 1998 [DIRS 100349], Table 3-2; Hunkeler and Boehni 1983 [DIRS 162221]; McGuire et al. 1998 
[DIRS 152193], Section 5.2.8, EPRI 2002 [DIRS 158069], Section 5.3.1; Frankel 1998 [DIRS 162216]; Newman and Franz 1984 [DIRS 162250]).

An alternative conceptual model for the localized corrosion penetration is a time-dependent growth law. The growth law model can be developed based on a combination of electrochemical and corrosion exposure measurements. A simple pitting model based on hemispherical pit growth yields a penetration law of the form (CRWMS M\&O 1998 [DIRS 100349], Table 3-2; Hunkeler and Boehni 1983 [DIRS 162221]; McGuire et al. 1998 [DIRS 152193], Section 5.2.8)

$$
D=k \cdot t^{n}
$$

where $D$ is the depth of penetration, $t$ is time, and $k$ is a growth constant. The growth constant will be dependent on the properties of the material, particularly its susceptibility to anodic dissolution in the acidic environment prevailing in a propagating localized corrosion site. The time exponent, $n$, would be about 0.5 for both diffusion-controlled (i.e., diffusion of metal ions out of the pit) and ohmically controlled (i.e., rate determined by the ohmic potential drop which develops in the electrolyte in the pit) pit growth (McGuire et al. 1998 [DIRS 152193], Section 5.2.8; Vetter and Strehblow 1974 [DIRS 162245]). The above model was used in a separate analysis for the repository by the Electric Power Research Institute (EPRI) (EPRI 2002 [DIRS 158069], Section 5.3.1).

The significance of this pit penetration law has been discussed by Frankel (1998 [DIRS 162216]) and leads to a pit growth current density $(i)$ proportional to the inverse square root of time (i.e., $i$ $\left.\propto t^{\prime \prime \prime}\right)$ in potentiostatic electrochemical experiments. Hunkeler and Boehni (1983 [DIRS 162221]) have shown that this growth law is obeyed for both the pitting and crevice corrosion of stainless steels. Newman and Franz (1984 [DIRS 162250]) have also observed a similar relationship for stainless steel.

When trying to adapt such a law for practical applications, two main problems arise: (1) insufficient penetration rate data are available, especially for relatively new materials such as Alloy 22, to determine values of $k$ and $n$; and (2) the factors that control the form of this apparently simple growth law are complex and, at best, only qualitatively understood. In order to determine values of $k$ and $n$, it is necessary to employ short-term experiments in which the pit growth process is accelerated electrochemically. In these experiments, those features of the propagation process that enhance growth (the development of critical chemistry; the evolution of pit geometry) are dominant. However, it is necessary to predict penetration behavior after long periods of exposure, when those factors that limit growth (IR drop, loss of critical chemistry, evolution of metallurgical factors, polarization of cathodic processes) are more important.

The literature data available for less corrosion-resistant materials (e.g., iron, copper, Ti Grade 2) (Hunkeler and Boehni 1983 [DIRS 162221]; Marsh et al. 1991 [DIRS 162234]; Mughabghab and Sullivan 1989 [DIRS 162235]; Sharland et al. 1991 [DIRS 162238]; and Ishikawa et al. 1991 [DIRS 162222]) clearly show that a penetration growth law of the form of Equation 6-38 is appropriate, and that a value of $n=0.5$, the theoretically predicted value, is justifiable. A key point with the materials discussed above (e.g., iron, carbon steel, copper and Titanium Grade 2) is that they are materials that would be expected to undergo rapid propagation. Providing it is not 
stifled by the accumulation of corrosion product deposits or slow cathodic kinetics, propagation would be limited only by diffusive or ohmic effects, leading to a value of $n$ approaching 0.5 .

By contrast, for highly corrosion-resistant materials such as Alloy 22 designed to resist localized corrosion, additional metallurgical features will be important in determining the value of $n$. One example of such a metallurgical influence that is pertinent to the case of Alloy 22 is the ability of molybdenum to decrease the pitting current densities in stainless steels, possibly by reducing the active dissolution rate within the pit (Frankel 1998 [DIRS 162216]; and Newman 1985 [DIRS 162251]). This prevents the maintenance of the critical pit or crevice chemistry to sustain propagation, leading to repassivation. Again, the $n$ value in the growth law in Equation 6-38 would effectively tend to zero. This is supported by the observation of Kehler et al. (2001 [DIRS 162231]), who showed that the depth of crevice penetration for Alloy 22 electrochemically driven in extremely saline $(5 \mathrm{~mol} / \mathrm{L} \mathrm{LiCl})$ solutions at $85^{\circ} \mathrm{C}$ was limited to less than $100 \mu \mathrm{m}$. The adoption of such a value considers that metallurgical features, such as the influence of molybdenum on pit/crevice propagation will suppress penetration.

Localized corrosion rate data are needed to obtain a value for $k$. The only presently available source of crevice corrosion rate data is that published by Haynes International (1997 [DIRS 100897]) and summarized in Table 22 of Gdowski (1991 [DIRS 100859]). These data were recorded in 10 weight percent $\mathrm{FeCl}_{3}$ (i.e., under extremely aggressive oxidizing conditions).

The localized corrosion growth law model of the form of Equation 6-38 is not used in the TSPA-LA because of lack of data to obtain the values of the model parameters, $n$ and $k$ for Alloy 22 for the exposure conditions relevant to the repository. The base-case model (timeindependent constant penetration rate model) is much more conservative than the growth law model. The base-case model should bound the penetration rate range by localized corrosion of the WPOB when it occurs.

\subsubsection{Effect of Microbial Activity on Corrosion}

Microbially influenced corrosion (MIC) is the contribution to the corrosion of a metal or alloy due to the presence or activity, or both, of microorganisms. MIC most often occurs due to the increase in anodic or cathodic reactions due to the direct impact of microorganisms on the alloy or by indirect chemical effects on the surrounding solution. Microorganisms can affect the corrosion behavior of an alloy either by acting directly on the metal or through their metabolic products. For example, some types of aerobic bacteria may produce sulfuric acid by oxidizing reduced forms of sulfur (elemental, sulfide, sulfite), and certain fungi transform organic matter into organic acids (Fontana 1986 [DIRS 100890], Section 8-10). Bacterial isolates from Yucca Mountain have been shown to be capable of iron oxidation, acid production, sulfate reduction and slime generation (Lian et al. 1999 [DIRS 110238]). However, acid production from sulfide oxidation in the repository is unlikely because the drift environment will remain oxic over the regulatory compliance period (BSC 2004 [DIRS 169991], Section 7.1). 


\subsubsection{Localized Corrosion}

Localized corrosion is a phenomenon in which corrosion progresses at discrete sites or in a nonuniform manner. The rate of localized corrosion penetration is generally higher than the rate of general corrosion penetration. The analysis assumes that crevice corrosion is representative of localized corrosion of the WPOB under the exposure conditions expected in the postclosure repository (Assumption 5.3). This is a conservative and bounding assumption because the initiation thresholds for crevice corrosion in terms of water chemistry and temperature are lower than those for pitting corrosion (Gdowski 1991 [DIRS 100859], Section 3.0; Haynes International 1997 [DIRS 100896]; Haynes International 1997 [DIRS 100897]), which is another form of localized corrosion.

Localized corrosion of the WPOB is analyzed in this report with two model components: an initiation model and a propagation model. In the localized corrosion initiation model, localized corrosion of the WPOB occurs when the open-circuit potential, or corrosion potential $\left(E_{\text {corr }}\right)$, is equal to or greater than a critical threshold potential $\left(E_{\text {critical }}\right)$, that is, $\Delta \mathrm{E}\left(=E_{\text {critical }}-E_{\text {corr }}\right) \leq 0$. The magnitude of $\Delta \mathrm{E}$ is an index of the localized corrosion resistance; that is, the larger the difference, the greater the localized corrosion resistance. This conceptual model of localized corrosion initiation is widely accepted by the corrosion community and has been published extensively (Böhni 2000 [DIRS 164137], Section B; Dunn et al. 2000 [DIRS 164495]; Dunn et al. 2003 [DIRS 164138]; Frankel 1998 [DIRS 162216]; Frankel 2002 [DIRS 164140]; Frankel and Kelly 2002 [DIRS 164141]; and Beavers et al. 2002 [DIRS 158781], Section 8.3). The localized corrosion initiation model components (i.e., $E_{\text {corr }}$ and $E_{\text {critical }}$ ) could be affected by the sample configuration (crevice, disk or rod), metallurgical condition (mill annealed or as-welded), and exposure conditions (temperature, $\mathrm{pH}$, chloride ion concentration, or nitrate ion concentration).

A series of the electrochemical corrosion tests were conducted to generate the data for the localized corrosion initiation model. The typical sequence for electrochemical testing is summarized in Appendix I. As discussed in Section 6.4.4.4, a set of long-term open-circuit potential measurements was used to measure the long-term corrosion potentials $\left(E_{\text {corr }}\right)$ of Alloy 22 in a wide range of exposure environments. A series of cyclic potentiodynamic polarization (CPP) tests were also performed to measure the critical potentials $\left(E_{\text {critical }}\right)$ of Alloy 22 for differing sample configurations and metallurgical conditions in a wide range of exposure environments.

Crevice corrosion initiation and propagation can be affected by the initial condition of the specimen surface prior to testing. Handbook data (Kain 1987 [DIRS 155193], p. 306) shows, for a variety of alloys, that fewer crevices initiate on as-received (mill condition) specimens than on specimens that have been wet ground to a 120 -grit surface finish. This behavior results from the ability to form a tighter crevice on ground surfaces promoting breakdown of the passive film (Kain 1987 [DIRS 155193], p. 303). In this report, as discussed in Appendix I, the samples used for analysis of crevice corrosion initiation had an equivalent 600 -grit surface finish. If the as-emplaced waste packages have a rougher surface finish, the localized corrosion initiation model developed in this report will be conservative relative to the expected behavior of the waste packages in the repository. 
In this report, localized corrosion of the WPOB is assumed to propagate at a (time-independent) constant rate (Assumption 5.4). This assumption is conservative because it is known that the localized corrosion rate decreases with time (Hunkeler and Boehni 1983 [DIRS 162221]; McGuire et al. 1998 [DIRS 152193], Section 5.2.8). Section 6.4.4.7 provides additional discussion on this issue.

\subsubsection{Overview of Approaches for Selection of Critical Potential for Localized Corrosion Initiation}

Localized corrosion of the WPOB occurs when the long-term corrosion potential $\left(E_{\text {corr }}\right)$ is equal to or greater than a certain critical potential $\left(E_{\text {critical }}\right)$, that is, $\Delta \mathrm{E}\left(=E_{\text {critical }}-E_{\text {corr }}\right) \leq 0 . E_{\text {corr }}$ and $E_{\text {critical }}$ could be affected by the sample configuration (crevice, disk, or rod), metallurgical condition (mill annealed, as-welded, or as-welded plus thermally aged), and exposure condition (temperature, $\mathrm{pH}$, chloride ion concentration, or nitrate ion concentration).

$E_{\text {critical }}$ can be defined as a potential above which the current density or corrosion rate of Alloy 22 increases significantly and irreversibly above the general corrosion rate of the passive metal (ASTM G 61-86 1987 [DIRS 127897]; Jones 1992 [DIRS 169906], Chapter 7). Above this potential local breakdown of the passive film can occur. The value of $E_{\text {critical }}$ cannot be measured as easily as the value of $E_{\text {corr }}$ because the value of $E_{\text {critical }}$ is also affected by the method used for its measurement.

The "true" value of $E_{\text {critical }}$ of a metal or alloy for a given set of conditions (including sample configuration, metallurgical condition, and exposure condition) would be the lowest potential at which the corrosion current (except for initial transients) does not decay with time and is significantly higher than the passive current density, when held potentiostatically. Therefore, one approach to defining $E_{\text {critical }}$ is to conduct a series of potentiostatic polarization (PSP) tests at predetermined potentials near the critical potentials initially measured by shorter-term cyclic potentiodynamic polarization (CPP) tests. CPP tests themselves have been widely used to obtain the critical potential (Jain et al. 2003 [DIRS 164087]; Dunn and Brossia 2002 [DIRS 162213]; Brossia et al. 2001 [DIRS 159836]) likely due to the much shorter test times required than for PSP tests. CPP tests were used in this report to determine the critical potential for the Alloy 22 WPOB.

In using the CPP technique to identify critical parameters for the initiation of localized corrosion, the potential-current curves are examined to look for values of the potential where there was a discernible, often abrupt, change of the current, much like a point of inflection on the potentialcurrent curve. The advantage of this approach is that the current change can be related to physical/chemical events occurring on the metal surface over the course of the polarization cycle. Because the potential is scanned initially in the oxidizing or anodic direction, and then in the reducing or cathodic direction, hysteresis of the curve is indicative of changes that have occurred on the metal surface during polarization, and the amount of this hysteresis is related to localized corrosion susceptibility (Jones 1992 [DIRS 169906], Chapter 7). Figure 6-33 shows a schematic of CPP curves showing different polarization behaviors for an alloy with a high resistance to localized corrosion. 


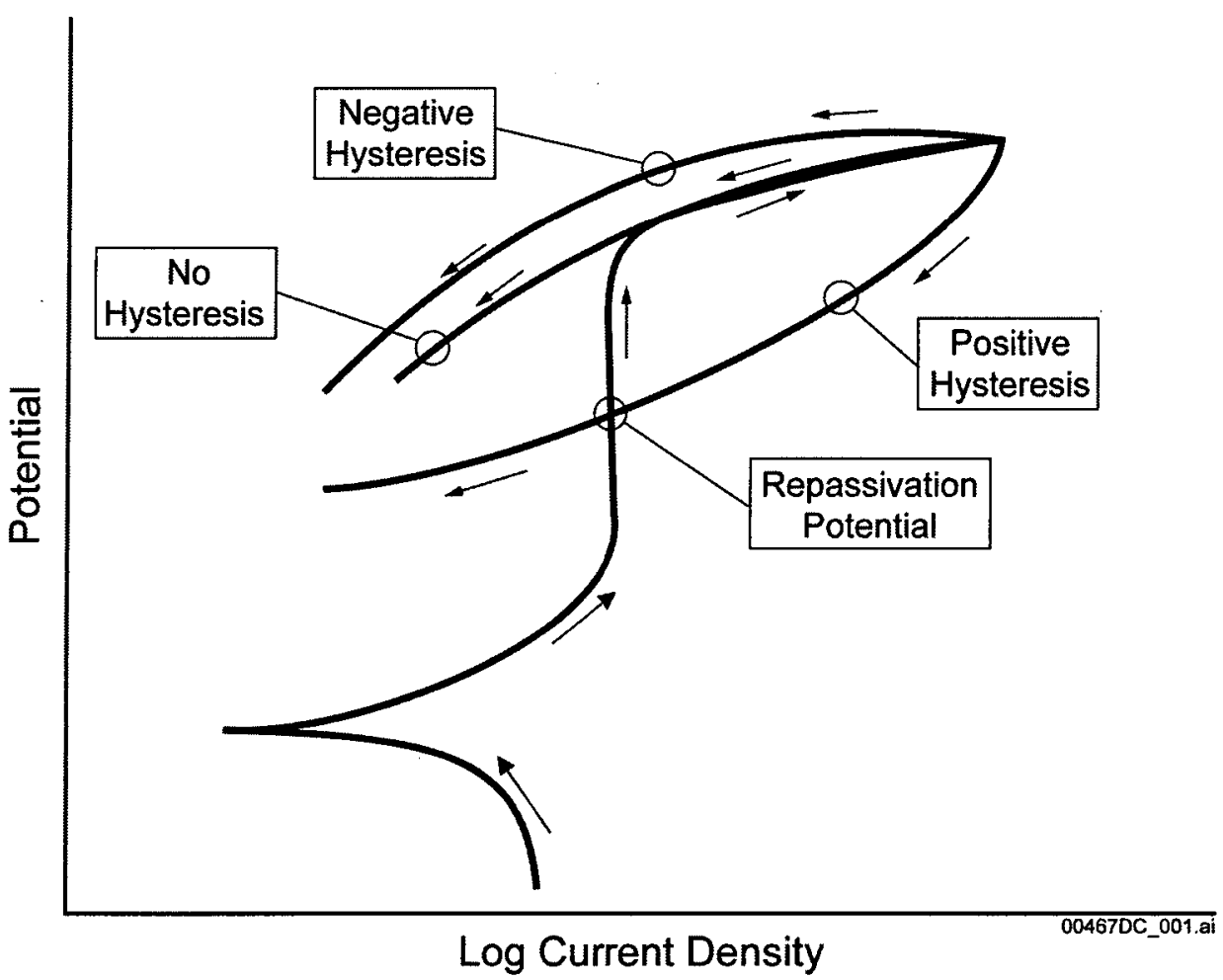

Figure 6-33. Schematic Potentiodynamic Polarization Curve Showing Likely Differing Behaviors of the Curves During Potentiodynamic Scanning of an Alloy with High Resistance to Localized Corrosion

To produce curves such as those shown in Figure 6-33, the electrochemical potential is continuously scanned from slightly below the open-circuit or corrosion potential following a relatively short period of exposure of the metal specimen to the environment. If the metal is passive (as in the case of Alloy 22), the anodic current tends to have a low and nearly constant value for a.wide range of potential; but eventually a potential is reached where there is a sharp increase in current. This change may be the beginning of oxygen evolution from water (if the applied potential is sufficient to electrolyze water). If this potential corresponds to oxygen evolution, passive film breakdown or localized corrosion would not initiate. On the other hand, this change in current may indicate the breakdown of the passive film, which could result from a number of electrochemical reactions.

After the potential scan is reversed, in cases where film breakdown due to localized corrosion has occurred, the metal surface is altered from its initial state (the passive film has broken down in some places), and the current shows, on reverse scan, a "positive" hysteresis. That is, compared to the forward scan, the current at a given potential is higher on the reverse scan. However, eventually the reverse-scan curve crosses over the curve generated during the forward scan. Where the reverse scan intersects the forward scan, repassivation is considered complete (the passive film has been repaired). The potential at the intersection is called the repassivation potential in this report (e.g., the pit repassivation potential $\left(E_{r p}\right)$ for a boldly exposed sample or the crevice repassivation potential $\left(E_{\text {rcrev }}\right)$ for a creviced sample geometry).

In some cases there is no hysteresis or only slightly positive or slightly negative hysteresis, meaning the reactions are reversible, and the current retraces the values from the forward scan, 
often crossing the forward scan more than once at potentials above the passive region. In some cases, a slightly positive hysteresis is observed with no traces of localized corrosion on the specimen surface and in other cases, crevice corrosion may be observed on a specimen that produced no hysteresis. The current on the reverse scan usually falls through zero, as it does at $E_{c o r r}$ during the increasing polarization. Almost always, this new apparent corrosion potential at the zero current is much more positive than the initial corrosion potential. If oxygen evolution has occurred, the solution has higher oxygen content and this would make the new corrosion potential more noble. If some additional oxidation of the alloy has occurred, this would also make the corrosion potential more noble on the reverse scan. If transpassive dissolution occurred, the reverse scan does not cross the forward scan; it traces down the forward scan. In the transpassive region, the entire metal surface has been depassivated (the passive film is absent). In these cases, the passive film has broken down, but not only in localized areas, so active general corrosion occurs. As the potential is scanned further in the reverse direction, this base material becomes passivated (a new passive film forms).

Many cyclic polarization curves obtained in concentrated solutions at elevated temperatures do not exhibit the same shapes as those observed in ideal curves, such as those illustrated in ASTM G 61-86 (1987 [DIRS 127897]) (i.e., Stainless Steel Type 304 and nickel-base Alloy $\mathrm{C}-276$ in 3.5 percent $\mathrm{NaCl}$ at room temperature). In some of the solutions, polarization curves of Alloy 22 did not show a well-defined passive region. These observations may indicate that the alloy is undergoing general corrosion, not localized corrosion.

Another complicating factor observed in many of the polarization curves was the appearance of one or more 'humps' in the passive region of the forward scan. These are due to changes in oxidation state of one or more of the metallic components in the passive film, and in some cases indicate changes in the morphology and structure of the film, with possible implications on localized corrosion susceptibility. Usually, humps were not observed during the reverse scan. For some test conditions in which no hump was present in the forward scan, humps were observed in the reverse scan.

There is no agreement among researchers about the definition of the repassivation potential ( $E_{r p}$ or $E_{r c r e v}$ for creviced specimens) in CPP tests. Many researchers identify the repassivation potential as the potential at which the reverse scan intersects the passive region of the forward scan (or the potential at which the forward current density equals reverse current density). A critical potential is not measured for the conditions where there is no positive hysteresis, because no localized corrosion has occurred. However, for conditions where the passive region in the forward scan is not clearly defined, the reverse scan never intersects the forward scan or the reverse scan crosses the forward scan more than once. In such cases, the repassivation potential can be subject to the judgment of an individual investigator and could result in inconsistent selection of the repassivation potential value.

The above approach has been shown to produce overly conservative repassivation potential values for Stainless Steel Type 304 materials in chloride solutions (Akashi et al. 1998 [DIRS 163903]). In other studies using the Tsujikawa method (Akashi et al. 1998 [DIRS 163903]) and its variation, the potential-step technique (Jain et al. 2003 [DIRS 164087]), which generally results in more reasonable values for the crevice repassivation potential than the CPP technique, Gruss et al. (1998 [DIRS 100893]) used a current-density threshold of $1 \mu \mathrm{A} / \mathrm{cm}^{2}$ 
in selecting the crevice repassivation potential. Other investigations have used a current-density threshold of $2 \mu \mathrm{A} / \mathrm{cm}^{2}$ to define the crevice repassivation potential (Jain et al. 2003 [DIRS 164087]; Dunn and Brossia 2002 [DIRS 162213]; Brossia et al. 2001 [DIRS 159836]). However, comparison of the repassivation potentials of Alloy 22 obtained with different test methods has shown that, when an appropriate criteria was employed in selecting the critical potential value, the repassivation potential obtained with the CPP method was similar to the repassivation potential obtained with the more time-consuming PSP method, the Tsujikawa method, or other similar methods (Jain et al. 2003 [DIRS 164087]).

Another study of Alloy 22 crevice corrosion behavior utilized the CPP technique and specimens containing multiple crevice assemblies (MCA) (Kehler et al. 1999 [DIRS 162230]; Scully et al. 1999 [DIRS 110246]; Kehler et al. 2001 [DIRS 162231]). In this study, the potential at which the current density permanently exceeded $1 \mu \mathrm{A} / \mathrm{cm}^{2}$ in the forward scan was selected as a threshold potential to define crevice corrosion initiation (or crevice stabilization). In this section, this potential is referred to as $E_{f l}$. The study also used two current-density thresholds in the reverse scan to define crevice repassivation potentials, $10 \mu \mathrm{A} / \mathrm{cm}^{2}$ and $1 \mu \mathrm{A} / \mathrm{cm}^{2}$. In this section, these potentials are referred to as $E_{r 10}$ and $E_{r l}$, respectively (Kehler et al. 1999 [DIRS 162230]; Scully et al. 1999 [DIRS 110246], Section 1.3; Kehler et al. 2001 [DIRS 162231]). It was shown that the crevice repassivation potentials were more stable and reproducible than the observed corrosion potentials. In many instances, the crevice corrosion initiation potential was associated with the transpassive dissolution and not with actual crevice corrosion initiation. The crevice repassivation potential defined at $1 \mu \mathrm{A} / \mathrm{cm}^{2}$ was more likely to be associated with deactivation of crevice corrosion that resulted from net cathodic electrochemical reactions in the crevice, and not necessarily associated with crevice repassivation. The crevice repassivation potential defined at $10 \mu \mathrm{A} / \mathrm{cm}^{2}$ obtained at slow scan rate was found to be more representative of crevice repassivation (Kehler et al. 1999 [DIRS 162230]; Kehler et al. 2001 [DIRS 162231]).

In most CPP scans of highly corrosion-resistant alloys, the passive current is on the order of 1 to $10 \mu \mathrm{A} / \mathrm{cm}^{2}$. The passive current density on the reverse scan is usually greater than in the initial (or forward) scan. This is because the newly formed (during the reverse scan) oxides over the crevice site are thin, defective, and can support charge transmission at higher rates than the typically thicker and less-defective oxides present during the forward scan. Moreover, the solution inside the crevice is acidified from hydrolysis of cations released into the crevice solution during the forward scan (Kehler et al. 1999 [DIRS 162230]; Kehler et al. 2001 [DIRS 162231]).

After review of the different approaches to obtaining the critical potential for localized corrosion initiation, the repassivation potential obtained from CPP tests was chosen as the basis for localized corrosion initiation analysis in this report. As discussed previously, in some cases the reverse scans showed slightly positive or slightly negative or no hysteresis at all, often crossing the forward scan more than once at potentials above the passive region, that is, at potentials in the highest section of the anodic polarization curves. In these latter cases, evidence of localized corrosion was observed in some instances and not in others. A highly conservative approach would be not to use these data in development of the model. This would be extremely conservative because environments in which localized corrosion was not observed (even under high anodic polarization) would not contribute to the model predictions. Also, environments in which localized corrosion was sometimes observed (e.g., in only 5 percent of tested specimens) 
would be reflected in the model as environments in which localized corrosion always occurred. At the same time, given the importance of localized corrosion to waste package performance, some conservatism is warranted. For the modeling purposes, in cases where the nitrate-to-chloride ion ratio of the testing solution was 0.50 , a crevice repassivation potential of $+600 \mathrm{mV} \mathrm{SSC}$ was used. In the cases where the nitrate-to-chloride ion ratio was less than 0.50 , the data from tests that did not produce a repassivation potential were not used. That is, for nitrate-to-chloride ratios of 0.50 and higher when a clear crossover on the passive region of potentials was not observed, the default repassivation potential was set to $+600 \mathrm{mV}$ SSC. For ratios of nitrate-to-chloride lower than 0.50 , when a clear cross over in the passive region was not observed, the repassivation potential data were not used. This last approach adds conservatism to the model predictions.

The value of $+600 \mathrm{mV} \mathrm{SSC}$ was selected since repassivation potentials higher than $+600 \mathrm{mV}$ SSC may not have physical meaning. At potentials of $+600 \mathrm{mV} \mathrm{SSC}$ or higher, the apparent breakdown of passivity of Alloy 22 may actually be oxygen evolution from water and/or transpassive dissolution processes. The values of potential for oxygen evolution and transpassive dissolution depend on the $\mathrm{pH}$ of the solution and the temperature. Above about $+600 \mathrm{mV} \mathrm{SSC}$, $\mathrm{Cr}^{3+}$ is not stable in the protective $\mathrm{Cr}_{2} \mathrm{O}_{3}$ oxide film formed on Alloy 22 and further oxidizes to $\mathrm{Cr}^{6+}$. For example, the Pourbaix diagram for pure chromium shows that at a $\mathrm{pH}$ of 7 and at ambient temperature $\left(25^{\circ} \mathrm{C}\right)$, the thermodynamically stable species would be $\mathrm{Cr}^{6+}$ at potentials higher than about $+600 \mathrm{mV}$ SHE (Pourbaix 1974 [DIRS 100817], p. 264). At $25^{\circ} \mathrm{C}$ the $\mathrm{Ag} / \mathrm{AgCl}$ reference electrode in saturated $\mathrm{KCl}$ solution is more noble than the standard hydrogen electrode scale by $199 \mathrm{mV}$ (Sawyer and Roberts 1974 [DIRS 162259], pp. 39 to 45, Table 2-4); therefore, $+600 \mathrm{mV}$ SHE is about $+400 \mathrm{mV}$ SSC. The synergistic effects of other elements in Alloy 22 such as nickel (Pourbaix 1974 [DIRS 100817], p. 333) and tungsten (Pourbaix 1974 [DIRS 100817], p. 282) tend to raise the potential stability range (i.e., the transpassive potential) for the passive film formed on Alloy 22. For example, the transpassive potential for Alloy 22 in SAW solution at $90^{\circ} \mathrm{C}$ has been reported to be about $+620 \mathrm{mV} \mathrm{SSC}$ (Chen et al. 2002 [DIRS 165441], Figure 10). Other investigators have reported similar Alloy 22 transpassive (or oxygen evolution or both) potentials (between about +860 and $+980 \mathrm{mV} \mathrm{SHE}$ or about 660 and $780 \mathrm{mV} \mathrm{SSC}$ ) in various acidic (pH 3) chloride-containing solutions (Jayaweera et. al 2003 [DIRS 162225], Section 9.2.2). Also, CPP tests in which it was determined that localized corrosion did occur always had repassivation potentials below $+600 \mathrm{mV} \mathrm{SSC}$ (Appendices V, VI, VII, and VIII). The above analysis indicates that crossover potentials above approximately $+600 \mathrm{mV} \mathrm{SSC}$ are more likely transpassive dissolution or oxygen evolution potentials and not true repassivation potentials. At high anodic potentials, surface modification of the tested specimens due to transpassive dissolution occurs; therefore, little hysteresis in the reverse scan is unavoidable. Therefore, it is reasonable to assign a "repassivation potential" value of $+600 \mathrm{mV}$ SSC to samples that did not undergo localized corrosion during the CPP tests as a method to account for the fact that localized corrosion of Alloy 22 is not observed in some experiments. Conservatively, only samples in exposure environments in which the nitrate-to-chloride ratio was 0.50 were assigned this effective repassivation potential (i.e., credit was not taken for every sample that did not display localized corrosion, for example, in solutions with a nitrate-tochloride ion ratio less than 0.50 ). This approach is conservative and defensible for the prediction of long-term localized corrosion susceptibility of the WPOB in the repository. 
As discussed in the following section, with few exceptions, the repassivation potentials of crevice samples (i.e., measurements of the crevice repassivation potential, $E_{\text {rcrev }}$ ) were used for localized corrosion initiation analysis. Investigators at the Center for Nuclear Waste Regulatory Analyses (CNWRA) used the same approach (also using crevice samples) (Brossia et al. 2001 [DIRS 159836], Table A-1).

\subsubsection{Cyclic Potentiodynamic Polarization Data Analysis for Crevice Repassivation Potential}

A series of cyclic potentiodynamic polarization (CPP) tests were performed for Alloy 22 samples over a wide range of exposure environments. Included in the tests were a variety of electrolyte solution chemistries, exposure temperatures, sample geometries and configurations (i.e., multiple crevice assembly (MCA), rod, and disc), and metallurgical conditions (i.e., mill annealed, as-welded, and as-welded plus thermally aged).

The CPP tests included simple salt solutions such as $\mathrm{NaCl}, \mathrm{CaCl}_{2}$, and mixed $\mathrm{CaCl}_{2}$ and $\mathrm{Ca}\left(\mathrm{NO}_{3}\right)_{2}$ solutions and mixed $\mathrm{NaCl}$ and $\mathrm{KNO}_{3}$ solutions. A wide range of concentrations up to near saturation were used. Descriptions of the electrochemical corrosion test procedures used to generate data for this report are provided in Appendix I. The CPP measurements were based on ASTM G 5-94 1994 [DIRS 117479]). Necessary deviations from the standard have been noted in the scientific notebooks corresponding to the DTNs used. Analyses of the CPP test data were performed to obtain repassivation potentials. Initial data screening and analysis are documented in DTNs: LL030409512251.051 [DIRS 163462] and LL040402212251.084 [DIRS 170097]. The repassivation potential data reported in these DTNs was supplemented as discussed below.

As discussed in Section 6.3 contact points between the waste package and structural components in the drift as well as with mineral deposits formed by evaporative concentration of solutions contacting the waste package could form crevices on the waste package surface. Therefore, only the crevice (MCA) sample data were considered for the repassivation potential model analysis with the exception of the repassivation potential data for samples DEA598, DEA599, and DEA600 (DTN: LL030409812251.054 [DIRS 164185]), which were disc samples exposed to high chloride concentrations $(12.6$ to $20.8 \mathrm{~m})$ and high temperatures $\left(120^{\circ} \mathrm{C}\right.$ and $\left.130^{\circ} \mathrm{C}\right)$. In the case of noncreviced samples, only a repassivation potential $\left(E_{r p}\right)$ is obtained, but in this report, the repassivation potential is treated in the same way as the crevice repassivation potential $\left(E_{\text {rcrev }}\right)$. The repassivation potential data were included in the data used to build the models because relatively few MCA data points exist at these high temperatures and solution concentrations. Use of these data improves the predictive capability of the models. Although MCA and disc samples have different geometries, and may be subject to slightly different local passive film breakdown mechanisms, there were no significant differences in the repassivation potential between the two types of samples at high temperatures and ion concentrations. Also, two CPP test data for MCA samples (DEA3125 and DEA3127 from DTN: LL030409812251.054 [DIRS 164185]) exposed to high chloride (19.2 and $20.8 \mathrm{~m}$ ) and high-temperature $\left(130^{\circ} \mathrm{C}\right)$ conditions with and without nitrate ions were also used in the model development.

These additional data (disc samples DEA598, DEA599, and DEA600; MCA samples DEA3125 and DEA3127) were analyzed following the same process as that used for the data in 
DTN: LL030409512251.051 [DIRS 163462]. The analyses to obtain the crevice repassivation potentials $\left(E_{r c r e v}\right)$ or repassivation potentials $\left(E_{r p}\right)$ of the above samples are illustrated graphically in Figure 6-34 to Figure 6-38. The resulting crevice repassivation potentials are shown in Table 6-7.

For the salt solutions in Table 6-7, the chloride and nitrate salts were considered to be completely dissociated. This approach is considered conservative as only a fraction of the ions are expected to be available to interact with the Alloy 22 surface due to strong interionic attractions in concentrated solutions. As noted in Table 6-7 and Appendix VI, the room temperature $\mathrm{pH}$ of the high chloride and high temperature solutions from DTN: LL030409812251.054 [DIRS 164185] was not reported. A measured room temperature $\mathrm{pH}$ of 4.14 for $5 \mathrm{M} \mathrm{CaCl}_{2}$ solutions tested at $120^{\circ} \mathrm{C}$ (from the scientific notebook associated with DTN: LL030400112251.043 [DIRS 163466] (i.e., Ilevbare 2003 [DIRS 171329], p. 141)) was used for $7 \mathrm{M} \mathrm{CaCl}_{2}$ solutions tested at $130^{\circ} \mathrm{C}$ (samples DEA3125 and DEA599). Also, as noted in Table 6-7 and Appendix VIII, a measured room temperature $\mathrm{pH}$ of 5.34 for $5 \mathrm{M} \mathrm{CaCl}_{2}$ plus $0.05 \mathrm{M} \mathrm{Ca}\left(\mathrm{NO}_{3}\right)_{2}$ solution at $120^{\circ} \mathrm{C}$ from DTN: LL030502212251.063 [DIRS 163456] (e.g., samples JE 0024 and JE 0025 in MCA E-chem Data Submittal Sheet 4-29-03-King.xls) was used for the $\mathrm{CaCl}_{2}$ plus $\mathrm{Ca}\left(\mathrm{NO}_{3}\right)_{2}$ salt solutions (Samples DEA3127, DEA598 and DEA600). Because pH has a small effect on the crevice repassivation potentials relative to temperature and chloride concentration (Section 6.4.4.3), the impact of the substituted $\mathrm{pH}$ values for the high chloride and high temperature solutions will be minor.

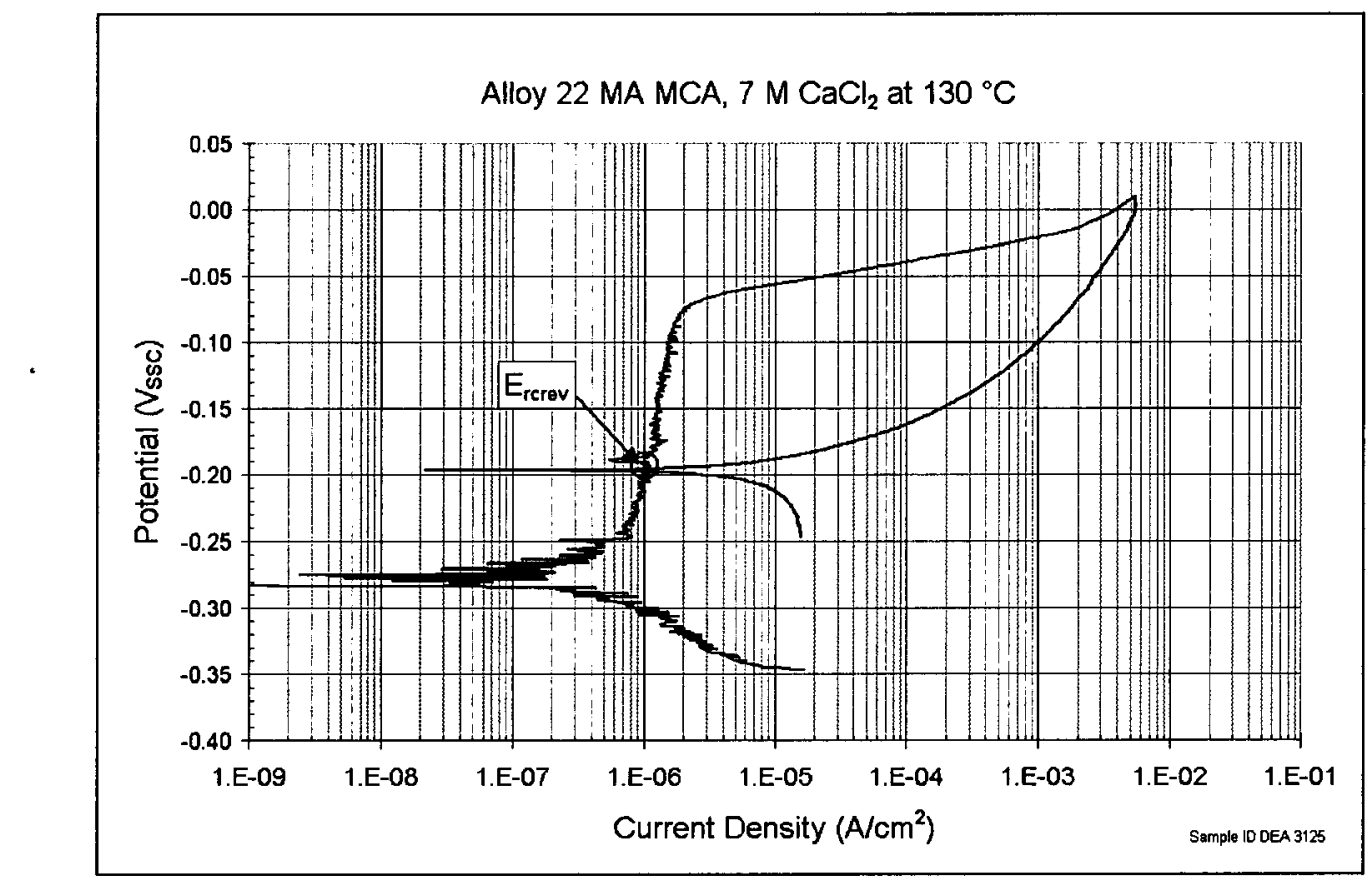

Source: DTN: LL030409812251.054 [DIRS 164185]; CP_DEA3125.x/s.

Output DTN: MO0409MWDULCMW.000.

Figure 6-34. Determination of Crevice Repassivation Potential of Mill-Annealed MCA Sample (Sample DEA 3125) Tested in $7 \mathrm{M} \mathrm{CaCl}_{2}$ Solution at $130^{\circ} \mathrm{C}$ 
Alloy $22 \mathrm{MA} M \mathrm{MCA}_{1}, 7 \mathrm{M} \mathrm{CaCl}_{2}+0.7 \mathrm{M} \mathrm{Ca}\left(\mathrm{NO}_{3}\right)_{2}$ at $130^{\circ} \mathrm{C}$

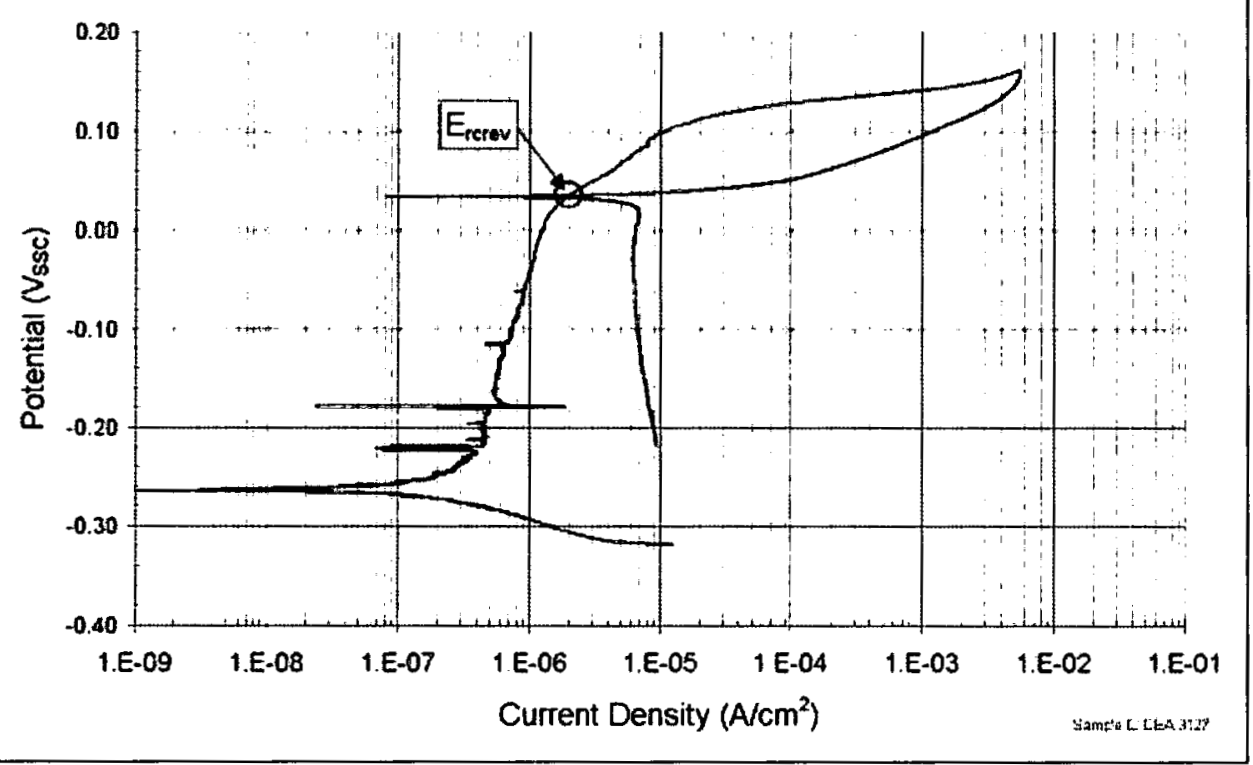

Source: DTN: LL030409812251.054 [DIRS 164185]; CP_DEA3127.x/s.

Output DTN: MO0409MWDULCMW.000.

Figure 6-35. Determination of Crevice Repassivation Potential of Mill-Annealed MCA Sample (Sample DEA 3127) Tested in $7 \mathrm{M} \mathrm{CaCl}_{2}+0.7 \mathrm{M} \mathrm{Ca}\left(\mathrm{NO}_{3}\right)_{2}$ Solution at $130^{\circ} \mathrm{C}$

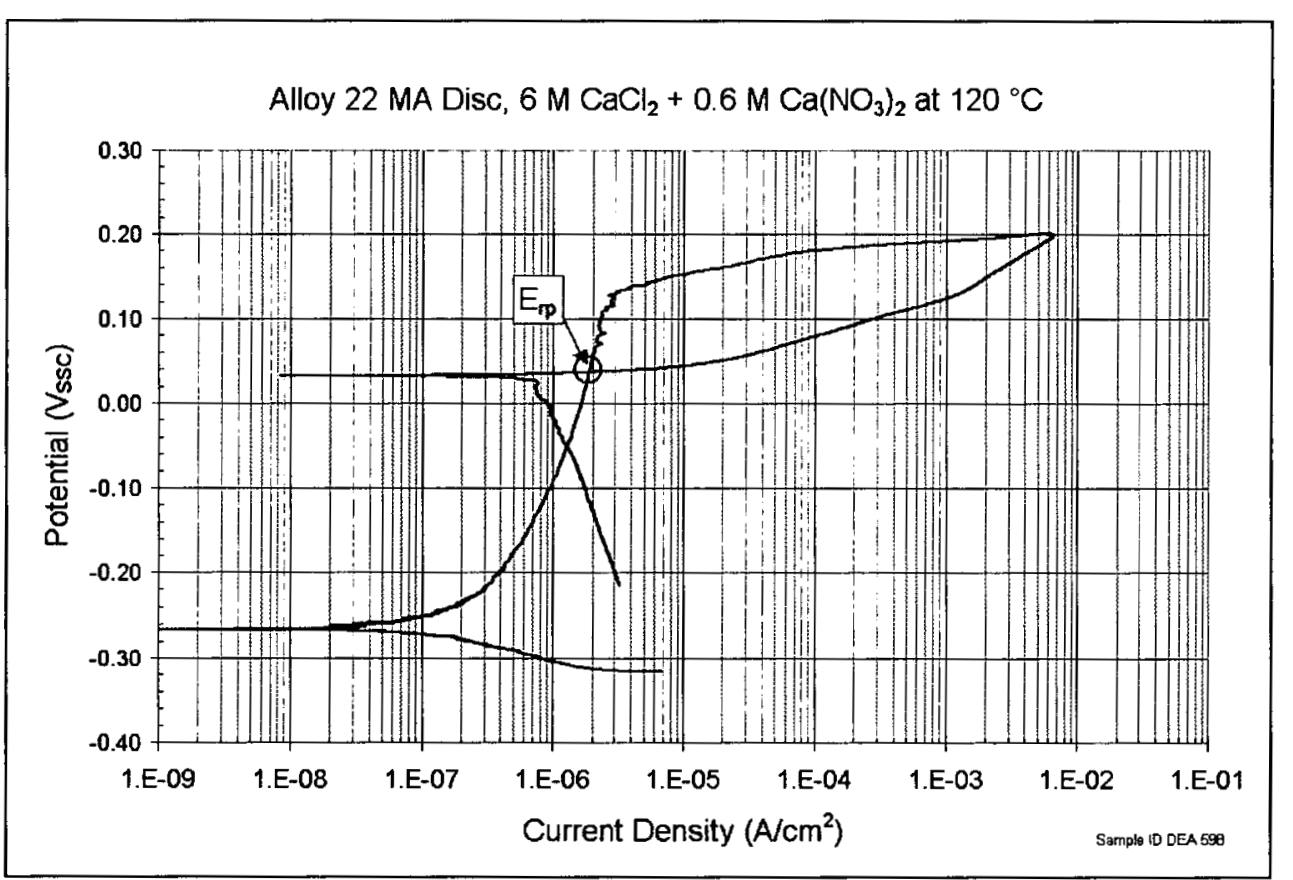

Source: DTN: LL030409812251.054 [DIRS 164185]; CP_DEA598.xIs.

Output DTN: MO0409MWDULCMW.000.

Figure 6-36. Determination of Repassivation Potential of Mill-Annealed Disc Sample (Sample DEA 598) Tested in $6 \mathrm{M} \mathrm{CaCl}_{2}+0.6 \mathrm{M} \mathrm{Ca}\left(\mathrm{NO}_{3}\right)_{2}$ Solution at $120^{\circ} \mathrm{C}$ 


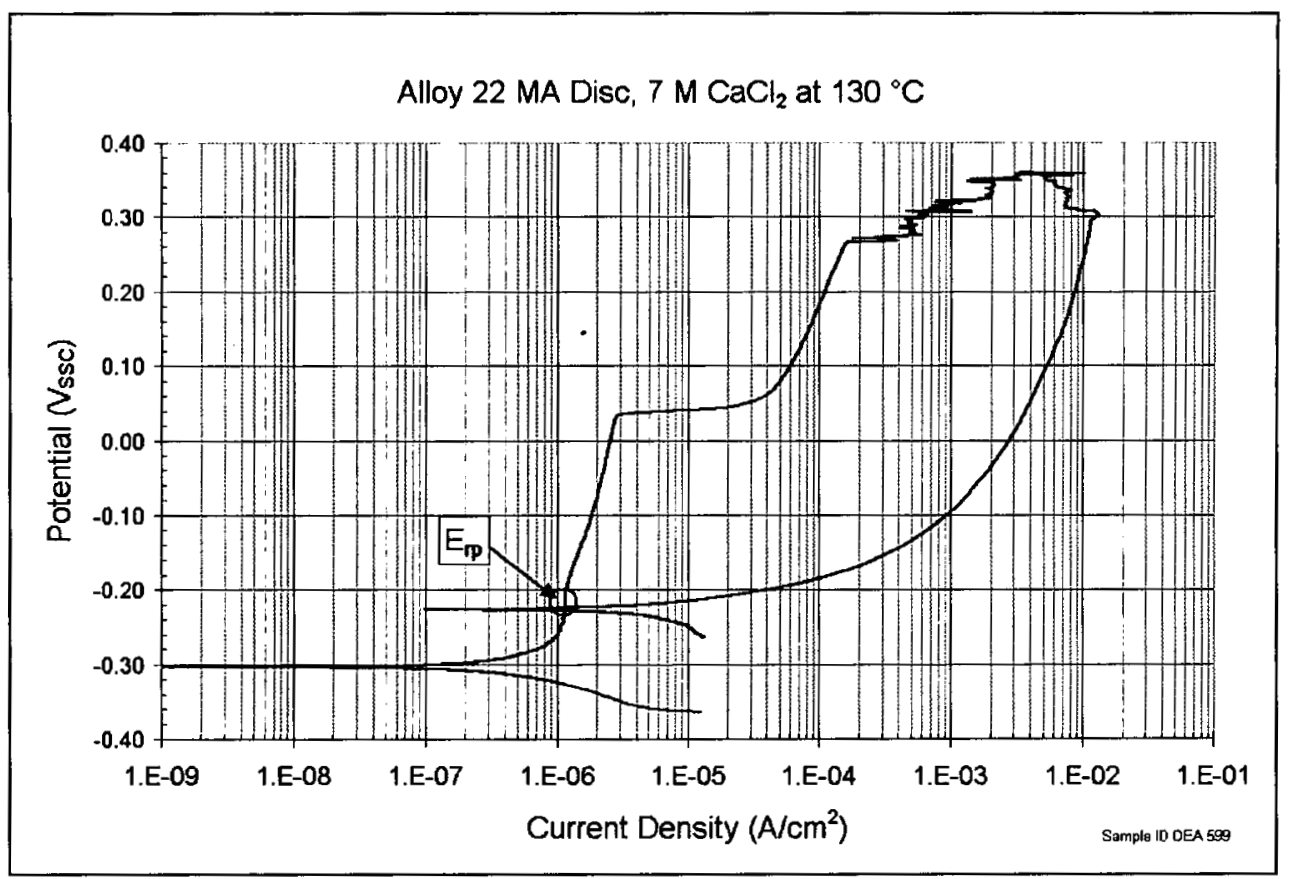

Source: DTN: LL030409812251.054 [DIRS 164185]; CP_DEA599.x/s.

Output DTN: MO0409MWDULCMW.000.

Figure 6-37. Determination of Repassivation Potential of Mill-Annealed Disc Sample (Sample DEA 599) Tested in $7 \mathrm{M} \mathrm{CaCl}_{2}$ Solution at $130^{\circ} \mathrm{C}$

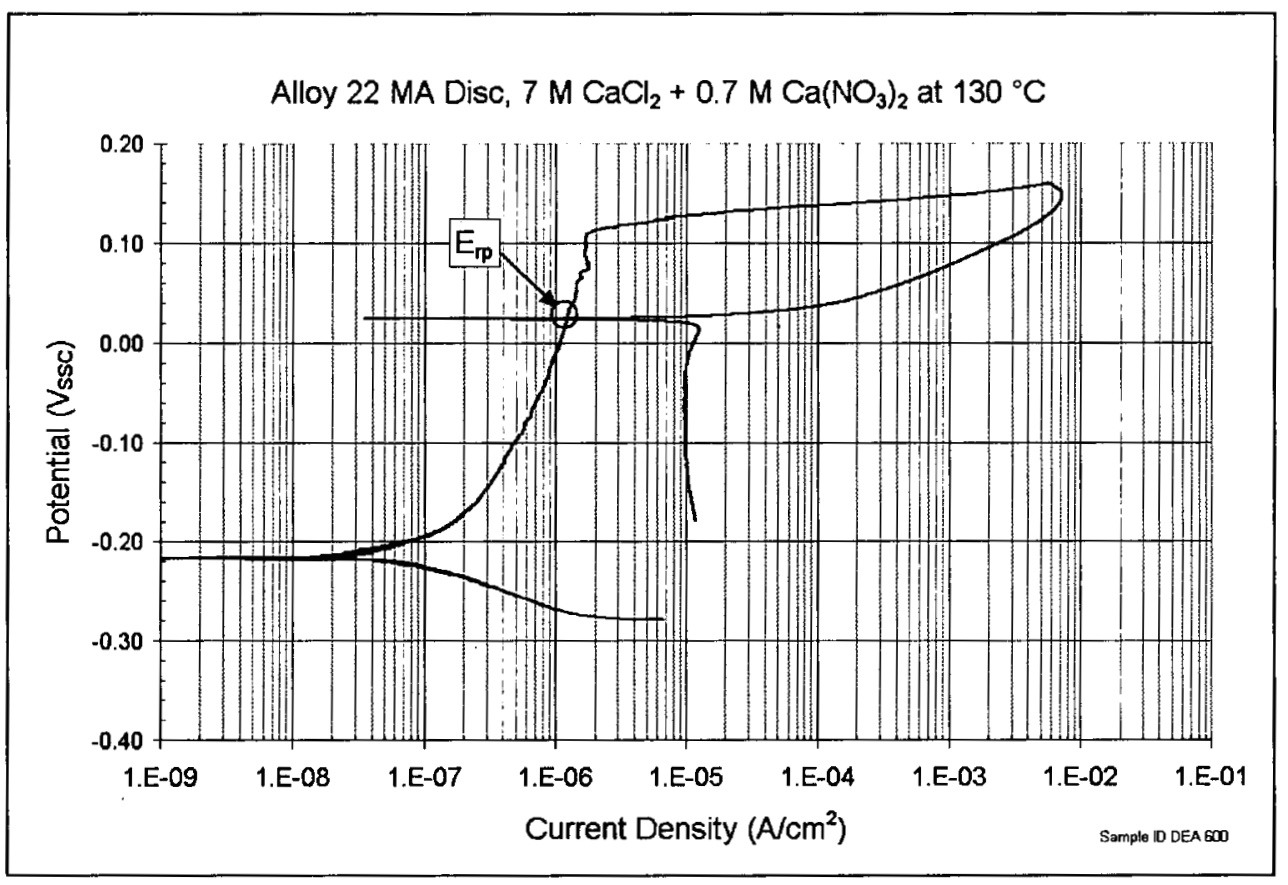

Source: DTN: LL030409812251.054 [DIRS 164185]; CP_DEA600.x/s.

Output DTN: MO0409MWDULCMW.000.

Figure 6-38. Determination of Repassivation Potential of Mill-Annealed Disc Sample (Sample DEA 600) Tested in $7 \mathrm{M} \mathrm{CaCl}_{2}+0.7 \mathrm{M} \mathrm{Ca}\left(\mathrm{NO}_{3}\right)_{2}$ Solution at $130^{\circ} \mathrm{C}$ 
Table 6-7. Crevice Repassivation Potentials Obtained from the Additional CPP Data

\begin{tabular}{|c|c|c|c|c|c|c|c|c|}
\hline$\underset{\text { ID }}{\text { Specimen }}$ & $\begin{array}{l}\text { Sample } \\
\text { Type a) }\end{array}$ & $\begin{array}{l}\text { Metallurgical } \\
\text { Condition a) }\end{array}$ & Electrolyte ${ }^{d)}$ & $\begin{array}{l}\text { Temp. } \\
\left({ }^{\circ} \mathrm{C}\right)\end{array}$ & $\mathrm{pH}^{\mathrm{b})}$ & $\begin{array}{c}{[\mathrm{Cl}]} \\
\text { (moles/kg }_{\text {water) }}^{\text {c) }}\end{array}$ & $\begin{array}{c}\left.\mathrm{CNO}_{3}\right] \\
\text { (moles/kg }^{\text {(mater) }}{ }^{c)}\end{array}$ & $\begin{array}{c}E_{\text {rcrev }} \\
(m V \text { SSC })^{e)}\end{array}$ \\
\hline DEA598 & Disc & MA & $6 \mathrm{M} \mathrm{CaCl}_{2}+0.6 \mathrm{M} \mathrm{Ca}\left(\mathrm{NO}_{3}\right)_{2}$ & 120 & 5.34 & 16.323 & 1.640 & 37 \\
\hline DEA3125 & MCA & MA & $7 \mathrm{M} \mathrm{CaCl}_{2}$ & 130 & 4.14 & 19.228 & 0.000 & -197 \\
\hline DEA599 & Disc & MA & $7 \mathrm{M} \mathrm{CaCl}_{2}$ & 130 & 4.14 & 19.228 & 0.000 & -229 \\
\hline DEA3127 & MCA & MA & $7 \mathrm{M} \mathrm{CaCl}_{2}+0.7 \mathrm{M} \mathrm{Ca}\left(\mathrm{NO}_{3}\right)_{2}$ & 130 & 5.34 & 20.815 & 2.073 & 32 \\
\hline DEA600 & Disc & MA & $7 \mathrm{M} \mathrm{CaCl}_{2}+0.7 \mathrm{M} \mathrm{Ca}\left(\mathrm{NO}_{3}\right)_{2}$ & 130 & 5.34 & 20.815 & 2.073 & 24 \\
\hline
\end{tabular}

Source: DTN: LL030409812251.054 [DIRS 164185].

NOTES: ${ }^{a} \mathrm{MCA}=$ multiple crevice assembly, $\mathrm{Disc}=$ disc sample, $\mathrm{MA}=$ mill annealed, $\mathrm{SSC}=$ silver-silver chloride reference electrode.

b A measured room temperature $\mathrm{pH}$ of 4.14 for $5 \mathrm{M} \mathrm{CaCl}_{2}$ solutions tested at $120^{\circ} \mathrm{C}$ (from the scientific notebook associated with DTN: LL030400112251.043 [DIRS 163466] (i.e., llevbare 2003 [DIRS 171329], p. 141)) was used for 7 $\mathrm{M} \mathrm{CaCl}_{2}$ solutions tested at $130^{\circ} \mathrm{C}$ (Samples DEA3125 and DEA599). Also a measured room temperature pH of 5.34 for $5 \mathrm{M} \mathrm{CaCl}_{2}$ plus $0.05 \mathrm{M} \mathrm{Ca}\left(\mathrm{NO}_{3}\right)_{2}$ solution tested at $120^{\circ} \mathrm{C}$ from DTN: LL030502212251.063 [DIRS 163456] was used for $\mathrm{CaCl}_{2}$ plus $\mathrm{Ca}\left(\mathrm{NO}_{3}\right)_{2}$ salt solutions (Samples DEA3127, DEA598, and DEA600). All pH values were measured at room temperature.

c Molal concentrations of chloride and nitrate ions of test solutions are from DTN: LL030703723121.031 [DIRS 164184].

d All solutions were considered to be fully dissociated.

- The $\mathrm{E}_{\text {rcrev }}$ values were obtained from the individual CPP curves plotted in Figures 6-33 through 6-37 following the same process as that used for the data in DTN: LL030409512251.051 [DIRS 163462].

As discussed in DTN: LL030409512251.051 [DIRS 163462], samples that showed abnormal polarization behavior (due to contamination or damage, or both during the sample fabrication process) were excluded from the analysis. In addition, Sample DEA3122 was excluded because the molal concentrations for chloride and nitrate ions and $\mathrm{pH}$ of the test solution used $\left(9 \mathrm{M} \mathrm{CaCl}_{2}\right.$ plus $0.9 \mathrm{M} \mathrm{Ca}\left(\mathrm{NO}_{3}\right)_{2}$ solution at $170^{\circ} \mathrm{C}$ ) were not available.

Also, data in DTNs: LL030409512251.051 [DIRS 163462] and LL030409812251.054 [DIRS 164185], which did not show the occurrence of localized corrosion (i.e., any "repassivation potential" reported would not represent actual repassivation of a sample undergoing localized corrosion), were not included in the model development. This is conservative since environments in which localized corrosion was not observed (even under polarization) will not contribute to the model results. One should note that some data in DTN: LL040402212251.084 [DIRS 170097] (discussed below) in which the nitrate-to-chloride ratio in the test solution was equal to 0.50 and localized corrosion may not have occurred under polarization, were used in model development. No data that did not show abnormal polarization behavior (due to contamination and/or damage during the sample fabrication process) in DTNs: LL030409512251.051 [DIRS 163462] and LL030409812251.054 [DIRS 164185] were obtained at nitrate-to-chloride ratios above 0.50 .

DTN: LL040402212251.084 [DIRS 170097] contains a data set of crevice repassivation potential measurements from as-welded MCA samples exposed to mixed $\mathrm{NaCl}$ and $\mathrm{KNO}_{3}$ solutions and mixed $\mathrm{CaCl}_{2}$ and $\mathrm{Ca}\left(\mathrm{NO}_{3}\right)_{2}$ solutions (as well as some others as discussed below). The $\mathrm{pH}$ of the high chloride and high temperature solutions was not reported and a measured room temperature $\mathrm{pH}$ of 5.34 for a $5 \mathrm{M} \mathrm{CaCl}_{2}$ plus $0.05 \mathrm{M} \mathrm{Ca}\left(\mathrm{NO}_{3}\right)_{2}$ solution tested at $120^{\circ} \mathrm{C}$ from DTN: LL030502212251.063 [DIRS 163456] was used for these $\mathrm{CaCl}_{2}$ plus $\mathrm{Ca}\left(\mathrm{NO}_{3}\right)_{2}$ salt solutions. The $\mathrm{pH}$ of these solutions was not reported because these solutions are not liquid at room temperature so the $\mathrm{pH}$ could not be measured. Also, several crevice repassivation 
potentials were not reported either because localized corrosion was not observed or the reverse scan crossed the forward scan above the passive region. In the cases where the nitrate-to-chloride ion ratio was 0.50 , a crevice repassivation potential of $+600 \mathrm{mV}$ SCC was used (samples JE3216, JE3207, JE3246, JE1772, JE3226, JE1775, JE3247, JE3285, JE3273, and JE3271). In the cases where the nitrate-to-chloride ion ratio was less than 0.50 , the data was not used (samples JE1774, JE3249, and JE3265).

Several data points were not used in the model development including mixed ion solutions of $\mathrm{NaCl}$ and $\mathrm{NaHCO}_{3}$ (samples JE3288 through JE3291 and JE3293 through JE3296), mixed ion solutions of $\mathrm{NaCl}, \mathrm{NaNO}_{3}$, and $\mathrm{MgSO}_{4}$ (samples JE3258 through JE3263). These data were not used since only the effects of the nitrate ion are accounted for in the model. DTN: LL040402212251.084 [DIRS 170097] also contained a limited data set at nitrate-tochloride ion ratios in excess of 0.50 (samples JE3252 through JE3255, JE3297 through JE3300, and JE3292) with few $\mathrm{pH}$ or crevice repassivation potentials reported. These data were not used in development of the functional forms used for evaluation of localized corrosion initiation between $20^{\circ} \mathrm{C}$ and $120^{\circ} \mathrm{C}$ (Section 6.4.4.3) due the small number of points and the lack of $\mathrm{pH}$ and crevice repassivation potential values. These data were used for corroboration in the evaluation of localized corrosion initiation at temperatures between $120^{\circ} \mathrm{C}$ and $160^{\circ} \mathrm{C}$ (Section 6.4.4.6.6).

Since the majority of the data were obtained from MCA samples, the critical potential model developed in this report would be more accurately referred to as a crevice repassivation potential model. The crevice repassivation potential data, including those additional data from DTN: LL030409812251.054 [DIRS 164185], that were considered for the critical potential model analysis are summarized in Appendix VI for those solutions not containing nitrate ions and Appendix VIII for those solutions containing nitrate ions. The appendices also provide the test conditions including sample geometry/configuration, metallurgical condition, test temperature, and electrolyte composition. For all the data in the appendices, chloride and nitrate salts were considered to be completely dissociated. This approach is considered conservative as only a fraction of the ions are expected to be available to interact with the Alloy 22 surface due to strong interionic attractions in concentrated solutions.

Investigators at the CNWRA used a similar approach to obtain the crevice repassivation potential data for Alloy 22. The data were reported by Brossia et al. (2001 [DIRS 159836], Table A-1). The test environments for the reported data are $0.005 \mathrm{M}$ to $4 \mathrm{M}$ chloride concentration and $80^{\circ} \mathrm{C}$ to $150^{\circ} \mathrm{C}$. The data set for mill-annealed and as-welded conditions was used in the critical potential model analysis. The data set is summarized in Appendix VII, along with the test conditions including sample geometry or configuration, metallurgical condition, test temperature, and electrolyte composition. For all the data in the appendix, chloride and nitrate salts were considered to be completely dissociated.

\subsubsection{Crevice Repassivation Potential Model for Waste Package Outer Barrier}

As described in Section 6.4.4.1, the WPOB is considered to be subject to localized corrosion when the corrosion potential $\left(E_{c o r r}\right)$ exceeds or is equal to the crevice repassivation potential $\left(E_{\text {rcrev }}\right)$, that is:

$$
E_{\text {corr }} \geq E_{\text {rcrev }}
$$


For the WPOB localized corrosion analysis in the postclosure repository environments, the crevice repassivation potential $\left(E_{r c r e v}\right)$ is expressed as follows:

$$
E_{\text {rcrev }}=E_{\text {rcrev }}^{o}+\Delta E_{r c r e v}^{N O_{3}^{-}}
$$

where $E_{r c r v}^{o}$ is the crevice repassivation potential in the absence of inhibitive nitrate ions, and $\Delta E_{r c r e v}^{N O_{3}^{-}}$is the crevice repassivation potential change resulting from the inhibiting effect of nitrate ion in solution.

Development of the crevice repassivation potential model for the WPOB was performed considering a set of multiple regression models as a function of the major exposure environment variables such as temperature, $\mathrm{pH}$, chloride ion concentration and nitrate ion concentration. The molal concentration unit (moles solute per $\mathrm{kg}$ water) was employed for the chloride and nitrate ion concentrations in the model development. This was to ensure an internal consistency with the total system performance assessment (TSPA), which also employs the molal concentration unit for the dissolved species. An advantage of using the molal concentrations is that the base of the concentration unit (i.e., mass of solvent, in this case water) and, thus, the concentration value, does not change with temperature.

The crevice repassivation potential data for Alloy 22 from the Project and CNWRA are listed in Appendices VI, VII, and VIII. The CNWRA data are from the data compilation reported by Brossia et al. (2001 [DIRS 159836], Table A-1). Because the crevice repassivation potential of both the mill-annealed (MA) and as-welded (ASW) Alloy 22 samples behaved similarly, the data for two metallurgical conditions were combined for the model development. A negligible effect of welding on the crevice repassivation potential was also observed by the CNWRA investigators (Brossia et al. 2001 [DIRS 159836], Section 3.2.2, Figure 3-19). The CNWRA data for the aged samples in the same data compilation table were not considered because of the high aging temperature $\left(870^{\circ} \mathrm{C}\right)$ employed for the sample preparation. The dominant secondary phase that forms at the above aging temperature, and is responsible for degradation of the corrosion resistance of the alloy, is the $\sigma$ phase, which is stable between $800^{\circ} \mathrm{C}$ and $930^{\circ} \mathrm{C}$ and forms rapidly (BSC 2004 [DIRS 169983], Section 6.6.1, Figures 76 and 77). The tetrahedrally closed-pack (TCP) phases more relevant to repository conditions are $\mathrm{P}$ and $\mu$ phases, which are stable over a wider temperature range than the $\sigma$ phase (BSC 2004 [DIRS 169983], Section 6.6.1, Figures 76 and 77). However, their formation kinetics are extremely slow at the temperatures that the waste packages are expected to experience in the repository (BSC 2004 [DIRS 169983], Section 8).

\subsection{Crevice Repassivation Potential Model in Absence of Inhibitive Nitrate Ions}

Among the functional forms that were considered, the following empirical functional form was considered to adequately describe the relationship between the crevice repassivation potential of the mill-annealed and as-welded Alloy 22 and the test environment parameters in the absence of inhibiting nitrate ions.

$$
E_{\text {rcrev }}^{o}=a_{o}+a_{1} T+a_{2} p H+a_{3} \log \left(\left[C l^{-}\right]\right)+a_{4} T \times \log \left(\left[C l^{-}\right]\right)
$$


where $E_{\text {rerev }}^{o}$ is the crevice repassivation potential (mV SSC) in the absence of inhibiting nitrate ions, $a_{o}, a_{1}, a_{2}, a_{3}$, and $a_{4}$ are constants, $T$ is the temperature $\left({ }^{\circ} \mathrm{C}\right)$, and $\left[\mathrm{Cl}^{-}\right]$is the molal ( $m$, moles $/ \mathrm{kg}$ water) chloride ion concentration.

The above functional form is similar to that for the crevice repassivation potential of Alloy 22 proposed by Brossia et al. (2001 [DIRS 159836], Section 3.2.2). Dependence of the critical potential on a logarithmic form of chloride concentration and a linear form of temperature were also used to model the critical potential by other investigators (Brossia et al. 2001 [DIRS 159836], Section 3.2.2; Frankel 1998 [DIRS 162216]; Frankel 2002 [DIRS 164140]; Kehler et al. 2001 [DIRS 162231]). The $\mathrm{pH}$ was not included in the functional forms from the above investigators because of a weak dependence of the critical potential on $\mathrm{pH}$. Relatively narrow $\mathrm{pH}$ ranges of the data used by the investigators may have led to difficulties in quantifying the effect of $\mathrm{pH}$. However, a reasonable $\mathrm{pH}$ range ( $\mathrm{pH} 4.1$ to 8, see Appendices VI and VII) of the database used in the current analysis allowed quantification of the $\mathrm{pH}$ effect. As will be discussed in Section 6.4.4.6, the crevice repassivation model shows a relatively weaker effect of $\mathrm{pH}$ relative to temperature and chloride concentration (Figure 6-61 and Figure 6-62).

Using the method of least squares, the above functional form was fit to the crevice repassivation potential data for the mill-annealed and as-welded conditions in Appendices VI and VII. The model fitting was performed using Mathcad version 11.1, and the Mathcad worksheet for the model fitting is given in the output DTN: MO0409MWDULCMW.000. The value of the coefficients and their uncertainty ( \pm 1 standard deviation) of the model parameters from the fitting were determined to be: $a_{0}=214.089 \pm 46.880, a_{1}=-3.696 \pm 0.476, a_{2}=25.284 \pm 5.641$, $a_{3}=-252.181 \pm 53.912$, and $a_{4}=1.414 \pm 0.547$. The covariance matrix resulting from the fitting procedure was determined to be:

$$
C V=\left[\begin{array}{ccccc}
2.198 \times 10^{3} & -1.516 \times 10^{1} & -8.325 \times 10^{1} & -1.805 \times 10^{3} & 1.590 \times 10^{1} \\
-1.516 \times 10^{1} & 2.267 \times 10^{-1} & -1.240 \times 10^{0} & 1.877 \times 10^{1} & -1.996 \times 10^{-1} \\
-8.325 \times 10^{1} & -1.240 \times 10^{0} & 3.183 \times 10^{1} & -3.237 \times 10^{1} & 7.425 \times 10^{-1} \\
-1.805 \times 10^{3} & 1.877 \times 10^{1} & -3.237 \times 10^{1} & 2.907 \times 10^{3} & -2.868 \times 10^{1} \\
1.590 \times 10^{1} & -1.996 \times 10^{-1} & 7.425 \times 10^{-1} & -2.868 \times 10^{1} & 2.995 \times 10^{-1}
\end{array}\right] \text { Eq. 6-33) }
$$

The correlation coefficient $R$ for the fit is 0.872 , and the coefficient of determination $R^{2}$ is 0.760 . $R^{2}$ is the ratio of the measures of variation explained by the regression model to the total variation present in the output variable under consideration. Values of $R^{2}$ vary between 0 (no variation explained and a very poor regression model) to 1 (perfect explanation of the model variation by the regression model). With the measure of a $R^{2}$ value of 0.760 , the regression model fits the experimental data reasonably well.

Variation in the crevice repassivation potential data for a given test condition are mostly due to the uncertainties associated with the test procedures and crevice repassivation potential selection criteria, in addition to some randomness in the localized corrosion process. Therefore, the entire variance of the model is due to uncertainty. ASTM G 61-86 (1987 [DIRS 127897]) specifies, "when the standard procedure for the CPP measurements is followed, an investigator's data should fall within the range of \pm 2 standard deviations of the mean because this includes $95 \%$ of 
all data provided random variations are the only source of error." While this criterion was specified for replicate CPP measurements obtained under the same exposure conditions, it is reasonable to apply this criterion to a model based on fitting these same measurements to a regression surface. Therefore, the uncertainty of the coefficients of the regression model is limited to \pm 2 standard deviations. Also, the approach and criteria for obtaining the crevice repassivation potentials from the CPP curves are highly conservative; therefore, limiting the uncertainty of the model parameters to \pm 2 standard deviations is sufficient to capture the model uncertainty.

For the waste package degradation analysis, variability in the crevice repassivation potential among waste packages is represented by the temporally and spatially varying waste package temperature and water chemistry contacting the waste package. In addition, the chemistry of water contacting waste package may vary depending on the thermal and hydrologic condition on the waste package surface, for example, the presence or absence of dripping water. In the absence of dripping water, the chemistry of water film forming on the waste package surface would be determined by the interactions of the chemical constituents in the dust on the surface with the water vapor in the humid-air around the waste package.

The model results and the Project's data are shown as a function of temperature and chloride concentration in Figure 6-39 and Figure 6-40 respectively. Note the model results are for the average values of the test environment parameters (temperature, chloride concentration and $\mathrm{pH}$ ) of the source data. The $E_{\text {rcrev }}$ data for the test conditions off the average values, therefore, are not bound within \pm 2 standard deviations of the model mean.

As shown in Figure 6-39, the crevice repassivation potential is a strong function of temperature and a relativity weaker function of chloride concentration. This strong dependence of the crevice repassivation potential of Alloy 22 on temperature was also reported by others (Brossia et al. 2001 [DIRS 159836], Section 3.2.2; Kehler et al. 2001 [DIRS 162231]). A logarithmic dependence of the crevice repassivation potential on chloride concentration (Figure 6-40) shows that the crevice repassivation potential decreases rapidly with chloride concentration for the low chloride concentration range, and the dependence becomes weaker as the chloride concentration increases. Although not shown here, it is seen from the value of the $\mathrm{pH}$ term coefficient that the crevice repassivation potential is a weak function of $\mathrm{pH}$.

The model results and the CNWRA data are shown as a function of temperature and chloride concentration in Figure 6-41 and Figure 6-42, respectively. As for the above discussion of the Project's data, the model results are for the average values of the test environment parameters (temperature, chloride concentration and $\mathrm{pH}$ ) of the source data. The $E_{\text {rcrev }}$ data for the test conditions off the average values are, therefore, not within \pm 2 standard deviations of the model mean. The behavior of the crevice repassivation potential data from CNWRA and their dependence on temperature and chloride concentration are consistent with Project data. 


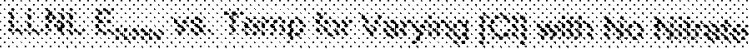

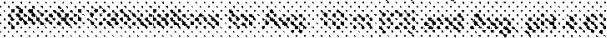

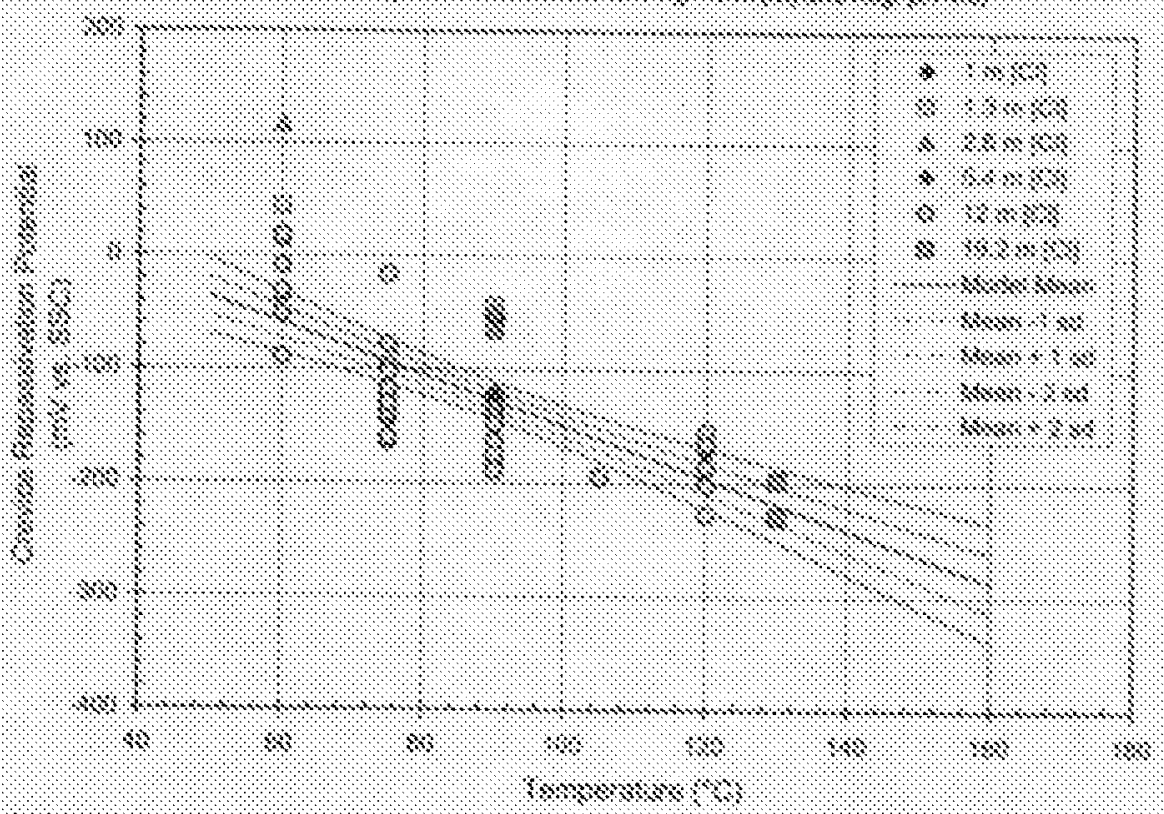

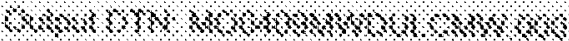

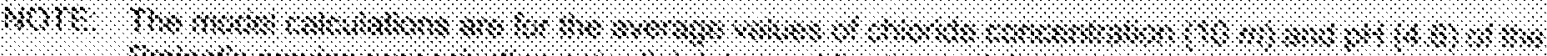

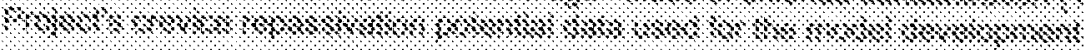

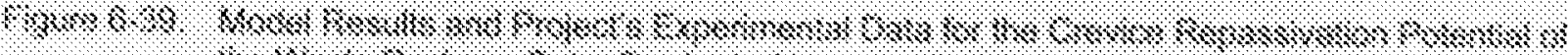

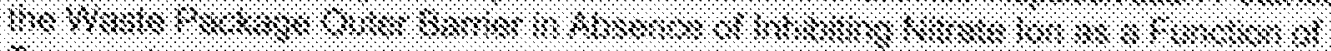
$3,1,1, n$

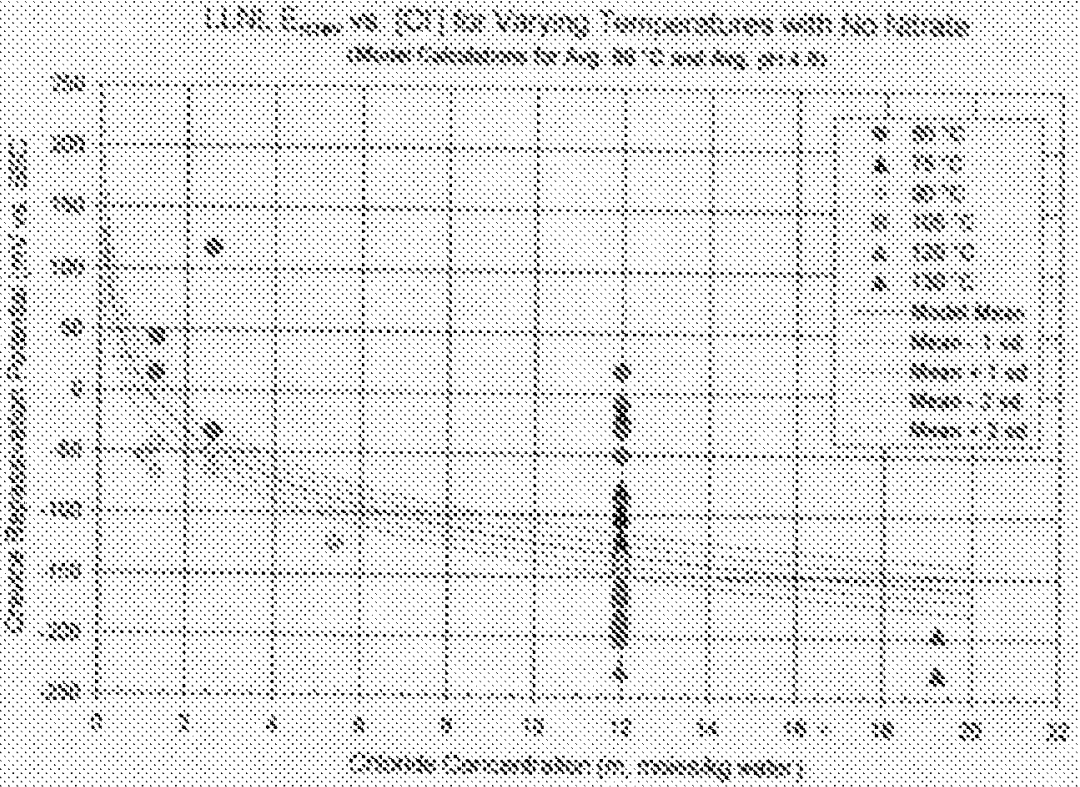

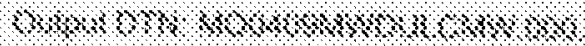

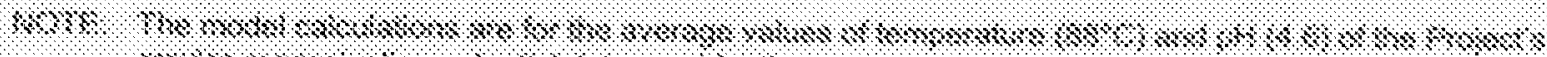

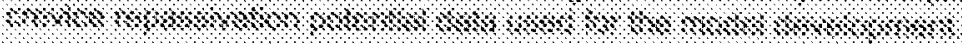

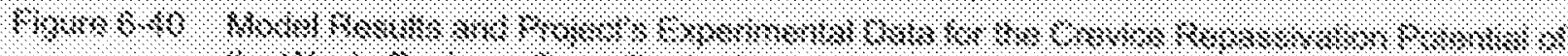

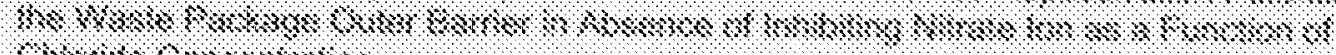
$3,06,0,3,13$ 


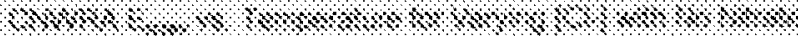

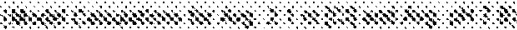

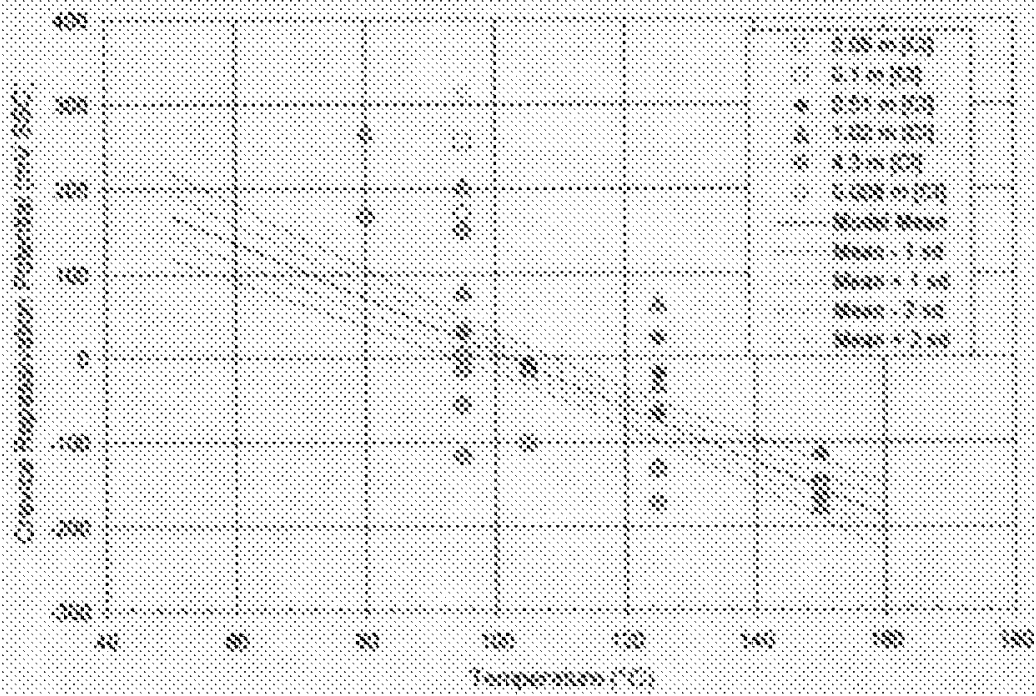

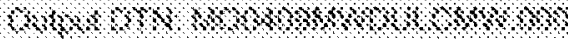

n

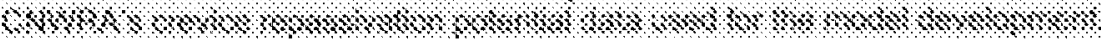

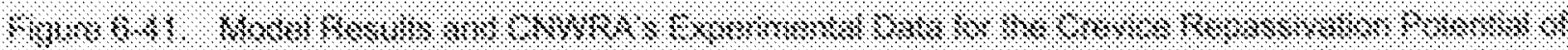

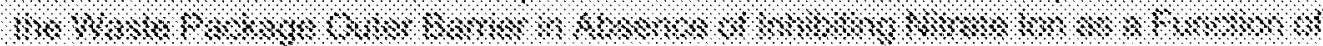

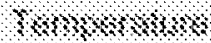

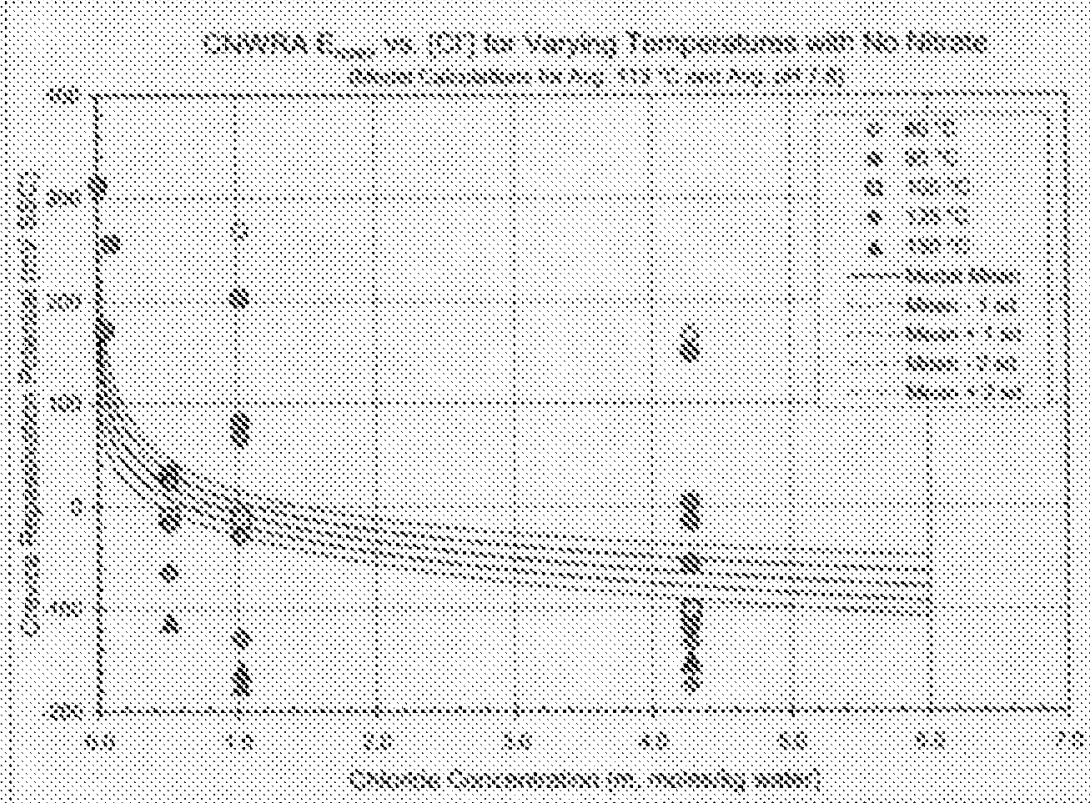

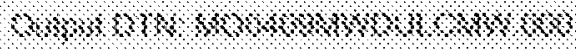

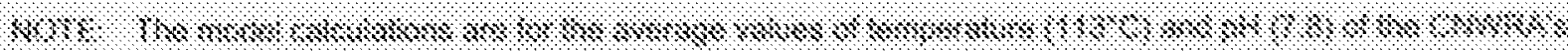

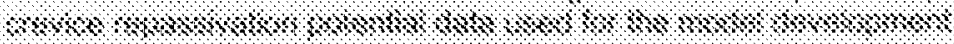

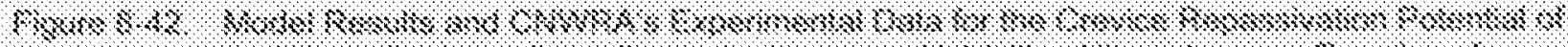

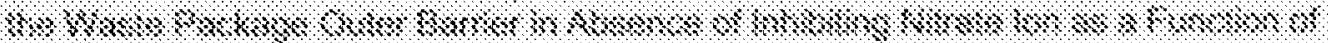
$3,1,0,1,0$ 


\subsection{Crevice Repassivation Potential Changes in Presence of Inhibitive Nitrate Ions}

The crevice repassivation potential data in Appendix VIII show that nitrate ions generally raise the crevice repassivation potential, decreasing the possibility of localized corrosion initiation. In this report, the effect of nitrate ions on the crevice repassivation potential is represented with an adjustment factor $\left(\Delta E_{r c r e v}^{N O_{3}^{-}}\right)$to the crevice repassivation potential in the absence of nitrate ions ( $E_{\text {rcrev }}^{o}$ ). That is, $\Delta E_{\text {rcrev }}^{N O_{3}^{-}}$is defined as the difference between the crevice repassivation potential with and without nitrate ions at the same chloride concentration and temperature. As can be seen in Appendix VIII, $\Delta E_{\text {rcrev }}^{\mathrm{NO}_{3}^{-}}$is obtained by first determining the crevice repassivation potential in a solution with a given chloride concentration and at a given temperature in the absence of nitrate ions (here taken to be the median value of $E_{\text {rcrev }}^{o}$ ), then subtracting this value from the crevice repassivation potential determined in a solution with the same chloride concentration and at the temperature in the presence of nitrate ions. As can be seen from Appendix VIII, nitrate ions generally raise the crevice repassivation potential by a significant amount.

The following functional form was found to adequately describe the effect of nitrate ions on the crevice repassivation potential.

$$
\Delta E_{r c r e v}^{N O_{3}^{-}}=b_{o}+b_{1}\left[\mathrm{NO}_{3}^{-}\right]+b_{2} \frac{\left[\mathrm{NO}_{3}^{-}\right]}{\left[\mathrm{Cl}^{-}\right]}
$$

where $\Delta E_{r c r v}^{N O_{3}^{-}}$is the change of the crevice repassivation potential due to the inhibiting effect nitrate ions, $\left[\mathrm{Cl}^{-}\right]$is the molal (moles/kg water) concentration of chloride ions, $\left[\mathrm{NO}_{3}^{-}\right]$is the molal concentration of nitrate ions, and $b_{o}, b_{l}$, and $b_{2}$ are the model coefficients. Using the method of least squares, the above functional form was fit to the $\Delta E_{r c r v}^{\mathrm{NO}_{3}^{-}}$data in Appendix VIII. The model coefficient fitting was performed using Mathcad version 11.1 (the Mathcad worksheet is provided in the output DTN: MO0409MWDULCMW.000).

The model coefficients and their uncertainty ( \pm 1 standard deviation) resulting from the fitting procedure were determined to be: $b_{o}=22.589 \pm 24.113, b_{1}=33.748 \pm 5.180$, and $b_{2}=749.745 \pm$ 95.491. The covariance matrix resulting from the fitting procedure was determined to be:

$$
C V=\left[\begin{array}{ccc}
5.814 \times 10^{2} & -1.737 \times 10^{0} & -1.542 \times 10^{3} \\
-1.737 \times 10^{0} & 2.683 \times 10^{1} & -2.291 \times 10^{2} \\
-1.542 \times 10^{3} & -2.291 \times 10^{2} & 9.119 \times 10^{3}
\end{array}\right]
$$

The correlation coefficient $R$ for the fit is 0.842 , and the coefficient of determination $R^{2}$ is 0.709 . With the measure of a $R^{2}$ value of 0.709 , the regression model fits the experimental data fairly well.

In the above model, the competing effect of the chloride and nitrate ions on the crevice repassivation potential is represented with the ratio of the ion concentrations (nitrate-to-chloride 
ion ratio). The model uses a linear relationship between $\Delta E_{r c r e v}^{\mathrm{NO}_{3}^{-}}$and the nitrate-to-chloride ion ratio. Because the effect of the measurement uncertainty of the CPP tests has already been captured in the crevice repassivation potential model in the absence of nitrate ions, the median values of the $\Delta E_{r c r e v}^{\mathrm{NO}_{3}^{-}}$model coefficients are used to determine the crevice repassivation potential $\left(E_{\text {rcrev }}\right)$ (Equation 6-31). Because the maximum nitrate and chloride ion concentrations in the data used for construction of the model were 18 and 36 molal ( $m$, moles per $\mathrm{kg}$ water), respectively, for ion concentrations above these values, these upper limits are imposed (i.e., if the exposure environment nitrate ion concentration is greater than 18 molal, the model is evaluated with a nitrate ion concentration of 18 molal. Similarly, because the maximum nitrate-to-chloride ion concentration ratio in the data used for construction of the model was 0.5 , this upper limit is imposed (i.e., if the exposure nitrate-to-chloride ion concentration ratio exceeds 0.5 , the value 0.5 is used in evaluating the model). Also, if only a negligible amount of nitrate ions are present (i.e., when the nitrate concentration is less than $0.001 \mathrm{~m}$ ), the crevice repassivation potential is conservatively represented by $E_{r c r e v}^{o}$ alone without use of $\Delta E_{r c r e v}^{{N O_{3}^{-}}^{2}}$.

Variability in $\Delta E_{\text {rcrev }}^{\mathrm{NO}_{3}^{-}}$among waste packages is represented by the temporally and spatially varying nitrate and chloride ion concentrations of solutions contacting the waste package. The solution chemistry contacting the waste packages may vary due to variations in the thermal and hydrologic conditions on the waste package surface and due to the presence or absence of dripping water.

Figure 6-43 shows the $\Delta E_{\text {rcrev }}^{N O_{3}^{-}}$model results (at $\mathrm{pH}$ of 5.38) and the $6 \mathrm{~m}$ chloride ion concentration data at nitrate-to-chloride ion ratios of 0.05 ( $0.3 \mathrm{~m}$ nitrate ion concentration), 0.15 ( $0.9 m$ nitrate ion concentration), and 0.50 ( $3 \mathrm{~m}$ nitrate ion concentration). Figure 6-44 shows the $\Delta E_{r c r e v}^{N O_{3}^{-}}$model results (at $27.1 \mathrm{~m}$ chloride ion and $\mathrm{pH}$ of 5.34) and the 20,24 , and $36 \mathrm{~m}$ chloride ion concentration data at nitrate-to-chloride ion ratios of $0.05(1,1.2$, and $1.8 m$ nitrate ion concentrations, respectively for the 20,24 , and $36 m$ chloride ion solutions $), 0.15(3,3.6$, and $5.4 \mathrm{~m}$ nitrate ion concentrations, respectively for the 20,24 , and $36 \mathrm{~m}$ chloride ion solutions), and $0.50(10,12$, and $18 m$ nitrate ion concentrations, respectively for the 20,24 , and $36 \mathrm{~m}$ chloride ion solutions). Note that the model results do not necessarily encompass the spread in the measured/calculated $\Delta E_{\text {rcrev }}^{N O_{3}^{-}}$values. This may be due to the use of only the median values of $E_{\text {rcrev }}^{o}$ during the development of the measured/calculated $\Delta E_{r c r e v}^{N O_{3}^{-}}$values. Nonetheless, the model is sufficient for its intended use (i.e., it provides an estimate of the beneficial effect of nitrate ions on the crevice repassivation potential). The curves in Figure 6-43 and Figure 6-44 change slope at $3 \mathrm{~m}$ nitrate ion and $13.55 \mathrm{~m}$ nitrate ion, respectively, because the nitrate-to-chloride ion ratio exceeds 0.50 at these concentrations and, as discussed above, the model is evaluated at a constant nitrate-to-chloride ion ratio of 0.50 under those conditions. This is a conservative approach to evaluating the repassivation potential because repassivation potentials increase with increasing nitrate concentration as shown in Figure 6-43 and Figure 6-44. 


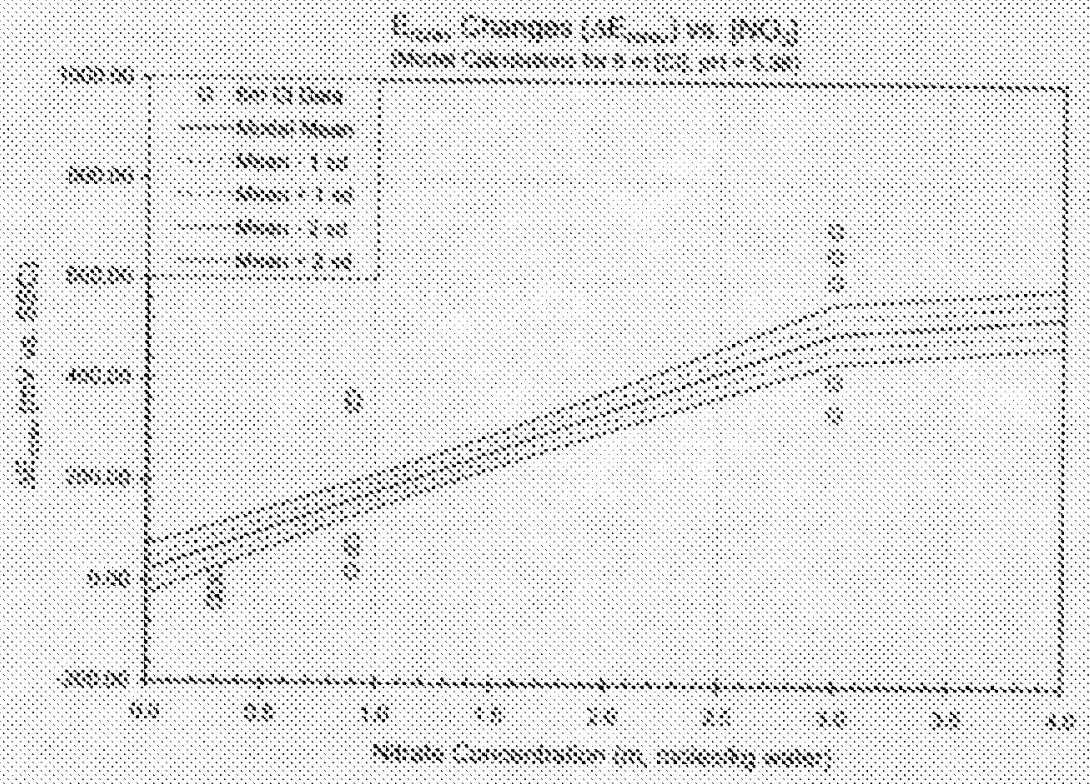

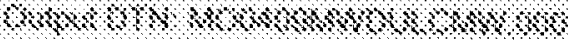

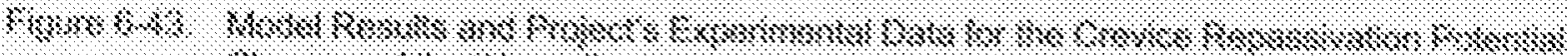

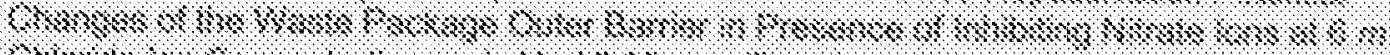

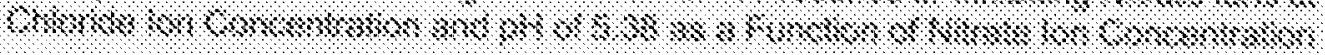

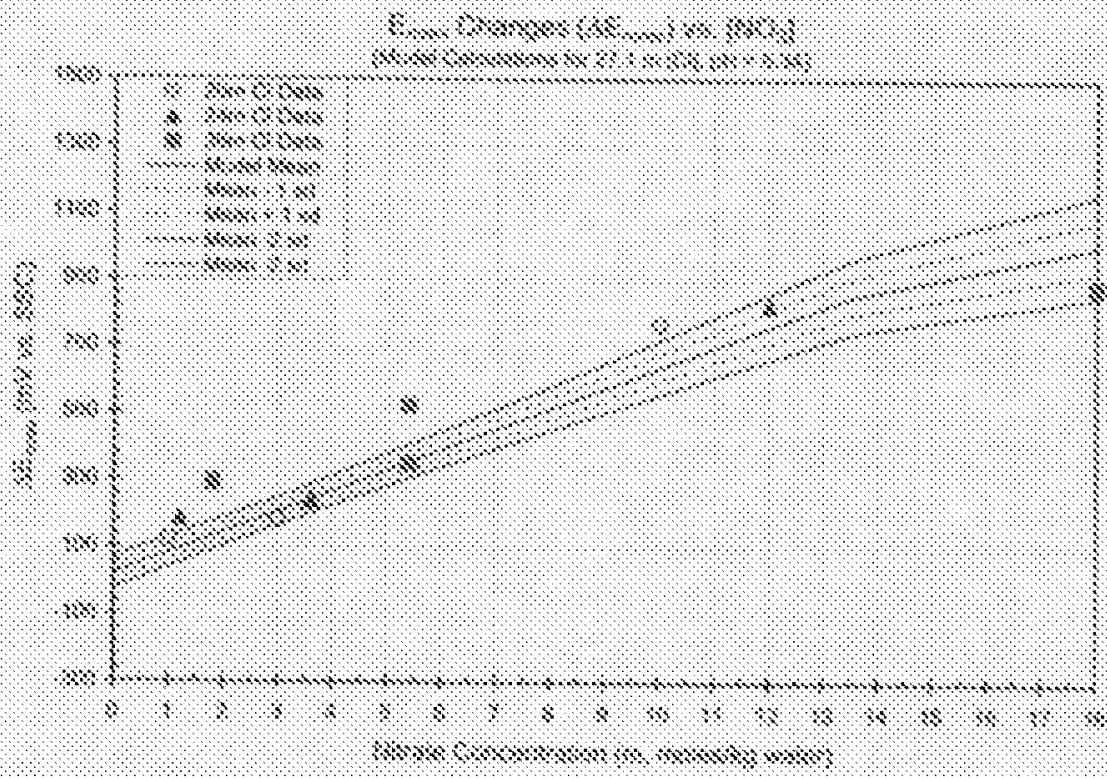

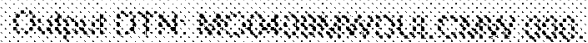

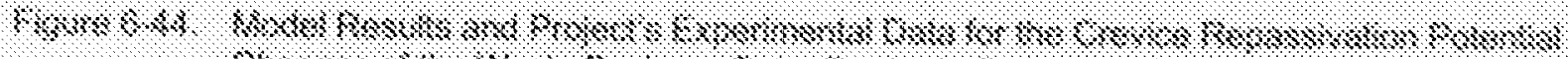

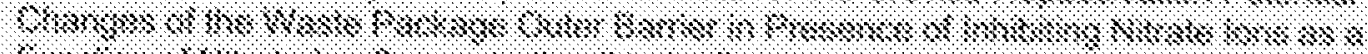

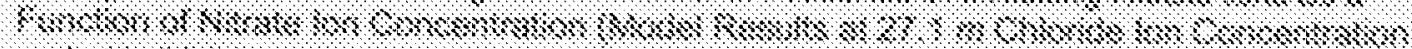
$3 \times 6 \times 1088 \%$ 


\subsubsection{Long-Term Open-Circuit Corrosion Potential Data Analysis}

Because the corrosion potential of Alloy 22 may change over time, it is important to know the most probable value of long-term corrosion potential $\left(E_{\text {corr }}\right)$ for Alloy 22 under different environmental conditions to evaluate the localized corrosion susceptibility of the WPOB in the repository. As discussed above, localized corrosion will only occur when $E_{c o r r}$ is equal to or greater than a critical potential (the crevice repassivation potential $\left(E_{\text {rcrev }}\right)$ in the current model). That is, if $E_{\text {corr }}<E_{\text {rcrev }}$, general or passive corrosion will occur.

The specimens used to evaluate $E_{\text {corr }}$ of Alloy 22 as a function of immersion time were machined from sheet and bar stock. There were two main groups of specimens, (1) Welded U-bend specimens and (2) Untested rod specimens. Welded plus aged samples in DTN: LL040402212251.084 [DIRS 170097] were not considered in this analysis because the aging treatment was more severe $\left(700^{\circ} \mathrm{C}\right.$ for 173 hours) than could reasonably be expected in the repository environment (Figure 6-2). Approximately half of the U-bend specimens tested for long-term corrosion potential were from the Long Term Corrosion Test Facility (LTCTF), and the other half of the U-bend specimens were not previously exposed to any electrochemical test condition. The U-bend specimens from the LTCTF already had a solution formed passive film and other surface alterations from exposure in the LTCTF. Both types of U-bend specimens were tested in the as-received (AR) or as-machined conditions, which corresponded to a root-meansquare roughness of $32 \mu$ inch. The U-bend specimens were fabricated from $3 / 4-i n . ~(19.05 \mathrm{~mm})$ wide and 1/16-in. $(1.588 \mathrm{~mm})$ thick metal strips according to ASTM G 30-94 (1994 [DIRS 137688]). The specimens were degreased in acetone and alcohol before testing. During the long-term open-circuit corrosion potential monitoring, the U-bend specimens were fully immersed in the electrolyte of interest. The rod specimens were all previously untested. Rod specimens were $1 / 4$ in. $(6.35 \mathrm{~mm})$ in diameter and 12 in. $(304.8 \mathrm{~mm})$ long. They were polished with 600-grit paper and degreased with acetone and alcohol before testing. The data and test details reported in DTNs: LL020711612251.017 [DIRS 161204] and LL040402112251.083 [DIRS 170283] are summarized in Appendix V.

The samples are identified by a combination of letters and numbers. In earlier tests (DTN: LL020711612251.017 [DIRS 161204]), these were either DUB or DEA followed by three or four digits. The letter D stands for Alloy 22, the second letter stands for the type of sample, that is, $U$ for $U$-bend specimen and E for electrochemical (or rod) specimen. The third letter could be either an A (mill annealed or not welded) or B (contains weld material). In the newer tests, some rod specimens were designated starting with the letters JE (for John Estill) or $\mathrm{KE}$ (for Kenneth Evans) followed by four digits. One specimen tested in BSW solution (Cell 4) was a double U-bend specimen and had a longer designation (ARC22 U20A and ARC22 U20B).

Many different electrolyte solutions were used in the tests (Appendix V for the type of solutions tested). These included four multi-ionic solutions and other simpler salt solutions. The solutions from the LTCTF tanks (i.e., SDW, SAW, and SCW) are referred to as "aged" solution (approximately 4.5-years old at the time the tests started). The multi-ionic solutions that were freshly prepared (not from the LTCTF tanks) are referred to as "fresh" solution. For some solution compositions, more than one temperature was used for testing (Appendix V). 
The volume of the electrolyte solution in each cell was two liters. The electrolyte solutions were naturally aerated; that is, a stream of air was circulated above the level of the solution. This stream of air exited the vessel through a condenser to avoid evaporation of the electrolyte. The electrochemical potentials are reported in the saturated silver silver chloride scale (SSC). At ambient temperature, the SSC scale with the reference electrode in a saturated $\mathrm{KCl}$ solution is $199 \mathrm{mV}$ more positive than the standard hydrogen electrode (SHE) (Sawyer and Roberts 1974 [DIRS 162259], pp. 39 to 45, Table 2-4). The values of the corrosion potentials for each specimen (electrode) were acquired using a commercial data acquisition unit that had the input resistance set at $10 \times 10^{9} \mathrm{ohm}$. Typically, the measurements were acquired every minute for the first week of testing and every hour after the first week. The data was logged into the internal memory of the data acquisition unit and simultaneously to a spreadsheet in an interfaced personal computer. Data back up was performed monthly.

The long-term corrosion potential behavior of some Alloy 22 samples in SDW, SAW, and SCW solutions from the LTCTF are shown in Figure 6-45. The data plotted in Figure 6-45 was taken weekly from the raw $E_{\text {corr }}$ versus time data contained in DTNs: LL020711612251.017 [DIRS 161204] and LL040402112251.083 [DIRS 170283]. Step-function discontinuities shown in the graphs indicate periodic changes of reference electrodes. Figure 6-45 shows that after an initial period of 300 to 400 days, the $E_{\text {corr }}$ did not change substantially with time. It is shown that the results of the welded U-bend samples (samples DUB052 and DUB159) and (nonwelded) rod samples (samples DEA2850, DEA2851, and DEA2852) in aged SAW at $90^{\circ} \mathrm{C}$ show no significant differences in their long-term open-circuit corrosion potential behaviors. Figure 6-45 shows two sets of $E_{\text {corr }}$ versus time curves for Alloy 22. In the acidic multi-ionic SAW solution the potentials tended to be in the +200 to $+400 \mathrm{mV}$ versus SSC range while in the alkaline multiionic solutions SDW and SCW, the potentials tended to be in the -100 to $+100 \mathrm{mV}$ versus SSC range. In the SAW solution, the potential was higher at $60^{\circ} \mathrm{C}$ and $90^{\circ} \mathrm{C}$ than at $25^{\circ} \mathrm{C}$.

Figure 6-46 compares the long-term corrosion potential evolution of freshly polished Alloy 22 rods in freshly prepared SAW at $90^{\circ} \mathrm{C}$ (Cell 9) with the corrosion potential evolution of the welded U-bend and rod samples in aged SAW at $90^{\circ} \mathrm{C}$ (Figure 6-45) for the first 150 days of testing. Initially the Alloy 22 rods in the fresh SAW solution had the corrosion potential on the order of about $-150 \mathrm{mV}$ versus SSC. Over approximately 50 days of testing, the corrosion potential increased rapidly to a more noble potential value of approximately $330 \mathrm{mV}$ versus SSC. Figure 6-45. shows that for the remainder of the testing time, the corrosion potential slowly reached a maximum value near $400 \mathrm{mV}$ versus SSC. This high value of $E_{\text {corr }}$ is probably due to the formation of a more protective chromium rich oxide film on the Alloy 22 electrodes. The test results show that, regardless of the initial condition of the metal surface or the age of the electrolyte solution, eventually Alloy 22 undergoes ennoblement in SAW. This ennoblement is probably promoted by both the $\mathrm{pH}$ value and the presence of nitrate in the solution (Estill et al. 2003 [DIRS 163849]). Such an ennoblement of Alloy 22 with time has also been reported elsewhere, and it was observed that the ennoblement was more significant in acidic solutions (Jayaweera et al. 2003 [DIRS 162225], Figures 9.12 and 9.13; Dunn et al. 2003 [DIRS 164138], Figures 8 and 9). 


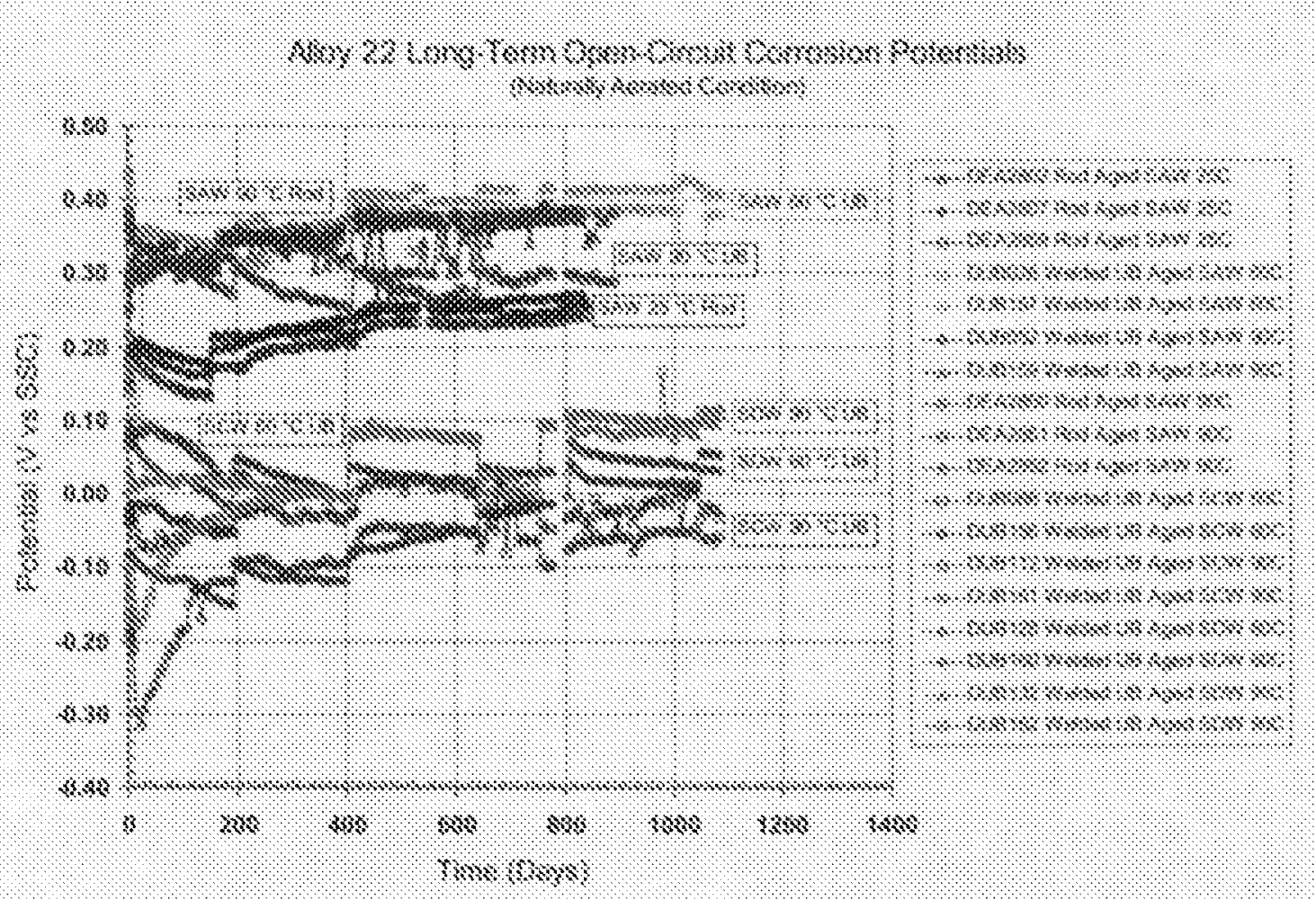

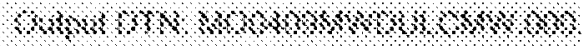

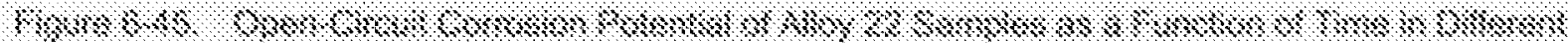

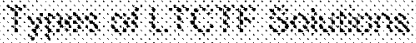

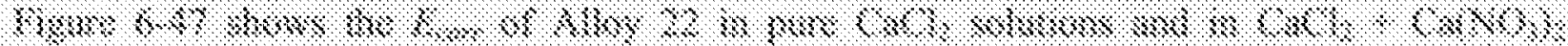

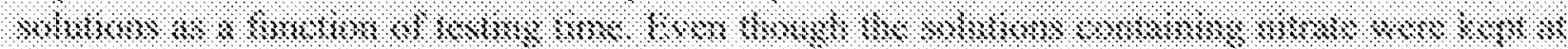

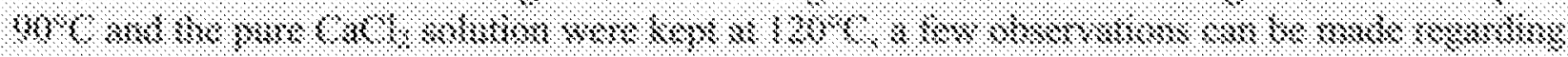

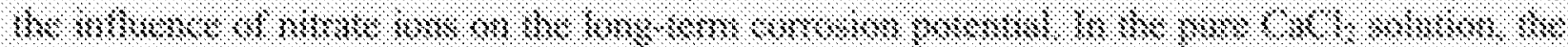

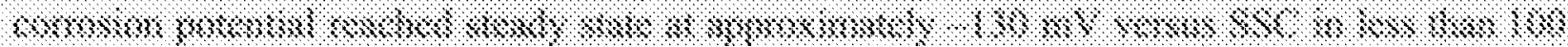

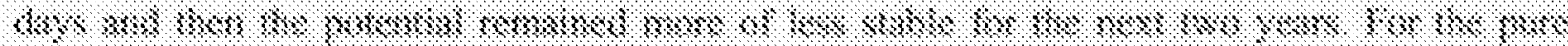

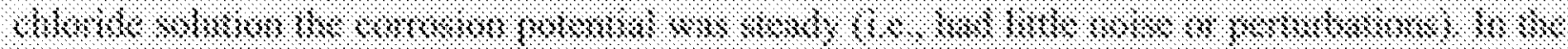

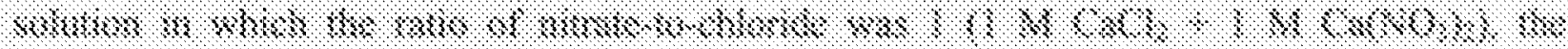

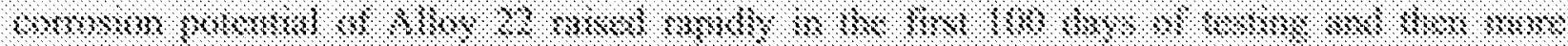

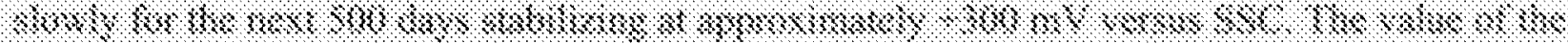

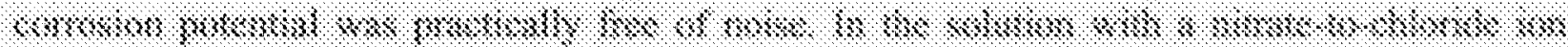

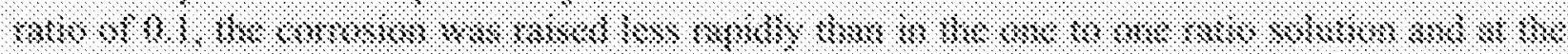

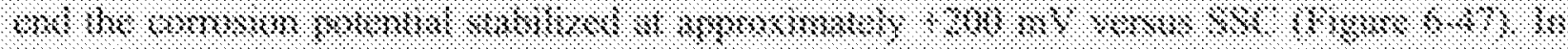

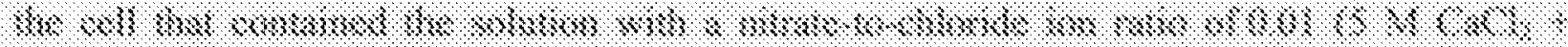

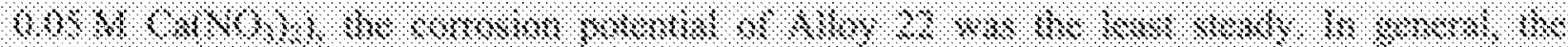

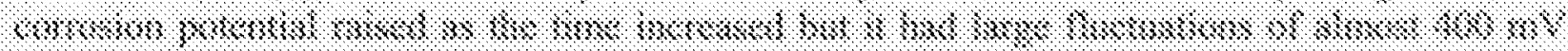

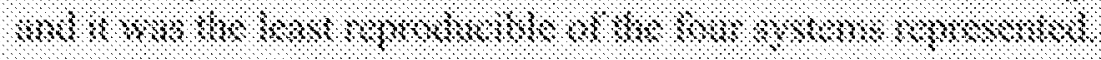




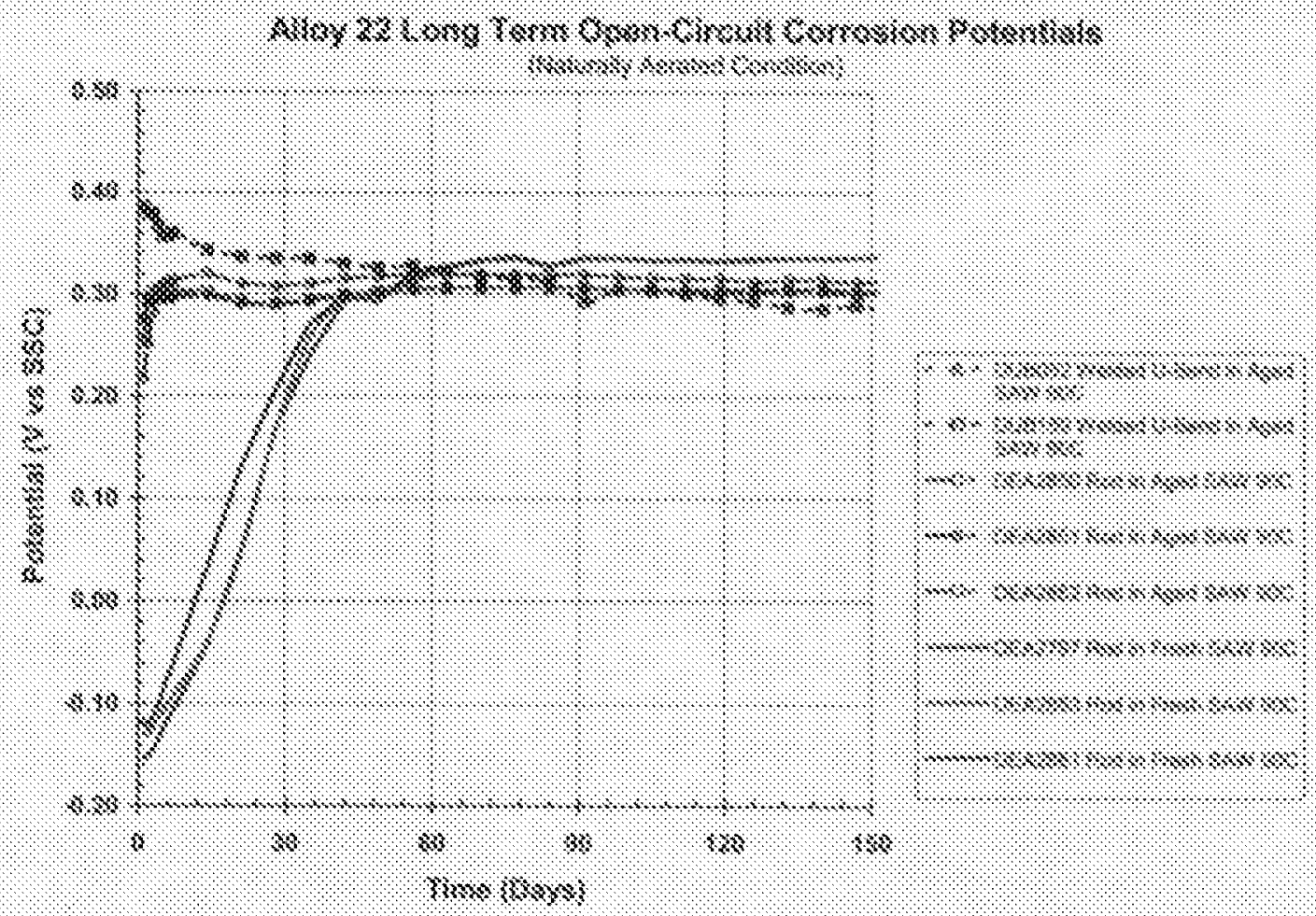

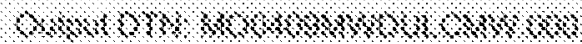

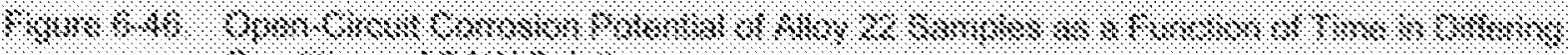

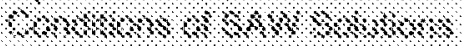

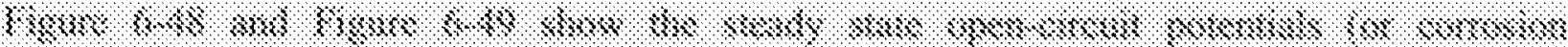

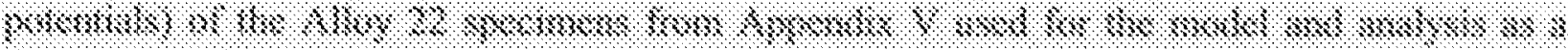

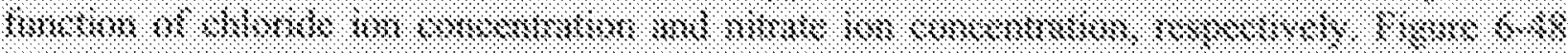

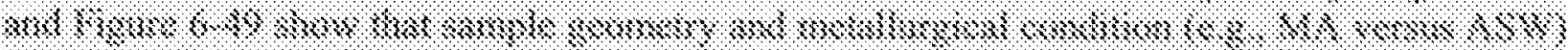

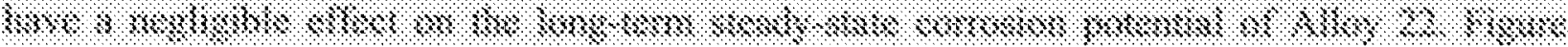

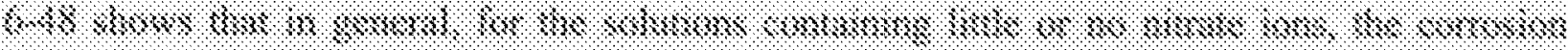

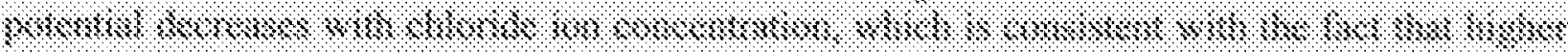

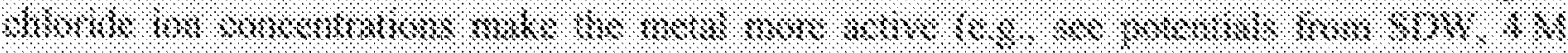

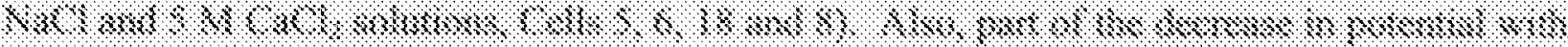

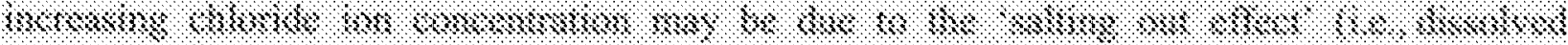

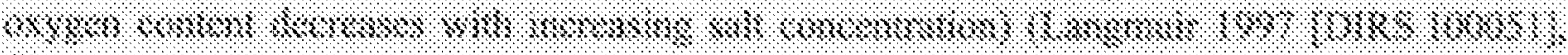
\%

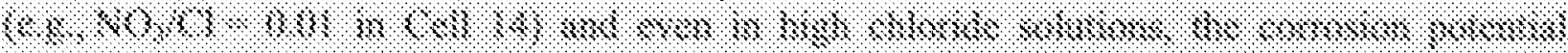

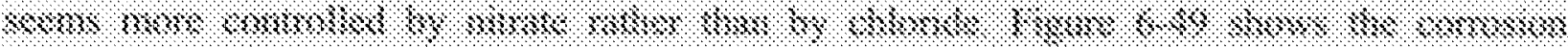

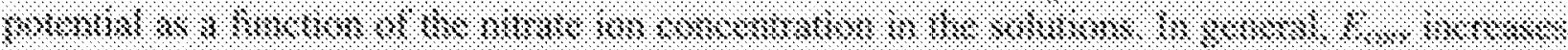

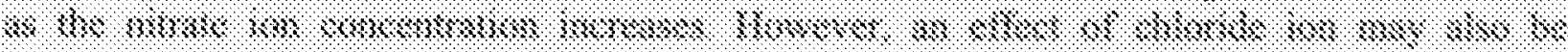

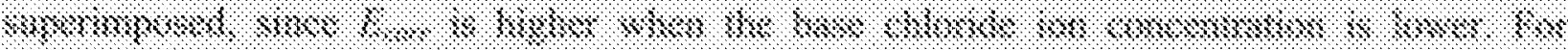

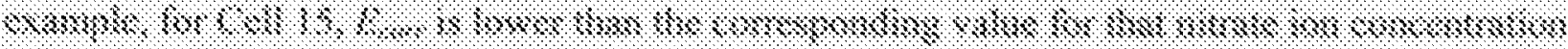

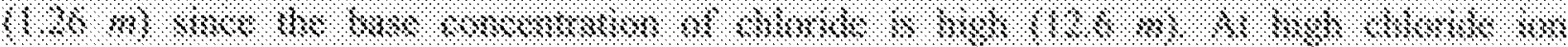

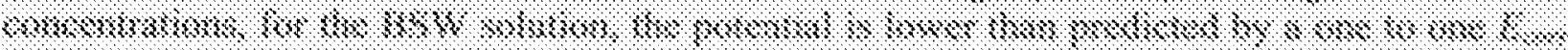




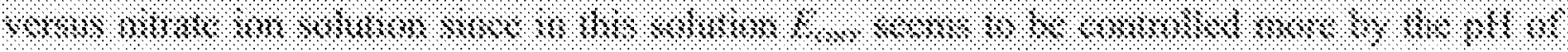
$3 \times 0 \times 1 \times 18$;

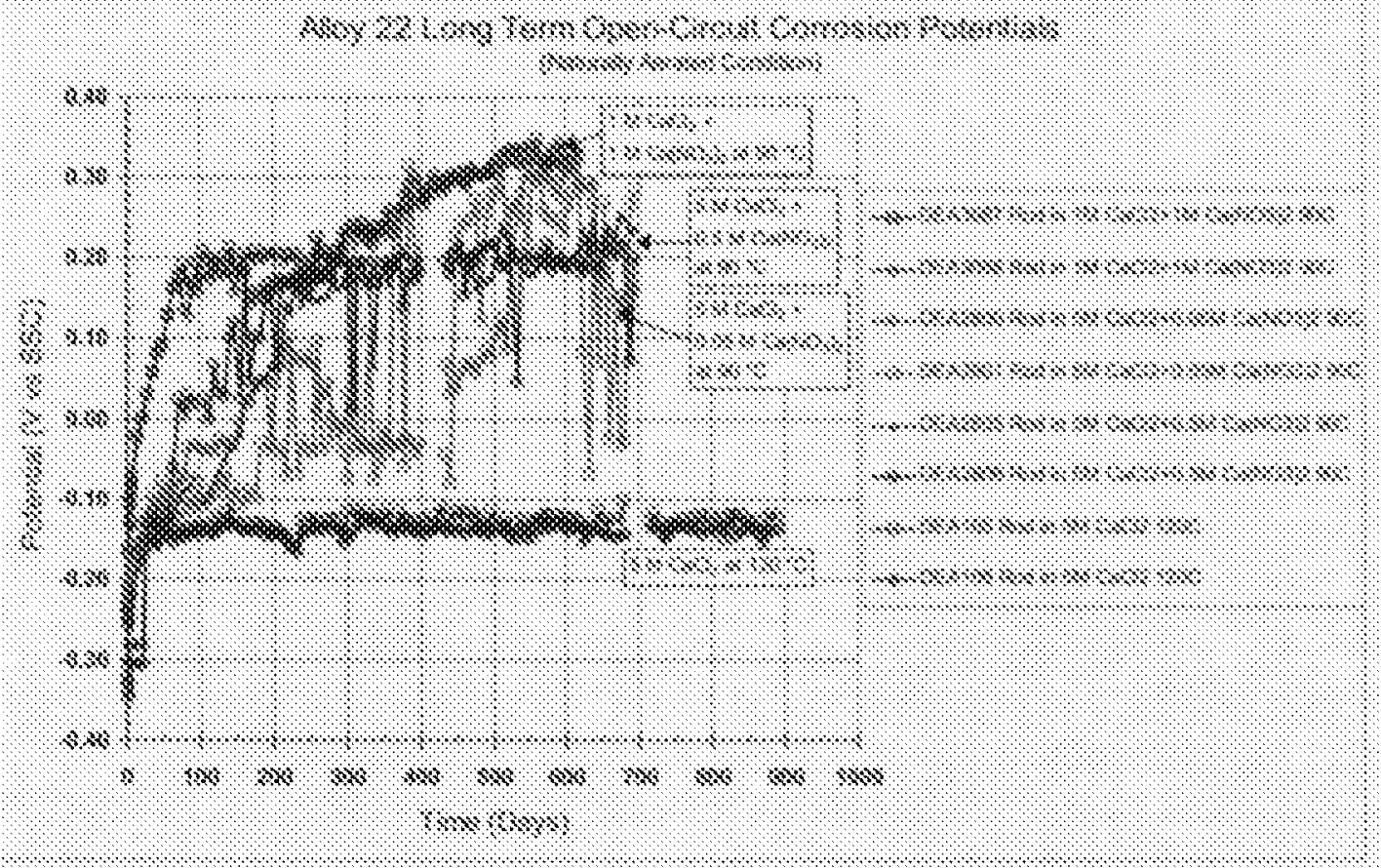

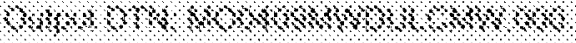

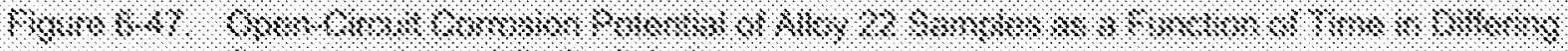

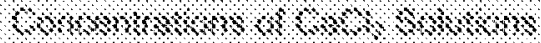

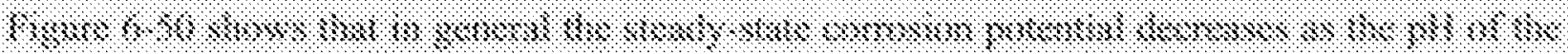

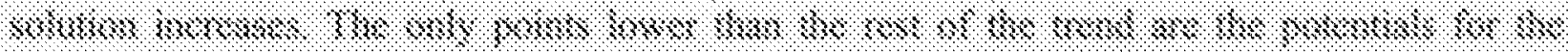

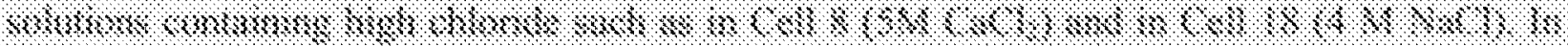

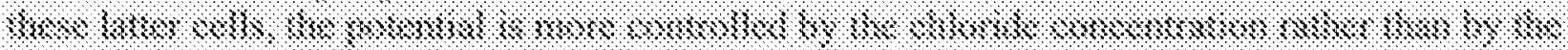

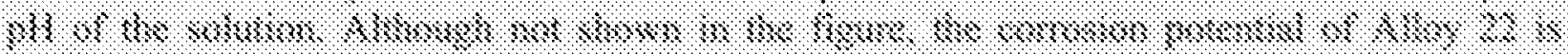

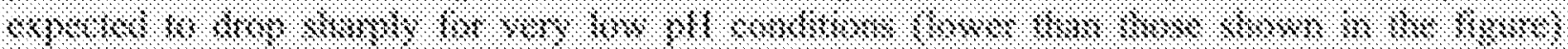

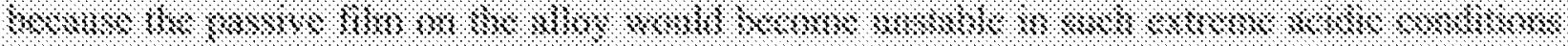

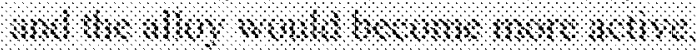

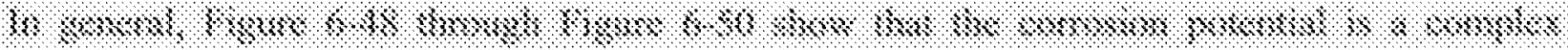

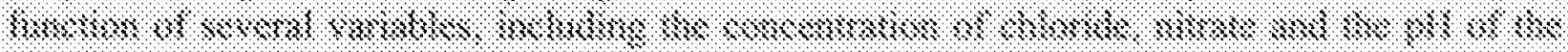

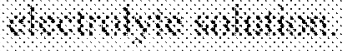


Calculation of the exposed surface area of the weight-loss and crevice samples using Equation 6-8 included the area directly under the crevice former for the crevice samples and the area directly under the sample spacer for the weight-loss samples. These areas were included because the test solutions penetrated and wet the areas under the crevice former or sample spacer. The inputs from DTN: LL030412512251.057 [DIRS 163712] used in the model analysis were the weight-loss measurements and characteristics of the sample and exposure conditions. The calculated corrosion rates of the weight-loss and crevice coupons are listed in Appendices II and III, respectively. The calculated corrosion rates may differ slightly from those in the input DTN due to differences in the number of significant digits used in the calculations.

As noted in Appendix II, Sample DCA177 is an outlier and was not included in the weight-loss data analysis and the WPOB general corrosion model analysis. The mean and standard deviation of all the 5-year crevice sample data including Sample DCA177 are $7.90 \mathrm{~nm} / \mathrm{yr}$ and $7.09 \mathrm{~nm} / \mathrm{yr}$, respectively (output DTN: SN0306T0506303.004). Applying the Grubbs test for outliers as specified in Standard Practice for Dealing with Outlying Observations (ASTM E 178-02 2002 [DIRS 169968]) the computed value for $T_{60}$ (there are $n=60$ general corrosion measurements of crevice samples listed in Appendix II) for the measured general corrosion rate of Sample DCA177 (46.68 nm/yr) is 5.469. From Table 1 in ASTM E 178-02 (2002 [DIRS 169968]), and using $n=60$, a $T_{60}$ value as large as 5.469 would occur by chance with probability less than 0.001 . Thus, the evidence is against the suspect value having come from the same population as the others (assuming the population is normally distributed). Therefore, the measured corrosion rate $(46.68 \mathrm{~nm} / \mathrm{yr})$ of Sample DCA177 is beyond five standard deviations from the mean. In addition, the sample was tested in the vapor phase over the SDW solution, which is the least corrosive condition among the test conditions of the long-term weight-loss tests. The above arguments provide sufficient justification for exclusion of the outlier. Sample DWA089 yielded a negative corrosion rate and was conservatively excluded in the weight-loss analysis.

Figure 6-10 and Figure 6-11 summarize the calculated corrosion rates for the Alloy 22 weightloss coupons and crevice coupons, respectively, exposed to the SAW, SCW, and SDW solutions at $60^{\circ} \mathrm{C}$ and $90^{\circ} \mathrm{C}$ for over five years. The mill-annealed (MA) and as-welded (ASW) samples were combined for the analyses. The average corrosion rates and two standard deviation ranges were calculated based on the use of a normal distribution and are presented in both figures. The 2-standard deviation range represents a 95 percent confidence level. Although the appearance and amount of deposits on the coupons exposed to different solutions were different, the calculated corrosion rates were not significantly different. The individual corrosion rates for the weight-loss coupons ranged from about 0 to $12 \mathrm{~nm} / \mathrm{yr}$ (Figure 6-16) with the lowest rates observed for the coupons in the SDW solution. The individual corrosion rates for the crevice coupons ranged from about 0 to $23 \mathrm{~nm} / \mathrm{yr}$ (Figure 6-21) with the highest rates observed in the SAW solution and, again, the lowest rates observed in the SDW solution. 


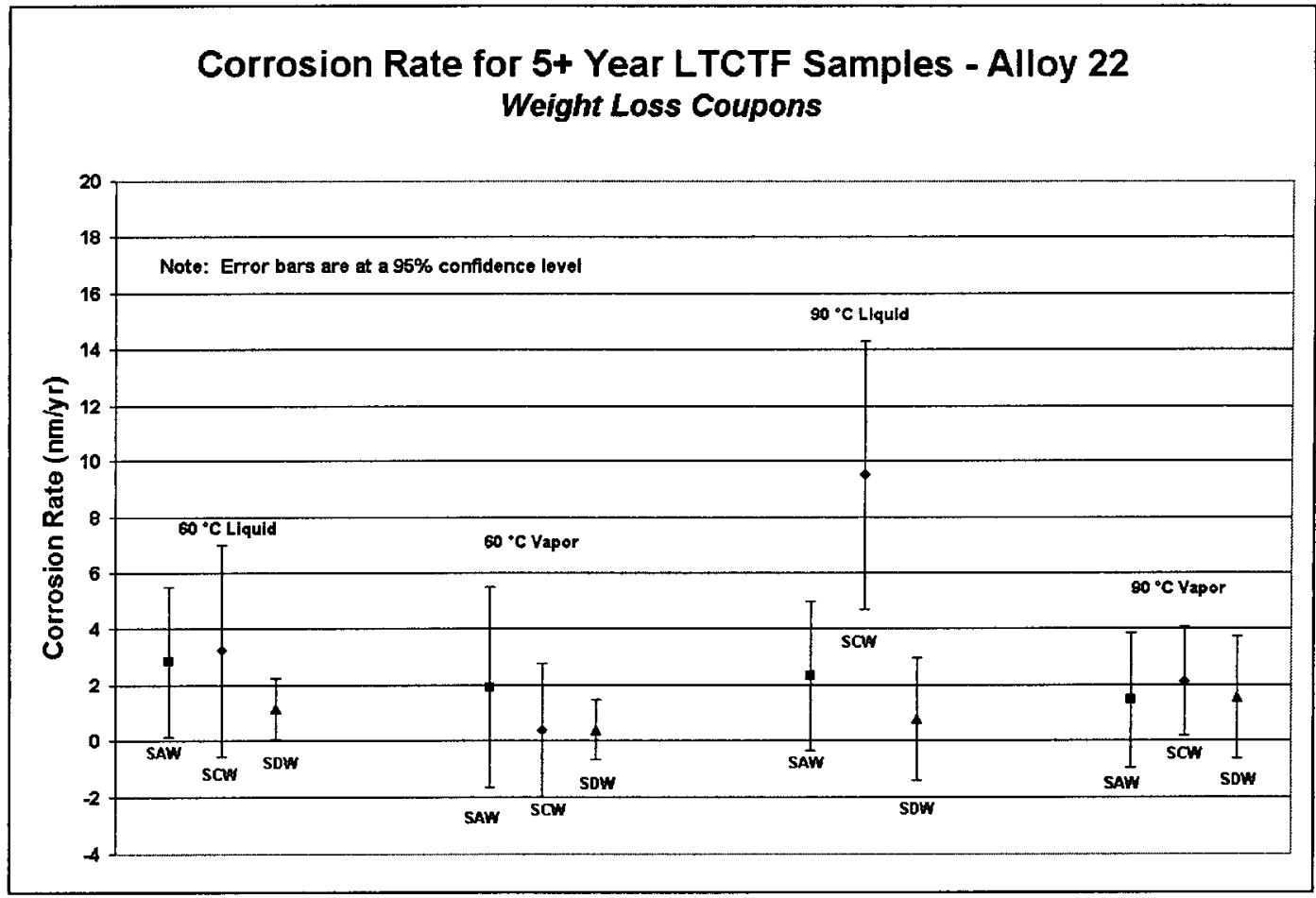

Output DTN: MO0409MWDUGCMW.000.

Figure 6-10. Corrosion Rates for Alloy 22 Weight-Loss Coupons in SAW, SCW, and SDW

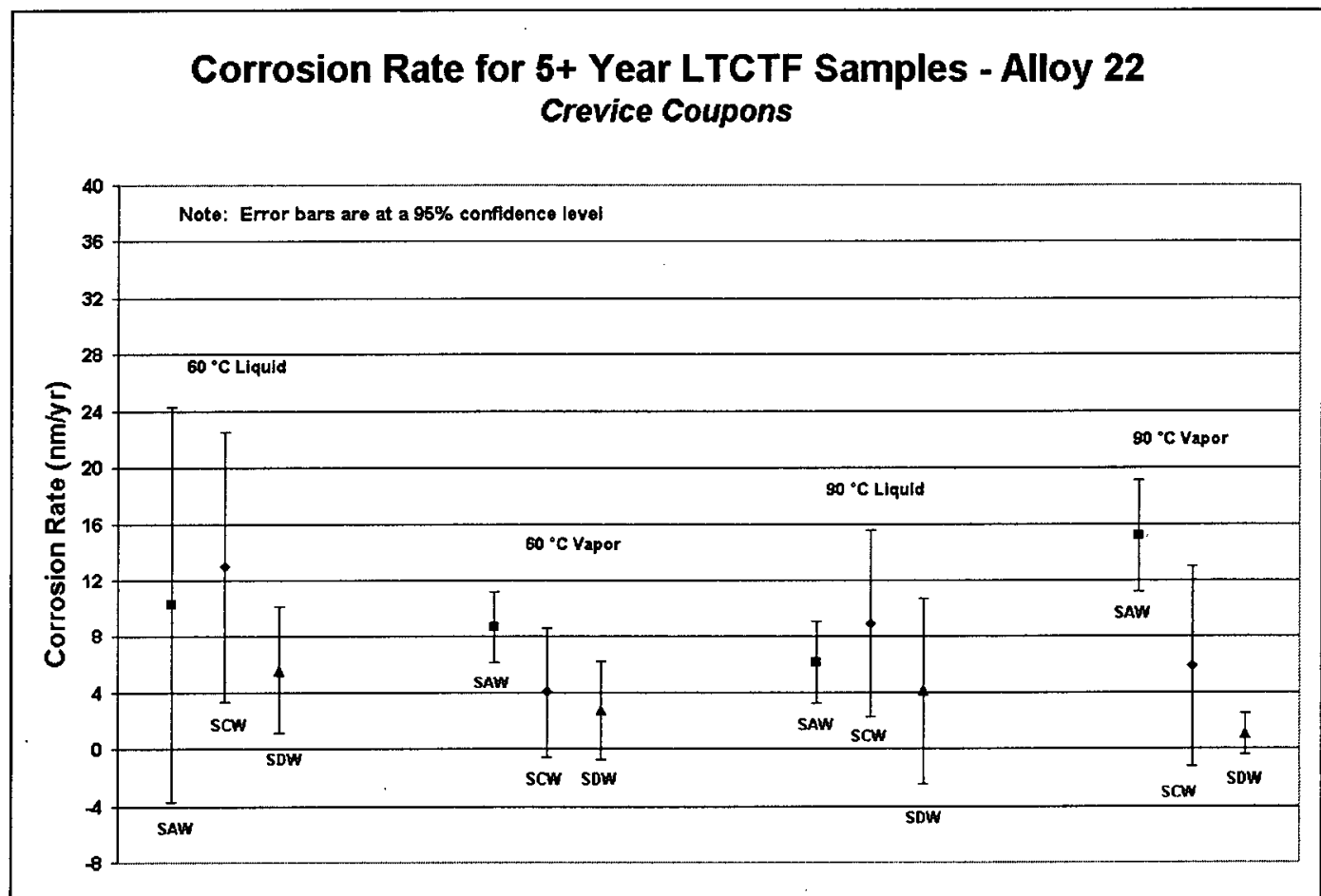

Output DTN: MO0409MWDUGCMW.000.

Figure 6-11. Corrosion Rates for Alloy 22 Crevice Coupons in SAW, SCW, and SDW 
In most cases, the crevice coupons exhibited corrosion rates 2 to 5 times higher than the weight-loss coupons in the same solutions. Stereomicroscopic and scanning electron microscopic (SEM) observations of weight-loss and crevice specimens indicated little or no sign of corrosion. The machining grooves remained uniform and sharp throughout each coupon. No crevice corrosion was observed on any of the tested coupons. It is possible that a different surface finish treatment used for the crevice samples may have led to measurement of different corrosion rates. However, it is noteworthy that among all test specimens, a maximum corrosion rate of only about $23 \mathrm{~nm} / \mathrm{yr}$ was observed (Figure 6-21).

For the weight-loss and crevice coupons, the corrosion rates were generally lower for specimens exposed to vapor than those immersed in liquid, regardless of the test temperature or electrolyte solution. Overall, the test coupons in the SAW solution at $90^{\circ} \mathrm{C}$ exhibited slightly lower corrosion rates than at $60^{\circ} \mathrm{C}$.

Similar to the weight-loss coupons, the corrosion rates for the crevice coupons exposed to liquid were lower at $90^{\circ} \mathrm{C}$ than at $60^{\circ} \mathrm{C}$, while the corrosion rates were generally higher at $90^{\circ} \mathrm{C}$ than at $60^{\circ} \mathrm{C}$ for the crevice coupons exposed to vapor. In general, for corrosion processes, the corrosion rate increases with temperature. However, in this study, since the determined corrosion rates were very low and the temperature range studied $\left(60^{\circ} \mathrm{C}\right.$ to $\left.90^{\circ} \mathrm{C}\right)$ was narrow, a clear dependence with the temperature could not be established for these data. For the weight-loss coupons, there was no effect of welds on the corrosion rate. The nonwelded crevice coupons exhibited slightly higher corrosion rates than their welded counterparts.

The general corrosion rates of the coupons were analyzed with "empirical" cumulative distribution functions (ECDFs) of the calculated rates. In constructing the ECDFs, the cumulative probability values of the general corrosion rate (except the upper and lower bounds) were calculated by the following plotting positions (Cleveland 1993 [DIRS 163885], Section 2.1):

$$
q_{i}=\frac{i-0.5}{n}
$$

where $q_{i}$ is the cumulative probability of the $i^{\text {th }}$ smallest event (e.g., general corrosion rate) and $n$ is the total number of events. The above plotting position formula is a traditional choice for probability plotting (Stedinger et al. 1993 [DIRS 105941], Section 18.3.2). The ECDFs developed using the above plotting position formula for the 5-year weight-loss analysis are to present the data trends and comparative analysis of different sets of data for varying sample geometry and exposure conditions.

The ECDFs for the general corrosion rates of the weight-loss specimens are shown in Figure 6-12 to Figure 6-16 for comparative analyses of the effect of various experimental conditions, such as solution chemistry, temperature, and metallurgical condition, on the general corrosion rate. The ECDFs for the general corrosion rates of the crevice coupons are shown in Figure 6-17 to Figure 6-21 for comparative analyses of the effect of various experimental conditions on the general corrosion rate. 


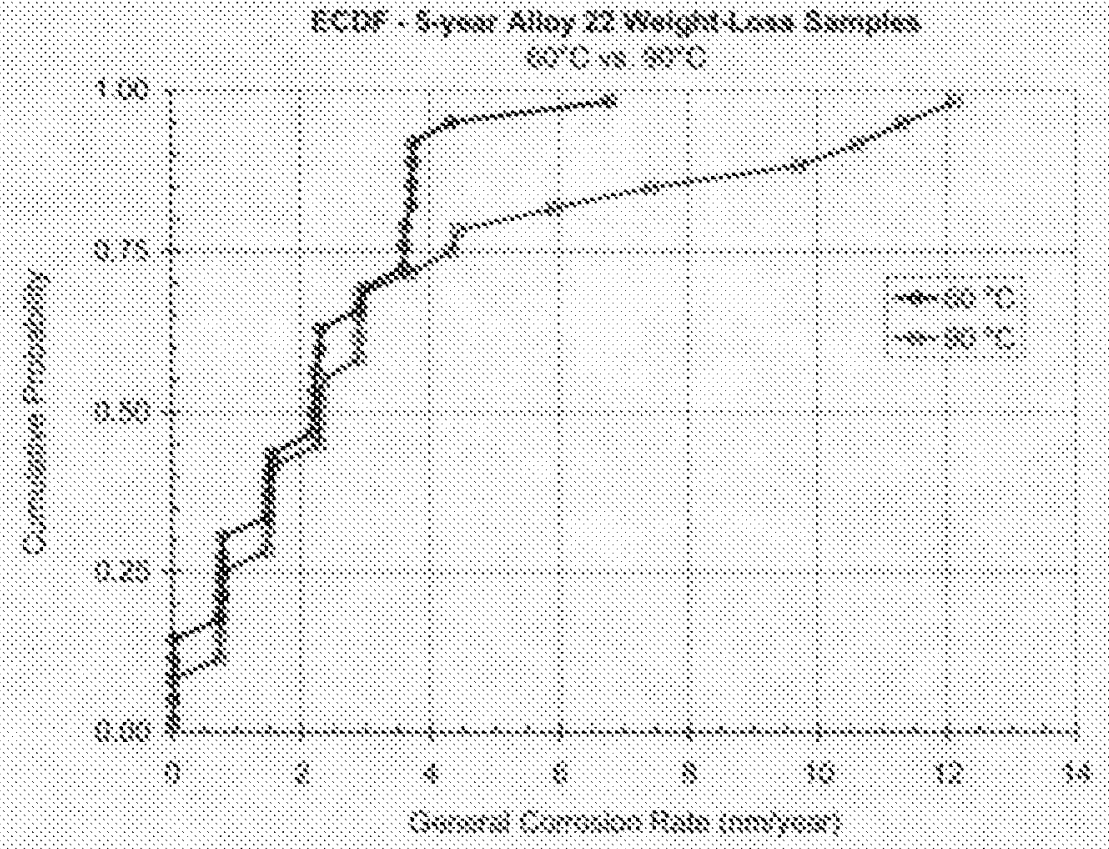

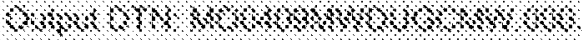

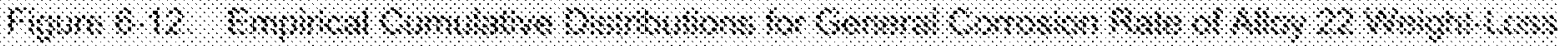

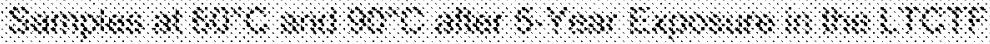

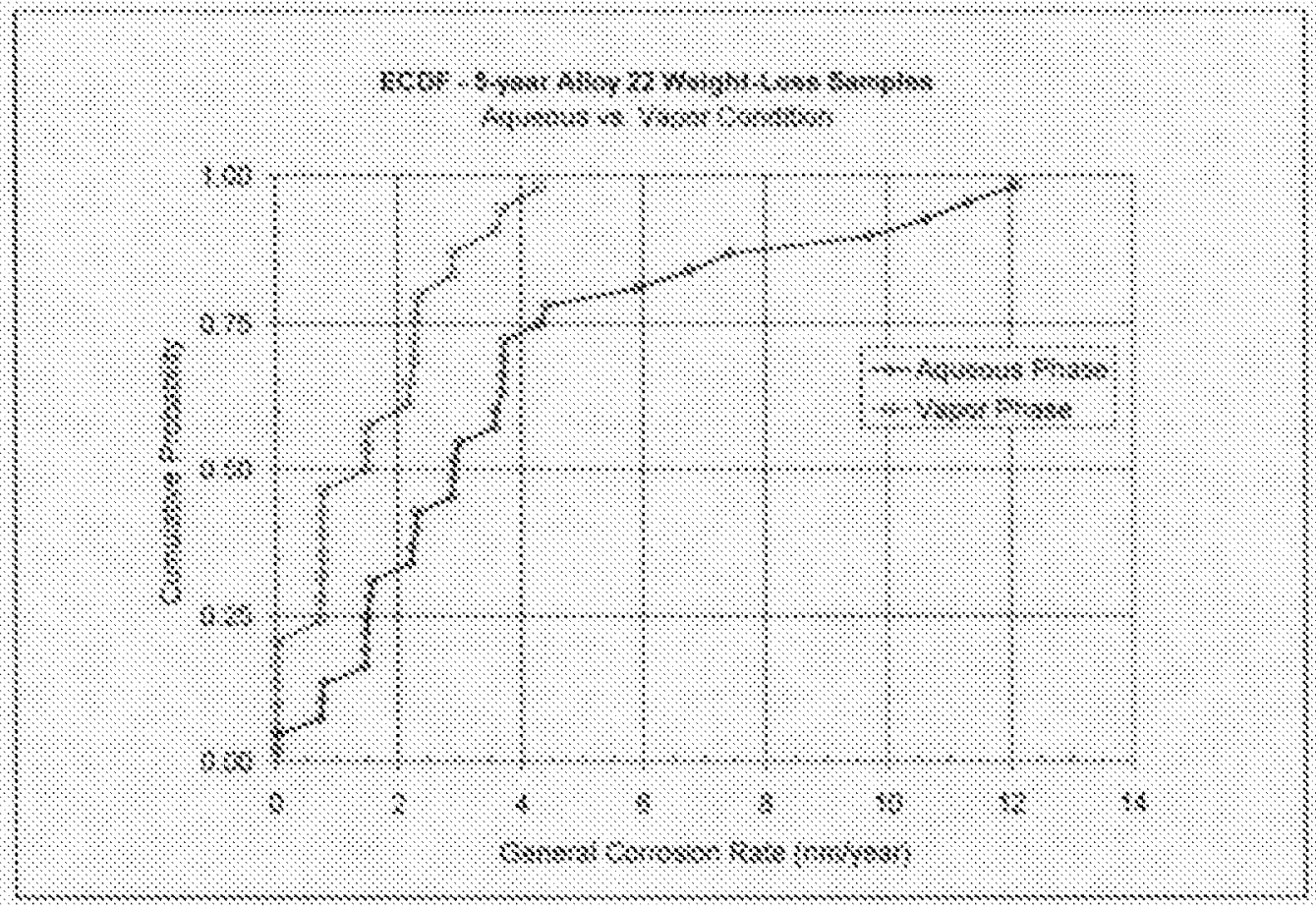

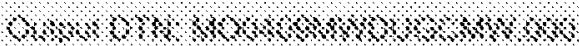

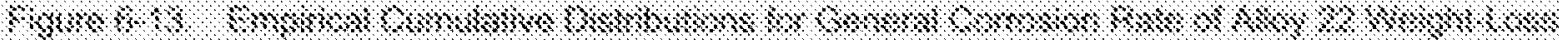

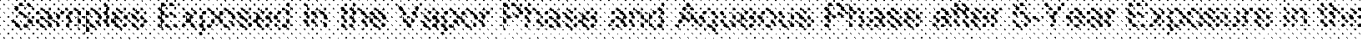
$13 \%$ 


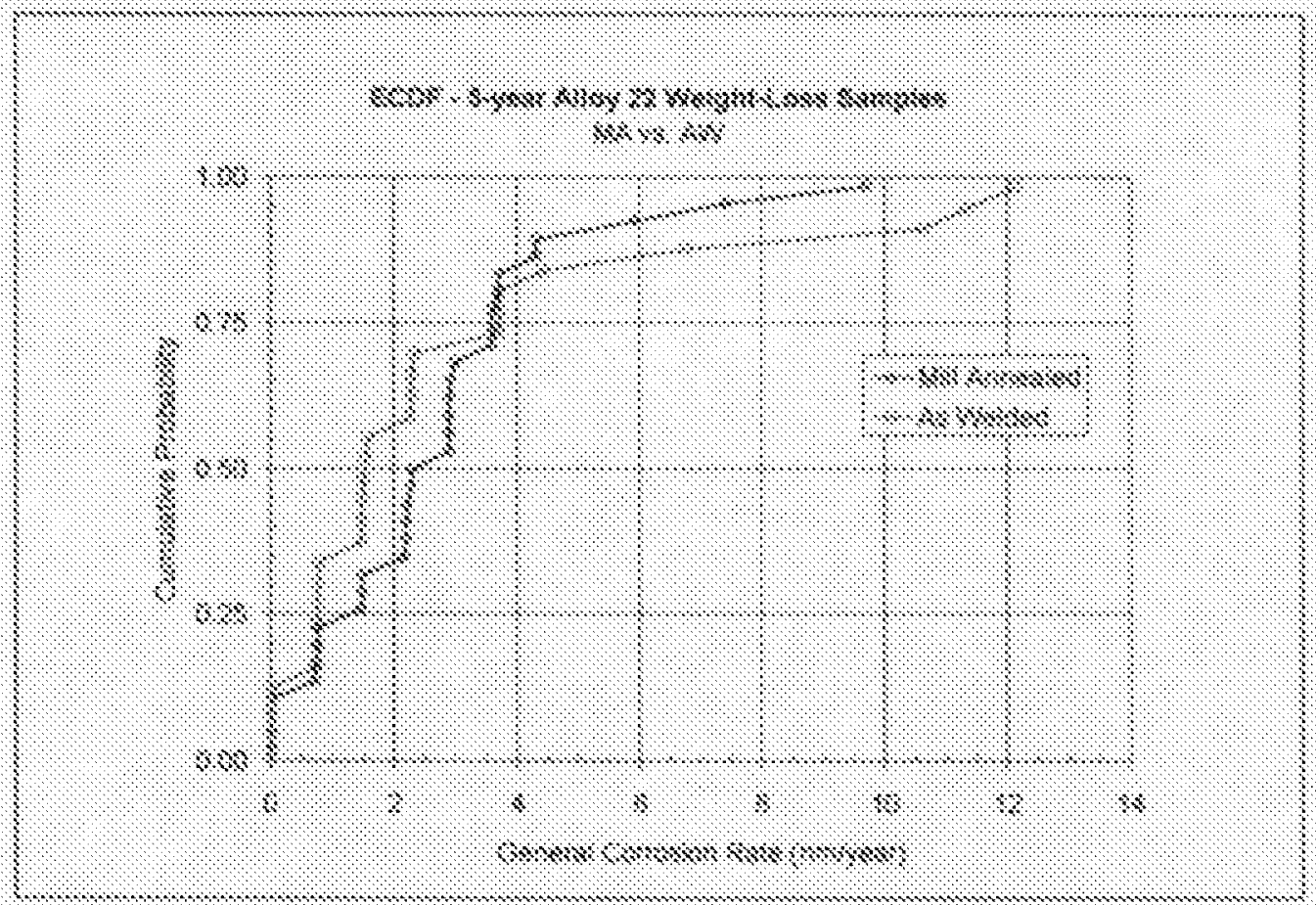

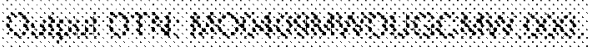

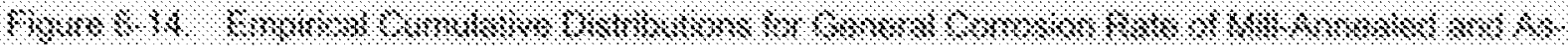

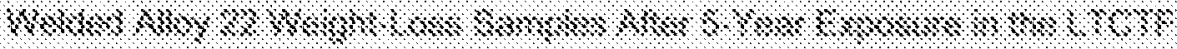

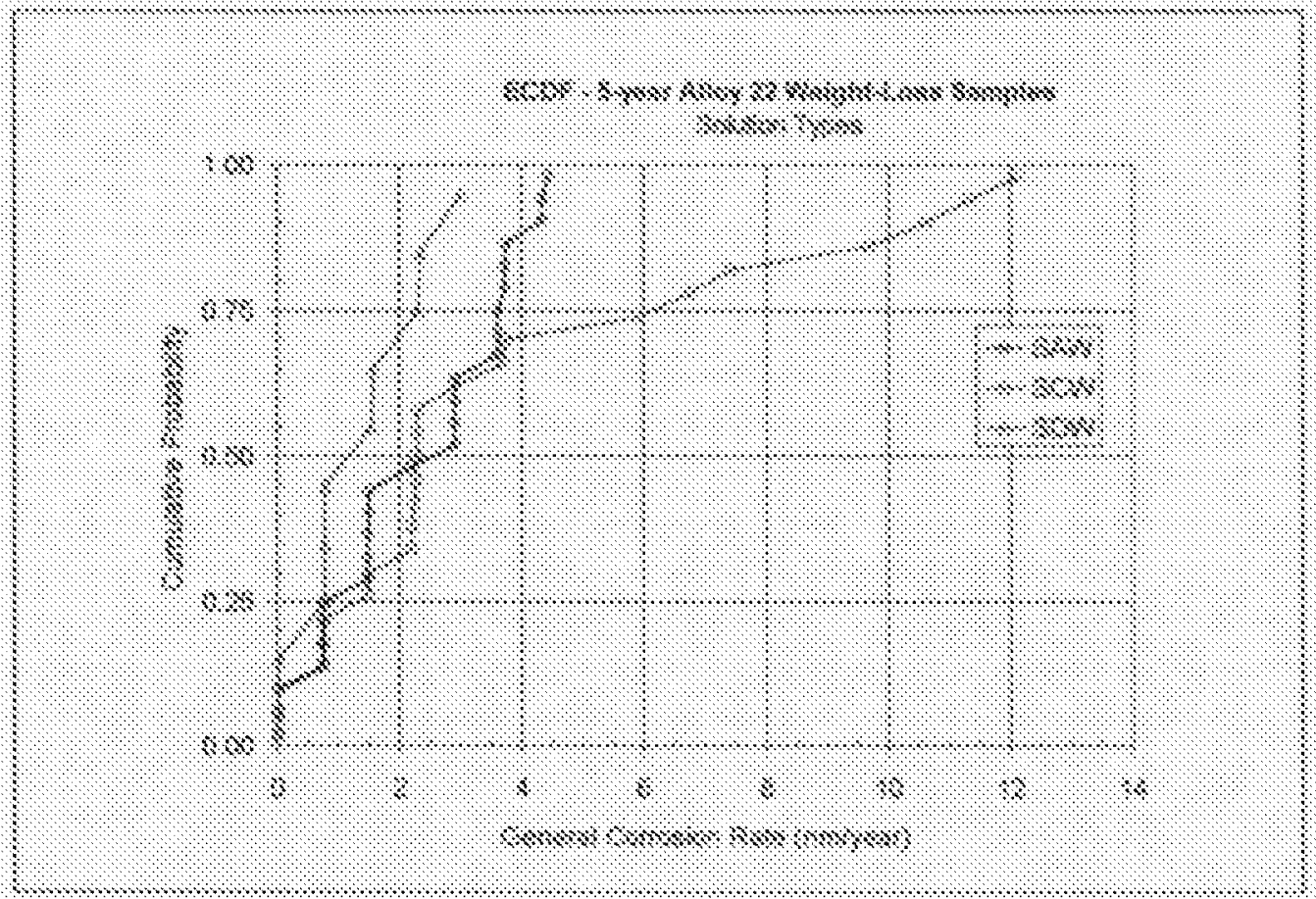

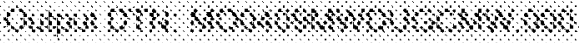

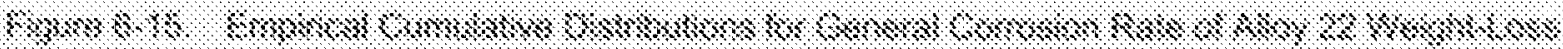

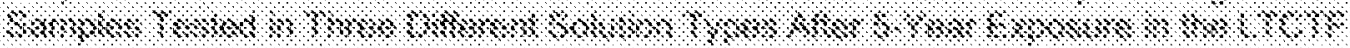




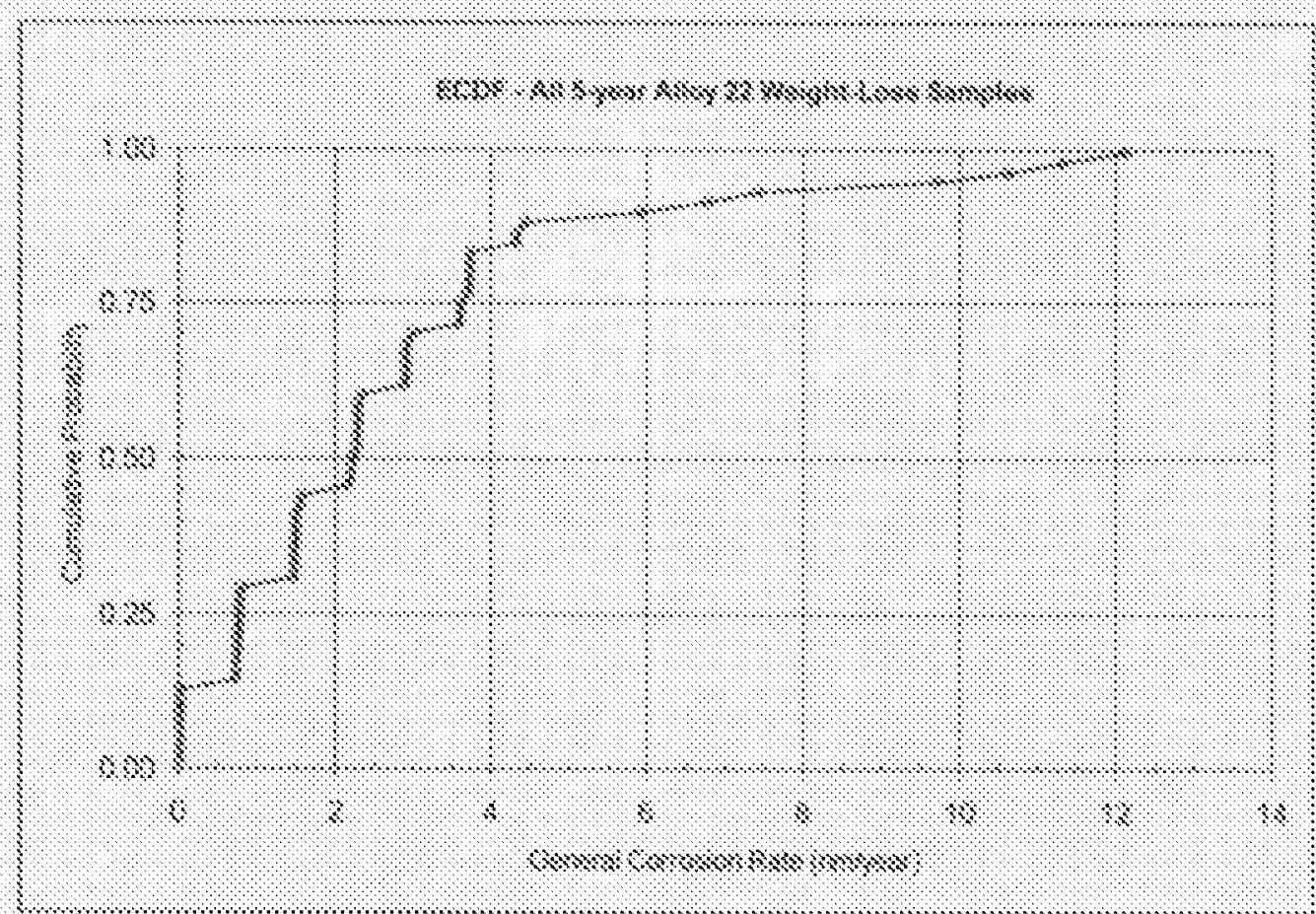

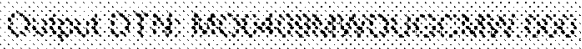

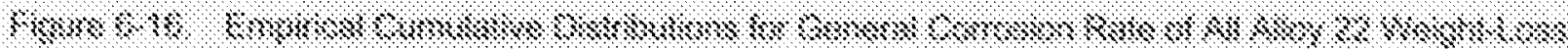

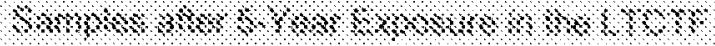

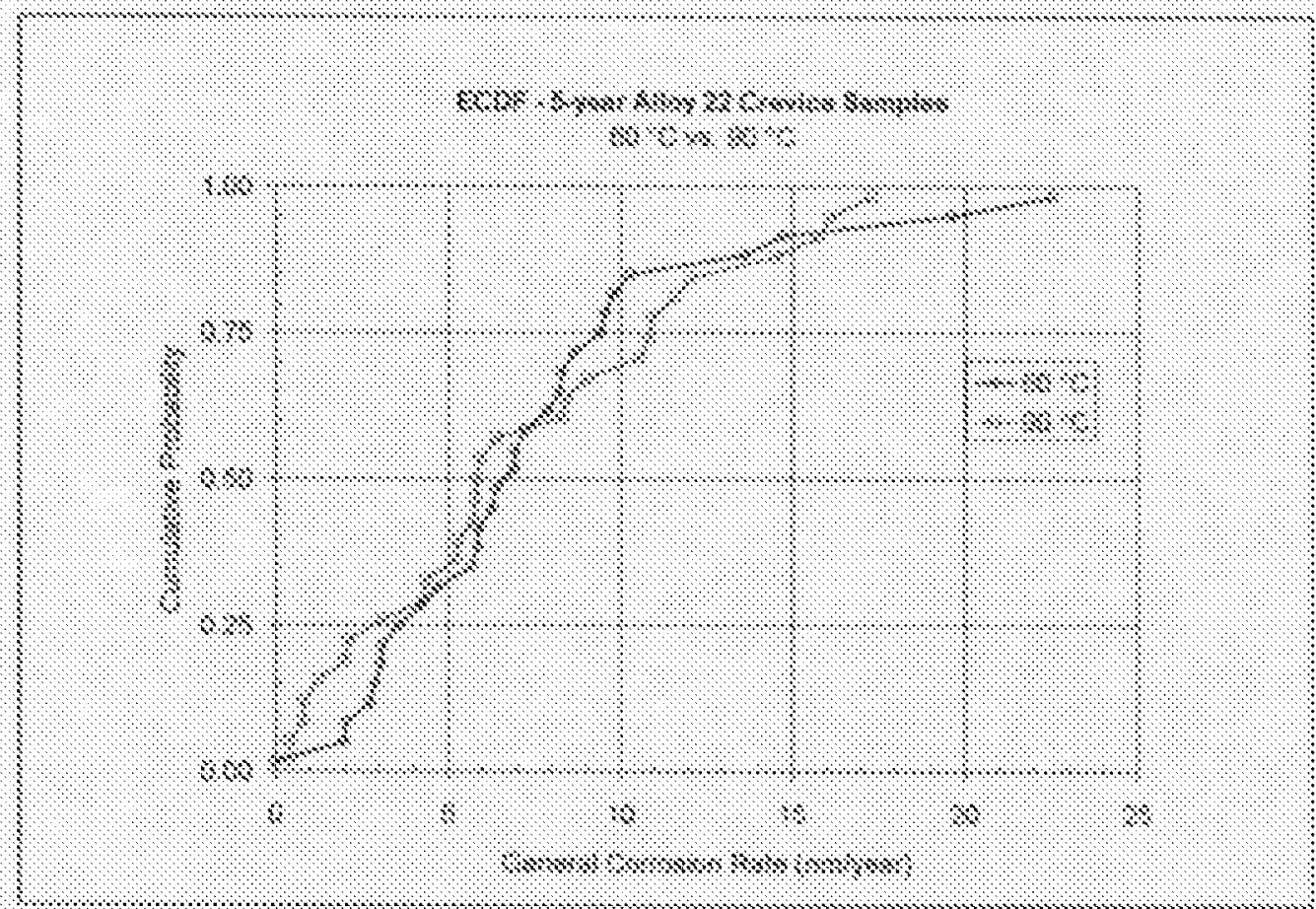

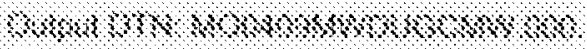

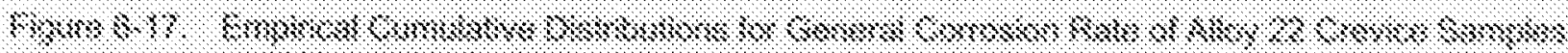

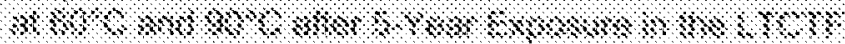




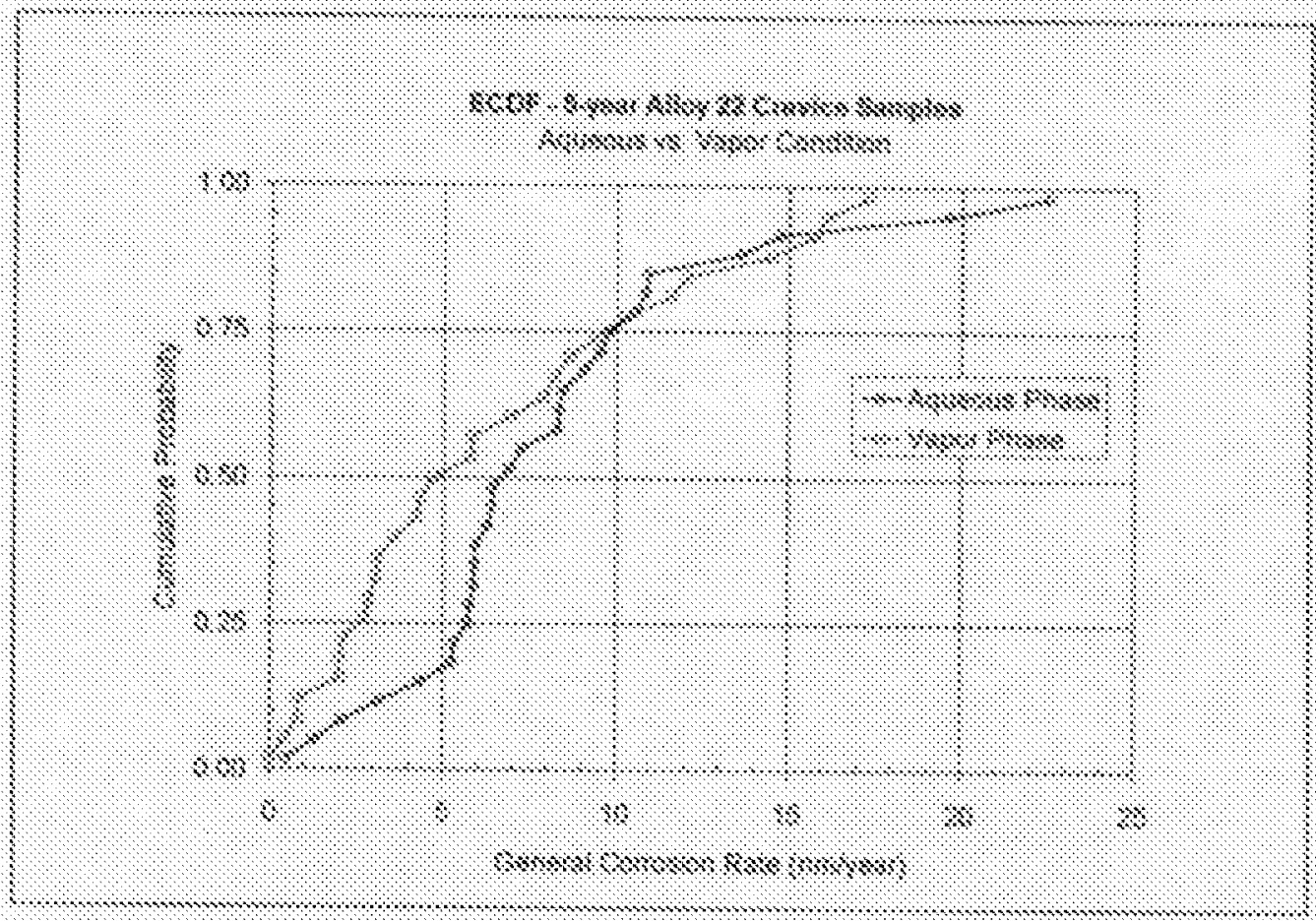

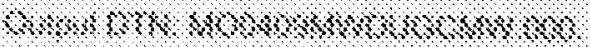

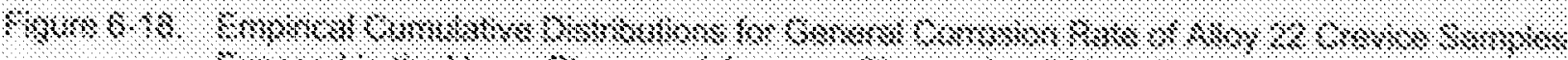

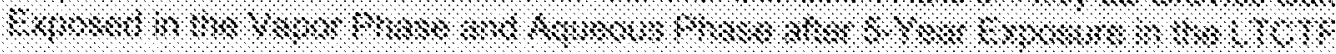

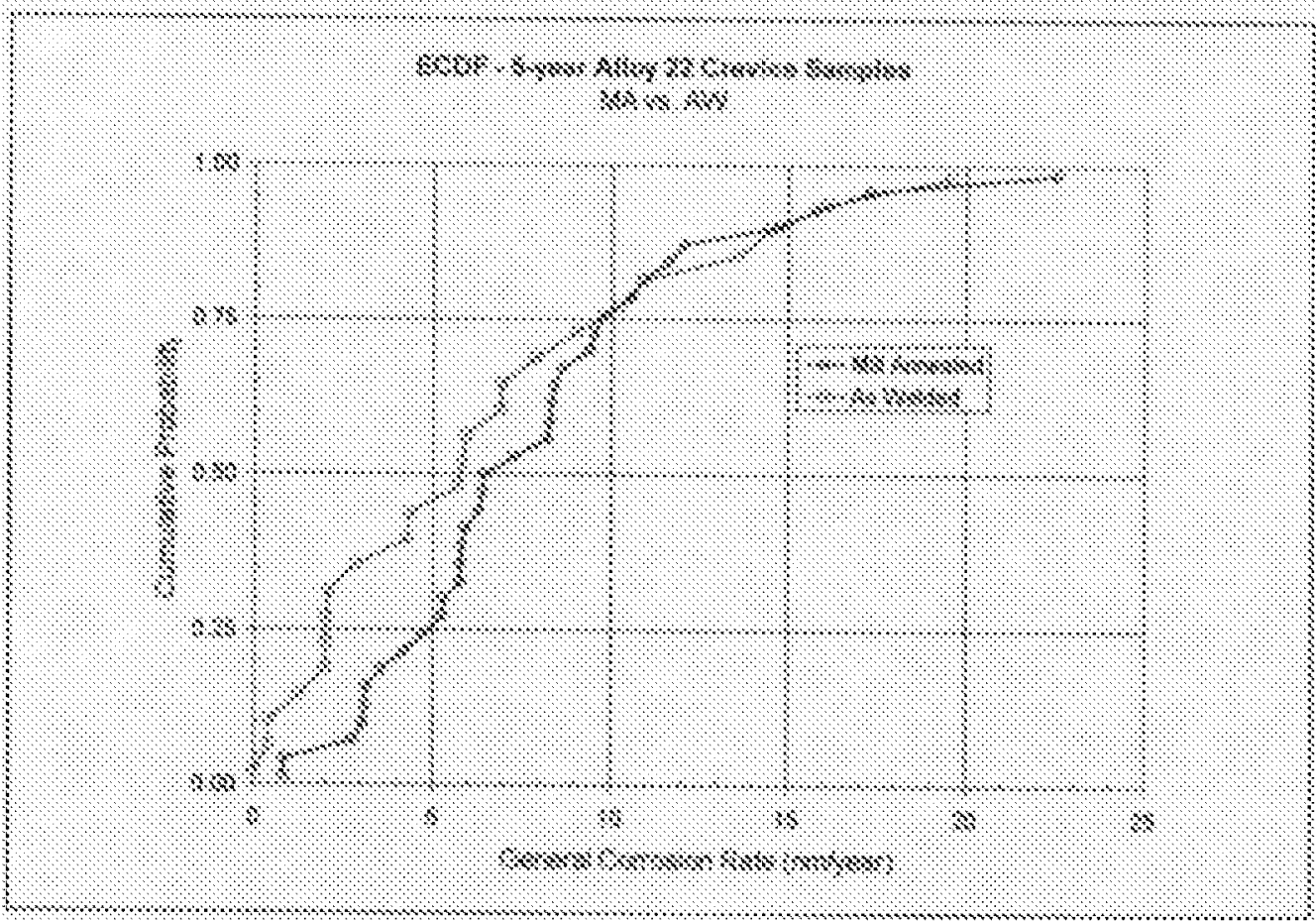

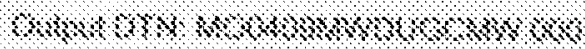

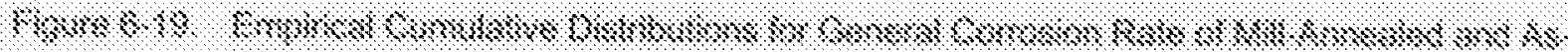

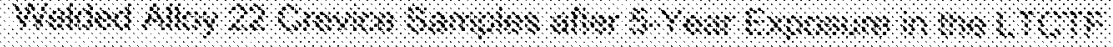




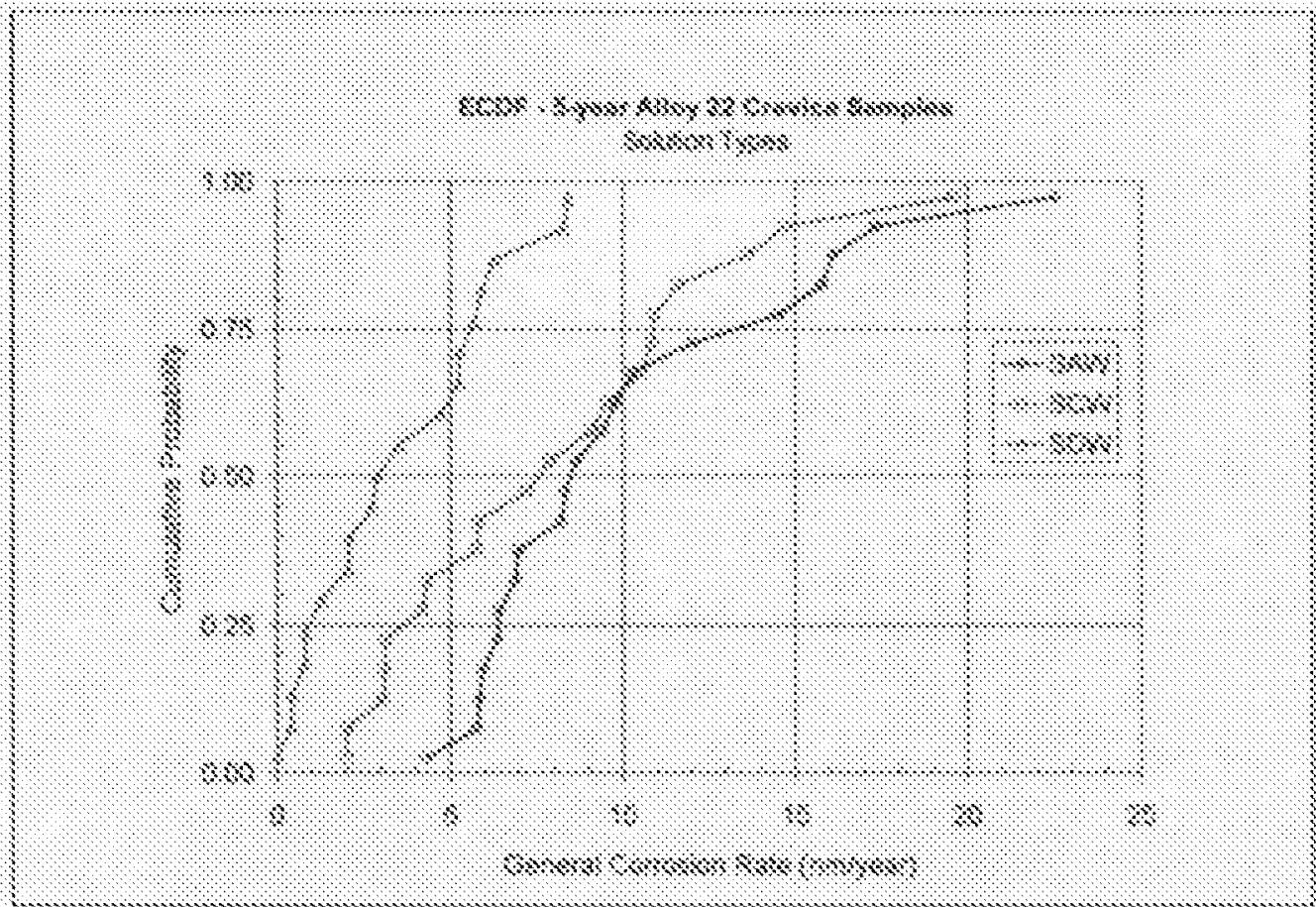

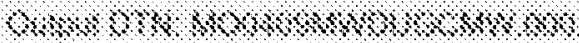



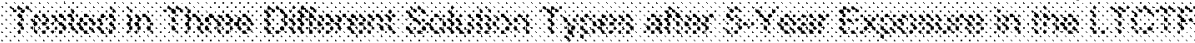

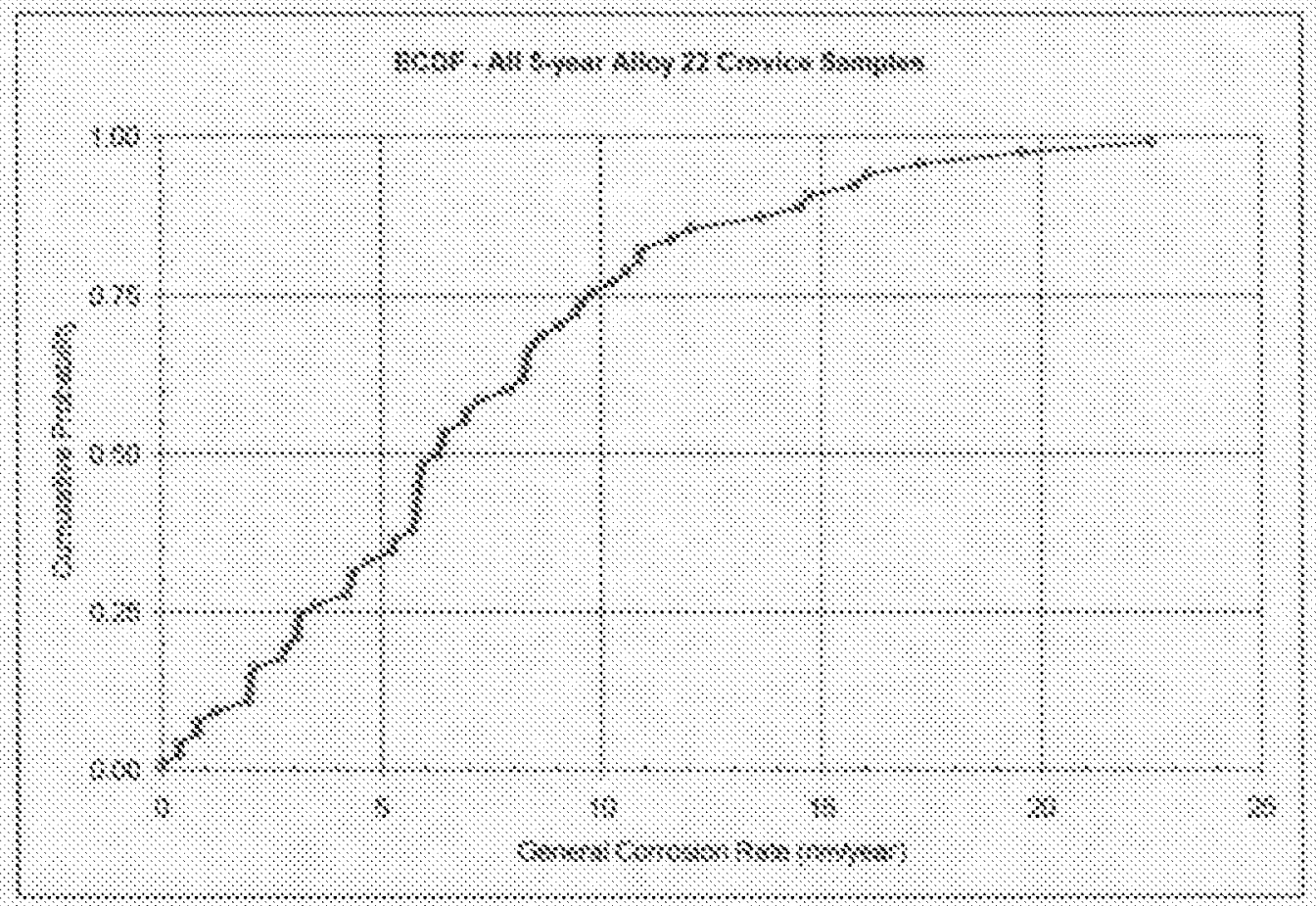

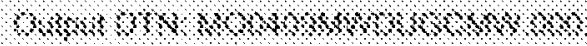

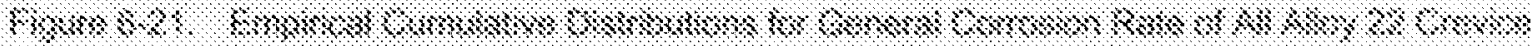

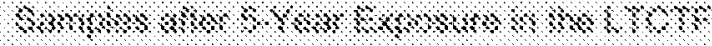




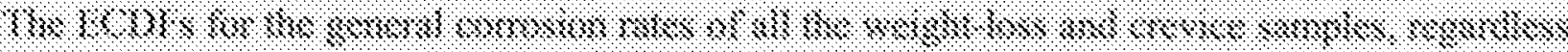

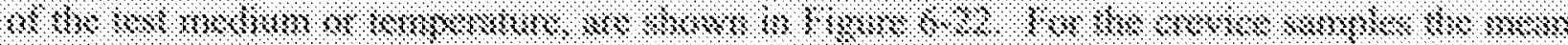

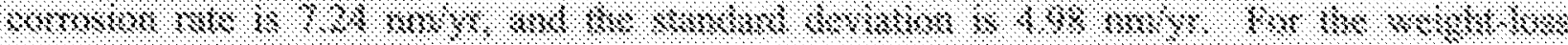

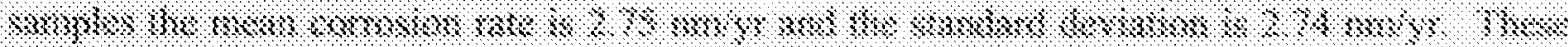

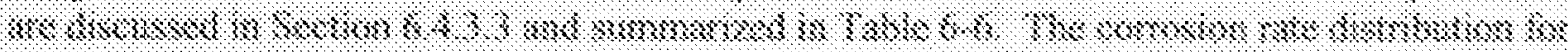

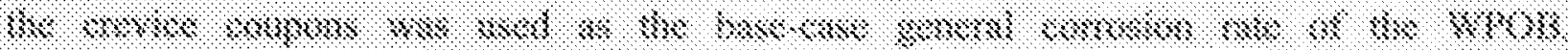

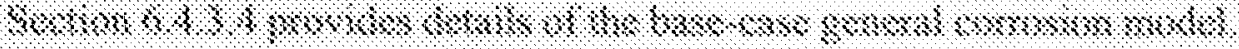

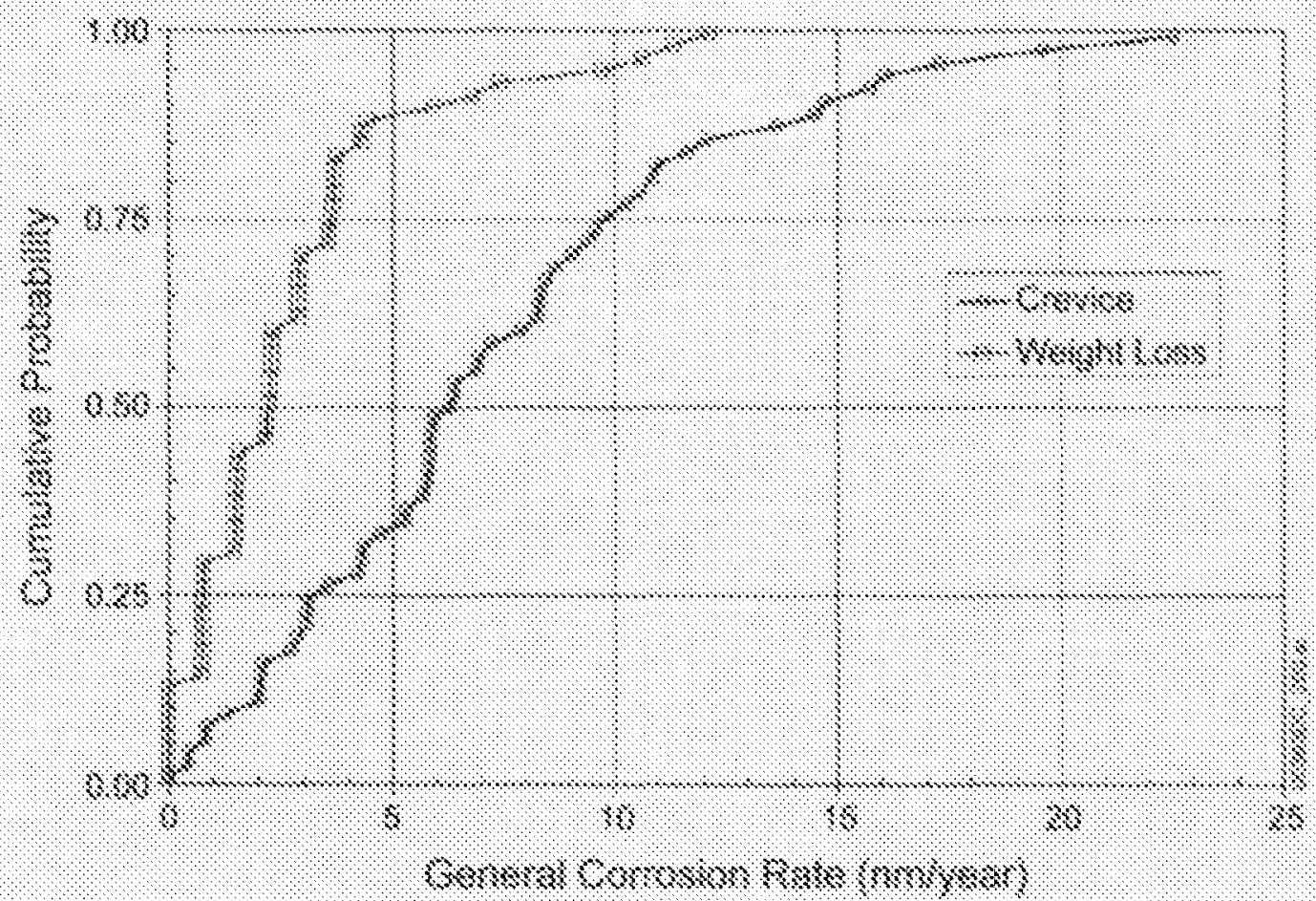

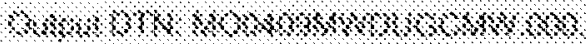

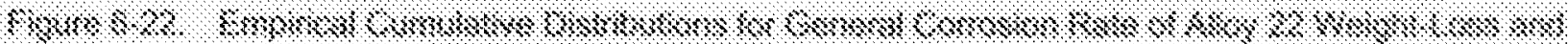

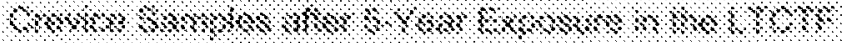

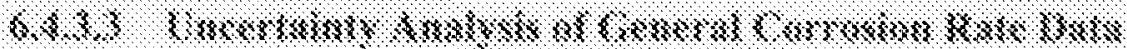

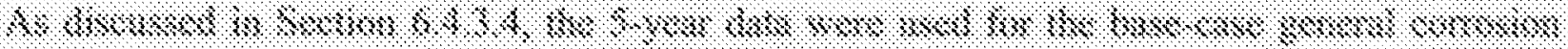

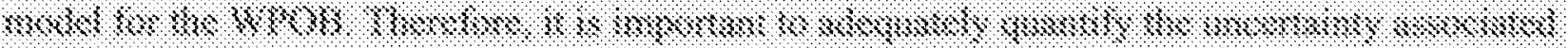

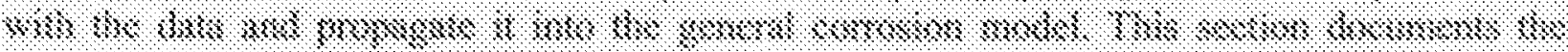

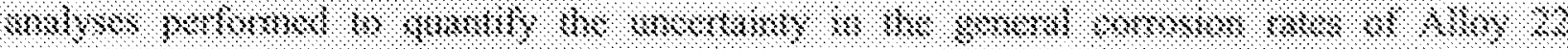

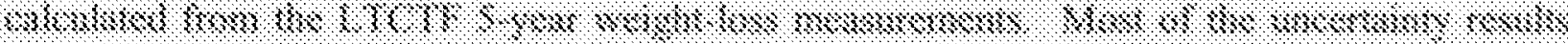

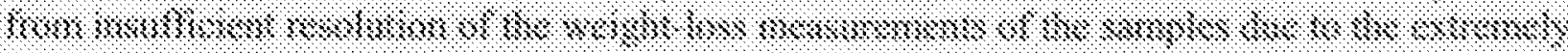

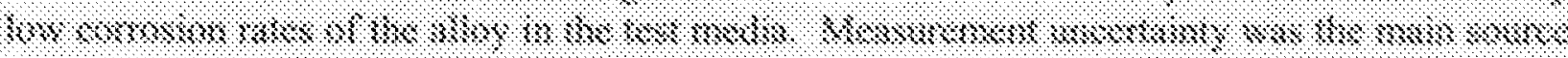

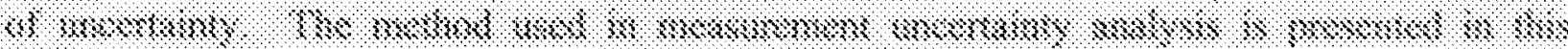

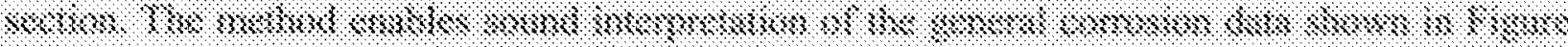

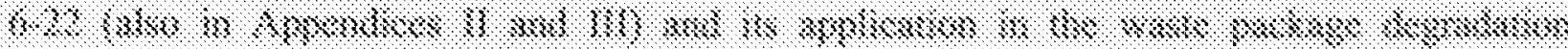
$3 \times k \times k m$ 
Consider a measurand $Y$, which is not measured directly, but determined from $N$ other quantities $X_{1}, X_{2}, \ldots, X_{N}$ through a functional relationship $f$ defined as follows:

$$
Y=f\left(X_{1}, X_{2}, \cdots, X_{N}\right)
$$

An estimate of the measurand $Y$, denoted by $y$, is obtained using input estimates $x_{1}, x_{2}, \ldots, x_{N}$ for the values of $N$ input quantities $X_{1}, X_{2}, \cdots, X_{N}$. The output estimate $y$, is given by:

$$
y=f\left(x_{1}, x_{2}, \cdots, x_{N}\right)
$$

The combined uncertainty of the measurement result $y$, designated by $\Delta y$, is given by Equation 6-12, the law of propagation of uncertainty (Taylor et al. 1994 [DIRS 162260], Appendix A):

$$
\Delta y=\sqrt{\sum_{i=1}^{N}\left(\frac{\partial f}{\partial x_{i}}\right)^{2} \Delta x_{i}^{2}+2 \sum_{i=1}^{N-1} \sum_{j=i+1}^{N} \frac{\partial f}{\partial x_{i}} \frac{\partial f}{\partial x_{j}} \Delta x_{i} x_{j}}
$$

In Equation 6-12, the partial derivatives $\frac{\partial f}{\partial x_{i}}$ are the sensitivity coefficients, $\Delta x_{i}$ is the standard uncertainty associated with the input estimate $x_{i}$, and $\Delta x_{i} x_{j}$ is the estimated covariance associated with $x_{i}$ and $x_{j}$.

Referring to Figure 6-9, the schematic of the Alloy 22 sample used in the weight-loss measurement in LTCTF, the exposed surface area of the sample is expressed as follows:

$$
A=2 a b+2 b c+2 a c-\left(\frac{\pi d^{2}}{2}\right)+\pi d c
$$

Referring to Equation 6-7 and letting the dependent variable $y$ be the 5-year general corrosion rate measured in the LTCTF, the equation for the general corrosion rate is expressed as follows:

$$
y=\frac{d p}{d t}=\frac{w}{\rho \times t\left(2 a b+2 b c+2 a c-\left(\frac{\pi d^{2}}{2}\right)+\pi d c\right)}
$$

where $y$ is the corrosion rate in $\mathrm{cm}$ per hour, $t$ is the time of exposure in hours, $w$ is the total weight-loss during the time, $t$, in grams, and $\rho$ is the density in grams per cubic centimeter. The combined uncertainty of the measurement result, $y$, the corrosion rate, is calculated using the law of propagation of uncertainty (Taylor et al. 1994 [DIRS 162260], Appendix A):

$$
\Delta y=\sqrt{\left(\frac{\partial y}{\partial w}\right)^{2} \Delta w^{2}+\left(\frac{\partial y}{\partial \rho}\right)^{2} \Delta \rho^{2}+\left(\frac{\partial y}{\partial t}\right)^{2} \Delta t^{2}+\left(\frac{\partial y}{\partial a}\right)^{2} \Delta a^{2}+\left(\frac{\partial y}{\partial b}\right)^{2} \Delta b^{2}+\left(\frac{\partial y}{\partial c}\right)^{2} \Delta c^{2}+\left(\frac{\partial y}{\partial d}\right)^{2} \Delta d^{2}}
$$


(Eq. 6-15)

where $w, \rho, t, a, b, c$, and $d$ were considered to be independent; hence, the covariance terms disappear.

The partial derivatives are:

$$
\begin{aligned}
& \frac{\partial y}{\partial w}=\frac{1}{\rho \times t\left(2 a b+2 b c+2 a c-\left(\frac{\pi d^{2}}{2}\right)+\pi d c\right)} \\
& \frac{\partial y}{\partial \rho}=-\frac{w}{\rho^{2} \times t\left(2 a b+2 b c+2 a c-\left(\frac{\pi d^{2}}{2}\right)+\pi d c\right)} \\
& \frac{\partial y}{\partial t}=-\frac{w}{\rho \times t^{2}\left(2 a b+2 b c+2 a c-\left(\frac{\pi d^{2}}{2}\right)+\pi d c\right)} \\
& \frac{\partial y}{\partial a}=-\frac{2 w(b+c)}{\rho \times t\left(2 a b+2 b c+2 a c-\left(\frac{\pi d^{2}}{2}\right)+\pi d c\right)^{2}} \\
& \frac{\partial y}{\partial b}=-\frac{2 w(a+c)}{\rho \times t\left(2 a b+2 b c+2 a c-\left(\frac{\pi d^{2}}{2}\right)+\pi d c\right)^{2}} \\
& \frac{\partial y}{\partial c}=-\frac{w[2(a+b)+\pi d]}{\rho \times t\left(2 a b+2 b c+2 a c-\left(\frac{\pi d^{2}}{2}\right)+\pi d c\right)^{2}} \\
& \frac{\partial y}{\partial d}=-\frac{w \pi(c-d)}{\rho \times t\left(2 a b+2 b c+2 a c-\left(\frac{\pi d^{2}}{2}\right)+\pi d c\right)^{2}}
\end{aligned}
$$

The maximum error in the corrosion rate is estimated by calculating numeric values of the partial derivatives from expected values of the independent variables, multiplying each partial derivative by the corresponding error (i.e., standard uncertainty) associated in the independent variables ( $\Delta w, \Delta \rho, \Delta t, \Delta a, \Delta b, \Delta c$, and $\Delta d)$, and summing the resulting products. 
The combined standard uncertainty in the corrosion rate was estimated with Equation 6-15 by calculating numeric values of the partial derivatives from expected values of the input variables and their estimated standard uncertainties. Those values and intermediate calculation steps are summarized in Table 6-5.

Upon examining the sensitivity coefficients in Equation 6-15, it was found that $\Delta y$ was most sensitive to the estimate of $\Delta w$. Because $\Delta w$ was most influential on $\Delta y$, a detailed description of how $\Delta w$ was calculated is given below. The Mettler AT200 balance was used to measure the weight of the specimens. The balance displays mass measurements to four decimal places. For instance, a four-digit readout might indicate a mass of 60.2675 grams for the weight of a specimen. If the balance employs standard round-off practice, then the displayed number is derived from a value that lies between 60.26745 grams and 60.26755 grams. The mass has an equal probability of lying between those two numbers. This would indicate that the error term has a uniform distribution. If $w_{1}=$ original weight of specimen and $w_{2}=$ final weight of specimen, then $w_{1}=\mu_{1}+10^{-4} \varepsilon_{1}$ and $w_{2}=\mu_{2}+10^{-4} \varepsilon_{2}$. That is, the mass $=$ true mass + error term, and $\varepsilon_{1} \sim U(-0.5,0.5)$, and $\varepsilon_{2} \sim U(-0.5,0.5)$. The weight-loss due to corrosion is:

$$
w=w_{1}-w_{2}=\left(\mu_{1}-\mu_{2}\right)+10^{-4}\left(\varepsilon_{1}-\varepsilon_{2}\right)
$$

where $\left(\mu_{1}-\mu_{2}\right)$ is the true difference in mass and $10^{-4}\left(\varepsilon_{1}-\varepsilon_{2}\right)$ is the error term. The error term $\left(\varepsilon_{1}-\varepsilon_{2}\right)$ has a triangular distribution (Papoulis 1965 [DIRS 162236], pp. 189 to 192) between -1 and 1 (i.e., $\left(\varepsilon_{1}-\varepsilon_{2}\right) \sim$ Triangular $(-1,1,0)$ ). For this distribution the standard deviation is:

$$
s=\frac{1}{\sqrt{6}}=0.41
$$

Therefore, $\Delta w=0.41 \times 10^{-4} \mathrm{~g}$. The summary of the measurement uncertainty analysis for the 5-year data based upon this method is shown in Table 6-6.

Table 6-5. Summary of Measurement Uncertainty Analysis for Corrosion Rates Based Upon WeightLoss Measurements after 5-Year Exposure in LTCTF

\begin{tabular}{|c|c|c|c|}
\hline \multicolumn{4}{|c|}{ Uncertainty Analysis of 5-Year Weight-Loss Measurement Data } \\
\hline Parameters & Units & Crevice Samples & Weight-Loss Samples \\
\hline$w$ & $\mathrm{~g}$ & 0.00187 & 0.00036 \\
\hline$\rho$ & $\mathrm{g} / \mathrm{cm}^{3}$ & 8.69 & 8.69 \\
\hline $\mathrm{t}$ & $\mathrm{hour}$ & 43,800 & 43,800 \\
\hline $\mathrm{a}$ & $\mathrm{cm}$ & 5.08 & 5.08 \\
\hline $\mathrm{b}$ & $\mathrm{cm}$ & 5.08 & 2.54 \\
\hline $\mathrm{c}$ & $\mathrm{cm}$ & 0.3048 & 0.3048 \\
\hline $\mathrm{d}$ & $\mathrm{cm}$ & 0.7925 & 0.7925 \\
\hline$\Delta \mathrm{w}$ & $\mathrm{g}$ & $4.100 \mathrm{E}-05$ & $4.100 \mathrm{E}-05$ \\
\hline$\Delta \rho$ & $\mathrm{g} / \mathrm{cm}$ & 0.1 & 0.1 \\
\hline$\Delta \mathrm{t}$ & $\mathrm{hour}$ & 24 & 24 \\
\hline$\Delta \mathrm{a}$ & $\mathrm{cm}$ & 0.00254 & 0.00254 \\
\hline$\Delta \mathrm{b}$ & $\mathrm{cm}$ & 0.00254 & 0.00254 \\
\hline
\end{tabular}




\begin{tabular}{|c|c|c|c|}
\hline \multicolumn{4}{|c|}{ Uncertainty Analysis of 5-Year Weight-Loss Measurement Data } \\
\hline Parameters & Units & Crevice Samples & Weight-Loss Samples \\
\hline$\Delta c$ & $\mathrm{~cm}$ & 0.00254 & 0.00254 \\
\hline$\Delta \mathrm{d}$ & $\mathrm{cm}$ & 0.00254 & 0.00254 \\
\hline Surface Area & $\mathrm{cm}^{2}$ & 57.5787 & 30.2239 \\
\hline$\partial \mathrm{y} / \partial \mathrm{w}$ & & $4.563 \mathrm{E}-08$ & $8.693 \mathrm{E}-08$ \\
\hline$\partial \mathrm{y} / \partial \rho$ & & $-9.819 \mathrm{E}-12$ & $-3.601 \mathrm{E}-12$ \\
\hline
\end{tabular}

Table 6-5. Summary of Measurement Uncertainty Analysis for Corrosion Rates Based Upon WeightLoss Measurements after 5-Year Exposure in LTCTF

\begin{tabular}{|c|c|c|c|}
\hline \multicolumn{4}{|c|}{ Uncertainty Analysis of 5-Year Weight-Loss Measurement Data } \\
\hline Parameters & Units & Crevice Samples & Weight-Loss Samples \\
\hline$\partial \mathrm{y} / \partial \mathrm{t}$ & & $-1.948 \mathrm{E}-15$ & $-7.145 \mathrm{E}-16$ \\
\hline$\partial \mathrm{y} / \partial \mathrm{a}$ & & $-1.596 \mathrm{E}-11$ & $-5.891 \mathrm{E}-12$ \\
\hline$\partial \mathrm{y} / \partial \mathrm{b}$ & & $-1.596 \mathrm{E}-11$ & $-1.115 \mathrm{E}-11$ \\
\hline$\partial \mathrm{y} / \partial \mathrm{c}$ & & $-3.011 \mathrm{E}-11$ & $-1.578 \mathrm{E}-11$ \\
\hline$\partial \mathrm{y} / \partial \mathrm{d}$ & & $2.271 \mathrm{E}-12$ & $1.586 \mathrm{E}-12$ \\
\hline$(\partial \mathrm{y} / \partial \mathrm{w})^{2} \Delta \mathrm{w}^{2}$ & & $3.500 \mathrm{E}-24$ & $1.270 \mathrm{E}-23$ \\
\hline$(\partial \mathrm{y} / \partial \mathrm{\partial})^{2} \Delta \rho^{2}$ & & $9.641 \mathrm{E}-25$ & $1.297 \mathrm{E}-25$ \\
\hline$(\partial \mathrm{y} / \partial \mathrm{t})^{2} \Delta \mathrm{t}^{2}$ & & $2.186 \mathrm{E}-27$ & $2.940 \mathrm{E}-28$ \\
\hline$(\partial \mathrm{y} / \partial \mathrm{a})^{2} \Delta \mathrm{a}^{2}$ & & $1.643 \mathrm{E}-27$ & $2.239 \mathrm{E}-28$ \\
\hline$(\partial \mathrm{y} / \partial \mathrm{b})^{2} \Delta \mathrm{b}^{2}$ & & $1.643 \mathrm{E}-27$ & $8.022 \mathrm{E}-28$ \\
\hline$(\partial \mathrm{y} / \partial \mathrm{c})^{2} \Delta \mathrm{c}^{2}$ & & $5.850 \mathrm{E}-27$ & $1.606 \mathrm{E}-27$ \\
\hline$(\partial \mathrm{y} / \partial \mathrm{d})^{2} \Delta \mathrm{d}^{2}$ & & $3.326 \mathrm{E}-29$ & $1.624 \mathrm{E}-29$ \\
\hline$\Delta \mathrm{y}$ & $\mathrm{cm} / \mathrm{hr}$ & $2.116 \mathrm{E}-12$ & $3.583 \mathrm{E}-12$ \\
\hline$\Delta \mathrm{y}$ & $\mu \mathrm{m} / \mathrm{yr}$ & $1.853 \mathrm{E}-04$ & $3.138 \mathrm{E}-04$ \\
\hline$\Delta \mathrm{y}$ & $\mathrm{nm} / \mathrm{yr}$ & 0.185 & 0.314 \\
\hline
\end{tabular}

Output DTN: MO0409MWDUGCMW.000.

Table 6-6. Summary of Measurement Uncertainty Analysis for Corrosion Rates Based on Weight-Loss Measurements after 5-Year Exposure in LTCTF

\begin{tabular}{|c|c|c|c|c|c|}
\hline & Sample Configuration & $\begin{array}{c}\text { Average } \\
\text { Weight Loss } \\
(\mathbf{g})\end{array}$ & $\begin{array}{c}\Delta \mathbf{y} \\
(\mathbf{n m} / \mathbf{y r})\end{array}$ & $\begin{array}{c}\text { Mean Corrosion } \\
\text { Rate } \\
(\mathbf{n m} / \mathbf{y r})\end{array}$ & $\begin{array}{c}\text { Standard } \\
\text { Deviation } \\
(\mathbf{n m} / \mathbf{y r})\end{array}$ \\
\hline & Weight-Loss Samples & 0.00036 & 0.314 & 2.75 & 2.74 \\
\hline & Crevice Samples & 0.00187 & 0.185 & 7.24 & 4.98 \\
\hline
\end{tabular}

Output DTN: MO0409MWDUGCMW.000.

The combined standard uncertainty is estimated to be approximately $0.185 \mathrm{~nm} / \mathrm{yr}$ in the case of crevice samples and $0.314 \mathrm{~nm} / \mathrm{yr}$ in the case of weight-loss samples. These estimates correspond to one standard deviation $(1 \sigma)$. Therefore, for the crevice samples, about 3 percent of the variation in the measured general corrosion rate is due to the measurement uncertainty, and 97 percent of it is from the variations of the corrosion rate among the specimens. For the weight-loss samples, most of the variation (about 89 percent) in the measured corrosion rate is due to variations among the specimens, and the rest is from measurement uncertainty. 
As discussed in the base-case conceptual model in Section 6.3, mineral deposits and scales could form on the waste package surface from evaporative concentration of the water in contact with the constituents such as dust and salts present on the surface. Crevices could form between the deposits and waste package. Therefore, the corrosion rate distribution determined from the creviced geometry samples was used for the base-case general corrosion rate of the WPOB (a conservative approach). Furthermore, because only a small amount (about 3 percent) of the total variation in the measured general corrosion rate of the crevice samples is due to the measurement uncertainty, all (100 percent) of the measured variation is considered to be due to the variability in the general corrosion processes for modeling purposes. The general corrosion rate variability is applied among modeled waste packages and among local areas on a waste package surface.

\subsubsection{Base-Case Temperature-Dependent General Corrosion Model}

The general corrosion (passive dissolution) of highly corrosion-resistant alloys such as Alloy 22 is governed by the transport properties of reacting species in the passive film and the dissolution rate of the passive film. The reacting species include metal ions, oxygen ions, vacancies and interstitials. These processes are influenced by the characteristics of the passive film, electrochemical potential across the film, and the chemistry of solution contacting the film (BSC 2001 [DIRS 155950], Section 7.3.5.1). Chromium and nickel oxides, which are the major constituents of the passive film of nickel-chromium-molybdenum alloys like Alloy 22 (Lorang et al. 1990 [DIRS 154718]), are stable and exhibit extremely low dissolution rates over a wide range of solution chemistry. The transport properties of the reacting species and reaction rates in the passive film are considered thermally activated processes. The general corrosion rate of the Alloy 22 waste package outer barrier is expected to have a certain level of temperature dependency (BSC 2001 [DIRS 155950], Section 7.3.5.1). The literature data summarized in a recent report by the Electric Power Research Institute (EPRI 2002 [DIRS 158069], Section 5.3.2) show such a temperature-dependence of Alloy 22 general corrosion rates in a wide range of test solution chemistries.

The general corrosion rate at a given temperature is represented by an Arrhenius relation in logarithmic form, i.e.:

$$
\ln \left(R_{T}\right)=C_{o}+\frac{C_{1}}{T}
$$

where $R_{T}$ is temperature-dependent general corrosion rate in $\mathrm{nm} / \mathrm{yr}, T$ is the absolute temperature in Kelvin, and $C_{o}$ and $C_{1}$ (in Kelvin) are constants.

The temperature-dependence term $\left(C_{1}\right)$ is determined from the corrosion rates obtained from the short-term polarization resistance data for Alloy 22 specimens tested for a range of sample configurations, metallurgical conditions, and exposure conditions (temperature and water chemistry). The intercept term, $C_{o}$, is determined from the value of $C_{1}$ and the distribution of measured general corrosion rates.

The polarization resistance of the samples was measured after 24-hour exposure of the samples in open-circuit potential in the test environments (Section 4.1.1.4, Appendix I). Polarization resistance is an acceptable technique for obtaining the temperature dependence of corrosion 


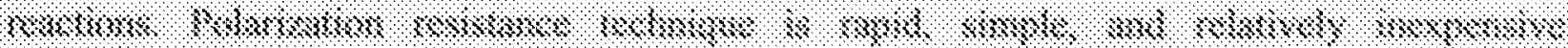

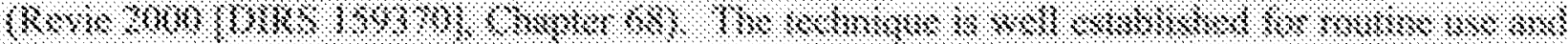

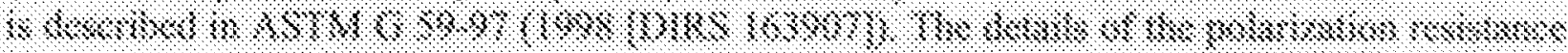

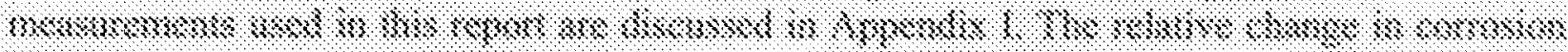

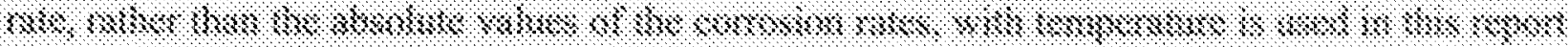

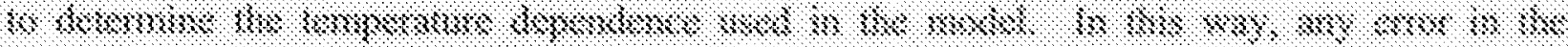

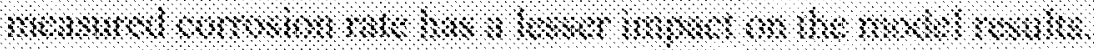

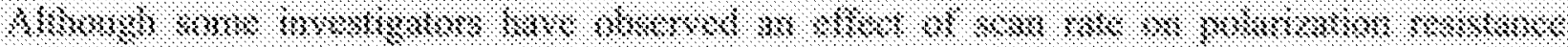

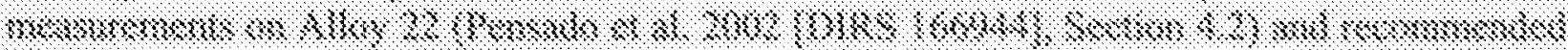

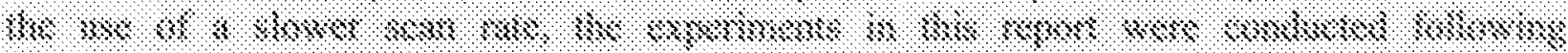

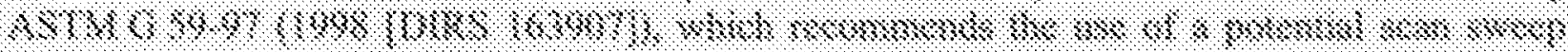

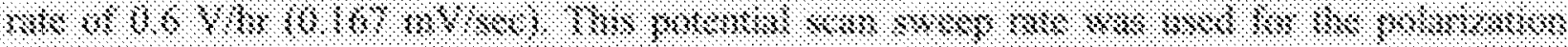

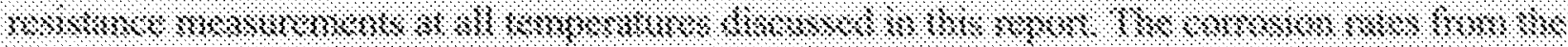

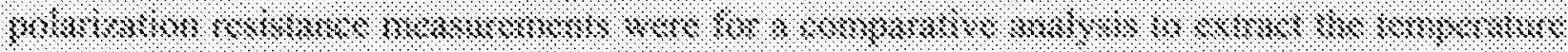

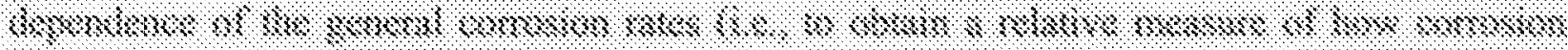

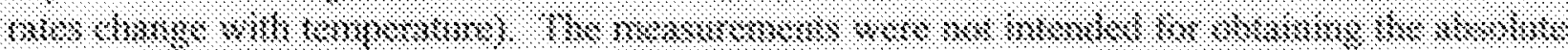

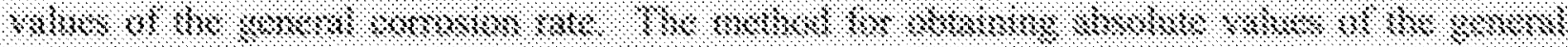

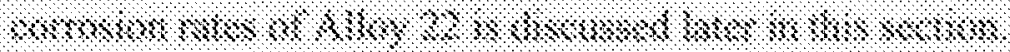

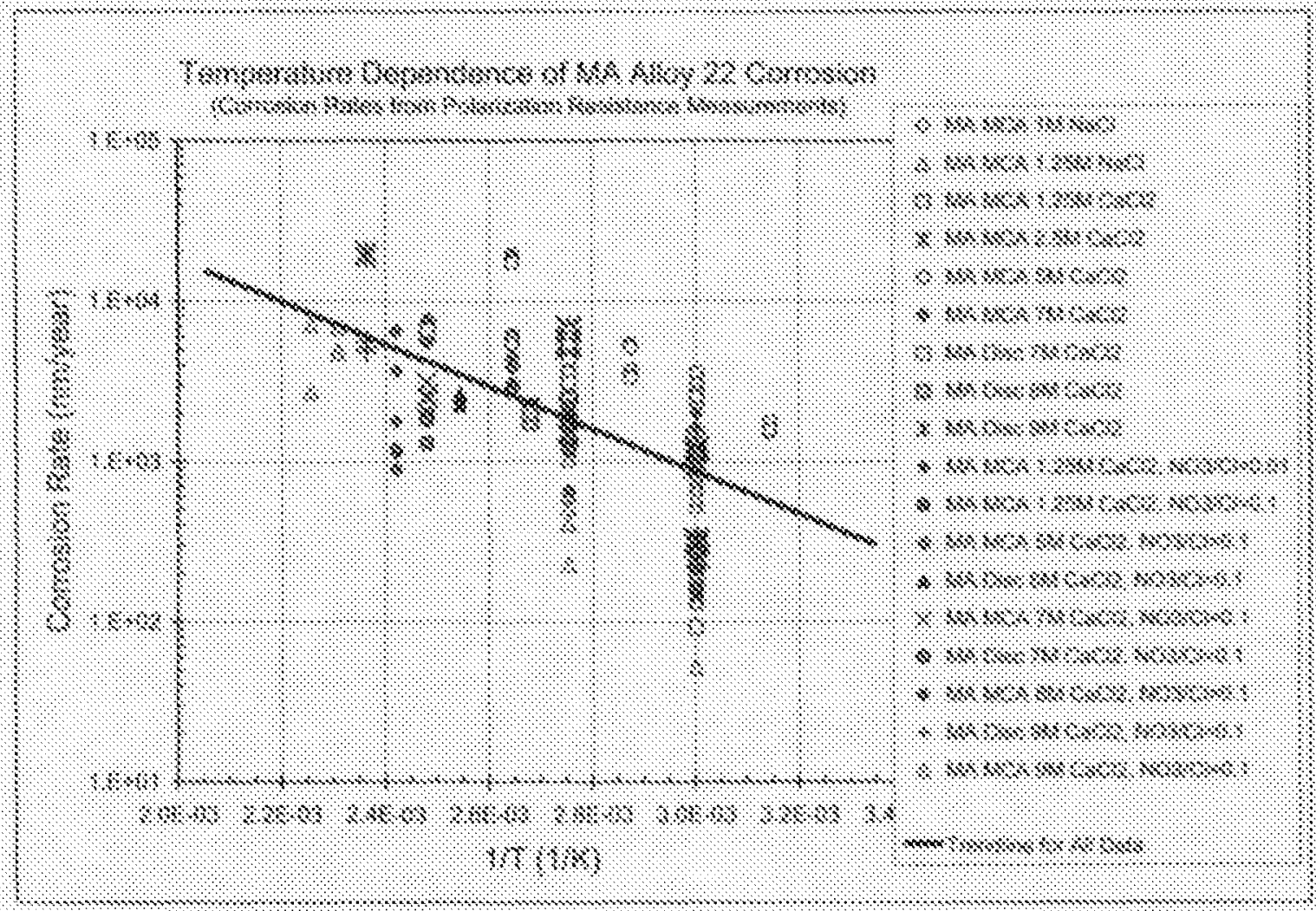

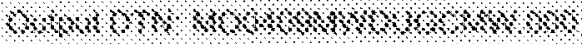

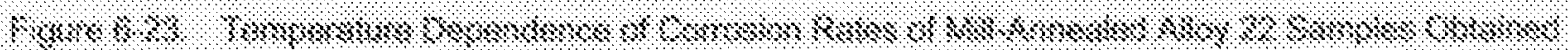

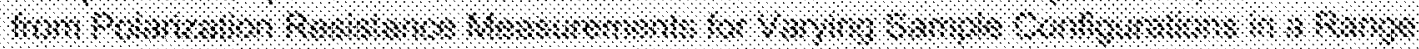

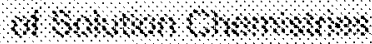




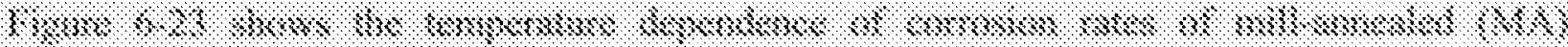

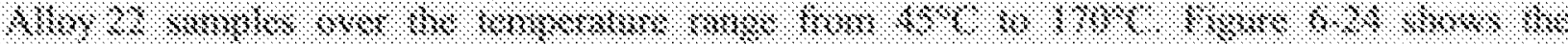

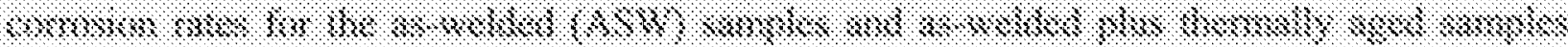

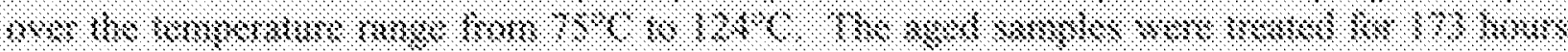

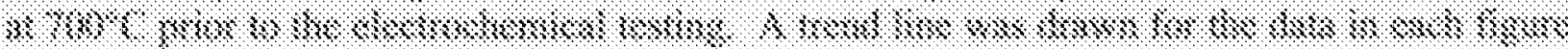

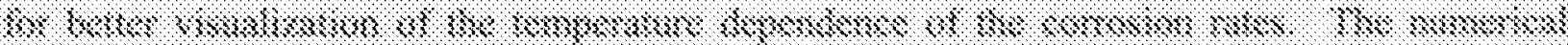

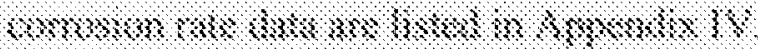

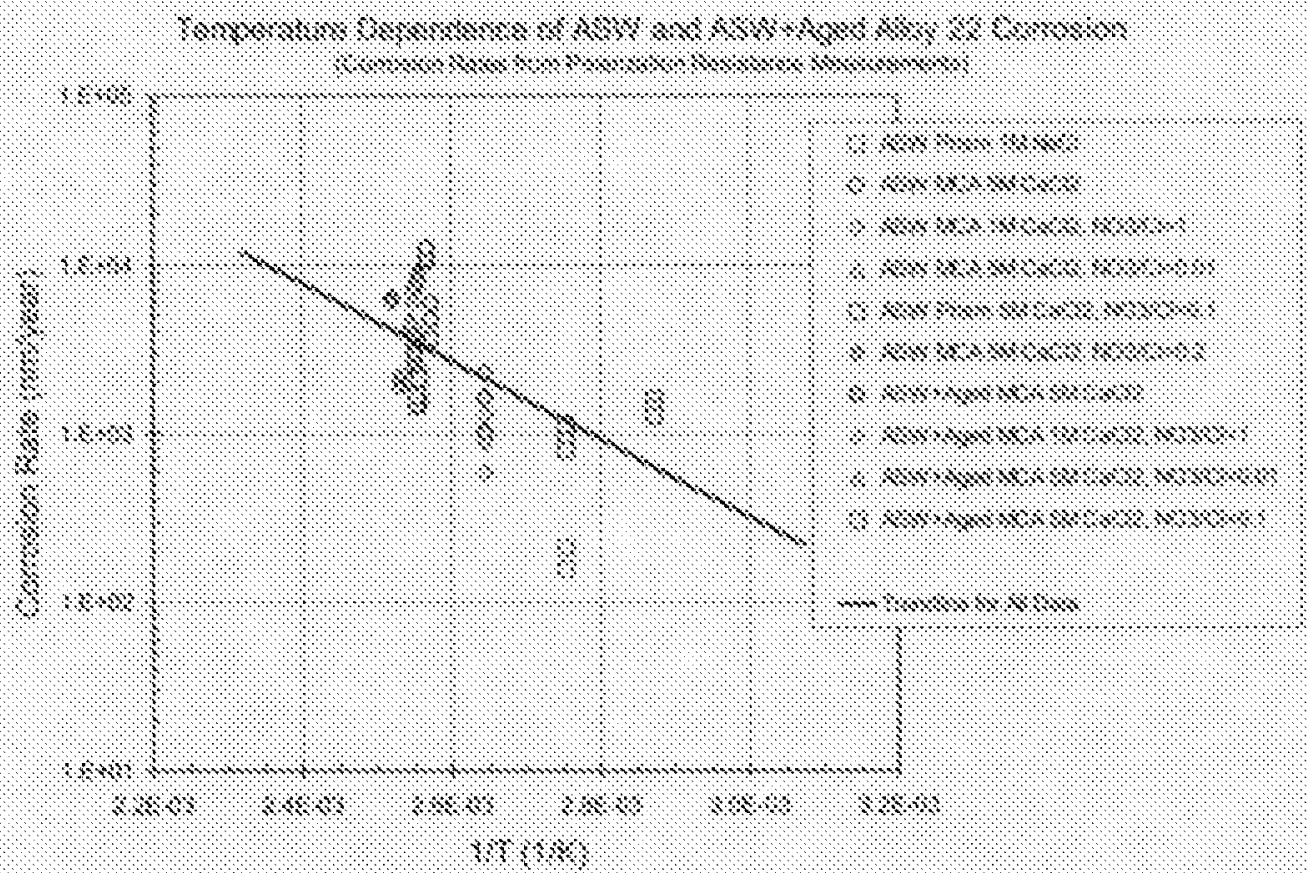

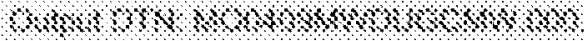

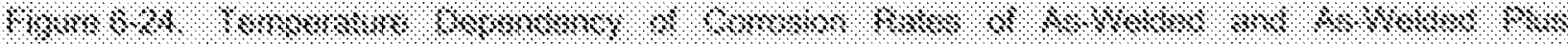

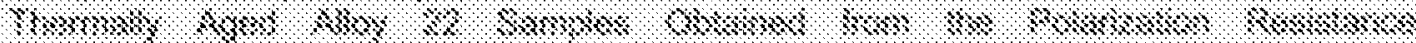

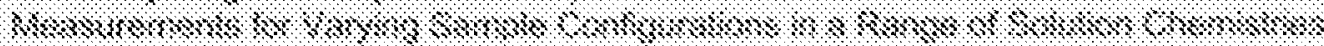

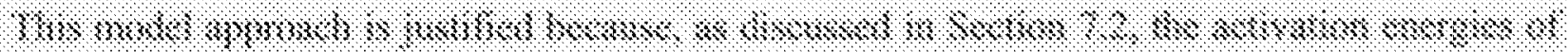

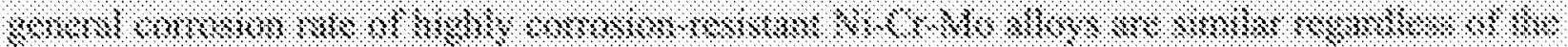

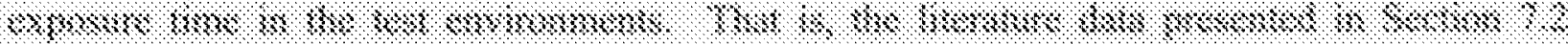

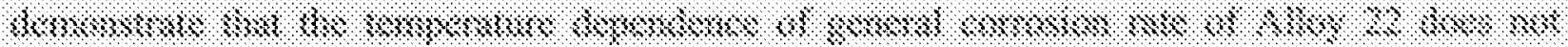

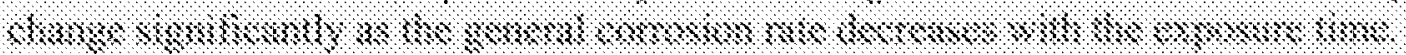

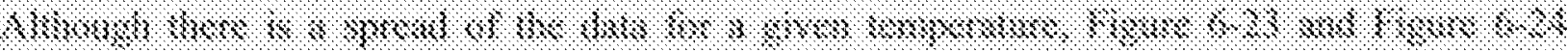

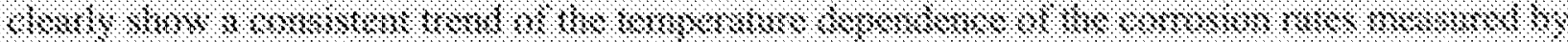

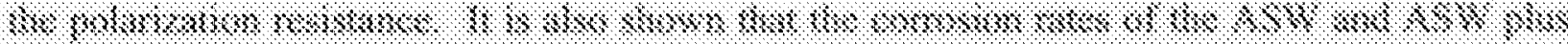

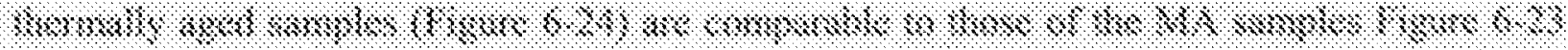

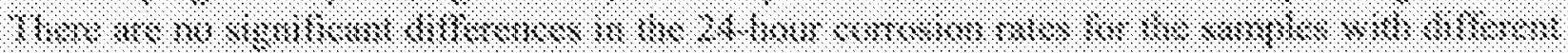

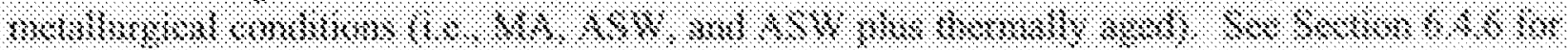

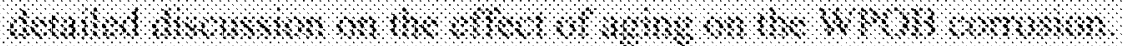


The corrosion rate data for all the samples with the three different metallurgical conditions were used together for the analysis of the temperature dependence of the WPOB general corrosion because their corrosion rates as obtained by the polarization resistance method are comparable. From fitting the data (listed in Appendix IV) to Equation 6-25, the temperature-dependence term, $C_{l}$, was determined. $C_{l}$ has a mean of $-3,116.47 \mathrm{~K}$ and a standard deviation of $296.47 \mathrm{~K}$. A normal distribution was used for the parameter in the regression analysis. Further analysis of the residuals showed that the errors are normally distributed. Rearranging Equation 6-25 to the form of the Arrhenius relation (i.e., $R_{T}=A \exp \left(-E_{a} / R T\right)$ ), $C_{1}$ can be expressed as $-E_{a} / R$, where $E_{a}$ is the activation energy $(\mathrm{J} / \mathrm{mol})$, and $R$ is the universal gas constant $\left(8.314 \mathrm{~J} \cdot \mathrm{mol}^{-1} \cdot \mathrm{K}^{-1}\right)$. This temperature dependence corresponds to an activation energy of $25.91 \pm 2.46 \mathrm{~kJ} / \mathrm{mol}$. The data for the MCA samples with their edges not polished were not included in the regression analysis for the temperature dependence (Section 4.1.1.4).

The general corrosion rate distribution determined from the weight-loss data of the 5-year crevice specimens measured at both $60^{\circ} \mathrm{C}$ and $90^{\circ} \mathrm{C}$ (Figure 6-17) represents the distribution of long-term general corrosion rates of the WPOB at $60^{\circ} \mathrm{C}$ for the purposes of this model. This approach is reasonably bounding because the general corrosion rates for crevice specimens are generally higher than those of noncreviced weight-loss specimen. The intercept term, $C_{o}$, can be determined from the temperature-dependence term, $C_{1}$, as follows:

$$
\begin{aligned}
& \ln \left(R_{o}\right)=C_{o}+\frac{C_{1}}{T_{0}} \\
& C_{o}=\ln \left(R_{o}\right)-\frac{C_{1}}{T_{0}}
\end{aligned}
$$

where $R_{o}$ is the general corrosion rate distribution from the weight loss of the 5-year crevice samples (Figure 6-22) in $\mathrm{nm} / \mathrm{yr}$ at $T_{0}=333.15 \mathrm{~K}\left(60^{\circ} \mathrm{C}\right)$. Substituting for $C_{o}$ in Equation 6-25, results in:

$$
\ln \left(R_{T}\right)=\ln \left(R_{o}\right)+C_{1}\left(\frac{1}{T}-\frac{1}{333.15}\right)
$$

The entire variance of the temperature dependence term, $C_{1}$, is due to uncertainty, and the uncertainty is limited to \pm 3 standard deviations. This treatment appropriately reflects the lack of knowledge in the exact value to the temperature dependence term and incorporates 99.7 percent of the data variance ( \pm 3 standard deviations).

The 5-year corrosion rate data for Alloy 22 were analyzed to determine the theoretical distribution that best fits the data. The following three goodness-of-fit tests were performed for the data analysis: the Chi-Square test, the Kolmogorov-Smirnov test, and the Anderson-Darling test. All the statistical tests indicated that the Weibull distribution best fits the 5-year corrosion rate data. Therefore, it was decided to use a Weibull distribution. To estimate the parameters of the Weibull distribution, the method of Maximum Likelihood Estimator was used. A Weibull distribution, with scale factor, $s=8.88 \mathrm{~nm} / \mathrm{yr}$; shape factor, $b=1.62$; and location factor, $l=0 \mathrm{~nm} / \mathrm{yr}$ best fits the corrosion rate distribution. 
Therefore, $R_{o}$ is expressed as an inverse Weibull CDF as follows (Evans et al. 1993 [DIRS 112115], p. 154):

$$
R_{o}=s\left[\ln \left(\frac{1}{1-p}\right)\right]^{1 / b}
$$

where $p$ is the cumulative probability, and $s$ and $b$ were defined and their values were given above. The cumulative distribution function of $R_{o}$ is given in Figure 6-25 along with the crevice sample data (Figure 6-22). The $99.99^{\text {th }}$ percentile rate (a reasonable upper-bound value) of $R_{o}$ is $35 \mathrm{~nm} / \mathrm{yr}$. This value is greater than the measured maximum rate of $23 \mathrm{~nm} / \mathrm{yr}$ for the crevice samples (Section 6.4.3.2).

The model outputs $\left(R_{T}\right)$ at temperatures of $25^{\circ} \mathrm{C}, 50^{\circ} \mathrm{C}, 75^{\circ} \mathrm{C}, 100^{\circ} \mathrm{C}, 125^{\circ} \mathrm{C}$ and $150^{\circ} \mathrm{C}$ are shown in Figure 6-26. The model results shown in the figure are for the mean value of the temperature dependence term $\left(C_{l}\right)$ and having the entire variance of $R_{o}$ due to the variability in the general corrosion process (Table 6-6 and associated discussions above for additional details on the basis for the corrosion rate variability). Figure 6-27 and Figure 6-28 show the cumulative distribution functions (CDFs) of the model outputs $\left(R_{T}\right)$ at temperatures of $25^{\circ} \mathrm{C}, 50^{\circ} \mathrm{C}, 75^{\circ} \mathrm{C}$, $100^{\circ} \mathrm{C}, 125^{\circ} \mathrm{C}$, and $150^{\circ} \mathrm{C}$ for the upper bound activation energy of $33.29 \mathrm{~kJ} / \mathrm{mol}$ and the lowerbound activation energy of $18.53 \mathrm{~kJ} / \mathrm{mol}$, respectively. The model results for the elevated temperatures assume the passivity of the alloy is maintained at those temperatures. Therefore, the cumulative distribution functions of the model calculated general corrosion rates in the figure represent the range of the variable general corrosion rates of the WPOB at each exposure temperature of interest, with the mean $C_{1}$ value.

The model results show that the uncertainty range of the $50^{\text {th }}$ percentile general corrosion rate at $25^{\circ} \mathrm{C}$ is from 1.7 to $3.2 \mathrm{~nm} / \mathrm{yr}$ (mean $2.4 \mathrm{~nm} / \mathrm{yr}$ ), and that of the $99^{\text {th }}$ percentile rate is from 5.6 to $10.4 \mathrm{~nm} / \mathrm{yr}$ (mean $7.6 \mathrm{~nm} / \mathrm{yr}$ ) (Figure 6-26 to Figure 6-28). For $100^{\circ} \mathrm{C}$, the uncertainty range of the $50^{\text {th }}$ percentile general corrosion rate is from 14.5 to $25.7 \mathrm{~nm} / \mathrm{yr}$ (mean $19.3 \mathrm{~nm} / \mathrm{yr}$ ), and that of the $99^{\text {th }}$ percentile rate is from 46.7 to $82.7 \mathrm{~nm} / \mathrm{yr}$ (mean $62.1 \mathrm{~nm} / \mathrm{yr}$ ) (Figure 6-26 to Figure 6-28). In addition, for $150^{\circ} \mathrm{C}$ and assuming the passivity of the alloy is maintained, the uncertainty range of the $50^{\text {th }}$ percentile general corrosion rate is from 29.4 to $91.3 \mathrm{~nm} / \mathrm{yr}$ (mean $51.8 \mathrm{~nm} / \mathrm{yr}$ ), and that of the $99^{\text {th }}$ percentile rate is from 94.6 to $294 \mathrm{~nm} / \mathrm{yr}$ (mean $167 \mathrm{~nm} / \mathrm{yr}$ ) (Figure 6-26 to Figure 6-28).

For a constant waste package surface temperature of $150^{\circ} \mathrm{C}$ and assuming the WPOB maintains the passivity, the total penetration depth by general corrosion over the regulatory period, using the $50^{\text {th }}$ percentile general corrosion rate of $51.8 \mathrm{~nm} / \mathrm{yr}$ for the mean activation energy $\left(25.91 \mathrm{~kJ} / \mathrm{mol}\right.$ ) (Figure 6-26), is only $518 \mu \mathrm{m}$ (about $0.5 \mathrm{~mm}$ ). Using the $99.99^{\text {th }}$ percentile rate (a reasonable upper bound rate) of $256 \mathrm{~nm} / \mathrm{yr}$ at $150^{\circ} \mathrm{C}$ for the mean activation energy (Figure 6-26), the total penetration depth by general corrosion is only $2,560 \mu \mathrm{m}$ (about $2.6 \mathrm{~mm}$ ). For the upper bound activation energy $(33.29 \mathrm{~kJ} / \mathrm{mol}$ ) (Figure 6-27), the total penetration depth by general corrosion over the regulatory period at an exposure temperature of $150^{\circ} \mathrm{C}$, using the $50^{\text {th }}$ percentile general corrosion rate $(91.3 \mathrm{~nm} / \mathrm{yr}$ ) is $913 \mu \mathrm{m}$ (about $0.9 \mathrm{~mm}$ ), and the total penetration depth using the $99.99^{\text {th }}$ percentile general corrosion rate $(451 \mathrm{~nm} / \mathrm{yr})$ is $4,510 \mu \mathrm{m}$ (about $4.5 \mathrm{~mm}$ ). This bounding analysis clearly demonstrates that the waste package performance is not limited by general corrosion. 


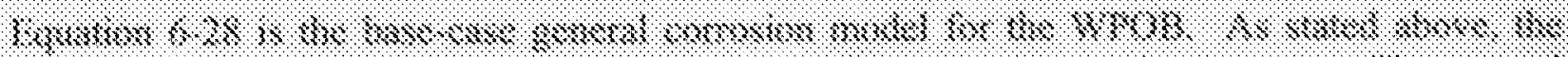

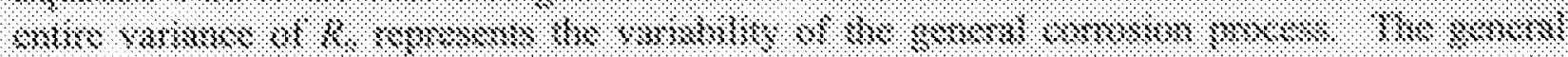

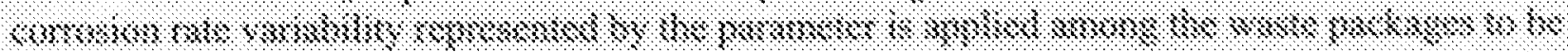

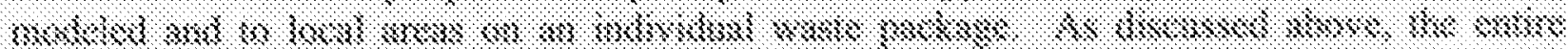

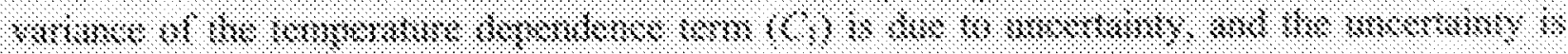

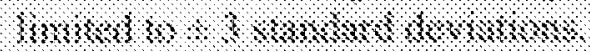

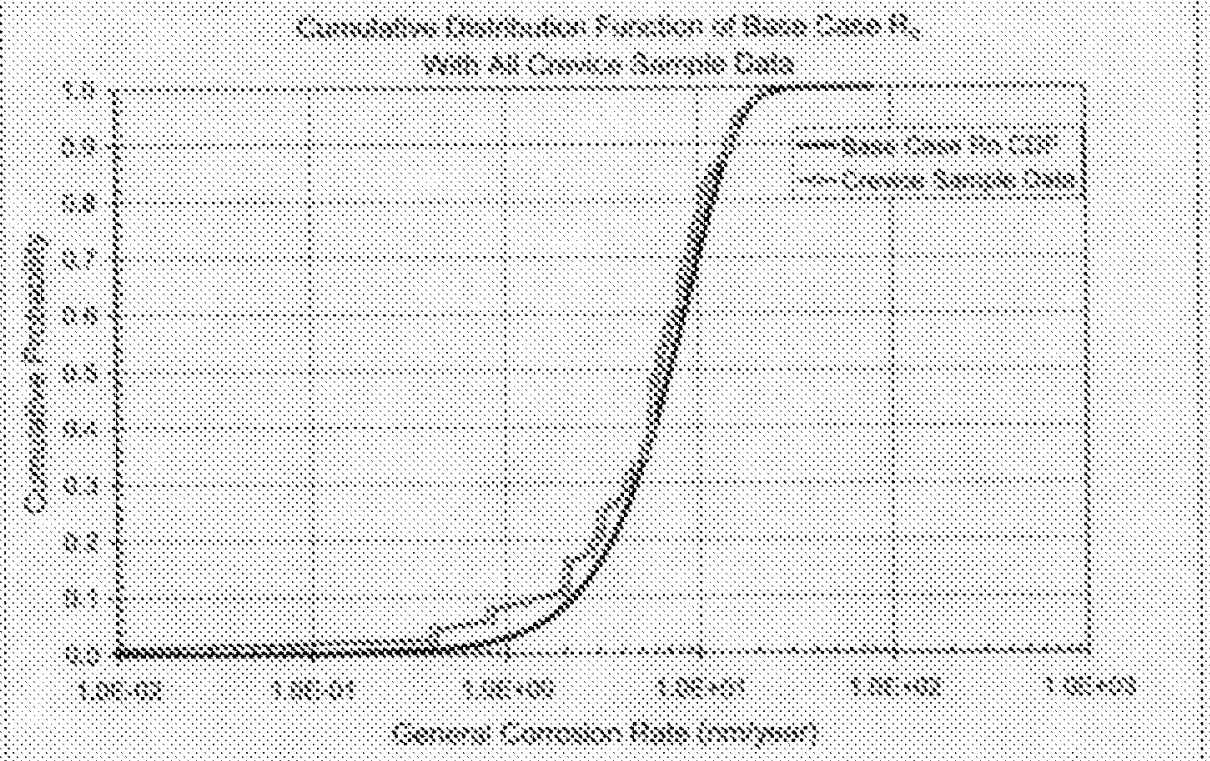

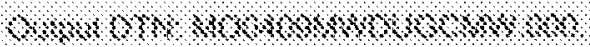

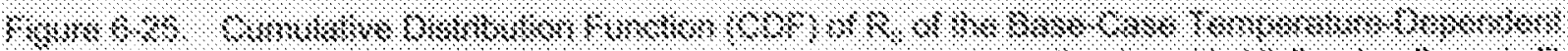

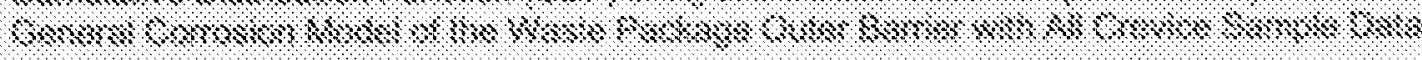




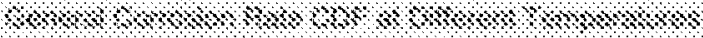

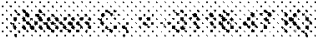

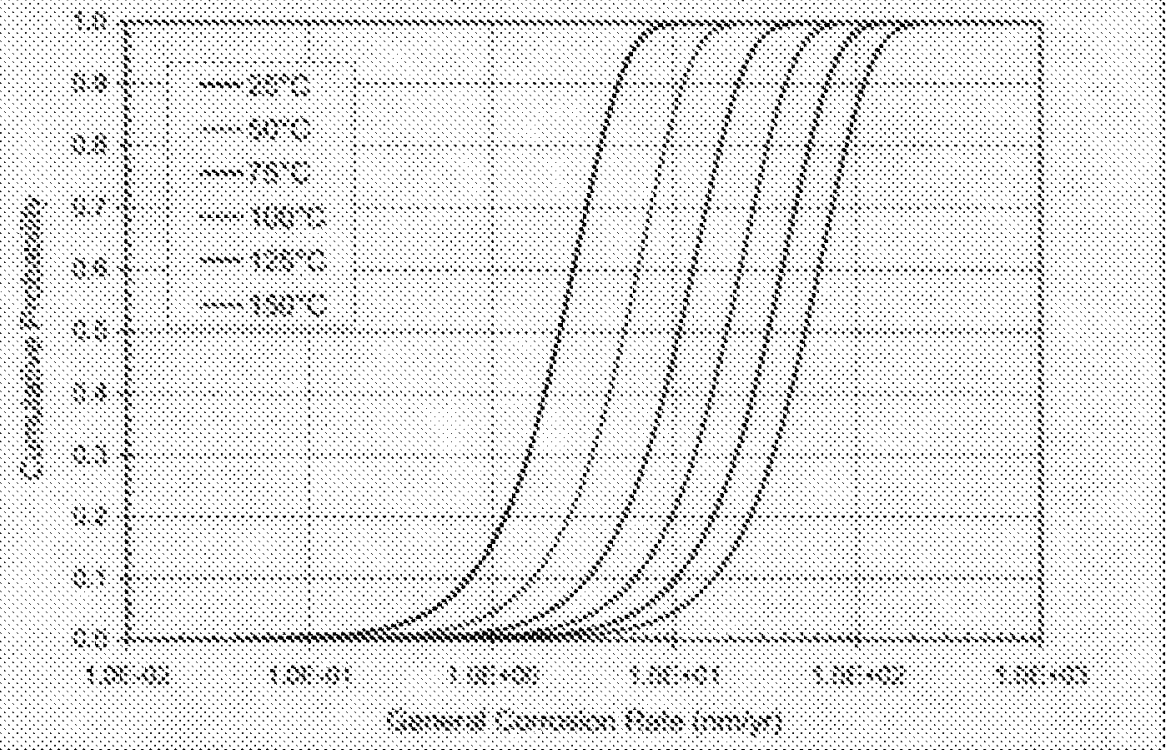

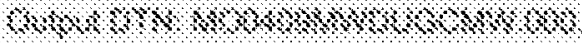

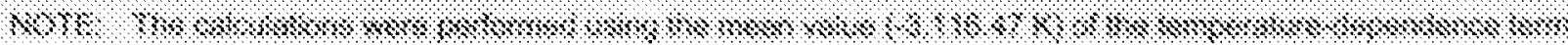

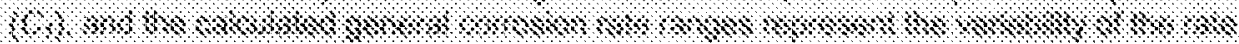

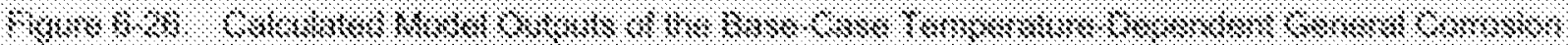

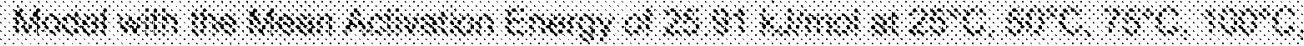

$308,0 \times 8 \%$ 


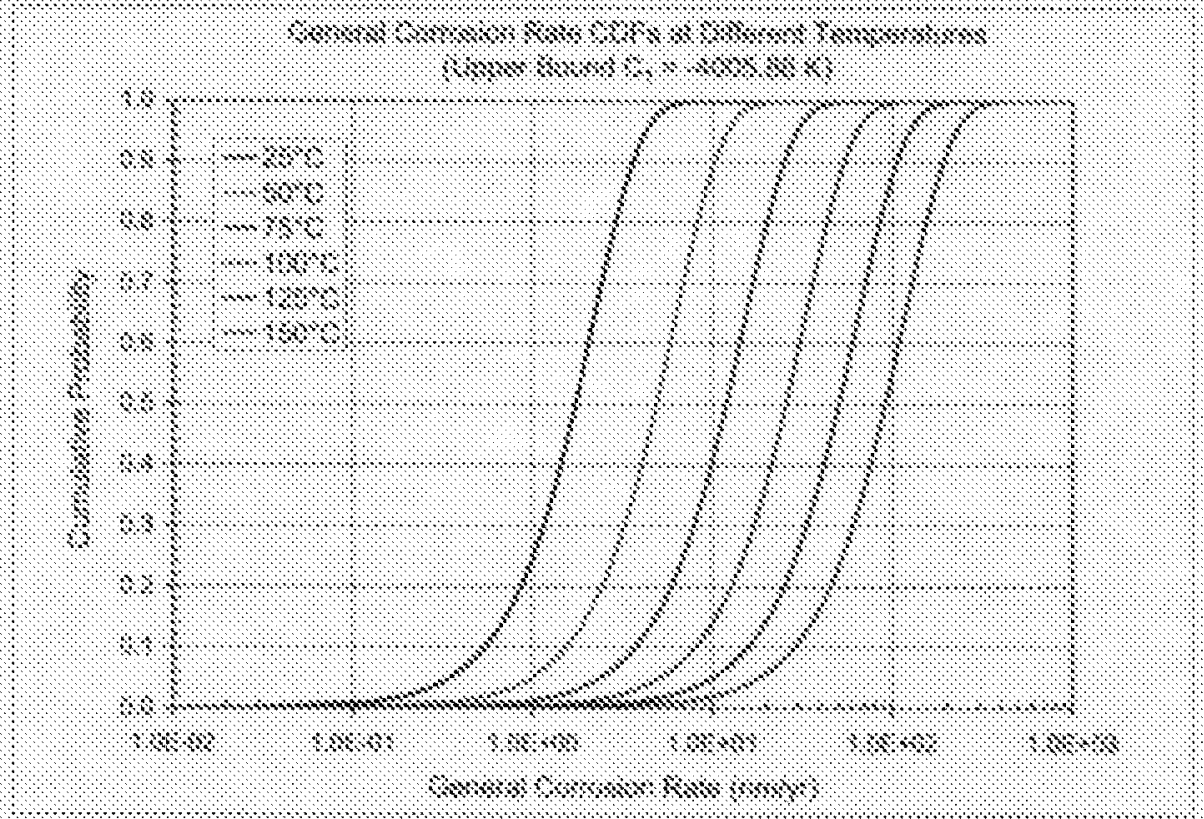

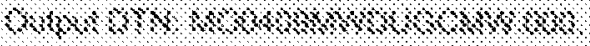

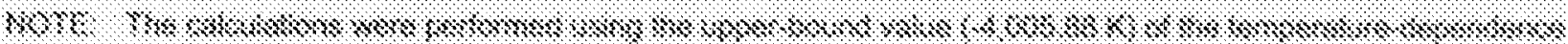

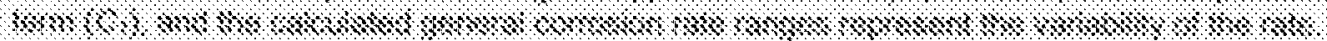

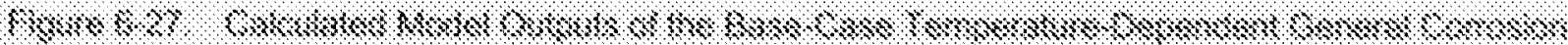

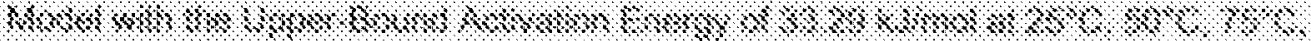

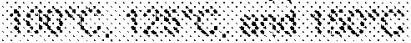

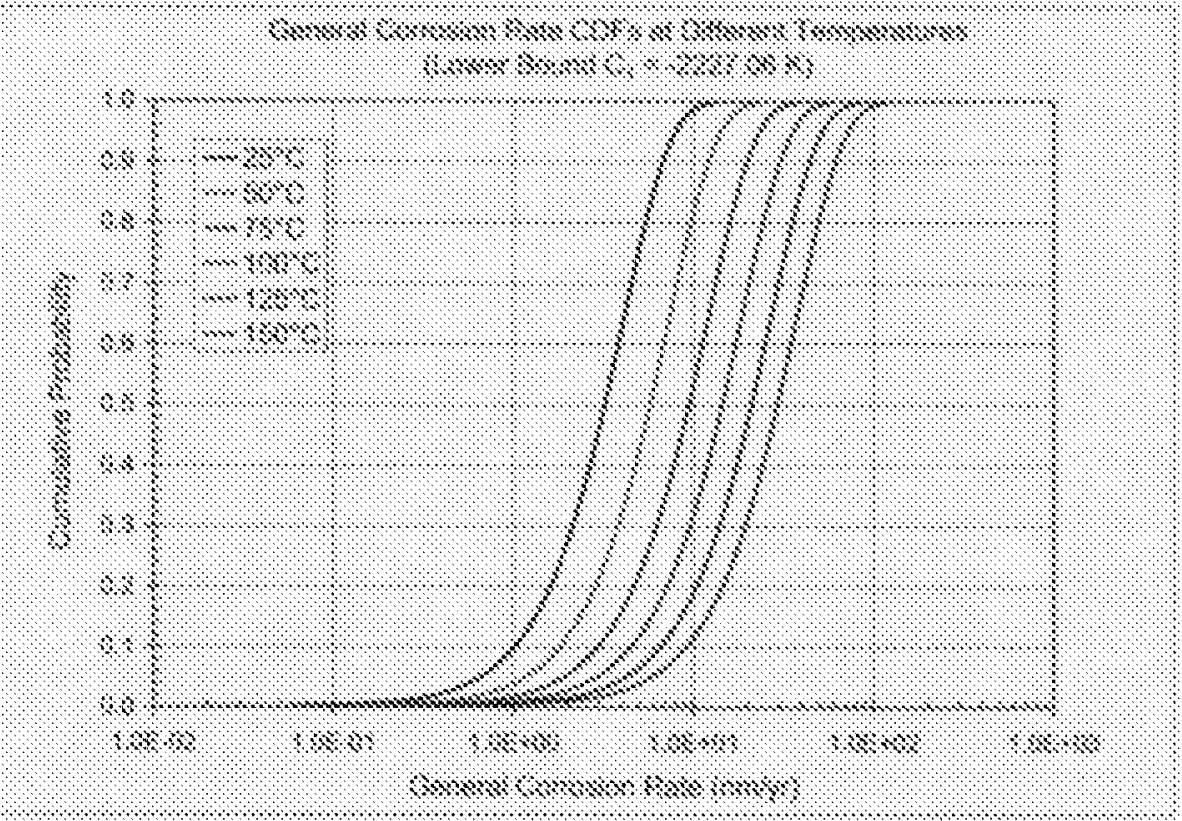

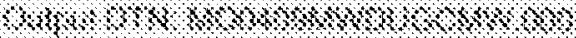

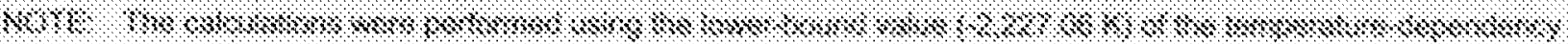

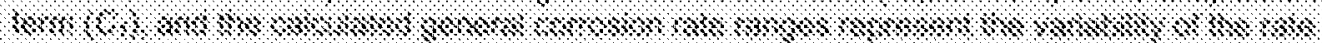

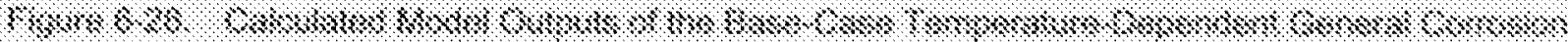

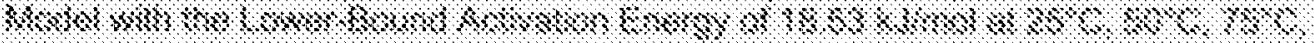

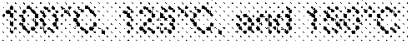




\subsubsection{Alternative Conceptual Model for General Corrosion}

Alternative conceptual models (ACMs) are based on modeling assumptions and simplifications different from those employed in the base-case model. An important reason for considering ACMs is to help build confidence that changes in modeling assumptions or simplifications will not change conclusions regarding subsystem and total system performance. Conceptual model uncertainty results from sparse observational data and a lack of available information to corroborate or refute plausible alternative interpretations of the subsystem and the processes occurring within the subsystem. This section discusses the ACMs for the general corrosion models of the WPOB. None of the general corrosion ACMs discussed in this section are included in the TSPA-LA.

\subsection{Time-Dependent General Corrosion Behavior of the Waste Package Outer Barrier}

As discussed in detail in Section 6.4.3.4, the base-case general corrosion model for the WPOB is based on the five-year weight-loss measurements of Alloy 22 crevice samples from the LTCTF. The general corrosion rate is temperature dependent, and for a given temperature, it is assumed constant (time independent) (Assumption 5.2). Therefore, for a given temperature, the depth of penetration or thinning of the WPOB by general corrosion is equal to the general corrosion rate at that temperature, multiplied by the time duration that the waste package surface is at that temperature. However, general corrosion rates of metals and alloys tend to decrease with time. Table 7-1, where general corrosion rates determined from potentiostatic polarization, polarization resistance, and weight-loss measurements are presented, shows a marked decrease in corrosion rate with time from 1 day to 2.3 years (the 5-year data point is excluded from this discussion for model validation purposes). Figure 7-1 shows that even if the 5-year data point were excluded from the figure, general corrosion rates for Alloy 22 decrease with time. Section 7.2 discusses that the trend of decreasing general corrosion rate with time is consistent with the expected corrosion behavior of passive alloys such as Alloy 22 under repository-type aqueous conditions.

The time-dependent general corrosion behavior of the WPOB was not included in the TSPA-LA because the constant (time-independent) rate model (for a given temperature) is more conservative and will bound the general corrosion behavior of the WPOB over the repository time period.

\subsection{Alternative Conservative Interpretation of Long-Term General Corrosion Rates of Crevice Samples}

As shown in Figure 6-22, the general corrosion rates of the crevice samples are higher than those of the "plain" weight-loss samples. This may have been caused by different surface polishing treatments between the two sample groups. Another supposition is that there might have been higher corrosion rates in the creviced area under the crevice former. Figure 6-29 provides a schematic diagram of the crevice sample geometry. One modeling assumption made in this section, is that the corrosion rate of the boldly exposed (noncrevice) area of the crevice samples can be represented by the average corrosion rate of the weight-loss geometry samples. Using 
this modeling assumption, the following data manipulations were made to estimate the corrosion rate of the crevice area under the crevice former.

- The average weight loss per unit area of weight-loss geometry samples was calculated.

- The "true" weight loss of the boldly exposed area of the crevice samples was estimated by multiplying the average weight loss per unit area (from the weight-loss geometry samples above) by the boldly exposed area of the crevice samples.

- The weight loss from the crevice area under the crevice former was determined by subtracting the "true" weight loss of the boldly exposed area from the total weight loss measured for the crevice samples.

- The corrosion rate of the crevice-area-only (the area under the crevice former) was calculated using the calculated weight loss from the crevice area.

The empirical CDF of the estimated corrosion rates of the crevice-area-only under the crevice former is shown in Figure 6-30, along with the empirical CDFs of the weight-loss samples and crevice samples for comparison. The median corrosion rate $\left(50^{\text {th }}\right.$ percentile rate $)$ of the crevice-area-only is $51 \mathrm{~nm} / \mathrm{yr}$ and is about 10 times the mean of the crevice samples. The maximum rate $\left(100^{\text {th }}\right.$ percentile rate $)$ of the crevice area only case is about $214 \mathrm{~nm} / \mathrm{yr}$, which is also about 10 times of the maximum rate of the crevice samples. Because the calculated corrosion rates of the crevice area under the crevice former are much higher than those of the boldly exposed area of the crevice samples, microscopic examinations would be able to show the differences in the corrosion fronts between the two areas (or at least at the edge of the area under the crevice former). However, examinations by optical microscopy and SEM did not reveal such differences.

As with the crevice sample case, it was decided to use a Weibull distribution for $R_{o}$. The method of Maximum Likelihood Estimator was used to estimate the parameters of the Weibull distribution. A Weibull distribution, with scale factor $s=82.3$, shape factor $b=1.36$, and location factor $l=0$, best fits the corrosion rate distribution for the crevice-area-only case. The cumulative distribution function of $R_{o}$ for the crevice-area-only case is given in Figure 6-31.

The measurement error for the crevice-area-only case was taken to be the same as the crevice sample case given in Table 6-6. Using the same mean value of the same temperaturedependence term $\left(C_{l}\right)$ (i.e., $\left.N(\mu=-3,116.47 K, \sigma=296.47 K)\right)$ and sampling for $R_{o}$ from the entire corrosion rate distribution for the conservative crevice-area-only case (Figure 6-31), the general corrosion rate distributions were calculated at $25^{\circ} \mathrm{C}, 50^{\circ} \mathrm{C}, 75^{\circ} \mathrm{C}, 100^{\circ} \mathrm{C}, 125^{\circ} \mathrm{C}$, and $150^{\circ} \mathrm{C}$ using the temperature-dependent model in Equation 6-28. The results are shown in Figure 6-32. For the $C_{l}$ value sampled at the mean, the CDFs in the figure represent the range of the variable general corrosion rates of the WPOB at each exposure temperature of interest for the conservative crevice-area-only case. Because it is not known what caused the higher corrosion rates for the crevice samples, the alternative conservative interpretation of the Alloy 22 general corrosion rate is not included in the waste package degradation analysis. 


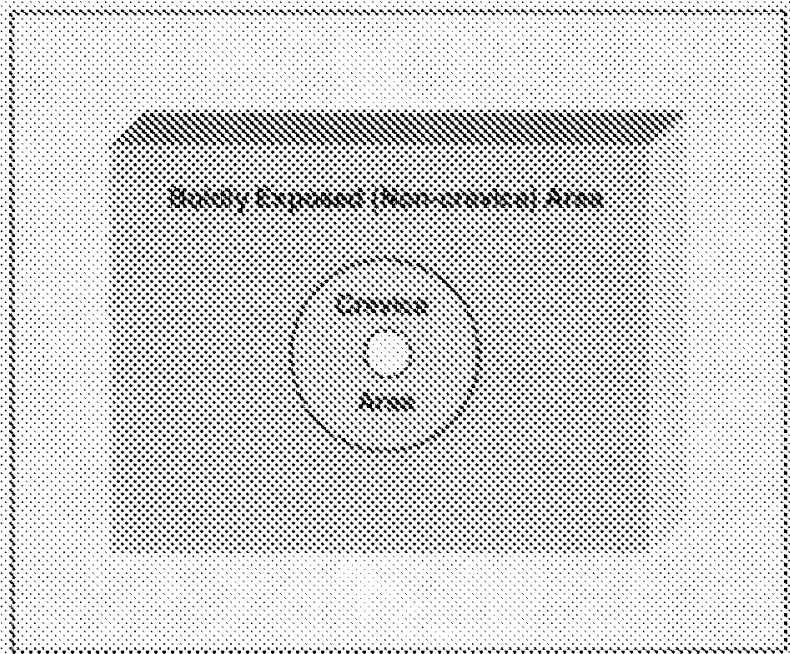

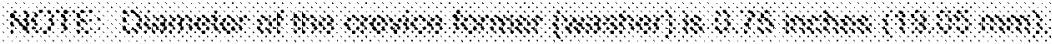

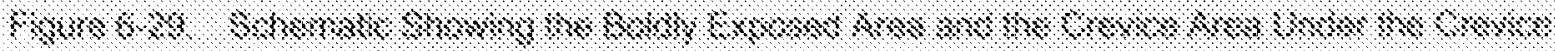

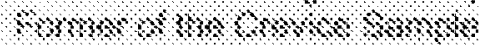

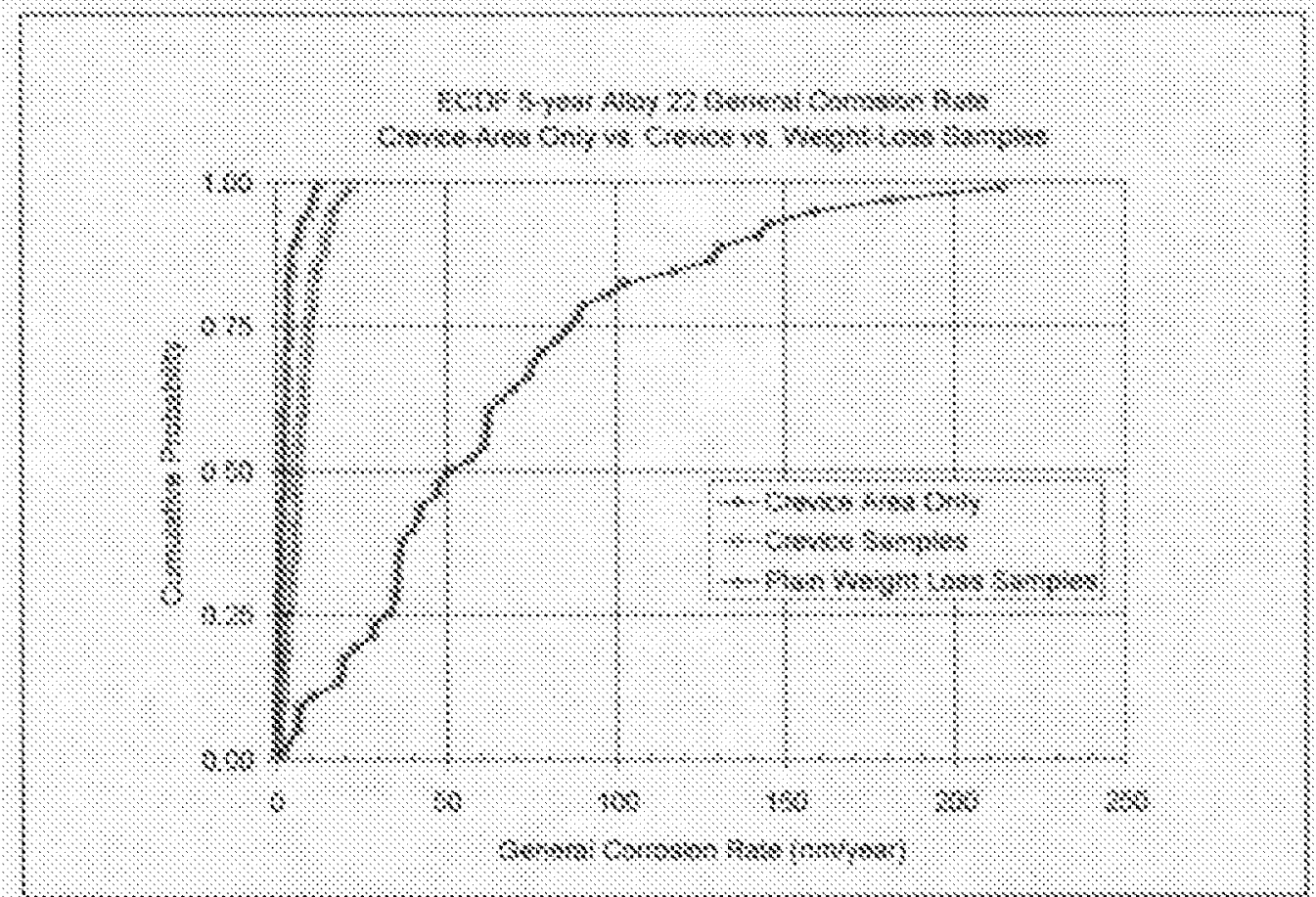

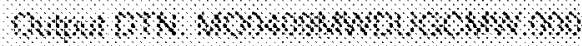

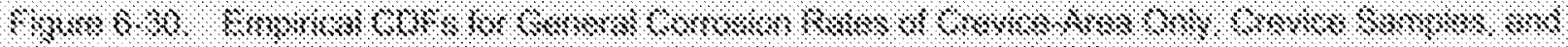

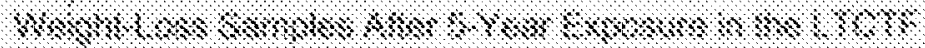




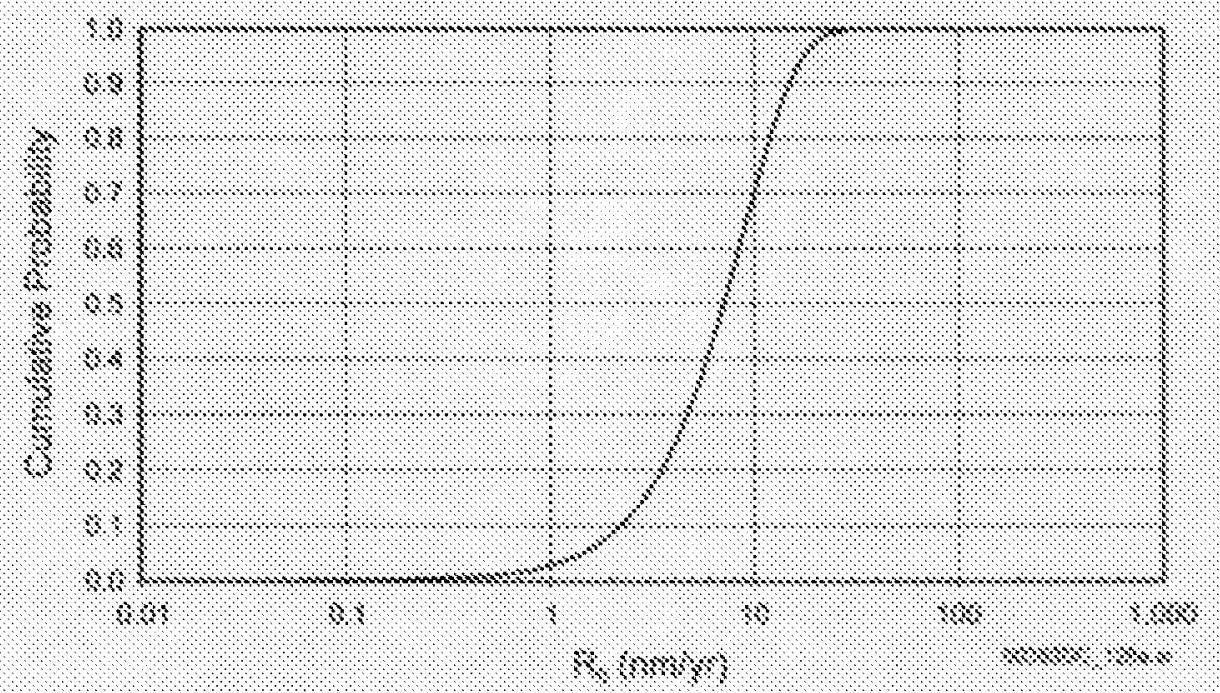

$0,6 \times 018 \times 4 \times 8 \times 8 \times 48 \times 8 \times 1 \% 8$

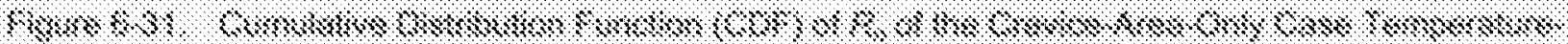

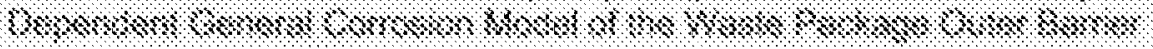

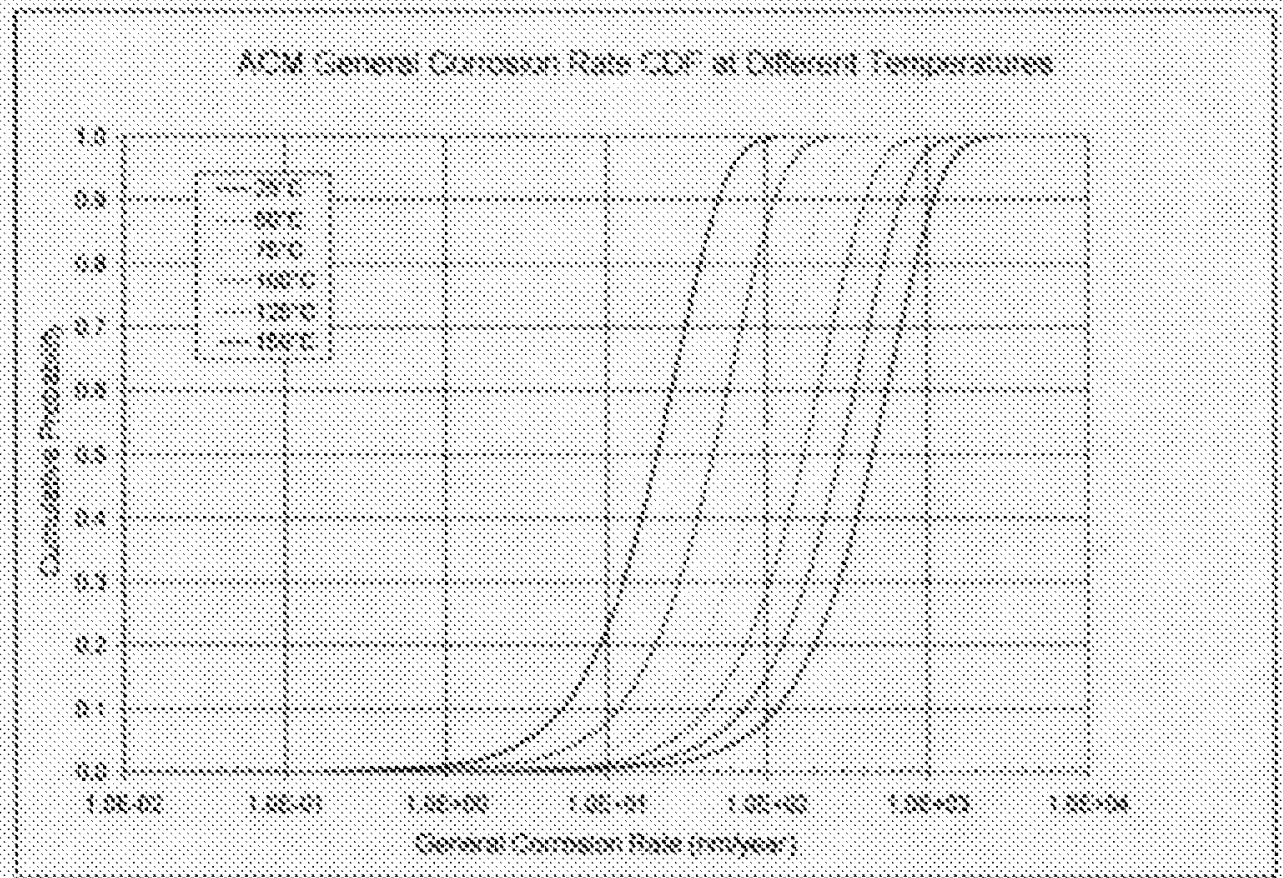

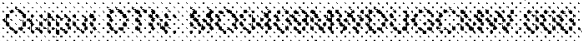

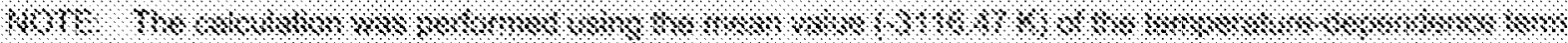

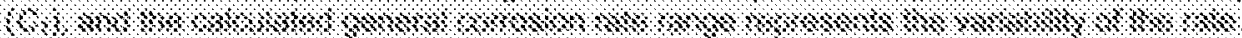

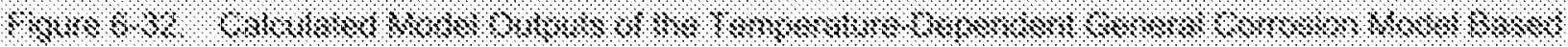

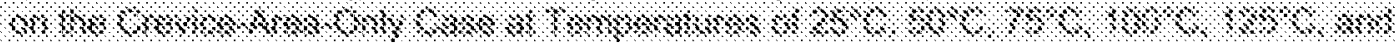
88 \% 
(a) All waste packages

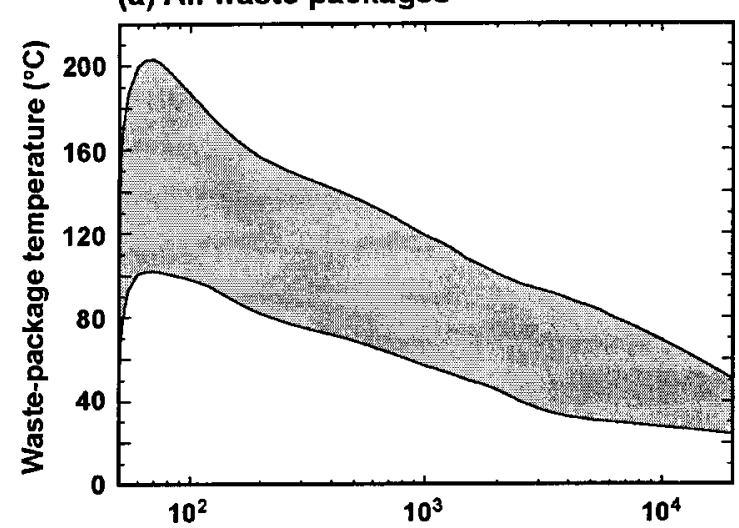

(c) All CSNF waste packages

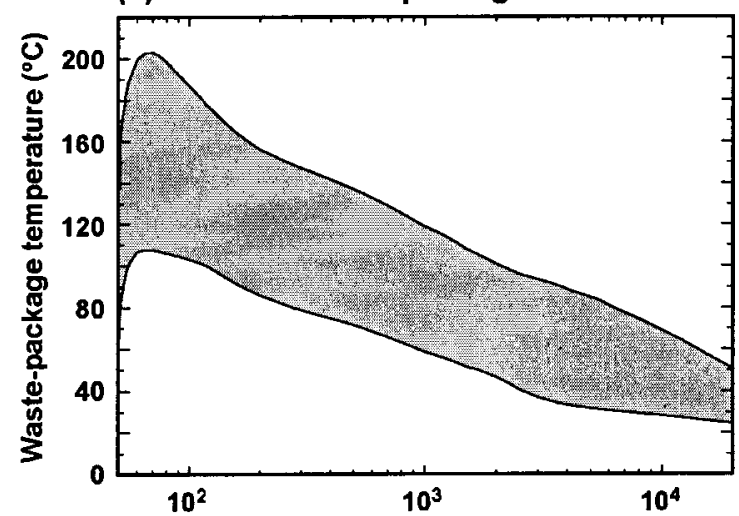

(e) All DHLW waste packages

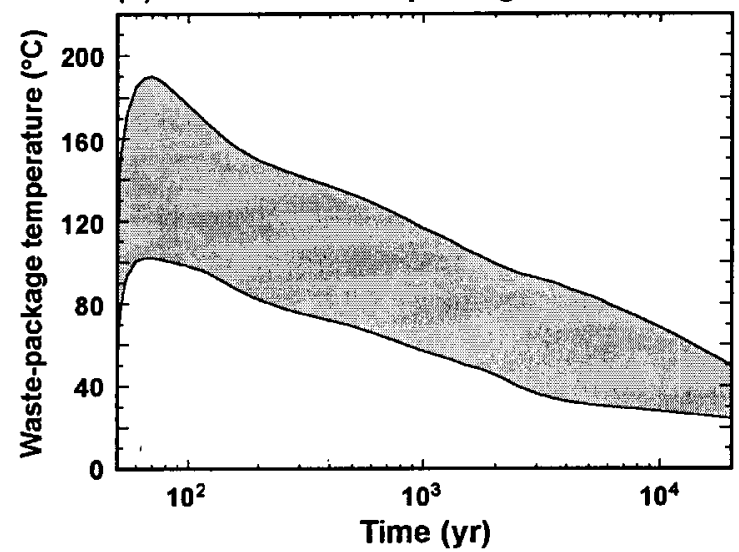

(b) All waste packages

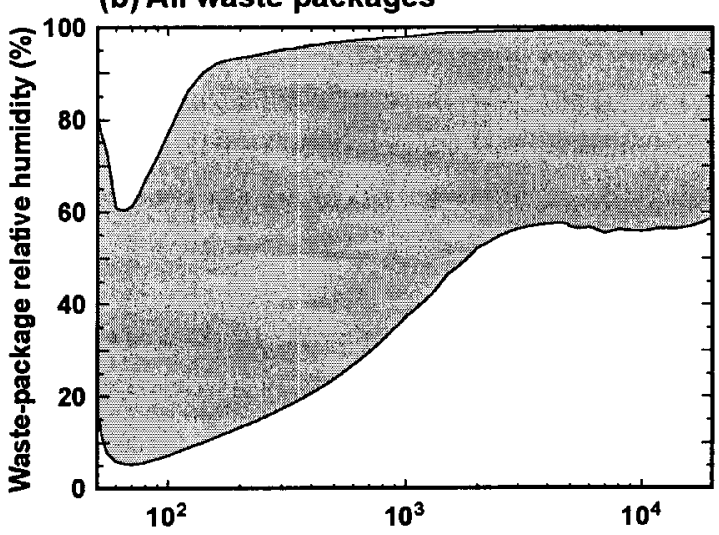

(d) All CSNF waste packages

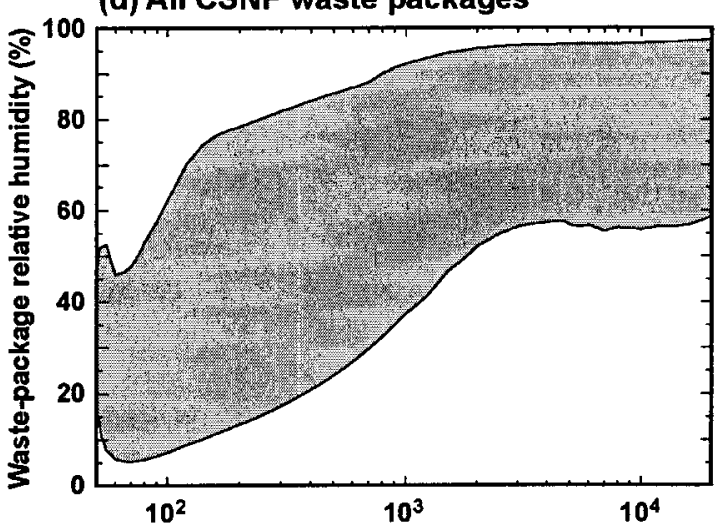

(f) All DHLW waste packages

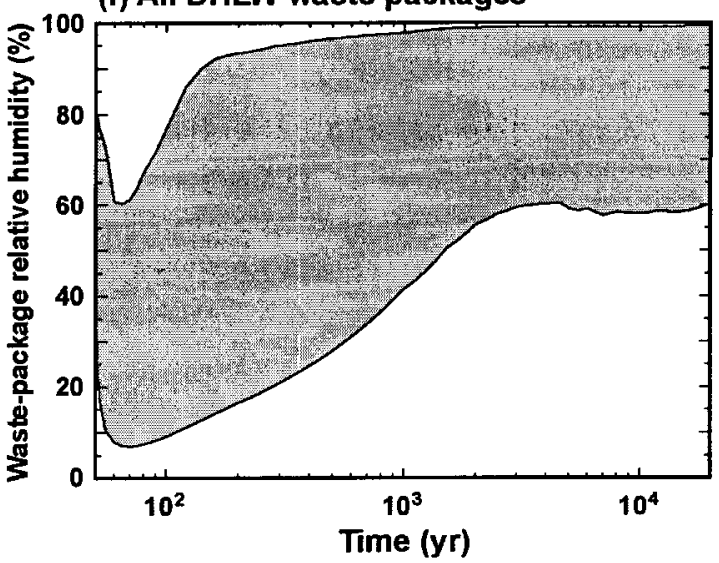

T\&RH_all_CSNF_DHLW_horsetail

Source: BSC 2004 [DIRS 169565], Figure 6.3-52.

NOTE: The ranges include the lower-bound, mean, and upper-bound infiltration flux cases and use of the mean thermal-conductivity values for all unsaturated zone layer units, including the host-rock units.

Figure 6-2. Waste Package Temperature and Relative Humidity Ranges 

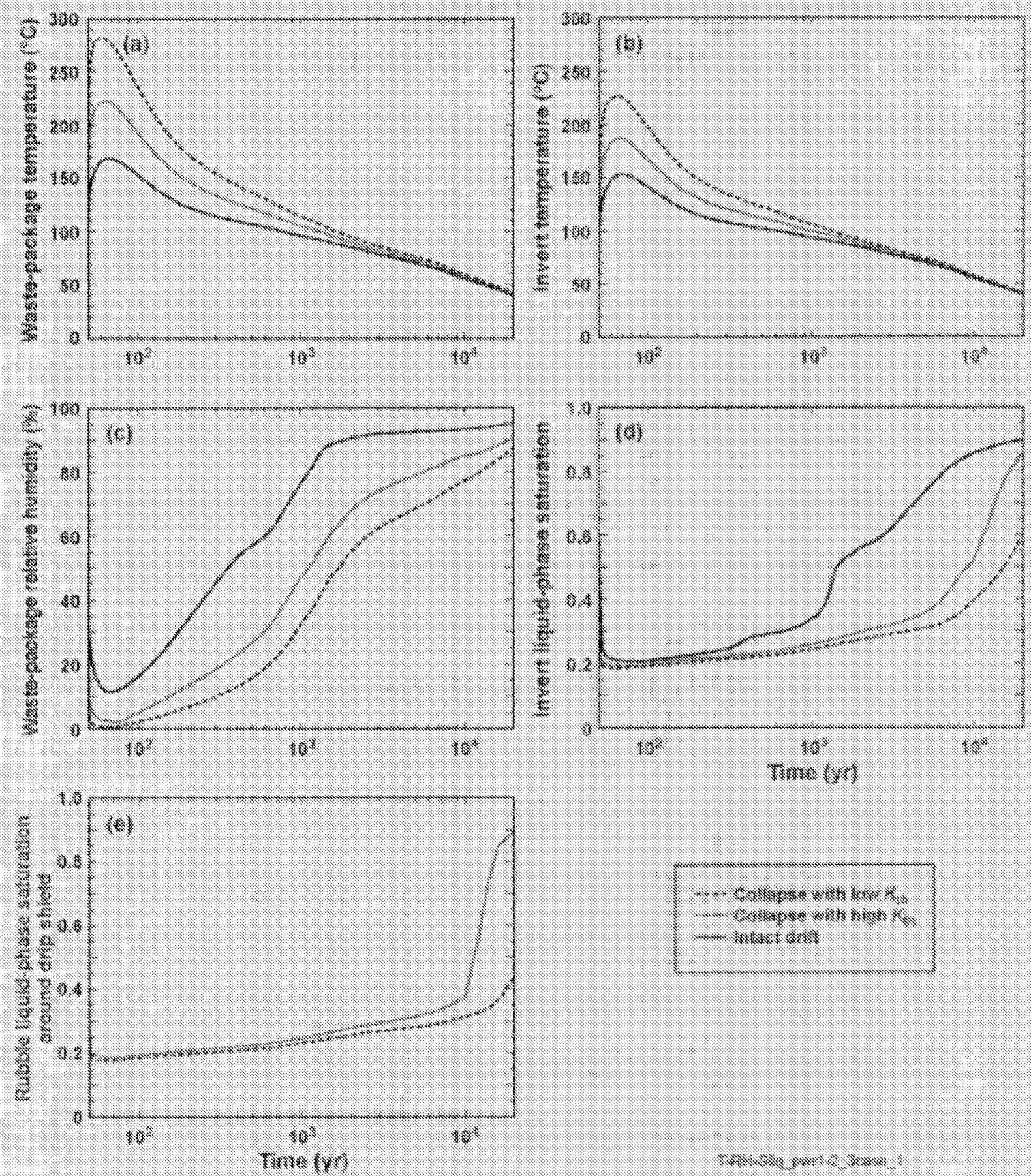

8.

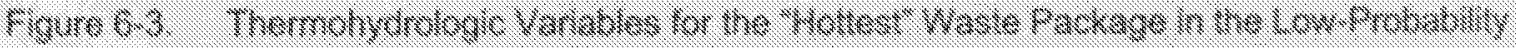

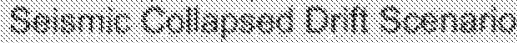


The curves for waste package temperature are bounding representations for the drip shield because the waste package (and the waste it contains) is the heat source in the repository environment. The repository conditions can be divided into three temperature regimes. The relevant attributes of each regime are summarized as follows (BSC 2004 [DIRS 169565]):

Dryout-Drift walls will first be dried by ventilation air during the preclosure period. During postclosure, heat generated by radioactive decay increases the temperature of waste packages and drift walls above the boiling point of water. Because no significant seepage is expected when drift wall temperatures are above the boiling point of water, no aqueous phase corrosion due to seepage will exist. However, depending on the surface temperature and relative humidity conditions, the existence of liquid-phase water on the waste package or drip shield is possible due to the presence of dust or salt deposits. In the presence of such a deposit, a thin-film liquid phase can be established at a higher temperature and lower relative humidity than otherwise possible. Thus, formation of deliquescent brines in the absence of seepage may occur, and corrosion of the waste package and drip shield is considered in the context of these solutions. Calcium chloride-type brines are possible and predicted to occur in this regime, but they occur in the host rock when temperatures are above boiling and seepage into the drift is prevented by the vaporization barrier effect.

Transition-Seepage into the drifts becomes possible as the waste package cools allowing the temperature of the drift wall to drop below the boiling point of water. If this occurs while the drip shield or waste package surface temperature, or both, is at or above the boiling point of the water, seepage waters will undergo evaporative concentration on the drip shield surface or the waste package surface at the time when the drip shield seepage diversion function is lost, thereby evolving into either carbonate- or sulfate-type brines. The drip shield, before it breaches, will mitigate seepage effects on the waste package. However, as in the dryout regime, formation of deliquescent brines could occur in this regime.

Low Temperature-As the waste package cools to a temperature below the boiling point of water, the in-drift relative humidity will increase, leading to less concentrated solutions contacting the waste package surfaces than at temperatures above the boiling point of water. With further cooling, the waste package surface temperature will drop and solutions contacting the waste package surface will be even less concentrated. Localized corrosion would not be expected under these conditions due to the low exposure temperatures and dilute solutions contacting the waste package surface.

\subsubsection{Relation of In-Drift Chemical Model Results to Corrosion Testing Environment}

The project has developed an understanding of the in-drift chemical environment for the three temperature regimes described in Section 6.3.1. The understanding is based on geochemical models and supporting data and analysis appropriate for the repository conditions. A description of the evolution of the chemical environment is provided in Engineered Barrier System: Physical and Chemical Environment (BSC 2004 [DIRS 169860], Section 6), which includes discussions of the relationship between the geochemical process model results, the range of expected in drift environments, and the chemical environments used in corrosion related testing. A brief summary of the chemical environment applicable to corrosion related testing follows. 
Brines that develop on the waste packages and drip shields are the result of either evaporative concentration of seepage water or deliquescence of deposited salts. Deposited salts are due to entrained matter in the ventilation air, dust and debris deposited within the drifts, or seepage waters evaporated to dryness. Seepage waters do not enter the drifts until host rock temperatures fall below $100^{\circ} \mathrm{C}$. Dust salts will deliquesce water from the atmosphere to form thin films on waste packages and drip shields above the normal boiling point of water (up to about $140^{\circ} \mathrm{C}$ ) (BSC 2004 [DIRS 169860], Section 6).

Corrosion testing to determine the response of waste package, drip shield, and other in-drift materials is carried out in environmental conditions consistent with those predicted by in-drift chemical modeling. Corrosion testing environments were chosen based on the three types of natural brines: (1) calcium chloride, (2) carbonate, and (3) sulfate. Initial studies focused on the carbonate-type brine, based on reasoning that carbonate-type waters, typified by J-13 well water from the saturated zone near Yucca Mountain, are the expected types of waters at the repository (Harrar et al. 1990 [DIRS 100814]).

The brine name reflects the chemical characteristic that distinguishes it from the other brines. Characterization of earth surface brines has guided, in part, the expected range of brine water chemistry in the repository. Some differences exist between brines formed at the earth's surface and brines formed in the repository. These differences are mainly due to the chemistry of seepage waters and surface waters giving rise to brines, and differences between the salt chemistry of dust and the dissolved salt content of such surface waters. Two important general factors specific to the repository brines are the presence of nitrate and more-effective mechanisms for magnesium removal. Nitrate will be present in the deliquescent brines owing to multiple potential sources (BSC 2004 [DIRS 161237], Section 6.7.2.8) and the generally high solubility of nitrate minerals (BSC 2004 [DIRS 161237], Section 4.1.1.7). Magnesium will not be significant owing to a combination of low source (for the dust, as well as for at least some groundwaters) and multiple removal mechanisms (e.g., $\mathrm{Mg}$ sequestration in secondary silicates), most of which are enhanced by elevated temperature (BSC 2004 [DIRS 161237], Sections 6.7.2.10 and 6.7.2.11).

Aqueous corrosion test solutions include several multiionic solutions based on a carbonate-based $\mathrm{J}-13$ well water and test solutions containing the major species expected to affect corrosion such as nitrate ions. The standardized solutions developed as relevant test environments are presented in Table 6-3. These solutions include Simulated Dilute Water (SDW), Simulated Concentrated Water (SCW), Simulated Acidified Water (SAW), and Basic Saturated Water (BSW) aqueous test solutions.

Table 6-3. Target Chemical Compositions of the Electrolyte Solutions ( $\mathrm{mg} / \mathrm{L}$ ) Employed in the LongTerm Weight-Loss Measurements

\begin{tabular}{|c|c|c|c|c|}
\hline \multicolumn{5}{|c|}{ Concentration (mg/L) } \\
\hline Ion & $\begin{array}{c}\text { Simulated Dilute } \\
\text { Water (SDW) }\end{array}$ & $\begin{array}{c}\text { Simulated Concentrated } \\
\text { Water (SCW) }\end{array}$ & $\begin{array}{c}\text { Simulated Acidified } \\
\text { Water (SAW) }\end{array}$ & $\begin{array}{c}\text { Basic Saturated } \\
\text { Water (BSW-SC) }\end{array}$ \\
\hline & $60^{\circ} \mathrm{C}$ and $90^{\circ} \mathrm{C}$ & $60^{\circ} \mathrm{C}$ and $90^{\circ} \mathrm{C}$ & $60^{\circ} \mathrm{C}$ and $90^{\circ} \mathrm{C}$ & - \\
\hline $\mathrm{K}$ & 34 & 3,400 & 3,400 & 90,846 \\
\hline $\mathrm{Na}$ & 409 & 40,900 & 37,690 & $231,224.1$ \\
\hline
\end{tabular}




\begin{tabular}{|c|c|c|c|c|}
\hline \multicolumn{5}{|c|}{ Concentration (mg/L) } \\
\hline Ion & $\begin{array}{c}\text { Simulated Dilute } \\
\text { Water (SDW) }\end{array}$ & $\begin{array}{c}\text { Simulated Concentrated } \\
\text { Water (SCW) }\end{array}$ & $\begin{array}{c}\text { Simulated Acidified } \\
\text { Water (SAW) }\end{array}$ & $\begin{array}{c}\text { Basic Saturated } \\
\text { Water (BSW-SC) }\end{array}$ \\
\hline $\mathrm{Mg}$ & 1 & $<1$ & 1,000 & 0 \\
\hline $\mathrm{Ca}$ & 0.5 & $<1$ & 1,000 & 0 \\
\hline $\mathrm{F}$ & 14 & 1,400 & 0 & 1,616 \\
\hline $\mathrm{Cl}$ & 67 & 6,700 & 24,250 & $177,695.4$ \\
\hline $\mathrm{NO}_{3}$ & 64 & 6,400 & 23,000 & $177,167.9$ \\
\hline $\mathrm{SO}_{4}$ & 167 & 16,700 & 38,600 & $16,907.3$ \\
\hline $\mathrm{HCO}_{3}$ & 947 & 70,000 & 0 & $107,170.7$ \\
\hline $\mathrm{Si}$ & $27\left(60^{\circ} \mathrm{C}\right)$ & $27\left(60^{\circ} \mathrm{C}\right)$ & $27\left(60^{\circ} \mathrm{C}\right)$ & 7058.9 \\
\hline Nominal pH & $49\left(90^{\circ} \mathrm{C}\right)$ & $49\left(90^{\circ} \mathrm{C}\right)$ & $49\left(90^{\circ} \mathrm{C}\right)$ & $>12$ \\
\hline
\end{tabular}

Source: DTN: LL040803112251.117 [DIRS 171362].

The following discussion on the relevance of the test media to the in-drift chemical environment is a summary of Engineered Barrier System: Physical and Chemical Environment Model (BSC 2004 [DIRS 169860], Section 6) and is used in this report as background information only.

Calcium-Chloride Brines-The calcium-chloride brines have near neutral $\mathrm{pH}$ and no significant bicarbonate/carbonate, fluoride, or sulfate content. These brines also contain other cations such as sodium, potassium, and magnesium and other anions such as nitrate. The endpoint of the evaporative concentration of this type of brine contains $\mathrm{Ca}-\mathrm{Cl} / \mathrm{NO}_{3}$ or a mixture of $\mathrm{Ca} / \mathrm{Mg}-\mathrm{Cl} / \mathrm{NO}_{3}$. The quantity of magnesium and calcium is limited due to the precipitation of calcium carbonates, sulfates, and magnesium silicates. Nitrate will be present; an endpoint brine of this type is dominated by calcium chloride and calcium nitrate. Formation of calcium-chloride brines is also limited in the repository. Brine generated by dust deliquescence is a potassium nitrate-sodium chloride brine with only a small probability of calcium due to the compositional nature of the dust leachate. Relative humidity dependence of the calcium-chloride brine composition is as follows. At low relative humidity, the aqueous solutions are dominated by calcium cations (very low sodium and potassium) and chloride and nitrate anions, because both calcium nitrate and calcium chloride are very soluble. At higher relative humidity, chloride and nitrate salts of sodium and potassium become soluble and dominate the aqueous solution compositions. This occurs at or above the deliquescence relative humidity for salts composed of these ions.

Corrosion test solutions corresponding to the calcium chloride type of brine include calcium chloride, calcium chloride plus calcium nitrate, and sodium chloride aqueous solutions. The sodium chloride test solutions simulate the moderate relative humidity scenario where calcium is a minor component in the aqueous solution.

Carbonate Brines-The carbonate brines are alkaline and do not contain significant calcium or magnesium content. In the early stages of the evaporative concentration, calcium precipitates predominately as carbonate mineral (calcite or aragonite) under equilibrium conditions. Magnesium precipitates as a minor component in the calcium carbonate species and as magnesium silicate. Potassium may be significant in some of these brines. Nitrate is expected to be an important component, and a brine of this type may evolve through a high extent of 
evaporation into one in which nitrate is actually the dominant anion. The carbonate brine is likely to be represented as alkali metal (sodium, potassium) carbonate brine. Relative humidity dependence of carbonate brine composition is as follows. At low relative humidity, the aqueous solutions are dominated by nitrate and chloride anions with nitrate ions dominating at the lowest relative humidity. At moderate relative humidity (greater than 70 -percent relative humidity), chloride ions dominate the solution composition. The nitrate-chloride solutions will have slightly elevated $\mathrm{pH}$ due to residual carbonate in solution. Significant amounts of carbonate and sulfate ion are not expected until the relative humidity is greater than 85 percent.

Corrosion test solutions corresponding to the carbonate type of brine include the Simulated Dilute Water (SDW), Simulated Concentrated Water (SCW), Basic Saturated Water (BSW), and under certain circumstances, Simulated Acidified Water (SAW) aqueous test solutions (Table 6-3). The BSW test solution is a highly concentrated alkaline solution with a boiling point of about $110^{\circ} \mathrm{C}$. The SCW test solution is a moderately concentrated alkaline solution and solutions in this concentration range form at relative humidity in the range of 90 to 95 percent. The SDW test solution is a dilute alkaline solution and solutions in this concentration range form at high relative humidity (greater than 99 percent). These may have characteristics of solutions at the drift wall, that is, typical of in-drift seepage waters.

Under conditions of extreme evaporative concentration (i.e., low relative humidity), this type of brine containing high nitrate and chloride content would evolve into nitrate-chloride brine with low carbonate content. The SAW test solution has characteristics of low carbonate brine and of solutions in equilibrium with relative humidity of nominally 90 percent. The calcium and magnesium addition to this test solution makes it more able to sustain lower $\mathrm{pH}$ values due to the hydrolysis properties of these cations.

Sulfate Brines-The sulfate brines have near-neutral $\mathrm{pH}$ and no significant bicarbonate/carbonate and calcium content. Calcium precipitates as carbonates and possibly as sulfates. In addition, they typically have only a small amount of magnesium, though some surface brines have been observed to have high magnesium (Drever 1997 [DIRS 140067], Table 15-1, Brines 1 to 3). The dominant cation is typically sodium. In the repository brines, potassium is comparable to sodium, and magnesium is insignificant. A brine of this type could evolve through a high extent of evaporation into one in which nitrate is the dominant anion.

Relative humidity dependence of the sulfate brine composition is as follows. At low relative humidity, the aqueous solutions are dominated by nitrate and chloride anions with nitrate ions dominating at the lowest relative humidity. At moderate relative humidity (greater than 70 percent relative humidity), chloride ions dominate the solution composition. However, unlike the carbonate brines, these brines have near neutral to slightly acidic pH because of the lack of a carbonate component. Significant amounts of carbonate and sulfate ion do not exist until the relative humidity is greater than 85 percent because of the increase in solubility of sulfate minerals (sodium and potassium sulfates). Magnesium sulfate is present in insignificant quantities in these brines.

The corrosion test solution corresponding to the sulfate type of brine is SAW (Table 6-3). This type of brine has near neutral to slightly acidic $\mathrm{pH}$ and, as noted, magnesium is not present in 
seepage waters to any significant extent. The SAW test solution has characteristics of solutions in equilibrium with nominally 90 percent relative humidity.

Two important general factors specific to the repository brines are the presence of nitrate and more-effective mechanisms for the removal of magnesium. Nitrate will be present in the deliquescent brines because of multiple potential sources (BSC 2004 [DIRS 161237], Section 6.7.2.8) and the generally high solubility of nitrate minerals (BSC 2004 [DIRS 161237], Section 4.1.1.7).

Magnesium ions will not be significant because of a combination of low concentration (for the dust, as well as for at least some groundwaters) and multiple removal mechanisms (e.g., $\mathrm{Mg}$ sequestration in secondary silicates), most of which are enhanced by elevated temperature (BSC 2004 [DIRS 161237], Sections 6.7.2.10 and 6.7.2.11).

Carbonate helps buffer the $\mathrm{pH}$ in any occluded geometry such as a crevice, and sulfate acts as a corrosion inhibitor. The compositions of these environments, as well as the BSW solution, are given in Table 6-3. Small amounts of carbonate form in the SAW and BSW solutions by interaction with gas phase carbon dioxide. The amount of carbonate formed was not determined experimentally because the small amounts will not affect the corrosion processes significantly.

BSW has a boiling point near $110^{\circ} \mathrm{C}$. The total concentration of dissolved salts in the starting liquid was more concentrated than that in the standard SCW solution. After evaporation of approximately 90 percent of the water from the starting solution, the residual solution reaches a maximum chloride concentration and has a boiling point of approximately $110^{\circ} \mathrm{C}$, with a pH of about 11 . The synthetic BSW solution composition can be slightly modified (mainly by adding sodium hydroxide) to cover a range of $\mathrm{pH}$ values, yielding solutions referred to as $\mathrm{BSW}-11,-12$, and -13 .

Deliquescence of dust deposited on the waste packages and drip shield is another means by which brines can form on these engineered barrier system components. In the absence of salts, condensed water can be present on smooth surfaces only if the relative humidity is 100 percent. At lower relative humidity, most of the water evaporates, with residual water existing on the surface as a very thin adsorbate layer due to deliquescence. Dissolved salts lower the relative humidity at which such dryout occurs. Salt minerals in a dry system lower the relative humidity required for an aqueous solution to form. When the dissolved salt composition of a solution is known, the relative humidity at which dryout occurs at a given temperature can be determined. Conversely, the relative humidity for a given salt or set of salt minerals at which deliquescence occurs at a specified temperature can also be found.

In all cases, the nitrate component, the most soluble species, dominates the solution composition at the deliquescent relative humidity or eutectic point of a mineral assemblage at elevated temperatures. At higher relative humidity, chloride minerals are soluble and could become a dominant ion. It is not until the relative humidity is much higher that the sulfate and carbonate compositions become appreciable. In essence, solutions are chloride and nitrate rich at low to moderate (less than 70 percent) relative humidity and, at higher relative humidity, sulfate and carbonate could be appreciable. 


\subsubsection{Waste Package Outer Barrier Degradation Conceptual Model}

A schematic representation of the base-case conceptual model is shown in Figure 6-1. The design functions of the drip shield are to prevent the water seeping into the emplacement drifts from dripping directly onto the waste package and to provide protection from rockfall damage to the waste package. Before the drip shield breaches, the chemistry of liquid water contacting the waste package would be determined by the chemistry of leachate from dust that settles on the waste package surface. As the thermal heat output from the radioactive waste decays with time, thermal-hydrologic conditions in the emplacement drift dynamically change. Concentrated brines could form on the waste package surface from evaporative concentration of dust leachate. The chemical evolution of the brines would be dependent on the humidity and temperature conditions. When the drip shield breaches and, therefore, no longer performs its seepagediversion design function, the waste package underneath the breached drip shield could be directly contacted by seepage water. In this case, the water chemistry contacting the waste package would be determined primarily by the chemistry of the seepage water. In either case, hygroscopic salts may be deposited on the waste package surface due to evaporative concentration of the leachate from the dust and aerosols in contact with humid air, or, after drip shield breach, due to the evaporative concentration of the seepage water that contacts the waste package surface. Such hygroscopic salts enable aqueous solutions to exist as thin water films at relative humidity $(\mathrm{RH})$ below 100 percent. The threshold $\mathrm{RH}\left(R H_{\text {threshold }}\right)$ at a given temperature, at which an aqueous solution can exist, is defined as the deliquescence point at that temperature. This threshold RH defines the lowest humidity condition necessary for aqueous electrochemical corrosion processes of a metal to occur at a given temperature.

In postclosure repository environments, crevices could form between the waste package surfaces and the structural components, other materials in the emplacement drift, and mineral deposits from evaporative concentration of the solutions in contact with the waste package surface. The general corrosion and localized corrosion models of the WPOB in this report are based on data from crevice geometry samples. This treatment is conservative because creviced geometries will not be formed over the entire waste package surface.

Dry oxidation of the WPOB occurs at any RH below the threshold $\mathrm{RH}\left(R H_{\text {threshold }}\right)$. This process results in the formation of an adherent, protective oxide film of uniform thickness. The rate of dry oxidation is generally limited by mass transport through the growing oxide film. As is discussed in Section 6.4.2, dry oxidation is not a performance limiting process of the WPOB under thermal conditions in the repository. Therefore, dry oxidation is not implemented in TSPA-LA.

General corrosion (or passive corrosion) is the uniform thinning of the WPOB at its open-circuit corrosion potential $\left(E_{\text {corr }}\right)$. As stated in Assumption 5.1, general corrosion of the WPOB is assumed to occur at any RH above a threshold $\mathrm{RH}\left(R H_{\text {threshold }}\right)$. The general corrosion rate is temperature dependent. However, in this modeling work, the corrosion rate is assumed constant for a given temperature, (i.e., time independent). Therefore, at a given temperature, the depth of penetration or thinning of the WPOB due to general corrosion is equal to the general corrosion rate at that temperature multiplied by the time duration when the waste package surface is at that temperature. This assumption is considered conservative because the general corrosion rate of metals and alloys tends to decrease with time (Assumption 5.2 and Section 6.4.3.5). 
The general corrosion model developed in this report uses a general corrosion rate distribution determined from weight-loss measurements of Alloy 22 crevice specimens that were exposed for over 5 years in a wide range of multi-ionic solutions at the Long Term Corrosion Test Facility (LTCTF) (Section 6.4.3.2). As discussed in Section 6.4.3.2, the sample configuration (crevice, disk, or rod), metallurgical condition (mill annealed or as-welded), and water chemistry within the expected range do not have a significant effect on the general corrosion behavior of Alloy 22 . Non-thermal stress mitigation processes, used to introduce a compressive stress layer in the outer lid closure weld region in order to delay the initiation of stress corrosion cracking, may introduce cold work into the material. Angeliu (2001 [DIRS 165442]) observed that unmitigated Stainless Steel Type $316 \mathrm{NG}$ weldments could contain up to 20 percent cold work due to weld shrinkage and differential thermal expansion. Therefore, the effects of cold work have been included in the analyses in this report. The temperature dependence of the general corrosion rate is represented with an Arrhenius relationship. The activation energy used in the Arrhenius relationship was determined from corrosion rates calculated from short-term polarization resistance measurements of Alloy 22 specimens with varying sample configurations and metallurgical conditions. The specimens were tested for a range of exposure conditions (temperature and water chemistry). As with the general corrosion rate from the long-term weight-loss measurements discussed above, the sample configuration (crevice, disk or rod), metallurgical conditions (mill annealed or aswelded), and water chemistry within the expected range do not have significant effect on temperature dependence of the general corrosion rate of the alloy (Section 6.4.3.4). The Electric Power Research Institute (EPRI 2002 [DIRS 158069], Section 5.3.2) presented a similar conceptual description by using literature data for similar corrosion-resistant Ni-Cr-Mo alloys.

Localized corrosion is a corrosion in which the attack progresses at discrete sites or in a nonuniform manner. The rate of localized corrosion is generally much higher than the rate of general corrosion. As stated in Assumption 5.3, the current analysis assumes crevice corrosion is representative of localized corrosion of the WPOB under the exposure conditions expected in the postclosure repository. This is a conservative and bounding assumption because initiation thresholds for crevice corrosion of Alloy 22 in terms of water chemistry and temperature are lower than for pitting corrosion (Gdowski 1991 [DIRS 100859], Section 3.0; Haynes International 1997 [DIRS 100896]; Haynes International 1997 [DIRS 100897]).

Localized corrosion of the WPOB is modeled with two model components: an initiation model and a propagation model. Localized corrosion of the WPOB occurs when the open-circuit corrosion potential $\left(E_{c o r r}\right)$ is equal to or greater than a critical potential $\left(E_{\text {critical }}\right)$, that is, $\Delta \mathrm{E}$ $\left(=E_{\text {critical }}-E_{\text {corr }}\right) \leq 0$. This conceptual model of localized corrosion initiation is widely accepted by the corrosion community (Böhni 2000 [DIRS 164137], Section B; Dunn et al. 2000 [DIRS 164495]; Dunn et al. 2003 [DIRS 164138]; Frankel 1998 [DIRS 162216]; Frankel 2002 [DIRS 164140]; Frankel and Kelly 2002 [DIRS 164141]; and Beavers et al. 2002 [DIRS 158781], Section 8.1). The crevice corrosion initiation model components (i.e., $E_{\text {corr }}$ and $\left.E_{\text {critical }}\right)$ are represented as a function of temperature, $\mathrm{pH}$, chloride ion concentration, and nitrate ion concentration.

When it occurs, localized corrosion of the WPOB is assumed to propagate at a constant (timeindependent) rate (Assumption 5.4). This assumption is highly conservative because it is known that the localized corrosion rate decreases with time, and this is particularly more likely under 
discontinuous and tortuous thin water films expected to form on the waste package surface in the postclosure repository. Section 6.4 .4 .8 provides a more detailed discussion.

The WPOB is subject to microbially influenced corrosion (MIC) when the relative humidity at the WPOB surface is above 90 percent. The MIC initiation threshold $\mathrm{RH}$ is based on the analysis documented in Evaluation of the Impact of Microbial Activities on Drift Chemistry (BSC 2004 [DIRS 169991], Section 7.1). The effect of MIC on general corrosion of the WPOB is represented by a general corrosion enhancement factor. The enhancement factor was determined from the comparative analysis of the corrosion rates measured from the short-term polarization resistance measurements of Alloy 22 specimens tested in abiotic and biotic conditions.

The waste package design and fabrication specification specifies that the WPOB base metal and all fabrication welds (except the closure lid welds) are fully annealed before the waste packages are loaded with waste (Plinski 2001 [DIRS 156800], Section 8.1).

According to the analysis documented in Aging and Phase Stability of Waste Package Outer Barrier (BSC 2004 [DIRS 169983], Section 6.6.5), phase instabilities are not expected in Alloy 22 base-metal and welded material due to the thermal hydrologic exposure profiles in the repository. In addition, Project data (Section 6.4.6) show that the corrosion properties of aged welds are comparable to those of unaged welds. Phase instabilities are not expected to occur and, even if they do, they are not expected to have a significant effect on the corrosion properties; therefore, the effects of aging and phase instability of the WPOB are not modeled in TSPA-LA.

Effects of oxidants, such as hydrogen peroxide generated from gamma radiolysis, on corrosion may be accounted for through the open-circuit corrosion potential $\left(E_{\text {corr }}\right)$. However, the radiolysis-enhanced corrosion has been screened out in FEPS Screening of Processes and Issues in Drip Shield and Waste Package Degradation (BSC 2004 [DIRS 169997], Section 6.2.32). Therefore, the radiolysis-enhanced corrosion of the WPOB is not considered in this waste package corrosion analysis.

The waste package is a double-walled container with an outer barrier constructed of the highly corrosion-resistant Alloy 22 (UNS 06022) and an inner vessel made of Stainless Steel Type 316 (UNS S31600). The inner vessel provides structural support to the thinner outer barrier (Plinski 2001 [DIRS 156800], Sections 1.0 and 4.0) while the outer barrier protects the inner vessel from significant corrosion degradation until failure of the outer barrier. Although the inner vessel could provide some delay of radionuclide release before it fails and could also retard the release rate of radionuclides from the waste package, no performance credit is taken for the corrosion resistance of the inner vessel (i.e., all corrosion performance is allocated to the WPOB). This model approach is used throughout the analysis and is conservative for the reasons noted above.

After penetration of the WPOB, a crevice can form in the interfacial region between the Alloy 22 outer cylinder and Stainless Steel Type 316 inner vessel. The formation of a low-pH crevice environment in this interfacial region is possible. A local acidic water chemistry could be developed through hydrolysis of dissolved metal ions in the crevice regions between the two 
barriers. Moreover, due to the limited availability of oxygen (dominant oxidizer for this condition) to such an occluded area, polarization of the creviced region due to differential aeration (between the creviced region and adjacent uncreviced region(s)) may occur. However, the analyses in this report show that Alloy 22 is highly resistant to crevice corrosion, especially in solutions with mixed ions. Alloy 22 will likely act as the cathode relative to the Stainless Steel Type 316 inner vessel under these exposure conditions.

\subsection{MODEL FORMULATION FOR BASE-CASE MODEL}

This section documents the analyses and models developed for general and localized corrosion of the WPOB under repository exposure conditions. The analyses and models also consider effects of microbiological processes (Section 6.4.5) and aging and phase instability (Section 6.4.6) of the WPOB for the repository conditions.

\subsubsection{Stability of the Passive Film in Repository Relevant Environments}

Corrosion performance of the WPOB depends on the integrity of the thin and adherent passive film formed on the alloy surface. The extremely low general corrosion rates and excellent resistance to localized corrosion of Alloy 22 in the repository intimately depend on the long-term stability of this passive film. This section discusses the conceptual understanding of passivity and passive film stability of Ni-Cr-Mo alloys by summarizing the data and information from the literature. Also discussed in this section is a summary of the experimental results of characterization for passive film on Alloy 22 tested in environments relevant to the conditions expected in the repository.

\subsubsection{Conceptual Description of Passivity and Passive Film Stability}

Passivity can be defined as the formation of a thin and adherent oxide or oxyhydroxide film that protects a metal or alloy from corrosive degradation. Corrosion performance of highly corrosion-resistant alloys such as Alloy 22 depends on the integrity of the passive film formed on the alloy surface in contact with the corrosive environment. Long-term stability of the passive film on the surface of the Alloy 22 waste package outer barrier is one of the key issues that determines the long-term performance of the waste packages in the repository. Extrapolation of the measured short-term corrosion rates over the regulatory period is based on the integrity of this passive film remaining stable over very long times. This section provides a brief description of the conceptual understanding of passivity and the passive film formation and stability on highly corrosion-resistant alloys such as Alloy 22.

\subsection{Passive Film Formed on Metals}

The passive film formed under aqueous condition is not a single layer, but rather has a stratified structure of at least two layers (Macdonald 1992 [DIRS 154720]; Macdonald 1999 [DIRS 154721]; Marcus and Maurice 2000 [DIRS 154738], p. 138). According to this bilayer model, the passive film consists of an inner layer of oxide and an outer layer of hydroxide or oxyhydroxide. The inner oxide layer plays the role of a barrier layer against corrosion, and the outer layer plays the role of an exchange layer (Marcus and Maurice 2000 [DIRS 154738], p. 138) as discussed further in Section 6.4.1.1.2. In general, the chemical composition and thickness of passive films depend on the nature of the metal, the $\mathrm{pH}$ of the electrolyte in which 
the metal is passivated, and the electrochemical potential (Macdonald 1992 [DIRS 154720]; Macdonald 1999 [DIRS 154721]; Marcus and Maurice 2000 [DIRS 154738], p. 138). For nickel, which can passivate in solutions over a wide range of $\mathrm{pH}$, the passive film is generally composed of nickel (II) cations with an inner layer of $\mathrm{NiO}$ and an outer layer of $\mathrm{Ni}(\mathrm{OH})_{2}$ (Marcus and Maurice 2000 [DIRS 154738], p. 140).

Passive films formed on metal surfaces are generally not electronic conductors, but rather semiconductors or insulators. The electronic structures of passive films can be determined by photoelectrochemical measurements. However, structural analyses are rather difficult, due to the nanometer thickness of passive films and the roughness of the surfaces because of dissolution. The passive film formed on nickel (in $0.05 \mathrm{M} \mathrm{H}_{2} \mathrm{SO}_{4}$ ) displays crystallites, the sizes of which are reduced with increasing potential (Marcus and Maurice 2000 [DIRS 154738], p. 146). The shape of these crystallites also changes with potential (Marcus and Maurice 2000 [DIRS 154738], Table 3-1). Another factor that must be considered is active dissolution, which occurs as long as the surface is not completely passivated. The dissolution rate increases with increasing potential. Dissolution processes may create new sites for oxide nucleation and can, thus, favor a higher density of oxide nuclei (Marcus and Maurice 2000 [DIRS 154738], p. 147). The passive film formed on nickel can be crystalline, with the surface exhibiting terraces and steps. The passive oxide film formed on chromium can have a nanocrystalline structure with oxide nanocrystals are cemented together by the chromium hydroxide outer layer, making the passive film extremely protective against corrosion-induced damage (Marcus and Maurice 2000 [DIRS 154738], p. 149).

\subsection{Passive Film Growth Mechanisms}

As summarized in the literature (Macdonald 1992 [DIRS 154720]; Macdonald 1999 [DIRS 154721]; Marcus and Maurice 2000 [DIRS 154738], Section 3.6), the barrier oxide layer (inner layer of the passive film) forms by generation of oxygen vacancies at the metal-film interface, balanced in the steady state by dissolution of the barrier layer at the barrier layer-outer layer interface. The outer layer forms via the hydrolysis and precipitation of cations transmitted through the barrier layer or by hydrolytic restructuring of the barrier layer-outer layer interface. The distinctly different origins of the barrier and outer layers are amply demonstrated by the fact that both layers may incorporate alloying elements from the alloy substrate, but only the outer layer incorporates species from the solution. Furthermore, with respect to a fixed external frame of reference, the barrier layer grows into the substrate metal, whereas the outer layer grows outwards into the solution (Macdonald 1992 [DIRS 154720]; Macdonald 1999 [DIRS 154721]; Marcus and Maurice 2000 [DIRS 154738], Section 3.6). Thus, while the growth of the barrier layer is due to the generation of oxygen vacancies at the metal-barrier layer interface, the growth of the outer layer is commonly (but not exclusively) due to the transmission of cations through the barrier layer, either through cation vacancies or as cation interstitials, and their eventual emission at the barrier layer-outer layer interface. The origin of the outer layer is not exclusively due to cation transmission, because it may also form via hydrolytic restructuring of the barrier layer at the barrier layer-outer layer interface (Macdonald 1992 [DIRS 154720]; Macdonald 1999 [DIRS 154721]; Marcus and Maurice 2000 [DIRS 154738], Section 3.6).

The electric current is carried by all charged species in the barrier layer, including cation vacancies, cation interstitials, and oxygen vacancies, which are generated and annihilated at the 
interfaces and by dissolution of the barrier layer, depending on whether a change in oxidation state occurs (Macdonald 1992 [DIRS 154720]; Macdonald 1999 [DIRS 154721]). The principal mode of transport of defects is migration under the influence of a strong electric field, the magnitude of which is postulated to be established by the potential differences across the film and interfaces and by buffering due to Esaki (band-to-band) tunneling within the barrier layer (Macdonald 1992 [DIRS 154720]; Macdonald 1999 [DIRS 154721]). Because a barrier layer exists on all passive metals, all barrier layers are oxygen vacancy conductors to an extent that, in the steady state, is determined by the dissolution rate of the film. However, other defects may dominate the structural and electronic defect structures of the barrier layer. For example, the defect structure of the barrier layer on nickel is dominated by cation vacancies, while that on zinc is dominated by cation interstitials, although oxygen vacancies exist in both cases.

\subsection{Surface-Enrichment of Chromium on Passivated Nickel-Base Alloys}

Chromium concentration plays a significant role in the passivation of austenitic nickel-base alloys, such as Alloy 22. The passive film on Alloy 22 can be described by the bilayer model (Macdonald 1992 [DIRS 154720]; Macdonald 1999 [DIRS 154721]; Marcus and Maurice 2000 [DIRS 154738], Section 3.6). The concentration of $\mathrm{Cr}^{+3}$ in the inner oxide layer is higher than the nominal chromium content of the alloy (Marcus and Maurice 2000 [DIRS 154738], p. 153; Lorang et al. 1990 [DIRS 154718], Figures 1 and 2). The mechanism of surface enrichment of chromium in the barrier layer has been developed within the framework of the point defect model (PDM) (Zhang and Macdonald 1998 [DIRS 154743]; 1998 [DIRS 154742]). The PDM is based on the selective oxidation of the elements at the alloy-barrier layer interface, differences in transport properties of the species in the barrier layer, and selective oxidation of the elements at the barrier layer-outer layer interface. In the specific case of the passive film on iron-chromium alloys, the enrichment of chromium in the barrier layer appears to entail the dissolution of iron and the oxidative segregation of chromium (Marcus and Maurice 2000 [DIRS 154738], p. 153). Iron atoms are detached from the surface and dissolve into solution as ions, whereas chromium atoms are rapidly oxidized. The passive film forms by the nucleation and growth of a $\mathrm{Cr}_{2} \mathrm{O}_{3}$-like phase (Marcus and Maurice 2000 [DIRS 154738], p. 153). However, the barrier layers on iron-chromium and nickel-chromium alloys are not pure chromium oxide $\left(\mathrm{Cr}_{2} \mathrm{O}_{3}\right)$, but contain significant amounts of other metal species, such as nickel, ferrous, and ferric ions (Lorang et al. 1990 [DIRS 154718]). Finally, it is important to note that the accurate description of alloy segregation phenomena requires solving the "moving boundary" problem, because the interfaces at which the segregation reactions occur and the phases bounded by these interfaces move differently with respect to a fixed external frame of reference. This is the approach adopted in the PDM (Zhang and Macdonald 1998 [DIRS 154743]; Zhang and Macdonald 1998 [DIRS 154742]).

\subsection{Role of Molybdenum}

There is a consensus that molybdenum reduces the rate of anodic dissolution in the active state (Marcus and Maurice 2000 [DIRS 154738], pp. 155 to 158). However, the mechanism by which molybdenum additions benefit the corrosion resistance of nickel-chromium-molybdenum alloys is not fully understood. Molybdenum tends to be located preferentially at local defects on the surface, which normally act as dissolution sites. The slowing down of the dissolution rate could be due to the strengthened metal-metal bonds when molybdenum is present (Marcus and 
Maurice 2000 [DIRS 154738], pp. 155 to 158). Further, the presence of molybdenum counteracts the deleterious effect of certain species such as sulfur, which can cause grainboundary attack in that it bonds to sulfur and then dissolves mitigating the detrimental effects of sulfur (Marcus and Maurice 2000 [DIRS 154738], p. 158).

An analytical prediction of the role played by molybdenum (or any alloying element) in inhibiting passivity breakdown on alloys is provided by the point defect model (PDM) (Urquidi and Macdonald 1985 [DIRS 154741]). The PDM accounts quantitatively (within the accuracy of the experimental data) for the impact of molybdenum on the pitting resistance of $18 \mathrm{Cr}-8 \mathrm{Ni}$ stainless steels (e.g., Stainless Steel Type 316, which contains molybdenum versus Stainless Steel Type 304, which contains no molybdenum). In this case, the highly oxidized molybdenum ions present substitutionally in the barrier layer lattice form immobile, positively charged centers. For example, recognizing that the barrier layer on chromium-containing stainless steels and nickel-base alloys is essentially $\mathrm{Cr}_{2} \mathrm{O}_{3}$, substitution of $\mathrm{Mo}^{6+}$ into a chromium cation vacancy would produce the immobile species $\left(\mathrm{Mo}_{\mathrm{Cr}}^{3 \circ}\right)$. These immobile species can interact electrostatically with the mobile, negatively charged cation vacancies $\left(V_{C r}^{3^{\prime}}\right)$, the condensation of which at the metal-film interface under breakdown conditions is responsible for passivity breakdown (Macdonald 1992 [DIRS 154720]; Macdonald 1999 [DIRS 154721]). Thus, the solute-vacancy interaction reduces the free cation vacancy concentration and diffusivity, which results in a positive shift in the breakdown voltage and a lengthening of the induction time (i.e., the alloy becomes more resistant to passivity breakdown). The electrostatic interaction is described rigorously in terms of ion-pairing theory that is commonly employed to describe ionic interaction in solutions. This solute-vacancy interaction model (Urquidi and Macdonald 1985 [DIRS 154741]) successfully accounts for the positive shift in the breakdown voltage upon adding molybdenum to the alloy without the need for arbitrary, adjustable parameters. Most importantly, the solute-vacancy interaction model accounts for why molybdenum must be present in the barrier layer at concentrations greater than about 2 percent for significant protection to be achieved.

Finally, complexing between $\mathrm{Mo}_{\mathrm{Cr}}^{3 \cdot}$ and a defect will only occur if the defect is negatively charged (i.e., if the defect is a cation vacancy) (Urquidi and Macdonald 1985 [DIRS 154741]). However, the $\mathrm{Cr}_{2} \mathrm{O}_{3}$ passive film on chromium containing alloys is normally n-type in electronic character because the dominant defect in the film is either a cation interstitial or an oxygen vacancy, both of which are formally positively charged (Urquidi and Macdonald 1985 [DIRS 154741]). Consequently, there should be little solute $\left(M_{C r}^{30}\right)$ - vacancy $\left(V_{o}^{* \bullet}\right.$ or $\left.M_{i}^{\chi+}\right)$ interaction and pairing, and molybdenum should have little consistent impact on the passive current density.

\subsubsection{Characterization of Alloy 22 Passive Film}

The passive film on Alloy 22 has been studied at $95^{\circ} \mathrm{C}$ in a high $\mathrm{pH}$ salt environment characteristic of concentrated Yucca Mountain groundwater (Andresen et al. 2003 [DIRS 170360], Section 3). Measurements of corrosion potential (CP) versus time, potentiostatic polarization (PSP) and cyclic potentiodynamic polarization (CPP) behavior were conducted to evaluate the passivity of these alloys. The passive films were also analyzed by $x$-ray 
photoelectron spectroscopy (XPS) and transmission electron microscopy (TEM) to obtain the chemical composition and cross-sectional view of the metal, oxide layers and interfaces.

\subsection{Summary of Experimental Procedures}

All materials were tested in the as-received condition. Specimens $(3 \mathrm{~mm}$ in diameter by $60 \mathrm{~mm}$ in length cylinders for CP, PSP, and CPP measurements, and $10 \mathrm{~mm} \times 10 \mathrm{~mm} \times 0.8 \mathrm{~mm}$ coupons for oxide analysis) were cut by electrodischarge machining and then wet-ground using a 600-grit SiC paper before testing. Specimens for electrochemical measurements were spot welded to a polytetrafluoroethylene insulated Alloy 600 wire and mounted in an insulated fitting. The testing solution used was diluted (by a factor of about 18×) Basic Saturated Water (BSW) (Table 6-3) with a $\mathrm{pH}$ of approximately 12.4 at $95^{\circ} \mathrm{C}$.

The chemicals were mixed with water that had been heated to the boiling point in an autoclave. All testing was performed in either a Hastelloy C-276 autoclave body or a commercial-purity titanium autoclave. Stainless steel components were present in all the autoclaves used. No signs of corrosion were observed on these stainless steel components, suggesting that the testing solution was not extremely aggressive. Solution was sampled from the autoclaves during the test.

To prevent evaporative loss of water, a four-foot-long tube-in-tube heat exchanger was used, with cooling water on the outside. The solution level in the test autoclave was monitored periodically by checking for continuity between the autoclave and an insulated stainless steel feed-through bar. No water addition was needed.

All potentials were measured with respect to a saturated calomel electrode (SCE) reference electrode. A Luggin probe with a porous zirconia membrane filled with the test solution was used to maintain the reference electrode at room temperature. A flag-shaped platinum sheet was employed as a counter electrode. All tests were performed at $95^{\circ} \mathrm{C} \pm 1^{\circ} \mathrm{C}$. CPP scans at $0.17 \mathrm{mV} / \mathrm{sec}$ were started at $50 \mathrm{mV}$ below the corrosion potential (obtained 1 hour after immersion in solution), then continued toward the more noble potential direction. The scans were reversed when a current density of $5 \mathrm{~mA} / \mathrm{cm}^{2}$ was reached. After the completion of each test, specimens were cleaned ultrasonically in deionized water, dried, and the specimen surface was examined with a scanning electron microscope (SEM). In addition, potentiostatic polarization (PSP) tests were conducted by applying various anodic potentials for 24 hours to measure the passive current and to characterize the oxide properties. The oxide composition and thickness were analyzed by XPS. The XPS data are quantitative for film composition, but the thickness of the oxide film is considered qualitative because precise calibrations of sputtering rate on an oxide of this composition was not performed (although very good estimates exist). No visual evidence of localized corrosion attack was observed after CPP or PSP measurements.

The cross-sectional TEM sample was prepared using a focused ion beam system. The bulk sample was placed into the focused ion beam system, and the region of interest was coated with a $1-\mu \mathrm{m}$-thick platinum layer using the in-situ metal deposition facilities of the focused ion beam system. The platinum layer was used to protect the underlying material. Staircase-shaped cuts were milled on either side of the region of interest using a gallium ion source. The ion current was reduced as the thickness of the section approached the desired dimension. The dimension of 


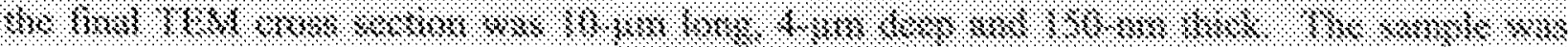

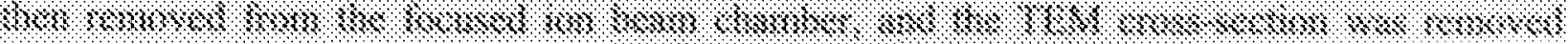

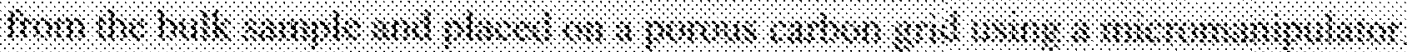

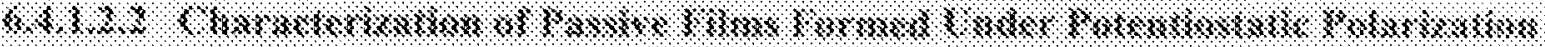

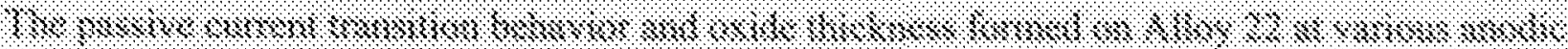

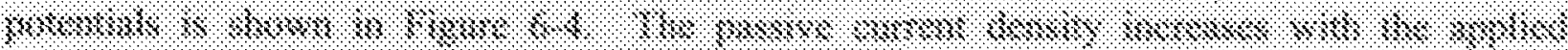

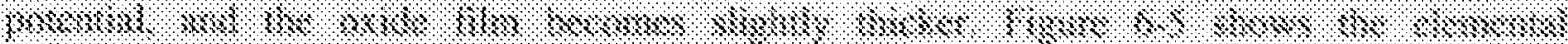

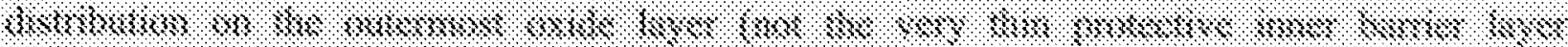

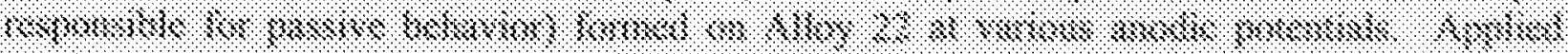

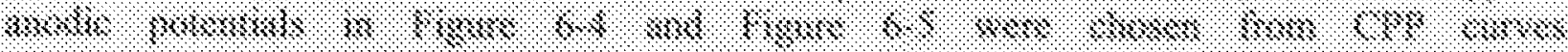

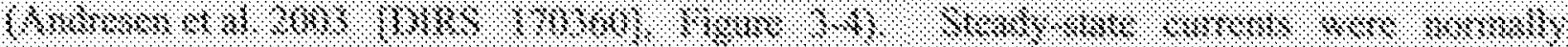

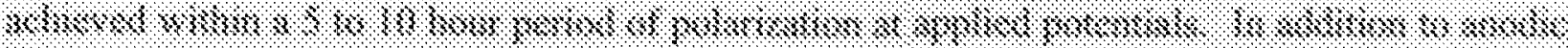

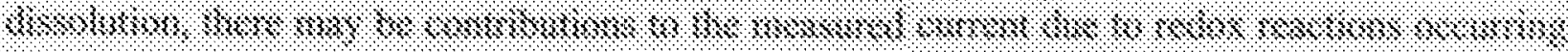

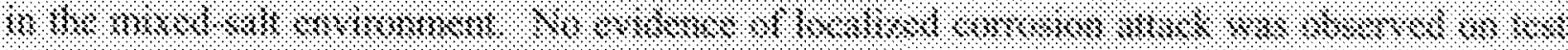

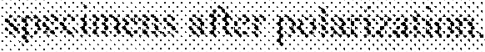

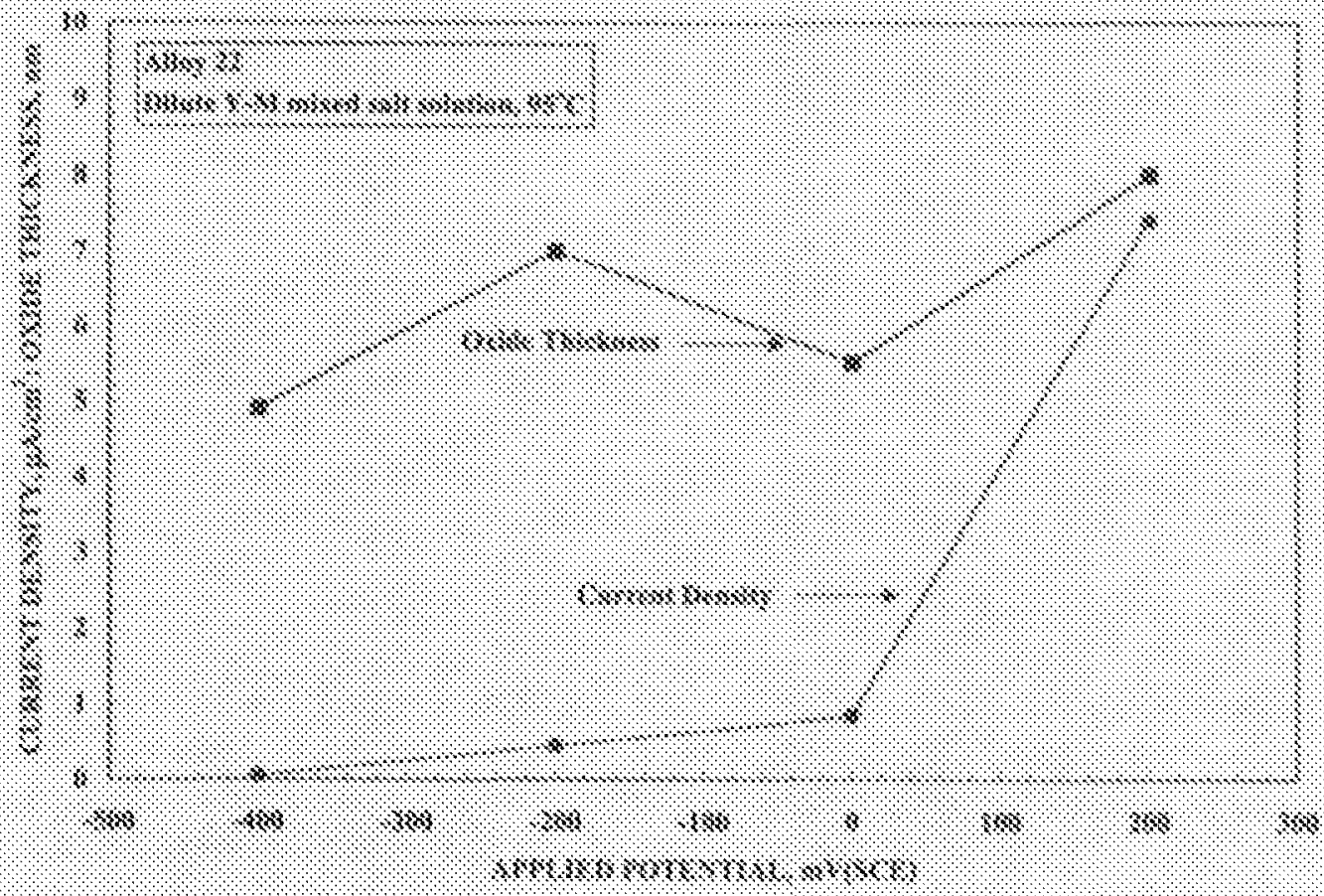

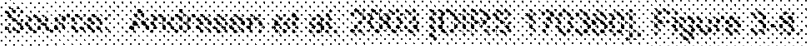

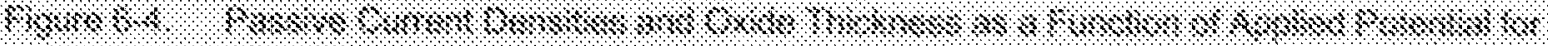
४०० 


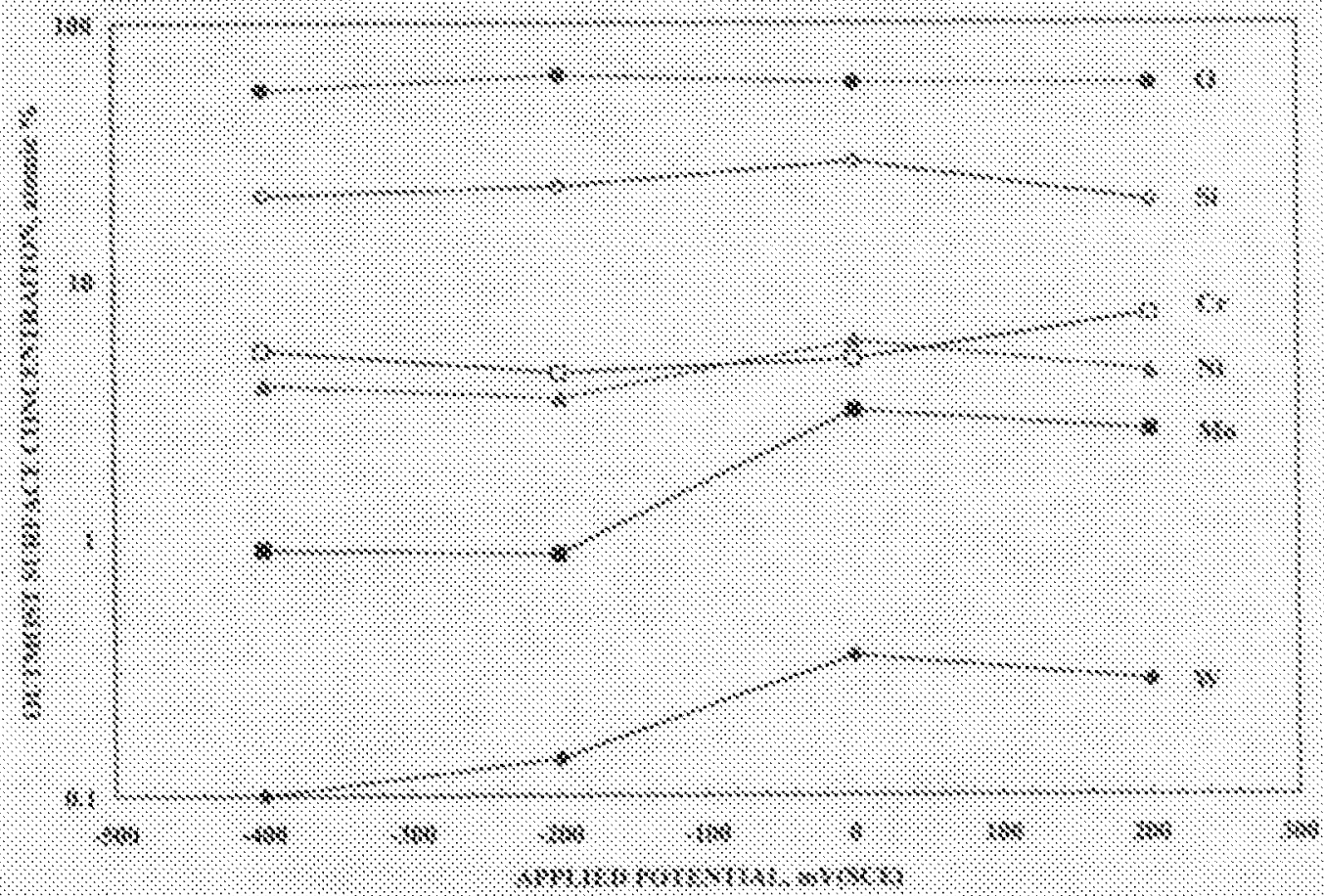

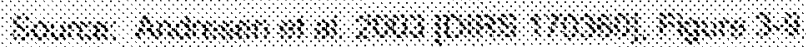

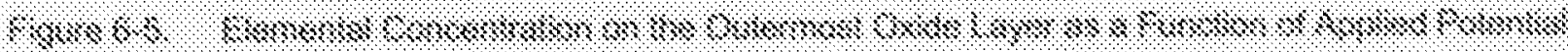

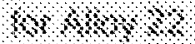

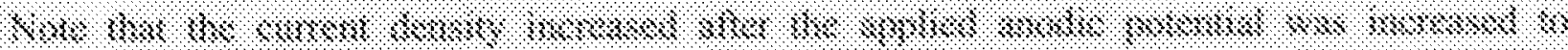

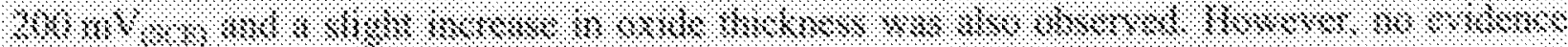

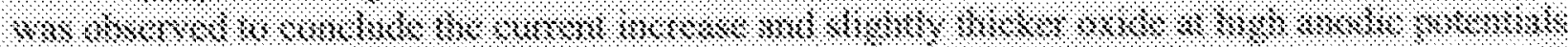

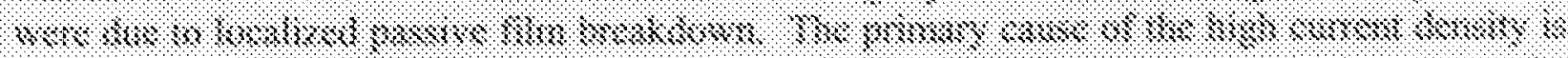

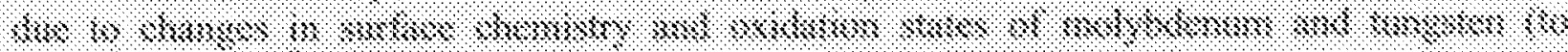
6 r

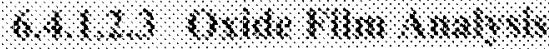

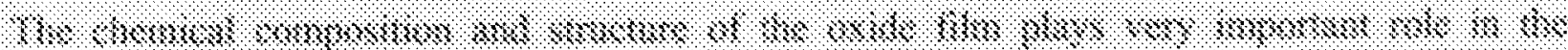

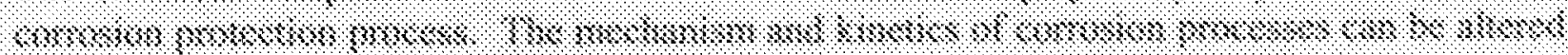

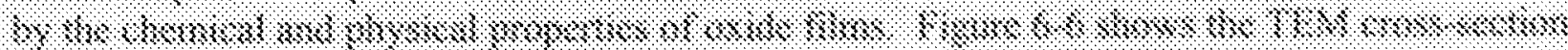

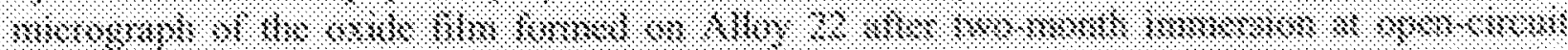

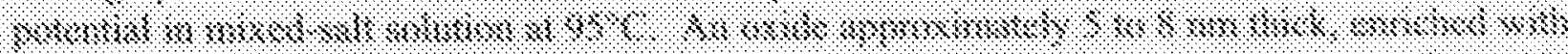

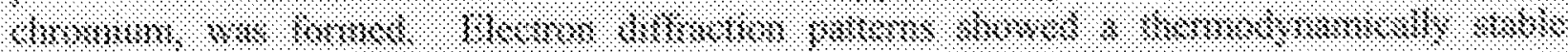

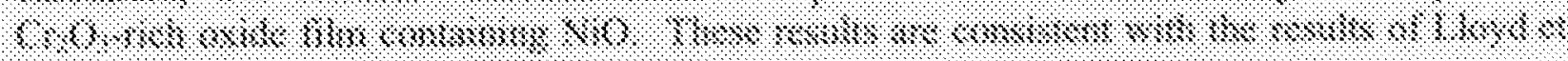

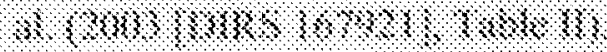




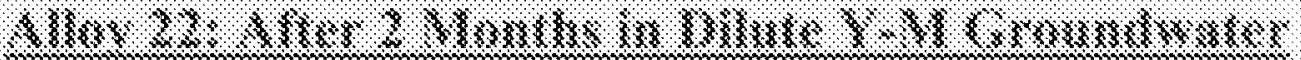

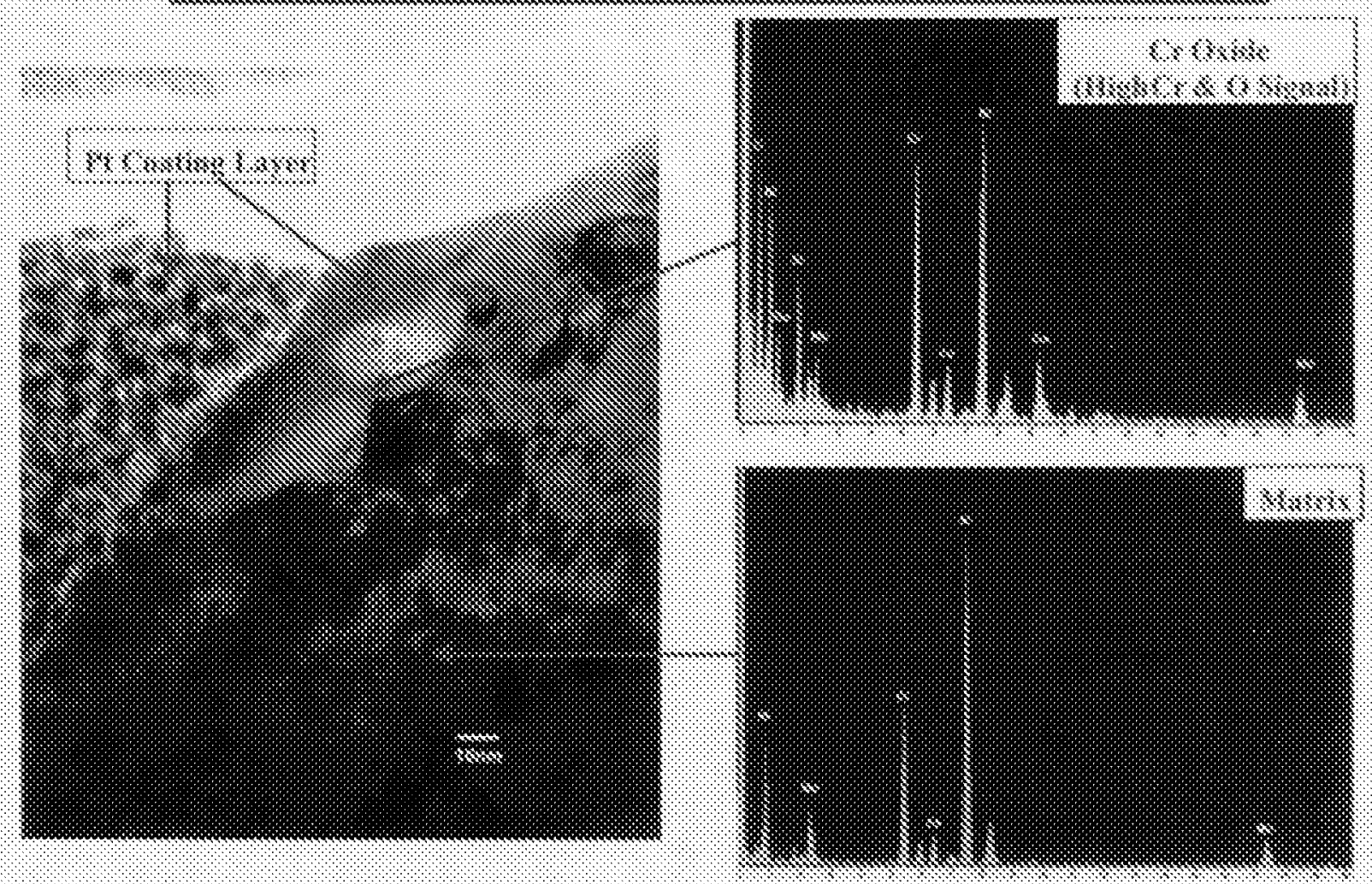

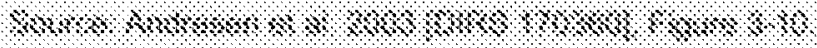

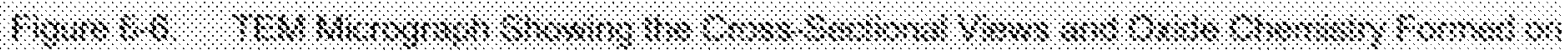

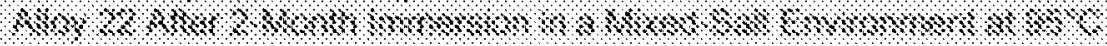

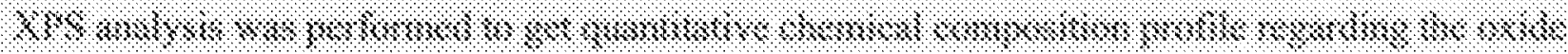

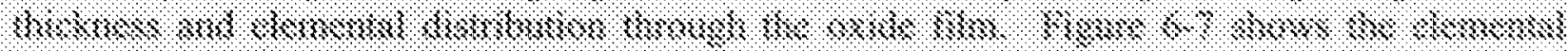

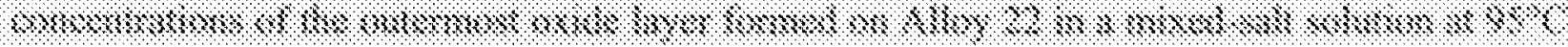

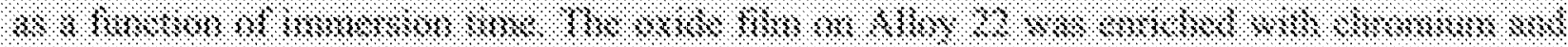

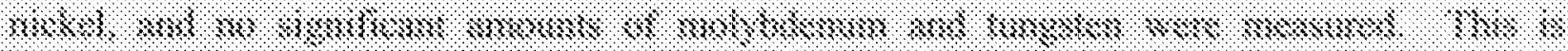

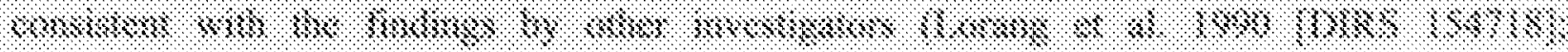

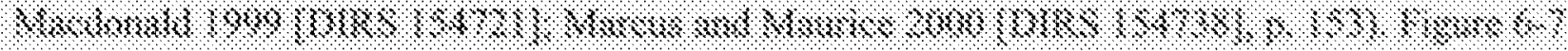

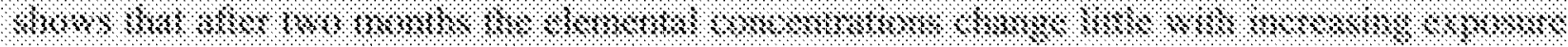

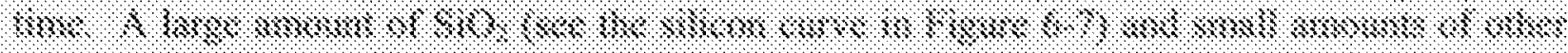

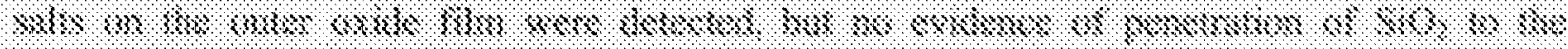

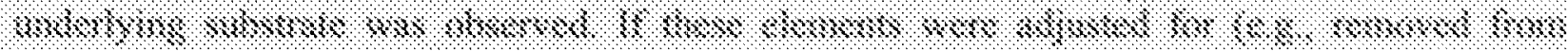

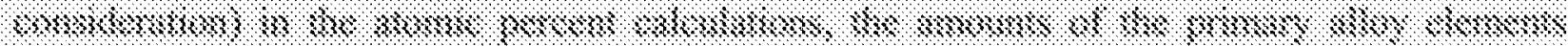

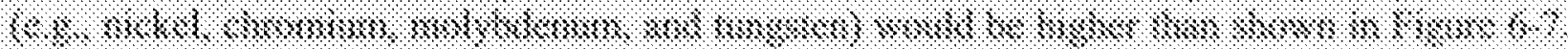

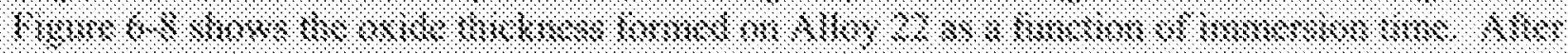

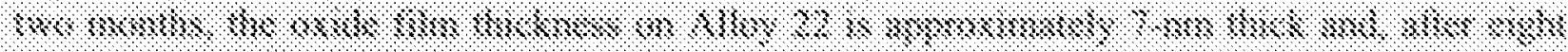

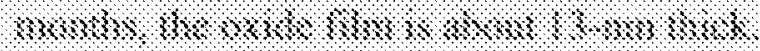




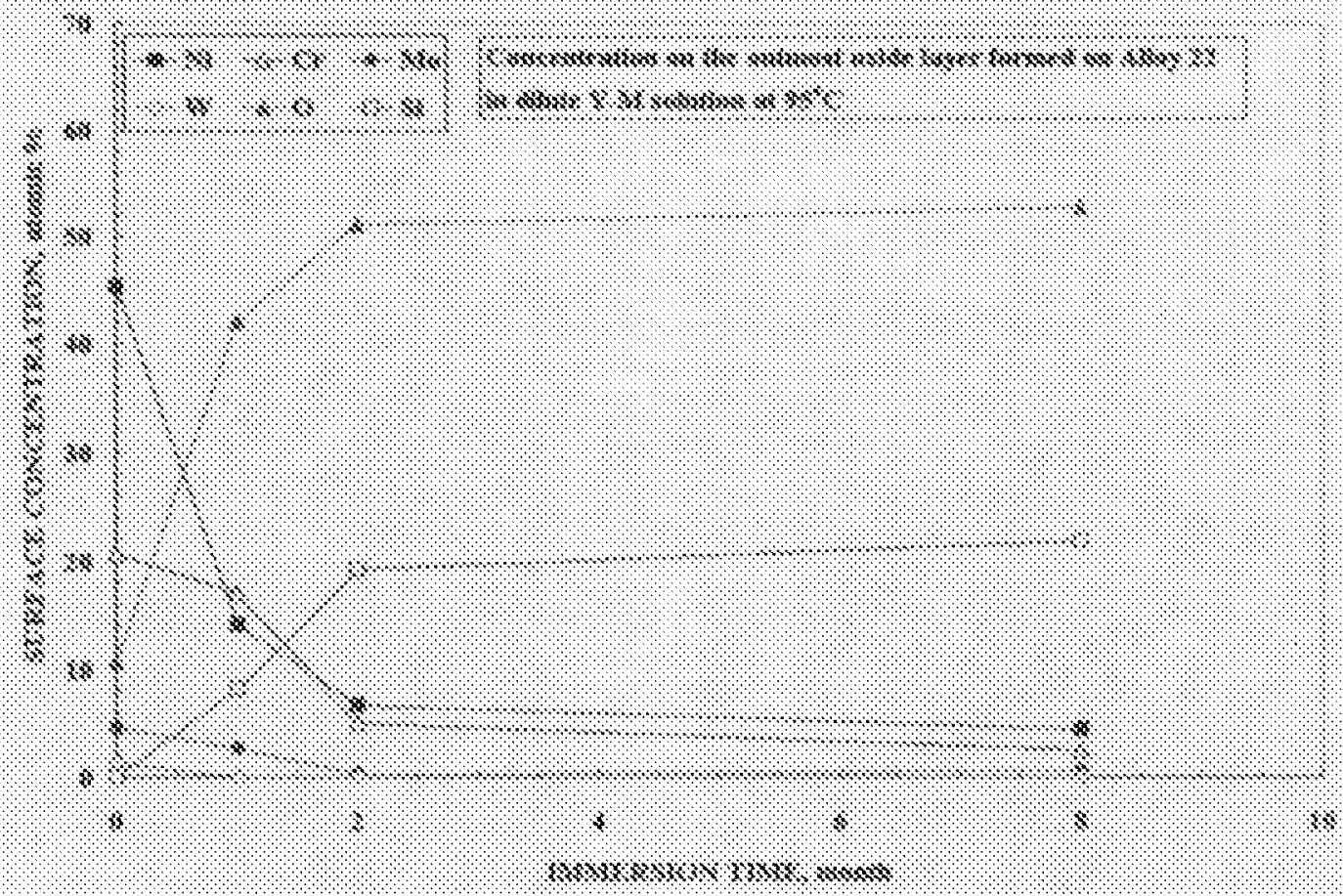

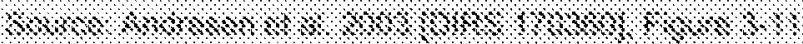

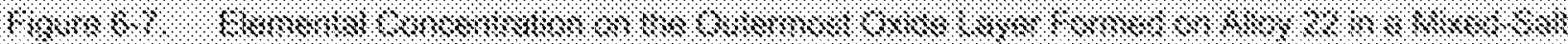

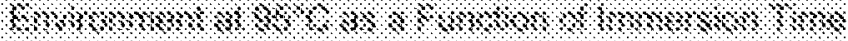

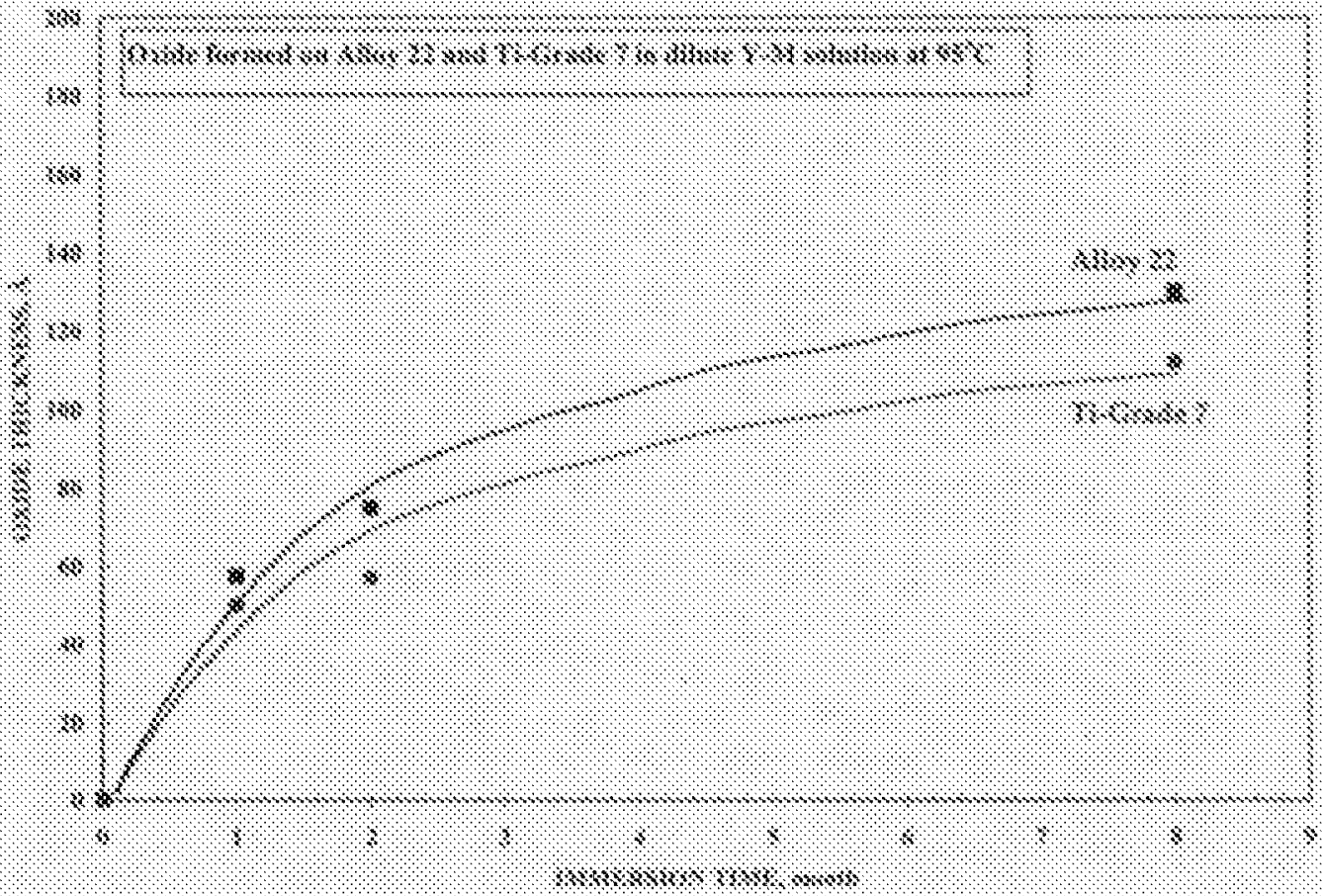

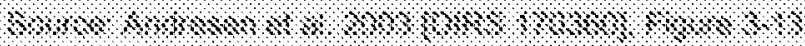

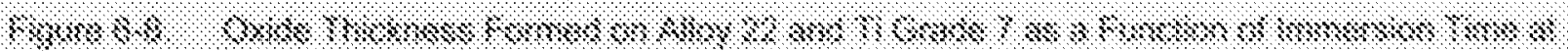

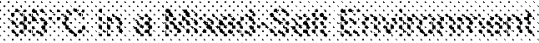




\subsubsection{Summary}

The conceptual understanding of the growth and stability of the passive film on Alloy 22 is described based on summarized data and information from the literature. The passivity of Alloy 22 is examined by measuring the corrosion potential and polarization behavior in a mixedsalt environment at $95^{\circ} \mathrm{C}$ with a pH of about 12.4. Steady-state corrosion potentials of Alloy 22 are measured. The surface analysis data indicated that the oxide layers responsible for passivity of Alloy 22 consist of chromium oxide $\left(\mathrm{Cr}_{2} \mathrm{O}_{3}\right)$ containing $\mathrm{NiO}$. After eight months of exposure to the mixed-salt solution at $95^{\circ} \mathrm{C}$ with a $\mathrm{pH}$ of about $12.4,12$ to $13 \mathrm{~nm}$ thick oxide films were formed on Alloy 22 specimen surfaces. In addition, the passive films formed on Alloy 22 at high anodic potentials ( $>0 \mathrm{mV}$ versus SCE) contained more molybdenum and tungsten than the ones formed at lower anodic potentials $(<0 \mathrm{mV}$ versus $\mathrm{SCE}$ ). No evidence of localized corrosion attack on Alloy 22 after potentiostatic polarization measurements was observed. No significant change in the open-circuit potential (OCP, or corrosion potential) of the test electrodes was observed. These data indicate that with increased time (1) the passive films become very protective and stable, (2) contributions from metal corrosion become extremely small, and (3) redox reactions from the species in solution are stable. Overall, the passive films formed on Alloy 22 can be concluded to be very stable over long time periods.

\subsubsection{Dry Oxidation}

Dry oxidation of Alloy 22 occurs at any $R H<R H_{\text {threshold, }}$ thereby forming an adherent, protective oxide film of uniform thickness. The dry oxidation model presented here considers uniform oxidation of the WPOB surface. The protective oxide film is primarily $\mathrm{Cr}_{2} \mathrm{O}_{3}$, as discussed in the previous section. The oxidation reaction for the formation of this oxide is (Welsch et al. 1996 [DIRS 114895]):

$$
4 / 3 \mathrm{Cr}+\mathrm{O}_{2} \longrightarrow 2 / 3 \mathrm{Cr}_{2} \mathrm{O}_{3}
$$

The rate of dry oxidation is considered to be limited by mass transport through this growing metal oxide film. Fick's first law (Jones 1992 [DIRS 169906], Section 12.3.1) is applied, considering a linear concentration gradient across the oxide film of thickness $x$ :

$$
J_{\text {oxide }}=-D_{\text {oxide }} \frac{\partial C}{\partial x} \approx-D_{\text {oxde }} \frac{\Delta C}{x}
$$

where $J_{\text {oxide }}$ is the molar flux of the reacting species in the oxide, $D_{\text {oxide }}$ is the diffusivity of the reacting species in the oxide, $\Delta C$ is the corresponding differential molar concentration. One can describe the oxide growth with a parabolic rate law as (Jones 1992 [DIRS 169906], Section 12.3.1):

$$
\frac{d x}{d t}=\frac{k_{p}}{x}
$$


where $x$ is the oxide thickness and $k_{p x}$ is the thickness-based parabolic rate constant and $t$ is the time. Integration of Equation 6-3 leads to:

$$
x=\sqrt{x_{0}^{2}+k_{p} \times t}
$$

where $x_{0}$ is the initial oxide thickness, $x$ is the oxide thickness at time $t$, and $k$ is a temperaturedependent parabolic rate constant. Dry oxidation of metal is a thermally activated process. Therefore, the rate constant, $k_{p}$ obeys the Arrhenius equation (Jones 1992 [DIRS 169906], Section 12.3.1):

$$
k_{p}=k_{p_{0}} e^{-E_{a} / R T}
$$

where $k_{p 0}$ is a constant, $E_{a}$ is the activation energy, and $R$ is the universal gas constant $(8.314 \mathrm{~J} / \mathrm{mol} \mathrm{K})$ (Lide 1991 [DIRS 131202], inside rear cover).

To facilitate an approximate calculation, published values of $k$ can be used (Welsch et al. 1996 [DIRS 114895]). The data in Figure 18 of this reference were fit to an Arrhenius relation such that all observed values of $k$ fall below a line defined by:

$$
\log \left[k\left(\frac{m^{2}}{\mathrm{sec}}\right)\right]=-3.5-\left(\frac{12500}{T}\right)
$$

where $T$ is defined as the absolute temperature in Kelvin. Recent measurements of the thickness of the Alloy 22 oxide film exposed to air at $550^{\circ} \mathrm{C}$ showed that the oxide film approaches a limiting thickness of about 0.025 to $0.050 \mu \mathrm{m}(25$ to $50 \mathrm{~nm})$ after about 333 days of exposure (DTN: LL030406412251.045 [DIRS 163469]). If this oxide film thickness is considered the depth of metal penetration (this is very conservative as the oxide-metal volume (PillingBedworth) ratio for chromia is about 2.02 (ASM International 1987 [DIRS 133378], p. 64)), then a penetration rate of 0.027 to $0.054 \mu \mathrm{m} / \mathrm{yr}(27$ to $54 \mathrm{~nm} / \mathrm{yr})$ is obtained. For that temperature, the value of $k$ corresponding to the upper limit from Equation $6-6$ is $2.06 \times 10^{-19} \mathrm{~m}^{2} / \mathrm{sec}$ $\left(6.51 \mu \mathrm{m}^{2} / \mathrm{yr}\right)$. Ignoring the initial oxide thickness in Equation 6-4, after one year, this corresponds to a growth of $2.55 \mu \mathrm{m}$ (about $2.55 \mu \mathrm{m} / \mathrm{yr}$ ). This shows that the model estimates are about 100 times greater than the measured thickness, and the above expression represents a very conservative upper bound.

Logarithmic growth laws may be more appropriate at lower temperatures than parabolic laws. However, the logarithmic law predicts that the oxide thickness (penetration) asymptotically approaches a small maximum value. In contrast, the parabolic law predicts continuous growth of the oxide, which is more conservative. As conservative estimates of the rate of dry oxidation based on parabolic growth kinetics are not life limiting and reliable data for determining the maximum oxide thickness for Alloy 22 are not available, the parabolic growth law was used for the WPOB.

For a temperature of $350^{\circ} \mathrm{C}$, the value of $k$ from Equation $6-6$ is $2.73 \times 10^{-24} \mathrm{~m}^{2} / \mathrm{sec}$ $\left(8.61 \times 10^{-5} \mu \mathrm{m}^{2} / \mathrm{yr}\right)$. After one year, this corresponds to a growth of $0.0093 \dot{\mu m}$ 
(about $9.3 \mathrm{~nm} / \mathrm{yr}$ ). As discussed in Section 6.4.3, this estimated dry-oxidation rate is comparable to the mean value of the general corrosion rate of Alloy 22 at lower temperatures. Assuming a constant oxide penetration rate of $0.0093 \mu \mathrm{m} / \mathrm{yr}$ (i.e., exposure to a constant temperature of $350^{\circ} \mathrm{C}$ ), the total penetration depth in the WPOB by dry oxidation in 10,000 years after permanent closure is only $93 \mu \mathrm{m}$, which is negligibly small (less than 1 percent of the total thickness of the WPOB $(20 \mathrm{~mm}))$.

On the basis of the above analysis, dry oxidation is not expected to be a performance limiting process of the WPOB under the exposure condition expected in the repository. Therefore, dry oxidation is not included in the waste package performance analysis.

\subsubsection{General Corrosion}

General corrosion (or passive corrosion) is the uniform thinning of the WPOB at its open-circuit corrosion potential $\left(E_{c o r r}\right)$. General corrosion can occur under immersed conditions or when a liquid film exists on the surface. At a given surface temperature, the existence of liquid water on the waste package surface depends upon the hygroscopic nature of salts, minerals, or both deposited on the surface. In the presence of such a deposit, a liquid-phase can be established at a higher temperature and lower $\mathrm{RH}$ than otherwise is possible. General corrosion of the WPOB occurs at any RH above a threshold RH ( $R H_{\text {threshold }}$ ) (Assumption 5.1). The general corrosion rate is temperature dependent. For a given temperature, the general corrosion rate is assumed constant (i.e., time-independent) (Assumption 5.2). Therefore, for a given temperature, the depth of penetration or thinning of the WPOB by general corrosion is equal to the general corrosion rate at that temperature, multiplied by the time duration that the waste package surface is at that temperature. This assumption is considered conservative because the general corrosion rate of metals and alloys is known to decrease with time (Section 6.4.3.5).

As is discussed in the following sections, general corrosion rates of the WPOB have been estimated from the weight-loss data of Alloy 22 samples after 5-year exposure in the Long Term Corrosion Test Facility (LTCTF) (Estill 1998 [DIRS 117697], Section 2.2). The LTCTF provides a comprehensive source of corrosion data for Alloy 22 in environments relevant to the repository. The LTCTF facility is described in detail in a previous publication by Estill (1998 [DIRS 117697], Section 2.2) and relevant experimental details are summarized in the next section. The 5-year weight-loss measurement data is documented in DTN: LL030412512251.057 [DIRS 163712].

\subsubsection{Long-Term Weight-Loss Measurements}

\subsection{Test Temperatures and Aeration}

The LTCTF is equipped with several nonmetallic tanks. Each tank has a total volume of approximately $2,000 \mathrm{~L}$ and is filled with approximately $1,000 \mathrm{~L}$ of aqueous test solution. The temperature of the solution in a particular tank is controlled at either $60^{\circ} \mathrm{C}$ or $90^{\circ} \mathrm{C}$. The test solution in each tank is covered with a blanket of air flowing at approximately $150 \mathrm{~cm}^{3} / \mathrm{min}$, and agitated. The reported test temperature corresponded to the liquid phase temperature. Details of the facility are described by Estill (1998 [DIRS 117697], Section 2.2). 


\subsection{Test Specimens}

Samples used for determination of weight-loss during long-term were mounted on insulating racks and placed in the tanks. Approximately half of the samples were submersed, half were in the saturated vapor above the aqueous phase, and a limited number were at the water line. Condensed water was present on specimens located in the saturated vapor.

Two coupon types were used for weight-loss measurements. These are identified as weight-loss coupons and crevice coupons. The nominal dimensions were $2 \mathrm{in.} \times 1 \mathrm{in} . \times 1 / 8 \mathrm{in}$. (approximately $50 \mathrm{~mm} \times 25 \mathrm{~mm} \times 3 \mathrm{~mm})$ and 2 in. $\times 2$ in. $\times 1 / 8$ in. $(50 \mathrm{~mm} \times 50 \mathrm{~mm} \times 3 \mathrm{~mm})$, respectively. The coupons had a 0.312 -in. $(7.9-\mathrm{mm})$ diameter hole in the center for sample mounting. For both coupon types (weight-loss and crevice), two metallurgical conditions were used (i.e., wrought material (only base metal) and welded material). The coupons were fabricated from Alloy 22 plate stock. All weight-loss coupons were affixed using an insulating $1 / 2$-in. (12.7-mm) diameter polytetrafluoroethylene or ceramic washer, while all crevice coupons were affixed using a $3 / 4$-in. (19.1-mm) diameter polytetrafluoroethylene or ceramic crevice former. The purpose of the crevice former was to create an environment that might induce corrosion at the contact interface, or under occluded conditions. Further details of the sample configuration are provided elsewhere (Estill 1998 [DIRS 117697], Section 2.2.5; DTN: LL030412512251.057 [DIRS 163712]).

\subsection{Test Media}

The weight-loss measurement testing includes a wide range of plausible generic test media, including Simulated Dilute Water (SDW), Simulated Concentrated Water (SCW), and Simulated Acidified Water (SAW). The compositions of three of these solutions are summarized in Table 6-3. Basic Saturated Water (BSW) is not used for long-term weight-loss testing (as no BSWcontaining tanks were installed) but is used in other testing activities discussed in this report; therefore, its composition is included in Table 6-3. The SCW test medium is three orders-ofmagnitude $(1,000 \times)$ more concentrated than $\mathrm{J}-13$ well water and is slightly alkaline (pH approximately 10). The SAW test medium is three orders-of-magnitude $(1,000 \times)$ more concentrated than J-13 well water and is acidic ( $\mathrm{pH}$ approximately 2.7 ). Concentrated solutions are intended to mimic the evaporative concentration of the electrolytes on the hot waste package surface (Estill 1998 [DIRS 117697], Section 2.2).

\subsection{Test Matrix}

The welded and nonwelded (wrought) coupons were tested under 12 different conditions (three electrolytes $x$ two temperatures $x$ two exposure conditions). The exposure time for each specimen was approximately 5 years. The specimen label and vessel numbers are shown in Table 6-4. Each sample was designated with three letters and three characteristic numbers. The letter $\mathrm{D}$ represents Alloy 22, the letter $\mathrm{C}$ indicates a crevice coupon, the letter $\mathrm{W}$ indicates a weight-loss coupon, the letter ' $A$ ' indicates that the coupon does not contain a weld seam, and the letter ' $B$ ' indicates that the coupon contains a weld seam along the middle of the specimen. Table 6-4 shows that a total of 134 test specimens were examined. Twelve welded crevice samples, representing each of the different test conditions, were used for surface analyses (designated (SA) in Table 6-4) and the remaining 122 samples were used for weight-loss determination. The weight-loss samples tested at the water line were included in the model 
analysis because there were no noticeable differences in the measured weight losses between the water line and liquid phase samples for the same exposure conditions (i.e., water chemistry and temperature).

Table 6-4. List of Examined Weight-Loss and Crevice Alloy 22 Coupons

\begin{tabular}{|c|c|c|c|c|c|c|}
\hline & $\begin{array}{l}\text { SAW } \\
60^{\circ} \mathrm{C}\end{array}$ & $\begin{array}{l}\text { SAW } \\
90^{\circ} \mathrm{C}\end{array}$ & $\begin{array}{l}\mathrm{SCW} \\
60^{\circ} \mathrm{C}\end{array}$ & $\begin{array}{l}\mathrm{SCW} \\
90^{\circ} \mathrm{C}\end{array}$ & $\begin{array}{l}\text { SDW } \\
60^{\circ} \mathrm{C}\end{array}$ & $\begin{array}{l}\text { SDW } \\
90^{\circ} \mathrm{C}\end{array}$ \\
\hline $\begin{array}{l}\text { Sample - } \\
\text { Exposure }\end{array}$ & Vessel 25 & Vessel 26 & Vessel 27 & Vessel 28 & Vessel 29 & Vessel 30 \\
\hline $\begin{array}{l}\text { Weight-Loss - } \\
\text { Vapor Phase }\end{array}$ & $\begin{array}{l}\text { DWA019 } \\
\text { DWA020 } \\
\text { DWA021 }\end{array}$ & $\begin{array}{l}\text { DWA059 } \\
\text { DWA060 } \\
\text { DWA061 }\end{array}$ & $\begin{array}{l}\text { DWA089 } \\
\text { DWA090 } \\
\text { DWA091 }\end{array}$ & $\begin{array}{l}\text { DWA129 } \\
\text { DWA130 } \\
\text { DWA131 } \\
\end{array}$ & DWA147 & DWA174 \\
\hline $\begin{array}{c}\text { Crevice - } \\
\text { Vapor Phase }\end{array}$ & $\begin{array}{l}\text { DCA019 } \\
\text { DCA020 } \\
\text { DCA021 }\end{array}$ & $\begin{array}{l}\text { DCA049 } \\
\text { DCA050 } \\
\text { DCA051 }\end{array}$ & $\begin{array}{l}\text { DCA079 } \\
\text { DCA080 } \\
\text { DCA081 }\end{array}$ & $\begin{array}{l}\text { DCA109 } \\
\text { DCA110 } \\
\text { DCA111 } \\
\end{array}$ & $\begin{array}{l}\text { DCA139 } \\
\text { DCA140 } \\
\text { DCA141 } \\
\end{array}$ & $\begin{array}{l}\text { DCA175 } \\
\text { DCA176 } \\
\text { DCA177 } \\
\end{array}$ \\
\hline $\begin{array}{l}\text { Weight-Loss - } \\
\text { Liquid Phase }\end{array}$ & $\begin{array}{l}\text { DWA022 } \\
\text { DWA023 } \\
\text { DWA024 }\end{array}$ & $\begin{array}{l}\text { DWA062 } \\
\text { DWA063 } \\
\text { DWA064 }\end{array}$ & $\begin{array}{l}\text { DWA092 } \\
\text { DWA093 } \\
\text { DWA094 }\end{array}$ & $\begin{array}{l}\text { DWA132 } \\
\text { DWA133 } \\
\text { DWA134 }\end{array}$ & DWA148 & DWA175 \\
\hline $\begin{array}{c}\text { Crevice - } \\
\text { Liquid Phase }\end{array}$ & $\begin{array}{l}\text { DCA022 } \\
\text { DCA023 } \\
\text { DCA024 }\end{array}$ & $\begin{array}{l}\text { DCA052 } \\
\text { DCA053 } \\
\text { DCA054 }\end{array}$ & $\begin{array}{l}\text { DCA082 } \\
\text { DCA083 } \\
\text { DCA084 }\end{array}$ & $\begin{array}{l}\text { DCA112 } \\
\text { DCA113 } \\
\text { DCA114 }\end{array}$ & $\begin{array}{l}\text { DCA142 } \\
\text { DCA143 } \\
\text { DCA144 }\end{array}$ & $\begin{array}{l}\text { DCA178 } \\
\text { DCA179 } \\
\text { DCA180 }\end{array}$ \\
\hline $\begin{array}{l}\text { Weight-Loss - } \\
\text { Waterline }\end{array}$ & DWA034 & DWA039 & DWA104 & DWA109 & DWA154 & DWA167 \\
\hline $\begin{array}{c}\text { Welded } \\
\text { Weight-Loss - } \\
\text { Vapor Phase }\end{array}$ & $\begin{array}{l}\text { DWB019 } \\
\text { DWB020 } \\
\text { DWB021 }\end{array}$ & $\begin{array}{l}\text { DWB059 } \\
\text { DWB060 } \\
\text { DWB061 }\end{array}$ & $\begin{array}{l}\text { DWB089 } \\
\text { DWB090 } \\
\text { DWB091 }\end{array}$ & $\begin{array}{l}\text { DWB129 } \\
\text { DWB130 } \\
\text { DWB131 }\end{array}$ & DWB147 & DWB174 \\
\hline $\begin{array}{c}\text { Welded } \\
\text { Crevice - } \\
\text { Vapor Phase }\end{array}$ & $\begin{array}{c}\text { DCB019 } \\
\text { DCB020 } \\
\text { DCB021 (SA) } \\
\end{array}$ & $\begin{array}{c}\text { DCB049 } \\
\text { DCB050 } \\
\text { DCB051 (SA) } \\
\end{array}$ & $\begin{array}{c}\text { DCB079 } \\
\text { DCB080 } \\
\text { DCB081 (SA) }\end{array}$ & $\begin{array}{c}\text { DCB109 } \\
\text { DCB110 } \\
\text { DCB111 (SA) }\end{array}$ & $\begin{array}{c}\text { DCB139 } \\
\text { DCB140 } \\
\text { DCB141 (SA) }\end{array}$ & $\begin{array}{c}\text { DCB175 } \\
\text { DCB176 } \\
\text { DCB177 (SA) }\end{array}$ \\
\hline $\begin{array}{c}\text { Welded } \\
\text { Weight-Loss- } \\
\text { Liquid Phase }\end{array}$ & $\begin{array}{l}\text { DWB022 } \\
\text { DWB023 } \\
\text { DWB024 } \\
\end{array}$ & $\begin{array}{l}\text { DWB062 } \\
\text { DWB063 } \\
\text { DWB064 } \\
\end{array}$ & $\begin{array}{l}\text { DWB092 } \\
\text { DWB093 } \\
\text { DWB094 }\end{array}$ & $\begin{array}{l}\text { DWB132 } \\
\text { DWB133 } \\
\text { DWB134 }\end{array}$ & DWB148 & DWB175 \\
\hline $\begin{array}{c}\text { Welded } \\
\text { Crevice - } \\
\text { Liquid Phase }\end{array}$ & $\begin{array}{c}\text { DCB022 } \\
\text { DCB023 } \\
\text { DCB024 (SA) }\end{array}$ & $\begin{array}{c}\text { DCB052 } \\
\text { DCB053 } \\
\text { DCB054 (SA) }\end{array}$ & $\begin{array}{c}\text { DCB082 } \\
\text { DCB083 } \\
\text { DCB084 (SA) }\end{array}$ & $\begin{array}{c}\text { DCB112 } \\
\text { DCB113 } \\
\text { DCB114 (SA) }\end{array}$ & $\begin{array}{c}\text { DCB142 } \\
\text { DCB143 } \\
\text { DCB144 (SA) }\end{array}$ & $\begin{array}{c}\text { DCB178 } \\
\text { DCB179 } \\
\text { DCB180 (SA) }\end{array}$ \\
\hline
\end{tabular}

Source: DTN: LL030412512251.057 [DIRS 163712] (for all but surface analysis (SA) designated samples).

NOTE: $\quad S A=$ Reserved for surface analyses (not subject to cleaning for weight-loss measurement or included in DTN: LL030412512251.057 [DIRS 163712]).

All tests were carried out under ambient pressure. After approximately five years of exposure to each solution, the specimens were removed from their respective test vessels to determine their weight-loss. In all of the tested conditions, the coupons were covered with deposits. Therefore, the coupons were cleaned prior to final weighing. Cleaning was carried out according to ASTM G 1-90 (1999 [DIRS 103515]). For specimens exposed to simulated concentrated water and simulated dilute water, the posttest specimens were descaled for 2 minutes in a cleaning solution prepared by diluting $150 \mathrm{~mL}$ of concentrated $\mathrm{HCl}$ acid $(37 \% \mathrm{HCl}$ of specific gravity of 1.19) with deionized water to make $1,000 \mathrm{~mL}$ of solution (TIP-CM-51 [DIRS 169585]). The cleaning temperature was ambient. For specimens exposed to simulated acidified water, the posttest specimens were first exposed for 2 minutes to the same $\mathrm{HCl}$ solution used for descaling samples exposed to simulated concentrated water and simulated dilute water (TIP-CM-51 [DIRS 169585]). The samples were then immersed at $95^{\circ} \mathrm{C}$ for 2 minutes in a solution prepared by dissolving $200 \mathrm{~g}$ of $\mathrm{NaOH}$ and $30 \mathrm{~g}$ of $\mathrm{KMnO}_{4}$ in deionized water to make $1,000 \mathrm{~mL}$ of solution. Finally, the specimens were immersed for 3 minutes in a solution prepared by dissolving $100 \mathrm{~g}$ of diammonium citrate in deionized water to make $1,000 \mathrm{~mL}$ of solution. Weight-loss measurements and scanning electron microscopy analysis showed that these 
cleaning methods removed all scale from tested samples, yet did not significantly affect untested foil samples (TIP-CM-51 [DIRS 169585]). Due to heavy deposits, some samples were cleaned multiple times before SEM photomicrographs indicated no remaining deposits. The only modifications to ASTM G 1-90 (1999 [DIRS 103515]) methods were an increased immersion time (found to be necessary for complete cleaning) and the use of a drying oven and desiccator to minimize air exposure (decreasing the amount of post cleaning film formation and increasing the measured weight-loss resulting in higher determined corrosion rates).

\subsubsection{Weight-Loss Data Analysis}

The general corrosion rate measurements are based upon ASTM G 1-90 (1999 [DIRS 103515]). The formula used to calculate the general corrosion rate from weight-loss data of the tested materials is shown in Equation 6-7:

$$
\text { Corrosion Rate }=\frac{87.6 \times 10^{9} \cdot \Delta w}{\rho \cdot A \cdot t}
$$

where $87.6 \times 10^{9}$ is the proportionality constant $\left(\mathrm{nm} \times \mathrm{cm}^{-1} \times\right.$ hour $\times$ year $\left.{ }^{-1}\right), \Delta w$ is the weightloss in grams after 5+ years, $\rho$ is the density of Alloy $22\left(8.69 \mathrm{~g} / \mathrm{cm}^{3}\right.$ ) (Haynes International 1997 [DIRS 100896], p. 13), $A$ is the exposed surface area of each coupon $\left(\mathrm{cm}^{2}\right)$, and $t$ is the exposure time (hours). The exposed surface area $A$ is calculated as follows:

$$
A=2 a b+2 b c+2 a c-\left(\frac{\pi d^{2}}{2}\right)+\pi d c
$$

where $a$ is the length of the specimen in $\mathrm{cm}, b$ is the width of the specimen in $\mathrm{cm}, c$ is the thickness of the specimen in $\mathrm{cm}$, and $d$ is the diameter of the hole in $\mathrm{cm}$ (Figure 6-9 provides a schematic of the sample coupon).

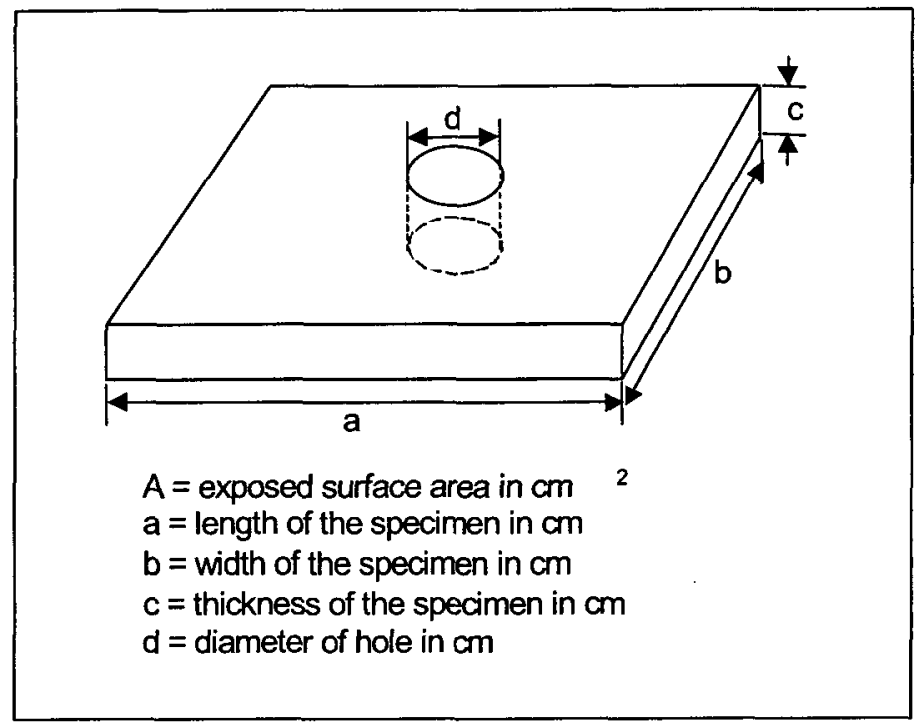

Figure 6-9. Schematic of Specimen Used in the Weight-Loss Measurements of Alloy 22 Samples in Long Term Corrosion Test Facility 
INTENTIONALLY LEFT BLANK 


\section{USE OF SOFTWARE}

Microsoft Excel 97 SR-2, bundled with Microsoft Office 1997, is a commercial off-the-shelf software program used in this report. The Excel computations performed in this report use only standard built-in functions and are documented in sufficient detail to allow an independent technical reviewer to reproduce or verify the results by visual inspection or hand calculation without recourse to the originator (Sections 6.4.3 to 6.4.6, and the Excel files included in the output DTNs: MO0409MWDULCMW.000 and MO0409MWDUGCMW.000). Therefore, use of this software is not subject to LP-SI.11Q-BSC, Software Management. Microsoft Excel 97 SR-2 is appropriate for this application as it offers the mathematical and graphical functionality necessary to perform and document the numerical manipulations used in this report. Microsoft Excel 97 SR-2 was executed on an Optiplex GX260 Workstation (CRWMS M\&O tag 152849, located in the Summerlin Offices, Las Vegas, Nevada) equipped with the Windows 2000 operating system.

Mathcad version 11.1 is a commercial off-the-shelf software program used in this model report. The Mathcad computations performed in this report use only standard functions and are documented in sufficient detail to allow an independent technical reviewer to reproduce or verify the results by visual inspection or hand calculation without recourse to the originator (Sections 6.4.3 and 6.4.4, and the Mathcad worksheet files included in the output DTNs: MO0409MWDULCMW.000 and MO0409MWDUGCMW.000). Therefore, use of this software is not subject to LP-SI.11Q-BSC, Software Management. This software is appropriate for this application as it offers the mathematical and graphical functionality necessary to perform and document the numerical manipulations used in this report. Mathcad version 11.1 was executed on an Optiplex GX260 Workstation (CRWMS M\&O tag 152849, located in the Summerlin Offices, Las Vegas, Nevada) equipped with the Windows 2000 operating system. 
INTENTIONALLY LEFT BLANK 


\section{INPUTS}

This section documents input data and parameters used in the models and analyses in this report. This section also documents inputs from other models or analyses used in this report. Criteria directly applicable to the analyses or models in this report are identified, and a list of the applicable codes and standards used in the analyses or models in this report is provided.

\subsection{DIRECT INPUT}

Inputs to this report are tracked in accordance with AP-SIII.10Q, Models, and AP-3.15Q, Managing Technical Product Inputs. The data used to develop the models in this report are not used to validate the models developed in this report.

\subsubsection{Data}

Table 4-1 lists the input data used in the analyses or models documented in this report, and identifies the DTNs and specific sections where the data were used. Additional details of the input data are described in the following sections. The test procedures for the various electrochemical corrosion tests that were employed to generate the input data documented in this section are summarized in Appendix I, along with a summary for the test conditions and parameters. The electrochemical corrosion tests summarized in Appendix I include polarization resistance and cyclic potentiodynamic polarization (CPP) tests. The long-term open-circuit potential tests are summarized in Section 6.4.4.4. A summary of the test procedures for the weight-loss measurements of the 5-year samples from the Long-Term Corrosion Test Facility (LTCTF) is given in Section 6.4.3.1. The treatment of input data uncertainty is addressed in the data analysis and model development throughout Section 6.4. 
General Corrosion and Localized Corrosion of Waste Package Outer Barrier

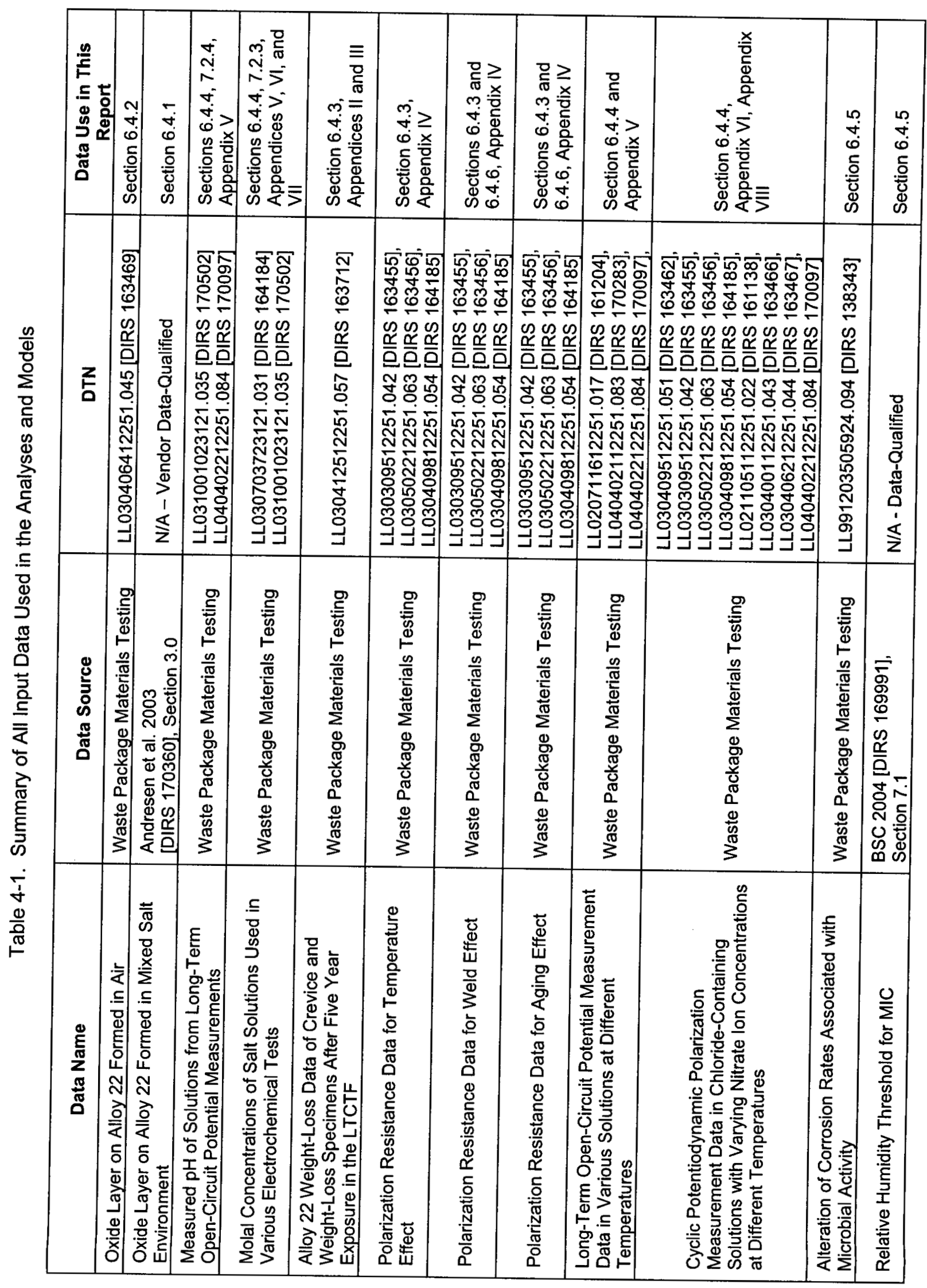




\begin{tabular}{|c|c|c|c|c|c|c|c|}
\hline 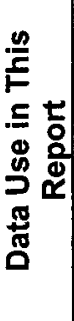 & 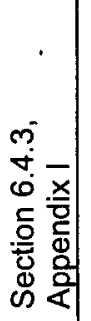 & $\begin{array}{l}\overline{\bar{x}} \\
\frac{. x}{\bar{z}} \\
\overline{0} \\
\frac{0}{2}\end{array}$ & 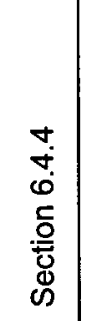 & 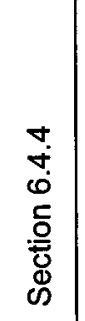 & 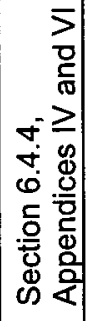 & $\begin{array}{l}\bar{x} \\
\frac{x}{\bar{g}} \\
\bar{\Phi} \\
\frac{0}{2} \\
\frac{\alpha}{\alpha}\end{array}$ & 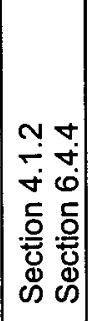 \\
\hline z & 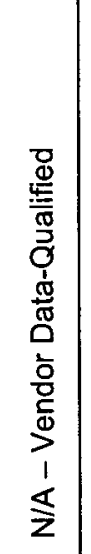 & 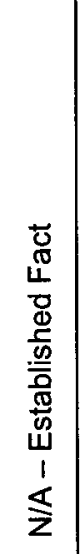 & 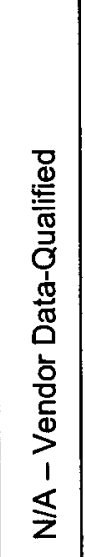 & 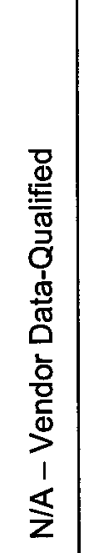 & 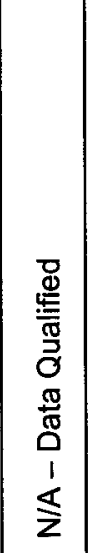 & 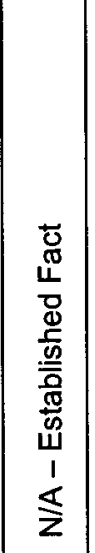 & 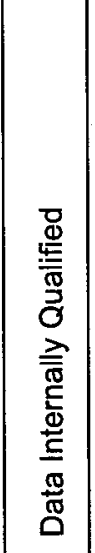 \\
\hline 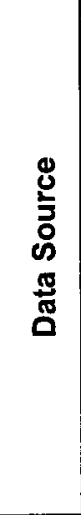 & 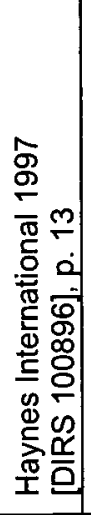 & 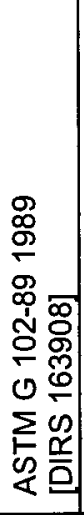 & 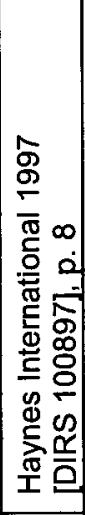 & 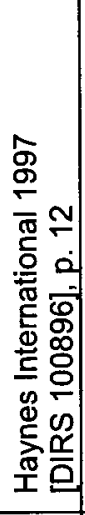 & 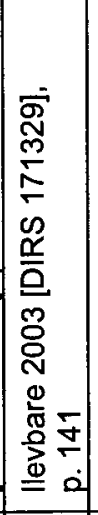 & 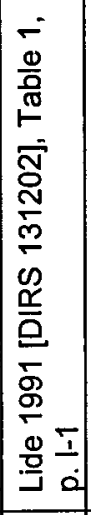 & 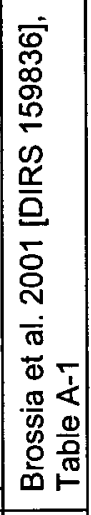 \\
\hline 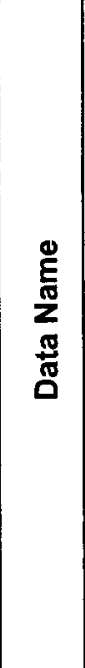 & 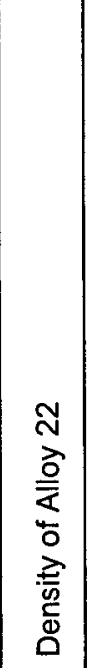 & 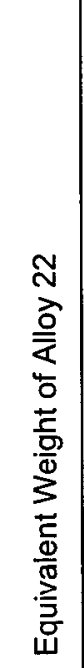 & 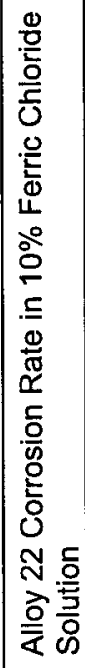 & 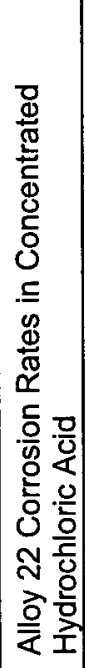 & 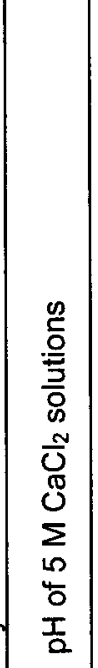 & 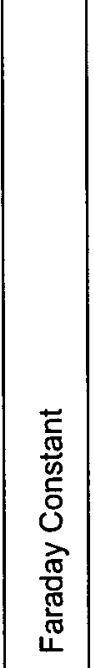 & 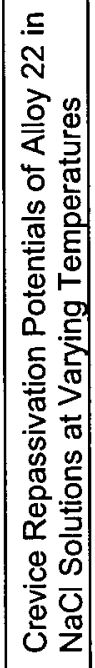 \\
\hline
\end{tabular}




\subsubsection{Passive Film Characterization}

Characterization of the passive film formed on Alloy 22 in various exposure environments is used to provide a technical basis for constructing the conceptual model of the structure and composition of the film. This information is also used as an aid in interpreting corrosion data and their implications on corrosion behavior. Table 4-2 lists the sources of the passive film characterization data and the associated DTNs (if applicable). Because these input data are used in a qualitative manner, no quantitative analysis for data uncertainty will be performed.

Table 4-2. Alloy 22 Passive Film Characterization Data

\begin{tabular}{|l|l|l|l|}
\hline \multicolumn{1}{|c|}{ Data Name } & \multicolumn{1}{|c|}{ Data Source } & \multicolumn{1}{c|}{ DTN } & \multicolumn{1}{c|}{$\begin{array}{c}\text { Data Use in } \\
\text { This Report }\end{array}$} \\
\hline Oxide Layer on Alloy 22 Formed in Air & $\begin{array}{l}\text { Waste Package Materials } \\
\text { Testing }\end{array}$ & $\begin{array}{l}\text { LL030406412251.045 } \\
\text { [DIRS 163469] }\end{array}$ & Section 6.4.2 \\
\hline $\begin{array}{l}\text { Oxide Layer on Alloy 22 Formed in Mixed } \\
\text { Salt Environment }\end{array}$ & $\begin{array}{l}\text { Andresen et al. 2003 } \\
\text { [DIRS 170360], Section 3.0 }\end{array}$ & $\begin{array}{l}\text { N/A - Vendor Data- } \\
\text { Qualified }\end{array}$ & Section 6.4.1 \\
\hline
\end{tabular}

\subsubsection{Corrosion Test Solution Composition}

Models and analyses of Alloy 22 degradation consider exposure conditions as well as metallurgical conditions (i.e., base metal versus weld, aged versus non-aged, etc.). Exposure condition parameters important to corrosion are temperature and composition of the solution contacting the metal. The species that significantly affect metal corrosion are hydrogen ions (e.g., pH), halide ions (e.g., chloride ions), corrosion-inhibiting ions (e.g., nitrate and sulfate ions), and dissolved oxygen. The compositions of the solutions employed in various corrosion tests for Alloy 22 are input to the Alloy 22 corrosion analyses and models. Table 4-3 lists the source of the corrosion test solution compositions and the associated DTNs. Because a single concentration measurement for each species for each solution condition (or each test condition) is reported and little uncertainty is expected in the measurements, no discussion or analysis of input data uncertainty is necessary.

Table 4-3. Compositions of Solutions Employed in Various Corrosion Tests of Alloy 22

\begin{tabular}{|c|c|c|c|}
\hline Data Name & Data Source & DTN & $\begin{array}{l}\text { Data Use in } \\
\text { This Report }\end{array}$ \\
\hline $\begin{array}{l}\text { Molal Concentrations of Salt Solutions } \\
\text { Used in Various Electrochemical Tests }\end{array}$ & $\begin{array}{l}\text { Waste Package } \\
\text { Materials Testing }\end{array}$ & $\begin{array}{l}\text { LL030703723121.031 [DIRS 164184] } \\
\text { LL031001023121.035 [DIRS 170502] }\end{array}$ & $\begin{array}{l}\text { Sections } \\
6.4 .4,7.2 .3 ; \\
\text { Appendices } \\
\text { V, VI, and } \\
\text { VII }\end{array}$ \\
\hline
\end{tabular}

\subsubsection{Long-Term Corrosion Weight-Loss Data}

Alloy 22 specimens with differing configurations and material conditions have been tested in the Project's Long-Term Corrosion Test Facility (LTCTF). Five-year weight-loss data are used to calculate the general corrosion rates used in the waste package outer barrier general corrosion model documented in this report (DTN: LL030412512251.057 [DIRS 163712]). The solution chemistries used in the LTCTF are listed in Table 6-3. The 5-year weight-loss data and the calculated rates are listed in Appendices II and III. The calculated general corrosion rates 
slightly differ from those in the input DTN due to differences in the number of significant digits used. Uncertainty in these data is analyzed, quantified, and propagated into the general corrosion model (Section 6.4.3.3). Table 4-4 lists the data source and associated DTNs for the 5-year Alloy 22 weight-loss data.

Table 4-4. Long-Term Corrosion Weight-Loss Data for Alloy 22

\begin{tabular}{|l|l|l|l|}
\hline \multicolumn{1}{|c|}{ Data Name } & \multicolumn{1}{|c|}{ Data Source } & \multicolumn{1}{|c|}{ DTN } & \multicolumn{1}{c|}{$\begin{array}{l}\text { Data Use in } \\
\text { This Report }\end{array}$} \\
\hline $\begin{array}{l}\text { Alloy 22 Weight-Loss Data of Crevice and } \\
\text { Weight-Loss Specimens After Five Year } \\
\text { Exposure in the LTCTF }\end{array}$ & $\begin{array}{l}\text { Waste Package } \\
\text { Materials Testing }\end{array}$ & $\begin{array}{l}\text { LL030412512251.057 } \\
\text { [DIRS 163712] }\end{array}$ & $\begin{array}{l}\text { Section 6.4.3 } \\
\text { Appendices II } \\
\text { and III }\end{array}$ \\
\hline
\end{tabular}

\subsubsection{Polarization Resistance Data}

The polarization resistance technique was used to measure the corrosion rate of Alloy 22 under various testing conditions. The corrosion rates measured by the technique are to be used for comparative analysis of the corrosion behavior under a wide range of test conditions. They are not used to obtain the absolute values of the corrosion rates. The polarization resistance data were used to determine the temperature dependence of the general corrosion rate (Section 6.4.3) and to evaluate the effect of welding and thermal aging on Alloy 22 corrosion rates (Section 6.4.6). The variables in the tests are exposure condition (e.g., temperature and water chemistry), sample configuration (e.g., crevice, rod, prism and disc), and metallurgical condition (e.g., mill annealed, as-welded, and as-welded plus thermally aged).

Table 4-5 lists the sources of the polarization resistance data of Alloy 22 that were used to analyze the effect of the exposure and metallurgical condition on the WPOB corrosion performance in the repository. The input data are listed in Appendix IV. The range of the test conditions for the polarization resistance data include temperature from $45^{\circ} \mathrm{C}$ to $170^{\circ} \mathrm{C}$, chloride concentration from $1 \mathrm{M}$ to $18 \mathrm{M}$, and nitrate concentration from zero to $2 \mathrm{M}$. Each of the DTNs listed in the table also contains the 24-hour open-circuit corrosion potential (or 24-hour corrosion potential) data and the cyclic potentiodynamic polarization (CPP) data. Details of the electrochemical testing techniques to measure the above corrosion properties of Alloy 22 are described in Appendix I. Uncertainty in the data was analyzed, quantified, and propagated into the model (Section 6.4.3.4).

The polarization resistance data for the electrolytes containing $\mathrm{NaF}$ and oxalic acid were not included in the quantitative model analysis because these chemical environments are not expected to be relevant to the repository conditions. The NaF data set includes pure $1 \mathrm{M} \mathrm{NaF}$ solutions and $0.5 \mathrm{M} \mathrm{NaF}+0.5 \mathrm{M} \mathrm{NaCl}$ solutions at $60^{\circ} \mathrm{C}$ and $90^{\circ} \mathrm{C}$. The seepage and dust deliquescence chemistries predicted in the repository are chloride dominated (BSC 2004 [DIRS 169860], Figures 6.13-2 through 6.13-12 and 6.13-18 through 6.13-23) meaning that solutions of pure $\mathrm{NaF}$ and solutions with one to one ratios of $\mathrm{NaF}$ and $\mathrm{NaCl}$ are not representative of the repository exposure environment. The testing solutions used in long-term weight-loss measurements at $60^{\circ} \mathrm{C}$ and $90^{\circ} \mathrm{C}$ include Simulated Concentrated Water (SCW, Table 6-3), which contains about $1,400 \mathrm{mg} / \mathrm{L}$ fluoride ions and $6,700 \mathrm{mg} / \mathrm{L}$ chloride ions. Therefore, the effects of fluoride ions on general corrosion are included in the general corrosion model developed in this report. The oxalic acid data set includes $0.01 \mathrm{M}, 0.1 \mathrm{M}$, and $1 \mathrm{M}$ oxalic 
acid solutions at $30^{\circ} \mathrm{C}, 60^{\circ} \mathrm{C}$, and $90^{\circ} \mathrm{C}$. The $\mathrm{pH}$ of these solutions varies from about 0.5 , for 1 $\mathrm{M}$ oxalic acid solution, to about 2.2 , for a $0.01 \mathrm{M}$ oxalic acid solution. Not only are these $\mathrm{pH}$ values lower than any in the repository, oxalic acid is not reported to be present in the repository environment as outlined in Engineered Barrier System: Physical and Chemical Environment (BSC 2004 [DIRS 169860], Figures 6.13-2 through 6.13-12 and 6.13-18 through 6.13-23).

The data for the multiple crevice assembly (MCA) samples with the surface condition labeled as "As Received (AR)" were not included in the model analysis in this report. This was because the surface of the edges of those MCA samples may have been damaged and contaminated with some "active" materials during the sample preparation. The active materials caused abnormal signals in the electrochemical corrosion tests under the action of externally applied potentials. Since the waste packages will not have damaged areas subjected to externally applied potentials in the repository, it is appropriate not to use these data in model development. Details of the conditions of those MCA samples are provided in scientific notebooks associated with the DTNs used.

Table 4-5. Polarization Resistance Measurement Data for Alloy 22

\begin{tabular}{|c|c|c|c|}
\hline Data Name & Data Source & DTN & $\begin{array}{l}\text { Data Use in } \\
\text { This Report }\end{array}$ \\
\hline $\begin{array}{l}\text { Polarization Resistance Data for Temperature } \\
\text { Effect }\end{array}$ & $\begin{array}{l}\text { Waste Package } \\
\text { Materials Testing }\end{array}$ & $\begin{array}{l}\text { LL030309512251.042 } \\
\text { [DIRS 163455], } \\
\text { LL030502212251.063 } \\
\text { [DIRS 163456], } \\
\text { LL030409812251.054 } \\
\text { [DIRS 164185] }\end{array}$ & $\begin{array}{l}\text { Section 6.4.3, } \\
\text { Appendix IV }\end{array}$ \\
\hline Polarization Resistance Data for Weld Effect & $\begin{array}{l}\text { Waste Package } \\
\text { Materials Testing }\end{array}$ & $\begin{array}{l}\text { LL030309512251.042 } \\
\text { [DIRS 163455], } \\
\text { LL030502212251.063 } \\
\text { [DIRS 163456], } \\
\text { LL030409812251.054 } \\
\text { [DIRS 164185] }\end{array}$ & $\begin{array}{l}\text { Sections } \\
6.4 .3 \text { and } \\
6.4 .6 \text {, } \\
\text { Appendix IV }\end{array}$ \\
\hline Polarization Resistance Data for Aging Effect & $\begin{array}{l}\text { Waste Package } \\
\text { Materials Testing }\end{array}$ & $\begin{array}{l}\text { LL030309512251.042 } \\
\text { [DIRS 163455], } \\
\text { LL030502212251.063 } \\
\text { [DIRS 163456], } \\
\text { LL030409812251.054 } \\
\text { [DIRS 164185] }\end{array}$ & $\begin{array}{l}\text { Sections } \\
6.4 .3 \text { and } \\
6.4 .6 \text {, } \\
\text { Appendix IV }\end{array}$ \\
\hline
\end{tabular}

\subsubsection{Long-Term Open-Circuit Corrosion Potential Data}

The open-circuit corrosion potential (also referred to as corrosion potential $\left(E_{\text {corr }}\right)$ ) is an important corrosion property of a metal/alloy. The corrosion potential of a metal or alloy may be affected by the sample configuration (e.g., boldly exposed and crevice), metallurgical condition (e.g., mill annealed, as-welded and aged), and exposure environment. For a given exposure environment, it can change over time depending mostly on the kinetics of electrochemical reactions involved. In this report, the corrosion potentials, along with the critical potentials for localized corrosion discussed in Section 6.4.4.3, were used for the localized corrosion initiation model. Section 6.4.4.3 provides details of the localized corrosion initiation model. 
As stated above, the corrosion potential may change over time, eventually approaching a steadystate value (Section 6.4.4.4). The initial changes can be significant, depending on the exposure environment and the sample surface conditions. Therefore, the long-term steady-state corrosion potentials were used for the corrosion potential model of Alloy 22. The test conditions varied in the long-term corrosion potential measurements are exposure environment (temperature and water chemistry), sample geometry (U-bend and rod), and metallurgical conditions (mill annealed and as-welded). Table 4-6 lists the data sources for the long-term open-circuit corrosion potential data of Alloy 22 for a range of exposure environments and metallurgical conditions. The range of the test conditions for the long-term open-circuit corrosion potential data include temperatures from $25^{\circ} \mathrm{C}$ to $120^{\circ} \mathrm{C}, \mathrm{pH}$ values from 2.8 to 10.9 , chloride concentration from very dilute to about $12.6 \mathrm{~m}$ (moles $/ \mathrm{kg}$ water), and nitrate concentration from zero to $2.6 \mathrm{~m}$.

Because of measurement noise in the long-term corrosion potentials, the average of the measured readings for the final week of exposure was used in the model. The acquired $E_{\text {corr }}$ data is contained in two separate DTNs: LL020711612251.017 [DIRS 161204] and LL040402112251.083 [DIRS 170283]. DTN: LL020711612251.017 [DIRS 161204] has the acquired data up to September 3, 2002, and the data acquired beyond that date is reported in DTN: LL040402112251.083 [DIRS 170283]. However, if a cell was terminated before September 3, 2002 (e.g., Cell 7), its data are reported only in DTN: LL020711612251.017 [DIRS 161204], and if testing was started after that date (Cells 16 and higher), the acquired data is reported only in DTN: LL040402112251.083 [DIRS 170283]. The final average values of $E_{\text {corr }}$ for each specimen from DTNs: LL040402112251.083 [DIRS 170283] and LL020711612251.017 [DIRS 161204] are listed in DTN: LL040402212251.084 [DIRS 170097]. Only the final $E_{\text {cor }}$ data in DTN: LL040402212251.084 [DIRS 170097], corresponding to testing times of 328 days and longer, were used for the development of the model in Section 6.4.4.5. Analyses in Section 6.4.4.4 indicate $E_{\text {corr }}$ values are reasonably stable for use in the model after 328 days. The data that were obtained accordingly are listed in Appendix V. Uncertainty in the data was analyzed, quantified, and propagated into the corrosion potential model. Details of the uncertainty analysis are documented in Section 6.4.4.5.

The long-term corrosion potential data for the electrolytes containing $\mathrm{NaF}$ and oxalic acid (Cells 11 and 12) reported in DTN: LL020711612251.017 [DIRS 161204] were not included in the quantitative model analysis because these chemical environments are not relevant to the repository conditions. The $\mathrm{NaF}$ data set includes pure $1 \mathrm{M} \mathrm{NaF}$ solutions at $90^{\circ} \mathrm{C}$ (Cell 12). The seepage and dust deliquescence chemistries are chloride dominated (BSC 2004 [DIRS 169860], Figures 6.13-2 through 6.13-12 and 6.13-18 through 6.13-23) meaning that solutions of pure $\mathrm{NaF}$ solutions are not representative of the repository exposure environments. The testing solutions used in long-term corrosion potential measurements at $60^{\circ} \mathrm{C}$ and $90^{\circ} \mathrm{C}$ include Simulated Concentrated Water (SCW, Table 6-3), which contains about 1,400 mg/L fluoride ions and $6,700 \mathrm{mg} / \mathrm{L}$ chloride ions. Therefore, the effects of fluoride ions are included in the long-term corrosion potential model developed in this report. The oxalic acid data set includes $0.1 \mathrm{M}$ oxalic acid solution at $30^{\circ} \mathrm{C}$ (Cell 11). The $\mathrm{pH}$ of this solution is about 1.5 . Not only is this $\mathrm{pH}$ value lower than any in the repository, oxalic acid is not present in the repository environment as outlined in Engineered Barrier System: Physical and Chemical Environment (BSC 2004 [DIRS 169860], Figures 6.13-2 through 6.13-12 and 6.13-18 through 6.13-23). 
Table 4-6. Long-Term Open-Circuit Corrosion Potential Measurement Data for Alloy 22

\begin{tabular}{|c|c|c|c|}
\hline Data Name & Data Source & DTN & $\begin{array}{l}\text { Data Use in } \\
\text { This Report }\end{array}$ \\
\hline \multirow{3}{*}{$\begin{array}{l}\text { Long-Term Open-Circuit Potential Measurement Data } \\
\text { in "Aged" LTCTF Solutions at Different Temperatures }\end{array}$} & \multirow{3}{*}{$\begin{array}{l}\text { Waste Package } \\
\text { Materials Testing }\end{array}$} & $\begin{array}{l}\text { LL020711612251.017 } \\
\text { [DIRS 161204] }\end{array}$ & \multirow{3}{*}{$\begin{array}{l}\text { Section 6.4.4, } \\
\text { Appendix V }\end{array}$} \\
\hline & & $\begin{array}{l}\text { LL040402112251.083 } \\
\text { [DIRS 170283] }\end{array}$ & \\
\hline & & $\begin{array}{l}\text { LL040402212251.084 } \\
\text { [DIRS 170097] }\end{array}$ & \\
\hline \multirow{3}{*}{$\begin{array}{l}\text { Long-Term Open-Circuit Potential Measurement Data } \\
\text { in "Fresh" LTCTF Solutions at Different Temperatures }\end{array}$} & \multirow{3}{*}{$\begin{array}{l}\text { Waste Package } \\
\text { Materials Testing }\end{array}$} & $\begin{array}{l}\text { LL020711612251.017 } \\
\text { [DIRS 161204] }\end{array}$ & \multirow{3}{*}{$\begin{array}{l}\text { Section 6.4.4, } \\
\text { Appendix } V\end{array}$} \\
\hline & & $\begin{array}{l}\text { LL040402112251.083 } \\
\text { [DIRS 170283] }\end{array}$ & \\
\hline & & $\begin{array}{l}\text { LL040402212251.084 } \\
\text { [DIRS 170097] }\end{array}$ & \\
\hline \multirow{3}{*}{$\begin{array}{l}\text { Long-Term Open-Circuit Potential Measurement Data } \\
\text { in BSW at Different Temperatures }\end{array}$} & \multirow{3}{*}{$\begin{array}{l}\text { Waste Package } \\
\text { Materials Testing }\end{array}$} & $\begin{array}{l}\text { LL020711612251.017 } \\
\text { [DIRS 161204] }\end{array}$ & \multirow{3}{*}{$\begin{array}{l}\text { Section 6.4.4, } \\
\text { Appendix V }\end{array}$} \\
\hline & & $\begin{array}{l}\text { LL040402112251.083 } \\
\text { [DIRS 170283] }\end{array}$ & \\
\hline & & $\begin{array}{l}\text { LL040402212251.084 } \\
\text { [DIRS 170097] }\end{array}$ & \\
\hline \multirow{3}{*}{$\begin{array}{l}\text { Long-Term Open-Circuit Potential Measurement Data } \\
\text { in } \mathrm{CaCl} \mathrm{Cl}_{2}+\mathrm{Ca}\left(\mathrm{NO}_{3}\right)_{2} \text { Solution with Varying Chloride } \\
\text { and Nitrate Concentration Ratios at Different } \\
\text { Temperatures }\end{array}$} & \multirow{3}{*}{$\begin{array}{l}\text { Waste Package } \\
\text { Materials Testing }\end{array}$} & $\begin{array}{l}\text { LL020711612251.017 } \\
\text { [DIRS 161204] }\end{array}$ & \multirow{3}{*}{$\begin{array}{l}\text { Section 6.4.4, } \\
\text { Appendix V }\end{array}$} \\
\hline & & $\begin{array}{l}\text { LL040402112251.083 } \\
\text { [DIRS 170283] }\end{array}$ & \\
\hline & & $\begin{array}{l}\text { LL040402212251.084 } \\
\text { [DIRS 170097] }\end{array}$ & \\
\hline
\end{tabular}

\subsubsection{Cyclic Potentiodynamic Polarization Data}

The cyclic potentiodynamic polarization (CPP) technique was used to measure a critical potential ( $\left.E_{\text {critical }}\right)$ for localized corrosion (pitting or crevice corrosion, or both) of Alloy 22 for a range of exposure conditions relevant to the repository. CPP tests were carried out for a variety of exposure environments (temperature and water chemistry), sample configurations (crevice or boldly exposed), and metallurgical conditions (mill annealed, as-welded, or aged). Table 4-7 lists the sources of the CPP data of Alloy 22 for a wide range of exposure and metallurgical conditions

The input DTNs listed in the table also contain other electrochemical measurement data such as 24-hour open-circuit corrosion potentials (or 24-hour corrosion potentials) and polarization resistance measurements (Section 4.1.1.4). Details of the electrochemical testing techniques used to measure the above corrosion properties of Alloy 22 are described in Appendix I. The sources of the data and associated DTNs are also listed in the table.

The CPP data for the electrolytes containing $\mathrm{NaF}$ and oxalic acid reported in DTNs: LL030309512251.042 [DIRS 163455] and LL030502212251.063 [DIRS 163456], were not included in the quantitative model analysis because these chemical environments are not relevant to the repository. The $\mathrm{NaF}$ data set includes pure $1 \mathrm{M} \mathrm{NaF}$ solutions and $0.5 \mathrm{M} \mathrm{NaF}+$ $0.5 \mathrm{M} \mathrm{NaCl}$ solutions at $60^{\circ} \mathrm{C}$ and $90^{\circ} \mathrm{C}$. The seepage and dust deliquescence chemistries predicted in the repository are chloride dominated (BSC 2004 [DIRS 169860], Figures 6.13-2 
through 6.13-12 and 6.13-18 through 6.13-23), which means solutions of pure $\mathrm{NaF}$ and solutions with one to one ratios of $\mathrm{NaF}$ and $\mathrm{NaCl}$ are not representative of the repository exposure environments. The testing solutions used in the cyclic polarization measurements at $60^{\circ} \mathrm{C}$ and $90^{\circ} \mathrm{C}$ include Simulated Concentrated Water (SCW, Table 6-3), which contains about $1,400 \mathrm{mg} / \mathrm{L}$ fluoride ions and $6,700 \mathrm{mg} / \mathrm{L}$ chloride ions. Therefore, the effects of fluoride ions on general corrosion are included in the analyses in this report. The oxalic acid data set includes $0.01 \mathrm{M}, 0.1 \mathrm{M}$, and $1 \mathrm{M}$ oxalic acid solutions at $30^{\circ} \mathrm{C}, 60^{\circ} \mathrm{C}$, and $90^{\circ} \mathrm{C}$. The $\mathrm{pH}$ of these solutions varies from about 0.5 , for $1 \mathrm{M}$ oxalic acid solution, to about 2.2 , for a $0.01 \mathrm{M}$ oxalic acid solution. Not only are these $\mathrm{pH}$ values lower than any expected in the repository, oxalic acid is not present in the repository environment as outlined in Engineered Barrier System: Physical and Chemical Environment (BSC 2004 [DIRS 169860], Figures 6.13-2 through 6.13-12 and 6.13-18 through 6.13-23).

The data for the multiple crevice assembly (MCA) samples with the surface condition labeled as "As Received" (AR) or as "Edges Not Polished" were not included in the quantitative model analysis of this model report. This was because the surface of the edges of those MCA samples may have been damaged or contaminated with some "active" materials during the sample preparation, or both. The MCA samples labeled "AR" did not have the edges properly polished, and this caused abnormal signals in the electrochemical corrosion tests under applied potentials.

Table 4-7. Cyclic Potentiodynamic Polarization Measurement Data for Alloy 22

\begin{tabular}{|c|c|c|c|}
\hline Data Name & Data Source & DTN & $\begin{array}{l}\text { Data Use in } \\
\text { This Report }\end{array}$ \\
\hline $\begin{array}{l}\text { Cyclic Potentiodynamic Polarization } \\
\text { Measurement Data in Fresh } \\
\text { Chloride-Containing Solutions with } \\
\text { Varying Nitrate lon Concentrations } \\
\text { at Different Temperatures }\end{array}$ & $\begin{array}{l}\text { Waste Package } \\
\text { Materials Testing }\end{array}$ & $\begin{array}{l}\text { LL030409512251.051 [DIRS 163462], } \\
\text { LL030309512251.042 [DIRS 163455], } \\
\text { LL030502212251.063 [DIRS 163456], } \\
\text { LL030409812251.054 [DIRS 164185], } \\
\text { LL021105112251.022 [DIRS 161138], } \\
\text { LL030400112251.043 [DIRS 163466], } \\
\text { LL030406212251.044 [DIRS 163467], } \\
\text { LL040402212251.084 [DIRS 170097] }\end{array}$ & $\begin{array}{l}\text { Section } 6.4 .4 \text {, } \\
\text { Appendices } \\
\text { VI and VIII }\end{array}$ \\
\hline
\end{tabular}

\subsubsection{Crevice Repassivation Potential Data}

The localized corrosion conceptual model assumes that crevices form on the waste package surface in the repository (Assumption 5.3 and Section 6.4.4). Thus, crevice corrosion was conservatively taken as the representative form of localized corrosion on the WPOB (Assumption 5.3). The crevice repassivation potentials $\left(E_{\text {rcrev }}\right)$ from the CPP curves described in Section 4.1.1.6 were selected as the critical potentials for localized corrosion. As discussed in Section 6.4.4.1, the crevice repassivation potential is a conservative measure of the critical potential for localized corrosion initiation. Input DTN: LL030409512251.051 [DIRS 163462] documents the analyses for obtaining the crevice repassivation potentials from the CPP curves. The final set of data developed from the analyses are listed in the tables contained in the files with the file name beginning with "TSES" under the "LL030409512251.051 TDMS" directory of the DTN data package. Crevice repassivation potentials were also obtained from DTN: LL040402212251.084 [DIRS 170097]. 
Only the crevice (i.e., MCA) sample data were used for repassivation potential model analysis with the exception of the repassivation potential data for samples DEA598, DEA599, and DEA600 from DTN: LL030409812251.054 [DIRS 164185], which were disc samples exposed to high-chloride concentrations $(12.6$ to $20.8 \mathrm{~m})$ and high temperatures $\left(120^{\circ} \mathrm{C}\right.$ and $\left.130^{\circ} \mathrm{C}\right)$. These data were included to increase the data population for the model analysis and improve the predictive capability of the model. As discussed in Section 6.4.4.2, there are no significant differences in the repassivation potential between the two types of samples for these conditions.

In addition, the MCA corrosion data that did not show occurrence of localized corrosion were conservatively excluded from the analysis, except when the nitrate-to-chloride ratio of the test solution was 0.50 , in which case a repassivation potential of $+600 \mathrm{mV}$ versus (silver-silver chloride reference electrode scale) SSC was used (Section 6.4.4.1). Section 6.4.4.2 and DTN: LL030409512251.051 [DIRS 163462] provide details on the data screening analysis. The crevice repassivation potential data used in the analyses are listed in Appendices VI and VIII.

Table 4-8 lists the sources of the crevice repassivation potential data of Alloy 22 used in the critical potential model analysis. Uncertainty in the data was analyzed, quantified, and propagated into the critical potential model. Details of the uncertainty analysis are documented in Section 6.4.4.3.

Table 4-8. Crevice Repassivation Potential Data for Alloy 22

\begin{tabular}{|c|c|c|c|}
\hline Data Name & Data Source & DTN & $\begin{array}{l}\text { Data Use in } \\
\text { This Report } \\
\end{array}$ \\
\hline \multirow{3}{*}{$\begin{array}{l}\text { Crevice Repassivation Potentials of Alloy } 22 \text { in } \mathrm{NaCl} \\
\text { and } \mathrm{CaCl}_{2} \text { Solutions with Varying Nitrate } \\
\text { Concentrations at Different Temperatures }\end{array}$} & \multirow{3}{*}{$\begin{array}{l}\text { Waste Package } \\
\text { Materials Testing }\end{array}$} & $\begin{array}{l}\text { LL030409512251.051 } \\
\text { [DIRS 163462] }\end{array}$ & \multirow{3}{*}{ Section 6.4.4 } \\
\hline & & $\begin{array}{l}\text { LL030409812251.054 } \\
\text { [DIRS 164185] }\end{array}$ & \\
\hline & & $\begin{array}{l}\text { LLO } 40402212251.084 \\
\text { [DIRS 170097] }\end{array}$ & \\
\hline
\end{tabular}

\subsubsection{8 pH of $5 \mathrm{M} \mathrm{CaCl}_{2}$ Solution}

The $\mathrm{pH}$ values for some $5 \mathrm{M} \mathrm{CaCl}_{2}$ solutions in which data were obtained were not measured. In these cases, a value of 4.14 was used (Section 6.4.4 and Appendices IV and VI). This $\mathrm{pH}$ value was from the scientific notebook associated with DTN: LL030400112251.043 [DIRS 163466] (i.e., Ilevbare 2003 [DIRS 171329], p. 141). This datum is appropriate for its intended use because it was obtained under the Project quality assurance process. Table 4-9 lists the source of the $\mathrm{pH}$ value used for the $5 \mathrm{M} \mathrm{CaCl}_{2}$ solutions and where they are used in this report. 
Table 4-9. $\mathrm{pH}$ of $5 \mathrm{M} \mathrm{CaCl}_{2}$ Solutions

\begin{tabular}{|c|c|c|c|}
\hline Data Name & Data Source & DTN & $\begin{array}{c}\text { Data Use in This } \\
\text { Report }\end{array}$ \\
\hline $\mathrm{pH}$ of $5 \mathrm{M} \mathrm{CaCl}_{2}$ solutions & llevbare 2003 [DIRS 171329], p. 141 & N/A - Data Qualified & $\begin{array}{c}\text { Section 6.4.4, } \\
\text { Appendices IV and VI }\end{array}$ \\
\hline
\end{tabular}

\subsubsection{Microbially Influenced Corrosion}

Microbially influenced corrosion (MIC) is the contribution to the corrosion of a metal or alloy due to the presence or activity, or both, of microorganisms. Nickel-based alloys such as Alloy 22 are highly resistant to microbially influenced corrosion (Lian et al. 1999 [DIRS 110238]).

The effect of MIC on the WPOB corrosion is represented with a general corrosion enhancement factor applied when the exposure relative humidity exceeds 90 percent (BSC 2004 [DIRS 169991], Section 7.1). Table 4-10 lists the source of the input data used to evaluate the MIC effect on the WPOB general corrosion rate. DTN: LL991203505924.094 [DIRS 138343] contains measurements for the corrosion potentials and corrosion rates of several engineering alloys, including Alloy 22, in the presence and absence of microbes relevant to the repository. The corrosion rates were obtained from the short-term polarization resistance tests. The MIC enhancement factor was determined from the comparative analysis of the corrosion rates of Alloy 22 samples in abiotic and biotic conditions. Uncertainty associated with the MIC enhancement factor is discussed in Section 6.4.5. The relative humidity threshold for MIC is based on analyses documented in Evaluation of the Impact of Microbial Activities on Drift Chemistry report (BSC 2004 [DIRS 169991], Section 7.1). In that report, it is noted that microbial activity is minimal when the activity of water (equivalent to the relative humidity fraction) falls below 0.90 and microbes have trouble thriving when the activity of water is less than 0.95 (BSC 2004 [DIRS 169991], Section 7.1).

Table 4-10. Input Data for Microbially Influenced Corrosion of Alloy 22

\begin{tabular}{|l|l|l|l|}
\hline \multicolumn{1}{|c|}{ Data Name } & \multicolumn{1}{|c|}{ Data Source } & \multicolumn{1}{c|}{ DTN } & \multicolumn{1}{c|}{$\begin{array}{c}\text { Data Use in } \\
\text { This Report }\end{array}$} \\
\hline $\begin{array}{l}\text { Alteration of Corrosion Rates Associated with } \\
\text { Microbial Activity }\end{array}$ & $\begin{array}{l}\text { Waste Package } \\
\text { Materials Testing }\end{array}$ & $\begin{array}{l}\text { LL991203505924.094 } \\
\text { [DIRS 138343] }\end{array}$ & Section 6.4.5 \\
\hline Relative Humidity Threshold for MIC & $\begin{array}{l}\text { BSC 2004 } \\
\text { [DIRS 169991], } \\
\text { Section 7.1 }\end{array}$ & N/A-Data-Qualified & Section 6.4.5 \\
\hline
\end{tabular}

\subsubsection{Density of Alloy 22 and Faraday Constant}

The density of Alloy $22\left(8.69 \mathrm{~g} / \mathrm{cm}^{3}\right)$ was used to calculate the general corrosion rates of Alloy 22 from the weight-loss measurements of the five-year samples from the LTCTF (Section 6.4.3) and for determination of corrosion rates from polarization resistance tests in Appendix I. The Faraday constant value $(96,486$ coulombs/mol) was used in this report to calculate the corrosion rates from the corrosion current measurements in Appendix I (Lide 1991 [DIRS 131202], Table 1, p. I-1). Table 4-11 lists these data and their sources. 
Table 4-11. Density of Alloy 22 and Faraday Constant

\begin{tabular}{|c|l|l|l|}
\hline \multicolumn{1}{|c|}{ Data Name } & \multicolumn{1}{|c|}{ Data Source } & \multicolumn{1}{c|}{ DTN } & \multicolumn{1}{c|}{$\begin{array}{c}\text { Data Use in } \\
\text { This Report }\end{array}$} \\
\hline Density of Alloy 22 & Haynes International 1997 [DIRS 100896], p. 13 & $\begin{array}{l}\text { N/A - Vendor Data- } \\
\text { Qualified }\end{array}$ & $\begin{array}{l}\text { Section 6.4.3; } \\
\text { Appendix I }\end{array}$ \\
\hline Faraday Constant & Lide 1991 [DIRS 131202], Table 1, p. l-1 & N/A - Established Fact & Appendix I \\
\hline
\end{tabular}

\subsubsection{Equivalent Weight of Alloy 22}

The equivalent weight for Alloy 22 (N06022) is calculated considering that the alloy dissolves stoichiometrically and depends on the valence of the dissolved elements. The equivalent weight of Alloy 22 is used in determining general corrosion rates from the polarization resistance data in Appendix I. Table 1 of ASTM G 102-89 (1989 [DIRS 163908]) presents several choices for the Alloy 22 equivalent weight. The actual choice of which equivalent weight to use has no impact on the analysis results presented in this report because only relative corrosion rates (e.g., between welded and nonwelded samples) are used, not their absolute values. The value of the equivalent weight used (23.28) was from Table 1 (column labeled "Third") in ASTM G 102-89 [DIRS 163908], Table 1. Table 4-12 lists the source of the equivalent weight of Alloy 22.

Table 4-12. Equivalent Weight of Alloy 22

\begin{tabular}{|c|l|c|l|}
\hline Data Name & \multicolumn{1}{|c|}{ Data Source } & DTN & Data Use in This Report \\
\hline Equivalent Weight of Alloy 22 & $\begin{array}{l}\text { ASTM G 102-89 (1989 } \\
\text { [DIRS 163908]), Table 1 }\end{array}$ & N/A - Established Fact & Appendix I \\
\hline
\end{tabular}

\subsubsection{Localized Penetration Rates of Alloy 22}

Corrosion rates of Alloy 22 in aggressive solutions are used to represent the localized corrosion penetration rates of Alloy 22 in Section 6.4.4. Table 4-13 lists the source of these corrosion rates of Alloy 22. The localized corrosion penetration rate values used are listed in Table 6-9.

Table 4-13. Localized Corrosion Penetration Rates of Alloy 22

\begin{tabular}{|l|l|l|l|}
\hline \multicolumn{1}{|c|}{ Data Name } & \multicolumn{1}{|c|}{ Data Source } & \multicolumn{1}{c|}{ DTN } & \multicolumn{1}{c|}{$\begin{array}{c}\text { Data Use in } \\
\text { This Report }\end{array}$} \\
\hline $\begin{array}{l}\text { Alloy 22 corrosion rate in 10\% ferric } \\
\text { chloride solution }\end{array}$ & $\begin{array}{l}\text { Haynes International 1997 } \\
\text { [DIRS 100897], p. 8 }\end{array}$ & $\begin{array}{l}\text { N/A - Vendor Data- } \\
\text { Qualified }\end{array}$ & Section 6.4.4 \\
\hline $\begin{array}{l}\text { Alloy 22 corrosion rates in concentrated } \\
\text { hydrochloric acid }\end{array}$ & $\begin{array}{l}\text { Haynes International 1997 } \\
\text { [DIRS 100896], p. 12 }\end{array}$ & $\begin{array}{l}\text { N/A - Vendor Data- } \\
\text { Qualified }\end{array}$ & Section 6.4.4 \\
\hline
\end{tabular}

\subsubsection{Other Model/Analyses Inputs}

An approach similar to that used for the Alloy 22 crevice repassivation potentials described in Section 4.1.1.7 was also used by the investigators at the Center for Nuclear Waste Regulatory Analyses (CNWRA) for the Nuclear Regulatory Commission (NRC) to obtain the crevice repassivation potentials for Alloy 22. The data were generated under CNWRA QA procedures and reported by Brossia et al. (2001 [DIRS 159836], Table A-1). On this basis, the data source is considered reliable and the personnel and organization generating the data operate under a QA 
program compatible with specifications of Quality Assurance Requirements and Description (BSC 2004 [DIRS 171539]). In Section 6.4.4, sufficient discussion of the extent to which the data demonstrate the properties of interest, prior uses of the data, and comparison to corroborating data are presented. Therefore, the data are suitable for the specific application for which they are used in this report and are qualified for use within this technical product. The test environments for the reported data set are $0.005 \mathrm{M}$ to $4 \mathrm{M}$ chloride concentration and $80^{\circ} \mathrm{C}$ to $150^{\circ} \mathrm{C}$. These data are also included in the critical potential model analysis and listed in Appendix VII. Table 4-14 lists the source of the CNWRA crevice repassivation potential data of Alloy 22 used in the critical potential model analysis. Uncertainty in the data was analyzed, quantified, and propagated into the critical potential model. Details of the uncertainty analysis are documented in Section 6.4.4.3.

Table 4-14. Crevice Repassivation Potentials for Alloy 22 from Other Source

\begin{tabular}{|c|l|l|l|}
\hline \multicolumn{1}{|c|}{ Data Name } & \multicolumn{1}{|c|}{ Data Source } & \multicolumn{1}{c|}{ DTN } & $\begin{array}{c}\text { Data Use in } \\
\text { This Report }\end{array}$ \\
\hline $\begin{array}{l}\text { Crevice Repassivation Potentials of Alloy 22 } \\
\text { in NaCl Solutions at Varying Temperatures }\end{array}$ & $\begin{array}{l}\text { Brossia et al. 2001 } \\
\text { [DIRS 159836], Table A-1 }\end{array}$ & $\begin{array}{l}\text { Data Internally } \\
\text { Qualified }\end{array}$ & Section 6.4.4 \\
\hline
\end{tabular}

\subsection{CRITERIA}

The technical work plan (TWP) (BSC 2004 [DIRS 171583], Table 3-1) identified the following acceptance criteria (AC) based on the requirements mentioned in Project Requirements Document (Canori and Leitner 2003 [DIRS 166275]) and Yucca Mountain Review Plan, Final Report (NRC 2003 [DIRS 163274]):

1. System Description and Demonstration of Multiple Barriers (NRC 2003 [DIRS 163274], Section 2.2.1.1.3; Canori and Leitner 2003 [DIRS 166275]; PRD-002/T-014; PRD-002/T-016)

Specific requirements involve identification of multiple barriers (natural and engineered), describing the capabilities of these barriers to isolate waste, and providing technical bases for capabilities descriptions consistent with the postclosure performance objectives. To comply with these requirements, the following acceptance criteria are identified:

- $\mathrm{AC1}$ : Identification of Barriers is Adequate

- AC2: Description of the Capability of Identified Barriers is Acceptable

- AC3: Technical Basis for Barrier Capability is Adequately Presented.

2. Degradation of Engineered Barriers (NRC 2003 [DIRS 163274], Section 2.2.1.3.1.3; Canori and Leitner 2003 [DIRS 166275], PRD-002/T-015)

Specific requirements include describing deterioration or degradation of engineered barriers and modeling degradation processes using data for performance assessment, including total system performance assessment (TSPA). Consideration of uncertainties and variabilities in model parameters and alternative conceptual models 
are also required. To fulfill these requirements, the following acceptance criteria are identified:

- AC1: System Description and Model Integration are Adequate

- AC2: Data are Sufficient for Model Justification

- AC3: Data Uncertainty is Characterized and Propagated Through the Model Abstraction

- AC4: Model Uncertainty is Characterized and Propagated Through the Model Abstraction

- AC5: Model Abstraction Output is Supported by Objective Comparisons.

The Yucca Mountain Review Plan Criteria are addressed in Section 8.4.

\subsection{CODES AND STANDARDS}

This section lists the codes and standards used in the model analyses documented in this report.

\subsubsection{Corrosion Degradation Analyses and Models}

- Standard Practice for Prediction of the Long-Term Behavior of Materials, Including Waste Forms, Used in Engineered Barrier Systems (EBS) for Geological Disposal of High-Level Radioactive Waste (ASTM C 1174-97 2002 [DIRS 105725]).

\subsubsection{Cyclic Polarization Measurements}

- Standard Reference Test Method for Making Potentiostatic and Potentiodynamic Anodic Polarization Measurements (ASTM G 5-94 1994 [DIRS 117479])

- Standard Test Method for Conducting Cyclic Potentiodynamic Polarization Measurements for Localized Corrosion Susceptibility of Iron-, Nickel-, or Cobalt-Based Alloys (ASTM G 61-86 1987 [DIRS 127897])

- Standard Practice for Making and Using U-Bend Stress-Corrosion Test Specimens (ASTM G 30-94 1994 [DIRS 137688]).

\subsubsection{General Corrosion Measurements}

- Standard Practice for Preparing, Cleaning, and Evaluating Corrosion Test Specimens (ASTM G 1-90 1999 [DIRS 103515])

- Standard Test Method for Conducting Potentiodynamic Polarization Resistance Measurements (ASTM G 59-97 1998 [DIRS 163907])

- Standard Practice for Conventions Applicable to Electrochemical Measurements in Corrosion Testing (ASTM G 3-89 1989 [DIRS 138911])

- Standard Practice for Calculation of Corrosion Rates and Related Information from Electrochemical Measurements (ASTM G 102-89 1989 [DIRS 163908]). 


\subsubsection{Composition of Alloy 22}

- Standard Specification for Low-Carbon Nickel-Molybdenum-Chromium and LowCarbon Nickel-Chromium-Molybdenum Steel Alloy Plate, Sheet, and Strip (ASTM B 575-94 1994 [DIRS 100497]).

\subsubsection{Standard Practice for Dealing with Outlying Observations}

- Standard Practice for Dealing with Outlying Observations (ASTM E 178-02 2002 [DIRS 169968]). 
INTENTIONALLY LEFT BLANK 


\section{ASSUMPTIONS}

This section documents the assumptions used to perform analyses and model development and, if necessary, their abstractions for general corrosion and localized corrosion of the waste package outer barrier (WPOB) for the exposure conditions expected in the postclosure repository. Where necessary, additional details of the assumptions are described in the section(s) in which the analyses and models are documented.

5.1 Assumption: Aqueous corrosion, including "humid-air" corrosion, general corrosion, and localized corrosion, is assumed to occur only if the relative humidity $(\mathrm{RH})$ exceeds a threshold RH ( $\left.R H_{\text {threshold }}\right)$. As discussed in Section 6.4.2, dry oxidation can occur at relative humidities below the threshold RH. Although no values for the threshold RH are presented in this report, the existence of a threshold $\mathrm{RH}$ is mentioned throughout this document.

Rationale: The critical relative humidity is the relative humidity below which water will not form on a clean metal surface and electrochemical processes will not occur (ASM International 1987 [DIRS 133378], p. 82). For clean metal surfaces, the relative humidity must exceed about 60 percent before a thin film of moisture will form on the metal surface, providing an electrolyte for ionic current transfer (ASM International 1987 [DIRS 133378], p. 82). Cleanliness, corrosion product build-up, and hygroscopic salts or contaminants can cause water absorption at lower relative humidities (ASM International 1987 [DIRS 133378], p. 80). On this basis, the existence of a threshold $\mathrm{RH}$ for initiation of corrosion processes is a reasonable assumption and consistent with data presented in corrosion handbooks.

Confirmation Status: This is a reasonable assumption and does not require further confirmation.

Use in Model: This assumption is used throughout this report.

5.2 Assumption: The general corrosion rate of Alloy 22, at a given temperature, is assumed constant (i.e., time independent).

Rationale: This assumption is considered conservative because the general corrosion rate of metals and alloys tend to decrease with time. This behavior is illustrated for Alloy 22 in Figure 7-1, which shows general corrosion rates of Alloy 22 at $90^{\circ} \mathrm{C}$ for exposure times up to 5 years decrease with increasing exposure time.

Confirmation Status: This is a conservative assumption because the general corrosion rate of metals and alloys tend to decrease with time; therefore, no additional confirmation of this assumption is necessary.

Use in Model: This assumption is used throughout this report.

5.3 Assumption: Localized corrosion can be either pitting corrosion on boldly exposed surfaces or "crevice corrosion" which takes place in occluded regions. In this report, the dominant form of localized corrosion is assumed to be crevice corrosion. 
Rationale: This is a conservative and bounding assumption because initiation thresholds for crevice corrosion, in terms of exposure parameters such as chemistry and temperature, are lower than those for pitting corrosion (Gdowski 1991 [DIRS 100859], Section 3.7; Agarwal 2000 [DIRS 163034], pp. 845 to 847). Additionally, crevice corrosion is applied to the entire waste package surface, though it is unlikely that crevice attack would occur over the entire surface area.

Confirmation Status: This is a conservative and bounding assumption; therefore, no additional confirmation of this assumption is necessary.

Use in Model: This assumption is used throughout this report.

5.4 Assumption: When localized corrosion occurs, the localized corrosion of the WPOB is assumed to propagate at a (time-independent) constant rate.

Rationale: This assumption is conservative because it is known that localized corrosion rates decrease with time (CRWMS M\&O 1998 [DIRS 100349], Table 3-2; Hunkeler and Boehni 1983 [DIRS 162221]; McGuire et al. 1998 [DIRS 152193], Section 5.2.8, EPRI 2002 [DIRS 158069], Section 5.3.1; Frankel 1998 [DIRS 162216]; Newman and Franz 1984 [DIRS 162250]).

Confirmation Status: This assumption is conservative because it is known that localized corrosion rates decrease with time; therefore, no additional confirmation of this assumption is necessary.

Use in Model: This assumption is used throughout this report.

5.5 Assumption: The localized corrosion data of the WPOB material (Alloy 22) that were generated in fully immersed conditions are assumed to be applicable to the localized corrosion processes of the waste package in contact with thin water films (under porous layers of dust and mineral precipitates) that have the same water chemistry as the fully immersed condition.

Rationale: A thin water film condition in the presence of the porous layers is expected to form on the waste package surface in the nominal-case postclosure repository. The localized corrosion data of the WPOB material (Alloy 22) generated in fully immersed conditions are applicable to the thin film case, because a similar stagnant boundary layer is formed in the creviced region in both cases; therefore, it is reasonable to expect that the crevice corrosion behavior should be similar in both. Corrosion potentials measured in short-term experiments could be lower due to short-term decreases in the oxygen content near the sample surface that would be replenished at a faster rate under thin water film conditions than in fully immersed conditions. The corrosion potentials used in model development were obtained from long-term experiments in which the oxygen concentration would have reached steady state levels.

Confirmation Status: This is a reasonable assumption and does not require further confirmation. 
Use in Model: This assumption is used throughout this report.

5.6 Assumption: The corrosion behavior of the stress-mitigated (laser peened) outer lid closure weld region does not significantly differ from the corrosion behavior of a closure lid weld region that has not undergone stress-mitigation.

Rationale: Laser peening involves the use of a laser pulse that induces a shock wave within the material being peened, resulting in the formation of a compressive surface layer. Laser peening causes less surface damage than conventional shot peening (Chen et al. 2002 [DIRS 165441]) particularly because in laser peening no physical contact is made with the part to be peened. Comparisons of the corrosion rates (measured by the polarization resistance technique in simulated acidified water at $90^{\circ} \mathrm{C}$ and potentiodynamic polarization curves) of unmitigated and laser-peened Alloy 22 samples have shown that laser peened samples exhibited lower corrosion rates than unmitigated samples (Chen et al. 2002 [DIRS 165441], Figure 9). Also laser peened samples exhibited lower passive current densities (in potentiodynamic polarization tests) than unmitigated samples (Chen et al. 2002 [DIRS 165441], Figure 10). The potentiodynamic polarization curves did not show the initiation of localized corrosion before the transpassive potential was reached. Conservatively, no credit is taken in this report for the increased resistance to corrosion processes imparted by the laser peening process.

Confirmation Status: This is a conservative assumption and does not require further confirmation.

Use in Model: This assumption is used throughout this report. 
INTENTIONALLY LEFT BLANK 


\section{MODEL DISCUSSION}

\subsection{ANALYSIS AND MODELING OBJECTIVES}

The purpose and scope of this report are to document the analyses and models for general and localized corrosion of the waste package outer barrier (WPOB). The purpose of the general corrosion model is to analyze degradation of the Alloy 22 outer barrier by general corrosion under the range of expected repository exposure conditions over the repository performance period. The purpose of the localized corrosion model is to analyze degradation of the Alloy 22 outer barrier by crevice corrosion under the range of expected repository exposure conditions over the repository performance period. The general and localized corrosion models include several submodels, which account for dry oxidation, aqueous general corrosion, crevice corrosion initiation, crevice corrosion growth, microbially influenced corrosion (MIC), and effect of aging and phase instability. This report is used in downstream waste package degradation analyses. Treatment of seismic and igneous events and their consequences on waste package outer barrier performance are not discussed in this report, although the general and localized corrosion models developed in this report are suitable for use in these scenarios.

\subsection{FEATURES, EVENTS, AND PROCESSES RELEVANT TO THIS REPORT}

The development of a comprehensive list of features, events, and processes (FEPs) potentially relevant to postclosure performance of the Yucca Mountain repository is an iterative process based on site-specific information, design, and regulations. To support TSPA-LA, the FEP list was reevaluated in accordance with The Enhanced Plan for Features, Events, and Processes (FEPs) at Yucca Mountain (BSC 2002 [DIRS 158966], Section 3.2). Table 6-1 provides a list of FEPs included in this report and provides specific references to where the FEPs are discussed within this report. Table 6-2 provides a list of FEPs excluded in this report and provides specific references to sections within this report where the FEPs are discussed. DTN: MO0407SEPFEPLA.000 [DIRS 170760] also describes these FEPs and FEPs Screening of Processes and Issues in Drip Shield and Waste Package Degradation (BSC 2004 [DIRS 169997]) for a complete list of all FEPs related to waste package and drip shield degradation.

Table 6-1. Included FEPs in This Report

\begin{tabular}{|c|l|c|}
\hline FEP Number & \multicolumn{1}{|c|}{ FEP Name } & $\begin{array}{c}\text { Section(s) Where } \\
\text { Described }\end{array}$ \\
\hline 2.1.03.01.0A & General corrosion of waste packages & $6.4 .3,6.4 .5$ \\
\hline $2.1 .03 .03 .0 \mathrm{~A}$ & Localized corrosion of waste packages & 6.4 .4 \\
\hline 2.1.03.05.0A & Microbially influenced corrosion (MIC) of waste packages & 6.4 .5 \\
\hline
\end{tabular}

Table 6-2. Excluded FEPs in This Report

\begin{tabular}{|c|c|c|}
\hline FEP Number & FEP Name & $\begin{array}{c}\text { Section(s) Where } \\
\text { Described }\end{array}$ \\
\hline 2.1.11.06.0A & Thermal sensitization of waste packages & 6.4 .6 \\
\hline
\end{tabular}




\subsection{BASE-CASE CONCEPTUAL MODEL}

This section summarizes the expected exposure conditions and their relationship to the test solutions used. The base-case conceptual model that was developed for the analyses and models of the general corrosion and localized corrosion of the waste package outer barrier (WPOB) under the expected exposure conditions for the nominal-case postclosure repository is also described. ASTM C 1174-97 (1998 [DIRS 105725]) was followed for development of the models for general and localized corrosion of the WPOB documented in this report. Semiempirical modeling approaches were adopted by incorporating mechanistic understanding of the degradation processes into the modeling process.

A schematic representation of the base-case conceptual model is shown in Figure 6-1. Additional details are described in the section in which the analyses and models are documented.

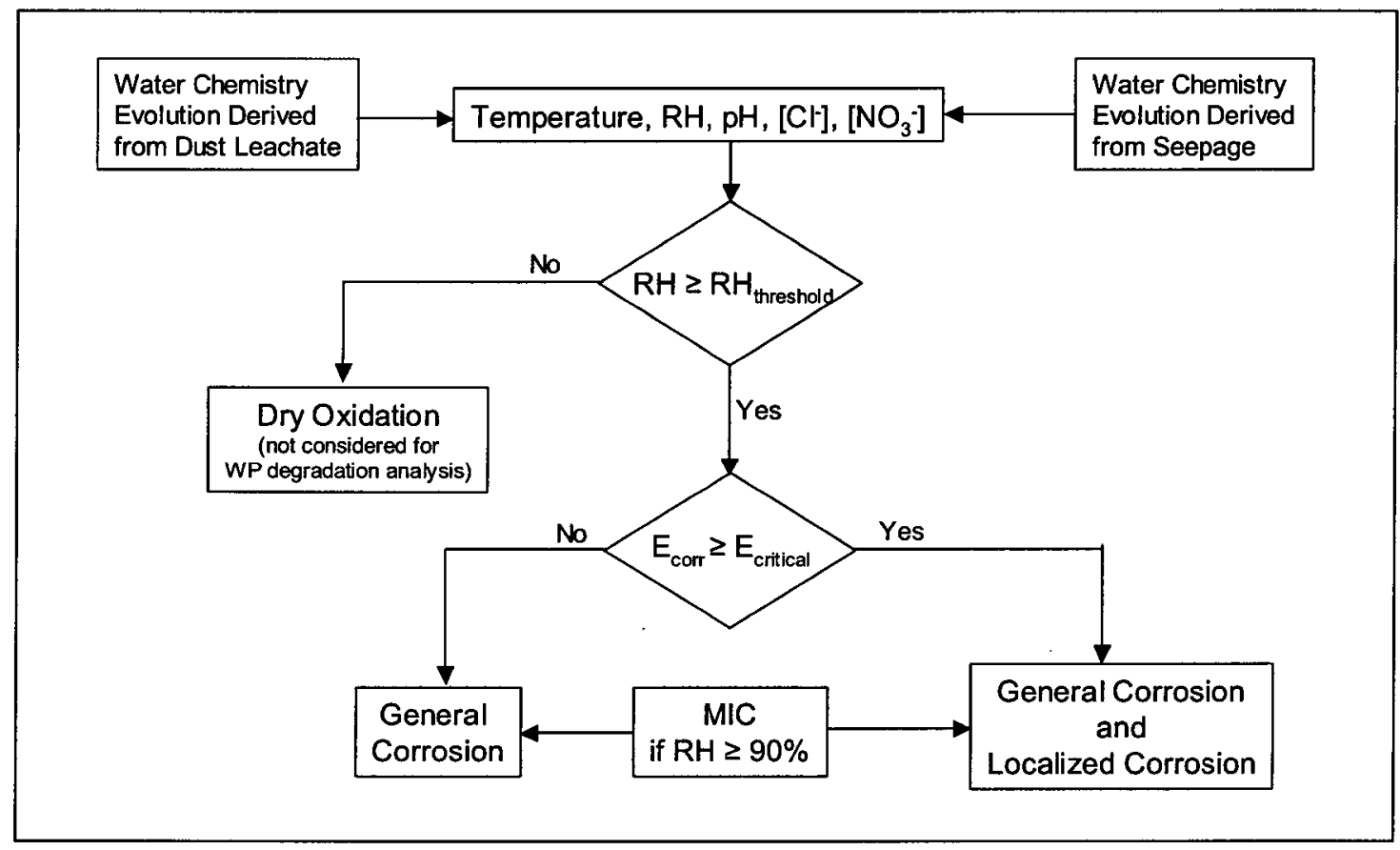

Figure 6-1. Schematic Representation of the Base-Case Conceptual Model for the General and Localized Corrosion Model of the Waste Package Outer Barrier

\subsubsection{Expected In-Drift Temperature And Relative Humidity}

Figure 6-2 shows a summary of the range of waste package temperature and relative humidity histories for all waste packages (a and b), all CSNF waste packages (c and d) and all DHLW waste packages (e and f) (BSC 2004 [DIRS 169565], Figure 6.3-52) for the TSPA-LA base case. The ranges include the lower-bound, mean, and upper-bound infiltration flux cases and use of the mean thermal-conductivity values for all unsaturated zone layer units, including the host-rock units. The influence of the low-probability-seismic collapsed-drift scenario on in-drift thermohydrologic conditions is shown in Figure 6-3 (BSC 2004 [DIRS 169565], Figure 6.3-56). 
DOC.20041004.0001

QA: QA

ANL-EBS-MD-000003 REV 02

October 2004

\section{General Corrosion and Localized Corrosion of} Waste Package Outer Barrier

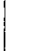

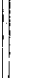

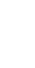

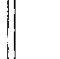

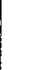




\section{DISCLAIMER}

This report was prepared as an account of work sponsored by an agency of the United States Government. Neither the United States Government nor any agency thereof, nor any of their employees, nor any of their contractors, subcontractors or their employees, makes any warranty, express or implied, or assumes any legal liability or responsibility for the accuracy, completeness, or any third party's use or the results of such use of any information, apparatus, product, or process disclosed, or represents that its use would not infringe privately owned rights. Reference herein to any specific commercial product, process, or service by trade name, trademark, manufacturer, or otherwise, does not necessarily constitute or imply its endorsement, recommendation, or favoring by the United States Government or any agency thereof or its contractors or subcontractors. The views and opinions of authors expressed herein do not necessarily state or reflect those of the United States Government or any agency thereof. 
QA: QA

General Corrosion and Localized Corrosion of Waste Package Outer Barrier

ANL-EBS-MD-000003 REV 02

October 2004 


\section{Type of Mathematical Model}

$\bigotimes$ Process Model

Abstraction Model

System Model

Describe Intenided Use of Mode

This report documents the analyses and models for general and localized corrosion of the waste package outer barrier (WPOB). The models are used to analyze degradation of the Alloy 22 outer barrier by general and localized corrosion processes under the expected repository exposure environments over the repository performance period.

\section{Title}

General Corrosion and Localized Corrosion of the Waste Package Outer Barrier

4. DI (including Rev. No., if applicable):

ANL-EBS-MD-000003 REV 02

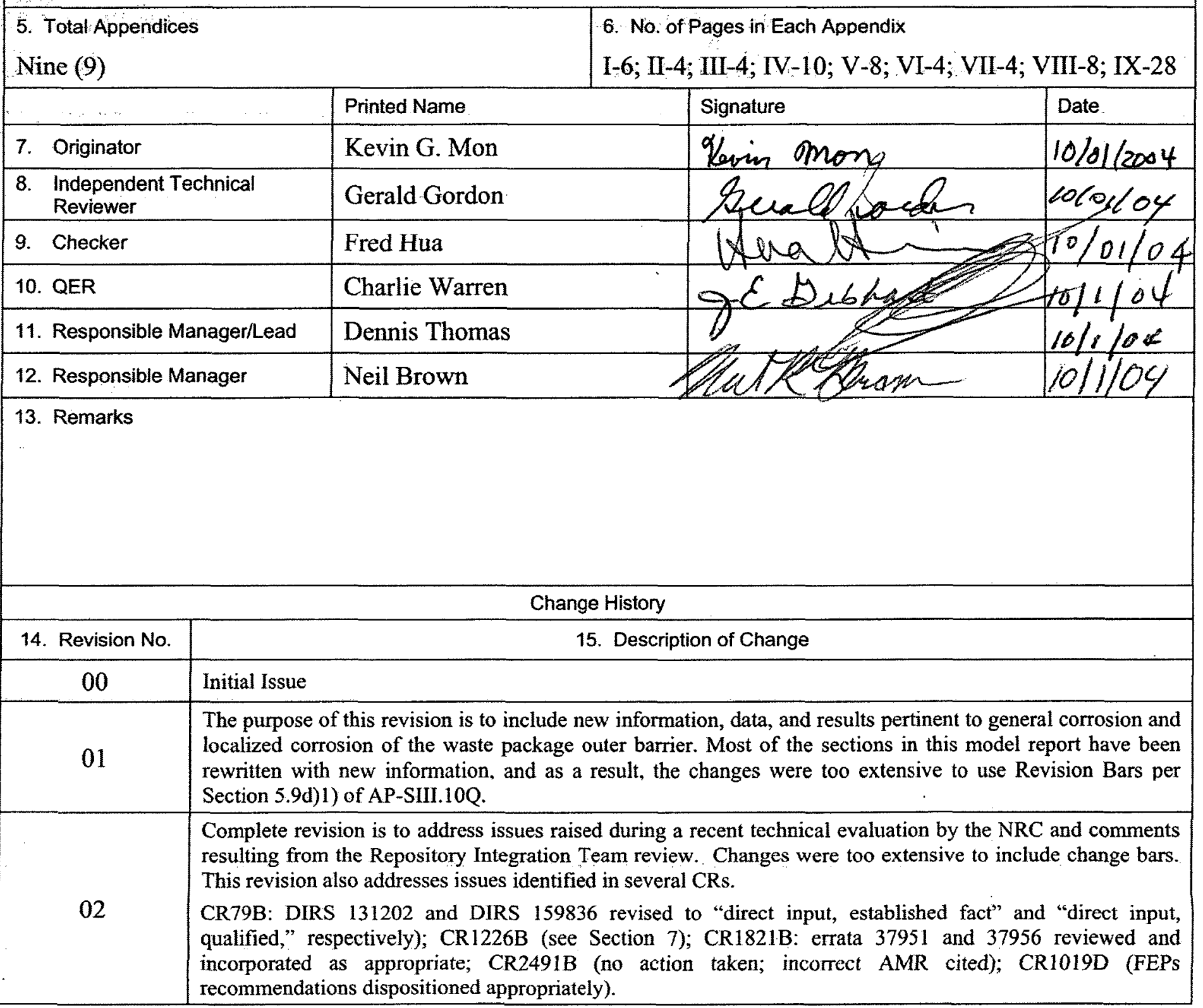


3. Title

General Corrosion and Localized Corrosion of the Waste Package Outer Barrier

4. DI (including Rev. No., if applicable):

ANL-EBS-MD-000003 REV 02

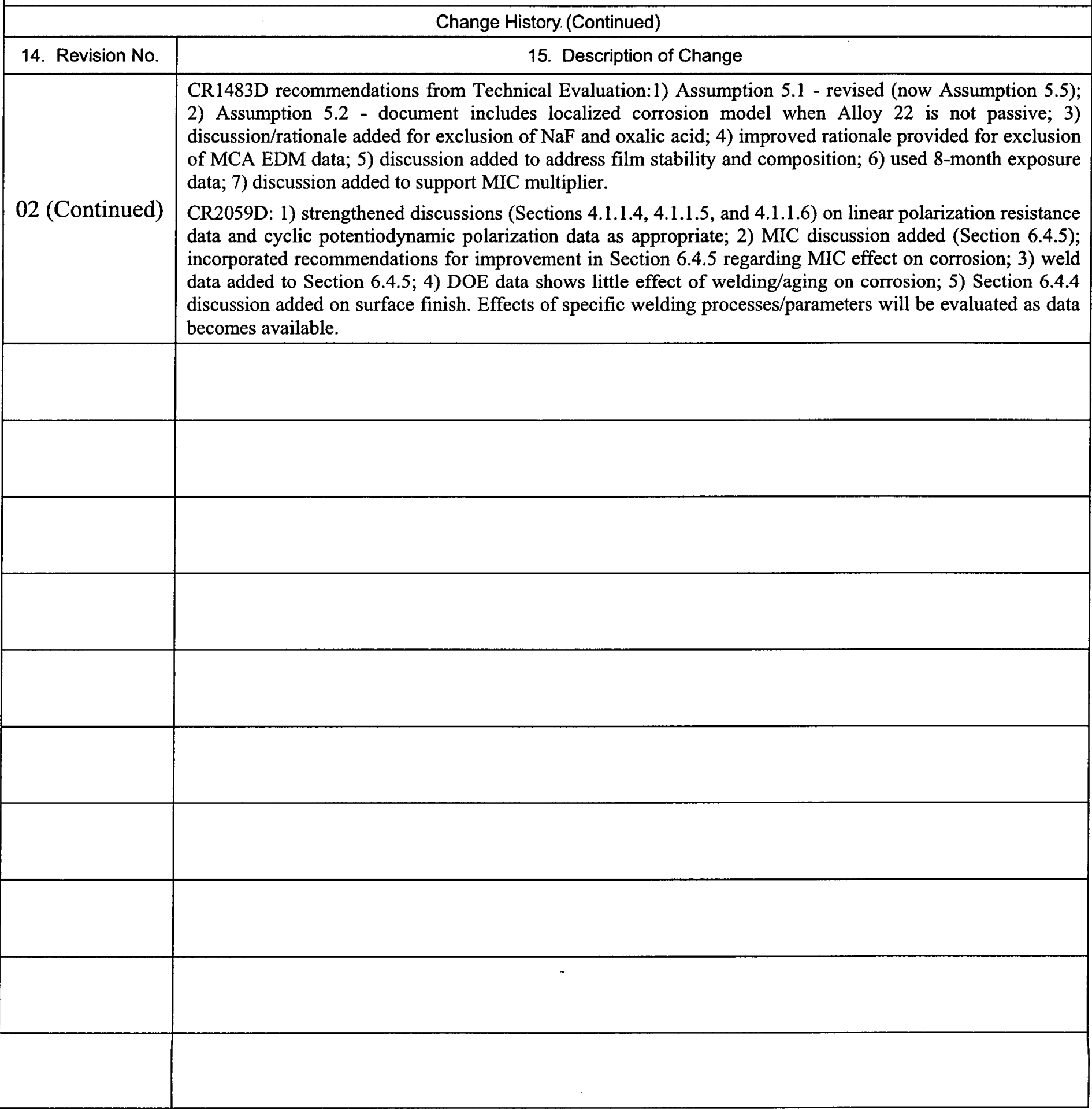




\section{CONTENTS}

Page

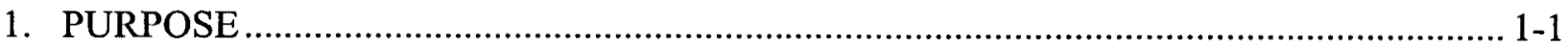

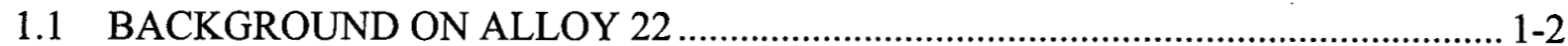

1.2 RANGES OF MODEL APPLICATION .................................................... 1-2

1.3 BARRIER CAPABILITIES ......................................................................... 1-4

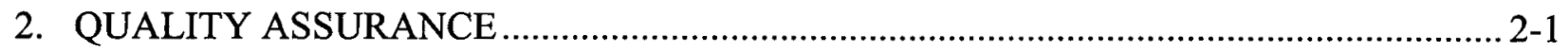

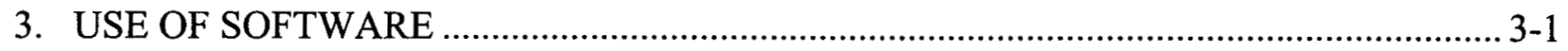

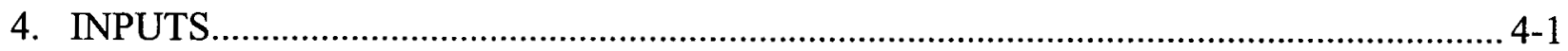

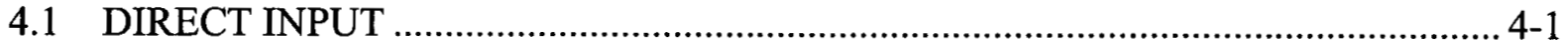

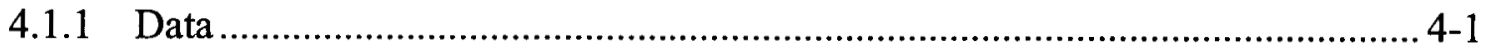

4.1.2 Other Model/Analyses Inputs ........................................................... 4-12

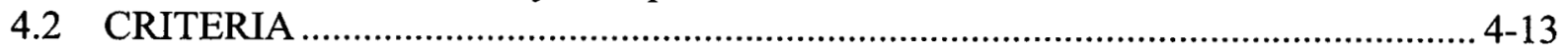

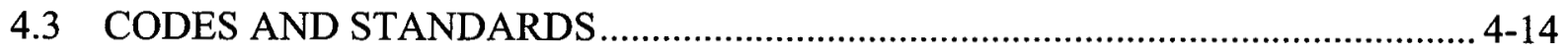

4.3.1 Corrosion Degradation Analyses and Models ............................................ 4-14

4.3.2 Cyclic Polarization Measurements .............................................................. 4-14

4.3.3 General Corrosion Measurements.......................................................... 4-14

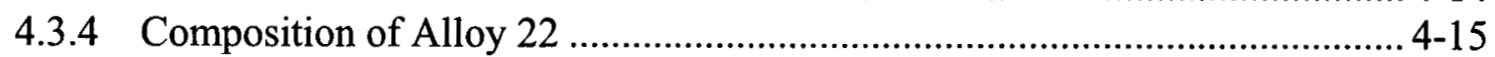

4.3.5 Standard Practice for Dealing with Outlying Observations.......................... 4-15

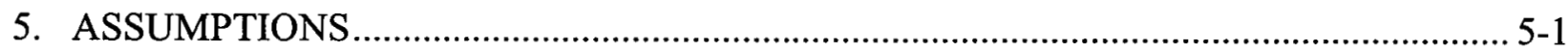

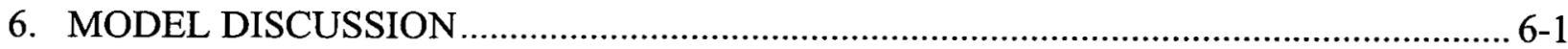

6.1 ANALYSIS AND MODELING OBJECTIVES …............................................ 6-1

6.2 FEATURES, EVENTS, AND PROCESSES RELEVANT TO THIS REPORT ......... 6-1

6.3 BASE-CASE CONCEPTUAL MODEL …........................................................... $6-2$

6.3.1 Expected In-Drift Temperature And Relative Humidity ................................ 6-2

6.3.2 Relation of In-Drift Chemical Model Results to Corrosion Testing

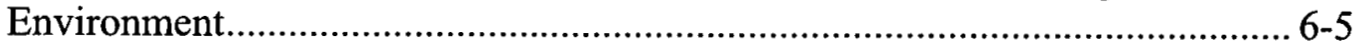

6.3.3 Waste Package Outer Barrier Degradation Conceptual Model ...................... 6-10

6.4 MODEL FORMULATION FOR BASE-CASE MODEL ….................................. $6-13$

6.4.1 Stability of the Passive Film in Repository Relevant Environments .............. 6-13

6.4 .2 Dry Oxidation .............................................................................. 6-22

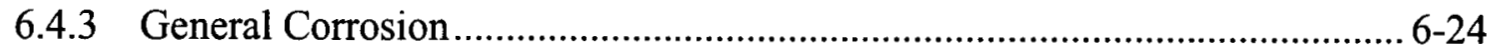

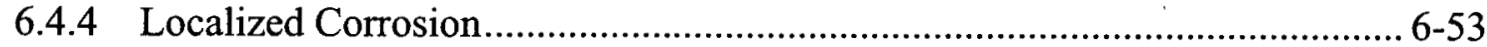

6.4.5 Effect of Microbial Activity on Corrosion.............................................. 6-102

6.4.6 Effect of Aging and Phase Instability on Corrosion .................................. 6-109

7. VALIDATION .................................................................................................. $7-1$

7.1 CONFIDENCE BUILDING DURING MODEL DEVELOPMENT TO ESTABLISH SCIENTIFIC BASIS AND ACCURACY FOR INTENDED USE....... 7-1

7.2 CONFIDENCE BUILDING AFTER MODEL DEVELOPMENT TO SUPPORT THE SCIENTIFIC BASIS OF THE MODEL. 


\section{CONTENTS (Continued)}

7.2.1 General Corrosion Model of the Waste Package Outer Barrier $7-3$

7.2.2 Corrosion Potential Model of the Waste Package Outer Barrier $7-8$

7.2.3 Critical Potential Model of the Waste Package Outer Barrier $7-11$

7.2.4 Localized Corrosion Initiation Model of the Waste Package Outer Barrier... 7-13

7.2.5 Localized Corrosion Penetration Model of the Waste Package Outer Barrier

7.3 SUMMARY OF MODEL VALIDATION

8. CONCLUSIONS.

8.1 BASE-CASE MODEL SUMMARY. $8-1$

8.2 WASTE PACKAGE OUTER BARRIER GENERAL CORROSION MODEL OUTPUTS

8.3 WASTE PACKAGE OUTER BARRIER LOCALIZED CORROSION MODEL OUTPUTS

8.3.1 Waste Package Outer Barrier Localized Corrosion Initiation Outputs............. 8-4

8.3.2 Localized Corrosion Propagation Model Outputs

8.4 YUCCA MOUNTAIN REVIEW PLAN ACCEPTANCE CRITERIA

8.4.1 System Description and Demonstration of Multiple Barriers (NRC 2003

[DIRS 163274], Section 2.2.1.1.3)

8.4.2 Degradation of Engineered Barriers (NRC 2003 [DIRS 163274], Section 2.2.1.3.1.3)

9. INPUTS AND REFERENCES

9.1 DOCUMENTS CITED......

9.2 CODES, STANDARDS, REGULATIONS, AND PROCEDURES.

9.3 SOURCE DATA, LISTED BY DATA TRACKING NUMBER

9.4 OUTPUT DATA, LISTED BY DATA TRACKING NUMBER

APPENDIX I: SUMMARY OF THE ELECTROCHEMICAL CORROSION TEST PROCEDURES TO GENERATE INPUT DATA FOR ANALYSES AND MODELS IN THIS REPORT

APPENDIX II: GENERAL CORROSION RATES OF ALLOY 22 CREVICE SAMPLES BASED ON WEIGHT-LOSS MEASUREMENTS AFTER 5-YEAR EXPOSURE IN THE LONG-TERM CORROSION TEST FACILITY.

APPENDIX III: GENERAL CORROSION RATE OF ALLOY 22 WEIGHT-LOSS SAMPLES BASED ON THE WEIGHT-LOSS MEASUREMENT AFTER 5-YEAR EXPOSURE IN THE LONG-TERM CORROSION TEST FACILITY. 


\section{CONTENTS (Continued)}

Page

APPENDIX IV: CORROSION RATE OF ALLOY 22 AS A FUNCTION OF TEMPERATURE CALCULATED FROM POLARIZATION RESISTANCE MEASUREMENTS

APPENDIX V: LONG-TERM STEADY-STATE CORROSION POTENTIAL MEASUREMENTS OF ALLOY 22 SAMPLES USED IN THE CORROSION POTENTIAL MODEL AND ANALYSIS.

APPENDIX VI: PROJECT'S CREVICE REPASSIVATION POTENTIAL DATA OF ALLOY 22 SAMPLES USED IN THE CRITICAL POTENTIAL MODEL AND ANALYSIS

APPENDIX VII: CNWRA CREVICE REPASSIVATION POTENTIAL DATA OF ALLOY 22 SAMPLES USED IN THE CRITICAL POTENTIAL MODEL AND ANALYSIS

APPENDIX VIII: PROJECT ALLOY 22 CREVICE REPASSIVATION POTENTIAL DATA USED IN THE CRITICAL POTENTIAL MODEL IN THE PRESENCE OF NITRATE IONS. VIII-1

APPENDIX IX: MATHCAD WORKSHEETS FOR REGRESSION ANALYSIS OF THE CORROSION POTENTIAL MODEL AND CREVICE REPASSIVATION POTENTIAL MODEL AND FOR LOCALIZED CORROSION SUSCEPTIBILITY ANALYSES. IX-1 
INTENTIONALLY LEFT BLANK 


\section{FIGURES}

Page

6-1. Schematic Representation of the Base-Case Conceptual Model for the General and Localized Corrosion Model of the Waste Package Outer Barrier

6-2. Waste Package Temperature and Relative Humidity Ranges.

6-3. Thermohydrologic Variables for the "Hottest" Waste Package in the LowProbability Seismic Collapsed Drift Scenario

6-4. Passive Current Densities and Oxide Thickness as a Function of Applied Potential for Alloy 22 ...

6-5. Elemental Concentration on the Outermost Oxide Layer as a Function of Applied Potential for Alloy 22.

6-6. TEM Micrograph Showing the Cross-Sectional Views and Oxide Chemistry Formed on Alloy 22 After 2-Month Immersion in a Mixed-Salt Environment at $95^{\circ} \mathrm{C}$

6-7. Elemental Concentration on the Outermost Oxide Layer Formed on Alloy 22 in a Mixed-Salt Environment at $95^{\circ} \mathrm{C}$ as a Function of Immersion Time

6-8. Oxide Thickness Formed on Alloy 22 and Ti Grade 7 as a Function of Immersion

Time at $95^{\circ} \mathrm{C}$ in a Mixed-Salt Environment

6-9. Schematic of Specimen Used in the Weight-Loss Measurements of Alloy 22 Samples in Long Term Corrosion Test Facility.

6-10. Corrosion Rates for Alloy 22 Weight-Loss Coupons in SAW, SCW, and SDW

6-11. Corrosion Rates for Alloy 22 Crevice Coupons in SAW, SCW, and SDW

6-12. Empirical Cumulative Distributions for General Corrosion Rate of Alloy 22

Weight-Loss Samples at $60^{\circ} \mathrm{C}$ and $90^{\circ} \mathrm{C}$ after 5-Year Exposure in the LTCTF

6-13. Empirical Cumulative Distributions for General Corrosion Rate of Alloy 22 Weight-Loss Samples Exposed in the Vapor Phase and Aqueous Phase after 5-Year Exposure in the LTCTF

6-14. Empirical Cumulative Distributions for General Corrosion Rate of Mill-Annealed and As-Welded Alloy 22 Weight-Loss Samples After 5-Year Exposure in the LTCTF

6-15. Empirical Cumulative Distributions for General Corrosion Rate of Alloy 22 Weight-Loss Samples Tested in Three Different Solution Types After 5-Year Exposure in the LTCTF

6-16. Empirical Cumulative Distributions for General Corrosion Rate of All Alloy 22 Weight-Loss Samples after 5-Year Exposure in the LTCTF.

6-17. Empirical Cumulative Distributions for General Corrosion Rate of Alloy 22 Crevice Samples at $60^{\circ} \mathrm{C}$ and $90^{\circ} \mathrm{C}$ after 5-Year Exposure in the LTCTF .

6-18. Empirical Cumulative Distributions for General Corrosion Rate of Alloy 22 Crevice Samples Exposed in the Vapor Phase and Aqueous Phase after 5-Year Exposure in the LTCTF

6-19. Empirical Cumulative Distributions for General Corrosion Rate of Mill-Annealed and As-Welded Alloy 22 Crevice Samples after 5-Year Exposure in the LTCTF.

6-20. Empirical Cumulative Distributions for General Corrosion Rate of Alloy 22 Crevice Samples Tested in Three Different Solution Types after 5-Year Exposure in the LTCTF 


\section{FIGURES (Continued)}

6-21. Empirical Cumulative Distributions for General Corrosion Rate of All Alloy 22 Crevice Samples after 5-Year Exposure in the LTCTF.

6-22. Empirical Cumulative Distributions for General Corrosion Rate of Alloy 22 Weight-Loss and Crevice Samples after 5-Year Exposure in the LTCTF

6-23. Temperature Dependence of Corrosion Rates of Mill-Annealed Alloy 22 Samples Obtained from Polarization Resistance Measurements for Varying Sample Configurations in a Range of Solution Chemistries

6-24. Temperature Dependency of Corrosion Rates of As-Welded and As-Welded Plus Thermally Aged Alloy 22 Samples Obtained from the Polarization Resistance Measurements for Varying Sample Configurations in a Range of Solution Chemistries

6-25. Cumulative Distribution Function (CDF) of $\mathrm{R}_{0}$ of the Base-Case TemperatureDependent General Corrosion Model of the Waste Package Outer Barrier with All Crevice Sample Data.

6-26. Calculated Model Outputs of the Base-Case Temperature-Dependent General Corrosion Model with the Mean Activation Energy of $25.91 \mathrm{~kJ} / \mathrm{mol}$ at $25^{\circ} \mathrm{C}, 50^{\circ} \mathrm{C}$, $75^{\circ} \mathrm{C}, 100^{\circ} \mathrm{C}, 125^{\circ} \mathrm{C}$, and $150^{\circ} \mathrm{C}$.

6-27. Calculated Model Outputs of the Base-Case Temperature-Dependent General Corrosion Model with the Upper-Bound Activation Energy of $33.29 \mathrm{~kJ} / \mathrm{mol}$ at $25^{\circ} \mathrm{C}, 50^{\circ} \mathrm{C}, 75^{\circ} \mathrm{C}, 100^{\circ} \mathrm{C}, 125^{\circ} \mathrm{C}$, and $150^{\circ} \mathrm{C}$

6-28. Calculated Model Outputs of the Base-Case Temperature-Dependent General Corrosion Model with the Lower-Bound Activation Energy of $18.53 \mathrm{~kJ} / \mathrm{mol}$ at $25^{\circ} \mathrm{C}, 50^{\circ} \mathrm{C}, 75^{\circ} \mathrm{C}, 100^{\circ} \mathrm{C}, 125^{\circ} \mathrm{C}$, and $150^{\circ} \mathrm{C}$

6-29. Schematic Showing the Boldly Exposed Area and the Crevice Area Under the Crevice Former of the Crevice Sample.

6-30. Empirical CDFs for General Corrosion Rates of Crevice-Area Only, Crevice Samples, and Weight-Loss Samples After 5-Year Exposure in the LTCTF

6-31. Cumulative Distribution Function (CDF) of $R_{o}$ of the Crevice-Area-Only Case Temperature-Dependent General Corrosion Model of the Waste Package Outer Barrier

6-32. Calculated Model Outputs of the Temperature-Dependent General Corrosion Model Based on the Crevice-Area-Only Case at Temperatures of $25^{\circ} \mathrm{C}, 50^{\circ} \mathrm{C}, 75^{\circ} \mathrm{C}$, $100^{\circ} \mathrm{C}, 125^{\circ} \mathrm{C}$, and $150^{\circ} \mathrm{C}$

6-33. Schematic Potentiodynamic Polarization Curve Showing Likely Differing Behaviors of the Curves During Potentiodynamic Scanning of an Alloy with High Resistance to Localized Corrosion

6-34. Determination of Crevice Repassivation Potential of Mill-Annealed MCA Sample (Sample DEA 3125) Tested in $7 \mathrm{M} \mathrm{CaCl}_{2}$ Solution at $130^{\circ} \mathrm{C}$

6-35. Determination of Crevice Repassivation Potential of Mill-Annealed MCA Sample (Sample DEA 3127) Tested in $7 \mathrm{MCaCl}_{2}+0.7 \mathrm{MCa}\left(\mathrm{NO}_{3}\right)_{2}$ Solution at $130^{\circ} \mathrm{C}$.

6-36. Determination of Repassivation Potential of Mill-Annealed Disc Sample (Sample DEA 598) Tested in $6 \mathrm{MCaCl}_{2}+0.6 \mathrm{MCa}\left(\mathrm{NO}_{3}\right)_{2}$ Solution at $120^{\circ} \mathrm{C}$ 6-61 


\section{FIGURES (Continued)}

Page

6-37. Determination of Repassivation Potential of Mill-Annealed Disc Sample (Sample DEA 599) Tested in $7 \mathrm{MCaCl}_{2}$ Solution at $130^{\circ} \mathrm{C}$

6-38. Determination of Repassivation Potential of Mill-Annealed Disc Sample (Sample DEA 600) Tested in $7 \mathrm{MCaCl}_{2}+0.7 \mathrm{MCa}\left(\mathrm{NO}_{3}\right)_{2}$ Solution at $130^{\circ} \mathrm{C}$

6-39. Model Results and Project's Experimental Data for the Crevice Repassivation Potential of the Waste Package Outer Barrier in Absence of Inhibiting Nitrate Ion as a Function of Temperature

6-40 Model Results and Project's Experimental Data for the Crevice Repassivation Potential of the Waste Package Outer Barrier in Absence of Inhibiting Nitrate Ion as a Function of Chloride Concentration.

6-41. Model Results and CNWRA's Experimental Data for the Crevice Repassivation Potential of the Waste Package Outer Barrier in Absence of Inhibiting Nitrate Ion as a Function of Temperature.

6-42. Model Results and CNWRA's Experimental Data for the Crevice Repassivation Potential of the Waste Package Outer Barrier in Absence of Inhibiting Nitrate Ion as a Function of Chloride Concentration...

6-43. Model Results and Project's Experimental Data for the Crevice Repassivation Potential Changes of the Waste Package Outer Barrier in Presence of Inhibiting Nitrate Ions at $6 \mathrm{~m}$ Chloride Ion Concentration and $\mathrm{pH}$ of 5.38 as a Function of Nitrate Ion Concentration

6-44. Model Results and Project's Experimental Data for the Crevice Repassivation

Potential Changes of the Waste Package Outer Barrier in Presence of Inhibiting Nitrate Ions as a Function of Nitrate Ion Concentration (Model Results at $27.1 \mathrm{~m}$ Chloride Ion Concentration and $\mathrm{pH}$ of 5.34)

6-45. Open-Circuit Corrosion Potential of Alloy 22 Samples as a Function of Time in Different Types of LTCTF Solutions

6-46. Open-Circuit Corrosion Potential of Alloy 22 Samples as a Function of Time in Differing Conditions of SAW Solutions

6-47. Open-Circuit Corrosion Potential of Alloy 22 Samples as a Function of Time in Differing Concentrations of $\mathrm{CaCl}_{2}$ Solutions

6-48. Long-Term Open-Circuit Corrosion Potential Versus Chloride Ion Concentration of

Alloy 22 Samples with Differing Sample Configurations and Metallurgical Conditions

6-49. Long-Term Open-Circuit Corrosion Potential Versus Nitrate Ion Concentration of Alloy 22 Samples with Differing Sample Configurations and Metallurgical Conditions.

6-50. Long-Term Open-Circuit Corrosion Potential Versus pH of Alloy 22 Samples with Differing Sample Configurations and Metallurgical Conditions.

6-51. Model Results and Experimental Data for Long-Term $E_{\text {corr }}$ of the Waste Package Outer Barrier as a Function of $\mathrm{pH}$..

6-52. Model Results and Experimental Data for Long-Term $E_{\text {corr }}$ of the Waste Package Outer Barrier as a Function of Nitrate Ion Concentration 6-82 


\section{FIGURES (Continued)}

Page

6-53. Model Results for Crevice Corrosion Susceptibility of the Waste Package Outer Barrier as a Function of Temperature for $10 \mathrm{~m}$ Chloride, $\mathrm{pH} \mathrm{7,} \mathrm{and} 0.5 \mathrm{~m}$ Nitrate $\left(\mathrm{NO}_{3} / \mathrm{Cl}\right.$ Ratio of 0.05$)$

6-54. Model Results for Crevice Corrosion Susceptibility of the Waste Package Outer Barrier as a Function of Temperature for $10 \mathrm{~m}$ Chloride, $\mathrm{pH} \mathrm{7}$, and $1.5 \mathrm{~m}$ Nitrate $\left(\mathrm{NO}_{3} / \mathrm{Cl}\right.$ Ratio of 0.15$)$.

6-55. Model Results for Crevice Corrosion Susceptibility of the Waste Package Outer Barrier as a Function of Temperature for $10 \mathrm{~m}$ Chloride, $\mathrm{pH} \mathrm{7,} \mathrm{and} 2.5 \mathrm{~m}$ Nitrate $\left(\mathrm{NO}_{3} / \mathrm{Cl}\right.$ Ratio of 0.25$)$.

6-56 Model Results for Crevice Corrosion Susceptibility of the Waste Package Outer Barrier as a Function of Temperature for $10 \mathrm{~m}$ Chloride, $\mathrm{pH} \mathrm{3}$, and $0.5 \mathrm{~m}$ Nitrate $\left(\mathrm{NO}_{3} / \mathrm{Cl}\right.$ Ratio of 0.05$)$.

6-57. Model Results for Crevice Corrosion Susceptibility of the Waste Package Outer Barrier as a Function of Temperature for $10 \mathrm{~m}$ Chloride, $\mathrm{pH} \mathrm{3}$, and $2.5 \mathrm{~m}$ Nitrate $\left(\mathrm{NO}_{3} / \mathrm{Cl}\right.$ Ratio of 0.25$)$.

6-58. Model Results for Crevice Corrosion Susceptibility of the Waste Package Outer

Barrier as a Function of Temperature for $10 \mathrm{~m}$ Chloride, $\mathrm{pH} \mathrm{3}$, and $5 \mathrm{~m}$ Nitrate $\left(\mathrm{NO}_{3} / \mathrm{Cl}\right.$ Ratio of 0.50$)$.

6-59. Model Results for Crevice Corrosion Susceptibility of the Waste Package Outer Barrier as a Function of Chloride Concentration for $95^{\circ} \mathrm{C}$, $\mathrm{pH} 7$, and $0.01 m$ Nitrate .. 6-88

6-60. Model Results for Crevice Corrosion Susceptibility of the Waste Package Outer Barrier as a Function of Chloride Concentration for $95^{\circ} \mathrm{C}$, pH 7, and $2.5 m$ Nitrate .... 6-89

6-61. Model Results for Crevice Corrosion Susceptibility of the Waste Package Outer Barrier as a Function of $\mathrm{pH}$ for $95^{\circ} \mathrm{C}, 10 \mathrm{~m}$ Chloride, and $0.01 \mathrm{~m}$ Nitrate $\left(\mathrm{NO}_{3} / \mathrm{Cl}\right.$ Ratio of 0.001 )

6-62. Model Results for Crevice Corrosion Susceptibility of the Waste Package Outer Barrier as a Function of $\mathrm{pH}$ for $95^{\circ} \mathrm{C}, 10 \mathrm{~m}$ Chloride, and $2.5 \mathrm{~m}$ Nitrate $\left(\mathrm{NO}_{3} / \mathrm{Cl}\right.$ Ratio of 0.25 )

6-63. Model Results for Crevice Corrosion Susceptibility of the Waste Package Outer Barrier as a Function of Nitrate Concentration for $95^{\circ} \mathrm{C}$, pH 7, and $10 m$ Chloride ..... 6-91

6-64. Model Results for Crevice Corrosion Susceptibility of the Waste Package Outer Barrier as a Function of Nitrate Concentration for $95^{\circ} \mathrm{C}$, $\mathrm{pH} 3$, and $10 m$ Chloride ..... 6-92

6-65. Maximum RH for "Wet" Conditions as a Function of Temperature, for Repository Ambient Pressure (left) and Standard Atmospheric Pressure (right)...

6-66. Comparison of Weight Changes of Alloy 22 Specimens Coated with a Thin Film of Calcium Chloride Exposed at $22.5 \% \mathrm{RH}$ at the Various Temperatures Shown

6-67. Comparison of Scanning Electron Microscopy-Imaged Alloy 22 Coupons (8,000× Magnification)............................................................................................... 6-107

6-68. Corrosion Rates Determined by Polarization Resistance Testing of Welded Alloy 22 Coupons 6-108

6-69. Comparison of Corrosion Rates from Polarization Resistance Measurements of Mill-Annealed (MA), As-Welded (ASW), and As-Welded Plus Aged Alloy 22 MCA Samples in $5 \mathrm{M} \mathrm{CaCl}_{2}$ Brines at Varying Temperatures 


\section{FIGURES (Continued)}

6-70. Comparison of Corrosion Rates from Polarization Resistance Measurements of Mill-Annealed (MA), As-Welded (ASW), and As-Welded Plus Aged Alloy 22 MCA Samples in $5 \mathrm{M} \mathrm{CaCl}_{2}+0.5 \mathrm{M} \mathrm{Ca}\left(\mathrm{NO}_{3}\right)_{2}$ Brines at Varying Temperatures..... 6-111

7-1. Decrease of the Mean General Corrosion Rate of Alloy 22 with Time. 7-8

8-1. Schematic Representation of General Corrosion and Localized Corrosion Model of the Waste Package Outer Barrier. 
INTENTIONALLY LEFT BLANK 


\section{TABLES}

Page

4-1. Summary of All Input Data Used in the Analyses and Models ................................... 4-2

4-2. Alloy 22 Passive Film Characterization Data .......................................................... 4-4

4-3. Compositions of Solutions Employed in Various Corrosion Tests of Alloy 22 ............. 4-4

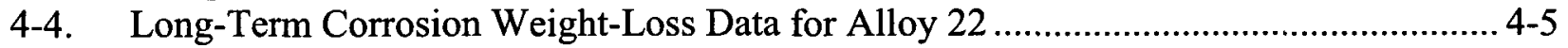

4-5. Polarization Resistance Measurement Data for Alloy 22 ......................................... 4-6

4-6. Long-Term Open-Circuit Corrosion Potential Measurement Data for Alloy 22............ 4-8

4-7. Cyclic Potentiodynamic Polarization Measurement Data for Alloy 22 ........................ 4-9

4-8. Crevice Repassivation Potential Data for Alloy 22 ........................................... 4-10

4-9. $\mathrm{pH}$ of $5 \mathrm{M} \mathrm{CaCl}_{2}$ Solutions .............................................................................. 4-11

4-10. Input Data for Microbially Influenced Corrosion of Alloy 22 ................................ 4-11

4-11. Density of Alloy 22 and Faraday Constant........................................................... 4-12

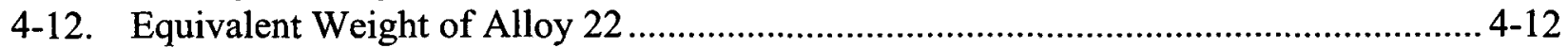

4-13. Localized Corrosion Penetration Rates of Alloy 22 .............................................. 4-12

4-14. Crevice Repassivation Potentials for Alloy 22 from Other Source ............................ 4-13

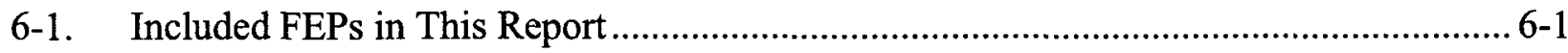

6-2. Excluded FEPs in This Report ................................................................................ $6-1$

6-3. Target Chemical Compositions of the Electrolyte Solutions $(\mathrm{mg} / \mathrm{L})$ Employed in the Long-Term Weight-Loss Measurements .................................................................6-6

6-4. List of Examined Weight-Loss and Crevice Alloy 22 Coupons................................. 6-26

6-5. Summary of Measurement Uncertainty Analysis for Corrosion Rates Based Upon Weight-Loss Measurements after 5-Year Exposure in LTCTF................................. 6-39

6-6. Summary of Measurement Uncertainty Analysis for Corrosion Rates Based on Weight-Loss Measurements after 5-Year Exposure in LTCTF.................................. 6-40

6-7. Crevice Repassivation Potentials Obtained from the Additional CPP Data................ 6-63

6-8. High Temperature and Concentrated Solution Data and Model Results ...................... 6-96

6-9. Distribution of Localized Corrosion Rates for Alloy 22 .......................................... 6-99

6-10. Alterations in Corrosion Rates and Potentials of Mill-Annealed Alloy 22 Associated with Microbial Degradation at Room Temperature ................................................ 6-106

7-1. Summary of Mean General Corrosion Rates of Alloy 22 at $90^{\circ} \mathrm{C}$ Versus Exposure Time.

7-2. Summary of Model Validation Analysis for the Corrosion Potential Model .............. 7-10

7-3. Summary of Model Validation Analysis for Crevice Repassivation Potential Model .. 7-12

7-4. Comparison of Model Prediction for Localized Corrosion Susceptibility with Experimental Observations of Alloy 22 Crevice Samples Tested for Over 5 Years in

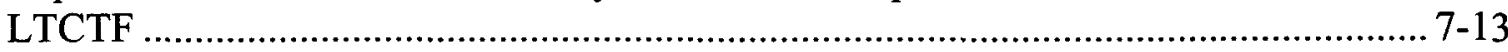

7-5. Compositions and pH Values of Salt Brines Used in Smailos 1993 [DIRS 168164]... 7-14

8-1. Summary of General Corrosion Model Output for Waste Package Outer Barrier ......... 8-4

8-2. Summary of Localized Corrosion Model Output for Waste Package Outer Barrier....... 8-8 
INTENTIONALLY LEFT BLANK 


\section{ACRONYMS}

$\mathrm{AC}$

ACM

AISI

AR

ASTM

ASW

BSC

BSW

CCT

CDF

CFR

CNWRA

CP

CPP

CPT

CRWMS

CSNF

DHLW

DIRS

DOE

DTN

EBS

ECDF

FEPs

HLW

KTI

LA

LLNL

LTCTF

$m$

$M$

MCA

MIC

NRC acceptance criteria

alternative conceptual model

American Iron and Steel Institute (numbering system)

as-received

American Society for Testing and Materials

as-welded

Bechtel SAIC Company

basic saturated water

critical crevice-corrosion temperature

cumulative distribution function

Code of Federal Regulations

Center for Nuclear Waste Regulatory Analyses

corrosion potential

cyclic potentiodynamic polarization

critical pitting temperature

Civilian Radioactive Waste Management System

commercial spent nuclear fuel

defense high-level waste

Document Input Reference System

U.S. Department of Energy

data tracking number

engineered barrier system

empirical cumulative distribution function

features events and processes

high-level waste

key technical issue

license application

Lawrence Livermore National Laboratory

Long Term Corrosion Test Facility

molal (moles $/ \mathrm{kg}$ water)

molar (moles/liter of solution)

multiple crevice assembly

microbially influenced corrosion

Nuclear Regulatory Commission 
OCP open circuit potential

OCRWM Office of Civilian Radioactive Waste Management

PDF probability density function

PDM Point Defect Model

PRD Project Requirements Document

PSP potentiostatic polarization

RH relative humidity

SAW Simulated Acidified Water

SCW Simulated Concentrated Water

SDW Simulated Dilute Water

SC

SCC

Safety category

SCE

sd stress corrosion cracking

SEM saturated calomel reference electrode scale standard deviation

SHE

SNF scanning electron microscope

SSC standard hydrogen reference electrode scale spent nuclear fuel silver-silver chloride reference electrode scale

TDMS Technical Data Management System TEM transmission electron microscopy TSPA total system performance assessment

TWP Technical Work Plan

UNS Unified Numbering System

WPOB waste package outer barrier

XPS $\quad \mathrm{x}$-ray photoelectron spectroscopy

YMP Yucca Mountain Project

YMRP Yucca Mountain Review Plan 


\section{PURPOSE}

The waste package design for the License Application is a double-wall waste package underneath a protective drip shield (BSC 2004 [DIRS 168489]; BSC 2004 [DIRS 169480]). The purpose and scope of this model report is to document models for general and localized corrosion of the waste package outer barrier (WPOB) to be used in evaluating waste package performance. The WPOB is constructed of Alloy 22 (UNS N06022), a highly corrosion-resistant nickel-based alloy. The inner vessel of the waste package is constructed of Stainless Steel Type 316 (UNS S31600). Before it fails, the Alloy 22 WPOB protects the Stainless Steel Type 316 inner vessel from exposure to the external environment and any significant degradation. The Stainless Steel Type 316 inner vessel provides structural stability to the thinner Alloy 22 WPOB. Although the waste package inner vessel would also provide some performance for waste containment and potentially decrease the rate of radionuclide transport after WPOB breach before it fails, the potential performance of the inner vessel is far less than that of the more corrosion-resistant Alloy 22 WPOB. For this reason, the corrosion performance of the waste package inner vessel is conservatively ignored in this report and the total system performance assessment for the license application (TSPA-LA). Treatment of seismic and igneous events and their consequences on waste package outer barrier performance are not specifically discussed in this report, although the general and localized corrosion models developed in this report are suitable for use in these scenarios. The localized corrosion processes considered in this report are pitting corrosion and crevice corrosion. Stress corrosion cracking is discussed in Stress Corrosion Cracking of the Drip Shield, the Waste Package Outer Barrier, and the Stainless Steel Structural Material (BSC 2004 [DIRS 169985]).

The waste package outer barrier general corrosion model (developed in this report) is to be used by downstream waste package degradation analyses to evaluate the extent of WPOB degradation by general corrosion under repository environmental conditions over the regulatory performance period $(10,000$ years after permanent closure). The waste package outer barrier general corrosion model considers several submodels, which account for dry oxidation, aqueous general corrosion, the effects of aging and phase instability, and microbially influenced corrosion (MIC). The purpose of the waste package outer barrier localized corrosion model is to be used by downstream waste package degradation analyses to evaluate the extent of WPOB degradation by localized corrosion under the expected repository environmental conditions over the regulatory performance period. The submodels included in the waste package outer barrier localized corrosion model are the crevice repassivation potential, long-term corrosion potential, and crevice corrosion propagation models. The analyses and models developed in this report are used by the waste package degradation analyses for TSPA-LA and are prepared in accordance with Technical Work Plan for: Regulatory Integration Modeling and Analysis of the Waste Form and Waste Package (BSC 2004 [DIRS 171583]).

The following scientific analyses or models reports provide direct or indirect inputs to this report: Abstraction of Drift Seepage, Aging and Phase Stability of Waste Package Outer Barrier, Engineered Barrier System: Physical and Chemical Environment Model, Environment on the Surfaces of the Drip Shield and Waste Package Outer Barrier, Evaluation of the Impact of Microbial Activities on Drift Chemistry, and Multiscale Thermohydrologic Model. This report provides direct or indirect inputs to the following scientific analyses or model reports: WAPDEG 
Analysis of Waste Package and Drip Shield Degradation, FEPs Screening of Processes and Issues in Drip Shield and Waste Package Degradation, and Total System Performance Assessment Model/Analysis For License Application.

Lists of data tracking numbers (DTNs) and their Q-status are included in the Document Input Reference System (DIRS) database report.

\subsection{BACKGROUND ON ALLOY 22}

Alloy 22 (UNS N06022) is the reference material for construction of the outer barrier of the waste package. This alloy consists, by weight, of 20.0 to $22.5 \%$ chromium, 12.5 to $14.5 \%$ molybdenum, $3.5 \%$ tungsten, 2.0 to $6.0 \%$ iron, 2.5 to $2.5 \%$ (maximum) cobalt, and balance nickel (ASTM B 575-94 1994 [DIRS 100497]). Other impurity elements include phosphorus, silicon, sulphur, manganese, and vanadium (CRWMS M\&O 1999 [DIRS 102933]; Treseder et al. 1991 [DIRS 110250]). The unusual localized corrosion resistance of Alloy 22 is mainly due to additions of molybdenum and chromium. Addition of these alloying elements to nickel alloys has been proven to significantly improve the resistance to localized corrosion of nickel alloys (Hack 1983 [DIRS 105750]; ASM International 1987 [DIRS 133378], pp. 641 to 657). The oxides of these elements are very stable at low $\mathrm{pH}$ values making Alloy 22 highly resistant to localized corrosion. High repassivation potentials, an indication of high resistance to localized corrosion, for Alloy 22 have been observed by some investigators (Gruss et al. 1998 [DIRS 100893]; Rebak et al. 2002 [DIRS 162237]), and others have found very low corrosion rates in crevice corrosion susceptibility testing solutions containing 10 weight percent $\mathrm{FeCl}_{3}$ (Gdowski 1991 [DIRS 100859], Section 3.0; Haynes International 1997 [DIRS 100896]; Haynes International 1997 [DIRS 100897]). Furthermore, no localized corrosion attack of Alloy 22 has been seen in crevices exposed to concentrated solutions (similar to those relevant to the repository) conducted in the Yucca Mountain Project (YMP) Long Term Corrosion Test Facility (LTCTF) (Estill 1998 [DIRS 117697]). Test media used in this facility include simulated acidified water (SAW), simulated dilute water (SDW), and simulated concentrated water (SCW). Table 6-3 provides compositions of these solutions.

\subsection{RANGES OF MODEL APPLICATION}

Treatment of seismic and igneous events and their consequences on waste package outer barrier performance are not specifically discussed in this report, although the general and localized corrosion models developed in this report are suitable for use in these scenarios. Dry oxidation of the WPOB has an insignificant impact on the waste package performance (Section 6.4.2). Therefore, this degradation mode is not included in the waste package degradation analysis. The effects of thermal aging and phase instability are not included in the waste package degradation analysis because thermal aging and phase instability processes are not relevant under the thermal conditions in the repository (BSC 2004 [DIRS 169983], Section 8).

The waste package outer barrier general corrosion model is applied when a stable aqueous water film exists on the waste package surface (Section 6.4.3.4). The general corrosion model (Section 6.4.3.4) was developed in this report using data in both mixed ionic environments and data from simple salt solutions including highly concentrated chloride brines and chloride brines containing nitrate ions. In Section 7.2, the general corrosion model is validated against data 
obtained at temperatures as high as $150^{\circ} \mathrm{C}$. Therefore, the general corrosion model should be applicable over all repository exposure environments. Microbially influenced corrosion (MIC) effects are applied when the relative humidity at the waste package surface is greater than 90 percent (Section 6.4.5).

The waste package surface may experience a wide range of exposure conditions during its service life. Crevices may be formed on the waste package surface at occluded regions such as in between the waste package and its supports and potentially beneath mineral scales, corrosion products, dust, rocks, and biofilms. The area between the layers of the waste packages also could be considered a creviced region after the outer layer is breached. The chemical environment in a creviced region may be more severe than the near field environment due to hydrolysis of dissolved metals in the creviced region. Metal ion hydrolysis can lead to the accumulation of hydrogen ions and a corresponding decrease in $\mathrm{pH}$. Electromigration of chloride ions (and other anions) into the crevice must occur to balance the charge within the creviced region (Jones 1992 [DIRS 169906], Chapter 7). In this report, the dominant form of localized corrosion is conservatively assumed to be crevice corrosion (Assumption 5.3) as opposed to pitting corrosion, which occurs on boldly exposed surfaces.

The empirical correlations used in the waste package outer barrier localized corrosion initiation model for the long-term corrosion potential $\left(E_{\text {corr }}\right)$ and crevice repassivation potential $\left(E_{\text {rcrev }}\right)$ are expressed as functions of temperature, $\mathrm{pH}$, chloride ion concentration, and nitrate ion concentration (Sections 6.4.4.3 and 6.4.4.5). Based on the range of environmental conditions in which the input data were obtained (Appendices V, VI, VII, and VIII) and the model validation activities (Sections 7.2.2 and 7.2.3), implementation of the waste package outer barrier localized corrosion initiation model can be summarized as follows.

Localized corrosion (like general corrosion) requires the presence of a liquid water film on the waste package surface. To implement the waste package outer barrier localized corrosion initiation model, the following criteria are applied in a stepwise fashion:

1. If aqueous brine chemistry causes the initiation of localized corrosion, then localized corrosion continues to propagate regardless of changes in the bulk chemical exposure environment. This is a conservative modeling assumption because no detailed model of the chemistry evolution of the crevice solution is available at this time.

2. If the exposure temperature exceeds $160^{\circ} \mathrm{C}$ and a water film is present on the waste package surface, then localized corrosion initiates.

Localized corrosion initiated as a result of this criterion is reevaluated in accordance with Criterion 3 (below) when the exposure temperature drops below $160^{\circ} \mathrm{C}$.

3. If the exposure temperature exceeds $120^{\circ} \mathrm{C}$, but is less than or equal to $160^{\circ} \mathrm{C}$ then,

a) If the nitrate-to-chloride ion ratio is 0.5 (or greater), no localized corrosion will occur, or

b) If the nitrate-to-chloride ion ratio is less than 0.5 , then localized corrosion initiates and continues to propagate regardless of changes in the bulk chemical exposure environment (Criterion 1). 
4. If the exposure temperature is greater than or equal to $20^{\circ} \mathrm{C}$, and less than or equal to $120^{\circ} \mathrm{C}$, then the empirical correlations for the long-term corrosion potential $\left(E_{\text {corr }}\right)$ and crevice repassivation potential $\left(E_{r c r e v}\right)$ (Sections 6.4.4.3 and 6.4.4.5) are evaluated in accordance with the following implementation rules. If localized corrosion is determined to initiate, then localized corrosion continues to occur regardless of changes in the bulk chemical exposure environment (Criterion 1).

a) If the nitrate-to-chloride ion ratio in the environment exceeds 0.5 , then evaluate $E_{\text {rcrev }}$ and $E_{\text {corr }}$ at a nitrate-to-chloride ion ratio of 0.5 .

b) If the molality of chloride ion in the environment exceeds 36 molal, then evaluate $E_{\text {rcrev }}$ and $E_{\text {corr }}$ at a molality of chloride ion of 36 molal.

If the molality of chloride ion is less than 0.001 molal, then evaluate $E_{\text {rcrev }}$ and $E_{c o r r}$ at a molality of chloride ion of 0.001 molal.

c) If the molality of nitrate ion in the environment exceeds 18 molal, then evaluate $E_{\text {rcrev }}$ and $E_{\text {corr }}$ at a molality of nitrate ion of 18 molal.

If the molality of nitrate ion is less than 0.001 molal, then $E_{\text {rcrev }}=E_{\text {rcrev }}^{o}$ (i.e., the crevice repassivation potential in the absence of nitrate ions) and evaluate $E_{\text {corr }}$ at a molality of nitrate ion of 0.001 molal.

d) If the $\mathrm{pH}$ in the environment exceeds 10.9 , then evaluate $E_{\text {rcrev }}$ and $E_{\text {corr }}$ at a $\mathrm{pH}$ of 10.9 .

If the $\mathrm{pH}$ in the environment is less than 2.8 , then initiate localized corrosion.

Nitrate ions inhibit localized corrosion initiation (Section 6.4.4.3). Carbonate and sulfate ions have an inhibitive effect on localized corrosion. Because the model only accounts for nitrate ions, model results for solutions with significant amounts of other potentially inhibitive ions in addition to nitrate ions are conservative.

\subsection{BARRIER CAPABILITIES}

10 CFR 63 [DIRS 156605] defines a barrier as "any material, structure, or feature that, for a period to be determined by NRC, prevents or substantially reduces the rate of movement of water or radionuclides from the Yucca Mountain repository to the accessible environment, or prevents the release or substantially reduces the release rate of radionuclides from the waste." 10 CFR 63.102 (h) [DIRS 156605] and 10 CFR 63.113 (a) [DIRS 156605] require the repository system to include multiple barriers, natural and engineered. The capability of a barrier is defined by its ability to achieve one or more of the functions described above (i.e., the extent to which it can prevent or delay the movement of water or radionuclides, or prevent or reduce the radionuclide release rate from the waste package).

In this document; the barrier considered is the waste package with particular emphasis on the performance of the waste package outer barrier. The waste package barrier contributes to waste isolation by keeping water away from the waste for its lifetime and, when breached, by reducing the contact of water with the waste and radionuclide release rate from the waste. 


\section{QUALITY ASSURANCE}

The Yucca Mountain Quality Assurance (QA) program applies to the development of this technical product. Technical Work Plan for: Regulatory Integration Modeling and Analysis of the Waste Form and Waste Package (BSC 2004 [DIRS 171583]) determined this activity is subject to Quality Assurance Requirements and Description (QARD) (DOE 2004 [DIRS 171539]) requirements. All waste package configurations have been determined to be important to waste isolation in accordance with AP-2.22Q and, therefore, are classified as Safety Category (SC) in Q-List (BSC 2004 [DIRS 168361], Appendix A; BSC 2004 [DIRS 170992]).

The inputs to this report were documented according to AP-3.15Q, Managing Technical Product Inputs. The methods used to control the electronic management of data as required by AP SV.1Q, Control of the Electronic Management of Information, were accomplished in accordance with the technical work plan (BSC 2004 [DIRS 171583]). The process for control of the electronic management of information on evaluation of work activities, processes, or process functions outlined in Section 5.0 of AP-SV.1Q was followed to ensure accuracy, completeness, and security of information and data used in preparation of this report. Examples of process controls mentioned in AP-SV.1Q are: (a) access to the information contained on personal computer is password protected; (b) secured backup copies are appropriately labeled and stored before changes are made and kept until the changes are confirmed and correct; (c) physical electronic media (tape, diskette, CD-ROM, etc.) are appropriately labeled; and (d) for nonphysical electronic media, transport mechanisms can be e-mail, TCP/IP, NetBios, etc. and methods of receipt verification may include visual inspection, transmission verification settings, check sums, application information integrity check, etc.

This document is prepared in accordance with AP-SIII.10Q, Models, and reviewed in accordance with AP-2.14Q, Document Review. 